\title{
3d virtual histology of neuronal tissue by propagation-based x-ray phase-contrast tomography
}

\author{
Dissertation \\ zur Erlangung des mathematisch-naturwissenschaftlichen \\ Doktorgrades \\ „Doctor rerum naturalium” \\ der Georg-August-Universität Göttingen \\ im Promotionsprogramm \\ Physics of Biological and Complex Systems \\ der Georg-August University School of Science (GAUSS)
}

vorgelegt von

Mareike Töpperwien

aus Buxtehude

Göttingen, 2018 


\section{Mitglieder des Betreuungsausschusses:}

Prof. Dr. Tim Salditt

Institut für Röntgenphysik, Georg-August-Universität Göttingen

Dr. Florian Rehfeldt

III. Physikalisches Institut, Georg-August-Universität Göttingen

Prof. Dr. Christine Stadelmann-Nessler

Institut für Neuropathologie, Universitätsmedizin Göttingen

\section{Mitglieder der Prüfungskommission:}

Referent: $\quad$ Prof. Dr. Tim Salditt

Institut für Röntgenphysik, Georg-August-Universität Göttingen

Korreferent: $\quad$ Dr. Florian Rehfeldt

III. Physikalisches Institut, Georg-August-Universität Göttingen

\section{Weitere Mitglieder der Prüfungskommission:}

Prof. Dr. Christine Stadelmann-Nessler Prof. Dr. Sarah Köster

Institut für Neuropathologie

Universitätsmedizin Göttingen

Prof. Dr. Tobias Moser

Institut für Auditorische Neurowissen-

schaften \& InnenOhrLabor

Universitätsmedizin Göttingen
Institut für Röntgenphysik

Georg-August-Universität Göttingen

Prof. Dr. Matthias Krüger

Institut für Theoretische Physik

Georg-August-Universität Göttingen

\section{Tag der mündlichen Prüfung:}

25. Mai 2018 




\section{Contents}

$\begin{array}{ll}\text { Introduction } & 1\end{array}$

1 Concepts of propagation-based phase-contrast imaging 5

1.1 From Maxwell's equations to free-space propagation . . . . . . 6

1.1.1 Vacuum wave equations ................... 6

1.1.2 Angular spectrum approach . . . . . . . . . 8

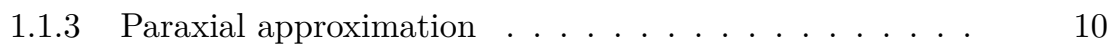

1.1.4 Fresnel diffraction ............... 11

1.1.5 Numerical implementation of propagation . . . . . . . 13

1.2 X-ray interactions with matter . . . . . . . . . . . 15

1.2.1 Wave equations in the presence of matter . . . . . . 15

1.2.2 The complex index of refraction . . . . . . . . . 16

1.2.3 The projection approximation ........... 18

1.3 Contrast formation ................... 21

1.3.1 Transport of intensity equation ........... 21

1.3.2 Contrast transfer function . . . . . . . . . . . 23

1.3.3 Imaging regimes . . . . . . . . . . . . . . . . . . . 25

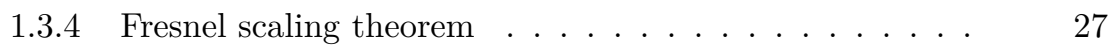

1.4 Coherence ..................... . . . . 30

1.5 Resolution estimate.................. 33

2 Solving the phase problem $\quad 37$

2.1 Direct-contrast regime . . . . . . . . . . . . . 37

2.1.1 Single material objects . . . . . . . . . . . 37

2.1.2 Modified Bronnikov algorithm ............ 39

2.1.3 Bronnikov-aided correction ............ 44

2.2 Holographic regime . . . . . . . . . . . . . . . . 46

2.2.1 Holographic phase retrieval . . . . . . . . . . . . . 46

2.2.2 CTF-based phase retrieval ............ 48 
3 Computed tomography $\mathbf{5 5}$

3.1 Mathematical concepts . . . . . . . . . . . 55

3.1.1 Fourier slice theorem . . . . . . . . . . . . 58

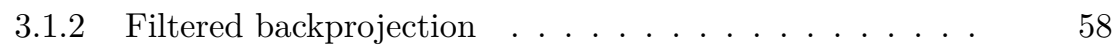

3.1.3 Cone-beam tomography . . . . . . . . . . . . 61

3.2 Artifacts in tomography . . . . . . . . . . . . . 62

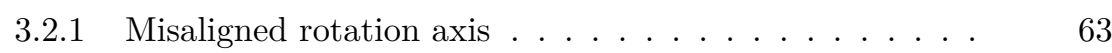

3.2 .2 Ring artifacts . . . . . . . . . . . . 64

3.2.3 Region-of-interest tomography ........... 66

3.2.4 Motion artifacts ................. 68

3.2.5 Geometry artifacts . . . . . . . . . . . 69

3.2.6 Beam hardening ................ 70

4 Experimental realization $\quad \mathbf{7 3}$

4.1 X-ray detectors . . . . . . . . . . . . . . . . . 73

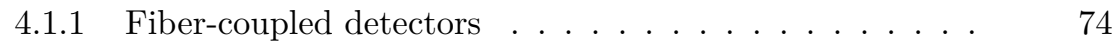

4.1.2 Lens-coupled detectors . . . . . . . . . . . . . . 75

4.2 GINIX setup at PETRAIII . . . . . . . . . . . . . . . . . 75

4.3 Empty-beam correction . . . . . . . . . . . . . 79

4.4 Laboratory setup JuLiA . . . . . . . . . . . . . . . . . 81

4.4.1 Experimental design . . . . . . . . . . . . . . . . 81

4.4.2 Phase retrieval at laboratory sources . . . . . . . . 85

4.5 Alignment procedures ................. 93

5 Imaging of unstained hydrated mouse cerebellum $\quad 97$

5.1 Basic anatomy of the cerebellum . . . . . . . . . . . 97

5.2 Methods . . . . . . . . . . . . . . . . . . 99

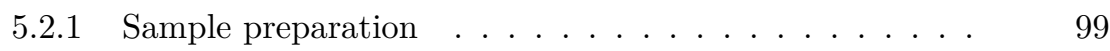

5.2 .2 Experimental setup . . . . . . . . . . . 100

5.3 Data analysis ..................... 101

5.3.1 Phase retrieval .................. 101

5.3.2 Reduction of low-frequency artifacts . . . . . . . . . . . 103

5.4 Results of the tomographic imaging . . . . . . . . . . . . 105

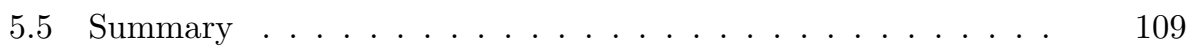

6 Contrast enhancement by metal staining $\quad 111$

6.1 Osmium-stained mouse retina . . . . . . . . . . . . . 111

6.1.1 Basic anatomy of the retina ........... 111 
6.1.2 Methods . . . . . . . . . . . . . . . . . . . . . . 113

6.1.2.1 Sample preparation . . . . . . . . . . . . 113

6.1.2.2 Experimental parameters . . . . . . . . . . 114

6.1.3 Results . . . . . . . . . . . . . . . . 116

6.2 Golgi-Cox stained mouse hippocampus . . . . . . . . . . . . 122

6.2.1 Basic anatomy of the hippocampus . . . . . . . . . 122

6.2.2 Methods . . . . . . . . . . . . . . . . . . . 124

6.2.2.1 Sample preparation . . . . . . . . . . . . 124

6.2.2.2 Experimental parameters at the laboratory setup 124

6.2.2.3 Experimental parameters at the GINIX setup 125

6.2 .3 Results . . . . . . . . . . . . . . . . . . . . . 127

6.2.3.1 Epon-embedded sample . . . . . . . . . . . 127

6.2.3.2 Hydrated sample . . . . . . . . . . . . . . . 134

6.3 Summary . . . . . . . . . . . . . . . . . . . . 139

7 Contrast enhancement by different embedding media 143

7.1 Evaporation-of-solvent preparation . . . . . . . . . . . . 144

7.1 .1 Methods . . . . . . . . . . . . . . . . . . . 144

7.1.1.1 Sample preparation . . . . . . . . . . . . 144

7.1.1.2 Experimental parameters . . . . . . . . . . 144

7.1 .2 Results . . . . . . . . . . . . . . . . . 146

7.2 Mouse cerebellum embedded in ethanol . . . . . . . . . . . 159

7.2 .1 Methods . . . . . . . . . . . . . . . . 159

7.2.1.1 Sample preparation . . . . . . . . . . 159

7.2.1.2 Experimental parameters . . . . . . . . . . 159

7.2 .2 Results . . . . . . . . . . . . . . . . . . 160

7.3 Mouse cerebellum embedded in paraffin . . . . . . . . . . 164

7.3 .1 Methods . . . . . . . . . . . . . . . . . . . . . 164

7.3.1.1 Sample preparation . . . . . . . . . . . . 164

7.3.1.2 Experimental parameters . . . . . . . . . 165

7.3 .2 Results . . . . . . . . . . . . . . . 166

7.4 Summary . . . . . . . . . . . . . . . . . . . . . 169

8 Quantitative 3d histology of human cerebellum 173

8.1 Sample preparation and experimental parameters . . . . . . . 174

8.2 Results . . . . . . . . . . . . . . . . . . . 176

8.2.1 Tomographic imaging at the GINIX setup . . . . . 176 
8.2.2 Automated cellular segmentation in the synchrotron results 180

8.2.2.1 Spherical Hough transform for 3d segmentation 181

8.2.2.2 Performance of the algorithm . . . . . . . . 187

8.2.2.3 Statistical measures . . . . . . . . . . . 191

8.2.3 Tomographic imaging at the laboratory setup . . . . . . 196

8.2.4 Automated cellular segmentation in the laboratory results 197

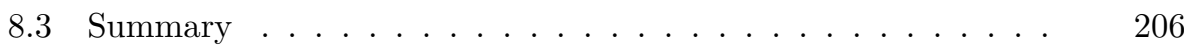

9 Outlook: 3d histology of neurodegenerative diseases 209

9.1 Multiple sclerosis . . . . . . . . . . . . . . . 209

9.2 Alzheimer's disease . . . . . . . . . . . . . . . 219

9.3 Ischemic stroke . . . . . . . . . . . . . . . 225

$\begin{array}{ll}\text { Conclusion } & 233\end{array}$

$\begin{array}{lr}\text { Appendix } & 237\end{array}$

A.1 Fourier transform and its properties . . . . . . . . . . 237

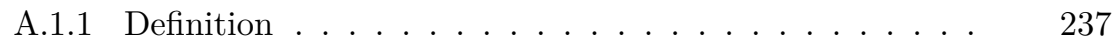

A.1.2 Linearity . . . . . . . . . . . . . . 237

A.1.3 Pseudo-differential-operators . . . . . . . . . . 237

A.1.4 Convolution theorem . . . . . . . . . . 238

A.1.5 Fourier transform of the Dirac Delta distribution . . . . 238

A.2 Step-by-step derivation of the contrast transfer function . . . . 239

A.3 Fringe visibility in the Young interferometer . . . . . . . . . . . 242

A.4 Regularization in the CTF-based reconstruction . . . . . . . . 245

A.5 Main processing steps in the tomographic reconstruction . . . . 248

List of publications $\quad 269$

$\begin{array}{ll}\text { Danksagung } & 271\end{array}$

$\begin{array}{ll}\text { Curriculum Vitae } & \mathbf{2 7 3}\end{array}$ 


\section{Introduction}

Understanding the cytoarchitecture of neuronal tissues is an essential step in order to decipher the underlying connection between tissue function and structure and to indicate the relevant structural changes in neurodegenerative diseases. This requires the acquisition of three-dimensional (3d) structural information about the tissue down to the (sub-)cellular scale, providing sufficient resolution, contrast and field of view while enabling the high throughput necessary for large biomedical studies. The gold standard in pathology is histological sectioning, which is a labor-intensive and invasive imaging technique [2]. Sample preparation requires chemical fixation, dehydration and subsequent paraffin-embedding, sectioning, deparaffinization and staining [16]. While it yields excellent results on the thin twodimensional sections, the $3 \mathrm{~d}$ architecture can only be determined after aligning these sections, resulting in a reconstructed volume with non-isotropic resolution which is susceptible to artifacts due to the mechanical slicing procedure [2]. Further established imaging techniques include light sheet fluorescence microscopy [38], serial block face electron microscopy [107, 108] or magnetic resonance imaging [40] which provide important data but need invasive or extensive sample preparation or lack the required spatial resolution or contrast.

Another promising approach for the non-invasive visualization of the $3 \mathrm{~d}$ cytoarchitecture is given by x-ray computed tomography (CT) which is predominantly used in daily clinical practice [75]. The weak interaction of hard x-rays with matter allows for non-destructive imaging of otherwise opaque samples while the short wavelength enables a high potential resolution. In classical CT, contrast formation is based on absorption of the x-rays as they pass through the sample. However, weakly absorbing objects like soft tissue from the central nervous system give nearly no contrast, diminishing the possible resolution. As x-rays are electromagnetic waves, transmission through the object not only decreases the amplitude but also leads to a shifted phase with respect to the incoming beam. The strength of this shift is determined by the real part $\delta(\mathbf{r})$ or decrement of the complex index of refraction $n(\mathbf{r})=1-\delta(\mathbf{r})+i \beta(\mathbf{r})$, while the complex part or extinction coefficient

gives rise to the absorption. For soft tissue and hard x-rays the refractive index decrement is up to a factor of 1000 larger compared to the extinction coefficient 
[125], and hence contrast can be increased by exploiting the phase shift in addition to the absorption for imaging. As the phase shift is not directly measurable, it has to be converted to object specific modulations in the intensity images. For this purpose various methods exist as, e.g., zone-plate microscopes with Zernike phase contrast [26, 151, 159], Talbot interferometry [111, 134, 152, 188], edge illumination $[64,116]$ or speckle-based imaging $[13,113,199]$, which are all full-field imaging techniques. In contrast to scanning techniques like ptychography $[36,72,189]$ acquisition time is significantly reduced which is an important factor in tomographic imaging, requiring several projection images of the sample from different angles, especially with regard to the stability of degrading biological specimens. While each of the aforementioned full-field techniques is also compatible with the low coherence provided by laboratory microfocus sources [64, 135, 164, 198], they all have their own advantages and drawbacks. X-ray microscopes using Zernike phase contrast provide an excellent resolution in the range of few 10 nanometers with high contrast but quantitativity of the data is missing as generally no further phase-reconstruction steps are carried out [5, 194]. Additionally, alignment of the necessary optical elements in the beam path might be time-consuming and imaging at a varying field of view and resolution requires substantial changes in the setup as, e.g., exchanging and realigning the objective lens. Talbot interferometry, edge illumination and speckle-based imaging provide excellent contrast and model-independent fully automatic reconstructions of the phase, amplitude and darkfield signal separately. Compared to other techniques, they provide a very high density resolution with accurately retrieved values of the complex index of refraction [197]. However, spatial resolution is limited to the range of few micrometers [137, 197, 199] and phase-stepping is necessary for high quality reconstructions, although also single-step approaches have been proposed, leading to the need of additional a priori knowledge or a decrease in spatial resolution [14, 15, 196]. Moreover, in Talbot interferometry and edge illumination radiation dose is increased due to absorbing optical elements behind the sample and imperfections in these elements as well as their alignment might alter the reconstruction quality [197]. Phase contrast based on free-space propagation behind the sample is well suited for microscopic resolutions in the range of 20-50 nm for test objects and biological cells, respectively [10, 28, 98, 120, 126]. As it does not depend on further optical elements behind the sample, experimental alignment is facilitated while the applied dose is reduced with respect to interferometric approaches [197]. Imaging of the sample at varying resolution and field of view can be easily achieved by exploiting a divergent beam geometry and changing the geometrical magnification of 
the setup [90]. Due to the high coherence needed for this imaging technique it was long assumed to be solely applicable at large-scale synchrotron facilities. However, with the advent of new microfocus sources [123], it could also be implemented at laboratory setups $[8,91,99]$. The main challenge for this technique is the phase reconstruction from the measured intensity images, providing quantitative information about the specimen. It is typically based on additional information about the sample from multiple measurement planes [28, 195] or a priori knowledge, e.g., the positivity of physical constants, a weakly absorbing or pure phase object or an estimate of the complex refractive index $[51,56,127]$.

To perform 3d virtual histology of tissue from the central nervous system at cellular and sub-cellular resolution, phase contrast based on free-space propagation is well suited and hence exploited in the course of this thesis. It is combined with computed tomography to obtain non-destructive and isotropic reconstructions of the $3 \mathrm{~d}$ density distribution in mm-sized samples of mice and humans and to decipher the underlying cytoarchitecture at varying resolution and field of view.

In the first chapter of the present thesis, the theoretical concepts of image formation in propagation-based imaging are presented, followed by an introduction to several phase-retrieval methods in chapter 2. The mathematical background of computed tomography as well as examples of typical artifacts and their circumvention are described in chapter 3 . In chapter 4 , the experimental realization of propagation-based phase-contrast tomography both at the synchrotron and a laboratory source is outlined, including an evaluation of different phase-retrieval approaches for data acquired under the non-ideal conditions of polychromaticity and partial coherence which are typical for laboratory setups. First experimental results on a hydrated brain slice from a mouse cerebellum are presented in chapter 5, representing a tissue preparation which is close to the native state. Methods for increasing the contrast in different parts of tissue from the central nervous system of a mouse via radiocontrast agents or different embedding media and their applicability at synchrotron as well as laboratory setups are evaluated in chapters 6 and 7 . In chapter 8, 3d virtual histology is performed on paraffinembedded human cerebellum obtained at routine autopsy, both at the synchrotron as well as laboratory setup. It is accompanied by an automated determination of the cellular distribution within the reconstructed volumes, enabling a subsequent statistical analysis based on several ten thousand to millions of cells. As an outlook, preliminary results from the application of propagation-based phase-contrast tomography for the visualization of cytoarchitectural changes in neurodegenerative diseases, namely multiple sclerosis, Alzheimer's disease and ischemic stroke, 
are presented in chapter 9 . The thesis is closed by a brief summary of the obtained results and an outlook on future experiments and challenges. 


\section{Concepts of propagation-based phase-contrast imaging}

In this chapter, the fundamental concepts of two-dimensional propagation-based imaging are derived. A generic experimental setup for this kind of imaging is depicted in Fig. 1.1. A (divergent) $\mathrm{x}$-ray beam is generated in the source and advances to the sample located in a distance $z_{01}$. During transmission of the x-rays through the specimen, phase shifts are induced which are translated into measurable intensity modulations due to interference effects upon free-space propagation. These are recorded further downstream in the detection plane at distance $z_{12}$ behind the sample.

The chapter starts with the theoretical framework governing the free-space propagation of the disturbed wave fronts behind the sample, leading to an expression for the complex wave front at any given propagation distance $z_{12}$, provided that the incident wave field is known. The interaction of x-rays with matter describes

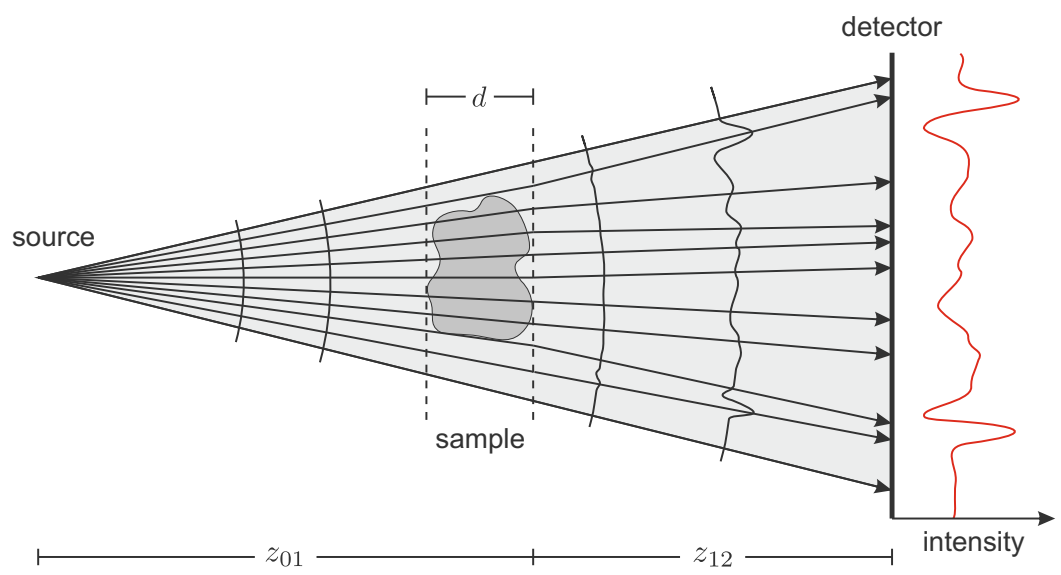

Figure 1.1: Generic setup of a propagation-based phase-contrast experiment. The x-rays are generated in the source and advance to the sample of thickness $d$ which is located in a distance $z_{01}$. Further downstream, phase shifts induced by the sample translate into measurable intensity modulations due to interference effects which are recorded by the detector placed at a distance $z_{12}$ behind the sample. 
the formation of this incident wave field directly behind the object, depending on its refractive index $n(\mathbf{r})$. By introducing the projection approximation, this interaction can be expressed in a single plane, which forms the basis for deriving simplified expressions for contrast formation at propagation distance $z_{12}$.

\subsection{From Maxwell's equations to free-space propagation}

\subsubsection{Vacuum wave equations}

The Maxwell's equations are the starting point to describe the temporal and spatial evolution of electromagnetic waves in free space. In vacuum they are given by [125]

$$
\begin{aligned}
\nabla \cdot \mathbf{E}(\mathbf{r}, t) & =0, \\
\nabla \cdot \mathbf{B}(\mathbf{r}, t) & =0, \\
\nabla \times \mathbf{E}(\mathbf{r}, t)+\partial_{t} \mathbf{B}(\mathbf{r}, t) & =\mathbf{0}, \\
\nabla \times \mathbf{B}(\mathbf{r}, t)-\varepsilon_{0} \mu_{0} \partial_{t} \mathbf{E}(\mathbf{r}, t) & =\mathbf{0},
\end{aligned}
$$

with the magnetic induction $\mathbf{B}(\mathbf{r}, t)$, the electric field $\mathbf{E}(\mathbf{r}, t)$, the vacuum electrical permittivity $\varepsilon_{0}$, the vacuum magnetic permeability $\mu_{0}$ and the Cartesian coordinates $\mathbf{r}=(x, y, z) \in \mathbb{R}^{3}$. Note that SI units as well as the convention to mark vector quantities in boldface type will be used throughout the thesis. To obtain the wave equation for the electric field $\mathbf{E}(\mathbf{r}, t)$, the curl of eqn. (1.3) is calculated and simplified via the vector identity $\nabla \times(\nabla \times \mathbf{f})=\nabla(\nabla \cdot \mathbf{f})-\nabla^{2} \mathbf{f}$, yielding

$$
\nabla(\nabla \cdot \mathbf{E}(\mathbf{r}, t))-\nabla^{2} \mathbf{E}(\mathbf{r}, t)+\nabla \times \partial_{t} \mathbf{B}(\mathbf{r}, t)=\mathbf{0}
$$

The first term of this equation vanishes due to the first Maxwell's equation (1.1). Interchanging the order of the operators $\nabla \times$ and $\partial_{t}$ in the third term and inserting eqn. (1.4) leads to

$$
\left(\varepsilon_{0} \mu_{0} \partial_{t}^{2}-\nabla^{2}\right) \mathbf{E}(\mathbf{r}, t)=\mathbf{0}
$$

which is known as the vacuum field equation for the electric field, or the d'Alembert wave equation [125].

In a similar line of reasoning the expression for the magnetic induction $\mathbf{B}$ can be derived. By taking the curl of eqn. (1.4) and using the same vector identity as 
before, one arrives at

$$
\nabla(\nabla \cdot \mathbf{B}(\mathbf{r}, t))-\nabla^{2} \mathbf{B}(\mathbf{r}, t)-\varepsilon_{0} \mu_{0} \nabla \times \partial_{t} \mathbf{E}(\mathbf{r}, t)=\mathbf{0}
$$

The first term vanishes due to the non-existence of magnetic monopoles, as stated in the second Maxwell's equation (1.2). Again interchanging the order of the operators $\nabla \times$ and $\partial_{t}$ and inserting eqn. (1.3) yields the vacuum field equation for the magnetic induction $\mathbf{B}(\mathbf{r}, t)$

$$
\left(\varepsilon_{0} \mu_{0} \partial_{t}^{2}-\nabla^{2}\right) \mathbf{B}(\mathbf{r}, t)=\mathbf{0}
$$

which is analogous to the relation for the electric field [125]. The two expressions show that all three components of the electric field and magnetic induction are uncoupled from one another in vacuum.

At this point, a transition from a vector to a scalar theory with the complex scalar quantity $\Psi(\mathbf{r}, t)$, solving the scalar d'Alembert equation

$$
\left(\varepsilon_{0} \mu_{0} \partial_{t}^{2}-\nabla^{2}\right) \Psi(\mathbf{r}, t)=0
$$

is considered. Although each of the three components of the electric and magnetic field obeys this equation, the validity of the transition to a scalar theory is not immediately apparent. However, it was shown that in free space, the electromagnetic field can indeed be derived from a single complex scalar function whose squared modulus corresponds to the optical intensity [17, 55, 102, 125, 191], and hence, only this function will be regarded in the following.

To determine the speed of propagation of an electromagnetic field, a monochromatic elementary plane wave $(\mathrm{PW})$ of the form $\Psi^{\mathrm{PW}}(\mathbf{r}, t)=e^{i \mathbf{k} \cdot \mathbf{r}-\omega t}$ is considered, where $\mathbf{k}=\left(k_{x}, k_{y}, k_{z}\right) \in \mathbb{R}^{3}$ is the wave vector with modulus $|\mathbf{k}|=: k=\frac{2 \pi}{\lambda}$ and wavelength $\lambda$. It is a solution to the scalar d'Alembert equation if $\varepsilon_{0} \mu_{0} \omega^{2}-k^{2}=0$. As the wave fronts of plane waves propagate with velocity $c=\frac{\omega}{k}$, this leads to

$$
c=\frac{1}{\sqrt{\varepsilon_{0} \mu_{0}}} .
$$

Experimentally determined values of the vacuum permittivity $\varepsilon_{0}$ and permeability $\mu_{0}$ revealed a propagation speed which is equal to the speed of light in vacuum, showing that light is actually an electromagnetic wave [125].

To obtain a stationary solution to the d'Alembert equation, the complex scalar 
quantity $\Psi(\mathbf{r}, t)$ is spectrally decomposed via a Fourier integral of the form [125]

$$
\Psi(\mathbf{r}, t)=\frac{1}{\sqrt{2 \pi}} \int_{\mathbb{R} \geq 0} \psi_{\omega}(\mathbf{r}) e^{-i \omega t} \mathrm{~d} \omega .
$$

The field can thus be interpreted as a superposition of monochromatic plane waves with weighting factors $\psi_{\omega}(\mathbf{r})$. Inserting this expression into eqn. (1.9), interchanging the order of integration and differentiation and performing the differentiation with respect to time, yields

$$
\int_{\mathbb{R} \geq 0}\left[\left(\nabla^{2}+k^{2}\right) \psi_{\omega}(\mathbf{r})\right] e^{-i \omega t} \mathrm{~d} \omega=0,
$$

where $c=\frac{1}{\sqrt{\varepsilon_{0} \mu_{0}}}$ and $k=\frac{\omega}{c}$ was used. To solve this equation, the expression in the square brackets has to vanish, leading to

$$
\left(\nabla^{2}+k^{2}\right) \psi_{\omega}(\mathbf{r})=0
$$

known as the Helmholtz equation [125]. It governs the spatial evolution of the stationary part $\psi_{\omega}(\mathbf{r})$ of a single monochromatic component of the electromagnetic field $\Psi(\mathbf{r}, t)$. To obtain a polychromatic solution, the single $\omega$-dependent solutions $\psi_{\omega}(\mathbf{r})$, fulfilling the Helmholtz equation, can be superimposed using eqn. (1.11). Simple solutions to the Helmholtz equation are given by stationary elementary plane waves of the form $\psi_{\omega}^{\mathrm{PW}}(\mathbf{r})=e^{i \mathbf{k} \cdot \mathbf{r}}$, analogous to the time-dependent plane waves solving the d'Alembert equation (1.9), or spherical waves (SW) $\psi_{\omega}^{\mathrm{SW}}(\mathbf{r})=\frac{1}{\left|\mathbf{r}-\mathbf{r}_{0}\right|} e^{i k\left|\mathbf{r}-\mathbf{r}_{0}\right|}$ with $\mathbf{r} \neq \mathbf{r}_{0}$. While in the case of plane waves the propagation direction is given by the direction of the wave vector $\mathbf{k}$, spherical waves propagate uniformly in all directions starting at the origin $\mathbf{r}_{0}$, with constant amplitude and phase at radial distance $r$.

\subsubsection{Angular spectrum approach}

In the following, an expression for the forward-propagation of an arbitrary monochromatic wave field $\psi_{\omega}(\mathbf{r})$ in vacuum is derived, closely following the considerations in [125]. Without loss of generality, propagation takes place along $z$, from a plane at $z=0$ to a parallel plane at $z>0$. As the wave propagates through vacuum, the Helmholtz equation (1.13) is valid, to which the elementary plane wave $\psi_{\omega}^{\mathrm{PW}}(\mathbf{r})=e^{i \mathbf{k} \cdot \mathbf{r}}$ is a solution. Considering the modulus of the wave vector $\mathbf{k}$, given by $k=\sqrt{k_{x}^{2}+k_{y}^{2}+k_{z}^{2}}$, and solving it for the component in $z$-direction leads 
to

$$
k_{z}=\sqrt{k^{2}-k_{x}^{2}-k_{y}^{2}}
$$

Note that only the positive square root is considered, accounting for the assumption of a forward-propagating wave with respect to the optical axis. Inserting this into the elementary plane wave equation gives

$$
\psi_{\omega}^{\mathrm{PW}}(\mathbf{r})=e^{i \mathbf{k}_{\perp} \cdot \mathbf{r}_{\perp}} e^{i z \sqrt{k^{2}-k_{\perp}^{2}}}
$$

where $\mathbf{k}_{\perp}=\left(k_{x}, k_{y}\right)$ with modulus $\left|\mathbf{k}_{\perp}\right|=: k_{\perp}=\sqrt{k_{x}^{2}+k_{y}^{2}}$ and $\mathbf{r}_{\perp}=(x, y)$ with modulus $\left|\mathbf{r}_{\perp}\right|=: r_{\perp}=\sqrt{x^{2}+y^{2}}$ were introduced.

By setting $z=0$ in the above equation, leading to

$$
\psi_{\omega}^{\mathrm{PW}}\left(\mathbf{r}_{\perp}, 0\right)=e^{i \mathbf{k}_{\perp} \cdot \mathbf{r}_{\perp}}
$$

the propagation of a single monochromatic plane wave from $z=0$ to $z>0$ can be identified as a multiplication of the unpropagated quantity with the factor

$$
H=e^{i z \sqrt{k^{2}-k_{\perp}^{2}}} .
$$

Hence, this term is called the free-space propagator [125].

This can be used to derive an expression for the propagation of an arbitrary monochromatic wave field $\psi_{\omega}(\mathbf{r})$. To this end, a decomposition of the unpropagated wave field at $z=0$ is obtained via the two-dimensional (2d) Fourier integral ${ }^{1}$

$$
\psi_{\omega}\left(\mathbf{r}_{\perp}, 0\right)=\frac{1}{2 \pi} \int_{\mathbb{R}^{2}} \hat{\psi}_{\omega}\left(\mathbf{k}_{\perp}, 0\right) e^{i \mathbf{k}_{\perp} \cdot \mathbf{r}_{\perp}} \mathrm{d} \mathbf{k}_{\perp}
$$

where $\hat{\psi}_{\omega}\left(\mathbf{k}_{\perp}, 0\right)$ denotes the lateral Fourier transform of $\psi_{\omega}\left(\mathbf{r}_{\perp}, 0\right)$. Note that Fourier transformed quantities will be marked with a circumflex throughout the thesis. This decomposition of the unpropagated wave field can be interpreted as a superposition of plane waves of the form $e^{i \mathbf{k}_{\perp} \cdot \mathbf{r}_{\perp}}$ with weighting factors $\hat{\psi}_{\omega}\left(\mathbf{k}_{\perp}, 0\right)$. The plane waves in turn can be considered as three-dimensional plane waves $e^{i \mathbf{k r}}$, solving the Helmholtz equation, evaluated at $z=0$. As seen before, these waves can be propagated in free space by multiplication with the free-space propagator $H$. Inserting this into eqn. (1.18) and therefore propagating each plane wave in

1 The Fourier transform is defined as in section A.1 in the appendix. 
the decomposition of the unpropagated wave field separately, yields

$$
\psi_{\omega}\left(\mathbf{r}_{\perp}, z\right)=\frac{1}{2 \pi} \int_{\mathbb{R}^{2}} \hat{\psi}_{\omega}\left(\mathbf{k}_{\perp}, 0\right) e^{i \mathbf{k}_{\perp} \cdot \mathbf{r}_{\perp}} e^{i z \sqrt{k^{2}-k_{\perp}^{2}}} \mathrm{~d} \mathbf{k}_{\perp} .
$$

By introducing the operator $\mathcal{F}_{\perp}$, denoting the 2 d lateral Fourier transform, the propagation of a monochromatic wave field from $z=0$ to $z>0$ can be rewritten as

$$
\psi_{\omega}\left(\mathbf{r}_{\perp}, z\right)=\mathcal{F}_{\perp}^{-1}\left[e^{i z \sqrt{k^{2}-k_{\perp}^{2}}} \mathcal{F}_{\perp}\left[\psi_{\omega}\left(\mathbf{r}_{\perp}, 0\right)\right]\right]=: \mathcal{D}\left[\psi_{\omega}\left(\mathbf{r}_{\perp}, 0\right)\right]
$$

where the diffraction operator

$$
\mathcal{D}=\mathcal{F}_{\perp}^{-1} e^{i z \sqrt{k^{2}-k_{\perp}^{2}}} \mathcal{F}_{\perp}
$$

was introduced. Note that for $k^{2}-k_{\perp}^{2}<0$, the free-space propagator (1.17) will be a real-valued quantity, leading to an exponential damping of the wave field instead of a propagation. In this case, the wave field is called an evanescent wave. If there are no evanescent waves present, the diffraction operator $\mathcal{D}$ is a unitary operator [112] and back propagation along a negative distance $z<0$ can be performed $[125]$.

\subsubsection{Paraxial approximation}

In the experimental geometries considered in this thesis, waves are well described by the paraxial approximation, in which it is assumed that the wave front normals of the wave field $\psi_{\omega}(\mathbf{r})$ form small angles with respect to a given optical axis. It therefore mainly varies perpendicular to the propagation direction and can be expressed via a separation ansatz

$$
\psi_{\omega}(\mathbf{r})=\psi_{\omega}^{\prime}(\mathbf{r}) e^{i k z}
$$

in which the wave is divided into an unscattered plane wave propagating along $z$, which is again defined as the optical axis, and a 'beamlike' envelope which slowly varies along $z$. Inserting this ansatz into the Helmholtz equation (1.13) yields

$$
\left(\nabla^{2}+k^{2}\right) \psi_{\omega}^{\prime}(\mathbf{r}) e^{i k z}=0
$$


Execution of the spatial derivative along $z$ and a subsequent elimination of the plane wave term via a simple division by the exponential results in

$$
\left(\nabla_{\perp}^{2}+\partial_{z}^{2}+2 i k \partial_{z}\right) \psi_{\omega}^{\prime}(\mathbf{r})=0
$$

where the lateral Laplacian operator $\nabla_{\perp}^{2}=\partial_{x}^{2}+\partial_{y}^{2}$ was introduced. As the envelope $\psi_{\omega}^{\prime}(\mathbf{r})$ varies slowly along $z$, the second derivative along this direction can be neglected, leading to the so-called paraxial wave equation [125]

$$
\left(\nabla_{\perp}^{2}+2 i k \partial_{z}\right) \psi_{\omega}^{\prime}(\mathbf{r})=0
$$

One particular solution to this equation is the Gaussian beam, as, e.g., discussed in [52], which is a good approximation for the wave field exiting x-ray waveguides (cf. section 4.2) [70, 93].

\subsubsection{Fresnel diffraction}

With the paraxial approximation outlined in the previous section, the free-space propagator (1.17) introduced in section 1.1.2 can be rewritten. In the case of a paraxial wave, the wave vector component along the optical axis is much larger compared to the perpendicular parts $\left(k_{\perp}^{2} \ll k^{2}\right)$. Therefore, the phase factor of the free-space propagator can be approximated via a first order Taylor expansion

$$
\sqrt{k^{2}-k_{\perp}^{2}} \simeq k-\frac{k_{\perp}^{2}}{2 k}
$$

Inserting this into the expression for the propagated wave field (1.20) yields

$$
\psi_{\omega}\left(\mathbf{r}_{\perp}, z\right)=\mathcal{F}_{\perp}^{-1}\left[e^{i z\left(k-\frac{k^{2}}{2 k}\right)} \mathcal{F}_{\perp}\left[\psi_{\omega}\left(\mathbf{r}_{\perp}, 0\right)\right]\right]=: \mathcal{D}^{(F)}\left[\psi_{\omega}\left(\mathbf{r}_{\perp}, 0\right)\right]
$$

with the Fresnel diffraction operator

$$
\mathcal{D}^{(F)}=\mathcal{F}_{\perp}^{-1} e^{i z\left(k-\frac{k_{\perp}^{2}}{2 k}\right)} \mathcal{F}_{\perp}
$$

The approximated form of the free-space propagator

$$
\hat{H}^{(F)}\left(\mathbf{k}_{\perp}, z\right)=e^{i z\left(k-\frac{k_{\perp}^{2}}{2 k}\right)}
$$


is called the (reciprocal) Fresnel propagator, where the term reciprocal indicates that it is valid for the propagation of Fourier transformed wave fields [125].

Via the convolution theorem $f\left(\mathbf{r}_{\perp}\right) \star g\left(\mathbf{r}_{\perp}\right)=2 \pi \mathcal{F}_{\perp}^{-1}\left(\mathcal{F}_{\perp}\left[f\left(\mathbf{r}_{\perp}\right)\right] \cdot \mathcal{F}_{\perp}\left[g\left(\mathbf{r}_{\perp}\right)\right]\right)$, where $\star$ denotes the convolution, a real space expression of the propagation can be obtained by rewriting eqn. (1.27) in terms of the real space representation of the Fresnel propagator $H^{(F)}\left(\mathbf{r}_{\perp}, z\right)$ [125]

$$
\begin{aligned}
\psi_{\omega}\left(\mathbf{r}_{\perp}, z\right) & =\mathcal{F}_{\perp}^{-1}\left[e^{i z\left(k-\frac{k_{\perp}^{2}}{2 k}\right)} \mathcal{F}_{\perp}\left[\psi_{\omega}\left(\mathbf{r}_{\perp}, 0\right)\right]\right] \\
& =2 \pi \mathcal{F}_{\perp}^{-1}\left[\mathcal{F}_{\perp}\{\underbrace{\frac{1}{2 \pi} \mathcal{F}_{\perp}^{-1}\left[e^{i z\left(k-\frac{k_{\perp}^{2}}{2 k}\right)}\right]}_{H^{(F)}\left(\mathbf{r}_{\perp}, z\right)}\} \cdot \mathcal{F}_{\perp}\left[\psi_{\omega}\left(\mathbf{r}_{\perp}, 0\right)\right]\right] \\
& =H^{(F)}\left(\mathbf{r}_{\perp}, z\right) \star \psi_{\omega}\left(\mathbf{r}_{\perp}, 0\right) .
\end{aligned}
$$

In order to obtain an explicit expression for the propagator, the inverse Fourier transform has to be executed

$$
\begin{aligned}
H^{(F)}\left(\mathbf{r}_{\perp}, z\right) & =\frac{1}{2 \pi} \mathcal{F}_{\perp}^{-1}\left[e^{i z\left(k-\frac{k_{\perp}^{2}}{2 k}\right)}\right] \\
& =\frac{e^{i k z}}{4 \pi^{2}} \int_{\mathbb{R}^{2}} e^{-\frac{i z}{2 k} k_{x}^{2}+i k_{x} x} \cdot e^{-\frac{i z}{2 k} k_{y}^{2}+i k_{y} y} \mathrm{~d}_{\perp} .
\end{aligned}
$$

Via completing the square this can be rewritten as

$$
H^{(F)}\left(\mathbf{r}_{\perp}, z\right)=\frac{e^{i k z}}{4 \pi^{2}} \int_{\mathbb{R}^{2}} e^{-\left(\sqrt{\frac{i z}{2 k}} k_{x}-\frac{i \sqrt{k}}{\sqrt{2 i z}} x\right)^{2}+\frac{i k}{2 z} x^{2}} \cdot e^{-\left(\sqrt{\frac{i z}{2 k}} k_{y}-\frac{i \sqrt{k}}{\sqrt{2 i z}} y\right)^{2}+\frac{i k}{2 z} y^{2}} \mathrm{~d} \mathbf{k}_{\perp} .
$$

Inserting the substitution $\mathbf{u}_{\perp}:=\sqrt{\frac{i z}{2 k}} \mathbf{k}_{\perp}-\frac{i \sqrt{k}}{\sqrt{2 i z}} \mathbf{r}_{\perp}$ with $\mathrm{d} \mathbf{u}_{\perp}=\frac{i z}{2 k} \mathrm{~d} \mathbf{k}_{\perp}$ and the solution of the Gauss integral $\int_{\mathbb{R}^{2}} e^{-\mathbf{r}_{\perp}^{2}} \mathrm{~d} \mathbf{r}_{\perp}=\pi$, the real space Fresnel propagator is given by [125]

$$
\begin{aligned}
H^{(F)}\left(\mathbf{r}_{\perp}, z\right) & =\frac{e^{i k z}}{4 \pi^{2}} e^{\frac{i k}{2 z} r_{\perp}^{2}} \int_{\mathbb{R}^{2}} e^{-\mathbf{u}_{\perp}^{2}} \frac{2 k}{i z} \mathrm{~d} \mathbf{u}_{\perp} \\
& =\frac{e^{i k z}}{i \lambda z} e^{\frac{i k}{2 z} r_{\perp}^{2}}
\end{aligned}
$$


where $k=\frac{2 \pi}{\lambda}$ was used.

Thus it is now possible to explicitly express the propagated wave field via the convolution in eqn. (1.32)

$$
\psi_{\omega}\left(\mathbf{r}_{\perp}, z\right)=\frac{e^{i k z}}{i \lambda z} \int_{\mathbb{R}^{2}} e^{\frac{i k}{2 z}\left(\mathbf{r}_{\perp}-\mathbf{r}_{\perp}^{\prime}\right)^{2}} \psi_{\omega}\left(\mathbf{r}_{\perp}^{\prime}, 0\right) \mathrm{d} \mathbf{r}_{\perp}^{\prime} .
$$

Another possibility to derive this equation is a small angle approximation of the Huygens-Fresnel principle, as detailed, e.g., in [53].

To further evaluate this expression for the propagation of a given wave field, eqn. (1.38) is rewritten as

$$
\psi_{\omega}\left(\mathbf{r}_{\perp}, z\right)=\frac{e^{i k z}}{i \lambda z} e^{\frac{i k}{2 z} r_{\perp}^{2}} \int_{\mathbb{R}^{2}} \psi_{\omega}\left(\mathbf{r}_{\perp}^{\prime}, 0\right) e^{\frac{i k}{2 z} r_{\perp}^{\prime 2}} e^{-\frac{i k}{z}\left(\mathbf{r}_{\perp} \cdot \mathbf{r}_{\perp}^{\prime}\right)} \mathrm{d} \mathbf{r}_{\perp}^{\prime}
$$

Hence, the propagated field is proportional to the $2 \mathrm{~d}$ lateral Fourier transform of the initial field multiplied by the phase factor $e^{\frac{i k}{2 z} r_{\perp}^{\prime 2}}$

$$
\psi_{\omega}\left(\mathbf{r}_{\perp}^{\prime}, 0\right) e^{\frac{i k}{2 z} r_{\perp}^{\prime 2}}
$$

This justifies the definition of the dimensionless Fresnel number [125]

$$
F:=\frac{a^{2}}{\lambda z}
$$

corresponding to the exponent of this phase factor which determines its influence on the propagated field, with $a$ being the characteristic length scale of lateral features within the incident field $\psi_{\omega}\left(\mathbf{r}_{\perp}^{\prime}, 0\right)$. For $F \ll 1$ it can be neglected and the propagated wave field is given by the lateral Fourier transform of the initial wave field, known as Fraunhofer diffraction [125]. For $F \simeq 1$ the phase factor is non-negligible and Fresnel diffraction occurs. In this regime, the size of the Fresnel number can be used to further distinguish between different imaging regimes with specific properties, as further evaluated in section 1.3.3.

\subsubsection{Numerical implementation of propagation}

As an analytical solution of the equations governing the free-space propagation of a known wave field is in general not available, the propagated wave field has to be obtained via numerical propagation. To this end, it can be either implemented via a multiplication with the reciprocal Fresnel propagator in Fourier space (eqn. (1.27)) or via a convolution with the real space Fresnel propagator (eqn. (1.38)). 
While these two notations are mathematically equivalent, their numerical implementations require different sampling criteria to be fulfilled.

To numerically implement Fresnel propagation of a given wave field it has to be represented on a discrete grid of pixels with sampling interval lengths $p_{x / y}$ in real space and $\Delta k_{x / y}$ in reciprocal space. The reciprocity relation of the discrete Fourier transform relates the two grids via

$$
p_{x / y} \Delta k_{x / y}=\frac{2 \pi}{N_{x / y}},
$$

where $N_{x / y}$ is the number of sampling points in $x$ and $y$ direction, respectively. For the implementation of the propagation in reciprocal space, the Fourier transform of the initial wave field has to be calculated which can be numerically performed via a fast Fourier transform (FFT) [31, 47]. Subsequently, the transformed wave field is multiplied with the reciprocal Fresnel propagator, thus requiring a proper sampling of this propagator in Fourier space in order to avoid artifacts. To ensure proper sampling according to the Nyquist-Shannon sampling theorem [158], which states that a continuous signal can be unambiguously represented if the sampling frequency is larger than twice the maximum frequency component contained within the signal, the relation

$$
\frac{\lambda z}{N_{x / y} p_{x / y}^{2}} \leq 1
$$

has to hold [50, 182]. Here, the local phase gradient of the propagator with respect to $\Delta k_{x / y}$ was interpreted as a 'frequency' in Fourier space. To obtain the propagated wave field, an inverse Fourier transform of the resulting expression must be performed at the end, which can again be implemented via an FFT.

In the implementation of the propagation via a convolution of the initial wave field with the real space Fresnel propagator, a different criterion has to be fulfilled as in this case the propagator is sampled in real space. With the same line of reasoning as above this leads to the relation [50, 182]

$$
\frac{\lambda z}{N_{x / y} p_{x / y}^{2}} \geq 1 .
$$

Therefore, despite being mathematically equivalent, the validity range of the numerical propagation in reciprocal and real space differs. For a given wavelength $\lambda$, pixel size $p_{x / y}$ and number of sampling points $N_{x / y}$, the reciprocal propagator is only properly sampled below a certain propagation distance $z$ whereas for 
the real space propagator the propagation distance $z$ has to lie above a certain threshold. Therefore, these propagators can be called the near-field and far-field Fresnel propagator, respectively. One way to extend the reciprocal implementation to larger propagation distances $z$ is to embed the incident wave field in an array with a higher number of sampling points $N_{x / y}$ by symmetrically extending the wave field via a replication of the border values in order to avoid edge artifacts.

Note that in both cases the criterion can be rewritten in terms of the Fresnel number $F_{x / y}=\frac{p_{x / y}^{2}}{\lambda z}$, showing that only the combination of these three variables is of importance for the numerical implementation of Fresnel propagation.

\subsection{X-ray interactions with matter}

In the previous section, propagation of a known incident wave field $\psi_{\omega}\left(\mathbf{r}_{\perp}, 0\right)$ in free space was derived, which is a good approximation for the propagation of $\mathrm{x}$ rays between the sample and the detector plane (cf. Fig. 1.1). To obtain structural information about the investigated sample, x-ray interactions in the presence of matter have to be considered. In the following, the influence of the sample on the incident wave field is examined and a simplified expression for this field under the assumption of sufficiently thin objects, known as the projection approximation, is introduced.

\subsubsection{Wave equations in the presence of matter}

The Maxwell's equations in their most general form are given by [125]

$$
\begin{aligned}
\nabla \cdot \mathbf{D}(\mathbf{r}, t) & =\rho(\mathbf{r}, t), \\
\nabla \cdot \mathbf{B}(\mathbf{r}, t) & =0, \\
\nabla \times \mathbf{E}(\mathbf{r}, t)+\partial_{t} \mathbf{B}(\mathbf{r}, t) & =\mathbf{0}, \\
\nabla \times \mathbf{H}(r, t)-\partial_{t} \mathbf{D}(\mathbf{r}, t) & =\mathbf{J}(\mathbf{r}, t),
\end{aligned}
$$

with the electric displacement $\mathbf{D}(\mathbf{r}, t)$, the magnetic induction $\mathbf{B}(\mathbf{r}, t)$, the electric field $\mathbf{E}(\mathbf{r}, t)$, the magnetic field $\mathbf{H}(\mathbf{r}, t)$, the charge density $\rho(\mathbf{r}, t)$ and the current density $\mathbf{J}(\mathbf{r}, t)$.

Analogous to section 1.1.1, the wave equations in the presence of matter can be derived from these equations. To this end, considerations are restricted to linear isotropic materials with $\mathbf{D}(\mathbf{r}, t)=\varepsilon(\mathbf{r}, t) \mathbf{E}(\mathbf{r}, t)$ and $\mathbf{B}(\mathbf{r}, t)=\mu(\mathbf{r}, t) \mathbf{H}(\mathbf{r}, t)$. Further, only static materials, i.e., materials with constant values of $\varepsilon$ and $\mu$ over 
time are assumed. As biological materials are generally non-magnetic, the magnetic permeability $\mu(\mathbf{r})$ equals the magnetic permeability of vacuum $\mu_{0}$. By additionally omitting the presence of current as well as charge densities $(\rho(\mathbf{r}, t)=0$ and $\mathbf{J}(\mathbf{r}, t)=0)$ and assuming that the sample varies slowly over length scales comparable to the wave length, i.e., that polarization effects can be neglected, wave equations can be derived in which all three components of the electric and magnetic field are uncoupled from each other. Hence, analogous to section 1.1.1, a transition to a scalar theory with the complex scalar wave field $\Psi(\mathbf{r}, t)$ can be made, resulting in [125]

$$
\left(\varepsilon(\mathbf{r}) \mu_{0} \partial_{t}^{2}-\nabla^{2}\right) \Psi(\mathbf{r}, t)=0 .
$$

Following the approach in section 1.1.1 and expressing the scalar wave field by a continuous superposition of monochromatic components $e^{-i \omega t}$, weighted with the $\omega$-dependent factor $\psi_{\omega}(\mathbf{r})$, yields the inhomogeneous Helmholtz equation [125]

$$
\left(\nabla^{2}+k^{2} n_{\omega}^{2}(\mathbf{r})\right) \psi_{\omega}(\mathbf{r})=0,
$$

in which the $\omega$-dependent index of refraction $n_{\omega}(\mathbf{r})=\sqrt{\frac{\varepsilon_{\omega}(\mathbf{r})}{\varepsilon_{0}}}$ was introduced. Analogous to section 1.1.3, an inhomogeneous wave equation in the paraxial approximation can be derived from the inhomogeneous Helmholtz equation by expressing the monochromatic wave field $\psi_{\omega}(\mathbf{r})$ via an unscattered plane wave $e^{i k z}$, in which $z$ is again the direction of the optical axis, and a slowly varying envelope $\psi_{\omega}^{\prime}(\mathbf{r})$, for which the second derivative along $z$ can be neglected. The resulting inhomogeneous paraxial wave equation is given by [125]

$$
\left(2 i k \partial_{z}+\nabla_{\perp}^{2}+k^{2}\left(n_{\omega}^{2}(\mathbf{r})-1\right)\right) \psi_{\omega}^{\prime}(\mathbf{r})=0 .
$$

\subsubsection{The complex index of refraction}

Due to the weak interaction of x-rays with matter, the index of refraction, introduced in the previous section, only varies marginally around unity and is therefore generally expressed as [1]

$$
n_{\omega}(\mathbf{r})=1+i \beta_{\omega}(\mathbf{r})-\delta_{\omega}(\mathbf{r}) .
$$

Here, the real-valued refractive index decrement $\delta_{\omega}(\mathbf{r})$, responsible for a phase shift in the illuminating wave field, and the likewise real-valued extinction coefficient 

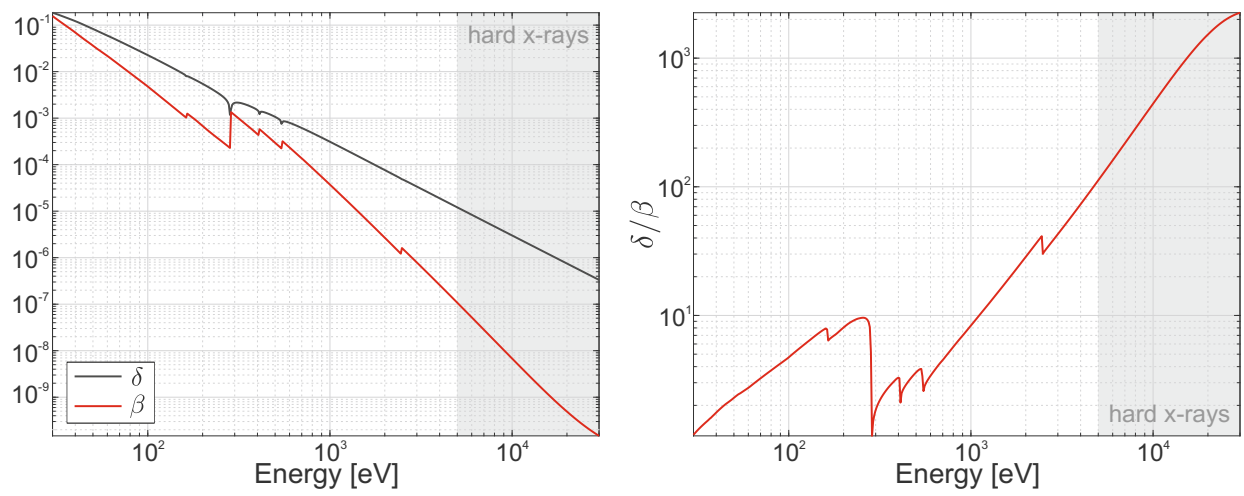

Figure 1.2: Energy-dependent index of refraction for a model protein with chemical formula $\mathrm{H}_{50} \mathrm{C}_{30} \mathrm{~N}_{9} \mathrm{O}_{10} \mathrm{~S}_{1}$ and density $1.35 \frac{\mathrm{g}}{\mathrm{cm}^{3}}[46,67]$, showing a general decrease in the interaction between $\mathrm{x}$-rays and matter for increasing photon energies, except at absorption edges. Towards higher energies, the ratio between the refractive index decrement $\delta_{\omega}$ and extinction coefficient $\beta_{\omega}$ increases significantly.

$\beta_{\omega}(\mathbf{r})$, leading to an absorption of the beam, were introduced. As the interaction primarily occurs between the x-rays and bound electrons in the electron shell of the atoms, $\delta_{\omega}(\mathbf{r})$ and $\beta_{\omega}(\mathbf{r})$ relate to the electron density. Typical values of these quantities for biological soft tissue as a function of the x-ray energy $E$ can be found in Fig. 1.2, in which a model protein with the chemical formula $\mathrm{H}_{50} \mathrm{C}_{30} \mathrm{~N}_{9} \mathrm{O}_{10} \mathrm{~S}_{1}$ and a mass density of $1.35 \frac{\mathrm{g}}{\mathrm{cm}^{3}}$ was considered to emulate the behavior of soft tissue [76]. The energy of the x-rays is correlated to the wave length $\lambda$ via [149]

$$
E=\frac{h c}{\lambda}
$$

with the Planck constant $h=6.626 \cdot 10^{-34} \mathrm{Js}$ and the vacuum wave velocity $c$. Note that in this thesis, only hard x-rays, i.e., x-rays with an energy $E \gtrsim 5 \mathrm{keV}$, are used.

As can be recognized in the left panel of Fig. 1.2, rapid changes of the extinction coefficient $\beta_{\omega}(\mathbf{r})$ can arise, leading to sharp discontinuities in the absorption spectrum, so-called absorption edges. These discontinuities occur at x-ray energies correlated to the binding energies of the shell electrons. Away from absorption edges, the refractive index decrement $\delta_{\omega}(\mathbf{r})$ can be expressed by means of the electron density $\rho_{e}(\mathbf{r})$ via $[28]$

$$
\delta_{\omega}(\mathbf{r}) \simeq \frac{2 \pi r_{e}}{k^{2}} \rho_{e}(\mathbf{r})
$$


with the Thompson scattering length $r_{e} \simeq 2.818 \cdot 10^{-15} \mathrm{~m}$. A similar relation between the extinction coefficient $\beta_{\omega}(\mathbf{r})$ and the electron density cannot be derived. However, for a single material element and away from absorption edges it relates to the atomic number $Z$ and the x-ray energy $E$ via [1]

$$
\beta_{\omega}(\mathbf{r}) \propto Z^{4} E^{-4}
$$

Note that for hard x-rays and soft tissue the refractive index decrement is orders of magnitude larger compared to the extinction coefficient (cf. the right panel of Fig. 1.2), leading to a stronger effect of the sample on the phase of the illuminating wave field than on its amplitude. This behavior increases the contrast in propagationbased imaging of biological samples compared to the classical absorption-based approach.

\subsubsection{The projection approximation}

In this section, an expression for a wave field traversing a sufficiently thin object of thickness $d$ will be derived. As a starting point the inhomogeneous paraxial wave equation (1.51) is considered. As the real and imaginary part of the refractive index, $\delta_{\omega}(\mathbf{r})$ and $\beta_{\omega}(\mathbf{r})$, respectively, are several orders of magnitude below unity, quadratic terms in $n_{\omega}(\mathbf{r})^{2}$ can be omitted, leading to

$$
n_{\omega}(\mathbf{r})^{2} \simeq 1+2 i \beta_{\omega}(\mathbf{r})-2 \delta_{\omega}(\mathbf{r})
$$

Inserting this into eqn. (1.51) yields

$$
\left(2 i k \partial_{z}+\nabla_{\perp}^{2}+2 k^{2}\left(i \beta_{\omega}(\mathbf{r})-\delta_{\omega}(\mathbf{r})\right)\right) \psi_{\omega}^{\prime}(\mathbf{r})=0
$$

The projection approximation, which corresponds to the limit of geometrical optics, assumes that scattering effects inside the specimen can be neglected, so that the wave field directly behind the specimen is solely determined by the accumulated phase shift and absorption along unscattered rays through the sample (cf. Fig. 1.3). The validity of this approximation can be estimated in terms of the ratio between the wavelength and the size of the smallest lateral feature of interest $\Delta r$ as well as the thickness of the specimen $d$, yielding [187]

$$
\sqrt{\frac{\lambda d}{2}}<\Delta r
$$




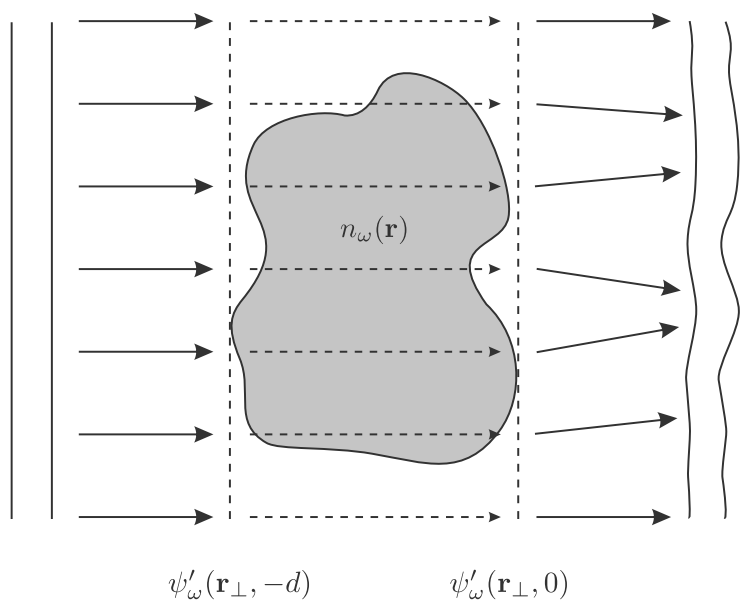

Figure 1.3: Illustration of the projection approximation. The x-ray beam hits the sample from the left and phase and amplitude changes are accumulated along unscattered rays (dashed lines), determining the exit wave field directly behind the sample. Subsequent free-space propagation then leads to interference effects of the disturbed wave fronts and thus to measurable intensity modulations, encoding the sample's amplitude and phase.

Reformulating this equation to

$$
d<\frac{2 \Delta r^{2}}{\lambda}
$$

gives an estimate for the maximum object thickness for which the projection approximation is still valid. Note that the definition of this criterion is not strict and prefactors vary in literature.

The projection approximation can be used to further simplify the inhomogeneous paraxial wave equation as it justifies the neglect of the transverse Laplacian in eqn. (1.57), leading to the differential equation

$$
\left(2 i k \partial_{z}+2 k^{2}\left(i \beta_{\omega}(\mathbf{r})-\delta_{\omega}(\mathbf{r})\right)\right) \psi_{\omega}^{\prime}(\mathbf{r})=0 .
$$

Integration along $z$ yields [125]

$$
\psi_{\omega}^{\prime}\left(\mathbf{r}_{\perp}, 0\right)=\psi_{0}^{\prime} \cdot e^{i k \int_{-d}^{0}\left(i \beta_{\omega}(\mathbf{r})-\delta_{\omega}(\mathbf{r})\right) \mathrm{d} z},
$$

with the given illumination function $\psi_{\omega}^{\prime}\left(\mathbf{r}_{\perp},-d\right)=\psi_{0}^{\prime}$. Inserting this into the ansatz for the paraxial wave field, $\psi_{\omega}(\mathbf{r})=\psi_{\omega}^{\prime}(\mathbf{r}) e^{i k z}$, gives an expression for the 
wave field directly behind the object or exit wave

$$
\psi_{\omega}\left(\mathbf{r}_{\perp}, 0\right)=e^{i k d} \cdot \psi_{0}^{\prime} \cdot e^{i k \int_{-d}^{0}\left(i \beta_{\omega}(\mathbf{r})-\delta_{\omega}(\mathbf{r})\right) \mathrm{d} z} .
$$

For convenience it can be written as the product of the probe function

$$
P_{\omega}\left(\mathbf{r}_{\perp}\right):=\psi_{\omega}^{\prime}\left(\mathbf{r}_{\perp},-d\right)=\psi_{0}^{\prime}
$$

and the object transmission function

$$
O_{\omega}\left(\mathbf{r}_{\perp}\right):=e^{-i k \bar{\delta}_{\omega}\left(\mathbf{r}_{\perp}\right)} \cdot e^{-k \bar{\beta}_{\omega}\left(\mathbf{r}_{\perp}\right)},
$$

where the effective refractive index decrement $\bar{\delta}_{\omega}\left(\mathbf{r}_{\perp}\right)=\int_{-d}^{0} \delta_{\omega}(\mathbf{r}) \mathrm{d} z$ and the effective extinction coefficient $\bar{\beta}_{\omega}\left(\mathbf{r}_{\perp}\right)=\int_{-d}^{0} \beta_{\omega}(\mathbf{r}) \mathrm{d} z$ were introduced.

Thus, the resulting exit wave reads

$$
\psi_{\omega}\left(\mathbf{r}_{\perp}, 0\right)=P_{\omega}\left(\mathbf{r}_{\perp}\right) O_{\omega}\left(\mathbf{r}_{\perp}\right) e^{i k d}
$$

From these equations, the phase and amplitude shifts imparted on the incident wave field when traversing the object can be deduced. According to eqn. (1.62), the phase shift $\phi_{\omega}\left(\mathbf{r}_{\perp}\right):=\phi_{\omega}\left(\mathbf{r}_{\perp}, 0\right)$ is given by

$$
\phi_{\omega}\left(\mathbf{r}_{\perp}\right)=-k \int_{-d}^{0} \delta_{\omega}(\mathbf{r}) \mathrm{d} z=-k \bar{\delta}_{\omega}\left(\mathbf{r}_{\perp}\right),
$$

whereas the intensity $I_{\omega}\left(\mathbf{r}_{\perp}\right)$, calculated by the square modulus of the exit wave $\left|\psi_{\omega}\left(\mathbf{r}_{\perp}, 0\right)\right|^{2}$, is

$$
I_{\omega}\left(\mathbf{r}_{\perp}, 0\right)=e^{-2 k \int_{-d}^{0} \beta_{\omega}(\mathbf{r}) \mathrm{d} z} I_{\omega}\left(\mathbf{r}_{\perp},-d\right)=e^{-2 k \bar{\beta}_{\omega}\left(\mathbf{r}_{\perp}\right)} I_{\omega}\left(\mathbf{r}_{\perp},-d\right) .
$$

Hence, if the probe $P_{\omega}\left(\mathbf{r}_{\perp}\right)$ is known a priori or can be reconstructed from the measurement, e.g., by near-field ptychography $[143,160]$, the projected refractive index of the object can be obtained by determining the amplitude and phase shift of the exit wave. The combination with tomography, which will be treated in section 3 , enables the reconstruction of the spatial distribution of $\delta_{\omega}(\mathbf{r})$ and $\beta_{\omega}(\mathbf{r})$. For the special case of a homogeneous object with constant refractive index decrement $\delta_{\omega}(\mathbf{r})=\delta_{\omega}$ and extinction coefficient $\beta_{\omega}(\mathbf{r})=\beta_{\omega}$, the expressions for the 
phase shift and intensity transform into

$$
\phi_{\omega}\left(\mathbf{r}_{\perp}\right)=-k \delta_{\omega} T\left(\mathbf{r}_{\perp}\right)
$$

and

$$
I_{\omega}\left(\mathbf{r}_{\perp}, 0\right)=e^{-\mu_{\omega} T\left(\mathbf{r}_{\perp}\right)} I_{\omega}\left(\mathbf{r}_{\perp},-d\right)
$$

with the projected thickness $T\left(\mathbf{r}_{\perp}\right)$ and the linear attenuation coefficient $\mu_{\omega}=2 k \beta_{\omega}$. The latter expression is known as Beer's law of absorption [125].

\subsection{Contrast formation}

In the previous sections it was shown that a quantitative analysis of the sample's electron density distribution requires the knowledge of the real part of the refractive index, the decrement $\delta_{\omega}(\mathbf{r})$. It can be determined from the phase shift of the object transmission function, a property which is not directly accessible using $\mathrm{x}$-ray detection systems. However, due to the interference of the disturbed wave fronts behind the sample, phase and amplitude information are encoded in the measurable intensity of the wave field. With the equations derived in the previous sections, governing free-space propagation and the interaction with matter, expressions for the intensity distribution of the wave field at a given propagation distance $z$ behind the sample can be obtained. This provides the basis for the reconstruction of the phase information from the intensity images, as further outlined in chapter 2.

\subsubsection{Transport of intensity equation}

To obtain an expression for the intensity $I_{\omega}(\mathbf{r})$ at propagation distance $z$, the wave field $\psi_{\omega}^{\prime}(\mathbf{r})$ is rewritten in terms of its real-valued intensity $I_{\omega}(\mathbf{r})=\left|\psi_{\omega}^{\prime}(\mathbf{r})\right|^{2}$ and phase distribution $\phi_{\omega}(\mathbf{r})$

$$
\psi_{\omega}^{\prime}(\mathbf{r})=\sqrt{I_{\omega}(\mathbf{r})} e^{i \phi_{\omega}(\mathbf{r})} .
$$

Inserting this into the paraxial wave equation (1.25), performing the differentiations and rearranging the terms leads to

$$
\nabla_{\perp}\left(I_{\omega}(\mathbf{r}) \nabla_{\perp} \phi_{\omega}(\mathbf{r})\right)=-k \partial_{z} I_{\omega}(\mathbf{r})
$$


for the imaginary part of the resulting equation, known as the transport of intensity equation (TIE) [125, 163]. This continuity equation states that the change in intensity $\partial I_{\omega}(\mathbf{r})$ upon propagation of a paraxial wave field along an infinitesimal distance $\partial z$ can be calculated provided that the intensity and phase distribution of the initial wave field are known.

The TIE can be further simplified for the special case of small propagation distances $z=\Delta z$ and weak objects. The intensity directly behind the object is given by $I_{\omega}\left(\mathbf{r}_{\perp}, 0\right)$. Inserting this into the TIE and approximating the partial derivative along $z$ as a difference quotient yields

$$
\nabla_{\perp} I_{\omega}\left(\mathbf{r}_{\perp}, 0\right) \cdot \nabla_{\perp} \phi_{\omega}\left(\mathbf{r}_{\perp}\right)+I_{\omega}\left(\mathbf{r}_{\perp}, 0\right) \nabla_{\perp}^{2} \phi_{\omega}\left(\mathbf{r}_{\perp}\right)=-k \frac{I_{\omega}\left(\mathbf{r}_{\perp}, \Delta z\right)-I_{\omega}\left(\mathbf{r}_{\perp}, 0\right)}{\Delta z} .
$$

The assumption of an object with weak and almost homogeneous absorption for which the first term in this equation, $\nabla_{\perp} I_{\omega}\left(\mathbf{r}_{\perp}, 0\right) \cdot \nabla_{\perp} \phi_{\omega}(\mathbf{r})$, can be neglected, leads to [125]

$$
\frac{I_{\omega}\left(\mathbf{r}_{\perp}, \Delta z\right)}{I_{\omega}\left(\mathbf{r}_{\perp}, 0\right)}=1-\frac{\Delta z}{k} \nabla_{\perp}^{2} \phi_{\omega}\left(\mathbf{r}_{\perp}\right)
$$

As this equation is only valid in the limit of small propagation distances, it can be referred to as the near-field TIE.

For the special case of pure phase objects, the intensity distribution behind the object corresponds to the intensity of the illuminating wave field. By assuming a plane wave with constant intensity $I_{0}$ as probe, this leads to the simplified expression

$$
\frac{I_{\omega}\left(\mathbf{r}_{\perp}, \Delta z\right)}{I_{0}}=1-\frac{\Delta z}{k} \nabla_{\perp}^{2} \phi_{\omega}\left(\mathbf{r}_{\perp}\right) .
$$

Note that in the near-field approximation, the intensity solely depends on the Laplacian, i.e., the curvature, of the phase distribution $\phi_{\omega}(\mathbf{r})$. Hence, phase contrast in this regime is mainly visible as edge enhancement. Importantly, the x-ray energy-dependent wave vector $k$ only acts as a scaling factor, so that for a polychromatic illumination the intensity distributions of the single energy contributions, which are incoherently superimposed in the detection plane, are comparable and phase effects will still occur in the resulting intensity image. As it is even applicable in the case of only partially coherent light [126, 190], this imaging modality is also compatible with microfocus laboratory sources (the partial coherence of 
laboratory sources will be further considered in section 1.4).

\subsubsection{Contrast transfer function}

The contrast transfer function gives an expression for the intensity at distance $z$ behind the object which is valid for an object with weak absorption and a slowly varying phase. It can be obtained via a propagation of the exit wave $\psi_{\omega}\left(\mathbf{r}_{\perp}\right):=$ $\psi_{\omega}\left(\mathbf{r}_{\perp}, 0\right)=P_{\omega}\left(\mathbf{r}_{\perp}\right) O_{\omega}\left(\mathbf{r}_{\perp}\right) e^{i k d}$, which is given by the convolution with the real space Fresnel propagator $H^{(F)}(1.37)$. For simplicity, this operation will be carried out in one dimension, without loss of generality along $x$, but the two-dimensional derivation would be analogous.

The Fourier transform of the intensity $I_{\omega}(x, z)=\left|\psi_{\omega}(x, z)\right|^{2}=\psi_{\omega}(x, z) \cdot \psi_{\omega}^{*}(x, z)$, with $\psi_{\omega}^{*}(x, z)$ being the complex conjugate of $\psi_{\omega}(x, z)$, yields

$$
\begin{aligned}
\hat{I}_{\omega}\left(k_{x}, z\right) & =\frac{1}{\lambda z} \frac{1}{\sqrt{2 \pi}} \int_{\mathbb{R}} \mathrm{d} x e^{-i k_{x} x} \int_{\mathbb{R}} \mathrm{d} x^{\prime} e^{\frac{i \pi\left(x-x^{\prime}\right)^{2}}{\lambda z}} \psi_{\omega}\left(x^{\prime}\right) \int_{\mathbb{R}} \mathrm{d} x^{\prime \prime} e^{-\frac{i \pi\left(x-x^{\prime \prime}\right)^{2}}{\lambda z}} \psi_{\omega}^{*}\left(x^{\prime \prime}\right) \\
& =e^{-\frac{i \lambda z k_{x}^{2}}{4 \pi}} \frac{1}{\sqrt{2 \pi}} \int_{\mathbb{R}} \mathrm{d} x^{\prime} e^{-i k_{x} x^{\prime}} \psi_{\omega}\left(x^{\prime}\right) \psi_{\omega}^{*}\left(x^{\prime}+\frac{\lambda z k_{x}}{2 \pi}\right) .
\end{aligned}
$$

A detailed description of the mathematical transformations involved in this step can be found in section A.2 in the appendix. A plane wave illumination with intensity $I_{0}$ leads to

$$
\frac{\hat{I}_{\omega}\left(k_{x}, z\right)}{I_{0}}=e^{-\frac{i \lambda z k_{x}^{2}}{4 \pi}} \frac{1}{\sqrt{2 \pi}} \int_{\mathbb{R}} \mathrm{d} x^{\prime} e^{-i k_{x} x^{\prime}} O_{\omega}\left(x^{\prime}\right) O_{\omega}^{*}\left(x^{\prime}+\frac{\lambda z k_{x}}{2 \pi}\right),
$$

with the object transmission function $O_{\omega}(x)=e^{-i k \bar{\delta}_{\omega}(x)-k \bar{\beta}_{\omega}(x)}$. The product of the transmission functions can be approximated to the first order by assuming a slowly varying phase $\left(\left|\Delta \phi_{\omega}(x)\right|=\left|k \Delta \bar{\delta}_{\omega}(x)\right| \ll 1\right)$ and weak absorption $\left(\xi_{\omega}(x):=\right.$ $\left.k \bar{\beta}_{\omega}(k) \ll 1\right)$

$$
\begin{aligned}
& O_{\omega}\left(x^{\prime}\right) O_{\omega}^{*}\left(x^{\prime}+\frac{\lambda z k_{x}}{2 \pi}\right)= e^{i\left(\phi_{\omega}\left(x^{\prime}\right)-\phi_{\omega}\left(x^{\prime}+\frac{\lambda z k_{x}}{2 \pi}\right)\right)-\left(\xi_{\omega}\left(x^{\prime}\right)+\xi_{\omega}\left(x^{\prime}+\frac{\lambda z k_{x}}{2 \pi}\right)\right)} \\
& \simeq 1+i\left(\phi_{\omega}\left(x^{\prime}\right)-\phi_{\omega}\left(x^{\prime}+\frac{\lambda z k_{x}}{2 \pi}\right)\right) \\
&-\left(\xi_{\omega}\left(x^{\prime}\right)+\xi_{\omega}\left(x^{\prime}+\frac{\lambda z k_{x}}{2 \pi}\right)\right) .
\end{aligned}
$$


Inserting this into eqn. (1.77) and performing several transformations (for a detailed description see again section A.2 in the appendix) yields the simple expression

$$
\frac{\hat{I}_{\omega}\left(k_{x}, z\right)}{I_{0}}=\sqrt{2 \pi} \delta_{D}\left(k_{x}\right)+2 \sin \chi \hat{\phi}_{\omega}\left(k_{x}\right)-2 \cos \chi \hat{\xi}_{\omega}\left(k_{x}\right)
$$

with the Dirac delta distribution $\delta_{D}$ and $\chi=\frac{\lambda z k_{x}^{2}}{4 \pi}$.

Due to the equivalent derivation in the $y$-direction it can be easily extended to

$$
\frac{\hat{I}_{\omega}\left(\mathbf{k}_{\perp}, z\right)}{I_{0}}=2 \pi \delta_{D}\left(\mathbf{k}_{\perp}\right)+2 \sin \chi \hat{\phi}_{\omega}\left(\mathbf{k}_{\perp}\right)-2 \cos \chi \hat{\xi}_{\omega}\left(\mathbf{k}_{\perp}\right) .
$$

This equation is known as the contrast transfer function (CTF) [28, 58, 195]. It states that directly behind the object $(z=0)$ the intensity distribution is given by the primary beam, represented by the Dirac delta distribution in reciprocal space, reduced by the attenuation coefficient $\hat{\xi}_{\omega}\left(\mathbf{r}_{\perp}\right)$, as also predicted by the Lambert-Beer law approximated for weakly absorbing objects. For propagation distances $z>0$, phase and amplitude, filtered by $\sin \chi$ and $\cos \chi$, respectively, contribute in an additive manner to the intensity in Fourier space. The two filter functions are referred to as the phase contrast transfer function (PCTF) and the amplitude contrast transfer function (ACTF). In Fig. 1.4 their behavior is shown as a function of the reduced spatial frequency $\sqrt{\lambda z} k_{\perp}$. As the propagation distance increases, contrast transfer of the phase map gets stronger due to the related rising slope of the $\sin \chi$ filter. The oscillatory nature of the trigonometric functions leads to specific spatial frequencies with modulus $k_{\perp}$, at which the measured intensity distribution contains no information on the object's phase or amplitude, as $\sin \chi$ or $\cos \chi$ vanishes, respectively.

For a pure phase object $\left(\xi_{\omega}\left(\mathbf{r}_{\perp}\right)=0\right)$, the CTF simplifies to

$$
\frac{\hat{I}_{\omega}\left(\mathbf{k}_{\perp}, z\right)}{I_{0}}=2 \pi \delta_{D}\left(\mathbf{k}_{\perp}\right)+2 \sin \chi \hat{\phi}_{\omega}\left(\mathbf{k}_{\perp}\right) .
$$

Assuming a small propagation distance $z$, so that the approximation $\sin \chi \simeq \chi$ holds, results in the near-field $C T F$

$$
\begin{aligned}
\frac{\hat{I}_{\omega}\left(\mathbf{k}_{\perp}, z\right)}{I_{0}} & \simeq 2 \pi \delta_{D}\left(\mathbf{k}_{\perp}\right)+\frac{\lambda z k_{\perp}^{2}}{2 \pi} \hat{\phi}_{\omega}\left(\mathbf{k}_{\perp}\right) \\
\stackrel{\mathcal{F}^{-1}}{\Rightarrow} \frac{I_{\omega}\left(\mathbf{r}_{\perp}, z\right)}{I_{0}} & =1-\frac{z}{k} \nabla_{\perp}^{2} \phi_{\omega}\left(\mathbf{r}_{\perp}\right) .
\end{aligned}
$$




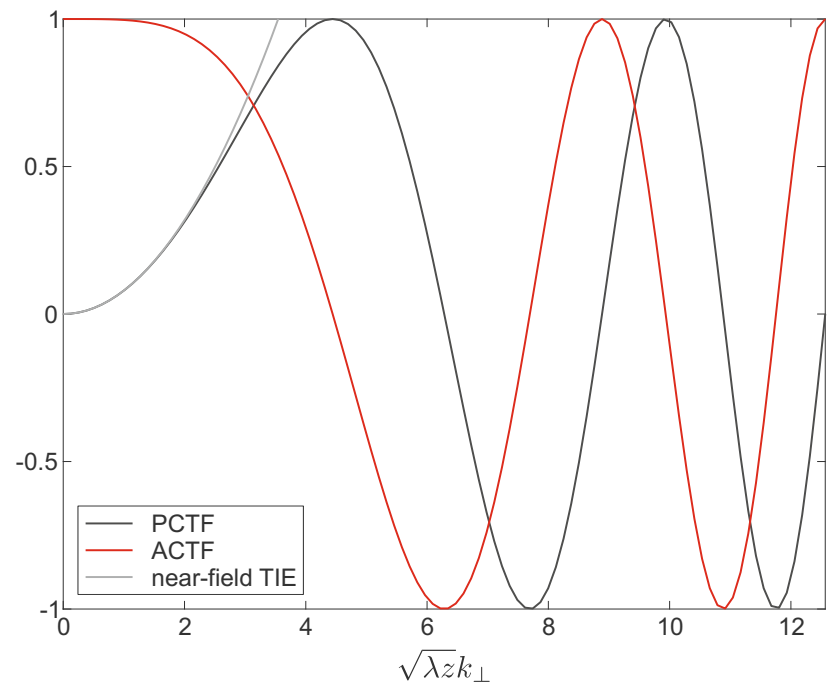

Figure 1.4: Phase and amplitude contrast transfer function (PCTF and ACTF, respectively) together with the near-field transport of intensity equation (TIE) for a pure phase object. With increasing propagation distance, contrast transfer of the phase distribution rises while the absorption shows the inverse behavior. Due to the oscillatory nature of the trigonometric functions, information about the object's amplitude or phase is missing in the measured intensity images at specific spatial frequencies $k_{\perp}$. In the limit of short propagation distances and pure phase objects, the near-field TIE and the PCTF are equivalent and follow a quadratic behavior.

In the second step, the inverse Fourier transform was performed by using the socalled pseudo-differential-operators (A.4), yielding $\mathcal{F}_{\perp}^{-1}\left[i k_{\perp} \hat{f}\left(\mathbf{k}_{\perp}\right)\right]=\nabla_{\perp} f\left(\mathbf{r}_{\perp}\right)$, and the wave length $\lambda$ was replaced by $\frac{2 \pi}{k}$. Thus, both the TIE and the CTF approach provide the same expression for the intensity distribution of a pure phase object at small propagation distances. This can also be recognized in Fig. 1.4, as the near-field TIE (light gray) and the PCTF (dark gray) coincide for small values of the reduced spatial frequency $\sqrt{\lambda z} k_{\perp}$.

\subsubsection{Imaging regimes}

To further evaluate the influence of the propagation distance on contrast formation, intensity distributions for several Fresnel numbers $F=\frac{a^{2}}{\lambda z}$ are simulated. As paraxial propagation solely depends on this combination of object feature size, wave length and propagation distance, it can be used to distinguish between different imaging regimes. In numerical simulations, discretization limits the size of the smallest feature to the size of a single pixel $p$, so that $F=\frac{p^{2}}{\lambda z}$ is assumed for 
the Fresnel number. The amplitude and phase distribution of the test object used for simulation is shown in Fig. 1.5 in the first row. In the upper part it consists of purely absorbing structures (the A's), mixed objects (the M's) with a constant $\frac{\beta}{\delta}$-ratio of 0.2 and purely phase-shifting structures (the P's) with different object sizes and surrounding media. The lower part emulates different kinds of biological tissue. In the image on the left, an original drawing of Ramon y Cajal depicting two Purkinje cells from a pigeon cerebellum [30], biological tissue with a specific staining of certain features using a radiocontrast agent, e.g., heavy metals with a large atomic number $Z$, is simulated with a $\frac{\beta}{\delta}$-ratio of 0.1 . The image on the right approximates unstained soft tissue with a $\frac{\beta}{\delta}$-ratio of 0.001 .

For the simulation, a pixel size of $1 \mu \mathrm{m}$, a wavelength of $1 \AA$ and a maximum projected object thickness of $10 \mu \mathrm{m}$ were assumed. Fig. 1.5 shows the results for the Fresnel numbers $F \in\{10000,0.5,0.03,0.001\}$. The first case corresponds to the limit of geometrical optics in which no free-space propagation occurs and the resulting intensity distribution solely depends on the absorbing structures of the test object. The mixed objects with a rather high $\frac{\delta}{\beta}$-ratio, i.e., the M's and the stained tissue, are already visible in this imaging regime, however, at low contrast. The unstained soft tissue shows almost no absorption and especially with noisy data, the imaging of this kind of tissue is not feasible in this regime.

For Fresnel numbers $F \simeq 1$, interference effects start to occur and phase contrast is visible in the form of edge enhancement, as predicted by the near-field TIE. As in this imaging regime the intensity distribution still resembles the original object, it is referred to as the direct-contrast regime. However, contrast transfer for low spatial frequencies is still weak (cf. Fig. 1.4) and especially objects embedded in a medium with comparable index of refraction (e.g., soft tissue embedded in water) are barely visible. Hence, to increase contrast in this regime, samples have to either be embedded in a medium with considerably lower index of refraction or stained with radiocontrast agents.

Smaller Fresnel numbers $F<1$ lead to an increased contrast in the intensity images. In this imaging regime, referred to as the holographic regime, oscillatory variations, so-called fringes, occur which lead to a decreased resemblance of the intensity to the original object. Thus, in order to obtain information about the original structure, the phase information has to be reconstructed from the intensity distributions using suitable phase-retrieval algorithms, further described in chapter 2. In this imaging regime, contrast transfer is high for all spatial frequencies and also embedded specimens deliver a considerable contrast, so that imaging of unstained hydrated tissue is possible. Note that especially for small Fresnel num- 
bers $(F=0.001)$, i.e., the deep holographic regime, the approximate structure of large scale features, e.g., the letters in the upper row of the test object, can still be recognized as these exhibit a larger Fresnel number, shifting the imaging mode more towards the near holographic regime.

\subsubsection{Fresnel scaling theorem}

In the considerations so far, the probe function $P_{\omega}\left(\mathbf{r}_{\perp}\right)$ was assumed to be a plane wave, whereas in many cases imaging experiments are carried out in a conebeam geometry using a divergent beam. However, by assuming a spherical wave $\frac{e^{i k r}}{r}$ as illumination and modifying the equations governing free-space propagation behind the object, an effective parallel-beam geometry can be derived, in which the previously found relations are still valid.

The starting point is a spherical wave with its origin at a distance $z_{01}$ before the sample, which is located at $z=0$ (cf. Fig. 1.6). Assuming the distance $z_{01}$ large enough for the paraxial approximation to hold, the wave function in the exit plane can be written as

$$
\begin{aligned}
\psi_{\omega}\left(\mathbf{r}_{\perp}, 0\right) & =\frac{e^{i k\left|\mathbf{r}-\mathbf{r}_{0}\right|}}{\left|\mathbf{r}-\mathbf{r}_{0}\right|} \cdot O_{\omega}\left(\mathbf{r}_{\perp}\right) e^{i k d} \\
& \simeq \frac{e^{i k z_{01}} e^{\frac{i k}{2 z_{01}} r_{\perp}^{2}}}{z_{01}} \cdot O_{\omega}\left(\mathbf{r}_{\perp}\right) e^{i k d}
\end{aligned}
$$

known as a parabolic beam. For simplicity, the constant phase factor $e^{i k z_{01}}$ will be omitted in the following. To obtain the wave field at a propagation distance $z=z_{12}$ behind the sample, this expression for the exit wave is propagated via a convolution with the real space Fresnel propagator (1.37)

$$
\psi_{\omega}\left(\mathbf{r}_{\perp}, z_{12}\right)=\frac{C\left(z_{12}\right)}{z_{01}} \int_{\mathbb{R}^{2}} e^{\frac{i k}{2} r_{\perp}^{\prime 2}\left(\frac{1}{z_{12}}+\frac{1}{z_{01}}\right)} e^{-\frac{i k}{z_{12}} \mathbf{r}_{\perp} \cdot \mathbf{r}_{\perp}^{\prime}} \cdot O_{\omega}\left(\mathbf{r}_{\perp}^{\prime}\right) e^{i k d} \mathrm{~d} \mathbf{r}_{\perp}^{\prime},
$$

where $C\left(z_{12}\right)=\frac{e^{i k z_{12}}}{i \lambda z_{12}} e^{\frac{i k}{2 z_{12}} r_{\perp}^{2}}$ was introduced. With the effective propagation distance

$$
\frac{1}{z_{\mathrm{eff}}}:=\frac{1}{z_{12}}+\frac{1}{z_{01}}=\frac{M}{z_{12}}
$$




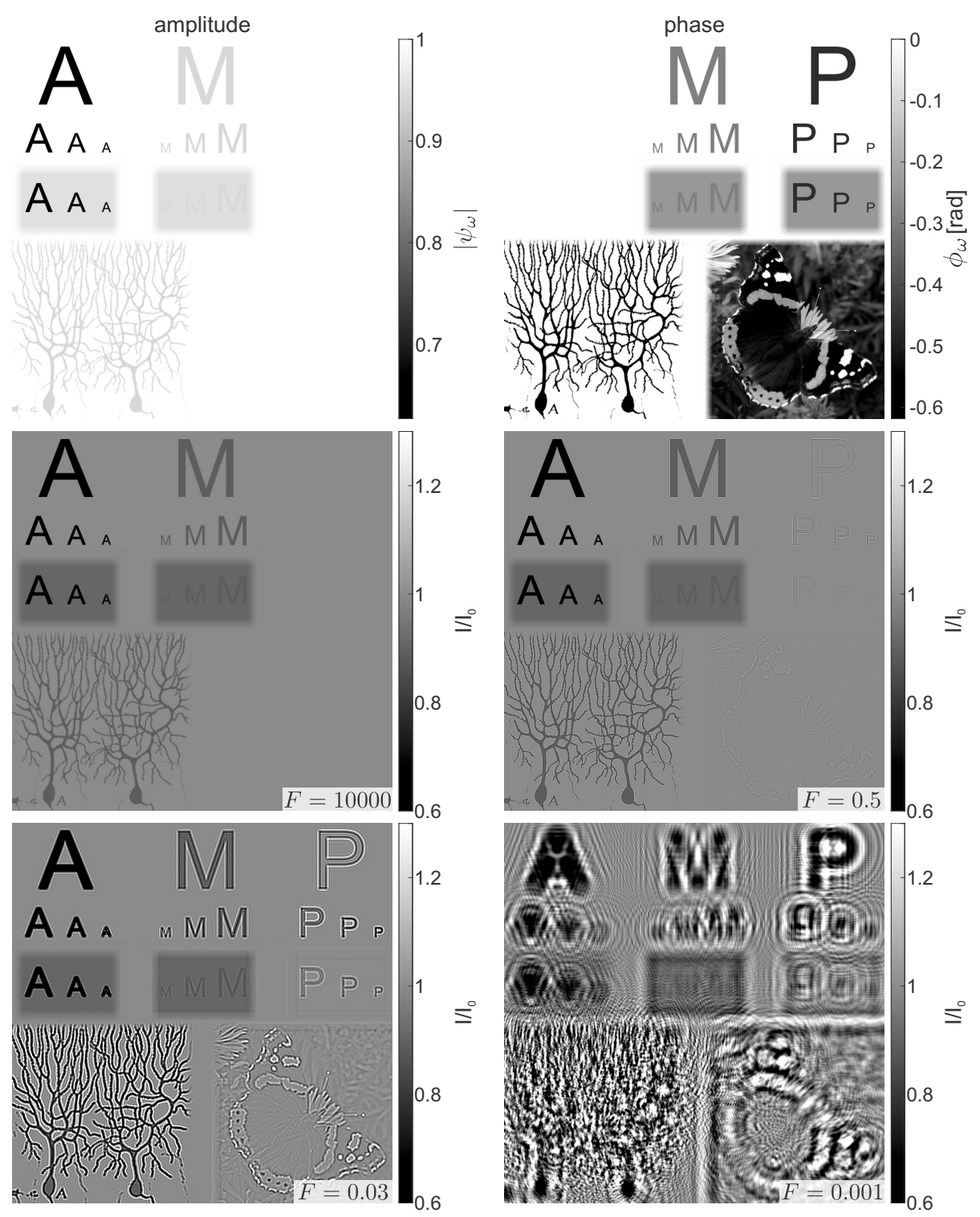

Figure 1.5: Simulated intensity distributions for different Fresnel numbers $F$. The amplitude and phase distribution of the phantom used for the simulations are shown at the top, containing purely absorbing ( $A$ 's) and purely phase shifting ( $P$ 's) structures as well as mixed objects with varying $\frac{\beta}{\delta}$-ratios (M's: 0.2, lower left: 0.1, lower right: 0.001). Large Fresnel numbers correspond to the regime of geometrical optics in which only absorption effects occur. With decreasing Fresnel number, the phase contrast transfer increases and typical interference patterns evolve, showing edge enhancement $(F \simeq 1)$ or oscillatory variations with multiple interference fringes $(F<1)$. 

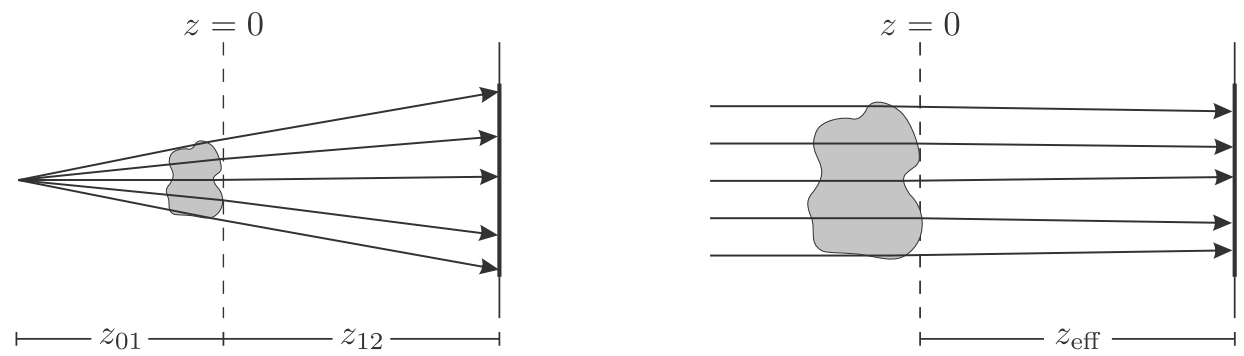

Figure 1.6: Illustration of the Fresnel scaling theorem. A cone-beam experiment using a divergent beam with a source-to-sample distance $z_{01}$ and a sample-to-detector distance $z_{12}$, leading to a geometrical magnification of $M=\frac{z_{01}+z_{12}}{z_{01}}$, can be described in an effective parallel-beam geometry with effective sample-to-detector distance $z_{\text {eff }}=\frac{z_{12}}{M}$ and effective pixel size $p_{\text {eff }}=\frac{p}{M}$. This enables imaging of the sample at varying resolution and field of view using the previously derived relations.

and the geometrical magnification

$$
M=\frac{z_{01}+z_{12}}{z_{01}},
$$

this can be transformed to

$$
\psi_{\omega}\left(\mathbf{r}_{\perp}, z_{12}\right)=\frac{C\left(z_{12}\right)}{z_{01}} \int_{\mathbb{R}^{2}} e^{\frac{i k}{2 z_{\mathrm{eff}}} r_{\perp}^{\prime 2}} e^{-\frac{i k}{z_{\mathrm{eff}}} \frac{\mathbf{r}_{\perp}}{M} \cdot \mathbf{r}_{\perp}^{\prime}} \cdot O_{\omega}\left(\mathbf{r}_{\perp}^{\prime}\right) e^{i k d} \mathrm{~d} \mathbf{r}_{\perp}^{\prime}
$$

According to eqn. (1.39), the propagated wave field at distance $z_{\text {eff }}$ and lateral position $\frac{\mathbf{r}_{\perp}}{M}$ obtained via illumination by a plane wave $P_{\omega}(\mathbf{r})=\frac{1}{z_{01}} e^{i k_{z} z}$, leading to the same intensity in the object plane as illumination by a spherical wave, is given by

$$
\psi_{\omega}^{\mathrm{PW}}\left(\frac{\mathbf{r}_{\perp}}{M}, z_{\mathrm{eff}}\right)=\frac{C\left(z_{\mathrm{eff}}\right)}{z_{01}} \int_{\mathbb{R}^{2}} e^{\frac{i k}{2 z_{\mathrm{eff}}} r_{\perp}^{\prime 2}} e^{-\frac{i k}{z_{\mathrm{eff}}} \frac{\mathbf{r}_{\perp}}{M} \cdot \mathbf{r}_{\perp}^{\prime}} \cdot O_{\omega}\left(\mathbf{r}_{\perp}^{\prime}\right) e^{i k d} \mathrm{~d} \mathbf{r}_{\perp}^{\prime} .
$$

Hence, with $C\left(z_{12}\right)=C\left(M \cdot z_{\mathrm{eff}}\right)=M \cdot C\left(z_{\mathrm{eff}}\right)$, the intensity distributions of the propagated wave fields are related via

$$
I_{\omega}\left(\mathbf{r}_{\perp}, z_{12}\right)=\frac{1}{M^{2}} I_{\omega}^{\mathrm{PW}}\left(\frac{\mathbf{r}_{\perp}}{M}, z_{\mathrm{eff}}\right)
$$

which is known as the Fresnel scaling theorem [125]. With a divergent beam as illumination, the propagation of the initial wave field can therefore be expressed as a plane wave propagation with the effective propagation distance $z_{\text {eff }}$ (cf. Fig. 1.6) and effective lateral variables $\mathbf{r}_{\perp \text {,eff }}=\frac{r_{\perp}}{M}$. This enables imaging of the sample at 
varying resolution and field of view, as the effective pixel size of the detector can be tuned by changing the magnification of the setup. The magnification also has an influence on the effective Fresnel number $F_{\text {eff }}$, determining the imaging regime in this cone-beam geometry. With $p_{\text {eff }}=\frac{p}{M}$ and $z_{\text {eff }}=\frac{z}{M}$ it converts to

$$
\begin{aligned}
F_{\text {eff }}=\frac{p_{\mathrm{eff}}^{2}}{\lambda z_{\mathrm{eff}}} & =\frac{\left(\frac{p}{M}\right)^{2}}{\lambda \frac{z}{M}} \\
& =\frac{1}{M} \frac{p^{2}}{\lambda z} .
\end{aligned}
$$

Hence, the Fresnel number scales with magnification such that a higher magnification and therefore higher resolution leads to a smaller Fresnel number, shifting the imaging mode more towards the (deep) holographic regime (cf. section 1.3.3).

\subsection{Coherence}

In the considerations so far, a fully coherent illumination was assumed for the free-space propagation of the wave between the object and the detection plane. However, full coherence is not required for propagation-based imaging and depending on the resolution a certain degree of partial coherence is sufficient. As seen in the previous sections, high resolution imaging shifts the imaging mode towards the holographic regime in which phase contrast is visible in the form of several interference fringes around the object. Hence, a relatively high degree of partial coherence is necessary in this regime to resolve the fine fringes. At moderate resolution, where imaging takes place in the direct-contrast regime, already a lower degree of partial coherence is sufficient for the edge-enhancement effects to occur $[126,190]$.

Coherence can be described by means of spatial coherence, which is a measure for the ability of two spatially separated points in a wave to interfere, and temporal coherence, evaluating the correlation of two points in a wave which are separated by time. Temporal coherence is determined by the spectral bandwidth $\frac{\Delta \lambda}{\lambda}$, i.e., the more monochromatic a wave field is, the higher is its temporal coherence. In the case of the direct-contrast regime, where contrast formation is based on the near-field TIE (1.73), the wavelength $\lambda$ acts as a scaling factor. Hence, the effects of temporal coherence are of minor importance. In the holographic regime, contrast formation can be described by the contrast transfer function (1.81). Here, the wavelength $\lambda$ enters the equation in the reduced spatial frequency $\sqrt{\lambda z} k_{\perp}$, influencing the phase and amplitude contrast transfer functions $\sin \chi$ and $\cos \chi$ 
with $\chi=\frac{\lambda z k_{\perp}^{2}}{4 \pi}$, respectively. This leads to a decrease in contrast as the contributions of the single wavelength components are superimposed. In the limiting case $\Delta \lambda=\frac{4 \pi^{2}}{z k_{\perp}^{2}}$, in which the extreme values of $\chi$ differ by $\pi$, they just cancel each other out. Hence, $\frac{\Delta \lambda}{\lambda} \ll \frac{4 \pi^{2}}{\lambda z k_{\perp}^{2}}$ has to hold for all spatial frequencies $k_{\perp}$, in order for the effects of partial temporal coherence to be neglected. This can be rewritten by means of resolution. Let $a$ be the smallest feature within the sample, corresponding to a spatial frequency $k_{\perp, \max }=\frac{2 \pi}{2 a}$. The temporal coherence needed to resolve it is thus given by

$$
\frac{\Delta \lambda}{\lambda} \ll \frac{4 a^{2}}{\lambda z}=4 F
$$

where $F$ is the Fresnel number [50, 147].

Besides temporal coherence, spatial coherence is of great importance for interference to occur. A common measure for spatial coherence is the visibility of fringes in the Young interferometer [125]. Consider the scenario depicted in Fig. 1.7, consisting of an extended incoherent uniform line source $S$ of length $l$ with quasimonochromatic illumination of mean wavelength $\bar{\lambda}$, lying perpendicular to the optical axis $z$. Incoherence in this case means that the wave field at any point on the source is uncorrelated with the wave field at any other point. In a distance $r_{S P}$ from the source, a screen is placed with two identical pinholes $p_{1}$ and $p_{2}$, separated by distance $b$, which lie symmetrically around the optical axis. The interference pattern produced by these pinholes is measured in the detection plane $D$ in a distance $r_{P D}$ from the screen. Fringe visibility in the detection plane is given by [125]

$$
V=\frac{|\sin \varrho|}{\varrho}
$$

with the effective variable $\varrho=\frac{\pi b l}{\lambda r_{S P}}$. A detailed derivation of this relation is given in section A.3 in the appendix. A plot of the visibility as a function of $\varrho$ is shown in Fig. 1.8. It is evident that only for $\varrho=0$ the visibility of the fringes reaches its maximum. In the range $\varrho \leq \pi$, a decrease in $\varrho$ leads to a monotonic decay of the fringe visibility until it is reduced to a minimum at $\varrho=\pi$ and no fringes can be resolved. Due to the oscillatory nature of the sine function, visibility is regained in the case of an ideal line source for larger values of $\varrho$, though at lower magnitude. For the discussion of coherence, only the range $\varrho \leq \pi$ is considered in the following. Assuming constant values of feature distance $b$, wavelength $\bar{\lambda}$ and distance $r_{S P}$ between the source and the screen, an increase in source size $l$ leads to a 


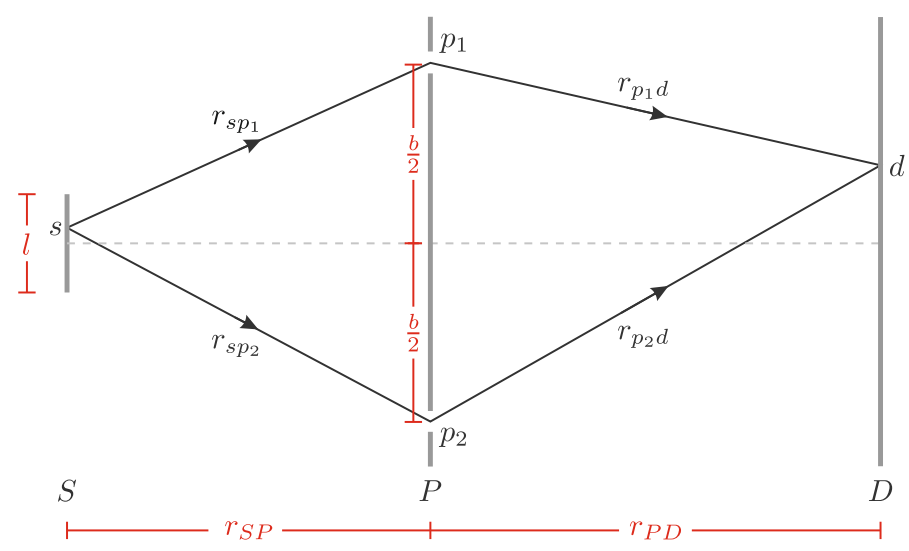

Figure 1.7: Sketch of a Young interferometer. A screen with two identical pinholes $p_{1}$ and $p_{2}$, separated by distance $b$ and located symmetrically around the optical axis, is placed at a distance $r_{S P}$ behind an extended line source of length $l$. The resulting interference pattern is recorded in the detection plane $D$ in a distance $r_{P D}$ from the screen. Rays starting from a point $s$ in the source plane and terminating at a point $d$ in the detection plane can either pass through pinhole $p_{1}$, leading to a path of length $r_{s p_{1}}+r_{p_{1} d}$, or through pinhole $p_{2}$ with a path length of $r_{s p_{2}}+r_{p_{2} d}$.

decrease in fringe visibility. Hence, a larger source size results in a smaller degree of spatial coherence. This can be pictured by assuming that the laterally extended source consists of single point radiators. The interference patterns caused by each point radiator are incoherently superimposed in the detection plane, leading to a blurring of the fringes up to the point where they cancel each other out completely. In the theoretical case of an infinitesimal source size and correspondingly maximum fringe visibility, the wave at the two spatially separated pinholes is said to be fully coherent, whereas for a fringe visibility of zero it is called fully incoherent. The intermediate states of fringe visibility correspond to partial coherence, where a higher fringe visibility indicates a higher degree of coherence at the location of the two pinholes.

Assuming a constant source size $l$ instead of distance $r_{S P}$ between source and screen, it is evident that with increasing $r_{S P}$ the fringe visibility rises. This indicates that the degree of spatial coherence can be increased by moving the screen further away from the source. Directly in front of the source, the beam is fully incoherent over the length scale $b$, whereas coherence is maximized at infinite distance to the source. Hence, the coherence properties of the beam are modified upon freespace propagation and the partial coherence needed for contrast formation can be reached by increasing the distance to the source. 


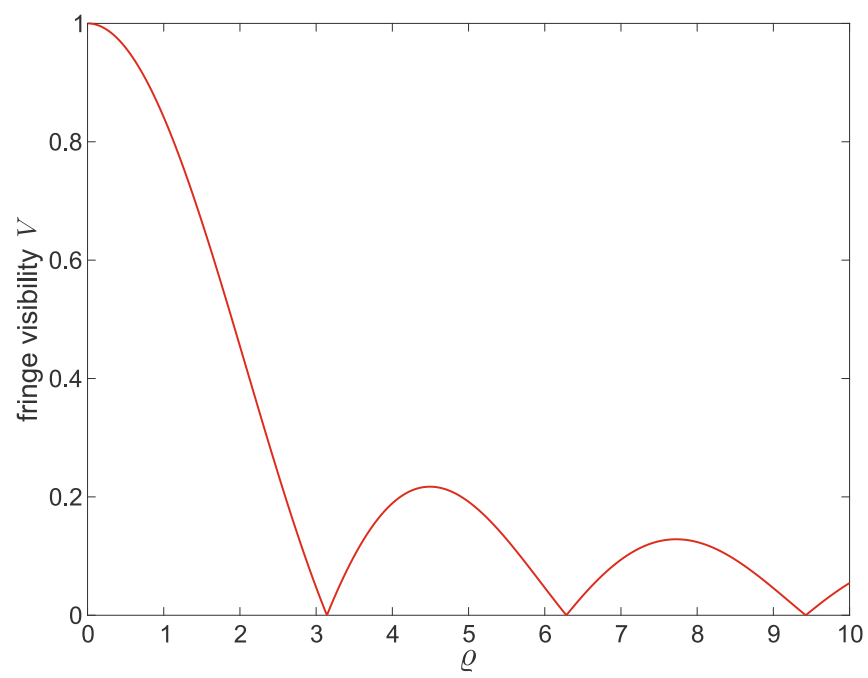

Figure 1.8: Fringe visibility in the Young interferometer as a function of the effective variable $\varrho=\frac{\pi b l}{\bar{\lambda} r_{S P}}$. Only for $\varrho=0$ the visibility of the fringes reaches its maximum, whereas in the range $\varrho \leq \pi$ a decrease in $\varrho$ leads to a monotonic decay of the fringe visibility with a minimum at $\varrho=\pi$, where no fringes can be resolved. Due to the oscillatory nature of the sine function, visibility is regained for larger values of $\varrho$, though at lower magnitude.

\subsection{Resolution estimate}

An important measure in propagation-based phase-contrast imaging is the possible resolution for a given instrument and investigated specimen.

The system resolution can be estimated via imaging of a suitable test pattern, i.e., a structure containing features of specific size. The smallest feature that can still be resolved defines the maximal resolution which might depend, e.g., on the source size and corresponding source blurring effects, the resolution of the detection system or vibrations in the setup. As the test pattern is generally made of metal components which produce a fairly high contrast and remain stable over long periods of time, the system resolution does not necessarily correspond to the resolution obtained in measurements of biological specimen due to a lower contrast or possible sample degradation.

For biological specimens, resolution could be estimated by considering the power spectral density, which is a measure of signal strength as a function of spatial frequency, and determining the transition to the noise level. The resolution is given by the highest spatial frequency for which the signal in the image lies above this level. As the estimated resolution highly depends on the determination of a 
corresponding threshold, this method is best suited for samples showing a distinct transition.

Another possibility is the determination of the edge steepness for features inside the sample. By taking a profile along the edge and fitting an error function to it, the resolution can be estimated via the full width at half maximum (FWHM) of the fit. For biological specimens, this method might pose a challenge as in a lot of cases no sharp edges are present in the tissue. Providing features with a high contrast can be found within the image, this resolution estimate is a measure for the overall system blur. However, if a specific feature within the sample contains an edge with a given FWHM, this does not necessarily mean, that each feature with a comparable size can be resolved, as the noise level as well as the contrast of these features also plays a role. Hence, the edge profile provides an upper limit for the resolution.

A more versatile method for the estimation of resolution is based on the Fourier shell correlation (FSC) which is a standard method in electron microscopy [65, 175]. The FSC is given by the normalized cross-correlation of two datasets over corresponding shells $\mathbf{k}_{i}$ in Fourier space and a measure of their consistency. To determine the resolution of a three-dimensional dataset $D$, it is divided into two independent datasets $D_{1}$ and $D_{2}$ and the FSC as a function of spatial frequency is calculated via

$$
\operatorname{FSC}\left(\mathbf{k}_{i}\right)=\frac{\sum_{\mathbf{k} \in \mathbf{k}_{i}} \hat{D}_{1}(\mathbf{k}) \hat{D}_{2}^{*}(\mathbf{k})}{\sqrt{\sum_{\mathbf{k} \in \mathbf{k}_{i}} \hat{D}_{1}^{2}(\mathbf{k}) \cdot \sum_{\mathbf{k} \in \mathbf{k}_{i}} \hat{D}_{2}^{2}(\mathbf{k})}}
$$

Falling below a certain threshold defines the resolution and different implementations of corresponding threshold curves are discussed in [176]. In the course of this thesis, the so-called 1/2-bit threshold curve is chosen as an estimate for resolution

$$
T_{1 / 2 \text {-bit }}\left(\mathbf{k}_{i}\right)=\frac{0.2071+1.9102 \cdot 1 / \sqrt{n\left(\mathbf{k}_{i}\right)}}{1.2071+0.9102 \cdot 1 / \sqrt{n\left(\mathbf{k}_{i}\right)}},
$$

where $n\left(\mathbf{k}_{i}\right)$ is the number of voxels (a cube in the three-dimensional volume with side length of 1 pixel) contained in a Fourier shell of radius $k_{i}=\left|\mathbf{k}_{i}\right|$. The crossover between the FSC and this threshold curve determines the spatial frequency for which enough information has been collected for interpretation [176]. In order to avoid artifacts originating from truncation of the real space volumes at the edges, which generally leads to an increase of the FSC at higher spatial frequencies, a multiplicative Kaiser-Bessel-window can be applied prior to the Fourier transform 
operation.

Note that the FSC criterion represents an estimate of the overall quality of the experiment and therefore a high noise level or low contrast of the object has a negative influence on the obtained value for the resolution. Noise reduction of the images via a linear filter in Fourier space, e.g., a Gaussian filter, leads to the same intersection point between FSC and threshold curve and hence the same resolution, while the estimation via edge steepness results in reduced values due to blurring effects. 



\section{Solving the phase problem}

In the previous chapter, the concepts of propagation-based imaging and in particular contrast formation were introduced. According to section 1.2, quantitative information about the density distribution of the sample is included in the object transmission function $O_{\omega}\left(\mathbf{r}_{\perp}\right)$ (1.64). Hence, for a given illumination $P_{\omega}\left(\mathbf{r}_{\perp}\right)$, the phase of the wave field directly behind the object is related to its integrated density distribution. However, the only measurable quantity is the intensity distribution in the detection plane $z$

$$
\frac{I_{\omega}\left(\mathbf{r}_{\perp}, z\right)}{I_{0}}=\left|\mathcal{D}^{(F)}\left[O_{\omega}\left(\mathbf{r}_{\perp}\right)\right]\right|^{2},
$$

here with the assumption of a plane wave illumination with intensity $I_{0}$. As phase information is lost upon this operation, it has to be reconstructed from the acquired images. Especially in the holographic regime, in which the resemblance between the intensity images and the original phase is small due to the occurring interference fringes, phase retrieval is of great importance (cf. Fig. 1.5). In the case of the direct-contrast regime, where phase contrast is visible as edge enhancement, the general shape of the sample is preserved but quantitativity, meaning that the gray values reflect the actual density distribution of the object, is lost. Therefore, also here phase retrieval is necessary in order to obtain quantitative information on the sample.

\subsection{Direct-contrast regime}

\subsubsection{Single material objects}

A widely used phase-retrieval approach for the direct-contrast regime was proposed by Paganin et al. [127]. In the case of a homogeneous object, i.e., an object consisting of a single material, with a constant refractive index decrement $\delta_{\omega}\left(\mathbf{r}_{\perp}\right)=\delta_{\omega}$ and extinction coefficient $\beta_{\omega}\left(\mathbf{r}_{\perp}\right)=\beta_{\omega}$, eqns. (1.68) and (1.69) showed that phase and intensity of the exit wave can be expressed as a function of projected thickness 
$T\left(\mathbf{r}_{\perp}\right)$, yielding

$$
\phi_{\omega}\left(\mathbf{r}_{\perp}\right)=-k \delta_{\omega} T\left(\mathbf{r}_{\perp}\right)
$$

and

$$
I_{\omega}\left(\mathbf{r}_{\perp}, 0\right)=I_{0} e^{-2 k \beta_{\omega} T\left(\mathbf{r}_{\perp}\right)}
$$

respectively. Inserting this into the transport of intensity equation (1.71) and approximating the partial derivative along $z$ via a difference quotient analogous to the procedure for the derivation of the near-field TIE in section 1.3.1, results in

$$
\nabla_{\perp}\left(I_{0} e^{-2 k \beta_{\omega} T\left(\mathbf{r}_{\perp}\right)} \nabla_{\perp}\left(-k \delta_{\omega} T\left(\mathbf{r}_{\perp}\right)\right)\right)=-k \frac{I_{\omega}\left(\mathbf{r}_{\perp}, z\right)-I_{\omega}\left(\mathbf{r}_{\perp}, 0\right)}{z} .
$$

With $e^{f(x)} \nabla_{\perp} f(x)=\nabla_{\perp} e^{f(x)}$ and the expression for the intensity at $z=0$ given above, this can be rewritten as

$$
\frac{I_{\omega}\left(\mathbf{r}_{\perp}, z\right)}{I_{0}}=\left(-\frac{\delta_{\omega} z}{2 \beta_{\omega} k} \nabla_{\perp}^{2}+1\right) e^{-2 k \beta_{\omega} T\left(\mathbf{r}_{\perp}\right)} .
$$

According to the pseudo-differential-operators (A.4), the lateral Laplace operator $\nabla_{\perp}^{2}$ transforms into a multiplication with the factor $-\left|\mathbf{k}_{\perp}\right|^{2}$ in reciprocal space. Hence, the above expression yields

$$
e^{-2 k \beta_{\omega} T\left(\mathbf{r}_{\perp}\right)}=\mathcal{F}_{\perp}^{-1}\left[\frac{\mathcal{F}_{\perp}\left[\frac{I_{\omega}\left(\mathbf{r}_{\perp}, z\right)}{I_{0}}\right]}{\frac{\delta_{\omega} z}{2 k \beta_{\omega}}\left|\mathbf{k}_{\perp}\right|^{2}+1}\right] .
$$

By taking the logarithm and exploiting the relation between projected thickness and phase given in eqn. (2.2), this can be converted into an expression for the phase in dependence of the measured intensity distribution $I_{\omega}\left(\mathbf{r}_{\perp}, z\right)$

$$
\phi_{\omega}\left(\mathbf{r}_{\perp}\right)=\frac{\delta_{\omega}}{2 \beta_{\omega}} \cdot \ln \mathcal{F}_{\perp}^{-1}\left[\frac{\mathcal{F}_{\perp}\left[\frac{I_{\omega}\left(\mathbf{r}_{\perp}, z\right)}{I_{0}}\right]}{\frac{\delta_{\omega} z}{2 k \beta_{\omega}}\left|\mathbf{k}_{\perp}\right|^{2}+1}\right]
$$

The spatial frequency $\mathbf{k}_{\perp}$ can be expressed via the dimensionless reciprocal variable $\mathbf{k}_{\perp}^{0}=p \cdot \mathbf{k}_{\perp}$, where $p$ is the pixel size in real space. As a consequence, the above expression for the phase only depends on the Fresnel number $F$ and the ratio 


$$
\kappa_{\omega}=\frac{\delta_{\omega}}{\beta_{\omega}}
$$

$$
\phi_{\omega}\left(\mathbf{r}_{\perp}\right)=\frac{\kappa_{\omega}}{2} \cdot \ln \mathcal{F}_{\perp}^{-1}\left[\frac{\mathcal{F}_{\perp}\left[\frac{I_{\omega}\left(\mathbf{r}_{\perp}, z\right)}{I_{0}}\right]}{\frac{\kappa_{\omega}}{4 \pi F}\left|\mathbf{k}_{\perp}^{0}\right|^{2}+1}\right] .
$$

In the following, this reconstruction scheme is referred to as single material object reconstruction (SMO). For the reconstruction of the phase distribution the exact knowledge of $\kappa_{\omega}$ is necessary. In the case of a too small ratio, the inversion of the Laplacian via the Fourier filter is insufficient, leading to an image in which edge enhancement is still visible, whereas a too large ratio overcorrects the effect, resulting in a blurred reconstruction of the phase. Hence, if $\kappa_{\omega}$ is unknown, it can be obtained from the measurement by adjusting the ratio until the image appears sharp while edge enhancement is removed. Note that in the case of polychromatic illumination, the obtained $\kappa_{\omega}$ is only an effective value and the reconstruction will get slightly blurred, as further discussed in the next section for a similar reconstruction approach.

In the case of an inhomogeneous object with nevertheless constant $\delta / \beta$-ratio, the assumptions for the SMO approach are still met. However, a substantial deviation of the single material object assumption will lead to a disturbed reconstruction of the phase, as for a given value of $\kappa_{\omega}$ parts of the sample with significantly different ratios will either be blurred or still include edge enhancement. Thus, $\kappa_{\omega}$ has to be chosen as a compromise between significantly reducing edge enhancement and preserving the sharpness of the reconstruction.

The application of this phase-retrieval approach to data obtained at a laboratory source is further quantified in section 4.4.2.

\subsubsection{Modified Bronnikov algorithm}

A different approach for phase retrieval in the direct-contrast regime is based on the assumption of a pure phase object, for which the simplified near-field TIE (1.74)

$$
\frac{I_{\omega}\left(\mathbf{r}_{\perp}, z\right)}{I_{0}}=1-\frac{z}{k} \nabla_{\perp}^{2} \phi_{\omega}\left(\mathbf{r}_{\perp}\right)
$$


is valid. By inverting the spatial derivative via pseudo differential operators (A.4), this equation can be solved for the phase

$$
\phi_{\omega}\left(\mathbf{r}_{\perp}\right)=\frac{k}{z} \cdot \mathcal{F}_{\perp}^{-1}\left[\frac{\mathcal{F}_{\perp}\left[\frac{I_{\omega}\left(\mathbf{r}_{\perp}, z\right)}{I_{0}}-1\right]}{\left|\mathbf{k}_{\perp}\right|^{2}}\right] .
$$

As in the case of the SMO approach, this can be expressed in dependence of the Fresnel number $F$, yielding

$$
\phi_{\omega}\left(\mathbf{r}_{\perp}\right)=2 \pi F \cdot \mathcal{F}_{\perp}^{-1}\left[\frac{\mathcal{F}_{\perp}\left[\frac{I_{\omega}\left(\mathbf{r}_{\perp}, z\right)}{I_{0}}-1\right]}{\left|\mathbf{k}_{\perp}^{0}\right|^{2}}\right] .
$$

Note that in this scheme a singularity occurs at $\mathbf{k}_{\perp}^{0}=\mathbf{0}$ and that small spatial frequency are assumed to have low contrast transfer due to the shape of the PCTF for this imaging regime (cf. Fig. 1.4). If this assumption is violated, e.g., in the case of a certain amount of residual absorption, the presented scheme will lead to blurred reconstructions, as low spatial frequencies are enhanced. This can be explained with the ACTF which is maximal at low frequencies and hence substantially increases the measured intensity in this frequency range, even at small residual absorption. As this effect is not inverted by the presented reconstruction scheme, low frequencies are overrepresented and the reconstructions get blurred. To overcome these limitations an absorption-dependent regularization parameter $\alpha$ is introduced

$$
\phi_{\omega}\left(\mathbf{r}_{\perp}\right)=2 \pi F \cdot \mathcal{F}_{\perp}^{-1}\left[\frac{\mathcal{F}_{\perp}\left[\frac{I_{\omega}\left(\mathbf{r}_{\perp}, z\right)}{I_{0}}-1\right]}{\left|\mathbf{k}_{\perp}^{0}\right|^{2}+\alpha}\right] .
$$

This phase-retrieval scheme is known as the Modified Bronnikov Algorithm (MBA) $[56,57]$. The correct choice of $\alpha$ depends on the strength of the absorption of the object and can be estimated by comparison with the SMO approach (2.8). For weak objects, the logarithm can be approximated as $\ln x \simeq x-1$ by a first order 
Taylor expansion, yielding

$$
\begin{aligned}
\phi_{\omega}^{(\mathrm{SMO})}\left(\mathbf{r}_{\perp}, z\right) & \simeq \frac{\kappa_{\omega}}{2} \cdot\left(\mathcal{F}_{\perp}^{-1}\left[\frac{\mathcal{F}_{\perp}\left[\frac{I_{\omega}\left(\mathbf{r}_{\perp}, z\right)}{I_{0}}\right]}{\frac{\kappa_{\omega}}{4 \pi F}\left|\mathbf{k}_{\perp}^{0}\right|^{2}+1}\right]-1\right) \\
& =\frac{\kappa_{\omega}}{2} \cdot \mathcal{F}_{\perp}^{-1}\left[\frac{\mathcal{F}_{\perp}\left[\frac{I_{\omega}\left(\mathbf{r}_{\perp}, z\right)}{I_{0}}-1\right]}{\frac{\kappa_{\omega}}{4 \pi F}\left|\mathbf{k}_{\perp}^{0}\right|^{2}+1}\right] .
\end{aligned}
$$

Hence, for $\alpha=\frac{4 \pi F}{\kappa_{\omega}}$ both algorithms provide the same reconstruction of the phase distribution.

In Fig. 2.1 the results of the MBA algorithm are shown for a simulated homogeneous test object with $\kappa_{\omega}=1000$. To emulate imaging of soft biological tissue with features at varying length scales, the lower right panel of the phantom in Fig. 1.5, depicting a butterfly on a flower, is chosen as the test object in the following. The maximum phase shift of the object is set to $-0.63 \mathrm{rad}$, leading to a minimal transmission of $99.94 \%$. A simulation of more realistic experiments is achieved by adding Poisson noise with a total flux of $5 \cdot 10^{4}$ photons/pixel to the intensity images. Figure 2.1 shows the resulting intensity image at a Fresnel number $F=1$, indicating an imaging regime in which the assumption of a small propagation distance is valid. The reconstructed phase using the regularization parameter $\alpha=\frac{4 \pi F}{\kappa_{\omega}} \simeq 0.0125$ is shown in (b). Apart from noise artifacts which are visible as cloudy structures overlaying the image, the reconstruction is sharp and without remaining edge enhancement. The comparison between the power spectral density (PSD) of the reconstruction and the original phase, depicted in (g), shows a high consistency.

For an unknown ratio between $\delta_{\omega}$ and $\beta_{\omega}$, the regularization parameter has to be chosen based on visual inspection. If $\alpha$ is chosen too high, the Fourier filter $\frac{1}{\left|\mathbf{k}_{\perp}^{0}\right|^{2}+\alpha}$ overcorrects for the absorption and is for low spatial frequencies dominated by the regularization parameter. Hence, those frequencies are suppressed and residual edge-enhancement effects occur in the reconstructed image (cf. Fig. 2.1(b) and (h)). For too small $\alpha$, lower spatial frequencies are amplified due to the insufficient regularization of the increased contrast transfer in this frequency range, leading to a blurred reconstruction of the phase (cf. Fig. 2.1(c) and (h)). Hence, the correct $\alpha$ yields a sharp representation of the phase without edge-enhancement artifacts, and the unknown $\delta / \beta$-ratio can then be obtained from this visually determined parameter.

Note that, as in the case of the SMO approach, the regularization parameter 


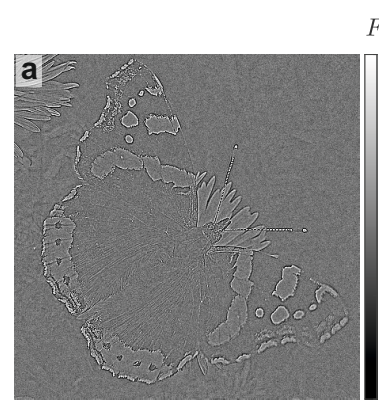

$$
F=1
$$

$F=0.03$
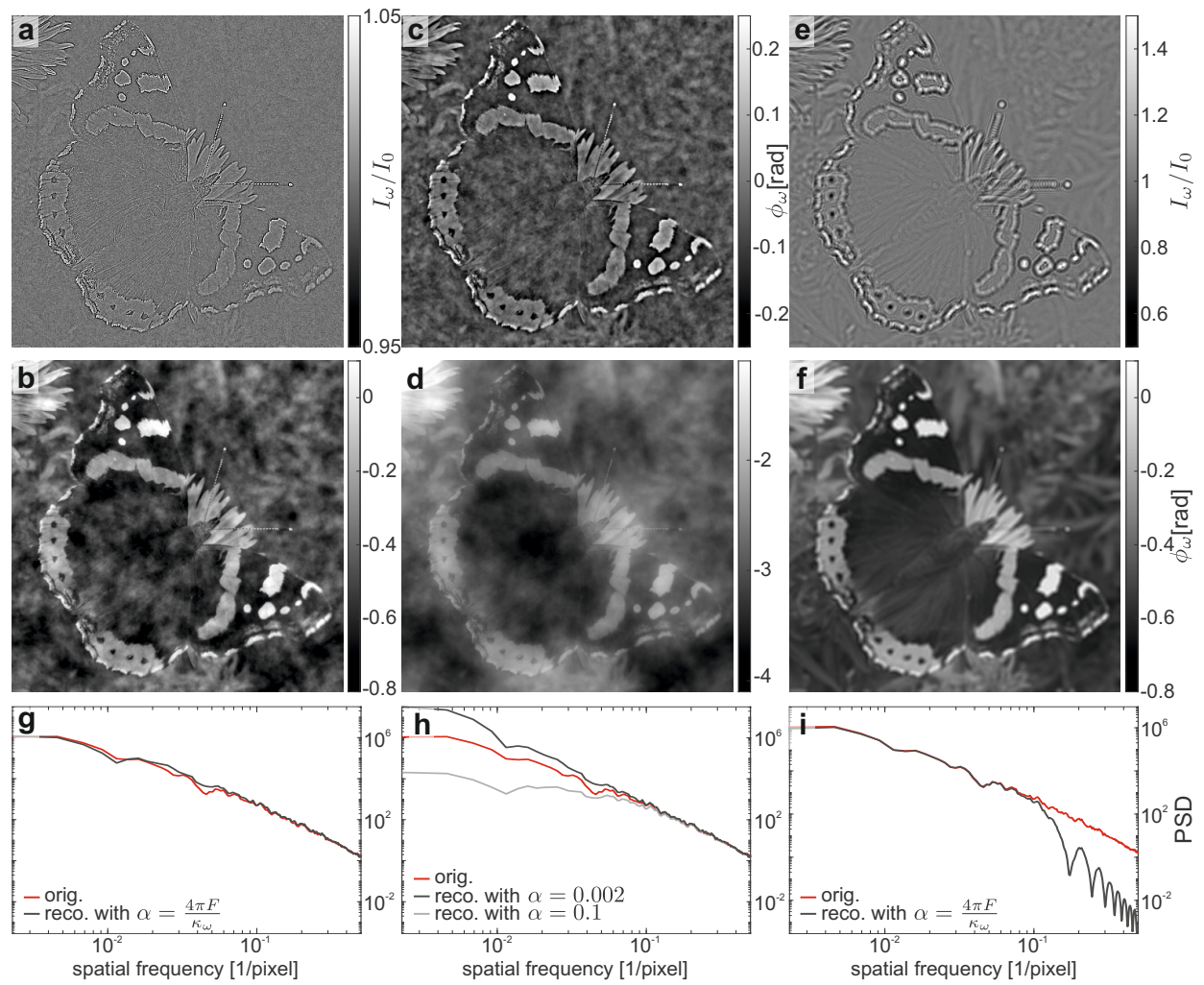

Figure 2.1: Performance of the MBA phase-retrieval algorithm. (a) Simulated intensity distribution of a homogeneous object with $\kappa_{\omega}=1000$ at a Fresnel number $F=1$, leading to contrast formation in the direct-contrast regime. (b) Reconstructed phase for the regularization parameter $\alpha=\frac{4 \pi F}{\kappa_{\omega}} \simeq 0.0125$, yielding a sharp representation of the object with cloudy artifacts caused by noise. (c) Reconstructed phase for the regularization parameter $\alpha=0.1$, in which low spatial frequencies are suppressed. (d) A regularization with $\alpha=0.002$ leads to an amplification of low spatial frequencies, resulting in a blurred reconstruction. (e) Simulated intensity for a Fresnel number $F=0.03$, resulting in contrast formation in the holographic regime. (f) Reconstructed phase with the regularization parameter $\alpha=\frac{4 \pi F}{\kappa_{\omega}} \simeq 3.8 \cdot 10^{-4}$. Due to the larger contrast transfer, the reconstruction yields a high contrast but the violation of the short propagation distance assumption results in a blurring of the image. ( $\mathrm{g}-\mathrm{i})$ The described behavior of the reconstructions is also reflected in the azimuthally averaged power spectral densities of the reconstructed phase distributions with respect to the original image.

is energy-dependent. For a polychromatic spectrum, the obtained value for $\alpha$ is therefore only an effective value. Additionally, the effect of the polychromaticity on contrast formation has to be considered. According to eqn. (1.73) the energydependent wave vector mainly acts as a scaling factor for the Laplacian of the 
phase, leading to comparable intensity distributions for the single energy contributions in the spectrum. However, for larger spatial frequencies, the contrast transfer varies more significantly, leading to a reduction of contrast in the superimposed intensity and therefore a blurring of the reconstruction. This can be explained by the phase contrast transfer function (cf. Fig. 1.4). For the direct-contrast regime, only the first part in which the PCTF and the near-field TIE coincide, is considered. A change in wave length will lead to a compression or stretching of the PCTF, which mainly influences the higher spatial frequencies and results in a deviation from the predicted $\left|k_{\perp}\right|^{2}$-behavior. A reconstruction of the phase via the MBA algorithm will therefore yield disturbed results in this part of the frequency range, an effect that increases with growing wavelength range $\Delta \lambda$. To demonstrate the influence of polychromaticity on the reconstructed phase, a homogeneous object of maximum thickness $10 \mu \mathrm{m}$ with the energy-dependent $\delta / \beta$-ratio presented in section 1.2 .2 is considered. The intensity distribution at propagation distance $z=0.01 \mathrm{~m}$ is simulated for the energy spectrum depicted in Fig. 2.2(c) with a pixel size of $1 \mu \mathrm{m}$. The reconstructed phase (Fig. 2.2(b)) shows a very high accuracy for the low spectral frequencies, as visible in the comparison of the power spectral densities, leading to a quantitative reconstruction of the principal shape of the object. As expected, however, it differs for higher spatial frequencies, resulting in a slight blurring of the image, which can be seen to some extent in the magnified regions in the center as well as in the power spectral densities in (d).

As a last point, the effect of larger propagation distances and hence smaller Fresnel numbers is considered. Figure 2.1(e) shows the simulated intensity for a Fresnel number $F=0.03$, leading to contrast formation in the holographic regime, as visible in the multiple interference fringes around the object. The MBA approach with a regularization parameter $\alpha=\frac{4 \pi F}{\kappa_{\omega}}$ yields a high contrast reconstruction of the phase without the typical cloudy structure caused by the noise, as the contrast transfer increases for smaller Fresnel numbers. However, higher spatial frequencies are not reconstructed correctly due to the invalidity of the near-field TIE in this frequency range (cf. Fig 1.4), resulting in a blurring of the image. The comparison between the PSD of the reconstructed and the original phase confirms this invalidity as the PSD of the reconstructed image clearly shows the zero crossings predicted by the PCTF for smaller Fresnel numbers which are not accounted for by the MBA approach.

As for the SMO, the application of the MBA approach to experimental data from a laboratory source is further discussed in section 4.4.2. 

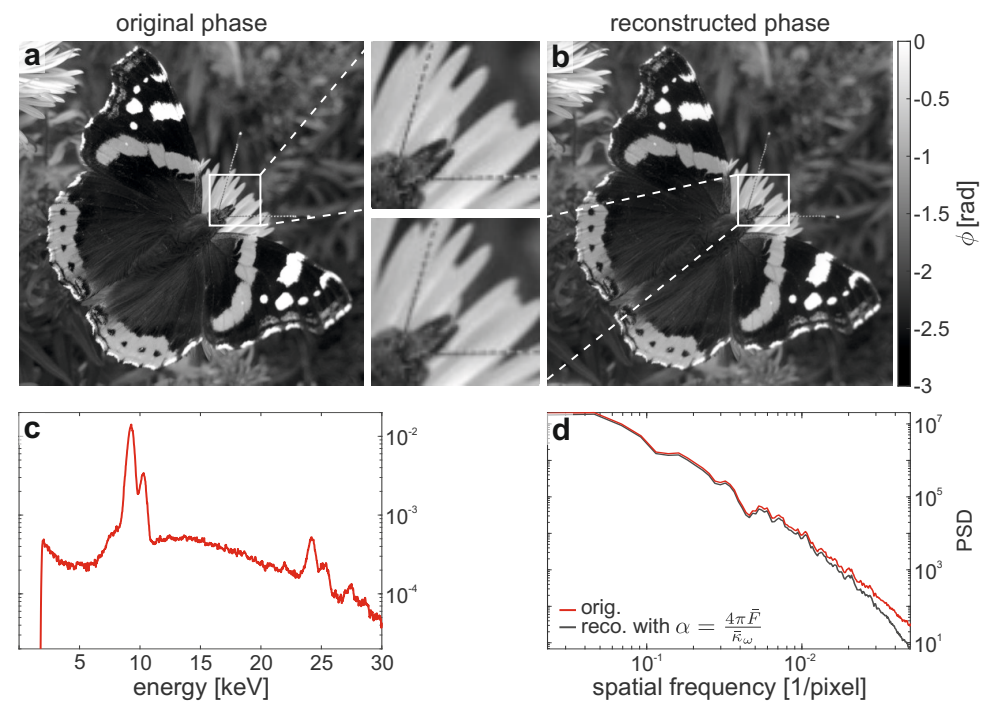

Figure 2.2: Influence of polychromaticity on the reconstruction with the MBA algorithm. (a) Original phase distribution of the test object in which the different energy contributions were averaged according to the normalized spectrum in (c). For the simulation, an object consisting of the model protein $\mathrm{H}_{50} \mathrm{C}_{30} \mathrm{~N}_{9} \mathrm{O}_{10} \mathrm{~S}_{1}$ with density $1.35 \frac{\mathrm{g}}{\mathrm{cm}^{3}}$ was used, leading to the energy-dependent refractive index in Fig. 1.2. (b) Reconstruction of the phase with the regularization parameter $\alpha=\frac{4 \pi \bar{F}}{\bar{\kappa}_{\omega}}$, in which both the Fresnel number $F$ and $\kappa$ were spectrally averaged, resulting in a quantitative but slightly blurred representation of the object. (d) In the azimuthally averaged power spectral densities, this blurring can be recognized as a deviation of the original object compared to the reconstruction at higher spatial frequencies.

\subsubsection{Bronnikov-aided correction}

The invalidity of the assumptions made for the phase-retrieval approaches so far, e.g., homogeneous object or weak absorption, as well as experimental imperfections as polychromatic illumination or low partial coherence, can lead to blurred reconstructions of the phase distribution (cf. section 4.4.2). A way to account for this is given by the Bronnikov-aided correction (BAC) [34]. It is based on the near-field TIE for weakly absorbing objects (1.73)

$$
\frac{I_{\omega}\left(\mathbf{r}_{\perp}, z\right)}{I_{\omega}\left(\mathbf{r}_{\perp}, 0\right)}=1-\frac{z}{k} \nabla_{\perp}^{2} \phi_{\omega}\left(\mathbf{r}_{\perp}\right)
$$


which can be solved for the intensity distribution $I_{\omega}\left(\mathbf{r}_{\perp}, 0\right)$ in the object plane

$$
I_{\omega}\left(\mathbf{r}_{\perp}, 0\right)=\frac{I_{\omega}\left(\mathbf{r}_{\perp}, z\right)}{1-\frac{z}{k} \nabla_{\perp}^{2} \phi_{\omega}\left(\mathbf{r}_{\perp}\right)} .
$$

Hence, the intensity in the exit plane is given by the measured intensity, consisting of both absorption and phase-contrast effects, divided by the hypothetical intensity image for a pure phase object with the phase distribution $\phi_{\omega}\left(\mathbf{r}_{\perp}\right)$. Although this distribution is unknown, an approximated phase $\phi_{\omega}^{\dagger}\left(\mathbf{r}_{\perp}\right)$ can be obtained via the MBA algorithm presented in the previous section. Inserting this into the above expression yields

$$
I_{\omega}\left(\mathbf{r}_{\perp}, 0\right)=\frac{I_{\omega}\left(\mathbf{r}_{\perp}, z\right)}{1-\gamma \nabla_{\perp}^{2} \phi_{\omega}^{\dagger}\left(\mathbf{r}_{\perp}\right)}
$$

where $\frac{z}{k}$ was replaced by the parameter $\gamma$ which regularizes the strength of the correction of the measured intensity image by the hypothetical intensity image of a pure phase object. Hence, the BAC approach is a two-step algorithm: in the first step, the phase has to be approximated via the MBA algorithm and an appropriate choice of the regularization parameter $\alpha$, which reduces most of the edge-enhancement effects though at the cost of resolution. In the second step, a sharp reconstruction of the intensity in the object plane is obtained by optimizing the regularization parameter $\gamma$ in order to avoid residual edge enhancement ( $\gamma$ too small) or an inversion of the edges ( $\gamma$ too large). Note that, similar to the MBA approach, the regularization parameters, which are in principal energy-dependent quantities, are in case of a polychromatic source effective values averaged over the whole energy spectrum.

In contrast to the MBA and SMO from the previous sections, the $\mathrm{BAC}$ algorithm reconstructs the intensity instead of the phase. However, as the algorithm implicitly assumes a phase-attenuation duality, an effective phase can be calculated via

$$
\phi_{\omega}\left(\mathbf{r}_{\perp}\right)=\frac{\kappa_{\omega}}{2} \ln I_{\omega}\left(\mathbf{r}_{\perp}, 0\right) .
$$

Due to the phase-attenuation duality and the increased contrast transfer via freespace propagation, the reconstructed intensity distribution has a much higher signal-to-noise ratio compared to an actual measurement of the object's (weak) absorption.

The application of the BAC algorithm to experimental data obtained at a laboratory source and in particular the comparison to the SMO and the MBA approach 
is further quantified in section 4.4.2.

\subsection{Holographic regime}

For the holographic regime other reconstruction algorithms have to be developed, as the assumption of a small propagation distance is not valid anymore. Hence, the application of the previously described algorithms will lead to disturbed reconstructions, as can be seen in the blurring of the image in Fig. 2.1(f). In the following, only the basic holographic phase retrieval, based on the original scheme of in-line holography proposed by Gabor in 1948 [48], and the CTF-based approach are considered $[28,195]$. Additional phase-retrieval algorithms include the Holo-TIE scheme [89, 92], where the transport of intensity equation is solved for an arbitrary propagation distance by approximating the partial derivative along $z$ via two measurements in close proximity, or iterative methods [45, 92, 103, 144], in which the phase distribution is reconstructed by alternatively propagating between the object and the measurement plane and applying according constraints (cf. Fig. 2.3). In particular, reconstruction starts by propagating the measured intensity image $\psi_{0}\left(\mathbf{r}_{\perp}, z\right)$ to the object plane, yielding $\varphi_{i}\left(\mathbf{r}_{\perp}, 0\right)$, and subsequently applying an object constraint, as, e.g., a support constraint for a spatially limited object. The resulting wave field $\varphi_{i}^{\prime}\left(\mathbf{r}_{\perp}, 0\right)$ is then propagated back to the detection plane, followed by the application of the modulus constraint, i.e., the replacement of the amplitude by the square root of the measured intensity $I_{\omega}\left(\mathbf{r}_{\perp}\right)=\left|\psi_{0}\left(\mathbf{r}_{\perp}, z\right)\right|^{2}$, resulting in $\psi_{i}\left(\mathbf{r}_{\perp}, z\right)$. Repetition of this scheme by a sufficient number of iteration steps leads to a refined reconstruction of the wave function in the object plane. Prominent examples of iterative reconstruction methods are, e.g., the errorreduction or Gerchberg-Saxton algorithm [45, 49], the modified hybrid input-out algorithm [51], near-field ptychography [143, 160] or multiple magnitude projections [63]. A comparison between different phase-retrieval approaches in the holographic regime, including Holo-TIE and iterative methods, applied to single cell imaging can be found in [92].

\subsubsection{Holographic phase retrieval}

In the original scheme proposed by Gabor [48], the intensity of the propagated wave is recorded on a photographic plate and the reconstruction is obtained by illuminating the developed intensity pattern with a wave identical to the original illumination. 


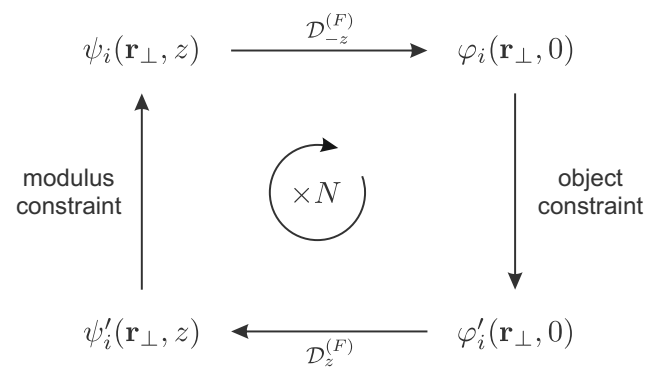

Figure 2.3: Illustration of iterative phase-retrieval algorithms. The wave field in the detection plane $\psi_{i}\left(\mathbf{r}_{\perp}, z\right)$ is propagated back to the object plane, yielding $\varphi_{i}\left(\mathbf{r}_{\perp}, 0\right)$. After application of the object constraint, the resulting wave field $\varphi_{i}^{\prime}\left(\mathbf{r}_{\perp}, 0\right)$ is propagated to the detection plane, leading to $\psi_{i}^{\prime}\left(\mathbf{r}_{\perp}, z\right)$. The subsequent modulus constraint then results in the next iteration $\psi_{i+1}\left(\mathbf{r}_{\perp}, z\right)$ of the wave field in the detection plane.

Consider a weak object, i.e., an object for which the object transmission function (1.64) can be expressed as $O_{\omega}\left(\mathbf{r}_{\perp}\right)=1+\delta O_{\omega}\left(\mathbf{r}_{\perp}\right)$, with $\delta O_{\omega}\left(\mathbf{r}_{\perp}\right) \ll 1$, which is illuminated by a plane wave with amplitude 1 . The propagated intensity can be determined via the Fresnel diffraction operator (1.28)

$$
\begin{aligned}
I_{\omega}\left(\mathbf{r}_{\perp}, z\right) & =\left|\mathcal{D}^{(F)}\left[1+\delta O_{\omega}\left(\mathbf{r}_{\perp}\right)\right]\right|^{2} \\
& =1+e^{-i k z} \delta O_{\omega}\left(\mathbf{r}_{\perp}, z\right)+e^{i k z} \delta O_{\omega}^{*}\left(\mathbf{r}_{\perp}, z\right)+\left|\delta O_{\omega}\left(\mathbf{r}_{\perp}, z\right)\right|^{2},
\end{aligned}
$$

where $\delta O_{\omega}\left(\mathbf{r}_{\perp}, z\right)$ denotes the propagation of the non-unity parts of the object transmission function. As the deviation of the object transmission function from unity is assumed to be small, the last summand in this equation will be neglected in the following.

According to Gabor, the original object can be reconstructed by illumination of the hologram imprinted on a photo plate with the same reference wave. As nowadays intensity images are acquired digitally, this operation can be numerically executed via a back propagation of the measured hologram, i.e., the application of the Fresnel diffraction operator along the distance $-z$

$$
\begin{aligned}
\mathcal{D}_{-z}^{(F)}\left[I_{\omega}\left(\mathbf{r}_{\perp}, z\right)\right] & \simeq \mathcal{D}_{-z}^{(F)}\left[1+e^{-i k z} \delta O_{\omega}\left(\mathbf{r}_{\perp}, z\right)+e^{-i k z} \delta O_{\omega}^{*}\left(\mathbf{r}_{\perp}, z\right)\right] \\
& =e^{-i k z}\left(1+\delta O_{\omega}\left(\mathbf{r}_{\perp}, 0\right)\right)+e^{i k z} \mathcal{D}_{-z}^{(F)}\left[\delta O_{\omega}^{*}\left(\mathbf{r}_{\perp}, z\right)\right]
\end{aligned}
$$

Thus, the reconstruction yields the original object transmission function overlaid by the term $\mathcal{D}_{-z}^{(F)}\left[\delta O_{\omega}^{*}\left(\mathbf{r}_{\perp}, z\right)\right]$, known as the twin image of the original object, which corresponds to the propagated wave at twice the propagation distance $z$ 

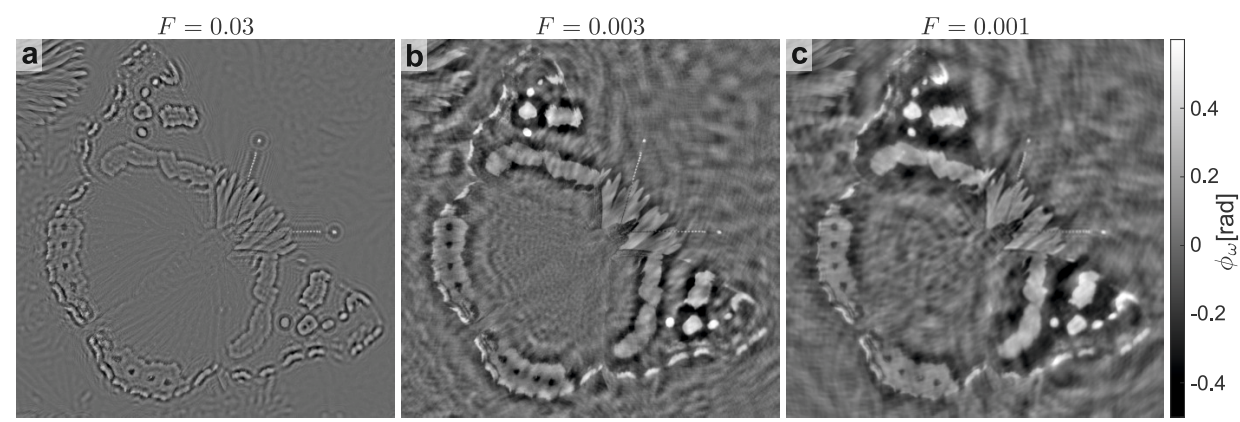

Figure 2.4: Holographic phase retrieval for different Fresnel numbers F. (a) A small Fresnel number leads to a large similarity between the original object and the twin image, resulting in a heavily disturbed reconstruction. (b,c) For smaller Fresnel numbers, the difference increases and the twin image mainly yields a disturbed background, whereas a sharp reconstruction of the object can be unambiguously identified.

[125]. This leads to disturbed reconstructions of the object when applying the holographic reconstruction scheme for phase retrieval, as illustrated in Fig. 2.4 for several Fresnel numbers. Note that for the simulation the same test object as in section 2.1.2 was used, again with additive Poisson noise with a total flux of $5 \cdot 10^{4}$ photons/pixel. For larger Fresnel numbers and correspondingly small propagation distances, the original object and twin image have a relatively large similarity, leading to a heavily disturbed reconstruction (cf. Fig. 2.4(a)). For smaller Fresnel numbers, the difference between the two images is large enough so that a sharp representation of the object can be unambiguously identified in the phase of the reconstructed wave field while the twin image mainly results in a disturbed background, as can be seen in (b) and (c). However, due to the heavy artifacts introduced by the twin image, this phase-retrieval method is only suitable to obtain a preliminary reconstruction of the object.

\subsubsection{CTF-based phase retrieval}

A more quantitative phase-retrieval method is based on the contrast transfer function $(1.81)[28,195]$. For pure phase objects $\left(\xi_{\omega}\left(\mathbf{r}_{\perp}\right)=0\right)$, it is given by

$$
\frac{\hat{I}_{\omega}\left(\mathbf{k}_{\perp}, z\right)}{I_{0}}=2 \pi \delta_{D}\left(\mathbf{k}_{\perp}\right)+2 \sin \chi \hat{\phi}_{\omega}\left(\mathbf{k}_{\perp}\right) .
$$

This can be used for phase retrieval by determining the phase distribution $\phi_{\omega}\left(\mathbf{k}_{\perp}\right)$ which minimizes the difference between the experimentally measured intensity 
$I_{\omega}^{(\exp )}\left(\mathbf{r}_{\perp}, z\right)$ and this theoretical prediction. Before minimizing an according error metric, the effect of the zero crossings in the CTF is further considered. Due to these zero crossings, information on specific spatial frequencies is missing in the measurement and those frequencies cannot be reconstructed from the intensity images. To overcome this limitation, images can be acquired at several propagation distances [195], leading to a shifting of the zero crossings in the corresponding contrast transfer functions. Hence, missing frequencies at a single distance can be accounted for by the information gathered at the other distances. To include these multiple distances, the sum of the differences between the measured and predicted intensity distributions has to be minimized by a suitable choice of the phase distribution $\phi_{\omega}\left(\mathbf{k}_{\perp}\right)$. To this end, a least square minimization is performed, leading to the error metric

$$
S_{c}=\frac{1}{N} \sum_{m=1}^{N} \int_{\mathbb{R}^{2}} d \mathbf{k}_{\perp}\left|\hat{I}_{\omega}^{(\exp )}\left(\mathbf{k}_{\perp}, z_{m}\right)-\hat{I}_{\omega}^{(\mathrm{th})}\left(\mathbf{k}_{\perp}, z_{m}\right)\right|^{2},
$$

where $N$ is the number of distances. To minimize this error metric with respect to the phase, the partial derivative $\frac{\partial S_{c}}{\partial \hat{\phi}_{\omega}}$ has to vanish, yielding

$$
\begin{aligned}
\frac{\partial S_{c}}{\partial \hat{\phi}_{\omega}}=\frac{4}{N} \sum_{m=1}^{N} \int_{\mathbb{R}^{2}} d \mathbf{k}_{\perp}( & -\sin \chi_{m} \hat{I}_{\omega}^{(\exp )}\left(\mathbf{k}_{\perp}, z_{m}\right)+\sin \chi_{m} 2 \pi \delta_{D}\left(\mathbf{k}_{\perp}\right) \\
& \left.+2 \sin ^{2} \chi_{m} \hat{\phi}_{\omega}\left(\mathbf{k}_{\perp}\right)\right) \\
\stackrel{!}{=} 0 . &
\end{aligned}
$$

This equation is valid for all spatial frequencies if the expression within the integral equals 0 , leading to [195]

$$
\hat{\phi}_{\omega}\left(\mathbf{k}_{\perp}\right)=\frac{\sum_{m=1}^{N} \sin \chi_{m} \cdot \mathcal{F}_{\perp}\left[I_{\omega}^{(\exp )}\left(\mathbf{r}_{\perp}, z_{m}\right)-1\right]}{\sum_{m=1}^{N} 2 \sin ^{2} \chi_{m}} .
$$

Due to the definition of the argument in the sine function, $\chi=\frac{\lambda z k_{x}^{2}}{4 \pi}$, the denominator of this expression vanishes for $\left|\mathbf{k}_{\perp}\right|=0$. Additionally, depending on the propagation distances $z_{m}$, it can also disappear for certain spatial frequencies $\left|\mathbf{k}_{\perp}\right|>0$ if the single factors of the denominator add up to zero. To avoid these singularities, a frequency-dependent regularization parameter $\alpha\left(\mathbf{k}_{\perp}\right)$ is introduced, 
resulting in

$$
\phi_{\omega}\left(\mathbf{r}_{\perp}\right)=\mathcal{F}_{\perp}^{-1}\left[\frac{\sum_{m=1}^{N} \sin \chi_{m} \cdot \mathcal{F}_{\perp}\left[I_{\omega}^{(\exp )}\left(\mathbf{r}_{\perp}, z_{m}\right)-1\right]}{\sum_{m=1}^{N} 2 \sin ^{2} \chi_{m}+\alpha\left(\mathbf{k}_{\perp}\right)}\right]
$$

where the transformation to real space was performed. The frequency-dependence is justified by the different nature of the singularities. For low frequencies, the contrast transfer resembles the behavior of the near-field TIE, and the regularization has to be performed in order to account for the singularity at $\left|\mathbf{k}_{\perp}\right|=0$ and residual absorption, similar to the MBA approach. For higher spatial frequencies, contrast transfer is high and regularization is only necessary if the different propagation distances are chosen such that the zero crossings are not accounted for. To ensure the difference in regularization for these two regimes, $\alpha\left(\mathbf{k}_{\perp}\right)$ is built according to $[27]$

$$
\alpha\left(\mathbf{k}_{\perp}\right)=\alpha_{1} \cdot f\left(\left|\mathbf{k}_{\perp}\right|\right)+\alpha_{2}\left(1-f\left(\left|\mathbf{k}_{\perp}\right|\right)\right)
$$

where the transition is realized via an error function

$$
f\left(\left|\mathbf{k}_{\perp}\right|\right)=\frac{1}{2}\left(1-\operatorname{erf}\left(\frac{\left|\mathbf{k}_{\perp}\right|-k_{\text {cut }}}{\sigma_{\text {cut }}}\right)\right) .
$$

Hence, the parameters $\alpha_{1}$ and $\alpha_{2}$ lead to a regularization of the low and high frequencies, respectively. The transition point $k_{\text {cut }}$ is usually chosen to be the first maximum of the PCTF and the width of the transition can be varied via $\sigma_{\text {cut }}$.

To extend the presented approach to weakly absorbing objects, the CTF (1.81) has to be considered again. Under the assumption of a homogeneous object, leading to a proportionality between the phase $\phi_{\omega}$ and the attenuation coefficient $\xi_{\omega}$ with proportionality factor $-\frac{\beta_{\omega}}{\delta_{\omega}}$, it can be rewritten as

$$
\begin{aligned}
\frac{\hat{I}_{\omega}\left(\mathbf{k}_{\perp}, z\right)}{I_{0}} & =2 \pi \delta_{D}\left(\mathbf{k}_{\perp}\right)+2 \sin \chi \hat{\phi}_{\omega}\left(\mathbf{k}_{\perp}\right)-2 \cos \chi \hat{\xi}_{\omega}\left(\mathbf{k}_{\perp}\right) \\
& =2 \pi \delta_{D}\left(\mathbf{k}_{\perp}\right)+2\left(\sin \chi+\frac{\beta_{\omega}}{\delta_{\omega}} \cos \chi\right) \hat{\phi}_{\omega}\left(\mathbf{k}_{\perp}\right) .
\end{aligned}
$$

Analogous to the derivation for a pure phase object, the minimization of the difference between the measured intensity images and the theoretical prediction leads 
to an expression for the phase in real space [169]

$$
\phi_{\omega}\left(\mathbf{r}_{\perp}\right)=\mathcal{F}_{\perp}^{-1}\left[\frac{\sum_{m=1}^{N}\left(\sin \chi_{m}+\frac{\beta_{\omega}}{\delta_{\omega}} \cos \chi_{m}\right) \cdot \mathcal{F}_{\perp}\left[I_{\omega}^{(\mathrm{exp})}\left(\mathbf{r}_{\perp}, z_{m}\right)-1\right]}{\sum_{m=1}^{N} 2\left(\sin \chi_{m}+\frac{\beta_{\omega}}{\delta_{\omega}} \cos \chi_{m}\right)^{2}+\alpha\left(\mathbf{k}_{\perp}\right)}\right] .
$$

As for the case of a pure phase object, a frequency-dependent regularization parameter $\alpha\left(\mathbf{k}_{\perp}\right)$, defined as before, is added to account for a vanishing denominator. However, due to the complementary behavior of the cosine function, the $\frac{\beta}{\delta}$-ratio already acts as a regularization for the lower frequencies, so that $\alpha_{1}$ can be set to zero.

In the following, the performance of the two algorithms applied to simulated data is investigated. As in the case of the MBA, the butterfly is used as a test object with maximum phase shift $-0.63 \mathrm{rad}$ and a $\beta / \delta$-ratio of 0.001 or 0.1 , leading to a minimum transmission of $99.94 \%$ and $94 \%$, respectively. To demonstrate the validity of the CTF for all propagation distances, two different sets of Fresnel numbers are considered. Additionally, Poisson noise with a total flux of $5 \cdot 10^{4}$ photons/pixel is added to the intensity images. The results of the two algorithms are depicted in Fig. 2.5, where the upper row corresponds to the assumption of a pure phase object and the lower row to a homogeneous and weakly absorbing object. For all reconstructions, $\alpha_{2}$ was set to zero without the introduction of artifacts, proving the appropriate choice of propagation distances. The regularization parameter $\alpha_{1}$ is determined automatically by minimizing the difference between the reconstructed and simulated phase. As the phase is unknown in a real experiment, $\alpha_{1}$ is generally chosen based on visual inspection, similar to the direct-contrast regime. The comparison between the two algorithm shows that both provide sharp reconstructions of the object with only minimal artifacts compared to the simple holographic reconstruction method presented in the previous section. Due to the validity of the approach for all imaging regimes, no significant deviations between the simulations at different Fresnel numbers are present apart from artifacts due to noise, as the contrast transfer decreases for larger Fresnel numbers. However, low spatial frequencies are better reconstructed using the homogeneous object approach, as visible in the power spectral densities shown in Fig. 2.5 (g-i), leading to a more quantitative reconstruction, i.e., areas in the object consisting of the same material are represented by the same gray values (see, e.g., the inner part of the butterfly).

This difference can be explained by the different nature of the filtering in reciprocal space. In the case of the pure phase object assumption it is of the form $\frac{\sin \chi}{2 \sin ^{2} \chi+\alpha}$, 

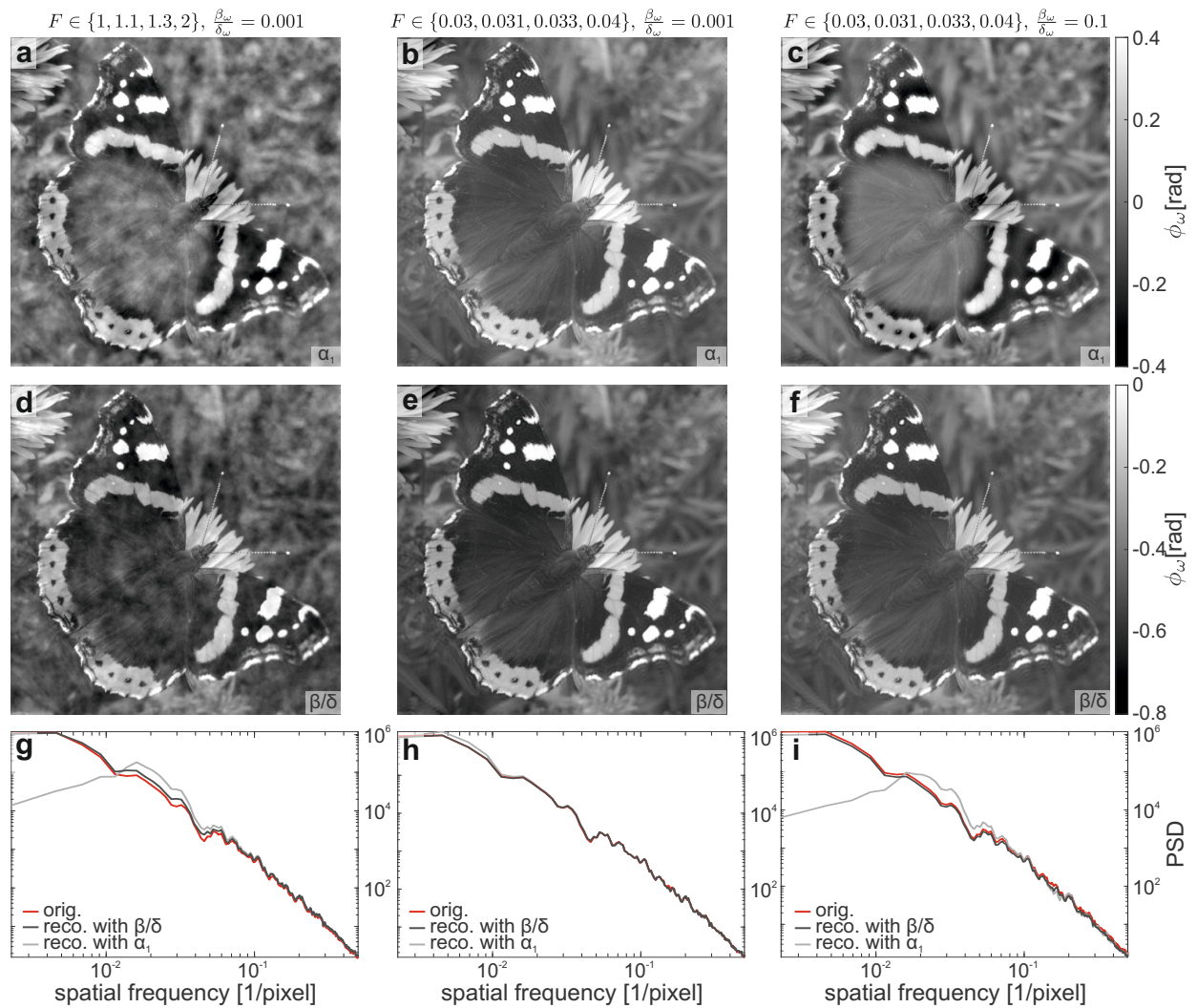

Figure 2.5: Simulation of the CTF-based phase retrieval. (a-c) Reconstructed phase under the assumption of a pure phase object at different Fresnel numbers and $\frac{\beta}{\delta}$-ratios. For larger Fresnel numbers, and hence contrast formation in the direct-contrast regime, the resulting reconstruction for an object with $\frac{\beta_{\omega}}{\delta_{\omega}}=0.001$ yields a sharp representation of the phase in which low spatial frequencies are suppressed. In the holographic regime, phase retrieval for the same object results in a more accurate reconstruction, while for an object with $\frac{\beta_{\omega}}{\delta_{\omega}}=0.1$, the low spatial frequencies are again suppressed. (d-f) Under the assumption of a weakly absorbing homogeneous object, phase retrieval yields accurate reconstructions for all simulated combinations of Fresnel numbers and $\frac{\beta}{\delta}$-ratios. ( $\left.g-i\right)$ The behavior of the different phase-retrieval approaches is reflected in the azimuthally averaged power spectral densities, showing the deviations at low spatial frequencies when assuming a pure phase object.

whereas for the homogeneous object the filter is given by $\frac{\sin \chi+\frac{\beta}{\delta} \cos \chi}{2\left(\sin \chi+\frac{\beta}{\delta} \cos \chi\right)^{2}+\alpha}$. The behavior of these functions as well as of the filter in the case of the MBA approach is shown in Fig. 2.6 for a Fresnel number $F=1$ and $\frac{\beta}{\delta}=0.001$, corresponding to the direct-contrast regime and to the reconstructions depicted in Figs. 2.1(b) and 2.5(a) and (d). Note that in all cases $\alpha_{2}$ is set to 0 . It is evident that in the case 


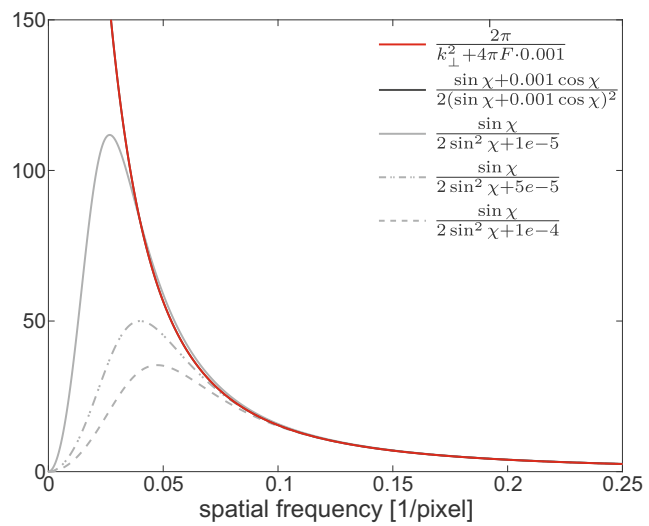

Figure 2.6: Fourier filters used in the different CTF approaches as compared to the $M B A$ algorithm for $F=1$ and $\frac{\beta}{\delta}=0.001$. For large spatial frequencies, all filter functions coincide, whereas in the small frequency range only the homogeneous object CTF and the $M B A$ are equivalent, while the pure phase CTF acts as a high-pass filter. The degree of this filtering is determined by the size of the regularization parameter $\alpha_{1}$. Note that due to the similarity of the homogeneous object $C T F$ and the MBA, the single filter functions are not distinguishable in the plot and only the red curve for the MBA approach is visible.

of a pure phase object the filter acts as a high-pass filter. If the intensity includes significant contributions at lower spatial frequencies, as is the case for residual absorption, this leads to the suppression of low frequencies in the reconstruction. A rather empirical approach to overcome this limitation and extend the applicability of this algorithm to weakly absorbing objects is presented in section A.4 in the appendix.

In the course of this thesis, the CTF derived for a homogeneous weakly absorbing object is used as the standard reconstruction method for imaging in the holographic regime. 



\section{Computed tomography}

In the previous chapters, considerations were restricted to the fundamentals of twodimensional (2d) x-ray propagation imaging, yielding the attenuation coefficient $\xi_{\omega}\left(\mathbf{r}_{\perp}\right)=-k \int_{-d}^{0} \beta_{\omega}(\mathbf{r}) \mathrm{d} z=-k \bar{\beta}_{\omega}\left(\mathbf{r}_{\perp}\right)$ and phase $\phi_{\omega}\left(\mathbf{r}_{\perp}\right)=-k \int_{-d}^{0} \delta_{\omega}(\mathbf{r}) \mathrm{d} z=$ $-k \bar{\delta}_{\omega}\left(\mathbf{r}_{\perp}\right)$ of the object's transmission function (1.64). Hence, $2 \mathrm{~d}$ imaging provides information about the projected index of refraction. The aim of computed tomography $(\mathrm{CT})$ is to reconstruct the $3 \mathrm{~d}$ distribution of the refractive index decrement $\delta_{\omega}(\mathbf{r})$ and extinction coefficient $\beta_{\omega}(\mathbf{r})$ from a set of such projections acquired at different rotation angles $\theta$. The mathematical framework was developed by Radon in 1917 [138] and the discussion of the principles presented below mainly follows [81].

\subsection{Mathematical concepts}

Consider the geometry depicted in Fig. 3.1. The function $f(x, z)$, where $f$ describes either $\delta_{\omega}$ or $\beta_{\omega}$, represents the investigated sample at a constant height $y$, therefore depicting one two-dimensional slice through the three-dimensional volume (cf. Fig. 3.1(f)). Let $\left(x^{\prime}, y^{\prime}, z^{\prime}\right)$ be the coordinate system of the laboratory frame, comprising source and detector, which is rotated around the object frame $(x, y, z)$ by the rotation angle $\theta$. Hence, it is written as

$$
\left(\begin{array}{l}
x^{\prime} \\
y^{\prime} \\
z^{\prime}
\end{array}\right)=\left(\begin{array}{ccc}
\cos \theta & 0 & \sin \theta \\
0 & 1 & 0 \\
-\sin \theta & 0 & \cos \theta
\end{array}\right)\left(\begin{array}{l}
x \\
y \\
z
\end{array}\right) .
$$

The inverse rotation can be performed by exchanging $\theta \rightarrow-\theta$. As in the previous chapter, the direction of the beam is defined by the $z^{\prime}$-axis. The projection $p_{\theta}\left(x^{\prime}\right)$ of the function $f(x, z)$ at a given angle $\theta$ is thus given by [81]

$$
\begin{aligned}
p_{\theta}\left(x^{\prime}\right)=\mathcal{R}_{\theta} f\left(x^{\prime}\right) & =\int_{\mathbb{R}} f(x, z) \mathrm{d} z^{\prime} \\
& =\int_{\mathbb{R}} f\left(x^{\prime} \cos \theta-z^{\prime} \sin \theta, x^{\prime} \sin \theta+z^{\prime} \cos \theta\right) \mathrm{d} z^{\prime},
\end{aligned}
$$



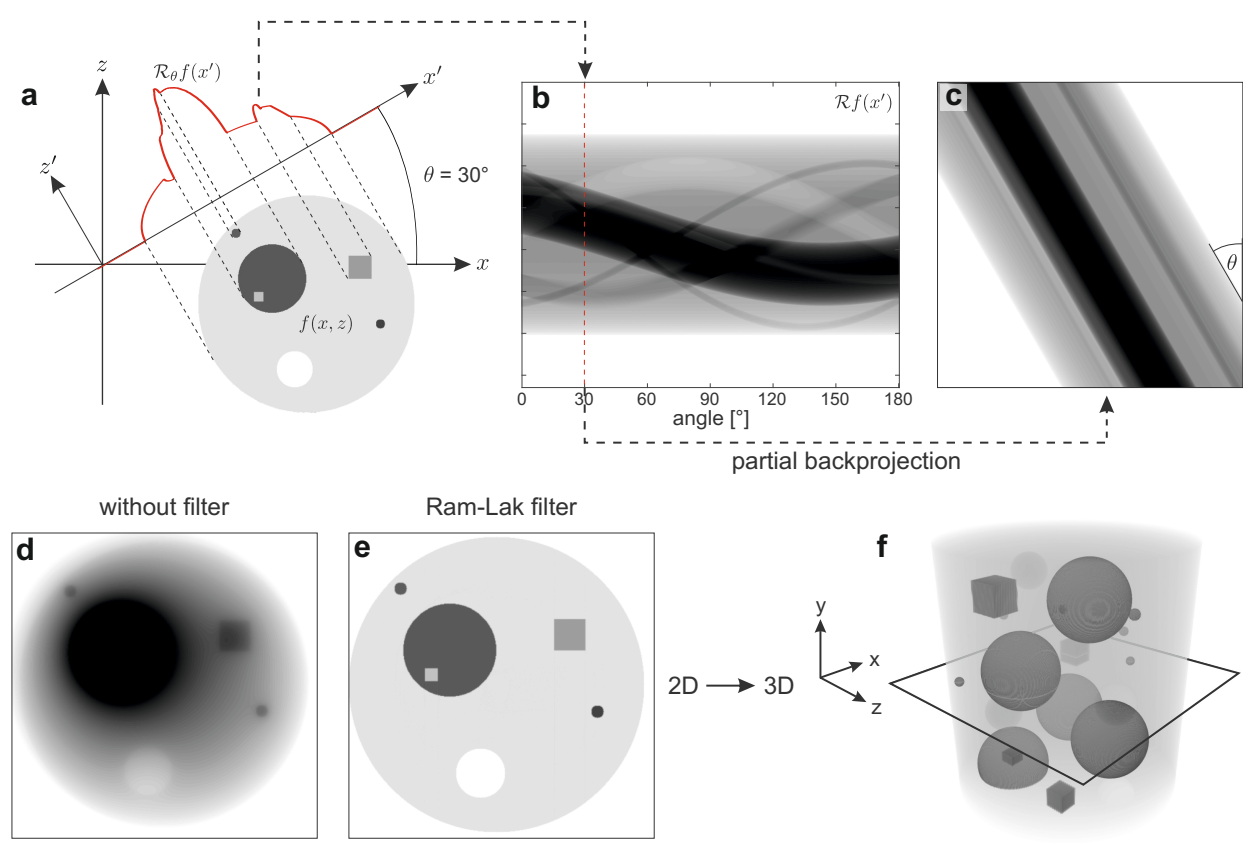

Figure 3.1: Illustration of the principle concepts in computed tomography. (a) An object $f(x, z)$ is illuminated under a given angle $\theta$, leading to the projection $p_{\theta}\left(x^{\prime}\right)$ of the object (red curve). Mathematically, this is performed by integrating over lines along $z^{\prime}$ at distance $x^{\prime}$ to the origin, yielding the Radon transform $\mathcal{R}_{\theta} f\left(x^{\prime}\right)$. For each parallel plane along the rotational axis $y$, a set of projections is recorded for a given angular range. (b) Plotting the acquired projections against the rotation angle yields the corresponding sinogram. (c) A partial backprojection is performed by smearing back the one-dimensional projection at the rotation angle $\theta$ into the two-dimensional space along the projection direction $z^{\prime}$. (d,e) A full backprojection without any additional filtering yields a blurred reconstruction of the object whereas the application of a Ram-Lak filter on each recorded projection results in a sharp representation of $f(x, z)$. (f) By subsequently reconstructing and combining two-dimensional slices at different positions along $y$, information about the $3 d$ structure of the entire object can be obtained.

with the two-dimensional Radon transform $\mathcal{R}_{\theta}$.

This operation can be alternatively expressed as [21]

$$
\mathcal{R}_{\theta} f\left(x^{\prime}\right)=\int_{\mathbb{R}^{2}} f(x, z) \delta_{D}\left(x \cos \theta+z \sin \theta-x^{\prime}\right) \mathrm{d} x \mathrm{~d} z,
$$

where $\delta_{D}$ again denotes the Dirac delta distribution. Hence, the Radon transform is performed by integrating over lines along $z^{\prime}$ with distance $x^{\prime}$ to the origin (in Fig. 3.1 this is indicated by the dashed lines). 
Consider the Radon transform of a single point located at $\left(x_{0}, z_{0}\right)$, with $f(x, z)=$ $\delta_{D}\left(x-x_{0}\right) \delta_{D}\left(z-z_{0}\right)$. Inserting this expression into eqn. (3.3) yields

$$
\begin{aligned}
\mathcal{R}_{\theta} f\left(x^{\prime}\right) & =\int_{\mathbb{R}} \delta_{D}\left(x^{\prime} \cos \theta-z^{\prime} \sin \theta-x_{0}\right) \delta_{D}\left(x^{\prime} \sin \theta+z^{\prime} \cos \theta-z_{0}\right) \mathrm{d} z^{\prime} \\
& =\frac{1}{|\cos \theta|} \delta_{D}\left(x^{\prime} \cos \theta-\sin \theta\left(-\frac{\sin \theta}{\cos \theta} x^{\prime}+\frac{z_{0}}{\cos \theta}\right)-x_{0}\right) \\
& =\delta_{D}\left(x^{\prime}-\sin \theta z_{0}-\cos \theta x_{0}\right)
\end{aligned}
$$

where the integral over $z^{\prime}$ was executed and the scaling property of the Dirac delta distribution (A.12) was used. This can be rewritten as

$$
\mathcal{R}_{\theta} f\left(x^{\prime}\right)=\delta_{D}\left(x^{\prime}-r \sin (\theta-\alpha)\right),
$$

with $r=\sqrt{x_{0}^{2}+z_{0}^{2}}$ and $\alpha=\operatorname{atan}\left(\frac{x_{0}}{y_{0}}\right)$ [149]. Hence, the Radon transform of a single point is following a sinusoidal curve. As an object can be regarded as a combination of single points and the Radon transform will thus be a superposition of sine curves, the resulting plot of the projections $p_{\theta}\left(x^{\prime}\right)$ against the angle $\theta$ is called a sinogram (cf. Fig. 3.1(b)).

The projections $p_{\theta}\left(x^{\prime}\right)$ are the measured quantity in (propagation-based) x-ray imaging, corresponding to the amplitude or reconstructed phase (after the application of suitable algorithms) of the object transmission function. Therefore, an inverse operator is required to obtain information about the original two-dimensional function $f(x, z)$. This operator is called the inverse Radon transform and its application yields

$$
f(x, z)=\mathcal{R}^{-1}\left[p_{\theta}\left(x^{\prime}\right)\right]
$$

It can be used to obtain information about the entire three-dimensional object by subsequently reconstructing and combining two-dimensional slices at different positions along $y$ (cf. Fig. 3.1(f)). The essential theorem for the inversion of the Radon transform is outlined in the following section. 


\subsubsection{Fourier slice theorem}

Consider the Fourier transform of the projection $p_{\theta}\left(x^{\prime}\right)$, as defined by eqn. (3.4), with respect to $x^{\prime}$

$$
\begin{aligned}
\mathcal{F}_{x^{\prime}}[R f]\left(k_{x}^{\prime}\right)=\hat{p}_{\theta}\left(k_{x}^{\prime}\right) & =\frac{1}{\sqrt{2 \pi}} \int_{\mathbb{R}^{3}} f(x, z) \delta_{D}\left(x \cos \theta+y \sin \theta-x^{\prime}\right) e^{-i k_{x}^{\prime} x^{\prime}} \mathrm{d} x \mathrm{~d} z \mathrm{~d} x^{\prime} \\
& =\frac{1}{\sqrt{2 \pi}} \int_{\mathbb{R}^{2}} f(x, z) e^{-i k_{x}^{\prime}(x \cos \theta+y \sin \theta)} \mathrm{d} x \mathrm{~d} z,
\end{aligned}
$$

where in the second step, the integration along $x^{\prime}$ was performed. This can be identified as the $2 \mathrm{~d}$ Fourier transform $\mathcal{F}_{\mathbf{r}}[f](\mathbf{q})$ with the reciprocal coordinate vector $\mathbf{q}=k_{x}^{\prime}(\cos \theta, \sin \theta)$. Hence, the above equation transforms into

$$
\hat{p}_{\theta}\left(k_{x}^{\prime}\right)=\mathcal{F}_{x^{\prime}}[\mathcal{R} f]\left(k_{x}^{\prime}\right)=\mathcal{F}_{\mathbf{r}}[f]\left(k_{x}^{\prime} \cos \theta, k_{x}^{\prime} \sin \theta\right)
$$

known as the Fourier slice theorem, which proves the invertibility of the Radon transform [117]. It states that the one-dimensional Fourier transform of the projection $p_{\theta}\left(x^{\prime}\right)=\mathcal{R}_{\theta} f\left(x^{\prime}\right)$ is equivalent to a line through the two-dimensional Fourier transform of the function $f(x, z)$ at the respective rotation angle $\theta$, as depicted in Fig. 3.2. According to the theorem, the original function $f(x, z)$ can be reconstructed by filling up the $2 \mathrm{~d}$ reciprocal space with projections recorded at all rotation angles $\theta$ and subsequently performing an inverse Fourier transform, called the direct Fourier method. However, as in the numerical implementation of the method the Fourier space is sampled on a rectangular grid, whereas the projections are known on a polar grid (cf. Fig. 3.2), interpolation between the two coordinate systems in reciprocal space can result in severe artifacts in real space [81]. Therefore, most practical implementations of the inverse Radon transform rely on a different reconstruction approach, leading to the filtered backprojection discussed in the next section.

\subsubsection{Filtered backprojection}

The partial backprojection of the Radon transform $\mathcal{R}_{\theta} f\left(x^{\prime}\right)=p_{\theta}\left(x^{\prime}\right)$ for a given rotation angle $\theta$ is defined as

$$
\mathcal{R}_{\theta}^{\#} p(x, z)=p_{\theta}(x \cos \theta+z \sin \theta)
$$




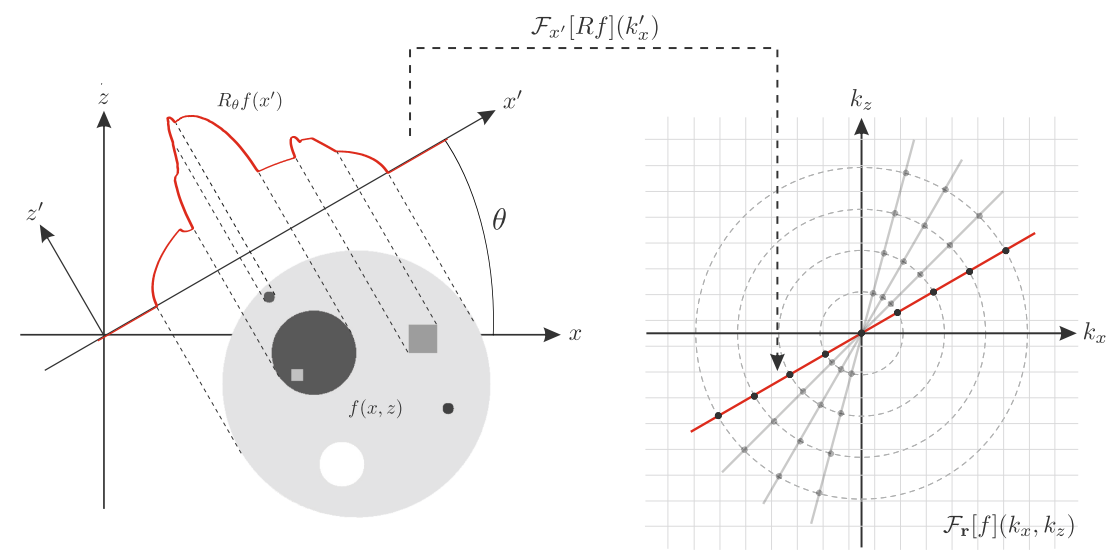

Figure 3.2: Illustration of the Fourier slice theorem. The 1d Fourier transform of the projection $\mathcal{R}_{\theta} f\left(x^{\prime}\right)$ at a given rotation angle $\theta$ is equivalent to a slice through the origin of the $2 d$ Fourier transform of the object $f(x, z)$ at the corresponding angle (red line). In the numerical implementation, the projections are known on a polar grid, indicated by the black dots, whereas the Fourier space is sampled on a rectangular grid, shown in light gray. The interpolation between these two coordinate systems can lead to severe artifacts.

Hence, each point $(x, z) \in \mathbb{R}^{2}$ on lines along $z^{\prime}$ with distance $x^{\prime}$ to the origin is assigned the value of the respective projection $p_{\theta}\left(x^{\prime}\right)$, i.e., the one-dimensional projection at the rotation angle $\theta$ is smeared back into the two-dimensional space along the projection direction $z^{\prime}$ (cf. Fig. 3.1(c)). The full backprojection is then defined via the integration of these partial backprojections over all angles $\theta$ [21]

$$
\mathcal{R}^{\#} p(x, z)=\int_{0}^{\pi} p_{\theta}(x \cos \theta+y \sin \theta) \mathrm{d} \theta .
$$

The result of this backprojection for the slice depicted in Fig. 3.1(a) is shown in (d). Due to the irregular sampling in Fourier space, i.e., the higher density of points for lower spatial frequencies (cf. Fig. 3.2), high spatial frequencies are underrepresented, leading to a blurred reconstruction of the $2 \mathrm{~d}$ object function $f(x, z)$.

To correct for this blurring and obtain a sharp representation of the object function, the object function is expressed via the inverse Fourier transform

$$
f(x, z)=\frac{1}{\sqrt{2 \pi}} \int_{\mathbb{R}^{2}} \hat{f}\left(k_{x}, k_{z}\right) e^{i\left(k_{x} x+k_{z} z\right)} \mathrm{d} k_{x} \mathrm{~d} k_{z} .
$$


A conversion to polar coordinates

$$
\left(\begin{array}{l}
k_{x} \\
k_{z}
\end{array}\right)=\left(\begin{array}{c}
k_{x}^{\prime} \cos \theta \\
k_{x}^{\prime} \sin \theta
\end{array}\right)
$$

with the differentials $\mathrm{d} k_{x} \mathrm{~d} k_{z}=k_{x}^{\prime} \mathrm{d} k_{x}^{\prime} \mathrm{d} \theta$, as determined via the modulus of the Jacobi determinant, yields

$$
f(x, z)=\frac{1}{\sqrt{2 \pi}} \int_{0}^{2 \pi} \int_{0}^{\infty} \hat{f}\left(k_{x}^{\prime} \cos \theta, k_{x}^{\prime} \sin \theta\right) e^{i\left(k_{x}^{\prime} \cos \theta x+k_{x}^{\prime} \sin \theta z\right)} k_{x}^{\prime} \mathrm{d} k_{x}^{\prime} \mathrm{d} \theta .
$$

With $\left(k_{x}^{\prime} \cos (\theta+\pi), k_{x}^{\prime} \sin (\theta+\pi)\right)=\left(-k_{x}^{\prime} \cos \theta,-k_{x}^{\prime} \sin \theta\right)$, the integration over $2 \pi$ can be split up and this expression transforms into

$$
f(x, z)=\frac{1}{\sqrt{2 \pi}} \int_{0}^{\pi} \int_{-\infty}^{\infty} \hat{f}\left(k_{x}^{\prime} \cos \theta, k_{x}^{\prime} \sin \theta\right) e^{i\left(k_{x}^{\prime} \cos \theta x+k_{x}^{\prime} \sin \theta z\right)}\left|k_{x}^{\prime}\right| \mathrm{d} k_{x}^{\prime} \mathrm{d} \theta .
$$

According to the Fourier slice theorem (3.12), the 2d Fourier transform of the function $f(x, z)$ along the slice defined by $\left(k_{x}^{\prime} \cos \theta, k_{x}^{\prime} \sin \theta\right)$ is given by the $1 \mathrm{~d}$ Fourier transform of the projection $p_{\theta}\left(x^{\prime}\right)$, yielding

$$
f(x, z)=\frac{1}{\sqrt{2 \pi}} \int_{0}^{\pi} \int_{-\infty}^{\infty} \hat{p}_{\theta}\left(k_{x}^{\prime}\right) e^{i\left(k_{x}^{\prime} \cos \theta x+k_{x}^{\prime} \sin \theta z\right)}\left|k_{x}^{\prime}\right| \mathrm{d} k_{x}^{\prime} \mathrm{d} \theta .
$$

By defining a filtered projection $p_{\theta}^{F}\left(x^{\prime}\right)$ as

$$
\begin{aligned}
p_{\theta}^{F}\left(x^{\prime}\right) & =\mathcal{F}^{-1}\left[\hat{h}\left(k_{x}^{\prime}\right) \hat{p}_{\theta}\left(k_{x}^{\prime}\right)\right] \\
& =\frac{1}{\sqrt{2 \pi}} \int_{\mathbb{R}} e^{i k_{x}^{\prime} x^{\prime}} \hat{h}\left(k_{x}^{\prime}\right) \hat{p}_{\theta}\left(k_{x}^{\prime}\right) \mathrm{d} k_{x}^{\prime},
\end{aligned}
$$

where the filter function $\hat{h}\left(k_{x}^{\prime}\right)$ was introduced, the expression in eqn. (3.19) can be identified as the full backprojection (cf. eqn. (3.14)) of the filtered projections $p_{\theta}^{F}\left(x^{\prime}\right)=p_{\theta}^{F}(x \cos \theta+z \sin \theta)$ with $h\left(k_{x}^{\prime}\right)=\left|k_{x}^{\prime}\right|$

$$
f(x, z)=\int_{0}^{\pi} p_{\theta}^{F}(x \cos \theta+z \sin \theta) \mathrm{d} \theta
$$

motivating the name filtered backprojection [81]. The filter $\hat{h}\left(k_{x}^{\prime}\right)=\left|k_{x}^{\prime}\right|$ is known as the ramp or Ram-Lak filter and it acts as a high pass on the projections, hence correcting for the irregular sampling in Fourier space. To suppress the enhancement of noise in the reconstructed slices, it can be combined with a suitable window 
function, which alters the behavior at high spatial frequencies [19, 81]. The result of the filtered backprojection with a Ram-Lak filter, which will be generally used throughout the thesis, for the same exemplary slice as before is depicted in Fig. $3.1(\mathrm{e})$, revealing a sharp reconstruction of the $2 \mathrm{~d}$ object function $f(x, z)$.

\subsubsection{Cone-beam tomography}

For the derivation of the filtered backprojection, a parallel-beam geometry was assumed. Therefore, each horizontal line in the detection plane is independent from its neighbors and corresponds to the projection of parallel slices through the object, lying perpendicular to the rotation axis $y$. That allows for the direct reconstruction of the three-dimensional object via a series of $2 \mathrm{~d}$ inverse Radon transforms from projections at constant height $y$. However, the experimental setups used in this thesis, further described in chapter 4 , are built in a cone-beam geometry with diverging x-ray sources.

For small cone angles, the deviations from the parallel-beam geometry are negligible and the theoretical relations derived in the previous sections are approximately still valid (cf. section 3.2). For larger cone angles, where each pixel in the detection plane, apart from the central slice, comprises information from several parallel slices through the sample, a different approach has to be used. The most commonly implemented method was developed by Feldkamp, Davis and Kress [44] and is termed FDK cone-beam reconstruction in the following. The central slice in the detection plane can be exactly reconstructed by a fan-beam reconstruction algorithm [44]. The other slices in the detection plane are then treated as the midplane of a tilted arrangement and by correcting for the difference between the actual rotation and the rotation in this tilted coordinate system, approximated for small cone angles, the cone-beam geometry can be treated as a fan beam. In practice, this algorithm is implemented via a modified filtered backprojection in which each point on the detector is filtered and weighted according to its relative position to the central point of the illumination cone. Due to the approximation for small cone angles, artifacts can appear at the rim of the reconstruction volume, depending on the considered geometry.

In contrast to parallel-beam geometry, where an angular range $\theta \in[0,180]^{\circ}$ is sufficient, the angular range has to be increased for a cone-beam setup to record a complete tomographic dataset for all voxels inside the reconstruction volume. The situation is depicted in Fig. 3.3 for the central slice, in which the tomographic measurement is represented as the rotation of an illumination cone (or rather an illumination fan for the $2 \mathrm{~d}$ case) with an opening angle $\eta=40^{\circ}$ around the object. 

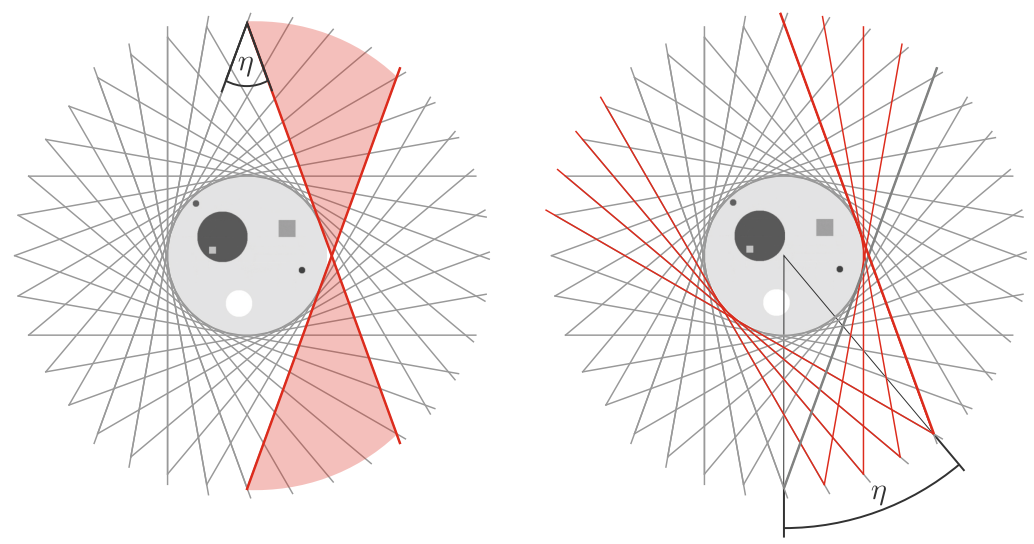

Figure 3.3: Illustration of the sampling in cone-beam tomography at the example of the central slice. The tomographic measurement is represented as the rotation of an illumination fan with an opening angle $\eta=40^{\circ}$ around the object. In the left panel, a scan with an angular range of $\theta \in[0,180]^{\circ}$ is presented, in which the sampling is incomplete in the red shaded area. The sampling can be completed by extending the angular range to $180^{\circ}+\eta$, as shown in the right panel (additional angles are depicted in red).

In the left panel, the situation is presented for a scan with an angular range of $\theta \in[0,180]^{\circ}$, in which the sampling is incomplete in the red shaded area and the voxels in the right half of the sample are not recorded from all sides. The sampling can be completed by extending the angular range to $180^{\circ}+\eta$, as depicted in the right panel of the Fig. 3.3. To correct for the redundancy of the data in oversampled regions of the sample, each projection has to be additionally weighted [130].

\subsection{Artifacts in tomography}

In the previous section, the fundamentals of tomographic imaging were described, leading to high quality reconstructions of the three-dimensional density distribution from projection images. However, imperfections in the recording process or data analysis can yield strong artifacts in the reconstruction, of which the most common are described in the following analogously to [88]. 


\subsubsection{Misaligned rotation axis}

For the tomographic reconstruction, both in the parallel and the cone-beam case, it is assumed that the axis of rotation is located in the vertical center line of the detection plane. For the simulation of the artifacts caused by a misaligned rotation axis, the same slice through the three-dimensional test volume as in the previous section is used (cf. Fig. 3.1). The volume consists of $512^{3}$ voxels and the projections are generated via the Matlab (MathWorks, USA) implemented function radon over an angular range $\theta \in[0,180]^{\circ}$ in $0.2^{\circ}$ steps. To evaluate the effect of a misaligned rotation axis with respect to the central line, all projections are shifted by 3 pixels (Fig. 3.4(a)) or -6 pixels (Fig. 3.4(b)), respectively. The tomographic reconstruction is carried out with the function iradon, performing a filtered backprojection with a standard Ram-Lak filter. It can be recognized that a misaligned rotation axis leads to artifacts at the edges of homogeneous features within the slice, i.e., the edges are not closed and half-ring structures appear. The direction and strength of these artifacts depends on the direction and strength of the misalignment. Note that the half-ring structures are caused by the angular range of $180^{\circ}$ and that in the case of a tomographic scan over the entire angular range $\theta \in[0,360]^{\circ}$, the effect would be visible as a doubling of the edges.

A misaligned rotation axis in the experimental data can be corrected by shifting all recorded projections by the distance of the actual tomographic axis to the central line of the detector. As this distance is generally unknown, the exact value has to be determined from the data, e.g., by comparing the projections at $0^{\circ}$ and $180^{\circ}$, as these should be in principle the same just flipped around the vertical axis, or by testing different shift values and determining the reconstruction in which the artifacts are minimized.

In the case of a tilted rotation axis, i.e., a rotation axis that is not positioned along the vertical center line of the detection plane but is tilted by an angle $\vartheta$, in the following denoted as the roll angle, a different form of artifacts occurs, as depicted in Fig. 3.4(c) for a roll angle of $\vartheta=2.5^{\circ}$. Features at the rim of the reconstructed slice are heavily deformed, while in the central part the artifacts appear less pronounced. In order to account for this kind of misalignment, all recorded projections have to be rotated by the corresponding value of the roll angle. As in the case of a shifted rotation axis, this value has to be obtained from the experimental data. According to Fig. 4.10, a tilted rotation axis can be considered as a rotation axis with a different shift in each parallel slice through the volume, provided that the roll angle $\vartheta$ is small. Hence, by optimizing this shift value for slices at different vertical positions in the detection plane, the angle can 

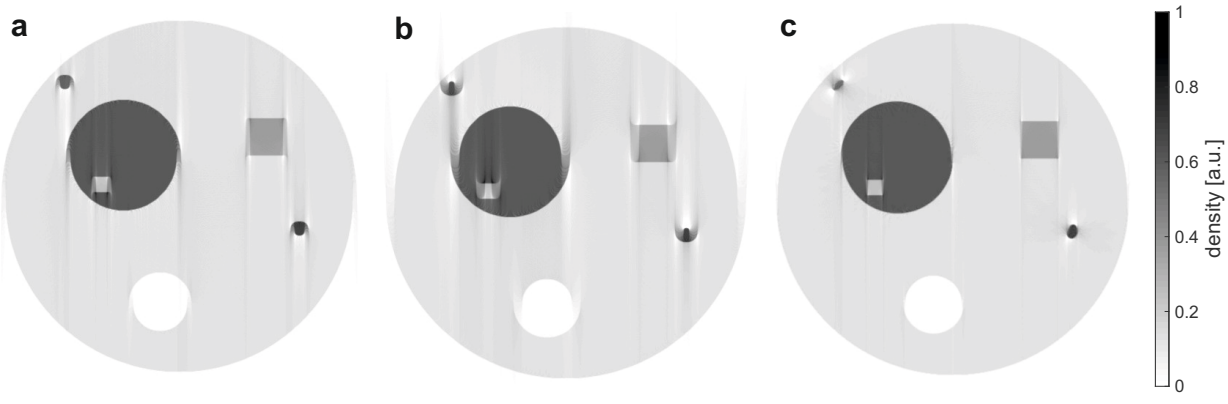

Figure 3.4: Influence of a misaligned rotation axis. For a shifted rotation axis by 3 pixels (a) and -6 pixels (b), respectively, artifacts occur at the edges of homogeneous features within the slice, i.e., the edges are not closed and half-ring structures appear. The direction and strength of these artifacts depends on the direction and strength of the misalignment. (c) A tilted rotation axis yields a deformed reconstruction of the object, which appears less pronounced in the central part of the slice.

be determined (cf. section 4.5).

\subsubsection{Ring artifacts}

A different kind of artifact is caused by pixels in the projections that systematically contain faulty intensity values. This can be caused, e.g., by a physically broken pixel, always leading to an incorrect value at the corresponding position in the detection plane or by hot pixels occurring during the measurement due to high energy photons. As each projection is corrected by a darkfield and an empty-beam image (cf. section 4.3), hot pixels in these correction images will lead to systematically altered pixels in all projections. In the first case, artifacts can be circumvented by masking out the pixels with modified sensitivity, whereas hot pixels can be corrected for by acquiring multiple darkfield and empty-beam images and averaging over them. Additionally, pixels that have a significantly different intensity value compared to their neighbors can be removed. Despite these corrections, systematically altered pixels can still be present, leading to horizontal stripes in the sinogram. During filtered backprojection, these lines are interpreted as a feature with constant distance to the rotation axis for all angles, hence resulting in a ring overlaying the actual structure of the $2 \mathrm{~d}$ reconstruction.

This situation is simulated by introducing 150 horizontal lines at random positions in the sinogram, each with a width of 1 pixel and a random variation of maximum $\pm 4 \%$ compared to the original intensity. The reconstruction of this altered sinogram without further corrections is shown in Fig. 3.5. Note that due to 
a
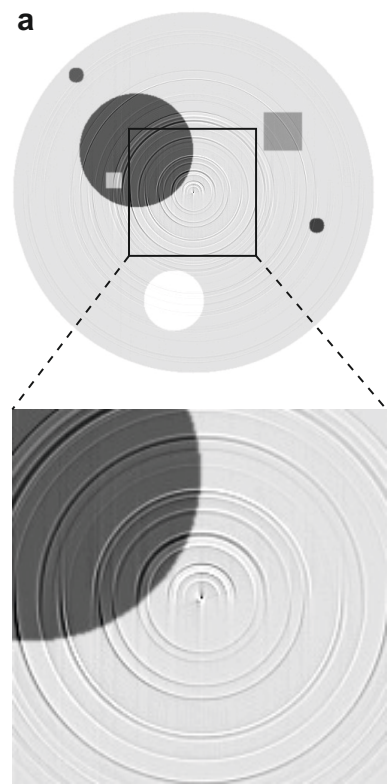

b
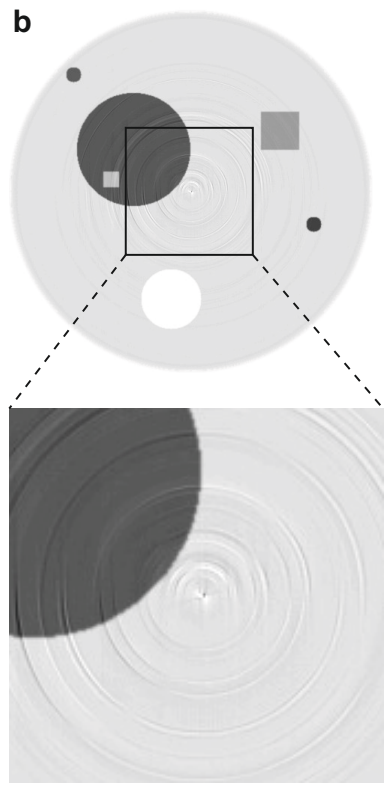
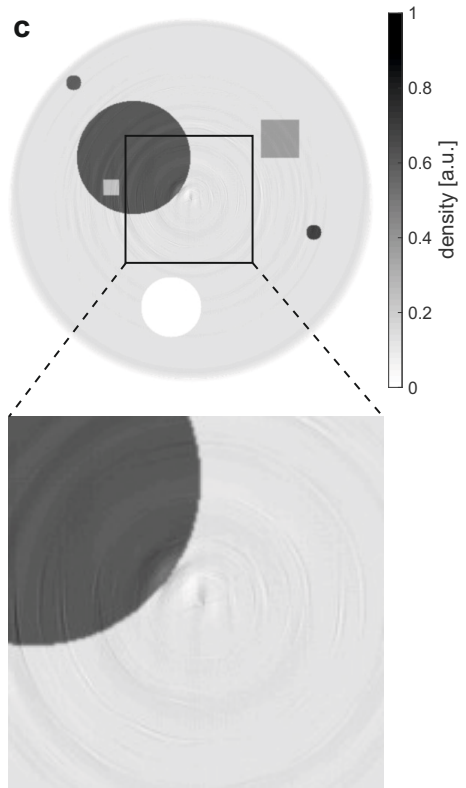

Figure 3.5: Performance of ring-removal algorithms. (a) The introduction of 150 horizontal lines at random positions in the sinogram, each with a width of 1 pixel and a random variation of maximum $\pm 4 \%$ compared to the original intensity, leads to strong ring artifacts in the reconstructed slice. (b) The application of the simple ring-removal algorithm based on the integration of the sinogram along the angle $\theta$ reduces these artifacts. However, small aberrations still remain. (c) The algorithm based on a wavelet decomposition and subsequent Fourier filtering also decreases the effect of rings in the image. As in the case of the simple algorithm, residual ring artifacts again remain in the resulting image. For this example, symlet 8 tap wavelets were used as the basis and $\sigma=1.5$ was chosen for the Gaussian filtering.

the angular range $\theta \in[0,180]^{\circ}$, the artifacts only consist of half rings that either occur in the upper or the lower half of the reconstructed slice, depending on the relative position to the central line in the sinogram. To account for these rings, different approaches are available that either aim at determining and subsequently correcting for inhomogeneities in the sensitivity of the detector pixels [172], removing the stripes in the sinogram prior to tomographic reconstruction or eliminating ring structures from the reconstructed slices [157].

In this thesis, ring removal is based on two algorithms, both following the second approach. The first starts by averaging the sinogram along the rotation angle $\theta$ [83]. As the sinogram typically consists of a superposition of different sine curves, averaging along the angle results in a rather smooth curve, whereas in the case of horizontal stripes peaks occur. By low-pass filtering of the obtained profile, mainly 
leading to a suppression of the high intensity peaks, and subtracting it from the original profile, the result will only have a significant contribution at the positions of the stripes. Hence, by subsequently correcting each line in the sinogram by subtracting this resulting profile, ring artifacts will be reduced (cf. Fig 3.5(b)). This rather simple approach works best for sharp and strong rings compared to the surrounding structures, as this simplifies the identification of peaks in the averaged profile.

A different approach is based on a wavelet decomposition of the sinogram in combination with a Gaussian filtering in Fourier space [115]. The wavelet decomposition allows for the identification and separation of horizontal features in the sinogram. This makes it possible to perform the subsequent filtering in Fourier space exclusively for these horizontal features, while not affecting the other components of the sinogram.

In Fourier space, horizontal features correspond to a vertical line through the origin. A multiplication with the Gauss function

$$
h\left(k_{\text {horz }}, k_{\text {vert }}\right)=1-e^{-\frac{k_{\text {horz }}^{2}}{2 \sigma^{2}}}
$$

where $k_{\text {horz }}$ and $k_{\text {vert }}$ denote the horizontal and vertical coordinate of the Fourier space, respectively, leads to a damping of this line and hence a reduction of horizontal features in the sinogram. The value of $\sigma$ defines the width of the Gaussian kernel and can account for deviations from a perfectly horizontal line in real space. The result for this approach is depicted in Fig. 3.5(c), showing a clear reduction of ring artifacts. However, both algorithms result in residual rings remaining in the image and their performance strongly depends on the individual datasets and the occurrence and strength of the pixel alterations.

\subsubsection{Region-of-interest tomography}

In a tomography setup with a divergent beam geometry, the geometrical magnification and hence effective pixel size can be varied, leading to measurements with changing resolution and field of view. Thus, an experiment can be carried out by recording an overview scan of the sample, determining interesting regions of interest and subsequently measuring it at higher magnification. In this case, the sample is usually larger than the field of view which can result in artifacts, as for the $2 \mathrm{~d}$ Radon transform, projections from a region of interest within the sample are not sufficient to quantitatively reconstruct the three-dimensional density distribution within this region [139]. Region-of-interest tomography, also called interior or local 

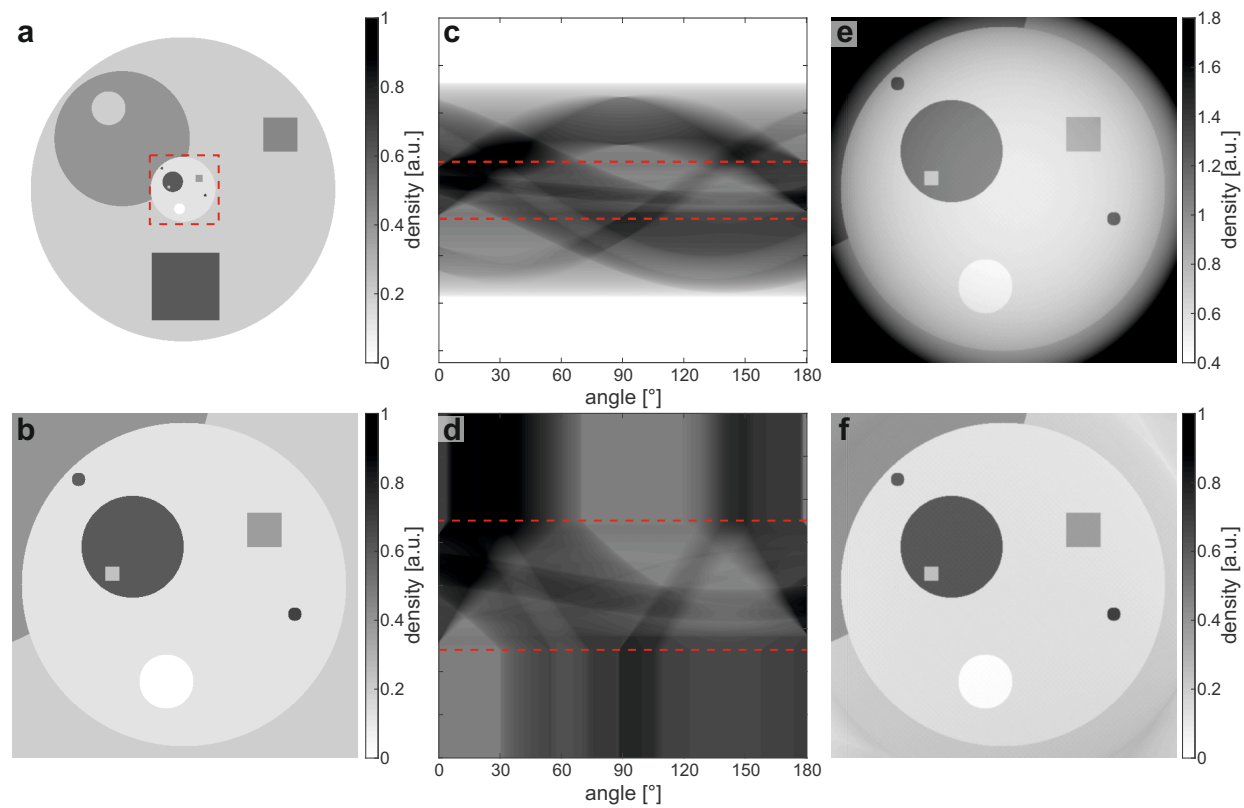

Figure 3.6: Artifacts introduced by region-of-interest tomography. (a) The original slice of size $512^{2}$ pixels is embedded in a larger phantom of size $2560^{2}$ pixels. The region of interest is marked by a red rectangle and a close-up is shown in (b). (c) The sinogram of the original object in (a) is cropped to the area marked by the red lines in order to simulate the sinogram acquired for the region of interest. The corresponding tomographic reconstruction is shown in (e), yielding strong cupping artifacts. (d) The effect of regionof-interest tomography can be corrected for by smoothly extending the sinogram via a replication of the edge values. The original sinogram is indicated by red lines. (f) Corresponding slice for the extended sinogram, revealing a reconstruction of the object in which low-frequency artifacts are reduced.

tomography, is simulated by embedding the previously considered slice in a larger phantom of size $2560^{2}$ pixels (cf. Fig. 3.6(a)), with the red rectangle marking the region of interest, shown at higher magnification in (b). The tomogram is emulated by generating the sinogram of the entire phantom for 900 angles within the range $\theta \in[0,180]^{\circ}$ and cropping it symmetrically around the central line to a width of 600 pixels (Fig. 3.6(c)). A direct reconstruction of this cropped sinogram via a filtered backprojection leads to the slice shown in Fig. 3.6(e), with low-frequency variations visible as a gradient towards the edges. Additionally, the obtained gray values strongly differ from the original data. A rather simple approach to overcome these artifacts is to smoothly extend the sinogram by replicating the edge values [96], as depicted in Fig. 3.6(d), which artificially increases the field of view. In the corresponding reconstruction in (f), the low-frequency artifacts are suppressed and 
the slice resembles the original, also with respect to the absolute gray values. Note, however, that these depend on the extent of the padding and therefore only the relative changes in density are truly accessible by region-of-interest tomography [96]. An alternative possibility to circumvent artifacts in local tomography might be given by the use of acquisition and reconstruction schemes based on the threedimensional Radon transform, but this still needs to be verified experimentally [178].

Additionally, the sampling required for an artifact-free reconstruction in regionof-interest tomography as well as tomography itself has to be considered. For an object of size $N_{x}$, where $N_{x}$ is the number of pixels perpendicular to the rotation axis necessary to image the entire sample, Nyquist sampling is fulfilled for a minimum of

$$
N_{\theta} \geq N_{x} \frac{\pi}{2}
$$

projections within the range $\theta \in[0,180]^{\circ}[21]$. In interior tomography, artifacts can be introduced by objects lying outside the field of view which are only present in projections from a restricted angular range and are therefore undersampled. To account for this, the number of projections has to be increased and it could be shown that the dimensions of the entire object have to be considered for optimal sampling, namely the above sampling criterion has to be fulfilled for the hypothetical case of the entire object being imaged at the reduced pixel size of the local tomography [96]. In practice, however, already a smaller number of projections yields rather satisfactory results, as, e.g., shown in the simulation in Fig. 3.6 where only 900 projections and therefore approximately a quarter of the required number was used.

\subsubsection{Motion artifacts}

Changes of the sample occurring during the recording of a tomographic scan can lead to severe artifacts which are only in few cases reversible. An example for the effect of a vertical motion of the sample on the reconstruction quality is given in Fig. 3.7. The motion model used for the simulation is depicted in (a) and the result of the filtered backprojection in (b), exhibiting strong artifacts visible as deformed features with open edges. For a known motion this can be corrected, e.g., by shifting the projections accordingly (in the case of rigid motion in vertical or horizontal direction) or performing a filtered backprojection along dynamically curved paths [146]. In the case of a global affine motion in cone-beam tomography, a method 
a

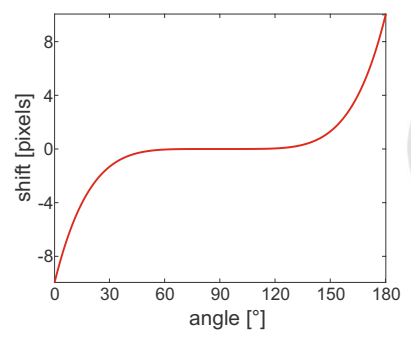

b

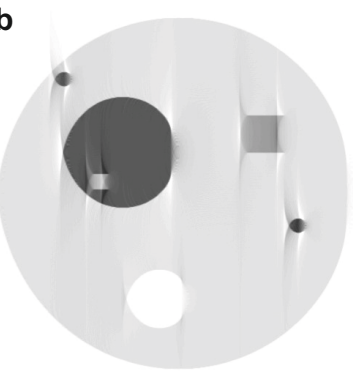

C

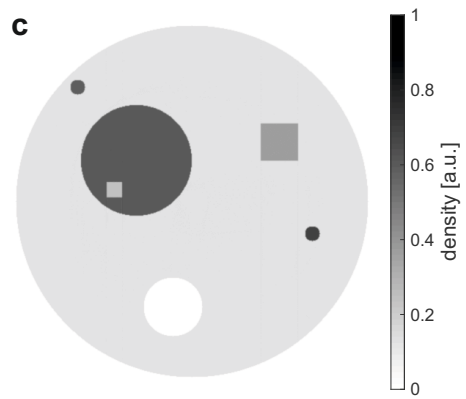

Figure 3.7: Effect of vertical motion of the object during the tomographic scan. Shifting all projections according to the motion model in (a) results in a heavily flawed reconstruction of the slice, in which single features are deformed with open edges (b). If the motion model is known, these artifacts can be corrected for by shifting the acquired projections accordingly, leading to the sharp reconstruction of the slice in (c).

for the detection and correction was presented in [177]. In most cases, however, the motion cannot be accounted for and artifacts can only be circumvented by changing the recording scheme, e.g., by decreasing the total scan time. However, due to limitations of the installed mechanical components and camera readout speed as well as the minimum dose required to reach a certain resolution [76], the minimal scan time is restricted and motion occurring on shorter time scales will yield disturbed reconstructions.

\subsubsection{Geometry artifacts}

As already described in section 3.1.3, a tomographic scan acquired in a divergent beam geometry has to be reconstructed using a cone-beam approach, as, e.g., given by the FDK algorithm. By reconstructing the data acquired in this geometry via a filtered backprojection assuming a parallel beam, artifacts are introduced in the reconstructed slices. To evaluate the effect with respect to the cone angle $\eta$, projections are simulated with the FDK implementation of the ASTRA tomography toolbox $[128,173,174]$ for cone angles of $0.25^{\circ}, 2.5^{\circ}$ and $25^{\circ}$, respectively. For each dataset, 900 projections over the angular range $\theta \in[0,180+\eta]^{\circ}$ are generated. The results of the parallel-beam reconstruction are depicted in Fig. 3.8(a-c). For small cone angles no deviations from the original slice can be observed whereas with increasing cone angles stronger artifacts are introduced, visible as deformed features with open edges similar to a misaligned rotation axis (cf. Fig. 3.4) or horizontal motion (cf. Fig. 3.7). The reconstruction with the FDK reconstruction algorithm, depicted in Fig. 3.8(d-f), leads to slices exhibiting a small amount of low-frequency 

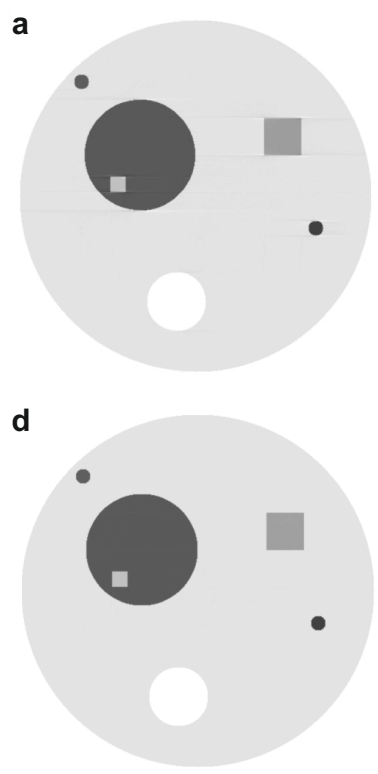

b

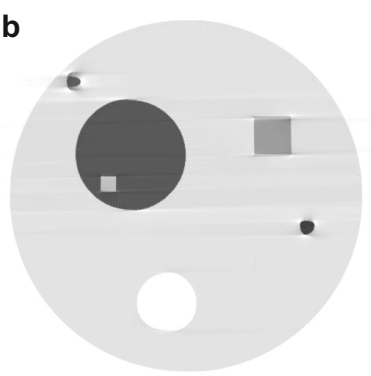

e

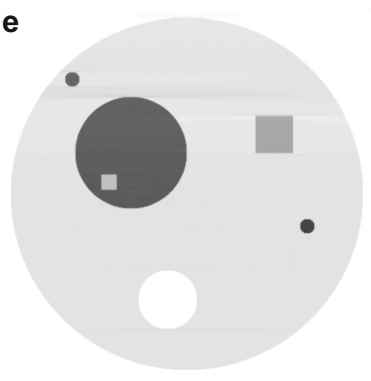

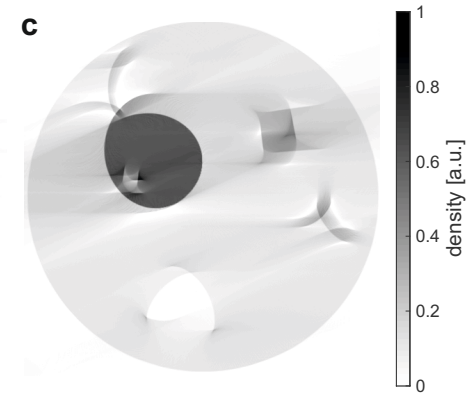

$\mathbf{f}$

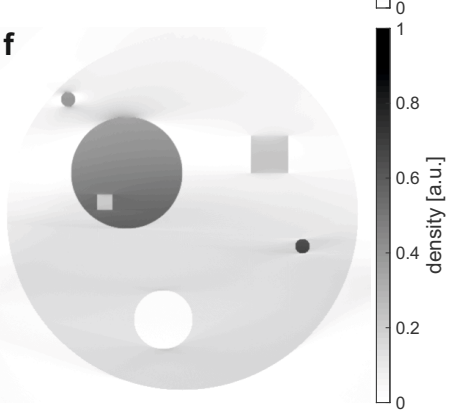

Figure 3.8: Artifacts introduced by an incorrectly assumed experimental geometry. For the simulation, a divergent beam with a cone angle of $0.25^{\circ}(\mathrm{a}, \mathrm{d}), 2.5^{\circ}(\mathrm{b}, \mathrm{e})$ and $25^{\circ}$ $(c, f)$ is assumed and the reconstruction is either carried out by a simple filtered backprojection using a parallel-beam geometry (upper row) or via an implementation of the FDK algorithm, taking the cone-beam geometry into account. For a small cone-angle, the difference between the two algorithms is negligible and both yield a satisfactory reconstruction. With increasing cone angle, the artifacts in the slices obtained by a parallelbeam assumption become more severe and are visible as deformed features with open edges similar to a misaligned rotation axis. The reconstructions with the FDK algorithm exhibit only a small amount of low-frequency artifacts, while high-frequency information can be reconstructed without distortions.

artifacts, while high-frequency information including the small features within the slice can be reconstructed without distortions. Note that for the successful reconstruction via the FDK algorithm, exact knowledge of the cone-angle and hence geometrical parameters as source-to-sample distance $z_{01}$, sample-to-detector distance $z_{12}$ and detector dimensions are required and that deviations will again introduce artifacts.

\subsubsection{Beam hardening}

In the case of polychromatic illumination with a large bandwidth $\Delta \lambda / \lambda$, as, e.g., generally produced by laboratory sources, artifacts can occur by imaging thick or 
strongly absorbing specimens, as low energies may be fully absorbed within the sample while higher energies are transmitted and contribute to the signal in the detection plane. Hence, the projected data deviates from the exponential decay predicted by the Lambert-Beer absorption law (1.69) which introduces artifacts, known as beam hardening (as the energy spectrum gets 'harder', i.e., shifts towards higher energies, while passing through the sample). They are typically visible as cupping, leading to an increased density towards the edges of the sample, or streaks along the direction of highest attenuation, especially visible for strongly absorbing features like metal components [33]. In the case of weakly absorbing unstained biological tissue, beam hardening is rarely observed. One possibility to reduce the artifacts introduced by beam hardening is the implementation of an appropriate choice of filters, leading to a decrease in spectral bandwidth $\Delta \lambda / \lambda$. 



\section{Experimental realization}

According to the generic experiment depicted in Fig. 1.1, a setup for propagationbased x-ray phase-contrast tomography mainly consists of three components: (i) a (partially) coherent x-ray source, (ii) the sample on a motorized sample stage and (iii) an x-ray detection device. In the following chapter, the setups used within this thesis are introduced and practical aspects regarding phase retrieval at a partially coherent polychromatic laboratory source as well as strategies for a correct tomographic alignment are discussed.

\subsection{X-ray detectors}

The resolution achievable by a detector is limited by its point spread function (PSF), which states how a single point will be mapped by the detection system, whereas the number of pixels combined with the effective pixel size restricts the field of view. There are several realizations of x-ray detectors available, each showing typical advantages and drawbacks. In a directly illuminated charged-coupled device $(C C D)$ with typical pixel sizes in the range of a few ten micrometers, $\mathrm{x}$-rays create electron-hole pairs due to the inner photoelectric effect and the accumulated charge is subsequently measured by the electronics. As this is performed in a pixel-by-pixel manner, the maximum frame rate is comparatively low [171]. The combination with a limited dynamical range, allowing only for short exposure times, makes this imaging device an ineffective choice for x-ray imaging with high flux sources $[7,171]$. In a photon-counting detector each pixel has its own additional electronics including amplifiers and energy discriminators, enabling a fast readout with zero readout noise and a high dynamical range while only photons above a certain energy threshold are considered. However, these additional electronics lead to large pixel sizes in the range of several tens of micrometers and insensitive inter-modular gaps [149].

In the course of this thesis, detection is performed via indirectly illuminated imaging devices, i.e., the x-rays are converted to visible light via a scintillation screen and subsequently recorded via classical CCD or CMOS (Complementary Metal 
Oxide Semiconductors) cameras. The light produced by the scintillator can be either transferred to the detection device via a fiber-optic plate or a lens-based system.

\subsubsection{Fiber-coupled detectors}

A fiber optic plate (FOP) consists of many micron-sized optical fibers, acting as waveguides, which efficiently transfer the visible light produced by the scintillator to the camera. Additionally, x-ray photons that are not converted to visible light are shielded by the FOP which reduces noise in the acquired image and prevents radiation damage in the sensor. In this thesis, three different realizations of fibercoupled detectors are used, denoted below by their laboratory names.

Pirra The 'Pirra' is a $2048 \times 2048$ pixels fiber-coupled sCMOS detector with a pixel size of $6.5 \mu \mathrm{m}$ (Photonic Science, United Kingdom). In contrast to a CCD device, CMOS cameras simultaneously convert each line of the detection device to a digital output and the speed is mainly limited by the time needed for the subsequent row-by-row readout. Scientific CMOS (sCMOS) cameras combine this fast readout with low noise and a high quantum efficiency of well over $80 \%[4,136]$. In the case of the 'Pirra' the maximum frame rate is given by $18 \mathrm{fps}$. For the conversion of x-rays into visible light it is equipped with a $15 \mu \mathrm{m}$ thick gadolinium oxysulfide $\left(\mathrm{Gd}_{2} \mathrm{O}_{2} \mathrm{~S}\right.$, gadox $)$ scintillator, leading to inhomogeneous images due to the polycrystalline structure of the scintillator.

Iris By using single-crystal scintillators, the inhomogeneities caused by the polycrystalline structure of a powder scintillator can be circumvented. This is realized in the 'Iris' detector which combines a $20 \mu \mathrm{m}$ lutetium aluminum garnet $\left(\mathrm{Al}_{5} \mathrm{Lu}_{3} \mathrm{O}_{12}\right)$ scintillator with a $2048 \times 2048$ pixels sCMOS camera chip (Hamamatsu Photonics, Japan). As in the case of the 'Pirra', each pixel has a size of $6.5 \mathrm{\mu m}$. The maximum readout at full resolution is $30 \mathrm{fps}$.

Talos The 'Talos' is a flat panel CMOS detector equipped with a $150 \mu \mathrm{m}$ thick gadox scintillator (PerkinElmer, USA). It consists of $1536 \times 1944$ pixels with a size of $75 \mathrm{\mu m}$. The thick scintillator leads to an efficient conversion of x-rays to visible light though at higher diffusion. The 'Talos' detector can be advantageously used for imaging of large fields of view in the range of several millimeters as the magnification needed to achieve the corresponding pixel sizes of a few micrometers 
requires relatively large sample-to-detector and hence propagation distances which lead to small Fresnel numbers and consequently a high contrast transfer [170].

\subsubsection{Lens-coupled detectors}

In order to achieve smaller pixel sizes for high resolution imaging, the fiber optic plate can be exchanged by a lens system which focuses the light onto the camera chip. By employing lenses with different magnifications, the pixel size of the detector can be varied.

Argos The 'Argos' is a $2504 \times 3326$ pixels CCD camera equipped with a 10 -fold magnification objective (XSight Micron, Rigaku, Czech Republic), converting the $5.4 \mu \mathrm{m}$ pixel size of the CCD chip to an effective pixel size of $540 \mathrm{~nm}$, and a thin single crystal scintillator which is designed to reduce diffusion and hence leads to a small detector PSF below $1 \mu \mathrm{m}[142,167]$. The combination of a thin scintillator, an inefficient transfer of light from the scintillator to the CCD chip and a small pixel size leads to comparatively long exposure times, especially at a laboratory setup.

\subsection{GINIX setup at PETRAIII}

A large part of the experiments presented in this thesis was carried out at the Göttingen Instrument for Nano Imaging with coherent X-rays (GINIX) [82] which is installed at the P10 beamline of the PETRAIII storage ring at DESY (cf. Fig. 4.1). By accelerating electrons perpendicular to their relativistic velocity, electromagnetic radiation in the energy range of x-rays is emitted. The brilliance $B$ of an x-ray source can be quantified via

$$
B=\frac{\text { photons }}{\mathrm{s} \cdot \mathrm{mrad}^{2} \cdot\left(\mathrm{mm}^{2} \text { source area }\right) \cdot(0.1 \% \text { bandwidth })}
$$

and hence takes into account the intensity, the divergence of the beam, the source size and the spectral bandwidth. The PETRAIII storage ring with a circumference of $2304 \mathrm{~m}$ and a maximum brilliance beyond $10^{21} \mathrm{ph} /\left(\mathrm{s} \mathrm{mrad}^{2} \mathrm{~mm}^{2}(0.1 \% \mathrm{BW})\right)$ (20 m long undulator with $29 \mathrm{~mm}$ magnetic period, photon energies $<10 \mathrm{keV}$ (1st harmonic) [185]) is currently the most brilliant storage ring in the hard x-ray range [35].

At the P10 beamline, radiation is produced by a $5 \mathrm{~m}$ long undulator with $29 \mathrm{~mm}$ 


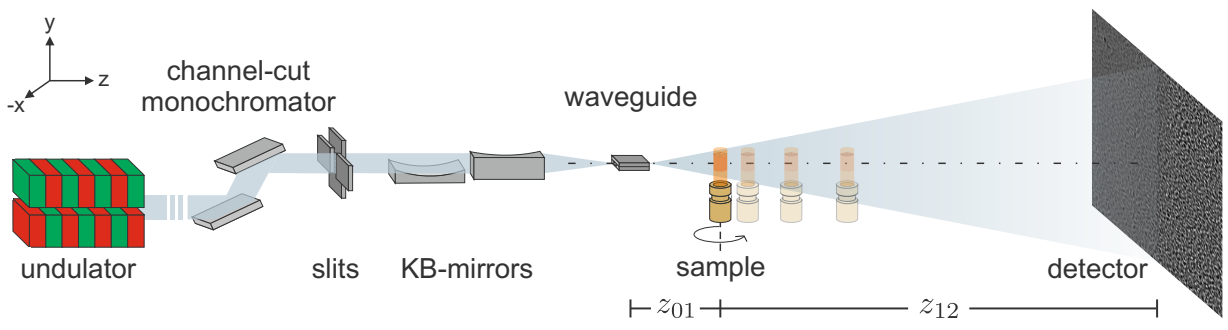

Figure 4.1: Sketch of the GINIX setup installed at the P10 beamline at PETRAIII. The $\mathrm{x}$-rays are generated in an undulator device and monochromatized by a doublecrystal Si(111) channel-cut monochromator. Subsequently, they are prefocused by a set of Kirkpatrick-Baez (KB) mirrors to a spot size of $\sim 200 \times 200 \mathrm{~nm}^{2}$ and refined by a waveguide placed in the focal plane of the mirrors. The sample is mounted on a fully motorized sample tower at a distance $z_{01}$ behind the waveguide and the evolving intensity distributions are recorded in the detection plane at distance $z_{12}$ behind the sample.

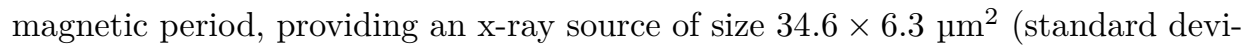
ation, $h \times v)$ with a divergence of $28.9 \times 1.6 \mu^{2} \mathrm{~d}^{2}$, which is installed in a low beta section of the storage ring. Note that the specified parameters are design values and that the actual measured values may slightly differ [153]. After two beamdefining pairs of slits (not shown in Fig. 4.1), the beam is monochromatized by a cryo-cooled double-crystal $\mathrm{Si}(111)$ channel-cut monochromator to a bandwidth of $\frac{\Delta \lambda}{\lambda} \simeq 10^{-4}$.

The GINIX endstation is installed on an optical table which can be moved into the beam downstream of the monochromator. The beam size is defined by a third pair of slits and the beam is subsequently focused by a set of Kirkpatrick-Baez (KB) mirrors, showing an elliptical shape [86]. In an ellipse the distances between any point on the boundary to the two focal points add to the same constant. Hence, by constructing the mirrors such that the first focal point lies in the x-ray source spot and the second some distance behind the mirrors, focusing can be reached due to constructive interference in the second focal point. The combination of two successive perpendicular mirrors leads to a two-dimensional focus, where the different focal lengths needed in order to create a single focal plane have to be taken into account [82]. The current set of KB-mirrors reaches focus sizes in the range of $200 \times 200 \mathrm{~nm}^{2}$ with a flux larger than $10^{11} \mathrm{ph} / \mathrm{s}$ [148]. Although imaging could be carried out in this configuration, the partial coherence and relatively large focal spot limit the achievable resolution. Additionally, small irregularities in the sub-nanometer range on the surface of the mirrors produce strong high-frequency 
aberrations in the illumination (cf. Fig. 4.2(c)), which lead to a violation of the requirements for empty-beam correction, as further discussed in the following section. Therefore, a waveguide is placed into the focal point of the KB-mirrors, acting as a coherence and spatial filter, as only a limited number of modes can propagate through the channel [70,93,122]. Additionally, it can focus the radiation to spot sizes well below $20 \mathrm{~nm}$ [94, 95, 118] and acts as a low-pass filter (cf. Fig. 4.2(d)), hence suppressing the high-frequency artifacts produced by the focusing mirrors. The waveguide is mounted on a hexapod positioner system (SmarPod (custommade model), SmarAct, Germany), including all six rotational and translational degrees of freedom, in order to precisely align it with respect to the incoming beam. Behind the waveguide the sample is placed on a fully motorized sample tower. It consists of an air-bearing rotation for tomographic measurements (UPR-160 AIR, Micos, Germany) and three translations above the rotation axis for a precise alignment of the sample into the field of view (SLS-1760 and SHL-1D20N-10, SmarAct, Germany). The positioning as well as alignment of the rotation axis, necessary to avoid artifacts as described in section 3.2.1, can be carried out with three additional translational motors below the rotation axis (HPS-170 and UPL-160, Micos, Germany) as well as two self-built cradles to account for a tilt along and perpendicular to the optical axis. The procedure for the alignment is further described in section 4.5. Behind the sample tower an on-axis-view (OAV) microscope (not depicted in Fig. 4.1) can be used for positioning of the sample with respect to beam. Due to the relatively small field of view in the detection plane, being in the range of $350 \times 350 \mathrm{\mu m}^{2}$ to $120 \times 120 \mathrm{\mu m}^{2}$ in the course of this thesis, an alignment of the sample without the optical pre-alignment with the microscope can be timeconsuming. Approximately $5 \mathrm{~m}$ behind the sample tower, the detector is placed on a motorized stage (LS-270 and UPL-160, Micos, Germany). Absorption of photons in between these two components is minimized by installing an evacuated flight tube in the setup. The long distance between the sample tower and the detector allows for imaging in the holographic range at magnifications that lead to pixel sizes in the range of a few ten to hundred nanometers by using one of the two fiber-coupled sCMOS detectors described in the previous section.

The setup can also be used in a parallel-beam geometry, allowing for larger fields of view at medium resolution. To this end, the focusing optics, namely the KBmirrors and the waveguide, are moved out of the beam, and the direct low divergent illumination of the undulator, monochromatized by the channel-cut monochromator, is used for imaging. As in this configuration the resolution is limited by the detector [8, 61, 167], the high resolution detector 'Argos' is installed in the setup 

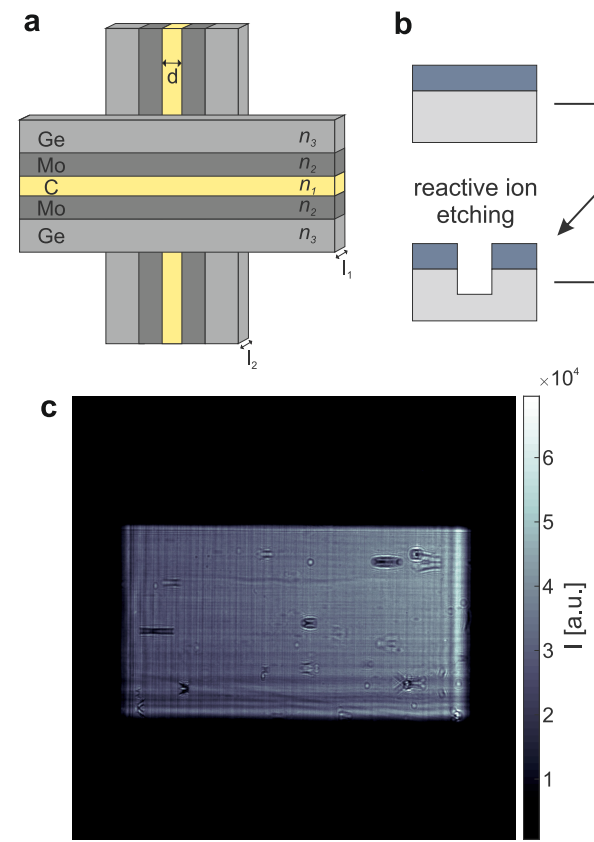

b

reactive ion etching

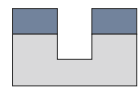

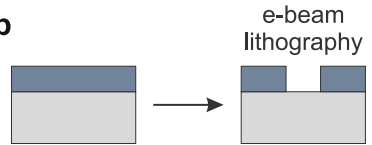
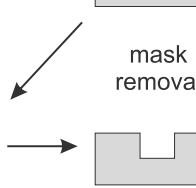
removal
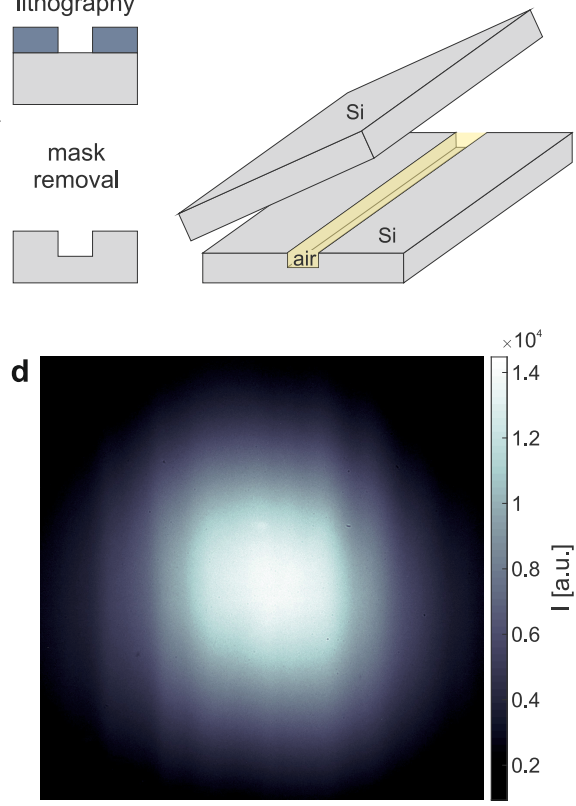

Figure 4.2: Effect of the waveguide on the illumination. (a,b) A waveguide can be either realized by combining two $1 d$ devices, consisting of a multilayer structure with carbon as guiding layer, or by etching a channel into a silicon wafer and bonding a second wafer on top, leading to a closed channel with air as guiding layer. By introducing these waveguides into the setup, the disturbed illumination (c), caused by small sub-nanometer irregularities on the surface of the KB-mirrors, is spatially filtered, resulting in a smooth illumination in which high-frequency variations are suppressed (d).

in close proximity to the sample, allowing for imaging in the direct-contrast or near holographic regime.

Waveguides In principle, an x-ray waveguide consists of a guiding layer surrounded by a cladding material thick enough to block the incoming beam. Due to the properties of x-rays, the refractive index of the cladding material has to be smaller compared to the guiding layer in order to allow for total reflection within the waveguide.

One way to fabricate a one-dimensional waveguide is to sputter different materials on a substrate (cf. Fig. 4.2(a)), leading to layers of varying refractive index which can be designed to optimize waveguide performance [94, 95]. Here, a layer of molybdenum is deposited on a germanium wafer, followed by a thin layer of carbon with width $d$, which acts as the guiding layer, and again molybdenum and 
a thin layer of germanium to create a closed channel. In a last step, a second germanium wafer is bonded on top of the multilayer to increase thickness and hence allow for blocking of the entire primary beam. This design allows for high aspect ratios between the width of the carbon layer $d$ and the thickness of the multilayer, depicted as $l_{1 / 2}$. Therefore, this design can also be used for relatively high photon energies, as here a thick cladding is needed to block the entire beam. However, as carbon also absorbs a substantial amount of the incoming radiation, the transmission of these waveguiding devices is lowered, especially when perpendicularly combining two multilayer devices in order to build a 2 d waveguide (cf. Fig. 4.2(a)). Together with the limited acceptance of the incoming beam due to the geometrical restrictions of the small channels, the flux for a typical photon energy of $13.8 \mathrm{keV}$ is reduced by $2-3$ orders of magnitude to the range of $10^{8}-10^{9} \mathrm{ph} / \mathrm{s}$.

Another approach to build a $2 \mathrm{~d}$ waveguide while providing a large transmission of x-rays is based on air filled channels etched into a silicon wafer (cf. Fig. 4.2(b)) $[71,118]$. To this end, a silicon wafer is coated with a resist which changes its solubility upon illumination by electrons. Via electron-beam lithography, channel structures with high aspect ratios can be imprinted in the resist and in a subsequent developing process removed from the mask. Afterwards, the channel structures are transferred to the silicon wafer via reactive ion etching. In a last step, the remaining resist is removed from the wafer and a second silicon wafer is bonded on top, leading to a closed channel with air as guiding layer. As the length of the waveguide is determined after this fabrication process by trimming the wafer to the desired dimensions, it can be adjusted with respect to the energy. The writing of the waveguide structures into the resist via electron-beam lithography also enables more elaborate designs of waveguides, as, e.g., a tapered geometry in which the width of the initially relatively large channel gradually decreases towards the waveguide exit [24]. This allows for a larger acceptance of the incoming beam and hence leads to a higher transmission. At an x-ray energy of $8 \mathrm{keV}$ straight channels yield a flux in the range of $10^{9} \mathrm{ph} / \mathrm{s}$, whereas for a tapered geometry up to $10^{10} \mathrm{ph} / \mathrm{s}$ can be transmitted through the waveguide.

\subsection{Empty-beam correction}

According to section 1.2.3, the probe $P_{\omega}\left(\mathbf{r}_{\perp}\right)$ in the object plane has to be known in order to reconstruct the projected refractive index from the measured intensity images. However, in most cases only the propagated probe can be obtained experimentally by measuring the so-called empty image, in which the sample is removed 
from the field of view, yielding

$$
I_{0}\left(\mathbf{r}_{\perp}, z\right)=\left|\mathcal{D}^{(F)}\left[P_{\omega}\left(\mathbf{r}_{\perp}\right)\right]\right|^{2} .
$$

Correcting the measured intensity $I_{\omega}\left(\mathbf{r}_{\perp}, z\right)$, given by the propagated product of the probe and object transmission function $O_{\omega}\left(\mathbf{r}_{\perp}\right)$, by this empty image leads to

$$
\begin{aligned}
I_{\text {corr }}\left(\mathbf{r}_{\perp}, z\right) & =\frac{I_{\omega}\left(\mathbf{r}_{\perp}, z\right)}{I_{0}\left(\mathbf{r}_{\perp}, z\right)} \\
& =\frac{\left|\mathcal{D}^{(F)}\left[O_{\omega}\left(\mathbf{r}_{\perp}\right) \cdot P_{\omega}\left(\mathbf{r}_{\perp}\right)\right]\right|^{2}}{\left|\mathcal{D}^{(F)}\left[P_{\omega}\left(\mathbf{r}_{\perp}\right)\right]\right|^{2}} .
\end{aligned}
$$

Under the assumption that the object transmission function and the probe function can be propagated independently

$$
\mathcal{D}^{(F)}\left[O_{\omega}\left(\mathbf{r}_{\perp}\right) \cdot P_{\omega}\left(\mathbf{r}_{\perp}\right)\right] \simeq \mathcal{D}^{(F)}\left[O_{\omega}\left(\mathbf{r}_{\perp}\right)\right] \cdot \mathcal{D}^{(F)}\left[P_{\omega}\left(\mathbf{r}_{\perp}\right)\right]
$$

this can be approximated as

$$
I_{\mathrm{corr}}\left(\mathbf{r}_{\perp}, z\right) \simeq \frac{\left|\mathcal{D}^{(F)}\left[O_{\omega}\left(\mathbf{r}_{\perp}\right)\right]\right|^{2} \cdot\left|\mathcal{D}^{(F)}\left[P_{\omega}\left(\mathbf{r}_{\perp}\right)\right]\right|^{2}}{\left|\mathcal{D}^{(F)}\left[P_{\omega}\left(\mathbf{r}_{\perp}\right)\right]\right|^{2}}=\left|\mathcal{D}^{(F)}\left[O_{\omega}\left(\mathbf{r}_{\perp}\right)\right]\right|^{2}
$$

Hence, as long as the assumption in eqn. (4.5) is fulfilled, the empty-beam corrected intensity images correspond to the propagated object transmission function which enables the reconstruction of the projected refractive index. In section 1.3.4 it was shown that for a parabolic wave this assumption holds true as propagation can be rewritten in an effective plane wave geometry. In [73] a mathematical approach towards the validity of eqn. (4.5) is presented. It is shown that with increasing smoothness of the illumination function the relative error made by the empty-beam correction decreases. Therefore, eqn. (4.5) is a good approximation for illumination by Gaussian or spatially filtered beams produced by a waveguide in the focal plane, whereas the illumination with a purely KB-focused beam, yielding high-frequency aberrations due to the roughness of the mirror surfaces, leads to strong artifacts in the empty-beam corrected intensities [73]. Note that these artifacts are largest for small object features, impeding high resolution imaging without further corrections of the resulting intensity images.

One possibility to overcome the limitations of the empty-beam correction is to simultaneously reconstruct the probe and the wave field in the object plane from the measurement, yielding the object transmission function via a simple division. 
However, in practice it is quite challenging to reconstruct the probe from the measurements and more elaborate acquisition or reconstruction schemes as, e.g., near-field ptychography including lateral and/or longitudinal shifts of the sample $[143,160]$ or divide and update [62], have to be used.

\subsection{Laboratory setup JuLiA}

\subsubsection{Experimental design}

Complementing the experiments carried out at the synchrotron, a laboratory setup with a microfocus source providing the necessary partial coherence for propagationbased imaging was used within this thesis (cf. Fig. 4.3). X-ray generation in conventional laboratory sources is based on the deceleration of electrons when approaching a metal anode, leading to x-ray photons with a continuous energy spectrum called 'bremsstrahlung', as well as the interaction between free electrons and those bound to an atom of the anode material, yielding characteristic radiation with element-specific discrete energy values (cf. Fig. 4.4).

As only $\sim 1 \%$ of the electron energy is converted to bremsstrahlung while the remaining part of the input energy leads to a heating of the anode material, melting can occur at too high electron impact, which limits the amount of photons produced by the x-ray source. This has an especially high influence for small source spots, needed for a sufficiently large partial coherence to enable propagationbased imaging, as in this case the maximum electron power is comparably low. Typically, low-power microfocus sources have electron-beam power densities of $\sim 200 \mathrm{~kW} / \mathrm{mm}^{2}$ while rotating anodes with a minimum spot size of $\sim 70 \mu \mathrm{m}$ can reach $\sim 300 \mathrm{~kW} / \mathrm{mm}^{2}[66,141]$. Note that in the case of the latter the effective source spot was considered and that the actual electron focal spot is a line focus with a typical aspect ratio of 1:10.

The laboratory setup JuLiA ('Just a Liquid Anode') consists of a microfocus x-ray source (JXS D2, Excillum, Sweden) with the metal alloy Galinstan $(68.5 \%$ $\mathrm{Ga}, 21.5 \% \mathrm{In}$ and $10 \% \mathrm{Sn}$ ) as anode, which is liquid at room temperature. It is constantly circulating at a pressure of 190 bar through the system, passing a chamber in which the electrons can encounter the metal and generate x-rays. To avoid contamination and instabilities in the jet, the entire circuit is kept at vacuum. Liquid metal jet sources can potentially achieve a $>100 \times$ increase in effective 


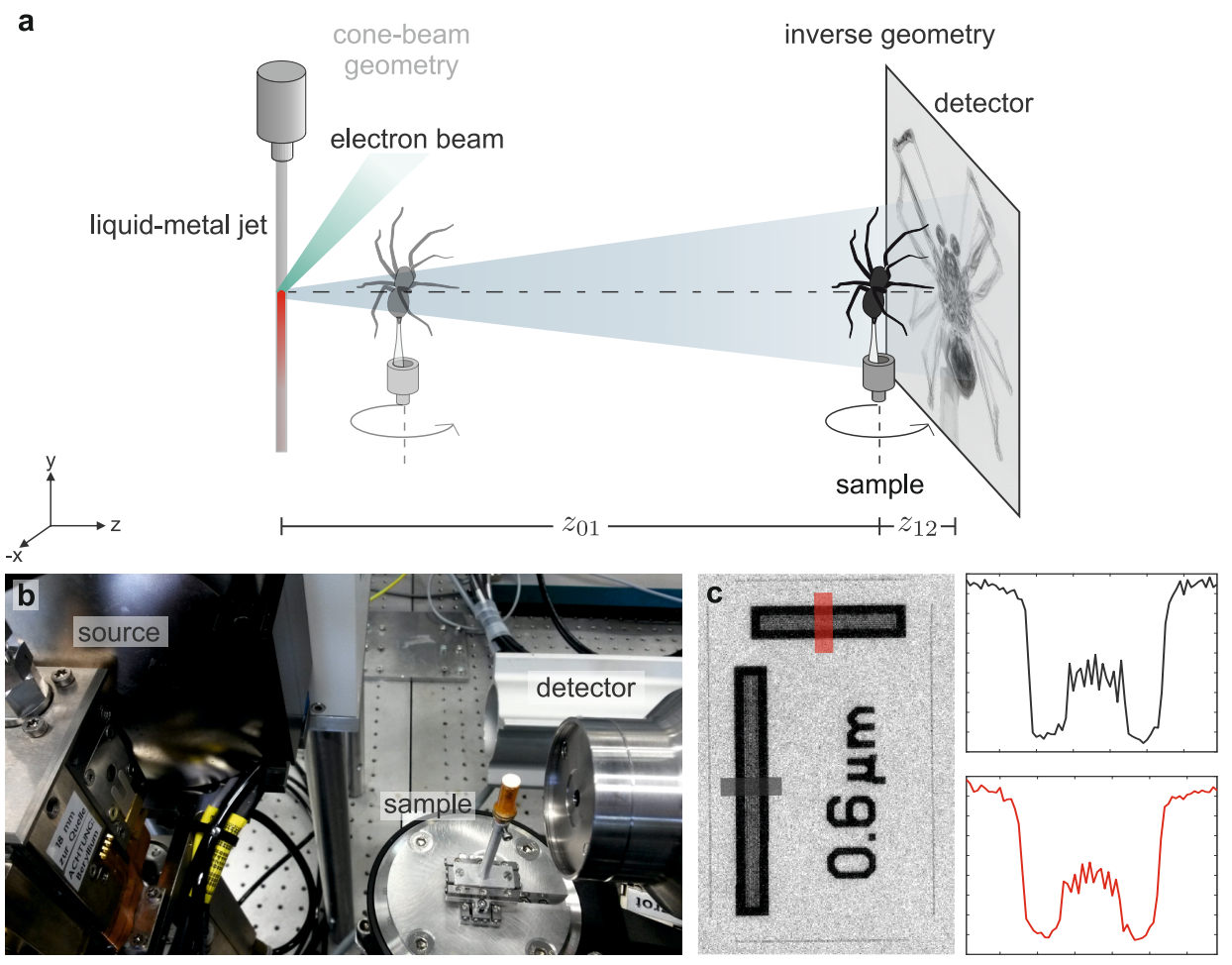

Figure 4.3: Sketch of the laboratory setup. (a) The x-rays are generated by a liquidmetal jet source with Galinstan as anode material. In a distance $z_{01}$ behind the source, the sample is positioned on a fully motorized sample stage and the intensity distributions are recorded in a distance $z_{12}$ behind the sample. Depending on the desired resolution and field of view, the setup can be either operated in a cone-beam $\left(z_{01} \ll z_{12}\right)$ or inverse geometry $\left(z_{01} \gg z_{12}\right)$. (b) Photograph of the setup in inverse geometry. (c) In the inverse geometry, the $600 \mathrm{~nm}$ lines and spaces of a test pattern can be resolved in $2 \mathrm{~d}$, as revealed in the profiles averaged over 50 pixels shown on the right.

brightness ${ }^{1}$ compared to conventional microfocus sources [66], whereby the current version reaches an approximate factor of 10 . This increase in brightness is on the one hand enabled by the continuous regeneration of the anode material, allowing for electron power densities that can vaporize the anode material, and on the other hand by the decrease in stationary heat development due to the high flow velocity of the liquid jet. The generated x-rays exit the source through a beryllium window which can be heated in order to remove metal debris, caused by, e.g., instabilities in the jet or evaporation of the metal due to a high heat load. In the current

1 The brightness is equivalent to the brilliance $B$, as defined in eqn. (4.1), without the normalization by the spectral bandwidth. 

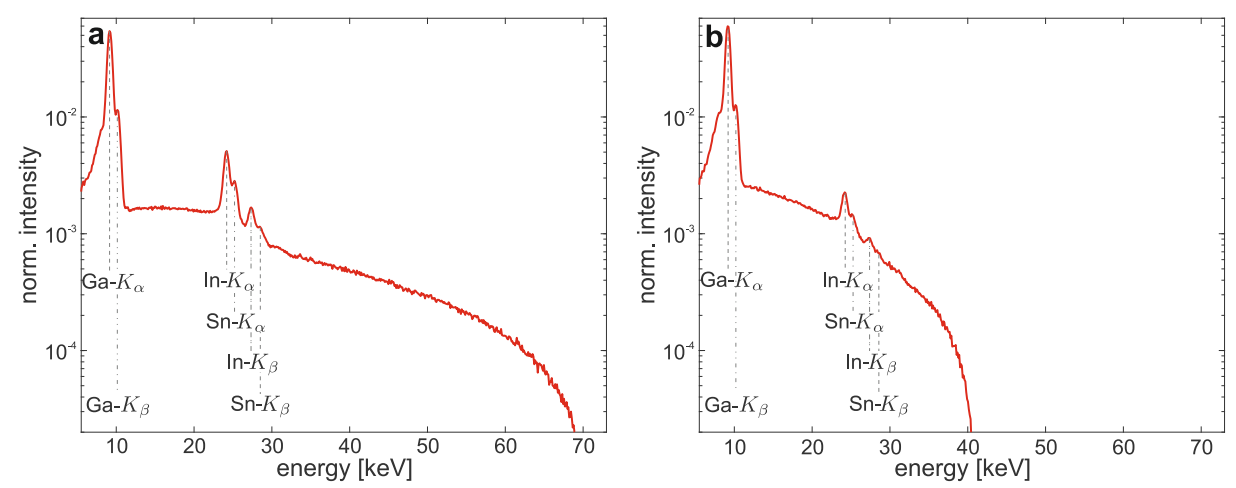

Figure 4.4: Energy spectra for acceleration voltages of $70 \mathrm{kV}$ (a) and $40 \mathrm{kV}$ (b), measured in a distance of $1.76 \mathrm{~m}$ to the source. The lower transmission of small energy contributions through air is corrected for, using tabulated values [12]. The characteristic peaks of the elements contained in the Galinstan anode, overlaying the bremsspectrum with a maximum energy given by the kinetic energy of the electrons, are clearly visible.

configuration the source can be operated at acceleration voltages of up to $70 \mathrm{kV}$, although in principle the maximum is at $160 \mathrm{kV}$. It is generally used at $40 \mathrm{kV}$ or $70 \mathrm{kV}$, leading to the x-ray spectra depicted in Fig. 4.4. The main energy is given by the characteristic Ga- $K_{\alpha}$ emission line at $9.25 \mathrm{keV}$, but also the higher contributions from indium and tin are clearly visible, especially at $70 \mathrm{kV}$.

The source size, influencing the degree of partial coherence (cf. section 1.4), is determined by the electron beam focus, reaching values down to $\sim 4 \mu \mathrm{m}$. Generally it is chosen asymmetrically with an aspect ratio of 1:4 (height:width), as only the height of the electron focus directly influences the size of the photon source spot in vertical direction as seen from the exit window. The increased width mainly leads to a higher photon flux as the generated photons are integrated along the beam direction (cf. Fig. 4.5 on the left). In the horizontal direction, the source spot is limited by the penetration depth of the electrons into the liquid jet with an approximate diameter of $190 \mathrm{\mu m}$. Therefore, the actual position of the electron spot on the metal jet also has an impact on the projected source size, as depicted in Fig. 4.5. If it is positioned off-center, the curvature of the jet leads to an increased horizontal spot size. However, due to the decreased self-absorption of x-rays in the metal jet, the photon flux is increased. Additionally, a more homogeneous illumination is generated as the self-absorption is approximately constant at each vertical position of the beam, whereas in the center position, photons created towards the center of the jet encounter a significantly larger self-absorption.

In a distance $z_{01}$ from the source, the sample is placed on a fully motorized sam- 


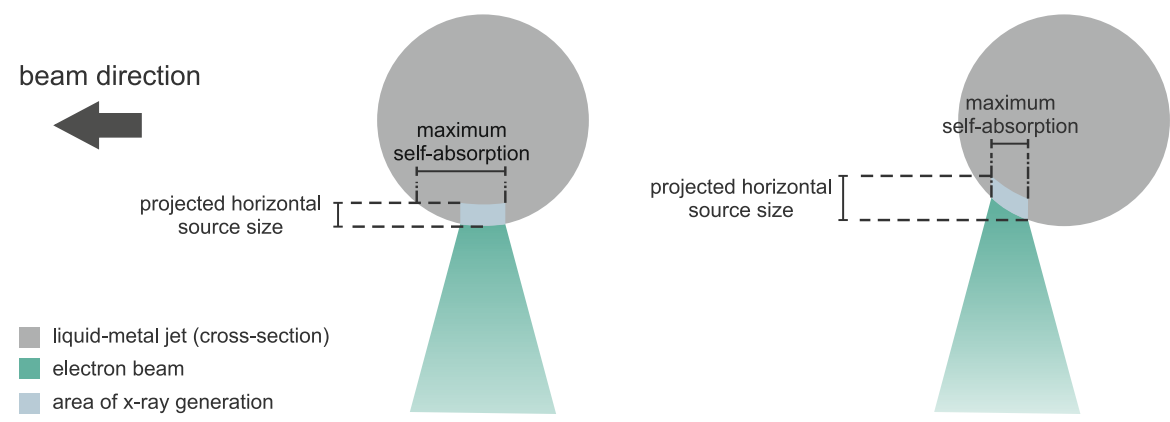

Figure 4.5: Illustration of the influence of the electron beam position along the optical axis. The projected source spot is minimal when the electron beam is positioned at the center of the metal jet, but the increased self-absorption leads to a decreased photon flux. If the electron beam is shifted towards the exit window, self-absorption is reduced, but the projected source spot is enlarged in horizontal direction.

ple stage, including a rotation for tomographic measurements (PRS-110, Micos, Germany), two translational motors above to align the sample with respect to the rotation axis (SLC-1730, SmarAct, Germany) and three additional linear stages below for positioning and alignment (PLS-85, Micos, Germany). The distance between the source and the sample can be varied over long distances, beyond the travel range of the according motor, as the entire sample stage is assembled on an aluminum profile, enabling a high flexibility. On a parallel aluminum profile the detector is placed on a motorized detector stage (5101.1 and 5103.1, Huber Diffraktionstechnik, Germany), allowing for a movement perpendicular to the optical axis. This can be used for the exact alignment of the rotation axis, as depicted in section 4.5 .

The source can be operated at two imaging modalities (cf. Fig. 4.3(a)). In the classical approach, termed 'cone-beam geometry' in the following, the sample is positioned at a small distance to the source as compared to the detector $\left(z_{01} \ll z_{12}\right)$, leading to a large magnification and hence small effective pixel size, similar to the synchrotron setup described in the previous section. The resolution of the imaging system, given by [61]

$$
\sigma_{\mathrm{sys}}=\sqrt{(M-1)^{2} M^{-2} \sigma_{\mathrm{src}}^{2}+M^{-2} \sigma_{\mathrm{det}}^{2}},
$$

where $\sigma$ denotes the standard deviation, is in this geometry limited by the source size and therefore restricted to $\sim 4 \mu \mathrm{m}$ (FWHM). By moving the sample close to the detector, leading to a small geometrical magnification of the imaging system, and installing the high resolution detector 'Argos' in the setup, the resolution can 
be increased as it is in this case limited by the detector point spread function. This configuration is hereinafter referred to as 'inverse geometry'. It enables the resolution of the $600 \mathrm{~nm}$ lines and spaces of an absorbing test pattern (JIMA RT RC-03, Japan Inspection Instruments Manufacturer's Association, Japan), as can be seen in Fig. 4.3(c). The small pixel size, restricting the field of view to approximately $1 \times 1.5 \mathrm{~mm}^{2}$, still leads to Fresnel numbers well below 1 and hence phase-contrast effects are visible despite the short propagation distance in the range of few centimeters.

By combining both geometries, the sample can be imaged on multiple length scales. An overview scan with a relatively large field of view in the range of several square millimeters can be obtained in the cone-beam geometry, enabling the selection of specific regions of interest. By subsequently changing the setup to the inverse geometry and aligning the sample accordingly, these regions can be imaged at higher resolution, providing insights into the three-dimensional sample structure in the micrometer range.

\subsubsection{Phase retrieval at laboratory sources}

Due to relatively short propagation distances and partial coherence, imaging at the laboratory is restricted to the direct-contrast regime and phase contrast is typically visible as edge enhancement. In section 2.1, approaches for phase reconstruction in this particular regime were introduced. Based on experimental data obtained for a biological test object, the performance of these algorithms is evaluated in the following. Note that these results are also published in [166] and that the figures shown here are based on those from the paper.

Sample preparation The sample is a wild type cobweb spider (Parasteatoda tepidariorum) which was sacrificed by placing it in a freezer for 20 minutes, followed by fixation in paraformaldehyde (PFA) for 1 hour at room temperature. Subsequently, it was dehydrated in an ascending series of ethanol (70\%, $80 \%$, $90 \%, 95 \%$ and pure ethanol, each for 30 minutes) and stained with $1 \%$ iodine for one day in order to enhance contrast. Absorption was minimized by removing the surrounding ethanol via critically point drying (CPD300, Leica, Germany), and the spider was finally glued to a pipette tip and enclosed by a sealed Kapton tube to mount it in the setup. 
Evaluation of the phase-retrieval algorithms The experiment was carried out in the inverse geometry at a source-to-sample distance of $z_{01}=158.75 \mathrm{~mm}$ and a sample-to-detector distance of $z_{12}=22.5 \mathrm{~mm}$, leading to an effective pixel size of $0.47 \mu \mathrm{m}$ by using the high resolution detector 'Argos'. As high energy photons cause several hot pixels in this configuration, the source was operated at an acceleration voltage of $40 \mathrm{kV}$, yielding the spectrum shown in Fig. 4.4(b), and an electron spot size of $10 \times 40 \mathrm{\mu m}^{2}$, enabling a power of $57 \mathrm{~W}$. For a $2 \mathrm{~d}$ overview of the entire spider, 28 overlapping projections were recorded at different lateral positions on the sample, each with an exposure time of $50 \mathrm{~s}$. For the tomographic scan, two measurements at adjacent positions in height were acquired in order to increase the field of view. Each scan was carried out by measuring 1000 projections over $180^{\circ}$ with an exposure time of $20 \mathrm{~s}$. Due to the low divergence of the beam in this configuration, an increase of the angular range for artifact-free cone-beam reconstructions was not necessary.

For a first evaluation of the phase-retrieval algorithms, the $2 \mathrm{~d}$ scan is considered. The 28 single projections were empty-beam corrected and subsequently virtually stitched together in Fiji [150]. Note that due to a fixed noise pattern of the detector, both the recorded projection and the empty beam have to be darkfield corrected, i.e., a detector image $I_{\text {dark }}$ is recorded in the absence of the x-rays and the result is subtracted from both images, yielding

$$
I_{\text {corr }}\left(\mathbf{r}_{\perp}, z\right)=\frac{I\left(\mathbf{r}_{\perp}, z\right)-I_{\text {dark }}}{I_{0}\left(\mathbf{r}_{\perp}, z\right)-I_{\text {dark }}} .
$$

To increase the signal-to-noise ratio (SNR), each projection was resampled by a factor of 2 , leading to an effective pixel size of $0.95 \mu \mathrm{m}$. The resulting raw projection is shown in Fig. 4.6(a), wherein the region marked by the rectangle, comprising a leg of the spider, is represented at higher magnification on the right. Phase contrast is visible in the form of edge enhancement, as confirmed by the line profile along an edge of the leg. Application of the SMO approach (cf. section 2.1.1) with a $\frac{\beta}{\delta}$-ratio of 0.015 , removing the edge enhancement while maximum sharpness is maintained, results in the projection in (b). Edge enhancement is clearly compensated at a higher SNR compared to the raw projection, which is especially visible in the magnified region and the line profile along the edge. However, this is accompanied with a decrease in sharpness and small structures as the roots of the hair are not as clearly resolved. The reconstruction with the MBA phase-retrieval algorithm (cf. section 2.1.2) in Fig. 4.6(c), using a regularization parameter $\alpha=0.04$, shows a similar behavior. Edge enhancement is removed and a high SNR can be 


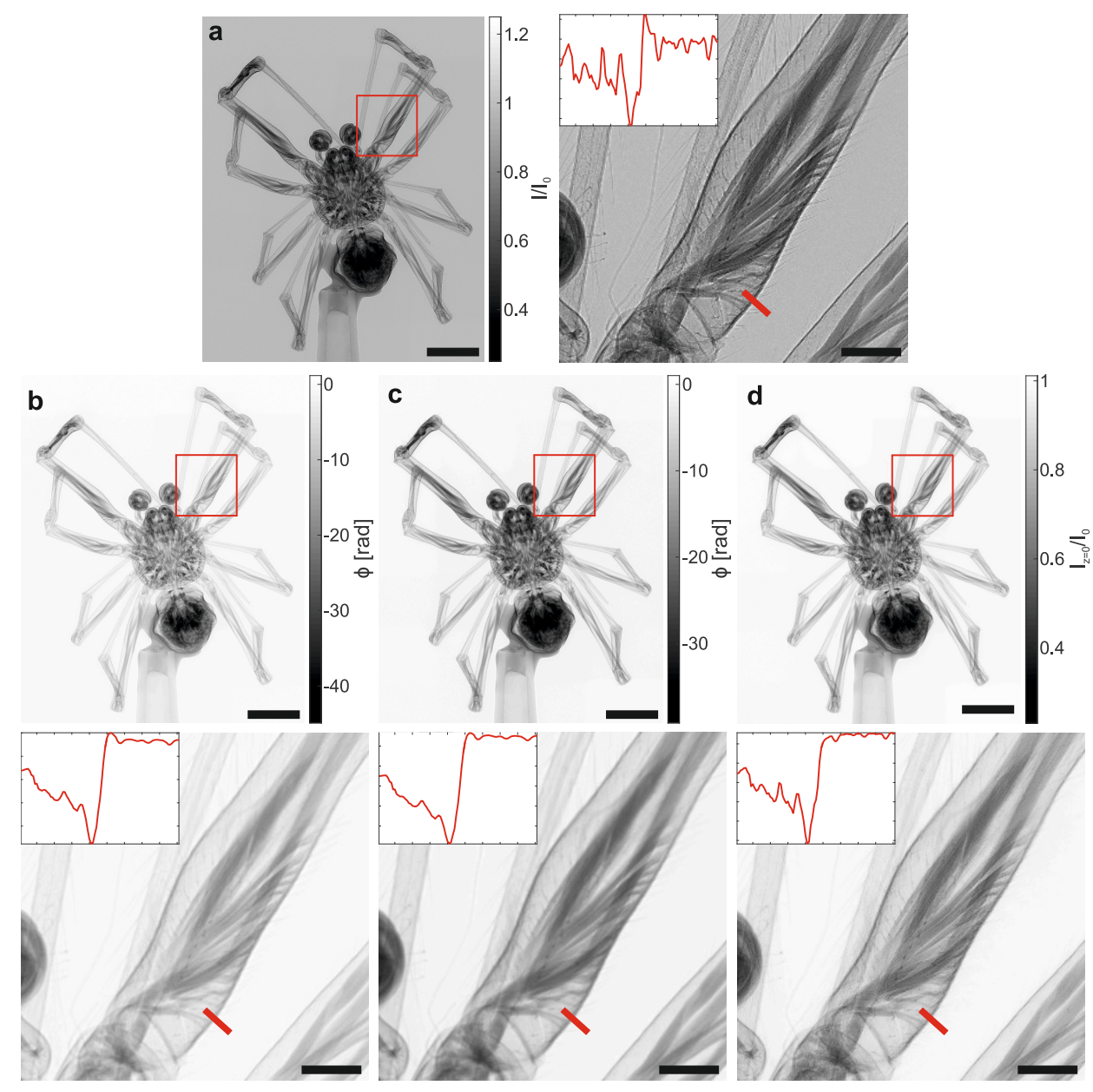

Figure 4.6: $2 d$ comparison of the different phase-retrieval algorithms for the directcontrast regime. (a) Raw projection of an iodine-stained spider, obtained in the inverse geometry by stitching of 28 adjacent projections in order to increase the field of view. The rectangle marks the region which is represented at higher magnification on the right. The profile along the edge of the spider leg reveals the typical edge enhancement as well as a relatively high noise level in the surrounding background. (b) Projection after application of the SMO phase-retrieval algorithm with a $\frac{\delta}{\beta}$-ratio of 0.015 . The edge enhancement is compensated and the noise level is clearly decreased, whereas the reconstruction is slightly blurred compared to the raw data. (c) The same behavior can be recognized in the reconstruction using the MBA approach with $\alpha=0.04$. (d) The reconstructed intensity using the BAC algorithm with regularization parameters $\alpha=0.02$ and $(\gamma \cdot F)=0.15$ yields a sharp reconstruction of the spider, while the edge enhancement is compensated and the noise level is again reduced, though not as significantly as in the case of the SMO and MBA. Scale bars: $1 \mathrm{~mm}$ (overview) and $200 \mu \mathrm{m}$ (magnified regions) 
reached, but the reconstruction is slightly blurred. By applying the BAC phaseretrieval algorithm with the regularization parameters $\alpha=0.02$ and $(\gamma \cdot F)=0.15$ (cf. section 2.1.3), the projection depicted in (d) can be reconstructed. As in the previous cases, edge enhancement is removed while the SNR increases, though not as drastically as for the SMO and the MBA. In exchange, the sharpness is better preserved and comparable to the raw data.

This behavior can be further evaluated in the tomographic scans covering the area of the thorax of the spider. To this end, phase retrieval was performed on each of the 1000 empty-beam corrected and resampled projections according to one of the three presented algorithms and the three-dimensional volumes were subsequently reconstructed via the FDK implementation of the ASTRA toolbox [128, 173, 174]. A summary of the main steps involved in the tomographic reconstruction of data acquired at the laboratory can be found in Fig. A.2 in the Appendix. Longitudinal virtual slices through the obtained volumes for the raw projections as well as each phase-retrieval approach are depicted in Figs. 4.7 and 4.8. Stitching of the subvolumes, acquired at two adjacent positions in height, was performed with the software Avizo (FEI Visualization Sciences Group, USA), which was also used for visualization of the $3 \mathrm{~d}$ data. Within the depicted slices, no artifacts due to the stitching are visible, proving the stability of the setup over long experiment times in the order of 15 hours. The result for the raw projections, shown in Fig. 4.7(a), reveals a sharp reconstruction of the object's density while lacking quantitativity of the gray values. This is especially evident for the edges of the sample, as here gray values get darker despite the sample consisting of the same material in this region, while at the outside of the sample a bright halo is visible. The resolution can be estimated via a profile along an edge, shown in (c), and a subsequent fit of an error function (cf. section 1.5), leading to a full width at half maximum (FWHM) of $1.47 \pm 1.46 \mu \mathrm{m}$. The uncertainty of the fit is caused by the edge enhancement, leading to a deviation of the line profile from a perfect error function, as well as the high steepness of the edge which results in only a small number of points that can be used for the fitting. An estimation of the signal-to-noise ratio, given by

$$
\mathrm{SNR}=\frac{\mu_{\mathrm{sig}}-\mu_{\mathrm{bkg}}}{\sigma_{\mathrm{bkg}}}
$$

with the mean signal value $\mu_{\mathrm{sig}}$, the mean background value $\mu_{\mathrm{bkg}}$ and the standard deviation of the background $\sigma_{\mathrm{bkg}}$, yields $\sim 4.5$ for the raw projections. The regions used for the calculation of the respective values were chosen manually. The result for the SMO approach is depicted in Fig. 4.7(b), revealing a quantitative 

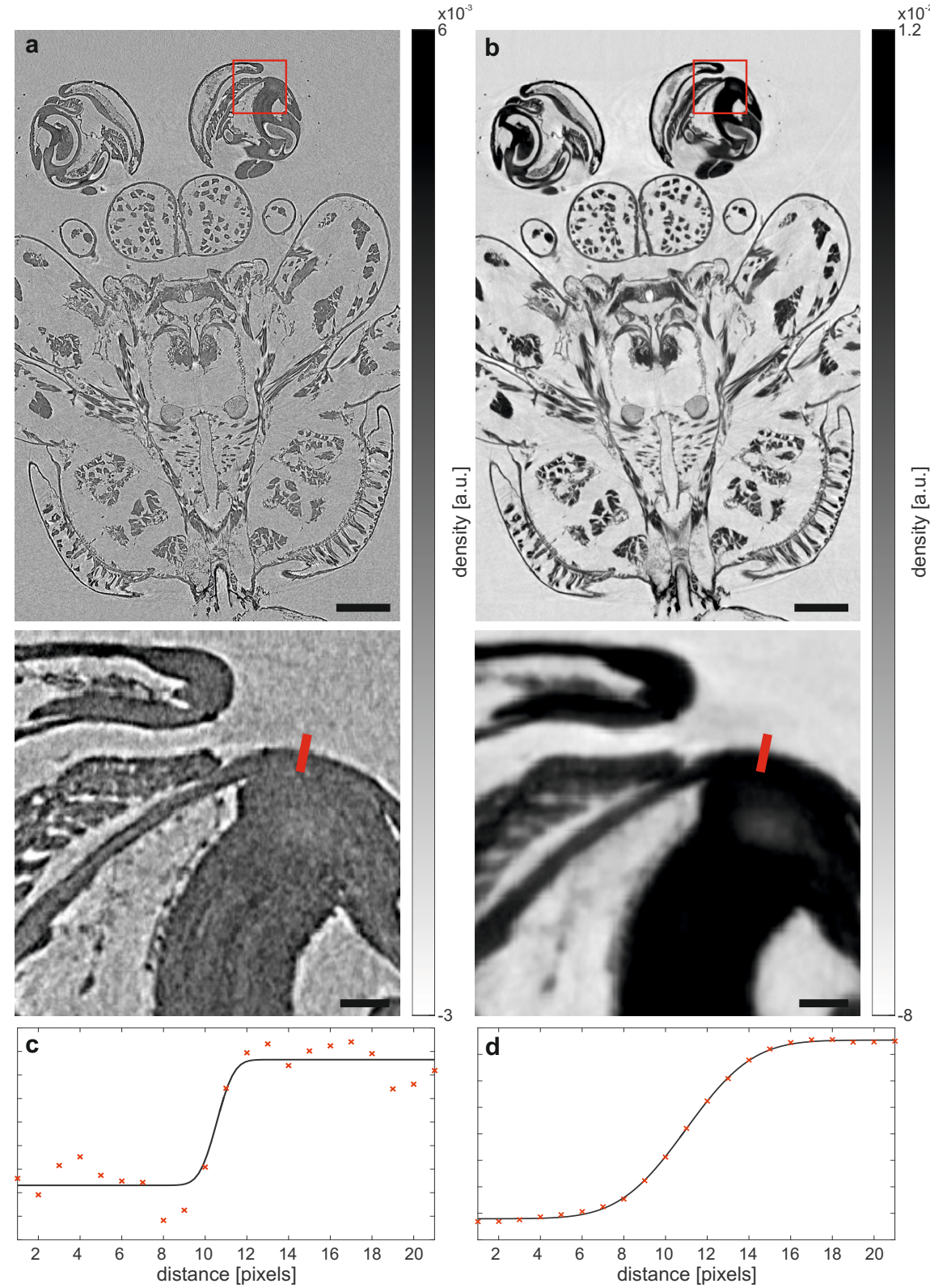

Figure 4.7: Evaluation of the different phase-retrieval approaches in 3d. (a) Slice through the stitched volume reconstructed from the raw projections. The rectangle marks the position of the magnified region shown below. (b) Corresponding slice through the volume obtained from the projections reconstructed according to the SMO approach. (c,d) The line profiles along the indicated edges reveal a FWHM of $1.47 \pm 1.46 \mu \mathrm{m}$ for the raw data and $5.9 \pm 0.3 \mu \mathrm{m}$ for the $S M O$ approach. Scale bars: $200 \mu \mathrm{m}$ (top) and $25 \mu \mathrm{m}$ (bottom) 

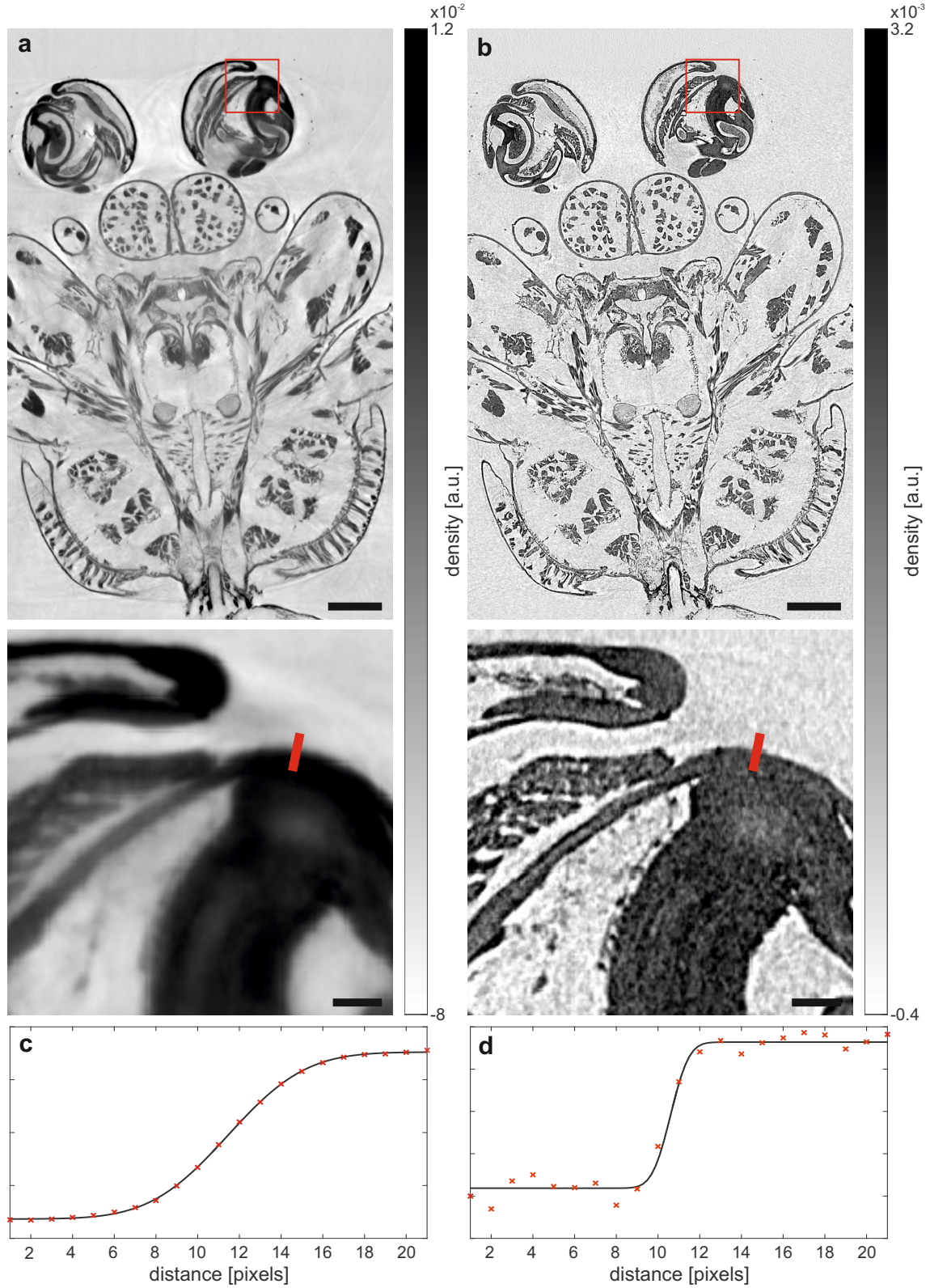

Figure 4.8: Continuation of the evaluation of the different phase-retrieval approaches in 3d. (a) Slice through the volume obtained using the MBA approach. (b) Corresponding slice through the volume reconstructed with the BAC algorithm. (c,d) The line profiles along the edges indicated in the magnified regions above reveal a FWHM of $6.9 \pm 0.2 \mathrm{\mu m}$ for the MBA approach and $1.6 \pm 0.8 \mu \mathrm{m}$ for the BAC. Scale bars: $200 \mu \mathrm{m}$ (top) and $25 \mu \mathrm{m}$ (bottom) 
reconstruction of the object at high $\mathrm{SNR}(\sim 74$ with the same regions for signal and background as before) in which areas consisting of the same material show similar gray values. However, as already observed in the $2 \mathrm{~d}$ projection, sharpness is decreased upon the application of the SMO which is already evident by visual inspection. An error function fit to the profile along the same line as for the raw data (Fig. 4.7(d)) provides a FWHM of $5.9 \pm 0.3 \mu \mathrm{m}$, hence revealing a decrease in resolution by an approximate factor of 4 . As in the $2 \mathrm{~d}$ case, the reconstruction using the MBA approach (cf. Fig. 4.8(a)) shows a similar behavior and a quantitative but slightly blurred reconstruction of the object can be obtained at high SNR $(\sim 54)$. The error function fit to the line profile over the edge of the object, shown in (c), reveals a FWHM of $6.9 \pm 0.2 \mu \mathrm{m}$. Hence resolution and SNR are even slightly worse compared to the SMO approach. Additional artifacts occur, e.g., in the form of a disturbed background. This difference in performance is most likely caused by the different assumptions for the phase-retrieval algorithms. In the SMO approach, a sample consisting of a single material is considered, an assumption which is relatively well met for an iodine stained spider, and the blurring of the reconstruction is mainly caused by the polychromaticity of the setup. In the case of the MBA, a pure phase object is the starting point for the derivation of the algorithm, an assumption which is not well met by the sample (according to Fig. 4.6(a) the minimum transmission lies in the range of approximately $40 \%$ ). Hence, additional artifacts occur in the reconstruction. As a last approach, the $\mathrm{BAC}$ algorithm is considered and the result of the tomographic scan is depicted in Fig. 4.8(b), showing a quantitative reconstruction of the object with drastically decreased edge-enhancement effects. The same level of detail as for the raw projections can be reached, as confirmed by an error function fit to the line profile in (d), revealing a FWHM of $1.6 \pm 0.8 \mu \mathrm{m}$, though at a lower signal-to-noise ratio of $\sim 12$ compared to the results obtained by the SMO and the MBA approaches. Note that also in this case, the fit has a relatively large uncertainty as the high steepness of the edge leads to only a small number of pixels that can be used for the fitting. An overview of the results obtained via the different phase-retrieval approaches is given in Tab. 4.1 .

For a better visualization of the $3 \mathrm{~d}$ density distributions, volume renderings of the datasets resulting from the different phase-retrieval algorithms are shown in Fig. 4.9. In these renderings, voxels belonging to the background are displayed in transparent whereas each voxel inside the sample is displayed in a different shade of gray, according to the respective value of the reconstructed density (higher densities lead to lighter gray values). Cuts through the volume provide a view into 

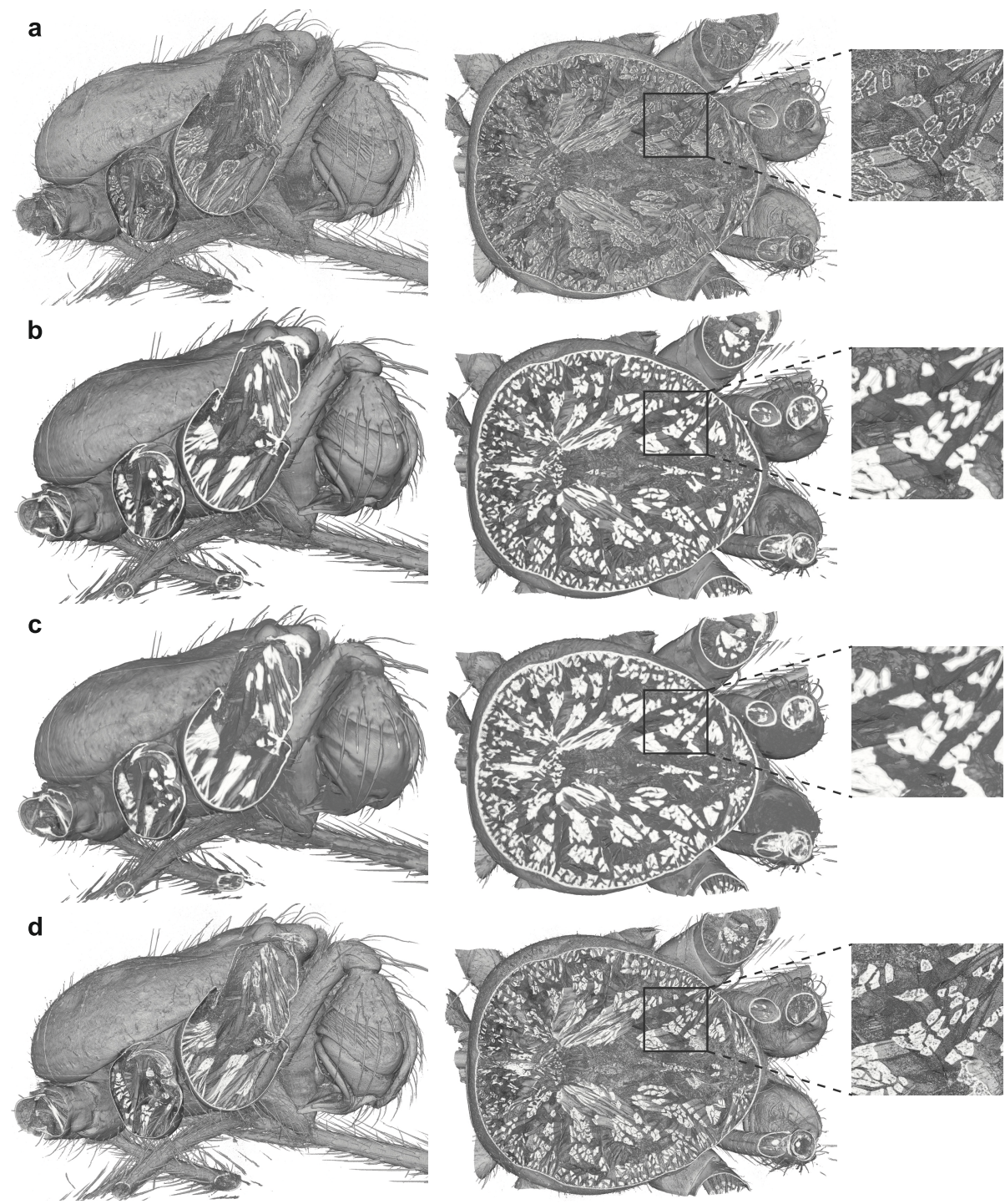

Figure 4.9: $3 d$ visualization of the reconstructed volumes via automated renderings of the density distribution obtained from the raw data (a) and projections reconstructed via the SMO (b), the MBA (c) and the BAC (d) approach, respectively. Cuttings into these renderings reveal the inner anatomy of the spider. In the case of the raw data, the spider is visualized at high detail, but due to the missing quantitativity structures appear hollow. The SMO and MBA approach lead to reconstructed volumes in which structures consisting of the same material are rendered completely. However, due to the decrease in resolution, small objects are not clearly separated. The BAC algorithm provides a sharp and quantitative representation of the inner anatomy, leading to a homogeneous rendering of structures consisting of the same material. 


\begin{tabular}{lcr}
\hline & error function FWHM & SNR \\
\hline raw projections & $1.47 \pm 1.46 \mu \mathrm{m}$ & $\sim 4.5$ \\
SMO & $5.9 \pm 0.3 \mu \mathrm{m}$ & $\sim 74$ \\
MBA & $6.9 \pm 0.2 \mu \mathrm{m}$ & $\sim 54$ \\
BAC & $1.6 \pm 0.8 \mu \mathrm{m}$ & $\sim 12$ \\
\hline
\end{tabular}

Table 4.1: Comparison between the edge steepness and the signal-to-noise ratio for the different phase-retrieval approaches.

the inner anatomy of the spider. In the case of the raw data, depicted in (a), the spider is rendered at high detail, revealing small structures as the hair on the outside of the body or the single muscle strands, as, e.g., apparent in the legs. However, due to the missing quantitativity, these structures appear hollow, as the gray value gradient towards the edges impedes automatic rendering (especially visible in the magnified regions on the right side). In the case of the SMO and MBA approach, muscles are rendered completely as structures consisting of the same material comprise similar gray values. However, due to the decrease in resolution, single muscle strands are not clearly separated and no inner substructure can be resolved. Only in the case of the BAC reconstruction, the inner anatomy of the spider can be visualized with the same amount of detail as the raw data, while due to the quantitativity, structures consisting of the same material are rendered homogeneously.

These results clearly demonstrate that in order to fully exploit the potential of the laboratory setup for high resolution phase-contrast imaging, phase retrieval has to be performed via the $\mathrm{BAC}$ algorithm, as only in this case, quantitative reconstructions without a loss in sharpness can be obtained. Hence, this reconstruction scheme is used by default for laboratory data throughout this thesis.

\subsection{Alignment procedures}

In order to avoid artifacts caused by a misaligned rotation axis (cf. section 3.2.1) it has to be aligned prior to the experiments. Especially the position of the axis with respect to the vertical center line of the detector and the pitch angle $\nu$, which describes a tilt of the rotation along the optical axis, have to be considered, whereas the roll angle $\vartheta$ can be corrected for afterwards. Fig. 4.10 illustrates the geometrical considerations for a misalignment of both the pitch and the roll angle. In the case of small pitch angles and a detector far away from the source, the correction 
a

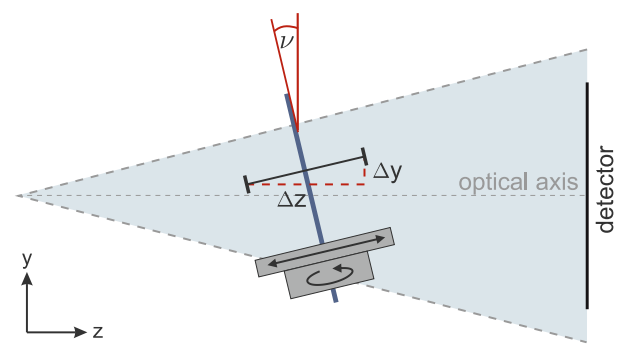

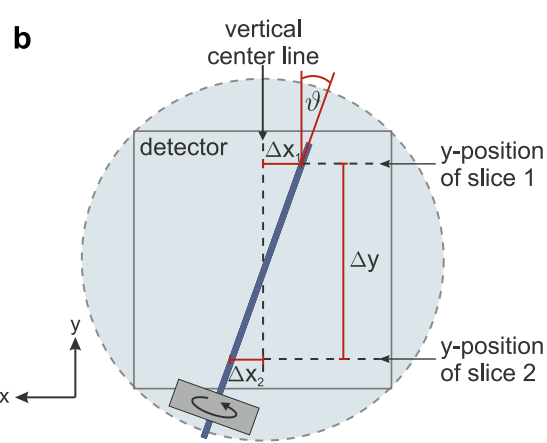

Figure 4.10: Illustration of the geometrical considerations for a misalignment of both the pitch and the roll angle of the rotation axis (depicted in blue). (a) For a misaligned pitch angle $\nu$, a translation of the sample along $z$, indicated by the black line, leads to a vertical shift along $y$. This can be used to calculate the pitch angle via $\nu=\operatorname{atan}\left(\frac{\Delta y}{\Delta z}\right)$. (b) A misaligned roll angle $\vartheta$ can be interpreted as a $y$-dependent shift of the rotation axis with respect to the vertical center line of the detector. Hence, by determining the actual position of rotation axis with respect to this line at two different positions $y_{1}$ and $y_{2}$, the roll angle can be calculated via $\vartheta=\operatorname{atan}\left(\frac{\Delta x_{1}-\Delta x_{2}}{\Delta y}\right)$.

can be equivalently performed by either tilting the cradle by the corresponding angle or by moving the detector such that the optical axis interferes with its central point, as deviations due to the curvature of the cone beam can be neglected in this case. Due to the high divergence of the beam at the laboratory, only limited by the dimensions of the exit window to an opening angle of approximately $10^{\circ}$, the detector can be used for the alignment as it will generally be fully illuminated. The relatively low divergence of the waveguide at the synchrotron leads to a fixed position of the detector in space. Hence, alignment has to be carried out using rotational motors below the axis.

It is easiest to perform the alignment with well-defined thin objects leading to a strong contrast in the x-ray images without disturbing effects of different magnifications within the sample due to a varying thickness. Thus, copper or gold grids, normally used for transmission electron microscopy, either with regular spacing or specific features as letters are well suited.

Assuming that the setup consists of a motor moving along the $x$-direction, which it situated beneath the rotation axis, and two perpendicular linear stages along $x$ and $z$ above, the alignment can be carried out as follows: 


\section{Alignment of the pitch angle $\nu$}

- Case 1: Moving the detector for alignment (laboratory setup)

- Center the object in the field of view of the detector.

- Move the sample stage along the optical axis with the $z$-motor above the rotation. A misaligned pitch angle will lead to a shift of the object along $y$ (cf. Fig. 4.10(a)), whereas in the case of an aligned angle, the only visible effect will be magnification. Hence, the part of the object located directly in the center of the detector, and therefore on the optical axis, stays at the same position. Note that for this alignment procedure it is assumed that the sample holder is mounted perpendicular to the translational motors above the rotation axis.

- Determine the point on the detector which corresponds to the position of the optical axis, hence the point in which no change in $y$ between the two images acquired at the different positions occurs. In the case of a regular test pattern, this is easily recognizable by considering the difference between the two images, as it only vanishes at the position of the optical axis.

- Move the detector stage such that this point coincides with the center point of the detector array. In this way, also the zero position of the rotation axis is aligned (a misalignment would lead to a shift between the two images along $x$ ).

- Case 2: Tilting the sample stage for alignment (synchrotron setup)

- Proceed as in the first case and acquire two images at different positions along $z$. Due to the smaller field of view it is easiest to consider a unique feature on the test pattern, center it in the detection plane and identify the direction of the tilt by determining the height difference of the feature position in the two images.

- Correct for this tilt by moving the cradle below the rotation axis, until the vertical position of the feature stays constant.

- The zero position of the rotation axis can be determined by finding the angle $\theta$ at which no horizontal shift of the feature occurs.

\section{Determination of the effective pixel size}

- If both the source-to-sample and the sample-to-detector distance are known, this step can be skipped, as in this case, the effective pixel size can be calculated from these quantities according to geometrical optics. 
- Move the sample along $x$ over a certain distance with a specified step size, e.g., over a distance of $1 \mathrm{~mm}$ in 10 steps, leading to a step size of $0.1 \mathrm{~mm}$.

- Determine the pixel shift between the recorded images with respect to the first and perform a linear regression. The slope gives the step size in pixels.

- Correlating the physical step size with the determined shift in pixels gives the effective pixel size $p_{\text {eff }}$.

\section{Alignment of the rotation axis to the vertical center line of the detector}

- To determine the horizontal shift of the rotation axis an image is acquired at $0^{\circ}$ and the position $\left(x_{1}, y_{1}\right)$ of a specific feature on the detector is determined.

- In an image acquired at $180^{\circ}$, this is repeated for the same feature, leading to a feature position $\left(x_{2}, y_{2}\right)$. Note that due to the rotation by $180^{\circ}$ the feature will be flipped along the vertical axis. With the horizontal number of pixels $N_{x}$ of the detector, the rotation axis shift $\Delta$ can be determined via

$$
\Delta=\frac{x_{1}+x_{2}}{2}-\frac{N_{x}}{2}
$$

- Correct for this misalignment by moving the motor along $x$, situated below the rotational axis, by $\Delta \cdot p_{\text {eff }}$.

Alignment of the roll angle $\vartheta$ The alignment of the roll angle $\vartheta$ is carried out after the measurement as part of the tomographic reconstruction. According to Fig. 4.10(b) a misaligned roll angle can be approximately interpreted as a changing position of the rotation axis with respect to the vertical position $y$ on the detector. Hence, it can be corrected for as follows:

- Determine the rotation axis shift $\Delta x_{1}$ at a vertical position $y_{1}$ by optimizing the reconstruction of the corresponding slice through the volume. Note that this optimization should be carried out with respect to the central part of the slice.

- Repeat this procedure for a different vertical position $y_{2}$, yielding the rotation axis shift $\Delta x_{2}$.

- The roll angle can be determined via

$$
\vartheta=\operatorname{atan}\left(\frac{\Delta x_{1}-\Delta x_{2}}{y_{1}-y_{2}}\right)
$$

- Tilt each acquired projection by this angle. 


\section{Imaging of unstained hydrated mouse cerebellum}

In this chapter, the capabilities of phase-contrast tomography to perform $3 \mathrm{~d}$ virtual histology on samples from the central nervous system and to decipher the underlying cytoarchitecture and connectivity are evaluated for unstained hydrated brain tissue. This sample preparation is on the one hand of great interest due to its proximity to the native structure of the brain, since apart from fixation, no further preparation steps need to be carried out, as, e.g., tissue dehydration, which is necessary for histological preparations and leads to tissue shrinkage. On the other hand it is very challenging due to the similarity in electron density between cellular structures and the surrounding hydrated tissue, requiring experimental settings optimized for contrast transfer.

Here, imaging of unstained hydrated brain tissue was performed on a $500 \mu \mathrm{m}$ thick slice from a mouse cerebellum. Due to the distinct structure of the cerebellum with the different layers as well as cell types, providing features of interest at varying size and abundance, it is well suited to evaluate the capabilities of the imaging method as well as experimental system.

\subsection{Basic anatomy of the cerebellum}

The cerebellum ('little brain') is located at the back of the brain (cf. Fig. 5.1(a) for the example of a mouse) and contains approximately $80 \%$ of the total number of neurons within the human brain, despite its relatively small size $(\sim 10 \%$ of the volume) [6, 68]. Among other things, it is important for the maintenance of upright posture and synergy of movements [156].

The cerebellum generally consists of the tightly folded cerebellar cortex, comprising three distinct layer, located above white matter containing a large amount of axon bundles. The histological appearance of the cerebellar cortex is virtually uniform throughout the entire cerebellum and a schematic overview is given in Fig. 5.1 (b-d). The innermost layer is called the granular layer (GL), the outermost layer the molecular layer (ML) and the intermediary mono-cellular layer contains the 
a

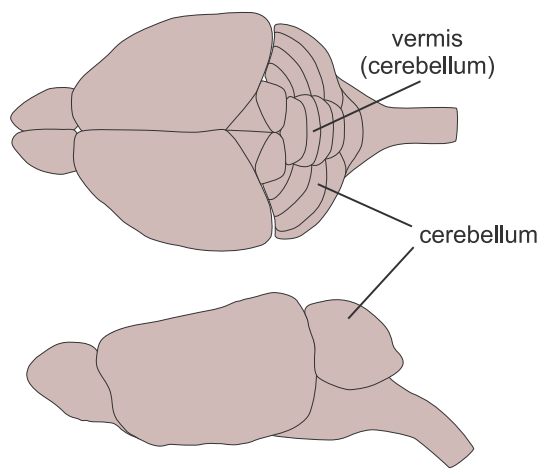

b

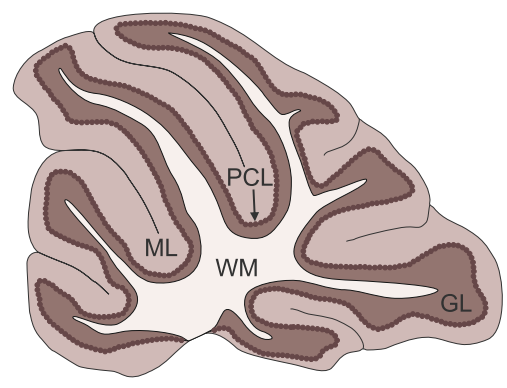

c

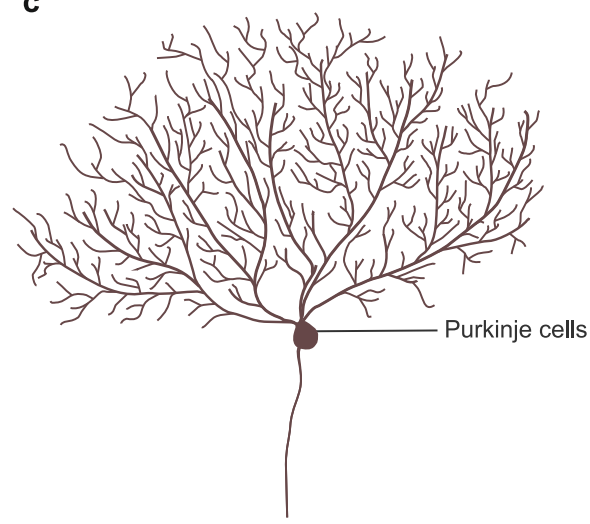

d

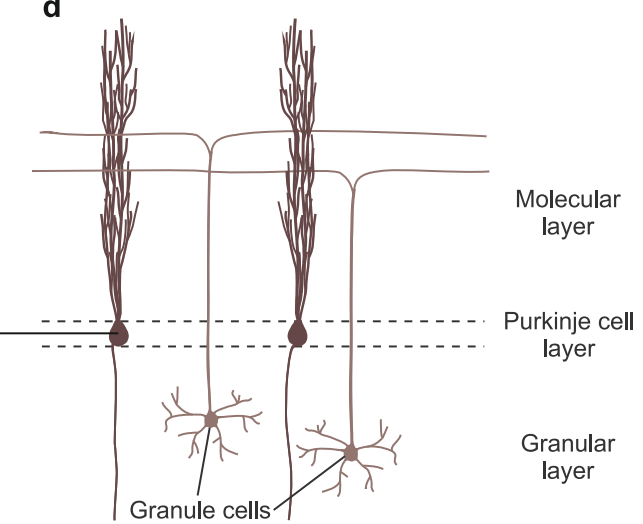

Figure 5.1: Sketch of the basic anatomy of a mouse cerebellum. (a) The cerebellum is located at the back of the brain, as shown here at the example of a mouse brain. (b) It consists of four distinct layers: the cerebellar cortex with the cell-rich granular layer (GL), the low-cell molecular layer (ML) and the mono-cellular Purkinje cell layer $(P C L)$ in between, is located above white matter (WM), containing a large amount of axon bundles. (c,d) The Purkinje cells comprise a highly branched dendritic tree which mainly evolves within $2 d$ planes into the molecular layer. They are arranged in parallel, enabling the synaptic connection to numerous granular cells whose axons bifurcate in the molecular layer and run perpendicular to the dendritic tree of the Purkinje cells. The drawing was inspired by [162] and [156].

Purkinje cells (PCL), named after Jan Evangelista Purkinyě, who first described these large nerve cells as well as the uniform structure of the cerebellar cortex in 1837 [183].

Granular layer The most abundant cell in this layer is the granule cell, containing three to five dendrites and an axon which ascends towards the cortex of the cerebellum and bifurcates in the molecular layer. Hence, each axon forms two 
strands that run parallel to the surface of the cortex in opposite directions (cf. Fig $5.1(\mathrm{~d})$ ). As this bifurcation occurs in each of the granule cells, leading to numerous axon strands running in parallel throughout the molecular layer, the granule cell axons are called parallel fibers. Additionally, the granular layer contains Golgi cells whose cell bodies are primarily found towards the edge of the Purkinje cell layer.

Purkinje cell layer The Purkinje cell layer is a mono-cellular layer situated at the interface between the granular and the molecular layer. It consists of large Purkinje cells, comprising a highly branched dendritic tree that protrudes into the molecular layer and primarily forms a flat disk, leading to an almost twodimensional cell shape (cf. Fig. 5.1(c) and (d)). The cells are arranged in a parallel manner with the parallel fibers of the granule cells running perpendicular to the dendritic tree, enabling synaptic contacts between a single Purkinje cells and numerous granule cells and vice versa (cf. Fig. 5.1(d)). The Purkinje cell is the sole output from the cerebellum and its axon emerges from the bottom of the cell body and traverses through the granular layer as well as the white matter (not shown in the sketch).

Molecular layer The molecular layer is almost devoid of cells and mainly contains the dendritic tree of the Purkinje cell layer as well as the parallel fibers of the granule cells. Besides these structures, stellate and basket cells are apparent at low cell density.

\subsection{Methods}

\subsubsection{Sample preparation}

A wild type mouse, provided by the group of Frauke Alves (MPI for Experimental Medicine, Göttingen, Germany), was sacrificed by an anesthetization with $\mathrm{CO}_{2}$ and a subsequent perfusion with $10 \%$ sucrose solution for 10 minutes. After dissection of the brain, it was fixed overnight in a solution containing $1 \%$ paraformaldehyde (PFA) and $1 \%$ glutaraldehyde (GTA). Prior to the experiments, the brain was cut into $500 \mu \mathrm{m}$ thick coronal slices and stored in phosphate-buffered saline (PBS). In order to mount the sample in the experimental setup, two aluminum rings with a diameter of $5 \mathrm{~cm}$ and a width of $500 \mu \mathrm{m}$ were covered with polypropylene foil on one side. By placing a brain slice from the region of the cerebellum as well as a small amount of PBS onto one of the foils and gluing a second ring on 
a

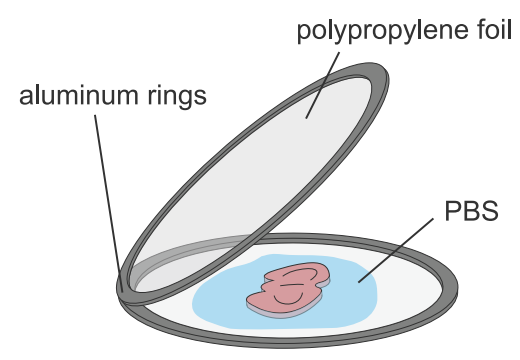

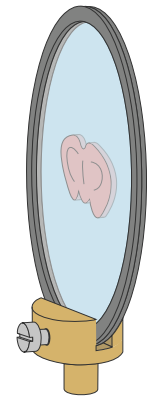

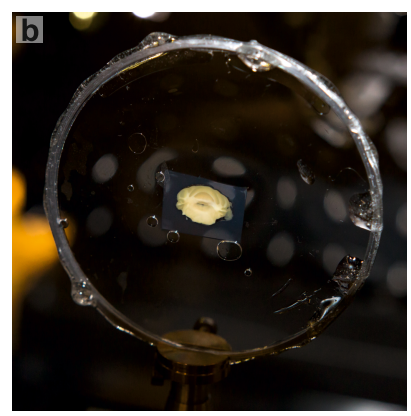

Figure 5.2: Sample preparation for the hydrated brain slice. (a) The $500 \mu \mathrm{m}$ thick slice is placed onto an aluminum ring which is coated with polypropylene foil on one side. By adding a small amount of PBS and gluing a second aluminum ring on top, a liquid chamber is built. The resulting chamber is then clamped into a sample holder which can be mounted in the setup. (b) Photograph of the resulting liquid chamber with the brain slice located approximately in the center.

top of it, a liquid chamber was built around the sample (cf. Fig. 5.2). The orientation of the aluminum rings determines the width of the chamber, as they can act as spacers between the polypropylene foils. In this case, it was set to $500 \mu \mathrm{m}$, resulting in a liquid chamber with a width equal to the width of the brain slice and thus, the sample was kept in place by the pressure of the polypropylene foils. Finally, the resulting liquid chamber was clamped into a brass sample holder for mounting in the setup.

\subsubsection{Experimental setup}

According to section 1.3.3, samples that are embedded in a medium with comparable index of refraction, as is the case for hydrated unstained neuronal tissue, are barely visible in the direct-contrast regime. Hence, imaging at the laboratory would lead to results with a low contrast and experiments were solely carried out at the GINIX endstation at the PETRAIII storage ring.

The energy was set to $8 \mathrm{keV}$ and a tapered waveguide, in the following denoted by its laboratory name 'Hercules II', was placed into the focus of the KB-mirrors for coherence and spatial filtering, providing a photon flux in the order of $10^{10} \mathrm{ph} / \mathrm{s}$. The 'Pirra' with a pixel size of $6.5 \mu \mathrm{m}$ was used for the detection of photons and it was positioned at a source-to-detector distance of $5.125 \mathrm{~m}$. Together with the source-to-sample distance $z_{01}=141 \mathrm{~mm}$, this leads to an effective pixel size $p_{\text {eff }}=178 \mathrm{~nm}$ and a Fresnel number $F=0.0015$. The high flux provided by the tapered waveguide enabled short exposure times and thus the tomogram could be 


\begin{tabular}{lc}
\hline & experimental setting \\
\hline GINIX run & 42 \\
energy & $8 \mathrm{keV}$ \\
detector & 'Pirra' \\
$z_{01}$ & $141 \mathrm{~mm}$ \\
$z_{02}$ & $5.125 \mathrm{~m}$ \\
$p_{\text {eff }}$ & $178 \mathrm{~nm}$ \\
field of view & $365 \times 365 \mathrm{~mm}^{2}$ \\
Fresnel number $F$ & 0.0015 \\
waveguide & 'Hercules II' \\
number of distances & $($ tapered channel) \\
angular range & 1 \\
number of projections & {$[0,180]^{\circ}$} \\
exposure time & 1662 \\
CTF $\frac{\delta}{\beta}$ & $0.2 \mathrm{~s}$ \\
CTF $\alpha_{2}$ & 500 \\
\hline
\end{tabular}

Table 5.1: Experimental parameters used for imaging of an unstained hydrated slice from a mouse cerebellum.

recorded in a continuous acquisition mode, i.e., the sample was rotated continuously while the detector captured images. To this end, the rotation speed was set to $0.45^{\circ} / \mathrm{s}$ and the exposure time was $0.2 \mathrm{~s}$, leading to 1662 projections recorded over an angular range of $180^{\circ}$. For clarity, all experimental settings are additionally listed in Tab. 5.1

\subsection{Data analysis}

\subsubsection{Phase retrieval}

Phase retrieval was performed on each of the empty-beam corrected projections prior to tomographic reconstruction. Note that in addition to the division by the empty beam, as described in section 4.3, a darkfield was subtracted, accounting for a fixed noise pattern of the detector. One exemplary empty-beam corrected projection is shown in Fig. 5.3(a). Due to the larger opening angle of a tapered waveguide as opposed to a straight channel, small variations in the position of the incident KB-focused beam will lead to larger deviations in the resulting wave field and hence to a decreased stability of the illumination. Therefore, low-frequency 

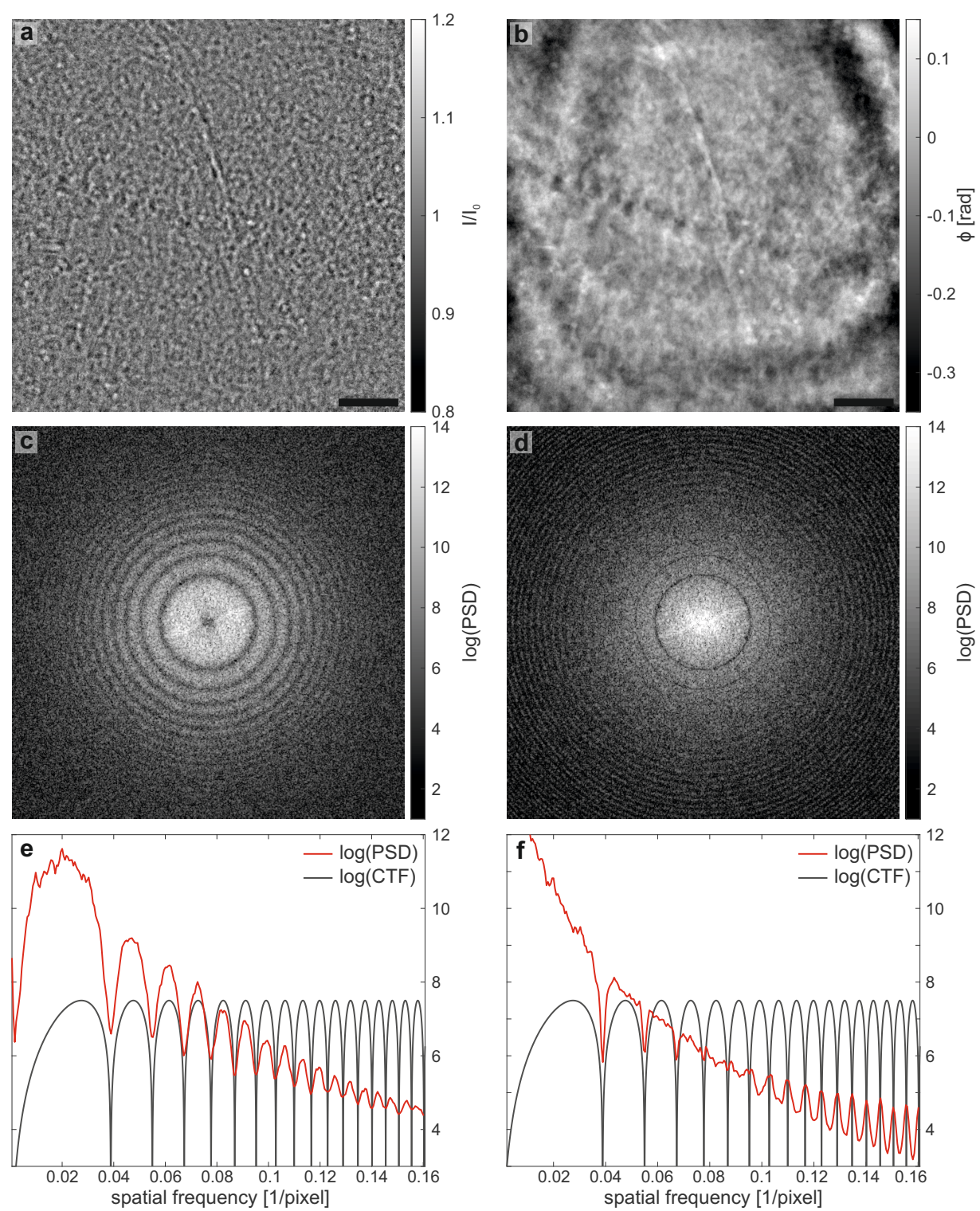

Figure 5.3: Evaluation of the CTF-based phase-retrieval algorithm. (a) Exemplary empty-beam corrected and high-pass filtered projection of the object. The corresponding single-distance reconstruction with $\frac{\delta}{\beta}=500$ and $\alpha_{2}=0.1$ is depicted in (b). (c) The power spectral density (PSD) of the projection exhibits the typical intensity modulations predicted by the CTF. (d) In the PSD of the reconstructed projection, residual modulations are visible. (e) The modulations of the azimuthally averaged PSD of the projection (red) coincide well with the predictions by the CTF. (f) The PSD of the reconstructed projection shows a reduction of these modulations. Scale bars: $50 \mu \mathrm{m}$ 
artifacts are introduced upon empty-beam correction and a high-pass filter was applied on each projection in order to minimize this effect. The power spectral density of the raw projection, depicted in Fig. 5.3(c), reveals the modulations expected from the zero crossings of the contrast transfer function (1.81). This can be further quantified by comparing the angular averaged power spectral density with the theoretically predicted curve for a weakly absorbing homogeneous object with a $\frac{\delta}{\beta}$-ratio of 500 (cf. Fig. 5.3(c)). The positions of the intensity modulations coincide with the predicted values, proving the validity of the CTF approach for a weakly absorbing object with slowly varying phase for imaging of unstained hydrated samples. Hence, the phase can be reconstructed according to the approach in eqn. (2.33) with the regularization parameters $\frac{\delta}{\beta}=500$ and $\alpha_{2}=0.1$, leading to the reconstruction in Fig. 5.3(b). Note that only one tomographic dataset at a single propagation distance was recorded, leading to a non-zero regularization parameter $\alpha_{2}$ for larger spatial frequencies. The resulting projection exhibits a quantitative reconstruction of the phase, better visible in the reconstructions of the three-dimensional density distribution shown below, while residual low-frequency artifacts are visible due to the unstable waveguide illumination. Compared to the raw projections, these artifacts are intensified in the reconstruction due to the phase-retrieval step, and they will lead to strong aberrations in the reconstructed volume, as detailed in the next section. The power spectral density of the reconstructed slice, depicted in Fig. 5.3(d), shows a reduction of the modulations, which is also visible in the angular averaged curve below. However, due to the missing information in the intensity images, artifacts remain in the reconstruction. Depending on the choice of regularization parameter $\alpha_{2}$, rings apparent in the raw image are either not fully accounted for, resulting in an underrepresentation of the corresponding frequencies (low spatial frequencies), or overcorrected, leading to an amplification (higher spatial frequencies).

\subsubsection{Reduction of low-frequency artifacts}

The tomographic reconstruction of the acquired dataset was obtained by performing a filtered backprojection of the reconstructed phase distributions with a standard Ram-Lak filter, using the function iradon implemented in Matlab. One exemplary orthogonal slice through the volume is depicted in Fig. 5.4(a). It is dominated by low-frequency artifacts due to the unstable waveguide illumination. In the reconstructed phase (cf. Fig. 5.3(b)), the effect of the varying illumination is visible as circular intensity modulations with increasing severity towards the edges. These modulations transform into low-frequency stripe artifacts in the sinogram 

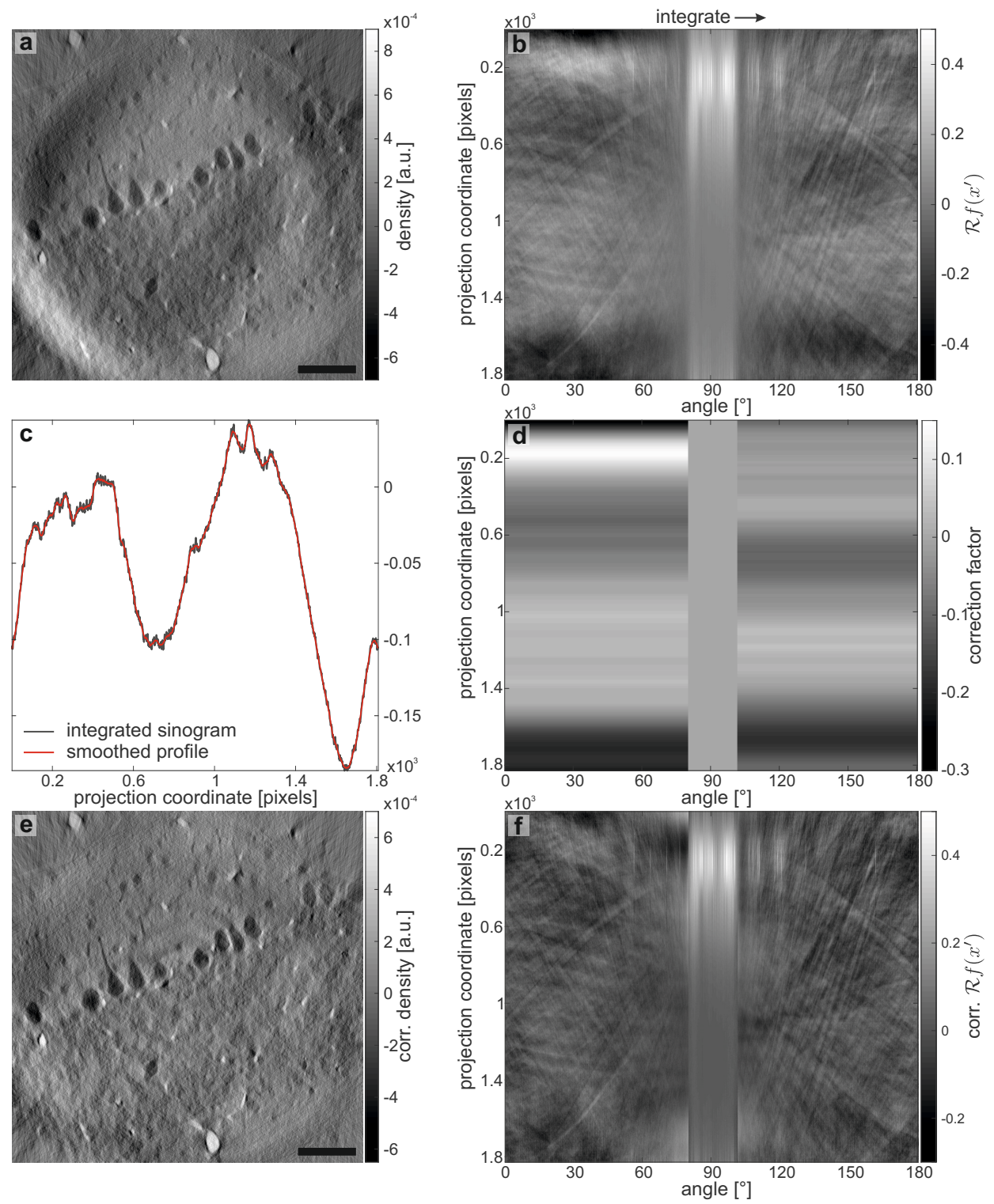

Figure 5.4: Reduction of low-frequency artifacts due to an unstable waveguide illumination. (a) Reconstructed slice from the uncorrected projections with low-frequency artifacts in the form of rings. (b) Sinogram corresponding to the slice in (a) with broad stripes causing these artifacts. (c) Integrated sinogram in which the intensity modulations can be identified via a smoothing of the resulting profile. (d) Correction matrix that was subsequently subtracted from the sinogram. Note that is was determined separately from the smoothed profiles for the first and the last half of the scan. (e) Reconstructed slice from the corrected sinogram shown in (f). Scale bars: $50 \mu \mathrm{m}$ 
with varying shape along the angular range due to the unstable illumination over time (cf. 5.4(b)). Note that the central part of the sinogram, comprising projections with constant intensity, corresponds to a sample orientation of $\sim 90^{\circ}$ at which the beam is completely absorbed as it passes through both aluminum rings. To avoid further artifacts, these projections are neglected in the tomographic reconstruction. Analogous to the development of sharp ring artifacts due to corrupted detector pixels described in section 3.2.2, the low-frequency stripes in the sinogram will lead to broad ring artifacts in the reconstructed slice, as visible in Fig. 5.4 (a). To account for these low-frequency variations, the sinogram was integrated along the rotation angle $\theta$, leading to the gray curve depicted in (c). The small modulations can be attributed to the integration over the sine functions of the object features whereas the large modulations are mainly caused by the intensity variations. Hence, smoothing the integrated sinogram, leading to the red curve in (c), yields a profile for the correction of the intensity modulations. Due to the changing illumination over time, this correction profile was determined separately for the first and the last half of the scan, resulting in the correction matrix in Fig. 5.4(d). Subtraction from the original sinogram yields the corrected slice in (e) and the corresponding sinogram in (f). Low-frequency artifacts are clearly reduced while quantitativity of the reconstruction is preserved, i.e., structures with a higher density are represented by darker gray values.

\subsection{Results of the tomographic imaging}

The results of the tomographic reconstruction of the entire dataset, in which the reduction of low-frequency artifacts was carried out for each slice individually as they depend on the illumination at the given position within the volume, are depicted in Fig. 5.5. Note that this reconstruction was carried out via the Matlab implementation of the filtered backprojection with a standard Ram-Lak filter and that no further ring-removal algorithms were applied to the sinograms. A summary of the main steps involved in the tomographic reconstruction of data acquired at the GINIX setup can be found in Fig. A.3 in the Appendix. Two exemplary slices through the volume are shown in (a) and (b) with an orientation parallel to the Purkinje cell layer. In order to obtain a higher signal-to-noise ratio, all slices were filtered with a Gaussian function with a standard deviation of 0.7 pixels, leading to a denoising of the images at the cost of resolution. The possibility to produce virtual slices in any arbitrary direction is one of the main advantages of $3 \mathrm{~d}$ histology, as in this case the slice orientation can be optimized in order to 
visualize specific features of the sample. In the slices depicted here, the different layers of the cerebellum, namely the cell-rich granular layer (GL), the low-cell molecular layer (ML), the mono-cellular Purkinje cell layer (PCL) and the white matter (WM) containing large axon bundles, are clearly resolved. Within these layers, single cells can be distinguished, especially the large Purkinje cells, and even sub-cellular details are visible as, e.g., the inner structure of the granule cells and larger branches of the dendritic tree as well as the nucleolus of the Purkinje cells (cf. Fig. 5.5(b), red arrow). However, overall contrast is comparatively low and a clear distinction between the small cells, especially in the granular layer, is challenging. A quantification of contrast can be obtained via the Weber contrast which is defined as [132]

$$
C=\frac{I_{\text {target }}-I_{\text {background }}}{I_{\text {background }}} .
$$

It is a measure for the contrast of specific targets compared to the background signal, in this case the contrast of the cells against the surrounding tissue. Due to the difference in cell types, contrast is determined both for the Purkinje cell layer and the granular layer. To this end, several cells with comparatively high contrast based on visual inspection are selected manually and the resulting contrast is averaged, yielding $C_{\mathrm{PCL}} \simeq 0.5$ and $C_{\mathrm{GL}} \simeq 0.19$, respectively. Note that due to the manual selection, a certain bias in the determined contrast values cannot be circumvented. For better comparability, the gray values were shifted so that the minimum is given by 0 , as different offsets, e.g., caused by region-of-interest tomography, yield the same visual contrast but influence the determined values.

In order to better visualize the $3 \mathrm{~d}$ structure of the Purkinje cell layer, a segmentation of selected cells was performed, including the large cell body and the parts of the dendritic tree that can be distinguished from background. This segmentation was carried out in the visualization software Avizo using the so-called Magic Wand tool, which performs a 3d region-growing algorithm that selects all connected voxels within a defined gray value range, starting from a user-specified seeding point. As this tool is prone to noise, leading to a high variety in gray values and hence artifacts in the segmentation, as the background might be interpreted as the structure of interest, all slices were filtered by a Gaussian filter with a standard deviation of 40 pixels. Although this leads to highly blurred data, resolution and signal-to-noise ratio were sufficient to segment the large branches of the dendritic tree automatically. A surface representation of the resulting segmentation is shown in Fig. 5.5(c) and (d), with a virtual slice depicting the orientation of the slices 


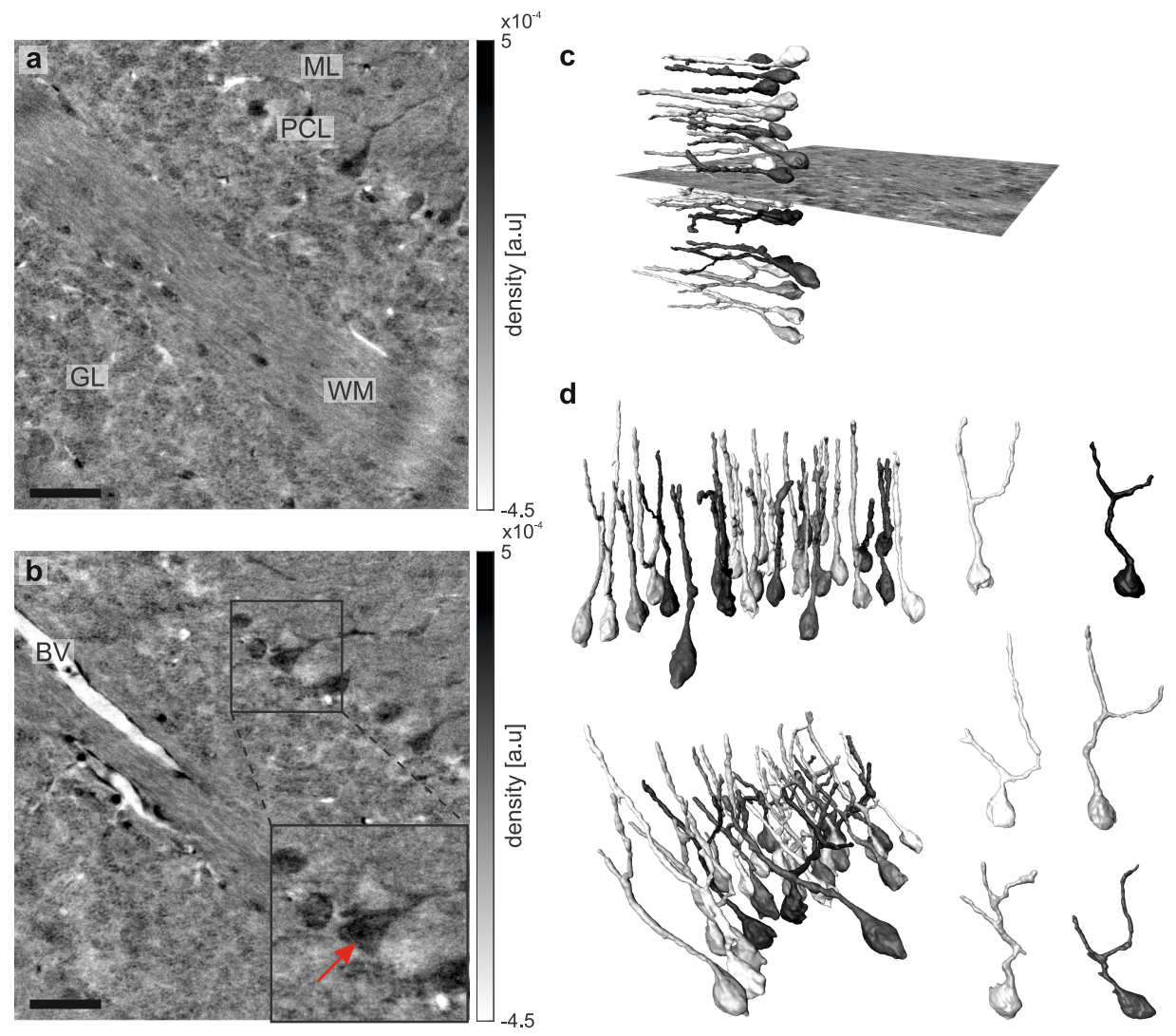

Figure 5.5: Results of the tomographic imaging of a slice from an unstained hydrated mouse cerebellum. $(a, b)$ Two exemplary virtual slices through the reconstructed volume with an orientation parallel to the Purkinje cell layer, as depicted in (c). For a higher signal-to-noise ratio, the slices were filtered by Gaussian filter with a standard deviation of 0.7 pixels. The typical layers of the cerebellum, the granular layer (GL), molecular layer $(M L)$, Purkinje cell layer (PCL) and white matter (WM), are clearly visible. Also blood vessels $(B V)$ can be recognized. Specific sub-cellular features as the nucleolus of the Purkinje cells (red arrow) as well as to some extent their dendritic tree are resolved. (d) A segmentation of the Purkinje cell layer reveals typical features as their parallel orientation and flat shape. However, only the thick branches of the dendritic tree can be segmented. Scale bars: $50 \mu \mathrm{m}$

in (a) and (b) with respect to the Purkinje cell layer. As the Purkinje cells lie on the interface between the cell-rich granular layer and the low-cell molecular layer, with their dendritic trees protruding into the latter, the interface between these two layers as well as their location can be deduced from the orientation of the segmented cells. Additionally, the typical shape of the Purkinje cells, comprising 


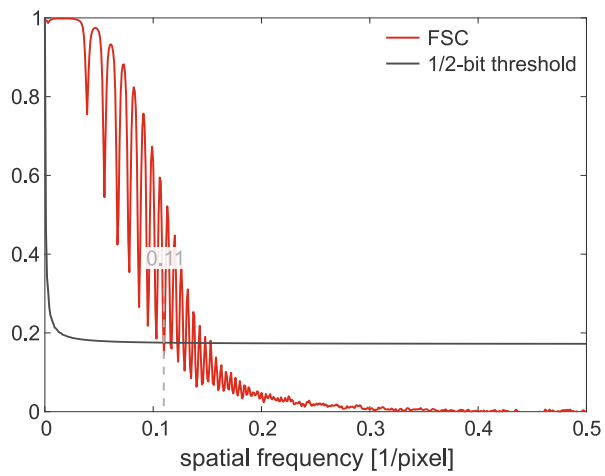

Figure 5.6: Fourier shell correlation of the central $1000^{3}$ voxels of the tomographic reconstruction of the hydrated unstained mouse cerebellum together with the 1/2-bit threshold curve. The intersection of the curves determines the resolution of the dataset.

a large dendritic tree which forms a nearly two-dimensional layer, as well as the parallel arrangement of cells within the entire layer can be recognized. However, the single cell segmentation shows that only the main part of the dendritic tree, close to the cell body, where branches are thickest, is resolved.

Resolution An estimate of the resolution can be either achieved by considering the Fourier shell correlation, yielding the overall quality of the experiment, or via an error function fit to the edge of a highly contrasted feature within the slices, measuring the system blur for the given experimental parameters (cf. section 1.5). To generate two independent datasets for the calculation of the FSC according to eqn. (1.97), the tomographic scan was divided into two subsets, consisting of every second projection and starting with the first or second, respectively. The FSC of the central $1000^{3}$ voxels of these reconstructions together with the $1 / 2$-bit threshold curve is depicted in Fig. 5.6. The intersection point at 0.11 cycles/pixel results in a half-period resolution of $809 \mathrm{~nm}$. Note that the oscillations within the FSC are caused by the zero crossings of the contrast transfer function which were not accounted for by measuring tomograms at several propagation distances. By fitting an error function to the edge profile along edges between manually selected Purkinje cell bodies and the surrounding tissue, a FWHM of $\sim 1.11 \mu \mathrm{m}$ can be determined. Hence, the half-period resolution, which is given by half of the FWHM, is $\sim 555 \mathrm{~nm}$. The large difference between the two values indicates that noise and a weak contrast of the sample features, which have a large influence on the result obtained via the FSC, significantly limit the resolution. 


\subsection{Summary}

In this chapter, the application of propagation-based phase-contrast tomography for imaging of unstained hydrated mouse cerebellum was evaluated. In order to achieve highest contrast and resolution, the sample was imaged in the holographic regime using the GINIX endstation at the P10 beamline at DESY. The results demonstrate that even for unstained hydrated tissue, exhibiting only small differences in electron density, imaging of sub-cellular details is possible. Phase retrieval based on the contrast transfer function is suitable for these samples, as the requirements of a weak absorption and a slowly varying phase are fulfilled, which was confirmed by the consistency of the experimental data and theoretical predictions. Even by exploiting only a single propagation distance for phase retrieval, quantitative reconstructions with minimal artifacts could be obtained. This enabled the automated gray value-based segmentation of the large Purkinje cells, including the cell body as well as to some extent the dendritic tree of selected cells, revealing the specific properties of this cell layer.

However, in order to achieve a clear distinction between single cells, an overall increase in tissue contrast has to be achieved, e.g., by lowering the photon energy as well as improving the sample mounting and waveguide performance with respect to stability and photon flux. This would enable a larger contrast due to a stronger interaction between the sample and the beam as well as a higher signal-tonoise ratio due to a larger amount of photons and longer possible exposure times without the introduction of artifacts resulting from sample movement or flawed empty-beam corrections.

With the current experimental settings, sample preparations aiming for higher electron density differences in the tissue can be exploited to increase the contrast. This can be achieved in two ways (cf. section 1.3.3): either specific features of the sample are stained with a radiocontrast agent, as, e.g., metals with a high atomic number, or the sample is embedded in a medium with significantly lower density compared to the tissue. 



\section{Contrast enhancement by metal staining}

In the following chapter, contrast enhancement by radiocontrast agents is considered for imaging of neuronal structures [20, 105, 200]. Radiocontrast agents have to provide a significantly higher electron density compared to the surrounding tissue. Hence, metals are well suited to increase the contrast, whereas typical staining agents used in histology, as, e.g., the hematoxylin and eosin (H\&E) stain, are too similar in electron density with respect to the surrounding tissue. Staining with metals is a standard procedure in electron microscopy, in which the lipids and cell membranes within the tissue are stained by osmium tetroxide $[9,80,108,140]$, or in histology, e.g., via the Golgi method, which was developed by Camillo Golgi and statistically stains a limited number of neurons $(\sim 1-5 \%)$ with silver [85, 100, 109, 129]. Santiago Ramon y Cajal used and improved the latter in order to visualize the anatomy and organization of the nervous system and both scientists were awarded the Nobel Prize in Physiology or Medicine in 1906 [54]. The exact mechanism underlying this reaction is, however, largely unknown [129].

In the following, both methods are evaluated on different tissue from the central nervous system, namely a strip of mouse retina which is stained by osmium tetroxide and a slice of mouse brain from the region of the hippocampus, on which the Golgi-Cox method, a more reliable version of the Golgi method, was applied.

\subsection{Osmium-stained mouse retina}

\subsubsection{Basic anatomy of the retina}

The eye, which is the organ of the visual system, is enclosed by three layers of tissue, of which the innermost is called the retina (cf. Fig. 6.1). The retina itself is likewise organized in distinct layers [156]. The outermost layer is the pigmented epithelium, which provides the light-sensitive photoreceptor cells, located in the neighboring layer, with nutrition. The photoreceptor cells comprise rods, which are 


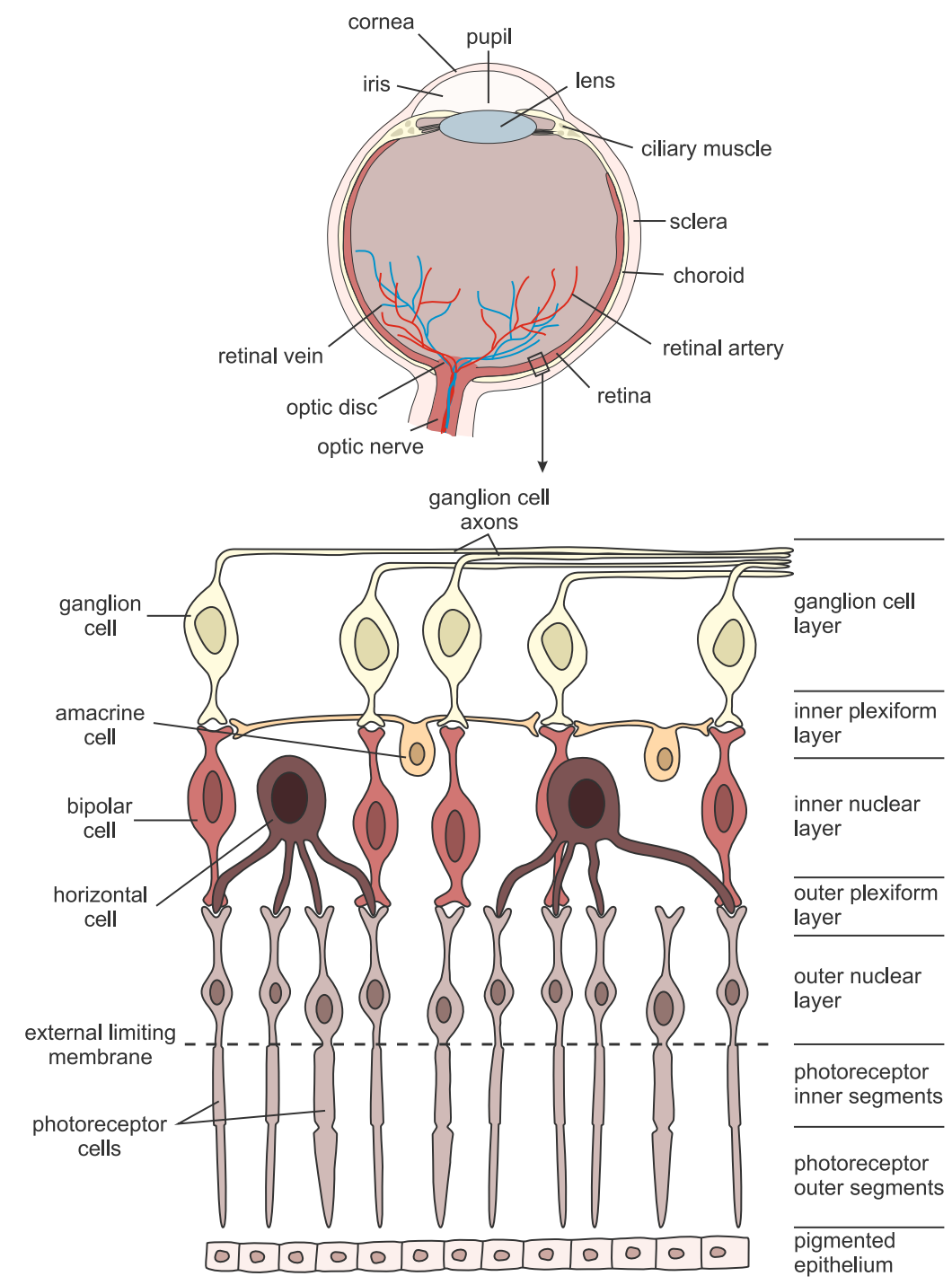

Figure 6.1: Basic anatomy of the retina. The eye is enclosed by three layers of which the innermost is called the retina (top panel). The retina itself is likewise organized in distinct layers (bottom panel), containing the pigmented epithelium, the light sensitive photoreceptor segments, the external limiting membrane, the outer nuclear layer, the outer plexiform layer, the inner nuclear layer, the inner plexiform layer and the ganglion cell layer. The output of the retina to the central nervous system is transmitted via the optic nerve, consisting of the ganglion cell axons. The drawing is based on [156]. 
specialized for night vision, and cones, which are responsible for daylight vision and generally less abundant than rods. Structurally, the photoreceptor cells can be divided into four parts: the outer segments, in which the incoming light is absorbed, the inner segments which mainly provide ATP, the cell bodies, which are located in the outer nuclear layer and are separated from the photoreceptor segments by the external limiting membrane, and the synaptic terminals. These can make contact to bipolar and horizontal cells in the outer plexiform layer. The cell bodies of these cells as well as of so-called amacrine cells are located in the inner nuclear layer. Their main function is to transmit the signal from photoreceptor cells to the ganglion cells and to interconnect the signal of different rods and cones. The synaptic connection to the ganglion cells is formed in the inner plexiform layer. In the outermost layer of the retina, the ganglion cell layer, the cell bodies of these ganglion cells are located. Their axons, bundled in the optic nerve, transmit the output of the retina to the central nervous system through the optic disc at the back of the eye. Due to the absence of photoreceptor cells in this area, it is insensitive to incoming light signals and hence called the blind spot. In addition to the optical nerve, blood vessels supplying the eye can pass through the optic disc.

\subsubsection{Methods}

\subsubsection{Sample preparation}

A wild-type mouse was sacrificed and its retina was acutely prepared and highpressure frozen without prior chemical fixation [110]. To this end, the retina was sliced into $200 \mu \mathrm{m}$ thick strips and subsequently transferred into a sample carrier containing 1-hexadecane. After placing a second sample carrier as a lid, it was inserted into the high-pressure freezer (BAL-TEC HPM 010, Central Microscopy Research Facility, USA). Afterwards, the sample was stored in liquid nitrogen to maintain cryogenic conditions. In order to fix as well as stain the sample with the osmium solution, a freeze substitution protocol was subsequently carried out with an automated freeze substitution device (Leica AFS2, Leica, Germany) [77]. In a first step, the liquid nitrogen was substituted by $0.1 \%$ tannic acid and the sample was kept at a constant temperature of $-90^{\circ} \mathrm{C}$. This step was followed by a washing of the sample in pre-cooled anhydrous acetone, which was carried out three times with $15 \mathrm{~min}$ intervals in between. Afterwards, the acetone was replaced by a solution containing $0.5 \%$ osmium tetroxide $\left(\mathrm{OsO}_{4}\right)$ and $0.1 \%$ uranyl acetate for fixation and staining. During the incubation time of the osmium solution, the 


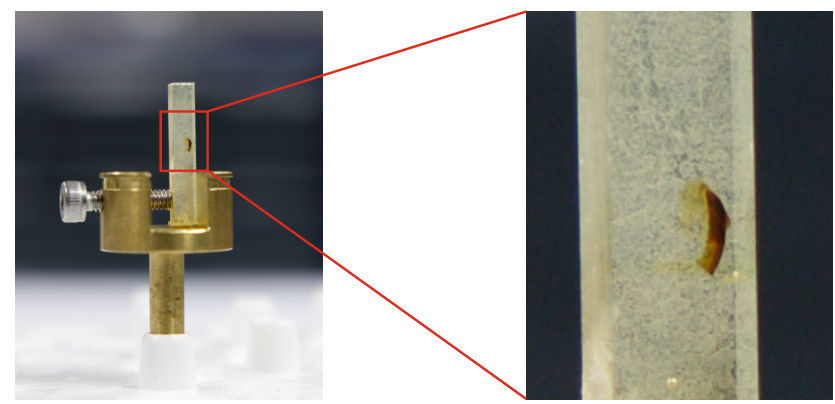

Figure 6.2: Photograph of the retina sample. The osmium-stained strip of mouse retina is embedded in epon and subsequently clamped into a brass pin in order to mount it in the experimental setup. The typical curvature of the retina is already visible in this photograph.

temperature was increased, up to $-20^{\circ} \mathrm{C}$ at a rate of $5^{\circ} \mathrm{C} / \mathrm{hr}$ and afterwards at a rate of $10^{\circ} \mathrm{C} / \mathrm{hr}$, until it reached $4^{\circ} \mathrm{C}$. To remove the sample from the osmium solution, the washing procedure with the anhydrous acetone described earlier was repeated.

In a last step, the sample was embedded in epon resin [77]. To this end, a substitution series was carried out, in which it was stored in dilutions of epon in acetone with increasing epon content $(50 \%, 90 \%, 100 \%)$. To ensure proper infiltration of the resin, it was first placed in the $50 \%$ solution for 3 hours, followed by the $90 \%$ solution overnight. Afterwards, the sample was transferred to fresh resin and incubated for one day. During the day, the epon was exchanged three times. As a last step, it was placed in a fresh amount of resin which was polymerized for $24 \mathrm{~h}$ at $60^{\circ} \mathrm{C}$. Note that the sample was kindly provided by Benjamin Cooper (MPI for Experimental Medicine, Göttingen, Germany).

For the tomographic experiments, excess epon around the $200 \mu \mathrm{m}$ strip of mouse retina was trimmed off in order to minimize absorption. To create smooth surfaces, leading to less artifacts in the reconstructed phase, as for the CTF slow variations are assumed, this reduction was performed with a wafer saw (Disco DAD-321, Gorgio Technology Sales/Service, USA). The resulting sample blocks were ultimately clamped into a brass pin for mounting in the experimental setup (cf. Fig. 6.2).

\subsubsection{Experimental parameters}

Experiments on the $200 \mu \mathrm{m}$ strip of mouse retina were performed at the GINIX endstation at the PETRAIII storage ring. The energy was set to $13.8 \mathrm{keV}$ and a crossed multilayer waveguide was used, providing a flux in the order of $9 \cdot 10^{8} \mathrm{ph} / \mathrm{s}$. 


\begin{tabular}{lcc}
\hline & medium resolution & high resolution \\
\hline GINIX run & 49 & 49 \\
energy $[\mathrm{keV}]$ & 13.8 & 13.8 \\
detector & 'Pirra' & 'Pirra' \\
$z_{01, \min }[\mathrm{mm}]$ & 145 & 50 \\
$z_{02}[\mathrm{~m}]$ & 5.19 & 5.19 \\
$p_{\text {eff }}[\mathrm{nm}]$ & 182 & 63 \\
field of view $\left[\mathrm{\mu m}^{2}\right]$ & $373 \times 373$ & $129 \times 129$ \\
Fresnel number $F$ & 0.0026 & 0.0009 \\
waveguide & multilayer & multilayer \\
number of distances & $(d=81 \mathrm{~nm})$ & $(d=81 \mathrm{~nm})$ \\
angular range $\left.{ }^{\circ}\right]$ & 1 & 4 \\
number of projections & {$[0,180]$} & {$[0,180]$} \\
exposure time $[\mathrm{s}]$ & $1800 / 900 / 900$ & 900 \\
CTF $\frac{\delta}{\beta}$ & 15 & 1 \\
CTF $\alpha_{2}$ & 0.05 & 15 \\
\hline
\end{tabular}

Table 6.1: Overview of the experimental parameters for imaging of an osmium-stained strip of mouse retina.

The 'Pirra' was installed as a detection device and placed at a source-to-detector distance of $5.19 \mathrm{~m}$. The retina was imaged at two source-to-sample positions, leading to different resolutions and fields of view. In the 'medium resolution' setting, it was placed at $145 \mathrm{~mm}$, leading to an effective pixel size of $182 \mathrm{~nm}$ and a field of view of $\sim 375 \times 375 \mathrm{\mu m}^{2}$, whereas in the 'high resolution' setting, a distance of $50 \mathrm{~mm}$ was chosen, resulting in an effective pixel size of $63 \mathrm{~nm}$ with a field of view of $\sim 130 \times 130 \mathrm{\mu m}^{2}$. For the overview scan, only a single propagation distance was acquired in order to decrease scan time while for the high resolution scan four propagation distances were taken into account to receive high quality reconstructions of the phase. In order to increase the field of view in the medium resolution setting, three adjacent tomograms were acquired with sufficient overlap to virtually stitch them together after reconstruction. All scans were performed over an angular range of $180^{\circ}$. In the case of the first position of the overview scan, 1800 projections were acquired with an exposure time of $1 \mathrm{~s}$ each, whereas in the other scans, including the high resolution measurement, 900 projections were recorded. The experimental parameters for all measurements are summarized in Tab. 6.1. 

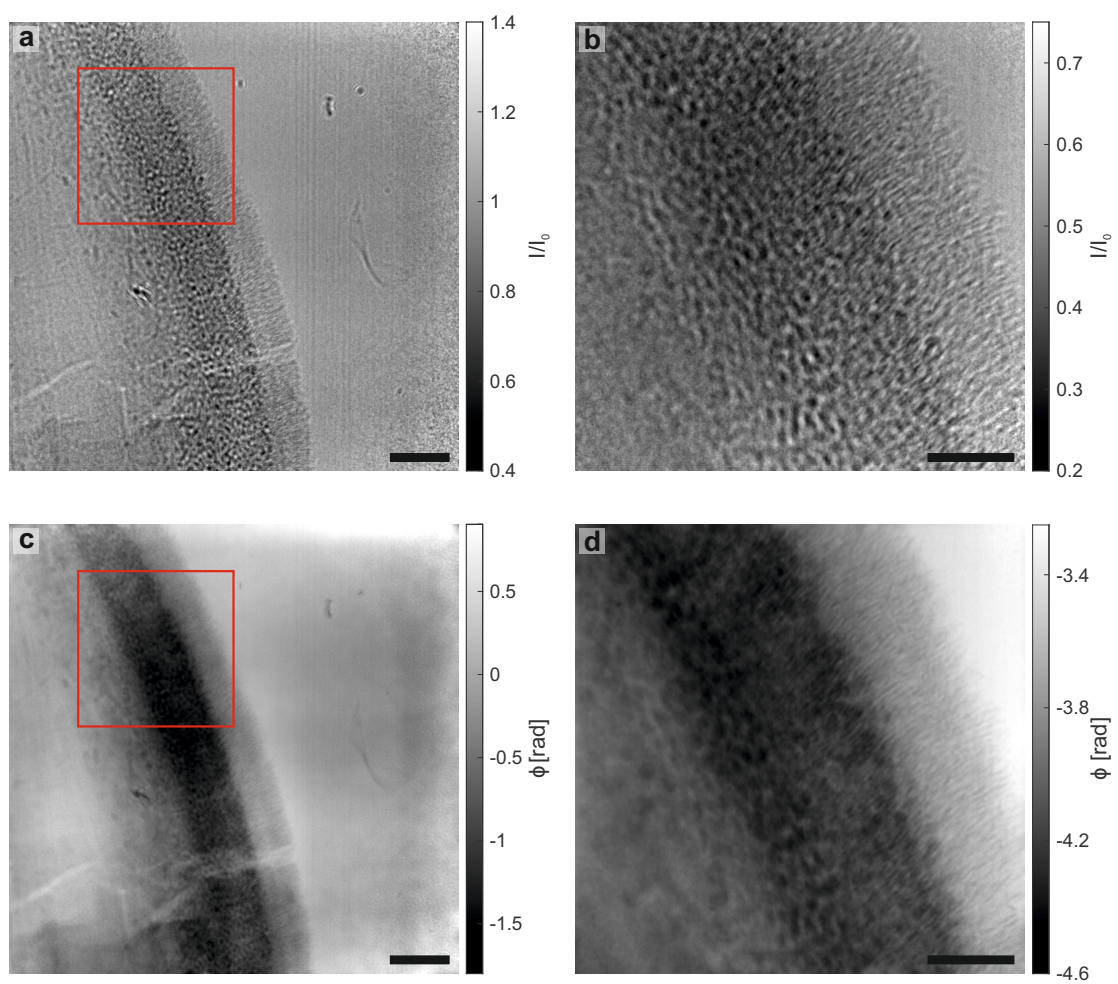

Figure 6.3: Projection data of the osmium-stained mouse retina, measured both in the medium and high resolution settings at the GINIX setup. (a) Projection acquired in the medium resolution setting at the first sample position with an approximate field of view of $380 \times 380 \mathrm{\mu m}^{2}$. The corresponding single-distance CTF reconstruction is depicted in (c). (b) Projection from the high resolution measurement with an approximate field of view of $130 \times 130 \mathrm{\mu m}^{2}$. The corresponding position within the overview scan is marked by the rectangle. (d) CTF-based reconstruction of the projection from the high resolution measurement taking four propagation distances into account. Already in these projections, different layers in the retina can be distinguished, especially the osmium-rich outer nuclear layer. Scale bars: $50 \mu \mathrm{m}(\mathrm{a}, \mathrm{c})$ and $25 \mu \mathrm{m}(\mathrm{b}, \mathrm{d})$

\subsubsection{Results}

Figure 6.3 shows two exemplary empty-beam corrected projections acquired in the medium (a) and high resolution setting (b), respectively. Compared to the projection of a hydrated brain slice, depicted in Fig. 5.3, the contrast enhancement due to osmium is already visible in these projections, since sample features can be easily distinguished from background. Note that in the case of the medium resolution setting, two further tomograms were acquired at adjacent positions towards the bottom of the depicted projection. In order to obtain a quantitative 
reconstruction of the phase, the CTF-based algorithm for a weakly absorbing object was applied on all datasets. For the medium resolution measurements, the regularization parameters $\frac{\delta}{\beta}=15$ and $\alpha_{2}=0.05$ were chosen for all positions, leading to the reconstruction depicted in (c). Hence, despite recording only a single propagation distance, a sharp representation of the phase can be obtained with quantitative gray values that already indicate the typical layers of the retina, especially the osmium-rich and thus dark region of the outer nuclear layer, in which even the cell bodies can be distinguished. For the high resolution dataset, projections recorded at the different propagation distances yield different magnifications and hence fields of view. Therefore, they were rescaled to the projection with the smallest pixel size and aligned to each other in order to perform multi-distance phase retrieval without artifacts. The alignment of the projections was carried out in Fourier space [59] and it proved most stable to perform it on single distance CTF reconstructions of the individual projections instead of the acquired holograms, as the resemblance between the images is in this case larger. For the phase-retrieval step, the parameters were set to $\frac{\delta}{\beta}=15$ and $\alpha_{2}=0.01$. As several propagation distances were considered for phase retrieval, regularization for high spatial frequencies is not as critical and hence, $\alpha_{2}$ could be chosen smaller compared to the single-distance overview scan. The result of this multi-distance phase retrieval for the projection shown in (b) is depicted in (d). As in the case of the overview scan, the algorithm provides a sharp and quantitative reconstruction of the object and the different layers can already be recognized in the projection. In addition to the granular structure of the osmium-rich outer nuclear layer, even the rod-like shape of the photoreceptor segments is already indicated in the projection.

The tomographic reconstruction was again carried out via the filtered backprojection implemented in Matlab with a standard Ram-Lak filter. In order to reduce ring artifacts in the slices, the simple ring-removal algorithm described in section 3.2.2 was applied to the sinograms, both in the medium and the high resolution dataset. The three individual volumes obtained in the medium resolution setting were manually aligned and subsequently merged with the visualization software Avizo. One longitudinal slice through the resulting volume is depicted in Fig. 6.4(a). No obvious artifacts due to the stitching are visible, proving the stability of the setup over relatively long time periods. Additionally, data quality is comparable within the individual scans, despite the larger number of acquired projections in the tomogram shown at the top of the slice. Hence, for the given sample dimensions and experimental settings, the recording of half as many projections is justified. Within the slice, the typical layers of the retina, the photoreceptor segments (POS/PIS), 


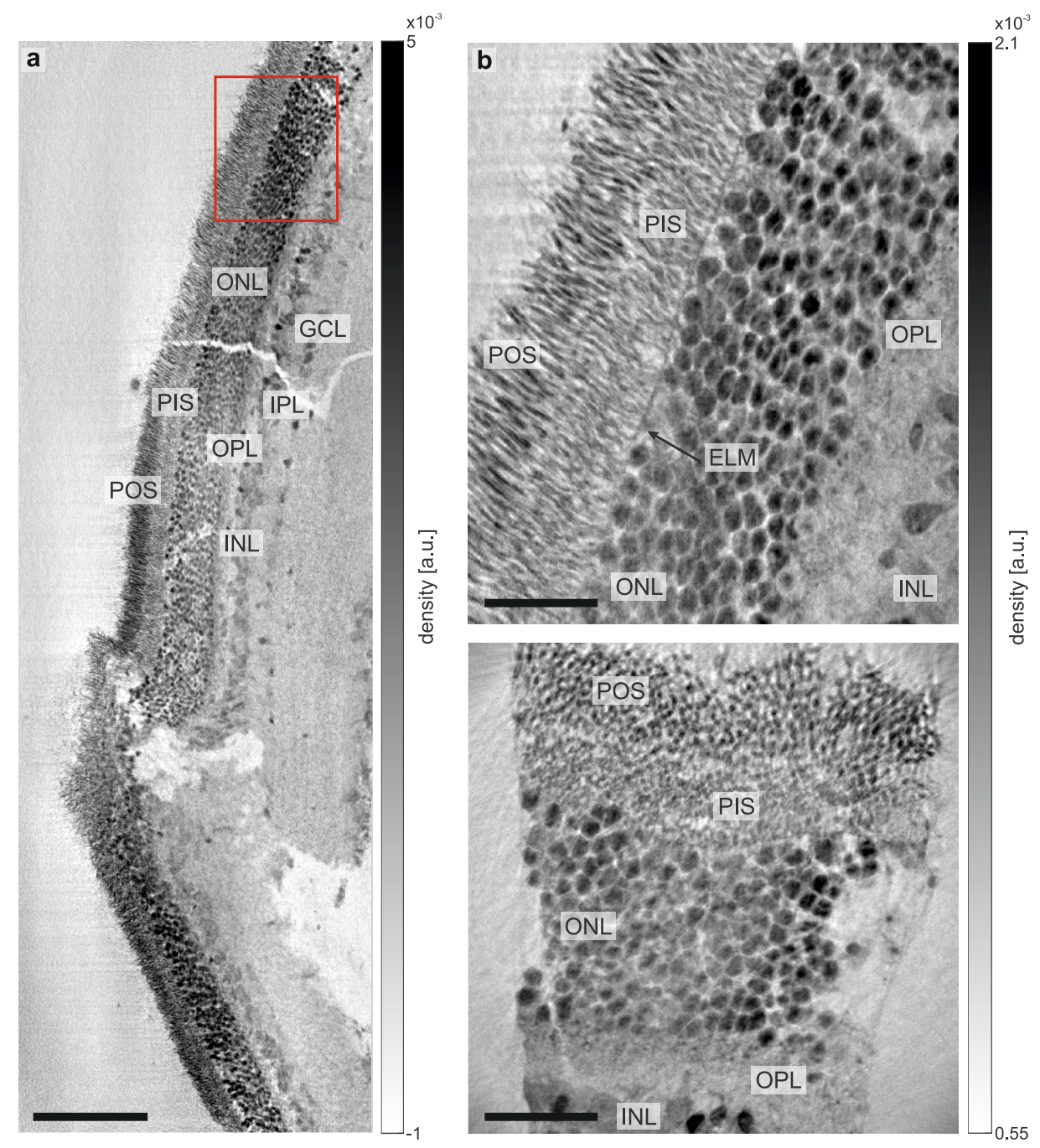

Figure 6.4: Virtual slices through the reconstructed volumes. (a) Longitudinal slice through the medium resolution dataset. In order to increase the field of view, three tomograms were recorded at adjacent positions and virtually stitched together. The typical layers of the retina are clearly visible: photoreceptor outer/inner segments (POS/PIS), outer nuclear layer $(O N L)$, outer plexiform layer $(O P L)$, inner nuclear layer (INL), inner plexiform layer (IPL) and ganglion cell layer (GCL). The rectangle marks the position of the high resolution scan. (b) Orthogonal slices through the high resolution dataset. In addition to the layers already visible in (a), which are resolved at higher detail here, the external limiting membrane (ELM) between the photoreceptor segments and the outer nuclear layer can be recognized. Scale bars: $100 \mu \mathrm{m}$ (a) and $25 \mu \mathrm{m}$ (b) 
the outer nuclear layer (ONL) including the single photoreceptor cell bodies, the outer plexiform layer (OPL), the inner nuclear layer (INL), the inner plexiform layer (IPL) and the ganglion cell layer (GCL) are clearly visible. However, resolution is not sufficient to identify the external limiting membrane whereas the pigmented epithelium was removed prior to epon embedding. It can also be recognized that the staining with osmium was not performed uniformly throughout the strip of retina. Towards the center of the sample, the osmium content is reduced, which is especially visible in the outer nuclear layer where the cell bodies located at the center appear less dark. This could be caused by the incubation times of the osmium, which were probably not sufficient to homogeneously penetrate the entire sample and hence, the outer regions of the retina were stained more strongly. Note that the cracks through the retina, visible around the center of the depicted slice, are artifacts that occurred during the sample preparation.

The rectangle marks the position of the high resolution scan, of which two orthogonal slices through the reconstructed volume are depicted in Fig. 6.4(b), again showing the distinct layers of the retina. The higher resolution of this dataset becomes immediately apparent, as even the external limiting membrane can be detected and individual photoreceptor segments are well distinguishable, both in the 'side view' depicted at the top as well as in the 'front view' at the bottom, in which a cut through these segments is shown. In this slice, the uneven staining can be additionally recognized in a direction perpendicular to that indicated in Fig. 6.4(a), as the inner part of the outer nuclear layer appears less dark compared to the edges.

The gain in contrast compared to the unstained hydrated brain slice considered in the previous chapter can be evaluated by estimating the Weber contrast with respect to the epon surrounding. Due to the varying amount of staining in the different layers as well as the uneven distribution of the osmium because of the non-uniform tissue penetration, the outer nuclear layer and the outer and inner photoreceptor segments are considered separately in strongly stained regions of the sample. This leads to contrast values of $C_{\mathrm{ONL}} \simeq 2.5, C_{\mathrm{POS}} \simeq 1.4$ and $C_{\mathrm{PIS}} \simeq 1.2$ in the high resolution measurement and $C_{\mathrm{ONL}} \simeq 2 / 2.4, C_{\mathrm{POS}} \simeq 1.9 / 1.9$ and $C_{\mathrm{PIS}} \simeq 1.2 / 1.5$, for the center and bottom part of the low resolution setting, respectively. Note that the varying values are caused by the different amounts of osmium content in the stained features along the sample. Hence, contrast is considerably increased compared to the hydrated unstained brain slice, especially for the highly stained outer nuclear layer. However, due to the different experimental parameters, as, e.g., the kind of central nervous tissue used as sample, photon 
energy, number of acquired projections and propagation distances as well as waveguide performance and exposure time, this is only a rough estimate.

To obtain a better impression on the 3 d density distribution, Fig. 6.5(a) shows a volume rendering of the stitched volume acquired at medium resolution which was created in Avizo. It depicts the approximate shape of the retina with the typical curvature due to location of the retina at the back of the eye ball. Additionally, a protrusion of the retina towards the bottom of the sample can be recognized which occurred during sample preparation (according to Fig. 6.4(a) this corresponds to a buckling of the retina at the according position). For the high resolution dataset, a segmentation of the photoreceptor segments as well as the outer nuclear layer was performed with the region growing Magic wand tool in Avizo (cf. Fig. 6.5(b)). To obtain a segmentation of the entire layer, several seeding points were chosen manually and the gray value range was varied accordingly. In the segmentation, the three-dimensional shape of the photoreceptor segments and cell bodies in the outer nuclear layer as well as the uniform spatial distribution of the photoreceptor cells in this part of the retina can be recognized. Although each pair of photoreceptor outer and inner segments is part of a photoreceptor cell which also includes a single cell body, a connection between the three layers is not visible, currently preventing the visualization of individual cells and their orientation with respect to each other.

Resolution An estimate for the resolution reached in the two datasets can be obtained via a Fourier shell correlation (cf. section 1.5). To this end, the tomographic datasets were divided into two subsets comprising every second projection and the FSC was calculated according to eqn. (1.97) for the central $1000^{3}$ voxels of the corresponding reconstructions. The resulting curves for the medium and high resolution datasets are depicted in Fig. 6.6 together with the 1/2-bit threshold curve (1.98). The intersection between these two determines the maximum spatial frequency which is resolved in the reconstruction, leading to half-period resolutions of $595 \mathrm{~nm}$ and $232 \mathrm{~nm}$ in the medium and high resolution dataset, respectively (see Tab. 6.2). The oscillations in the FSC from the medium resolution dataset are caused by the zero crossings of the CTF which are not accounted for, as only one propagation distance was acquired, leading to artifacts in the corresponding spatial frequencies of the retrieved phase maps. 


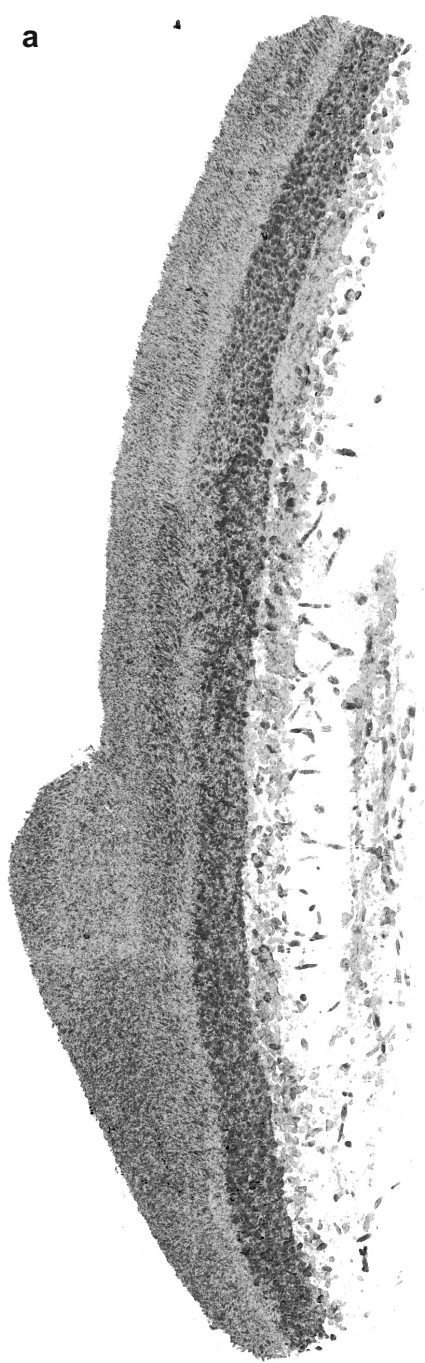

b
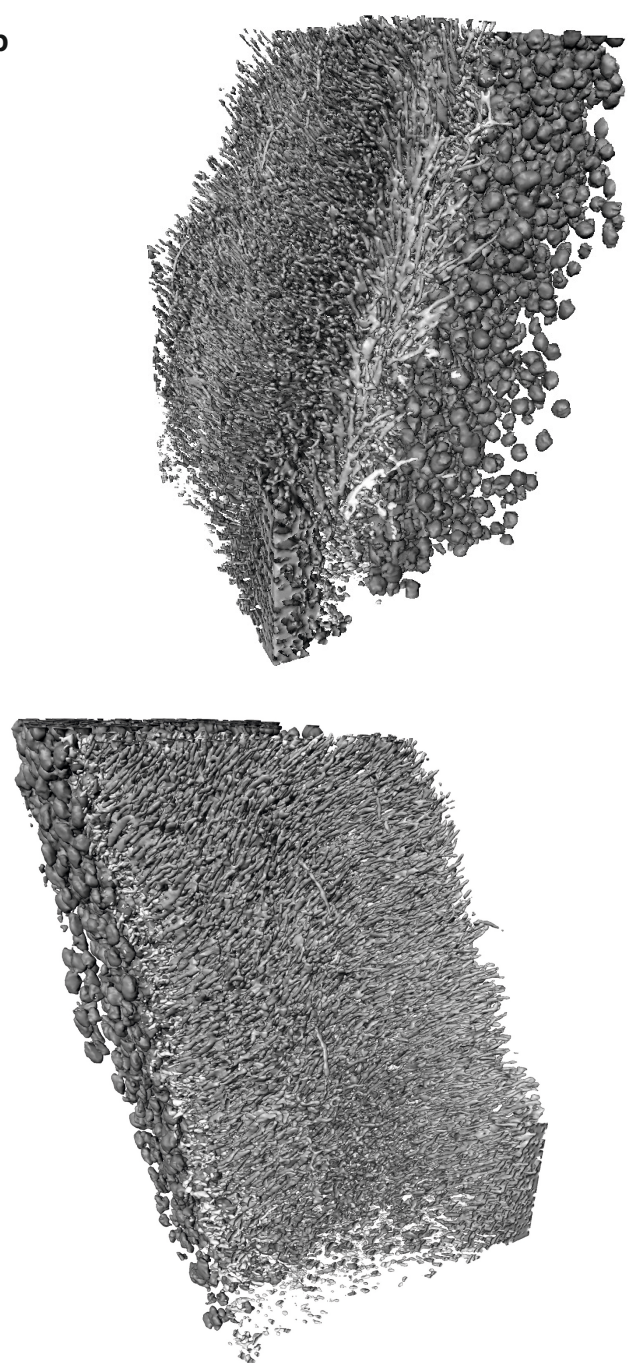

Figure 6.5: Volume representation of the retina. (a) The volume rendering of the medium resolution dataset depicts the approximate shape of the retina with the typical curvature due to its location at the back of the eye. (b) The segmentation of the high resolution dataset clearly shows the typical rod-shaped photoreceptor outer (medium gray) and inner (light gray) segments, as well as the round cell bodies in the outer nuclear layer (dark gray). Although each photoreceptor cell consists of a single cell body from the ONL and a pair of outer and inner photoreceptor segments, a connection between these layers is not visible in the data. 

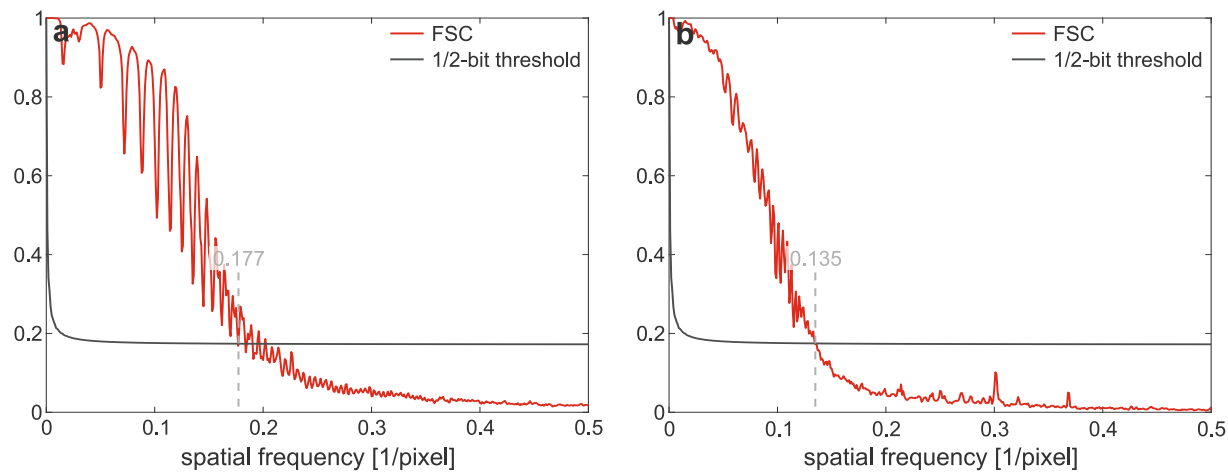

Figure 6.6: Fourier shell correlations of the central $1000^{3}$ voxels of the tomographic reconstructions from the medium (a) and high resolution (b) scan of a strip of osmiumstained mouse retina, together with the 1/2-bit threshold curves. The corresponding half-period resolutions are listed in Tab. 6.2.

\begin{tabular}{lcc}
\hline & Figure & half-period resolution \\
\hline medium resolution & $6.4(\mathrm{a})$ & $514 \mathrm{~nm}$ \\
high resolution & $6.4(\mathrm{~b})$ & $233 \mathrm{~nm}$ \\
\hline
\end{tabular}

Table 6.2: Half-period resolutions for the medium and high resolution tomographic scans of an osmium-stained strip of mouse retina, determined via a Fourier shell correlation of the central $1000^{3}$ voxels in the reconstructed volume.

\subsection{Golgi-Cox stained mouse hippocampus}

The data presented in this section is published in [165] and the presented figures are based on those from the paper.

\subsubsection{Basic anatomy of the hippocampus}

The hippocampus (named after the Greek word for seahorse due to the large resemblance in shape) is a major component of the brain in humans as well as other mammals. Each of the two hemispheres of the brain contains a hippocampus which plays an important role in learning and memory formation [156]. The primary cell in the hippocampus is the pyramidal cell, located in the C-shaped pyramidal cell layer (cf. Fig. 6.7). In addition to the pyramidal cell layer, the hippocampus consists of a number of distinct layers, as the peripheral stratum oriens as well as the stratum radiatum and stratum lacunosum-moleculare, containing the apical dendrites of the pyramidal cells. The hippocampus can be divided into four subre- 


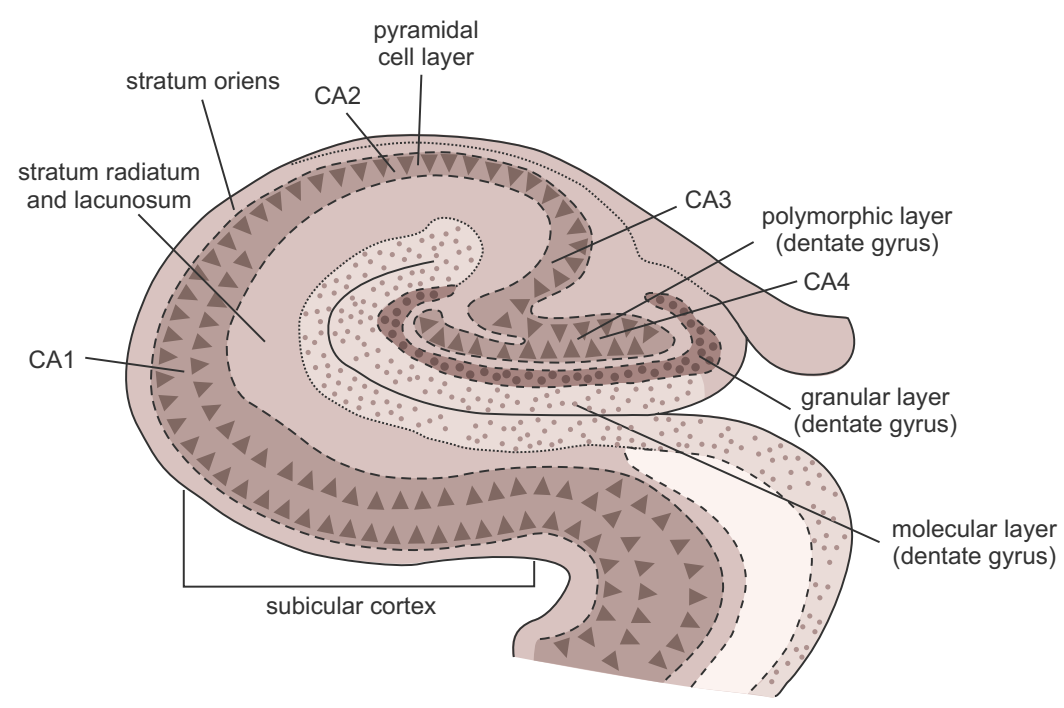

Figure 6.7: Basic anatomy of the hippocampus. The hippocampus consists of distinct layers, including the $C$-shaped pyramidal cell layer as well as the apposing stratum oriens, stratum radiatum and stratum lacunosum. It can be separated into four subfields: CA1CA4, of which the latter is located within the polymorphic layer of the dentate gyrus. The dentate gyrus is a formation within the hippocampus, which is likewise C-shaped and intertwines with the pyramidal cell layer of the hippocampus. In addition to the polymorphic layer, it consists of the granule cell layer, containing the primary cell type of the dentate gyrus, the granule cell, as well as the molecular layer. The drawing is based on $[156]$.

gions, called CA1, CA2, CA3 and CA4. The pyramidal cells located closest to the subicular cortex (cf. Fig. 6.7) are referred to as the CA1 field, whereas the CA4 field is located deep within the so-called dentate gyrus.

The pyramidal cell layer is interlocked with the likewise C-shaped dentate gyrus, which also consists of three distinct layers. The principal cell type is the granule cell, located in the granule cell layer, whose axons make synaptic contact with the pyramidal cells in the CA3 region. The polymorphic cell layer or hilus of the dentate gyrus (CA4 field) consists of modified pyramidal cells and is located deep within the granular layer. The third layer of the dentate gyrus is the molecular cell layer, which mainly contains axons and lies adjacent to the molecular layer of the hippocampus (stratum lacunosum). 


\subsubsection{Methods}

\subsubsection{Sample preparation}

A wild-type mouse, provided by the group of Paul Lingor (Department of Neurology, University Medical Center, Göttingen, Germany), was sacrificed by an anesthetization with $\mathrm{CO}_{2}$ and a subsequent perfusion with $4 \%$ paraformaldehyde to ensure tissue preservation. The brain was dissected and directly prepared for the Golgi-Cox staining procedure via the FD Rapid GolgiStain ${ }^{\mathrm{TM}}$ Kit (FD Neurotechnologies, Inc., USA). Prior to the last step of the staining procedure, the brain was cut into $500 \mu \mathrm{m}$ thick coronal slices and afterwards either embedded in epon (Serva, Germany) or stored in PBS. For the epon embedding, water content was removed via an ascending acetone series, followed by an infiltration with epon and its polymerization at $60^{\circ} \mathrm{C}$ for 24 hours (cf. previous section for more details).

For the experiments, the epon-embedded samples were trimmed at the edges to minimize epon content around the tissue and hence decrease absorption of the $\mathrm{x}$ rays. The hydrated samples were mounted as described in section 5.2 .1 by building a liquid chamber with a width of $500 \mu \mathrm{m}$ between two aluminum rings that are covered with polypropylene foil and have a diameter of $5 \mathrm{~cm}$.

Experiments on the epon-embedded brain slice were performed both at the laboratory setup as well as the GINIX endstation, while the hydrated brain slice was only imaged at the synchrotron due to the mounting of the sample between the two aluminum rings. The large circumference of these rings impedes a measurement of the sample in the inverse geometry, which is needed for high resolution, and additionally, the comparatively unstable sample preparation is incompatible with the associated long exposure times.

\subsubsection{Experimental parameters at the laboratory setup}

To achieve highest resolution and image the sample at sub-cellular detail, the laboratory setup was used in the inverse geometry, i.e., the source-to-sample distance was large compared to the sample-to-detector distance. In this geometry, resolution is limited by the detector PSF and hence, the 'Argos' detector with a pixel size of $0.54 \mu \mathrm{m}$ was installed in the setup. The source-to-sample distance was set to $158.75 \mathrm{~mm}$ and the detector was placed in a distance of $22.5 \mathrm{~mm}$ behind the sample, resulting in a magnification of 1.15 and an effective pixel size of $0.47 \mu \mathrm{m}$ with a field of view of $1.18 \times 1.57 \mathrm{~mm}^{2}$. The source was operated at a maximum acceleration voltage of $40 \mathrm{keV}$, leading to the spectrum shown in Fig. 4.4(b), and an electron beam power of $57 \mathrm{~W}$ at a projected spot size of $\sim 10 \times 10 \mu \mathrm{m}^{2}$. The 


\begin{tabular}{lccc}
\hline & laboratory & P10 med. resolution & P10 high resolution \\
\hline GINIX run & - & 51 & 51 \\
energy [keV] & $0-40 / 9.25$ & 13.8 & 13.8 \\
detector & 'Argos' & 'Iris' & Iris' \\
$z_{01, \min [\mathrm{mm}]}$ & 158.75 & 145 & 50 \\
$z_{02}[\mathrm{~m}]$ & 0.181 & 5.19 & 5.19 \\
$p_{\text {eff }}[\mathrm{nm}]$ & 470 & 182 & 63 \\
field of view $\left[\mathrm{\mu m}^{2}\right]$ & $1177 \times 1563$ & $373 \times 373$ & $129 \times 129$ \\
Fresnel number & 0.085 & 0.0026 & 0.0009 \\
waveguide & - & multilayer & multilayer \\
$\#$ distances & 1 & $(d=81 \mathrm{~nm})$ & $(d=81 \mathrm{~nm})$ \\
angular range $\left[{ }^{\circ}\right]$ & {$[0,180]$} & 4 & $40,180]$ \\
$\#$ projections & 2000 & {$[0,180]$} & 1000 \\
exposure time $[\mathrm{s}]$ & 100 & 1000 & 0.2 \\
BAC $\alpha$ & 0.03 & 0.4 & - \\
BAC $(\gamma \cdot F)$ & 0.15 & - & - \\
CTF $\frac{\delta}{\beta}$ & - & - & 6 \\
CTF $\alpha_{2}$ & - & 6 & 0.00001 \\
\hline
\end{tabular}

Table 6.3: Overview of the experimental parameters for imaging of a Golgi-Cox stained brain slice embedded in epon, which was measured both at the laboratory setup as well as the GINIX endstation.

electron beam was focused with a ratio of 1:4 (height:width) and deflected towards the exit window in order to increase photon flux (cf. Fig. 4.5).

For the tomographic scan, 2000 projections were recorded over an angular range of $180^{\circ}$ with an exposure time of $100 \mathrm{~s}$ each. These long exposure times were enabled by the sample preparation, as the embedding of the brain slice in epon should impede sample alterations throughout the measurement. The experimental parameters are summarized in Tab. 6.3.

As the sample was too large for the field of view of the camera, 15 adjacent projections were additionally recorded in $2 \mathrm{~d}$ with an exposure time of $50 \mathrm{~s}$ and subsequently stitched together to correlate the position of the tomogram to the entire brain slice.

\subsubsection{Experimental parameters at the GINIX setup}

Epon-embedded sample Experiments on the epon-embedded brain slice were performed at a photon energy of $13.8 \mathrm{keV}$ with a crossed multilayer device placed in the focal spot of the KB-mirrors, providing a flux in the order of $3 \cdot 10^{9} \mathrm{ph} / \mathrm{s}$. 
Intensity images were recorded with the 'Iris' detector, which was placed at a source-to-sample distance of $5.19 \mathrm{~m}$. The brain slice was recorded at two source-tosample distances, leading to different resolutions and fields of view. In the 'medium resolution' setting, it was placed at $145 \mathrm{~mm}$, leading to an effective pixel size of $182 \mathrm{~nm}$ and a field of view of $\sim 375 \times 375 \mu^{2}$, whereas in the 'high resolution' setting, the source-to-sample distance was $50 \mathrm{~mm}$ with an effective pixel size of $63 \mathrm{~nm}$ and a field of view of $\sim 130 \times 130 \mathrm{\mu m}^{2}$. In both settings, tomographic scans were carried out at four propagation distances with 1000 projections acquired over an angular range of $180^{\circ}$. The exposure times for the medium and the high resolution setting were set to $0.2 \mathrm{~s}$ and $0.4 \mathrm{~s}$, respectively. All experimental parameters are summarized in Tab. 6.3.

Hydrated sample For the hydrated sample, the energy was likewise set to $13.8 \mathrm{keV}$ and a crossed multilayer device was placed into the focus of the KBmirrors, providing an approximate flux of $9 \cdot 10^{8} \mathrm{ph} / \mathrm{s}$. Images were recorded with the 'Pirra' detector which was placed at a distance of $5.19 \mathrm{~m}$ behind the waveguide. As in the case of the epon-embedded sample, the hydrated brain slice was imaged at two resolution settings, with a source-to-sample distance of $145 \mathrm{~mm}$ in the case of the 'medium resolution', leading to an effective pixel size of $182 \mathrm{~nm}$ and a field of view of $\sim 375 \times 375 \mathrm{\mu m}^{2}$, and a source-to-sample distance of $65 \mathrm{~mm}$ in the 'high resolution', resulting in an effective pixel size of $81 \mathrm{~nm}$ and a field of view of $\sim 165 \times 165 \mathrm{\mu m}^{2}$. For an overview of the sample, 420 overlapping projections were acquired in the medium resolution and subsequently stitched together. The tomographic scans were performed by recording 1500 projections over an angular range of $180^{\circ}$ with an exposure time of $1 \mathrm{~s}$. In the case of the medium resolution, measurements were carried out at a single propagation distance, whereas for the high resolution, scans were acquired at four propagation distances. However, a comparison between the projections acquired at different distances indicated that the silver slowly dissipated from the cells over time, probably due to radiation damage. Hence, only the first propagation distance with the smallest effective pixel size was considered in the following to minimize artifacts in the reconstruction. For a better overview, all experimental parameters are listed in Tab. 6.4. 


\begin{tabular}{lcc}
\hline & P10 medium resolution & P10 high resolution \\
\hline GINIX run & 49 & 49 \\
energy [keV] & 13.8 & 13.8 \\
detector & 'Pirra' & 'Pirra' \\
$z_{01}[\mathrm{~mm}]$ & 145 & 65 \\
$z_{02}[\mathrm{~m}]$ & 5.19 & 5.19 \\
$p_{\text {eff }}[\mathrm{nm}]$ & 182 & 81 \\
field of view $\left[\mathrm{\mu m}^{2}\right]$ & $375 \times 375$ & $165 \times 165$ \\
Fresnel number & 0.0026 & 0.0012 \\
waveguide & multilayer & multilayer \\
$\#$ distances & $(d=81 \mathrm{~nm})$ & 1 \\
angular range $\left[{ }^{\circ}\right]$ & 1 & $10,180]$ \\
$\#$ projections & {$[0,180]$} & 1500 \\
exposure time $[\mathrm{s}]$ & 1500 & 1 \\
CTF $\frac{\delta}{\beta}$ & 1 & 15 \\
CTF $\alpha_{2}$ & 15 & 0.2 \\
\hline
\end{tabular}

Table 6.4: Overview of the experimental parameters for imaging of a hydrated Golgi-Cox stained brain slice measured at the GINIX setup.

\subsubsection{Results}

\subsubsection{Epon-embedded sample}

Laboratory setup Figure 6.8(a) shows the overview of the sample obtained from 15 individual projections recorded at the laboratory setup and virtually stitched together. For a better signal-to-noise ratio, all projections were resampled by a factor of 2 . Already in this 2 d overview the sparsely stained neurons are well discernible due to the high contrast of the silver. From morphology, the region of the hippocampus is well recognizable and the position of the tomogram, marked by the largest rectangle, lies well within this structure. One exemplary projection from the tomographic scan is depicted in (b), with part of the slice shown at higher magnification below. Note that this part corresponds to the field of view in the medium resolution synchrotron dataset. The projection is dominated by absorption effects due the large $Z$-value of the silver and phase contrast is barely visible. Hence, the reconstruction with the BAC algorithm (not shown) has only a small impact. Tomographic reconstruction of the laboratory data was performed via the FDK implementation of the ASTRA toolbox and one exemplary slice through the obtained volume is depicted in Fig. 6.9(a), with part of the slice shown at higher magnification on the right. It is evident that motion has occurred during the mea- 

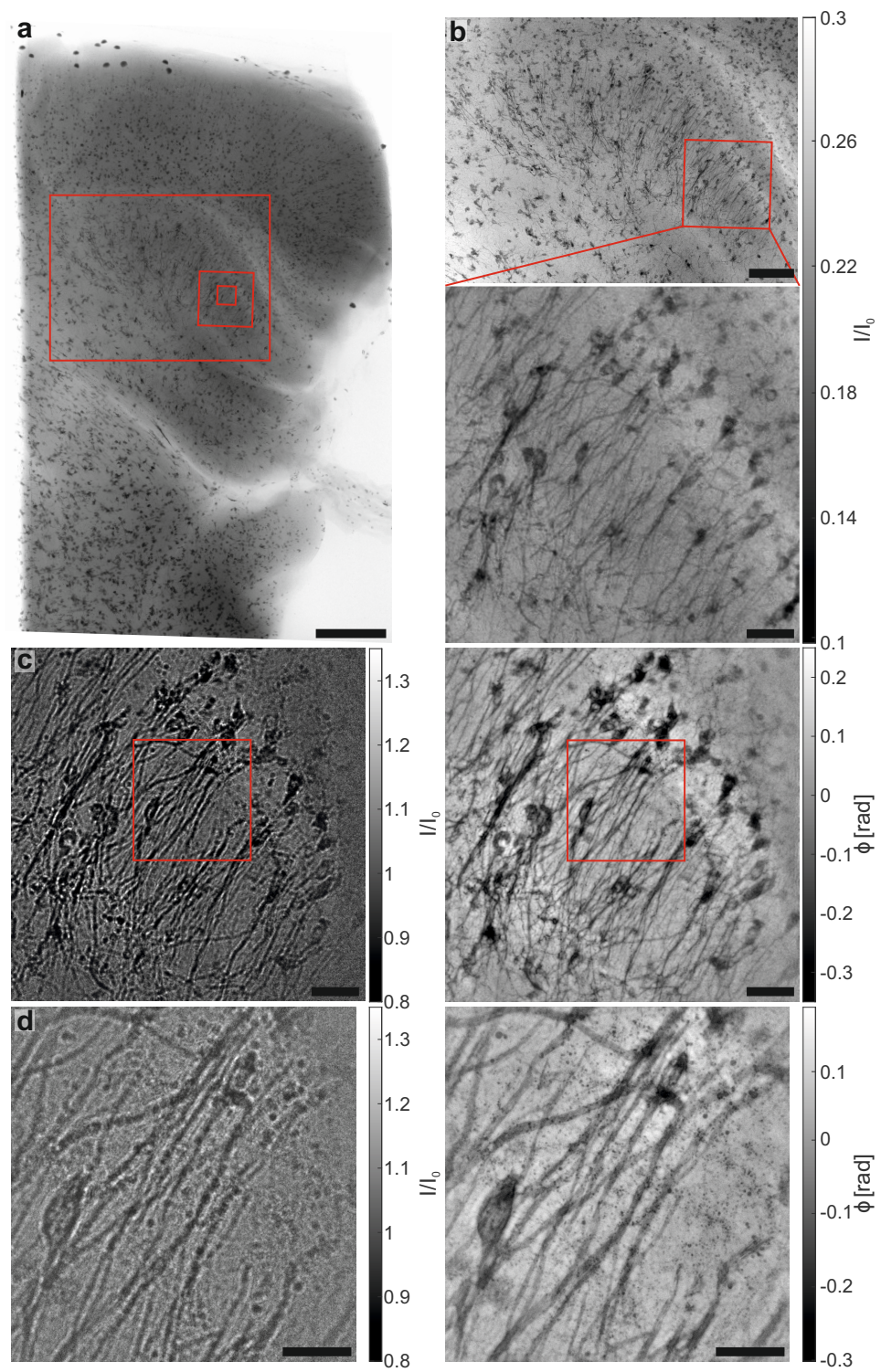

Figure 6.8: Empty-beam corrected projections of the epon-embedded Golgi-Cox stained brain slice. (a) Stitched projection of the entire sample. The rectangles mark the positions of the tomograms acquired at the laboratory (largest rectangle) as well as the GINIX setup in the two resolution settings. (b) Projection acquired at the laboratory setup. The magnified region at the bottom depicts the position of the tomogram obtained at the synchrotron (medium resolution). (c) Projection of the synchrotron dataset at medium resolution with the corresponding reconstructed phase (right). The rectangle marks the position of the high resolution scan depicted in (d). Scale bars: $500 \mu \mathrm{m}$ (a), $200 \mu \mathrm{m}$ (b, top), $50 \mu \mathrm{m}$ (b, bottom) and (c) and $25 \mu \mathrm{m}(\mathrm{d})$ 
surement, as artifacts, resembling those in section 3.2.4, are present in the slice. In view of the stable sample preparation it is most likely that these arose due to temperature drifts and the associated thermal expansion of the components in the setup, which mainly consist of aluminum. However, by assuming that these movements are merely translational, the artifacts can be largely reduced.

Translational motion correction Translational motion can occur both in horizontal and vertical direction, which is accounted for separately in the following. For the vertical motion, the so-called linogram can be used for correction. To this end, all projections were integrated along the horizontal dimension and the resulting profile, plotted against the rotation angle $\theta$, is depicted in cf. Fig. 6.9(b). Due to the small magnification in the inverse geometry, features of the sample should stay at the same height throughout the measurement, leading to straight horizontal lines in the linogram. However, if vertical motion occurred, the single lines within the sinogram will show slight deviations. By aligning each profile to the first one, a model for the vertical motion can be obtained (shown in (f)) and corrected by shifting the projections accordingly. The resulting slice is depicted in 6.9 (b) and the quality is already improved noticeably. To further reduce artifacts, the horizontal movement has to be corrected for as well. For this purpose, the sinogram can be taken into account. Due to the small cone angle of approximately $0.25^{\circ}$, the influence of the cone-beam geometry on the shape of the sinogram is negligible. Hence, the single lines, corresponding to specific features of the sample, should follow a sine function, whereas deviations are caused by horizontal motion of the sample. To account for horizontal motion, specific points on well recognizable curves within the sinogram were selected based on visual inspection and fitted to a sine curve, as shown in Fig. 6.9(d) for one exemplary feature. The horizontal motion model, shown in (f), can be obtained by determining the deviations to this sine curve. For higher accuracy, this was repeated for three different features and the mean of the resulting curves, depicted in red, was used for the motion correction. Note that in contrast to methods as the projection alignment by tomographic consistency [60, 124], the presented approach is less susceptible to artifacts caused by objects lying outside the field of view. The resulting slice after both the linogram and sinogram correction is shown in Fig. 6.9(e). Motion artifacts are significantly decreased and the single objects appear as closed features. The remaining white halo around the dense objects is caused by beam hardening due to the polychromatic illumination (cf. section 3.2.6) as well as missing wedge. This is caused by the asymmetric shape of the sample, with one side length be- 

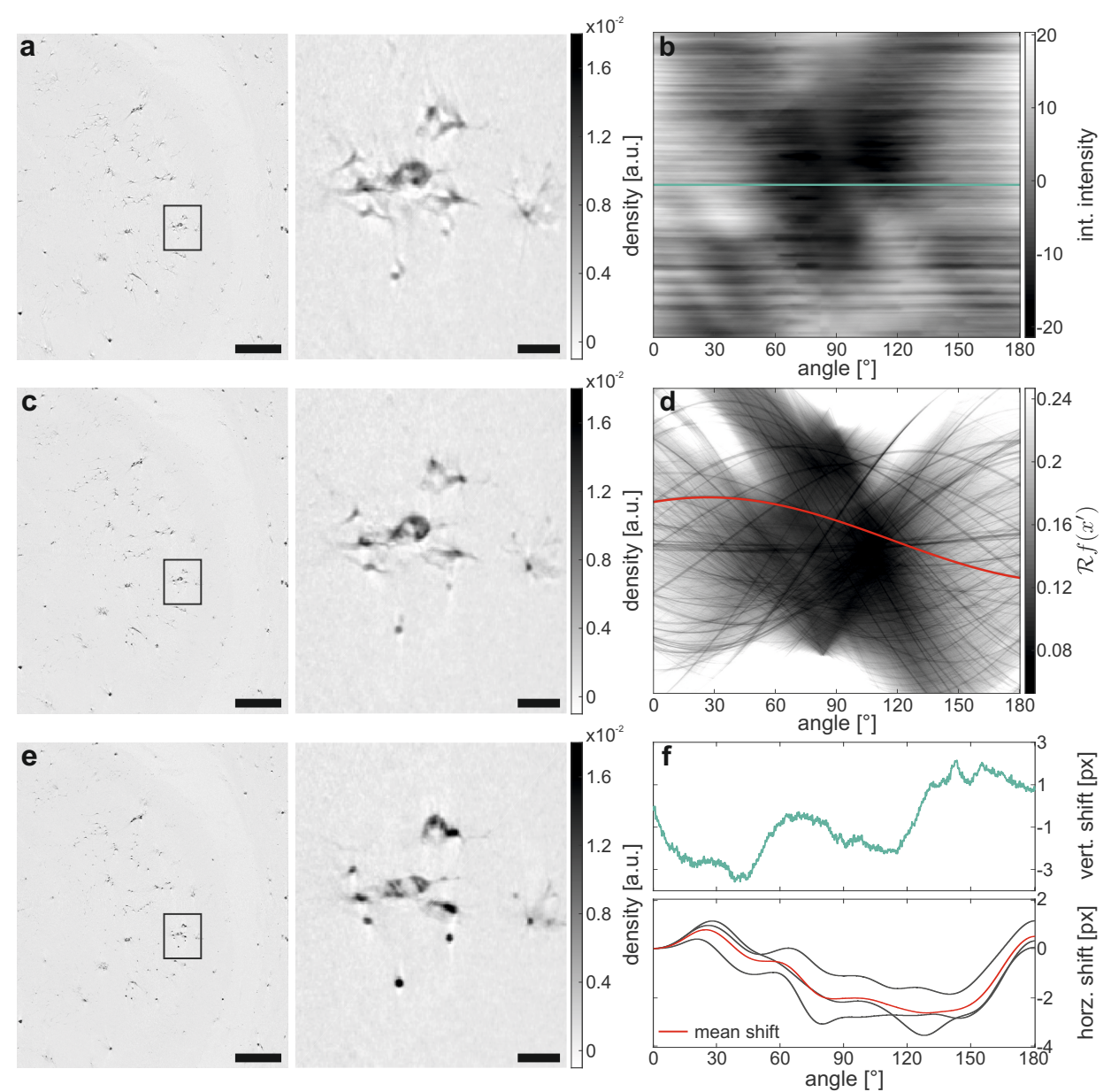

Figure 6.9: Translational motion correction for the laboratory data. (a) Reconstructed slice without any motion correction, in which artifacts are clearly visible. (b) High-pass filtered linogram of the tomographic scan, in which vertical motion is visible as deviations from straight horizontal lines. The strength of these deviations is shown in (f) at the top. (c) Reconstructed slice after the linogram alignment, in which artifacts are already reduced. (d) Exemplary sinogram of the tomographic scan after the linogram alignment. Horizontal motion of the sample is visible as deviations of the curves from a perfect sine function. The deviations for three exemplary features as well as their mean is depicted in (f) at the bottom. (e) Reconstructed slice after both the linogram and sinogram correction, showing a further reduction of motion artifacts. Scale bars: $200 \mu \mathrm{m}$ (left) and $25 \mu \mathrm{m}$ (right) 


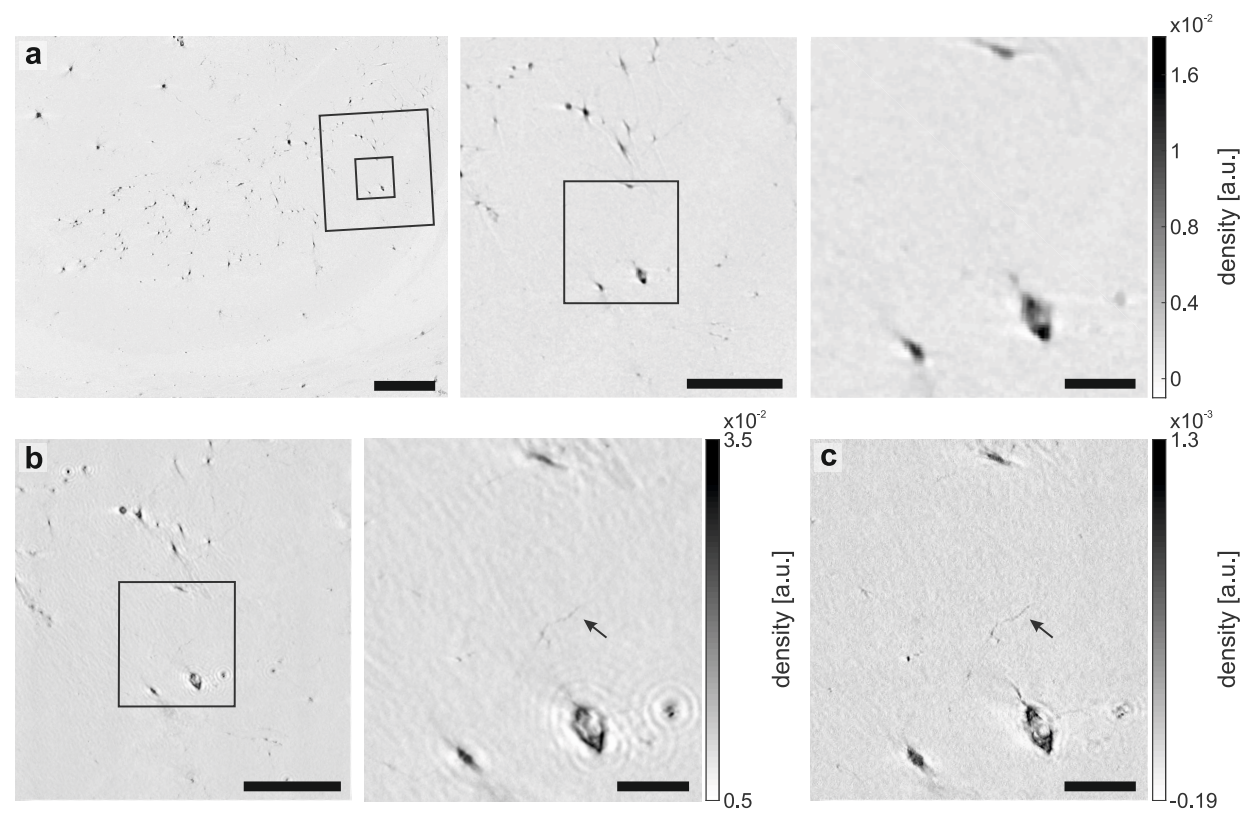

Figure 6.10: Comparison of slices through the reconstructed density of the eponembedded brain slice, obtained at the laboratory as well as GINIX setup. (a) Slice through the volume obtained from the laboratory dataset. Parts of the slice, corresponding to the positions of the medium and high resolution scan indicated by the rectangles, are depicted at higher magnification on the right. (b) Slice through the volume obtained in the medium resolution setting at the synchrotron. The artifacts around the edges of the cells are caused by an imperfect phase retrieval. The rectangle marks the region of the high resolution scan, shown at higher magnification on the right. The arrow indicates parts of the cell that are not resolved in the laboratory data. (c) Corresponding slice through the volume obtained in the high resolution setting, revealing the internal silver distribution at higher detail. Scale bars: $200 \mu \mathrm{m}$ (a, left), $100 \mu \mathrm{m}$ (a, center) and (b, left) and $25 \mu \mathrm{m}$ (a, right), (b, right) and (c)

ing determined by the slice thickness and the other one by the spatial extent of the brain slice, leading to an uneven absorption of the sample throughout the tomographic scan.

3d visualization of the laboratory data Figure 6.10(a) depicts a longitudinal slice through the reconstructed volume after translational motion correction. The rectangles indicate the positions of the datasets obtained at the synchrotron. For comparison, the corresponding regions from the slice obtained at the laboratory setup are depicted at higher magnification on the right. The dark structures are the neurons which were stained by silver atoms. In these stained structures, 
the large features can be assigned to the cell bodies of the neuronal cells whereas the smaller structures belong to axons, which are cut transversely, or unbound silver molecules. An estimate of the contrast within the slice can again be obtained according to the Weber contrast ratio, yielding $C \simeq 4.8$ for the strongly stained features. Note, however, that compared to the previous experiments, contrast formation takes place in the direct-contrast regime where contrast transfer is significantly lower than in the holographic regime. Hence, the obtained values are not entirely comparable.

Due to the small slice thickness of $0.95 \mu \mathrm{m}$, the shape of the stained cells cannot be reconstructed directly, as it emerges over several adjacent slices. Hence, an automatic gray value-based segmentation of the cells was performed in Avizo to better visualize the $3 d$ structure. Figure 6.11(a) depicts the result for the pyramidal layer of the hippocampus. The long axons of the pyramidal cells are well resolved and the round shape of the cell layer can be recognized. The rectangle marks a single cell which is shown in higher magnification in (b). Both the cell body and the axon are clearly resolved, while smaller structures such as the dendrites are not visible. Additionally, it cannot be unequivocally clarified whether the junction at the upper part of the cell belongs to the cell itself or whether it is formed by a connecting cell which is not stained entirely.

GINIX endstation Figure 6.8(c) and (d) depict two exemplary empty-beam corrected projections recorded in the medium and high resolution setting, respectively. Note that in contrast to the laboratory measurement, phase-contrast effects are clearly visible due to the higher degree of coherence and smaller Fresnel numbers. On the right, the reconstructed phase distributions are shown, using the CTF-based approach for weakly absorbing objects with regularization parameters $\frac{\delta}{\beta}=6$ and $\alpha_{2}=0.5$ and $\alpha_{2}=0.00001$, respectively. In order to consider all four propagation distances, the single projections were again scaled to the same effective pixel size and aligned to each other. The resulting images depict a sharp and quantitative reconstruction of the phase, in which the cell bodies as well as axons of the pyramidal cells are already well visible. However, due to the strong interaction of the silver atoms with the illuminating beam, the slowly varying phase as well as weakly absorbing object assumption are violated, leading to artifacts around the edges of the strongly stained objects. This is even better visible in the reconstructed slices depicted in Fig. 6.10(b) and (c), which were obtained via the Matlab implementation of the filtered backprojection using a standard RamLak filter. The imperfect CTF phase retrieval leads to residual fringes around the 

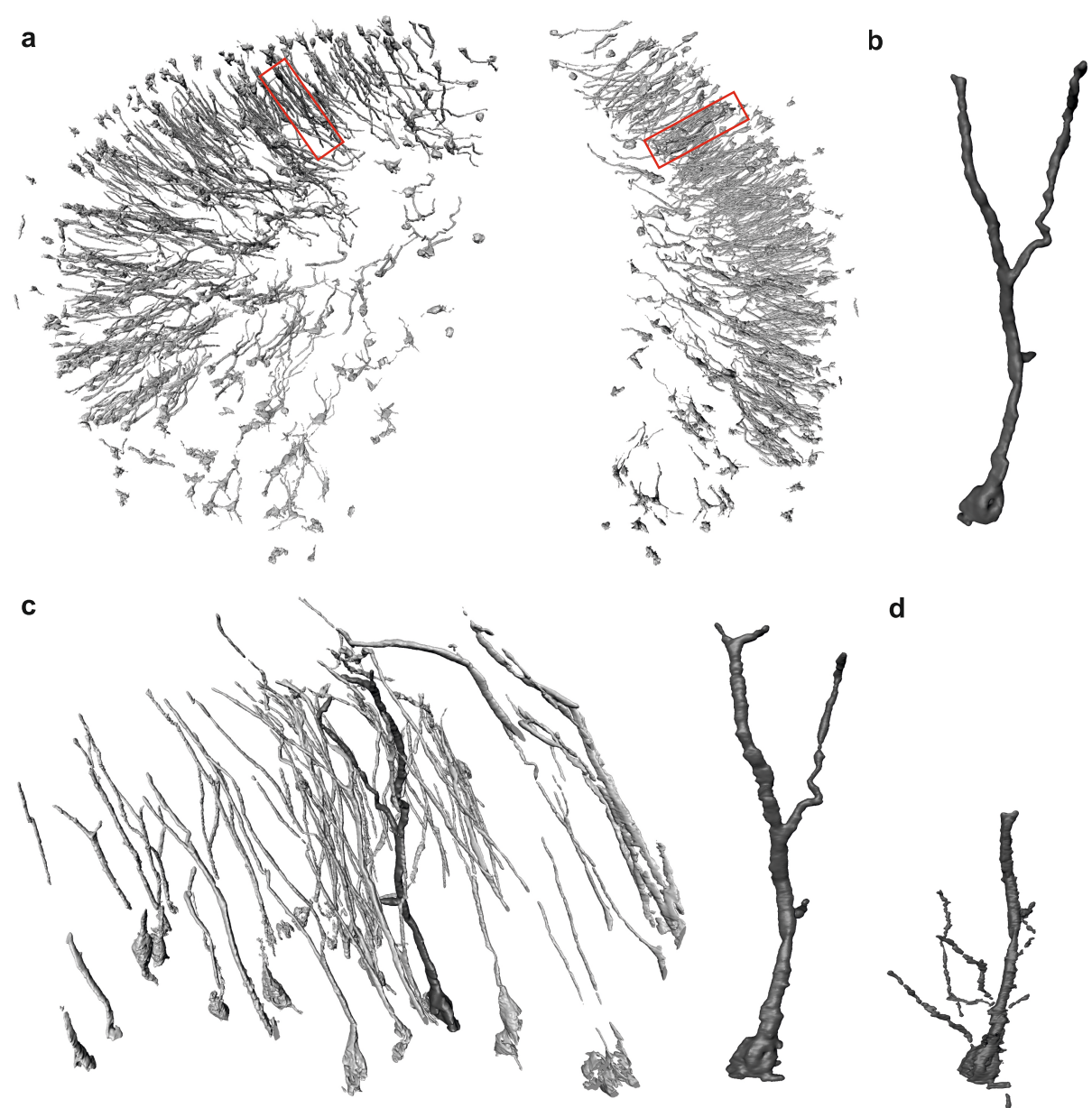

d

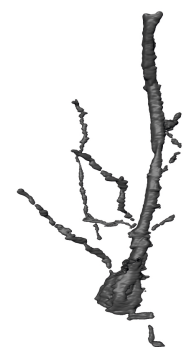

Figure 6.11: $3 d$ visualization of the epon-embedded sample. (a) Segmentation of the pyramidal cell layer of the hippocampus as obtained by the laboratory setup. The segmentation was performed automatically via a gray-value threshold and subsequent refinement. (b) Segmentation of a single cell within the pyramidal cell layer. The corresponding position of the cell within the layer is marked in (a). (c) Gray value-based segmentation of the part of the pyramidal cell layer which was included in the field of view of the medium resolution synchrotron dataset. For comparison, a segmentation of the same cell as in the laboratory data is depicted on the right. Despite the higher resolution of the synchrotron measurement, the cell is depicted in approximately the same amount of detail. (d) Semi-automatic segmentation of the corresponding cell from the high resolution dataset obtained at the synchrotron. Only by manually adding structures to the segmentation that seem to belong to the cell, an advantage of the higher resolution becomes apparent on the level of the $3 d$ cellular shape. 
edges. Additionally, a white halo is visible which can again be attributed to the missing wedge caused by the asymmetrical shape of the epon block. Nevertheless, the comparison between the results obtained at the laboratory as well as at the synchrotron clearly shows the benefit in resolution of the latter while the laboratory dataset can cover a significantly larger field of view. The inner structure of the cell bodies is resolved in higher detail and parts of the cell which are not resolved at the laboratory measurement can be discerned (see arrow in Fig. 6.10(b) and (c)). The contrast of the stained cells with respect to the surrounding tissue can be estimated at $C \simeq 2.9$ in the medium resolution and $C \simeq 2.2$ in the high resolution dataset.

To obtain a better impression of the $3 \mathrm{~d}$ structure of the sample, a gray valuebased segmentation is also carried out for the synchrotron datasets (depicted in Fig. 6.11(c) and (d)). As in the case of the laboratory, the cell bodies as well as axons of the pyramidal cells, which are included in the field of view of the medium resolution dataset, can be well visualized. However, also at this resolution, smaller structures as the dendrites are not visible. Even for the high resolution dataset, the automatic segmentation delivers approximately the same amount of detail on the outer shape of the cell as the measurements at lower resolution. Only by manually adding objects that have no distinct connection to the cell body but seem to emerge from it, an advantage of the higher resolution becomes apparent.

Hence, despite the higher resolution of the synchrotron dataset, the gain in the obtained volumes is mainly restricted to the inner structure of the stained cells, whereas the outer shape is depicted in approximately the same amount of detail throughout the setups and configurations. This is most likely caused by an incomplete staining of the neurons and in particular a leaking of bound silver during the epon embedding, in which the sample is rinsed several times in different solutions.

\subsubsection{Hydrated sample}

As epon embedding represents a considerable alteration of the sample environment, presumably causing the leaking of silver and hence incomplete staining of the cells, a hydrated Golgi-Cox stained brain slice was measured as well. As this sample preparation is not compatible with the long measurement times associated with the laboratory setup in inverse geometry, imaging was only carried out at the synchrotron. The result of the $2 \mathrm{~d}$ overview scan of the sample, comprising 420 overlapping projections, is depicted in Fig. 6.12, clearly showing the hippocampal formation including the pyramidal cell layer as well as dentate gyrus. The tomographic scans were carried out in the region of the pyramidal cell layer, as 


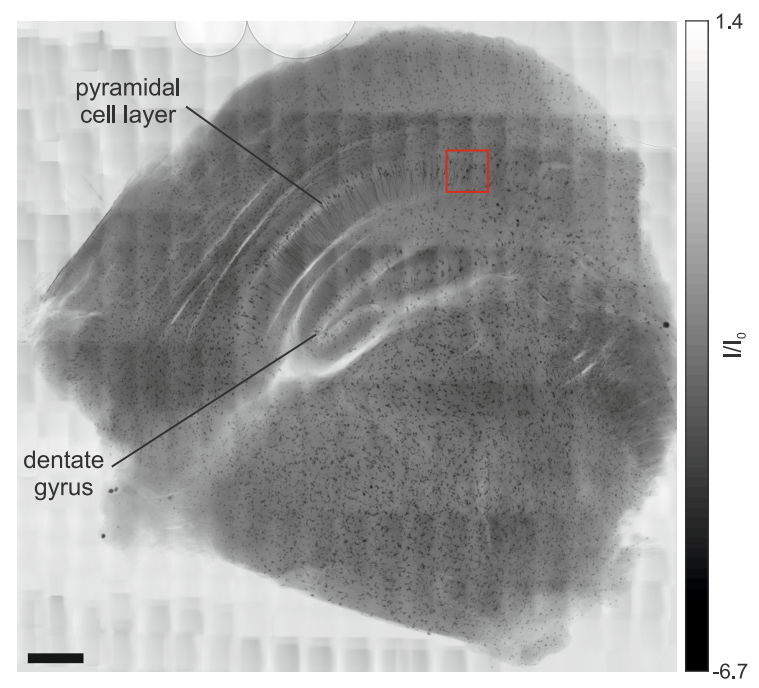

Figure 6.12: Overview of the hydrated Golgi-Cox stained brain slice. To obtain this overview of the sample, 420 overlapping projections were acquired at medium resolution and subsequently stitched together. The hippocampal formation including the pyramidal cell layer as well as the dentate gyrus are clearly visible. The position of the tomogram acquired at the medium resolution, lying within the pyramidal cell layer, is indicated by the rectangle. Scale bar: $500 \mu \mathrm{m}$

indicated by the rectangle. Figure 6.13(a) and (b) show exemplary projections acquired both in the medium as well as high resolution setting. Sub-cellular structures as the cell bodies as well as axons and dendrites can already be recognized in $2 \mathrm{~d}$. Phase retrieval was performed based on the single-distance CTF for a weakly absorbing sample, with regularization parameters $\frac{\delta}{\beta}=15$ and $\alpha_{2}=0.2$ for both scans, leading to the projections in (b) and (d). Even by considering only a single propagation distance, quantitative reconstructions of the phase can be obtained, with residual fringes still present around the edges of the cells, comparable to the epon-embedded sample.

The tomographic reconstructions were carried out with the Matlab implementation of the filtered backprojection with a standard Ram-Lak filter. Prior to the reconstruction, the simple ring-removal algorithm was applied on the sinograms (cf. section 3.2.2). Longitudinal slices through the resulting volumes are depicted in Fig. 6.14. For comparison, the region of the high resolution scan is marked by a rectangle and the corresponding part of the medium resolution scan is shown at higher magnification on the right. The benefit of the higher resolution becomes immediately apparent, as the edges of the reconstructed cells are sharper and the 

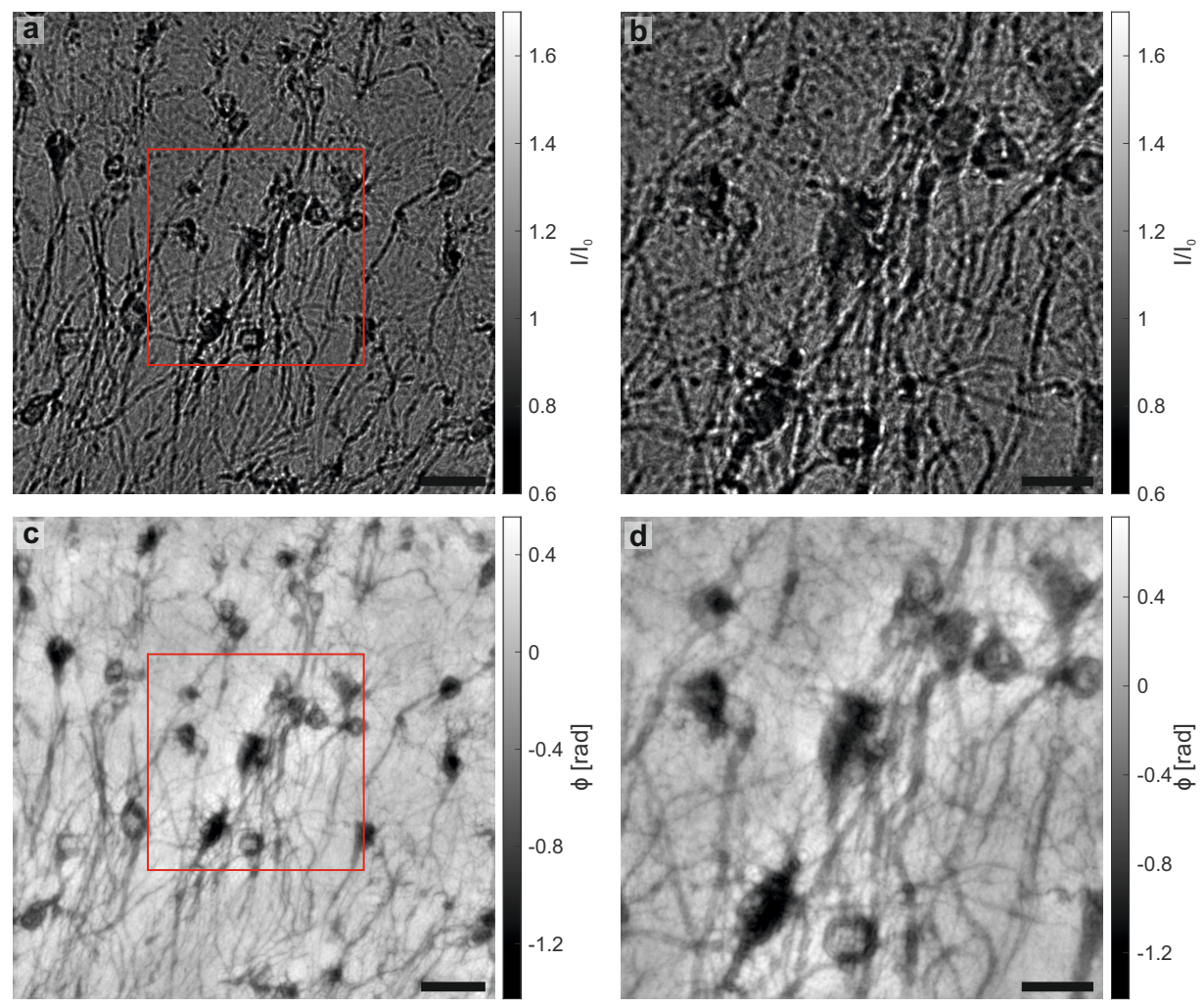

Figure 6.13: Projections of the hydrated brain slice, acquired both at medium and high resolution. (a) Empty-beam corrected projection from the medium resolution scan, in which the single cells within the tissue are well visible. The corresponding reconstruction, obtained via a single-distance CTF approach, is depicted in (c). The position of the high resolution scan is indicated by the rectangle. (b) Empty-beam corrected projection acquired at the high resolution setting. Again, a single-distance CTF approach was applied for phase retrieval, yielding the result in (d). Scale bars: $50 \mu \mathrm{m}(\mathrm{a}, c)$ and $25 \mu \mathrm{m}$ $(b, d)$

inner structure within the cell body can be reconstructed at higher detail. Additionally, small dendrites emerging from the cell body become visible, as indicated by the arrows in (b). Apart from these dendrites, visible due to the higher resolution, new, relatively large clusters of silver molecules appear around the cell body, which should have been resolved in the medium resolution scan as well. Hence, they are probably caused by the dissipation of bound silver molecules during the scan which also impeded the consideration of all four propagation distances acquired in the high resolution setting. Fringes around the highly absorbing cell bodies are caused by the imperfect phase retrieval, already indicated in the $2 \mathrm{~d}$ projections. 


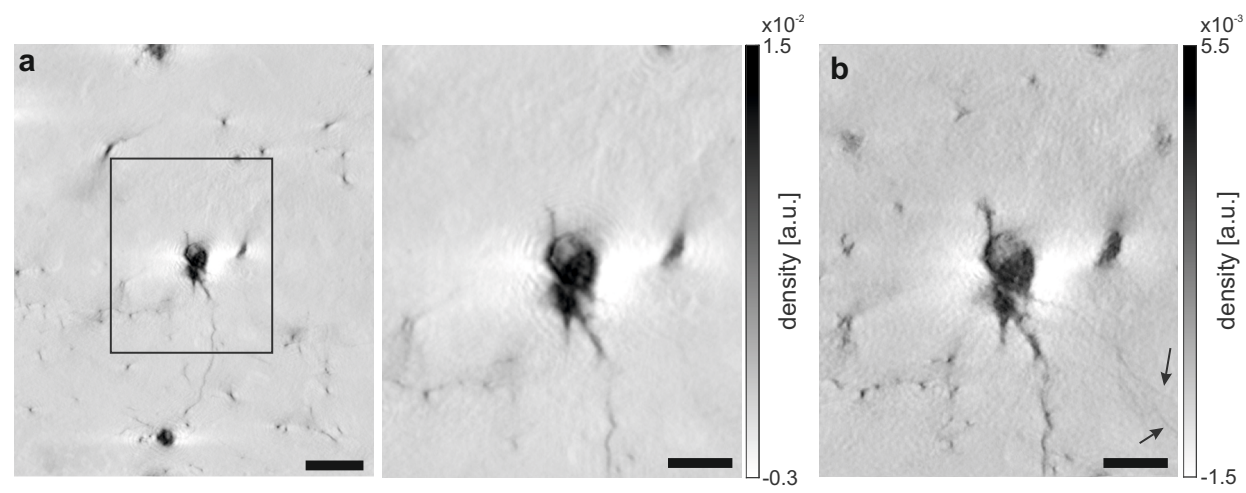

Figure 6.14: Slices through the reconstructed density from the medium and high resolution scan. (a) Longitudinal slice from the medium resolution scan, showing two cell bodies in close proximity as well as sub-cellular structures as axons. For comparison, the position of the high resolution scan is marked by a rectangle and the corresponding part of the slice is depicted in higher magnification on the right. (b) Corresponding slice through the volume reconstructed from the high resolution scan, showing the cell at larger detail, as, e.g., small dendrites emerging from the cell body can be resolved (arrows). Scale bars: $50 \mu \mathrm{m}$ (a, left) and $25 \mu \mathrm{m}$ (a, right) and (b)

As in the case of the epon-embedded sample, they occur due to the violation of the slowly varying phase and weakly absorbing object assumption. Additionally, a white halo is visible, which can be explained by a missing wedge due to the highly absorbing aluminum rings, as indicated in the sinograms depicted in section 5.3.2 for a similar sample mounting. The contrast of stained features within the slice compared to the surrounding tissue can be estimated at $C \simeq 3.2$ and $C \simeq 2.2$ for structures with a high silver content in the medium and high resolution dataset, respectively.

For a better impression of the $3 \mathrm{~d}$ shape of the stained cells, a (semi)-automatic rendering was performed for both the medium and high resolution scan, as depicted in Fig. 6.15. To this end, the gray value-based region-growing tool Magic Wand, implemented in Avizo, was used by manually defining seeding points within the cell structures. Same color of the segmentation indicates a connection which is either functional or just due to the local proximity of the cells. For the high resolution dataset, structures which were not segmented automatically but belong to the cell according to visual inspection were added manually. Note, however, that compared to the epon-embedded sample, the automatic gray value-based segmentation worked much better and the manual refinement had to be carried out only for a few structures. Both in the medium and the high resolution scan, the cells can be depicted in high detail and in addition to the axons, already visible in the 

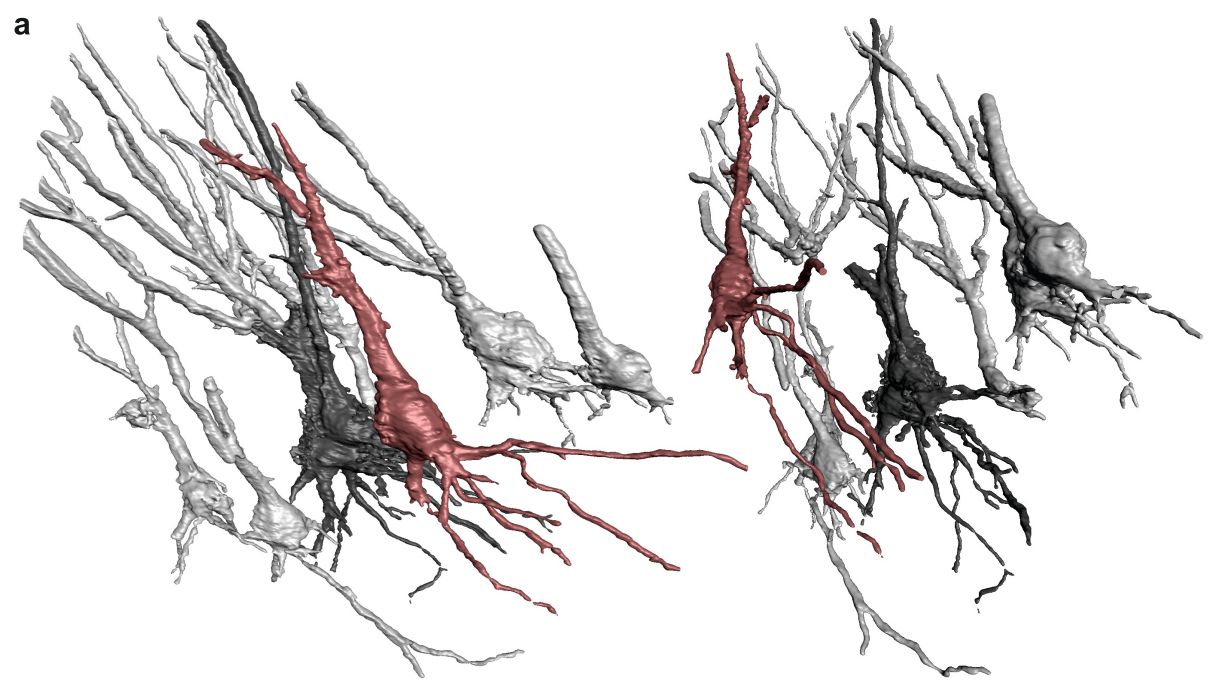

b
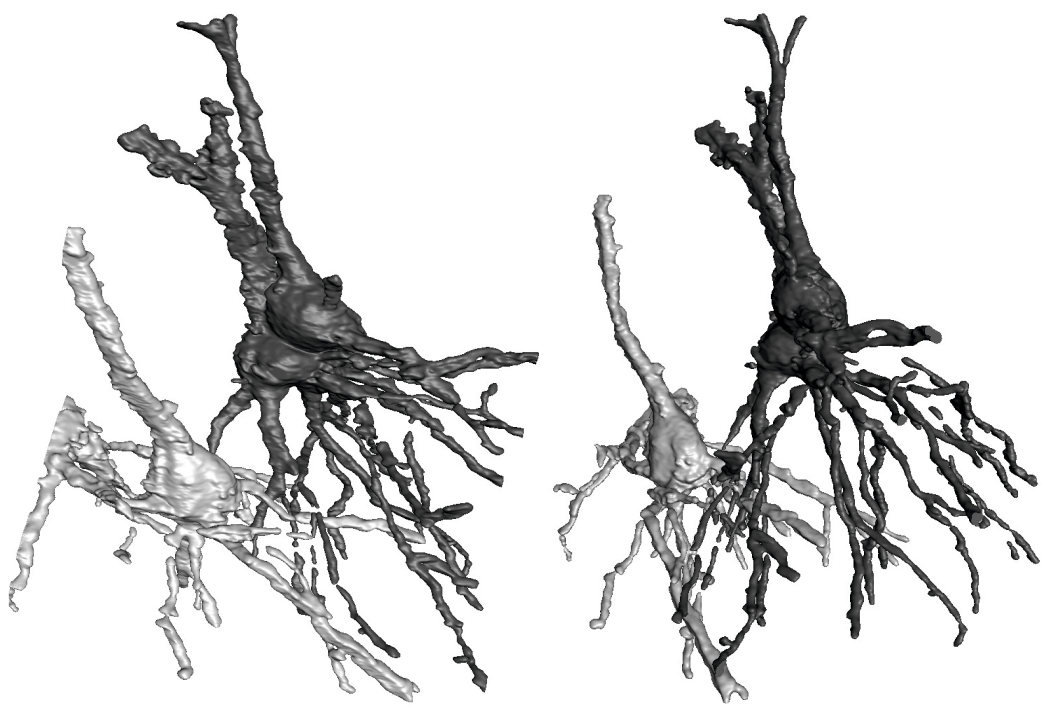

Figure 6.15: $3 d$ visualization of the stained cells within the hydrated brain tissue. (a) Automatic region growing-based segmentation of selected cells within the medium resolution dataset. The same color indicates a connection between the cells at a certain point. (b) (Semi)-automatic segmentation of the stained cells in the high resolution dataset, revealing the cellular structure, especially the dendrites, at higher detail. As some connections could not be segmented automatically, the corresponding structures were added manually. 


\begin{tabular}{lccc}
\hline & Figure & method & half-period resolution \\
\hline epon (laboratory) & $6.10(\mathrm{a})$ & FSC & $1.2 \mu \mathrm{m}$ \\
epon (med. resolution) & $6.10(\mathrm{~b})$ & FSC & $493 \mathrm{~nm}$ \\
epon (high resolution) & $6.10(\mathrm{c})$ & FSC/edge & $1.17 \mu \mathrm{m} / \sim 200 \mathrm{~nm}$ \\
hydr. (med. resolution) & $6.14(\mathrm{a})$ & FSC & $487 \mathrm{~nm}$ \\
hydr. (high resolution) & $6.14(\mathrm{~b})$ & FSC & $386 \mathrm{~nm}$ \\
\hline
\end{tabular}

Table 6.5: Half-period resolutions for the medium and high resolution tomographic scans of a Golgi-Cox stained mouse hippocampus, determined via a Fourier shell correlation of the central $1000^{3}$ voxels in the reconstructed volume.

epon-embedded samples, even dendrites emerging from the cell body are visible. Especially in the high resolution scan, more and finer dendrites can be segmented automatically or based on visual inspection, which is a necessary step towards addressing questions of neuronal connectivity in $3 \mathrm{~d}$.

Resolution To estimate the resolution achieved with the different experimental settings and preparation procedures, the Fourier shell correlation (cf. section 1.5) was calculated for the central $1000^{3}$ voxels of two independent tomographic reconstructions, which were obtained by using only every second projection from the entire scan. The resulting curves together with the $1 / 2$-bit threshold curve, which determines the maximum spatial frequency resolved in the $3 \mathrm{~d}$ volume, are depicted in Fig. 6.16. Note that due to the comparatively high noise level, the FSC curve for the high resolution measurement of the epon-embedded brain slice corresponds to a very low resolution, although visual inspection of Fig. 6.10 reveals the advantage within the stained objects in the reconstructed slices. Hence, an error function was additionally fitted to a profile along manually chosen edges between silver stained features and the surrounding tissue. The resulting half-period resolutions are listed in Tab. 6.5. The relatively small gain in resolution for the hydrated brain slice measured at high resolution compared to the medium resolution setting is most likely caused by the sample changes occurring during the measurement, which already impeded the use of four propagation distances for phase retrieval.

\subsection{Summary}

In this chapter, the contrast enhancement in propagation-based phase-contrast tomography of neuronal tissue due to staining with metals was evaluated. To this end, an osmium-stained retina embedded in epon was imaged at the synchrotron, 

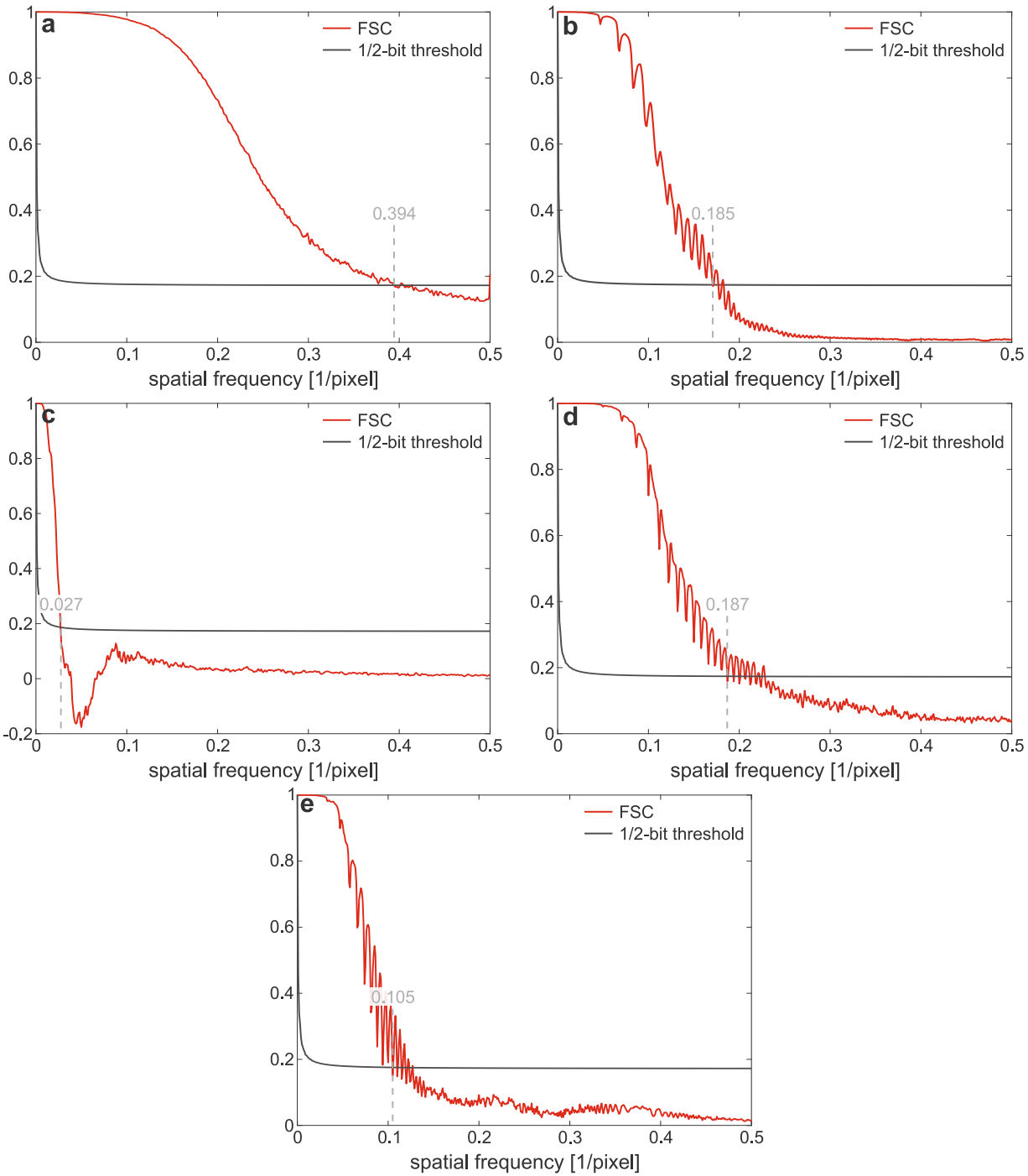

Figure 6.16: Fourier shell correlations of the central $1000^{3}$ voxels of the tomographic reconstructions from the epon-embedded sample, measured at the laboratory (a), in the medium (b) and the high resolution setting (c), as well as the hydrated sample, both at medium (d) and high resolution (e). To estimate the resolution, the intersection between the FSC and the 1/2-bit threshold curve was determined. The corresponding half-period resolutions are listed in Tab. 6.5. 
while a silver-stained brain slice from mouse, which was either hydrated or embedded in epon, was investigated both at the synchrotron as well as at the laboratory setup in the case of the latter. The labeling with osmium tetroxide, which stains lipid-containing structures, as, e.g., cell membranes, results in an excellent overall tissue contrast but restricts the sample size due to its high absorption, whereas the statistical staining of specific cells with silver leads to a high visibility of these cells while the surrounding tissue remains mostly hidden. For both staining methods, contrast is superior compared to the hydrated unstained brain slice imaged in chapter 5, with Weber contrast values that are factors of 2-25 larger, depending on the considered features and tissue preparations. Even at the laboratory setup, contrast and resolution are high enough to image single cells at sub-cellular detail. Note, however, that the obtained contrast values are only roughly comparable and that the different experimental parameters, as, e.g., the kind of central nervous tissue used as sample, contrast transfer in the different imaging regimes, photon energy, number of acquired projections and propagation distances as well as waveguide performance and exposure time, have to be taken into account as well. Phase retrieval according to the CTF-based approach yields sharp and quantitative results, with some residual artifacts around the outer edges of the sample due to the violation of the slowly varying phase and weakly absorbing object assumption. Hence, algorithms with less restrictions on the sample, e.g., the Holo-TIE or iterative approaches, could lead to superior results in the future. However, resolution is nevertheless high enough to resolve both the retina with its distinct layers as well as single cells within the pyramidal layer of the mouse hippocampus at sub-cellular detail. Issues of neuron/photoreceptor cell distribution, shape, orientation and spatial arrangement can be clearly addressed in three dimensions. In contrast to the unstained hydrated brain slice, even small structures as dendrites are resolved. Hence, imaging of metal-enhanced tissue can be probably used to decipher connectivity in the future, especially if the sample preparation is further optimized, e.g., by reaching a more complete staining as well as a stable sample environment so that no radiation damage or motion artifacts occur. The importance of the complete staining was best seen at the example of the epon-embedded silver-stained brain slice, where the epon embedding procedure most likely caused a leakage of the bound silver so that only parts of the cell were stained. The negative effects of motion could be observed for the laboratory setup, where thermal expansion caused strong artifacts in the reconstructed slices. Here, a method for compensating translational motion was introduced which significantly reduced these artifacts and could possibly be extended to rotational motion perpendicular 
to the optical axis. However, the correction for internal changes occuring during the measurements, as, e.g., a leakage of silver molecules, is not as easy. In addition to an optimized sample preparation, mechanical stability has to be increased to achieve the level of neuronal connectivity, as even for the stable epon-embedded tissues, the resolution lies in the range of 3-4 pixels. 


\section{Contrast enhancement by different embedding media}

According to section 1.3.3, contrast transfer can be enhanced by either staining specific features with radiocontrast agents, as shown in the previous chapter, or by embedding the sample in a different surrounding medium, leading to an increase in overall tissue contrast. As propagation-based imaging is sensitive to relative changes in electron density, a medium with significantly smaller density with respect to the tissue of interest can lead to superior contrast in the reconstructed images [41, 69, 84, 161].

In order to maximize contrast, the surrounding medium an be replaced by air, resulting in a maximum difference in electron density. To prevent significant sample changes due to the accompanying drying procedure, Jürgen Goldschmidt (Leibniz Institute for Neurobiology, Magdeburg, Germany) developed the 'evaporation-ofsolvent' method, which removes the water content from the sample while preserving the overall tissue structure. Due to the resulting large difference in electron density, phase retrieval in the holographic regime is, however, challenging as, e.g., the assumptions for the CTF-based algorithm are not fulfilled. Hence, imaging of these samples is in the following solely carried out at the laboratory setup and thus in the direct-contrast regime. Note, however, that synchrotron setups, in which contrast formation takes place in the direct-contrast regime as well, as is generally the case for setups exploiting a parallel-beam geometry, are also well suited for imaging of these kinds of samples [167].

For imaging in the holographic regime, embedding media with densities between water $\left(0.997 \mathrm{~g} / \mathrm{cm}^{3}\right.$ at $\left.25^{\circ} \mathrm{C}[180]\right)$ and air $\left(0.0013 \mathrm{~g} / \mathrm{cm}^{3}\right.$ at $0^{\circ} \mathrm{C}$ and $1013 \mathrm{hPa}$ [181]) can be exploited. Conveniently, the intermediate steps in a standard paraffin embedding procedure, namely ethanol $\left(0.789 \mathrm{~g} / \mathrm{cm}^{3}\right.$ at $20^{\circ} \mathrm{C}$ [179]) and the wax itself $\left(\sim 0.9 \mathrm{~g} / \mathrm{cm}^{3}[97]\right)$, can be used, as the corresponding preparation steps are well established. In addition, after non-destructive imaging of the sample by means of phase-contrast tomography, a defined region of interest could be subsequently investigated via standard histological sectioning, combining information on the entire $3 \mathrm{~d}$ density with insights gained from more specific staining agents 
used in histology [25, 69].

\subsection{Evaporation-of-solvent preparation}

The results presented in the following are published in [167] and the majority of figures is based on those from the paper.

\subsubsection{Methods}

\subsubsection{Sample preparation}

Adult mice were deeply anesthetized (induced with $2 \%$ isoflurane, $0.75 \% \mathrm{O}_{2}$ and $0.55 \% \mathrm{~N}_{2} \mathrm{O}$ and a subsequent intraperitoneal injection of $150 \mu \mathrm{l}$ of a ketamine/xylazine solution containing $12.5 \mathrm{mg}$ ketamine/ml and $0.5 \%$ xylazine) and transcardially perfused with a short flush of saline, followed by a $4 \%$ PFA solution for 15 minutes as well as ethanol for 30 minutes. After perfusion, brains were dissected and stored in ethanol overnight at $7^{\circ} \mathrm{C}$. Subsequently, the brains were transferred to fresh ethanol and remained in this solution for one to three days. After replacing the ethanol with xylene and leaving the sample in this solution overnight at room temperature, it was stored in a fresh batch of xylene for another one to two days. Finally, the sample was placed on tissue paper under a hood and the xylene evaporated overnight at room temperature, resulting in a sample, in which the water content was replaced by air (cf. Fig. 7.1(a)). Due to this evaporation of the xylene, the method is termed 'evaporation-of-solvent'. For the tomographic experiments, selected regions were separated from the dried brains, provided by the group of Jürgen Goldschmidt (Leibniz Institute for Neurobiology, Magdeburg, Germany), in order to decrease absorption due to excess parts of the sample (cf. 7.1(b) (top panel)). The remaining samples, namely the right hemisphere, cerebellar vermis, olfactory bulb or brain stem of a mouse brain (Fig. 7.3(a)), were squeezed carefully into a pipette tip, which was glued to a brass pin and sealed with hematocrit in order to maintain experimental conditions and minimize artifacts due to external factors as changing temperature or humidity (cf. 7.1(b) (bottom panel)).

\subsubsection{Experimental parameters}

Experiments were carried out at the laboratory setup JuLiA both in cone-beam and inverse geometry, in order to first gain an overview of the sample and subsequently zoom into specific regions of interest. 

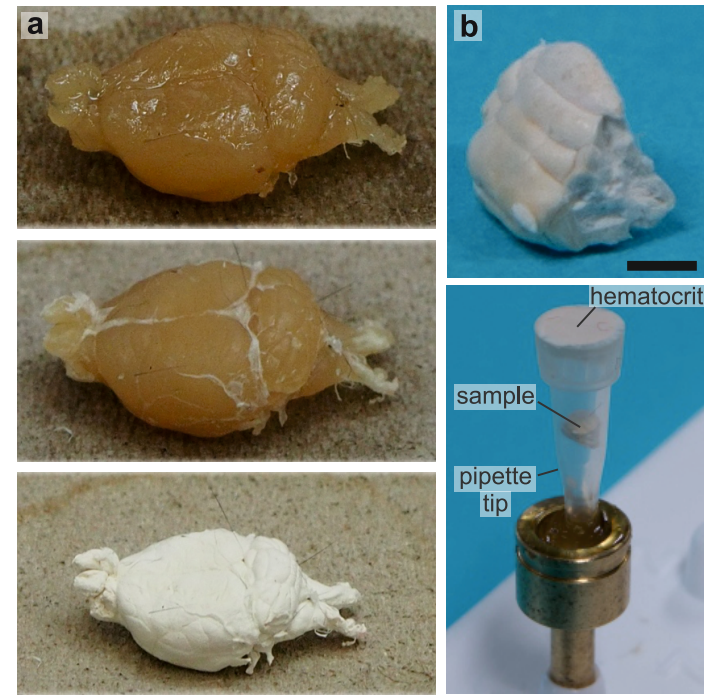

Figure 7.1: Steps in the sample preparation following the evaporation-of-solvent method. (a) Images of the same mouse brain at different time points in the sample preparation, starting directly after removing the brain from the xylene solution as well as after $12 \mathrm{~min}$ and $2 \mathrm{~h}$, respectively. (b) Detached mouse cerebellar vermis imaged at the laboratory setup. For the experiments, the sample was squeezed into a pipette tip, which was glued to a sample holder and sealed by a layer of hematocrit. Scale bar: $1 \mathrm{~mm}$

Cone-beam geometry In the cone-beam geometry, comparatively large fields of view can be reached by installing the 'Talos' detector with a pixel size of $75 \mu \mathrm{m}$ in the setup. The right hemisphere of a mouse brain was measured at a source-tosample distance of $100 \mathrm{~mm}$ and a sample-to-detector distance of $1.767 \mathrm{~m}$, leading to a magnification of $\sim 187$ and an effective pixel size of $4.02 \mu \mathrm{m}$ with a field of view of $6.17 \times 7.8 \mathrm{~mm}^{2}$. The source was operated at an acceleration voltage of $70 \mathrm{kV}$ and an electron spot size of $4 \times 16 \mathrm{\mu m}^{2}$, allowing for a maximum electron beam power of $40 \mathrm{~W}$. In order to reduce source blurring effects, the beam was located at the center of the metal jet (cf. Fig. 4.5).

For the tomographic scan, 916 projection were recorded over an angular range $\theta \in[0,183]^{\circ}$, with the extra $3^{\circ}$ being necessary due to the opening angle of the divergent beam. Each projection was recorded with an exposure time of $1 \mathrm{~s}$. For a better overview, the experimental parameters are summarized in Tab. 7.1.

Inverse geometry In order to obtain high resolution scans of selected regions of interest from the mouse brain, the setup was also operated in inverse geometry, with the 'Argos' detector placed in close proximity to the sample. The source- 


\begin{tabular}{lccc}
\hline & Fig. 7.3 & Figs. 7.5 and 7.8 & Fig. 7.6 \\
\hline geometry & cone beam & inverse & inverse \\
energy [keV] & $0-70 / 9.25$ & $0-40 / 9.25$ & $0-40 / 9.25$ \\
detector & 'Talos' & 'Argos' & 'Argos' \\
$z_{01}[\mathrm{~mm}]$ & 100 & 158.75 & 158.75 \\
$z_{02}[\mathrm{~m}]$ & 1.867 & 0.181 & 0.177 \\
$p_{\text {eff }}[\mu \mathrm{m}]$ & 4.02 & 0.47 & 0.48 \\
field of view $\left[\mathrm{mm}^{2}\right]$ & $6.17 \times 7.8$ & $1.18 \times 1.57$ & $1.2 \times 1.6$ \\
e-spot size $\left[\mathrm{\mu m}^{2}\right]$ & $4 \times 16$ & $10 \times 40$ & $10 \times 40$ \\
e-beam power $[\mathrm{W}]$ & 40 & 57 & 57 \\
Fresnel number & 1.27 & 0.084 & 0.11 \\
angular range $\left[{ }^{\circ}\right]$ & {$[0,183]$} & {$[0,180]$} & {$[0,180]$} \\
number of projections & 916 & 1000 & 1000 \\
exposure time $[\mathrm{s}]$ & 1 & 50 & 50 \\
\hline
\end{tabular}

Table 7.1: Overview of the experimental parameters for imaging of different parts of a mouse brain prepared according to the evaporation-of-solvent method.

to-sample distance was set to $158.75 \mathrm{~mm}$, whereas the detector was located at $22.6 \mathrm{~mm}$ behind the sample for imaging of the hippocampal formation as well as cortical region, olfactory bulb and brain stem, and at $18.3 \mathrm{~mm}$ behind the sample for imaging of the cerebellar vermis. The resulting effective pixel sizes were $0.47 \mu \mathrm{m}$ and $0.48 \mu \mathrm{m}$ with fields of view of $1.18 \times 1.57 \mathrm{~mm}^{2}$ and $1.2 \times 1.6 \mathrm{~mm}^{2}$, respectively. In order to reduce hot pixels in the acquired projections due to high energy photons, the source was operated at $40 \mathrm{kV}$ acceleration voltage, $57 \mathrm{~W}$ electron beam power and an electron spot size of $10 \times 40 \mathrm{\mu m}^{2}$, slightly deflected towards the exit window to increase photon flux (cf. Fig. 4.5).

For all samples, tomographic measurements were carried out by acquiring 1000 projections over an angular range of $180^{\circ}$ with an exposure time of $50 \mathrm{~s}$ each. A summary of the experimental parameters is given in Tab. 7.1.

\subsubsection{Results}

The first sample, the right hemisphere of a mouse brain, was measured in conebeam geometry in order to obtain an overview of the entire specimen. One exemplary projection of this scan is shown in Fig. 7.2(a) with the corresponding reconstruction of the intensity distribution directly behind the object in (b). This reconstruction was obtained via the $\mathrm{BAC}$ approach and the regularization parameters listed in Tab. 7.2. The effect of phase contrast is clearly visible as edge 

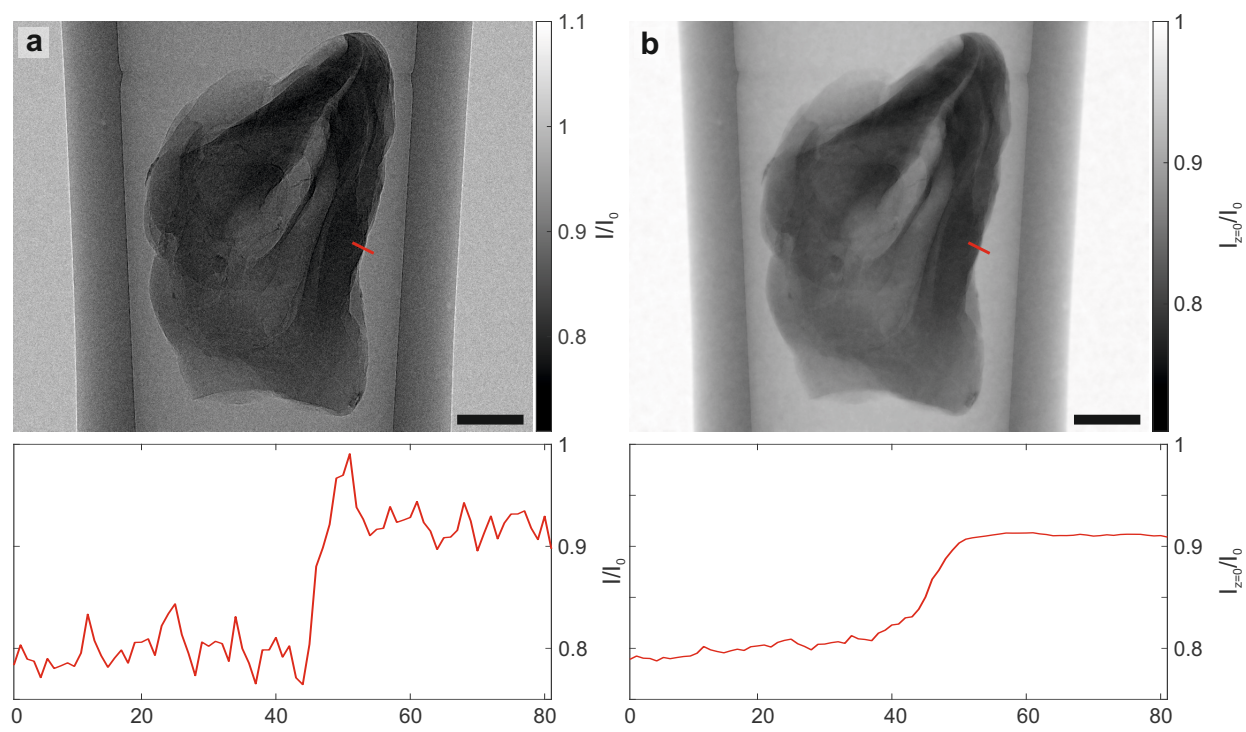

Figure 7.2: Exemplary projection of the right hemisphere of a mouse brain, recorded in cone-beam geometry. (a) Empty-beam corrected projection, in which phase contrast is visible in the form of edge enhancement. The edge of the pipette tip, which acts as the sample holder, is visible around the sample. (b) Corresponding reconstruction of the intensity distribution directly behind the object using the BAC phase-retrieval approach. The application of this algorithm corrects for the edge-enhancement effects while preserving the sharpness of the projection at higher signal-to-noise ratio. The gain in SNR can be visualized via the line profiles along an edge of the sample, depicted below, where regions of constant density, e.g., the air around the tissue, show a significantly smoothed curve compared to the raw data. Scale bars: $1 \mathrm{~mm}$

\begin{tabular}{lcc}
\hline & $\mathrm{BAC} \alpha$ & $\mathrm{BAC}(\gamma \cdot F)$ \\
\hline right hemisphere (Fig. 7.3) & 0.02 & 0.17 \\
cortex/hippocampus (Fig. 7.5) & 0.02 & 0.22 \\
cerebellum (Fig. 7.6) & 0.03 & 0.2 \\
olfactory bulb (Fig. 7.8) & 0.02 & 0.19 \\
brain stem (Fig. 7.8) & 0.015 & 0.21 \\
\hline
\end{tabular}

Table 7.2: Overview of the reconstruction parameters for the BAC phase-retrieval algorithm for imaging of different parts of a mouse brain prepared according to the evaporation-of-solvent method.

enhancement around the object and already in the $2 \mathrm{~d}$ projection, the sample, enclosed by the pipette tip, can be recognized at high contrast. In the reconstructed projection, edge enhancement is removed at higher signal-to-noise ratio compared to the raw projections, which is visible in the profiles along an outer 


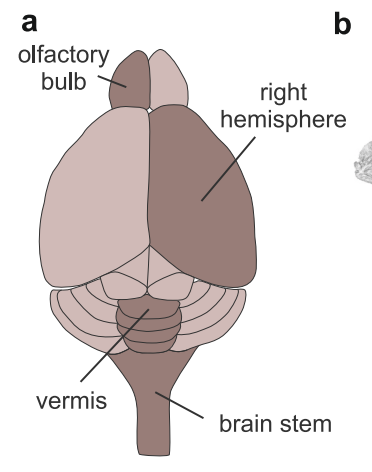

b
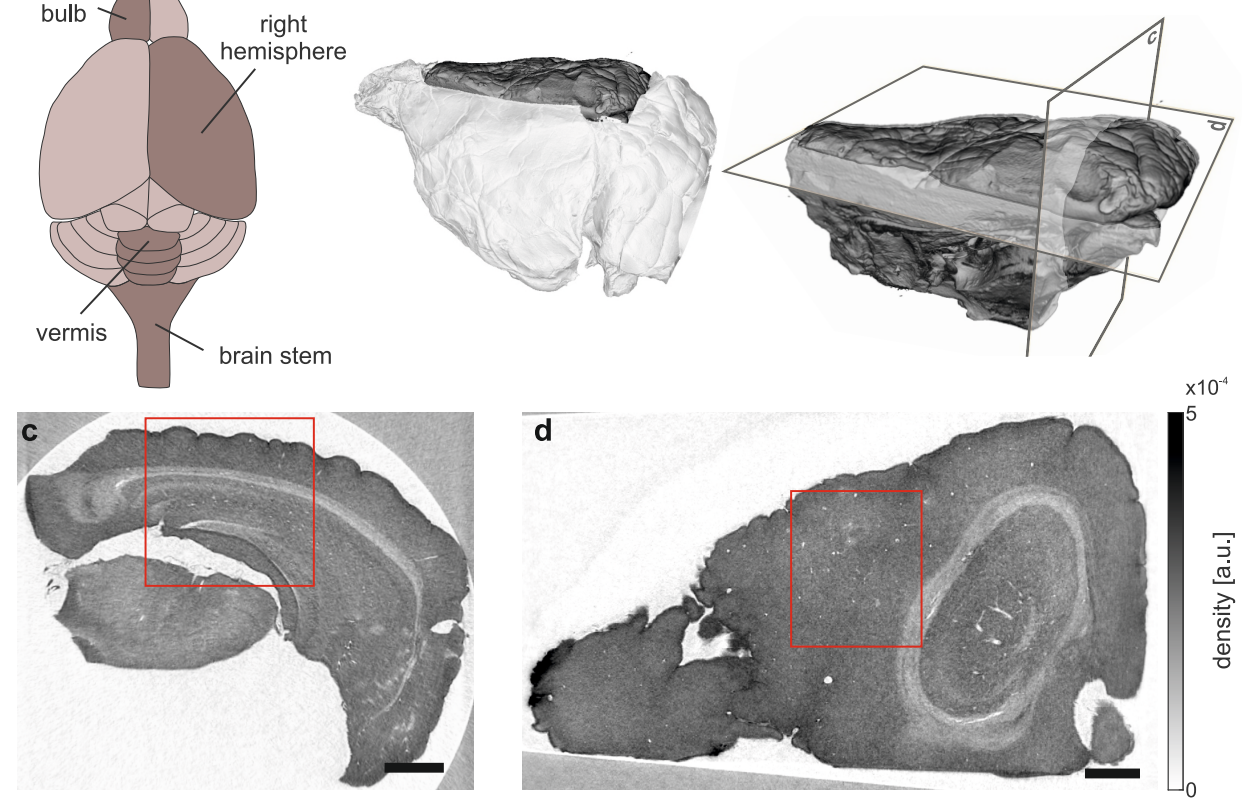

Figure 7.3: Overview scan of the right hemisphere of a mouse brain. (a) Sketch of a mouse brain, in which the regions measured in the following, namely the olfactory bulb, right hemisphere, cerebellar vermis and brain stem, are highlighted. (b) Volume rendering of the right hemisphere of a mouse brain, shown with respect to the entire brain (left) as well as individually (right). The planes indicate the positions of the virtual slices depicted in (c) and (d). (c) Coronal slice through the reconstructed volume obtained from the overview measurement. The rectangle marks the region of the hippocampus which was measured at higher resolution in inverse geometry, depicted in Fig. 7.5(a). (d) Horizontal slice through the $3 d$ density distribution, with a rectangle marking the region of the cortex which was measured at higher resolution as well (Fig. 7.5(b)). Scale bars: $500 \mu \mathrm{m}$

edge shown below. Prior to the tomographic reconstruction, ring removal was performed according to the simple scheme presented in section 3.2.2. The tomographic reconstruction itself was carried out with the FDK implementation of the ASTRA toolbox. A rendering of the resulting volume, created with Avizo and shown with respect to an entire mouse brain as well as individually, is depicted in Fig. 7.3(b). The transparent planes indicate the positions of the coronal and horizontal virtual slices in (c) and (d). Prominent structures as the hippocampal formation are already visible in this overview, facilitating the selection of specific regions of interest for the scans in inverse geometry, which are aiming for the visualization of 

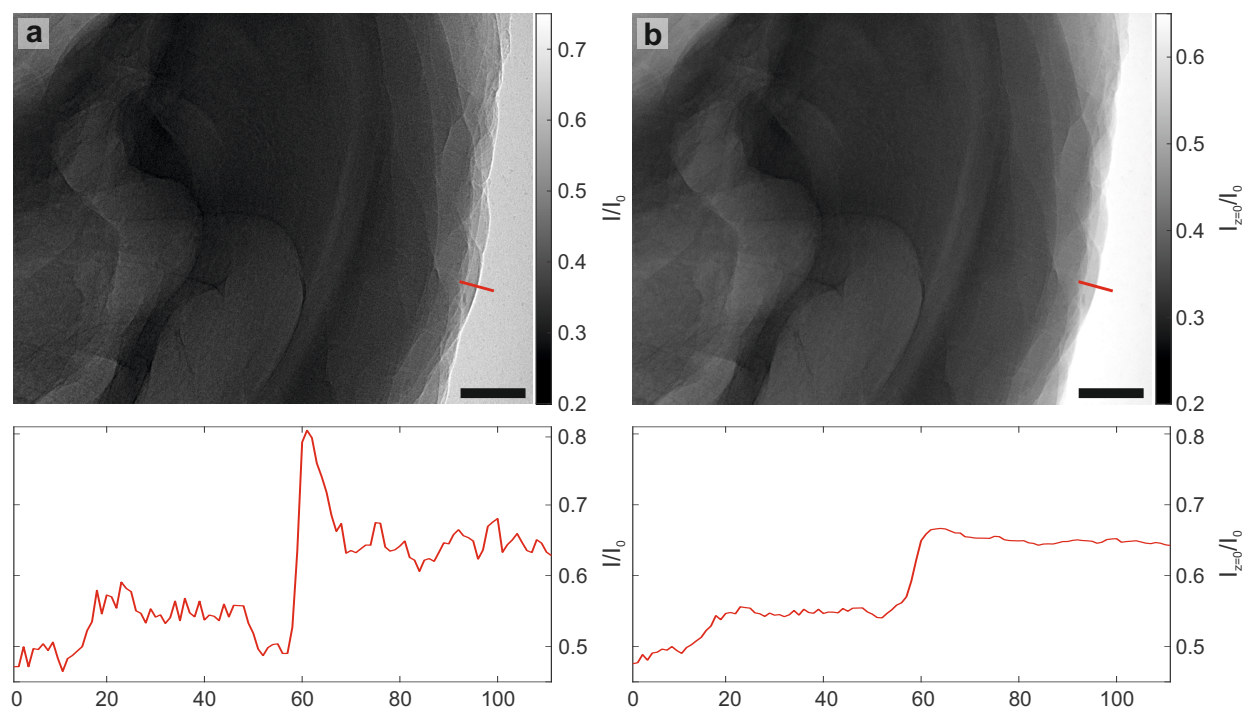

Figure 7.4: Exemplary projection from the region of the hippocampus recorded in inverse geometry. (a) Empty-beam corrected projection with a line profile along an outer edge to illustrate the phase-contrast effects. For a better signal-to-noise ratio, the projections were resampled by a factor of 2 prior to tomographic reconstruction. (b) Corresponding reconstruction of the intensity distribution using the $B A C$ algorithm. The advantage of phase retrieval is clearly visible in the line profile shown below, as edge enhancement is reduced while simultaneously the signal-to-noise ratio is increased. Scale bars: $200 \mu \mathrm{m}$

the sample at cellular detail. The rectangles mark the positions of these scans, located within the hippocampus as well as the cortical region. One exemplary projection acquired in the region of the hippocampus is depicted in Fig. 7.4(a). In contrast to the overview scan, only a small part of the sample is included in the field of view. Additionally, due to the lower acceleration voltage of $40 \mathrm{kV}$ in the inverse geometry, which reduces hot pixels in the 'Argos' detector caused by high energy photons, transmission of the x-rays is decreased. As this leads to higher restrictions on the maximum sample dimensions, the substitution of water content in the sample by air yields another advantage apart from a higher contrast, as this already decreases absorption of the x-rays and hence enables larger samples compared to other preparation techniques. The corresponding reconstruction using the BAC algorithm and the parameters specified in Tab. 7.2 is shown in Fig. 7.4(b). As in the case of the overview scan, the phase-retrieval step yields intensity distributions with reduced edge enhancement at higher signal-to-noise ratio.

After applying the simple ring-removal algorithm on the data obtained in the hippocampal region as well as the wavelet-based algorithm on the data from the 
cortex, tomographic reconstruction was carried out with the FDK implementation of the ASTRA toolbox. In order to increase the signal-to-noise ratio, the individual projections were resampled by a factor of 2 in this as well as the following scans. Two exemplary slices through the resulting volumes are shown in Fig. 7.5. Note that in order to further reduce the noise within the images, though at the cost of blurring, the slices from this as well as the following datasets were filtered with a Gaussian function with a standard deviation of 1 pixel. Cellular details are clearly visible within the depicted slices in a resolution comparable to histology. Hence, by exchanging the water content within the sample and therefore significantly increasing the differences in electron density, single cells within mm-sized reconstruction volumes can already be visualized at the laboratory setup. In contrast to histology, this cellular resolution is reached isotropically within the entire volume and the sample can be virtually sliced in every arbitrary direction. The thickness of the virtual sections is determined by the voxel size of the reconstructed volume, which in this case is $0.95 \mu \mathrm{m}$ due to the resampling. To emulate the contrast of $30 \mu \mathrm{m}$ thick histological sections in both datasets, maximum intensity projections over 31 successive slices were generated in Avizo via the module Image Ortho Projections and are shown at the bottom of Fig. 7.5. Although already visible in the thin virtual sections, prominent features within the two investigated brain regions can be clearly distinguished, as, e.g., the pyramidal cell layer as well as the granular and polymorphic layer of the dentate gyrus within the region of the hippocampus or the barrel field in the region of the cortex, a major target for somatosensory inputs.

To further evaluate the imaging capabilities of the laboratory setup in combination with the evaporation-of-solvent method, additional prominent examples of central nervous tissue were scanned. In order to facilitate a comparison between the results obtained for a hydrated unstained slice from a mouse cerebellum at the synchrotron setup (cf. chapter 5), a tomogram was acquired on a detached cerebellar vermis (cf. Fig. 7.1). The intensity distributions for each empty-beam corrected projection were reconstructed via the BAC algorithm using the parameters listed in Tab. 7.2. To reduce ring artifacts in the resulting virtual slices, the wavelet-based ring-removal algorithm was applied on the sinograms prior to tomographic reconstruction via the FDK implementation of the ASTRA toolbox. Three orthogonal slices through the obtained volume are depicted in Fig. 7.6, with the regions indicated by the rectangles shown at higher magnification on the right. The different layers of the cerebellum, namely the cell-rich granular layer, the low-cell molecular layer, the intermediate Purkinje cell layer as well as the white 

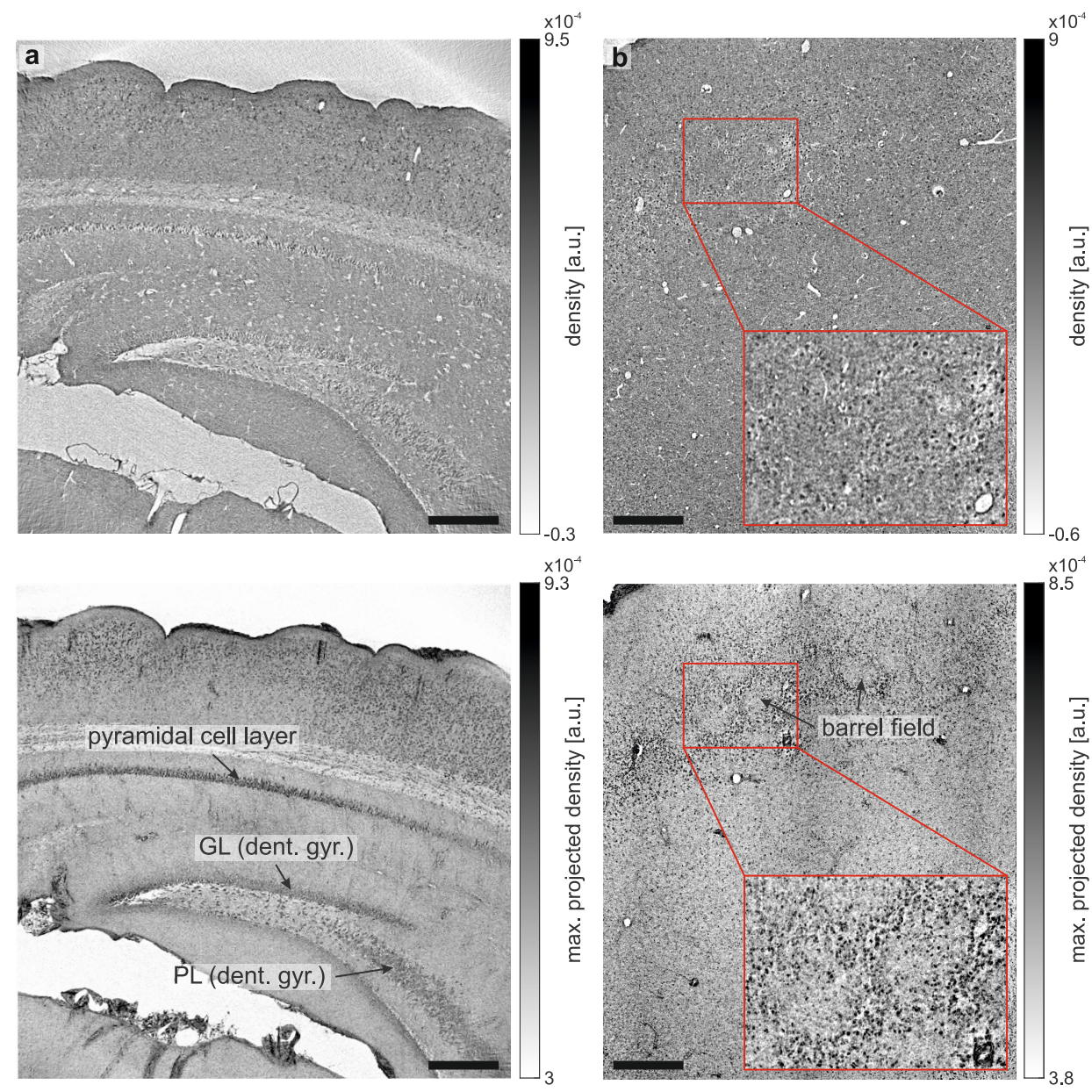

Figure 7.5: Slices through the reconstructed density from measurements of the hippocampal formation as well as cortex of a mouse brain in inverse geometry. (a) Coronal slice through the reconstructed density from the first scan, showing the hippocampal region at cellular resolution. The position of the scan with respect to the entire right hemisphere is depicted in Fig. 7.3. A maximum intensity projection of 31 successive slices, imitating a $30 \mu \mathrm{m}$ thick histological section, shows the pyramidal cell layer as well as the granular $(G L)$ and polymorphic layer $(P L)$ of the dentate gyrus at high contrast. (b) Horizontal slice through the reconstructed volume from the second scan, located in the cortical region, as depicted in Fig. 7.3. Especially in the maximum intensity projection shown below, prominent features as the barrel field are clearly visible. Scale bars: $200 \mu \mathrm{m}$ 

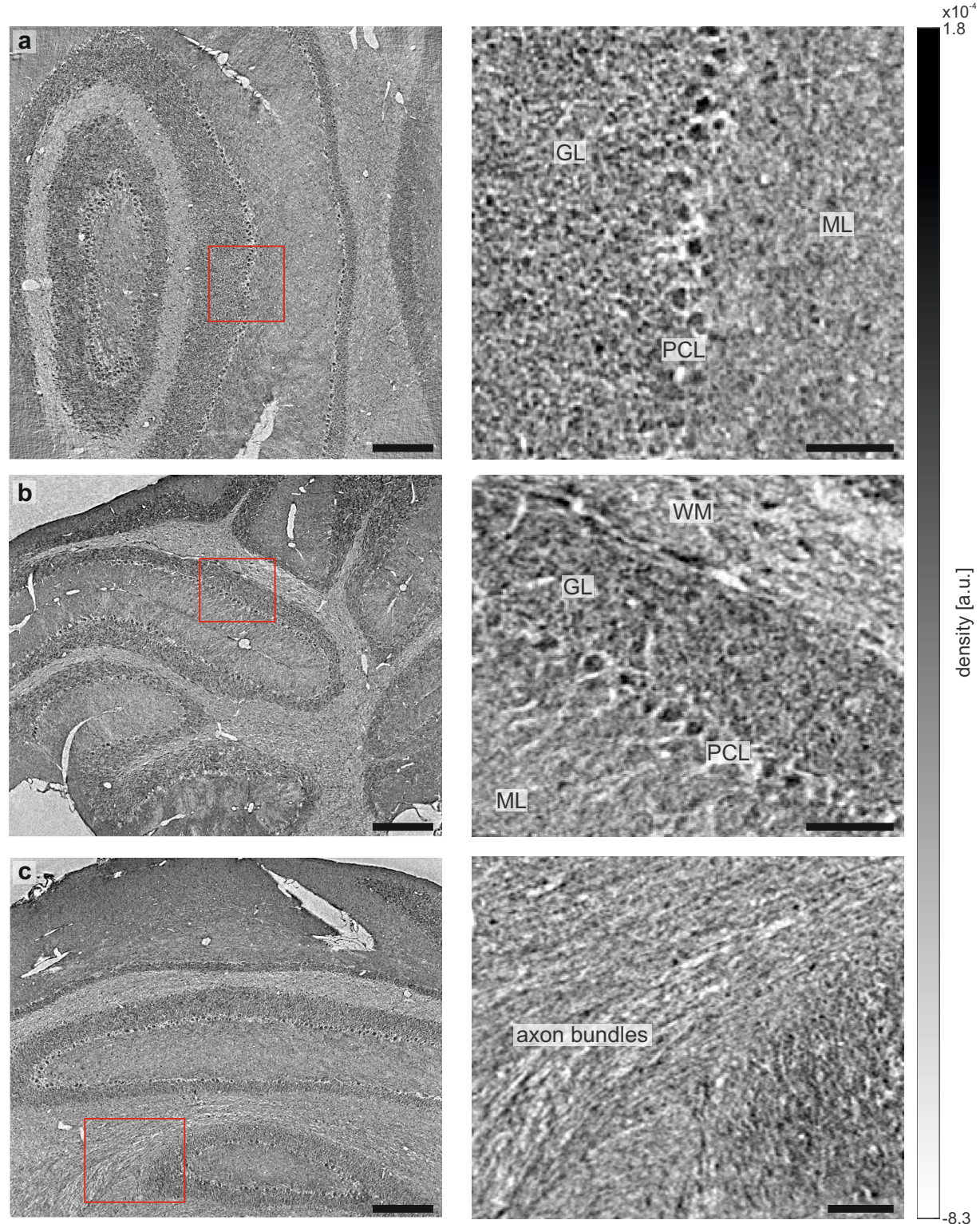

Figure 7.6: Virtual slices through the reconstruction of a cerebellar vermis measured in inverse geometry. (a) Transverse slice through the sample, with a magnification of the region marked by a rectangle on the right. $(b, c)$ Longitudinal slices through the sample, again with magnified regions shown on the right. In all slices the typical layers of the cerebellum, the granular layer (GL), molecular layer (ML) as well as Purkinje cell layer $(P C L)$ and white matter are resolved at cellular detail. Scale bars: $200 \mu \mathrm{m}$ (left) and $50 \mathrm{\mu m}$ (right) 
matter are clearly visible. Within these slices, single cells can be resolved, again at an isotropic resolution comparable to classical histological sections. According to the slice depicted in (c), even large axon bundles within the white matter can be resolved to some extent. Further sub-cellular details as the axons or dendrites are not visible. However, considering the magnified region shown in (b), the large dendritic tree might be indicated within the white 'tree-like' features emerging from the cell bodies of the Purkinje cells, which are protruding into the molecular layer. If these would indeed follow the shape of the Purkinje cell dendrites, it would mean that within the EOS preparation protocol, the dendritic structures were actually removed from the sample, leaving behind hollow regions in the shape of the corresponding features. Hence, these sub-cellular details would only be visible as a negative structure. However, resolution within the laboratory data is not sufficient to unambiguously determine whether these structures can indeed be identified as cellular components or whether they are artifacts caused by the drying procedure. In order to quantify the contrast reached within the cerebellum, the Weber values were again determined for the Purkinje cells as well as the granular layer with respect to the surrounding tissue, yielding $C_{\mathrm{PCL}} \simeq 0.65$ and $C_{\mathrm{GL}} \simeq 0.36$, respectively. Hence, despite the lower contrast transfer compared to imaging in the holographic regime at the synchrotron, as well as the lower coherence and signalto-noise ratio, comparable contrast values can be reached in the Purkinje cell layer whereas for the granular layer, contrast is even enhanced by an approximate factor of 2. Note, however, that due to the large differences in data acquisition, the comparability of the two datasets is limited and a fair comparison would have to be carried out between measurements obtained at the same setup. As unstained hydrated tissue measured at the laboratory would yield almost no contrast in the reconstructed slices, the actual gain estimated by the difference in Weber contrast is even larger than the values obtained here.

For a better visualization of the $3 d$ cytoarchitecture of the sample, an automated volume rendering is depicted in Fig. 7.7(a) and (b), revealing the typical tight folding of the cerebellum with the three-layered cerebellar cortex located above the white matter. As the resolution allows for the segmentation of single cells, the cellular distribution can be visualized in $3 \mathrm{~d}$ as well, as shown in (c) for a small exemplary subvolume. The location and size of this subvolume with respect to the entire sample is indicated in (b). The cellular segmentation is only depicted for a small part of the sample as it was performed semi-manually, a time-consuming procedure which impedes execution for the entire dataset. Within this process, the Purkinje cells were segmented via the gray value-based region-growing tool Magic 

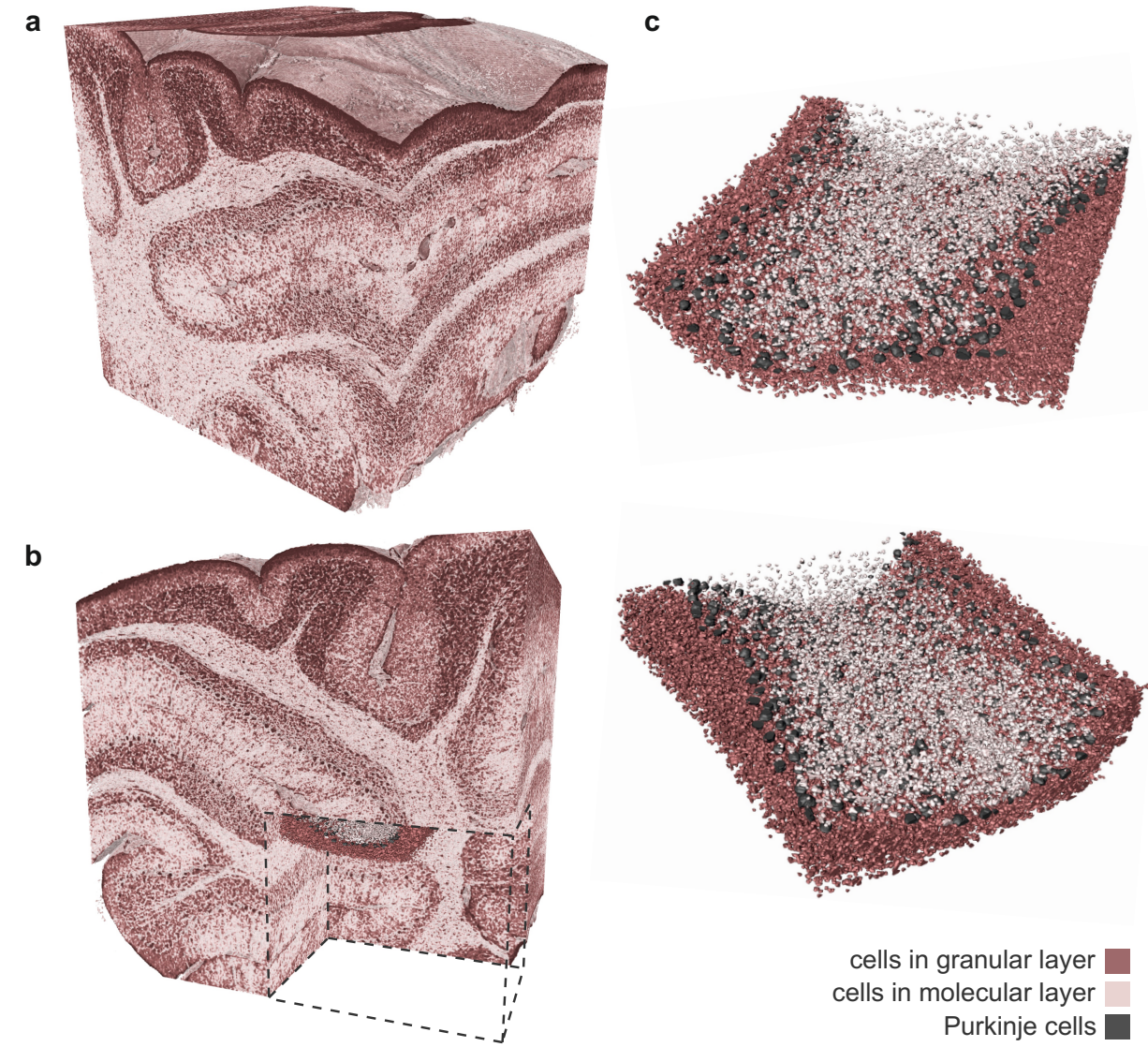

Figure 7.7: $3 d$ visualization of the cerebellar vermis recorded in inverse geometry. $(a, b)$ Automatic volume renderings of the sample, yielding a first impression on the $3 d$ density distribution. The cut in the lower panel indicates the position of the cellular segmentation shown in (c). (c) Semi-manual cellular segmentation of a small part of the sample.

Wand by manually determining seeding points within the individual cells. For the granular as well as molecular layer a threshold-based Brush tool was used on every slice through the subvolume that, within a manually selected region, only marks pixels lying in a specified gray value range. Although the cellular segmentation is only shown for a small subregion of the sample, data quality is high enough to extend it towards the entire sample. However, the great effort involved in semimanual segmentation necessitates the development of automated algorithms for this task.

To conclude the evaluation of the evaporation-of-solvent method for neuronal imaging at the laboratory with cellular resolution, the left part of the olfactory bulb as 
well as the brain stem of a mouse brain were considered (cf. Fig. 7.3(a)). To this end, the corresponding structures were removed from the brain in order to reduce absorption of excess material around the actual regions of interest. Prior to the tomographic reconstruction via the FDK implementation of the ASTRA toolbox, the intensity distribution in the exit plane was retrieved via the BAC algorithm and the parameters listed in Tab. 7.2. Additionally, the wavelet-based ring-removal algorithm was applied on the sinograms to minimize artifacts in the reconstructed slices. Two virtual slices through the resulting volumes are depicted in Fig. 7.8, again accompanied by a maximum intensity projection over 31 consecutive slices to emulate the contrast of $30 \mu \mathrm{m}$ thick histological sections.

As for the structures considered before, the anatomical features expected from histological studies are clearly resolved at cellular detail. Within the virtual slice through the olfactory bulb, distinct layers can be recognized, namely the glomerular layer, containing the spherical glomeruli, the external plexiform layer, the mitral cell layer, internal plexiform layer and granular layer [101]. In the results from the brain stem, different features as the air-filled blood vessels as well as thick fiber bundles, which are visible as round structures of lower electron density, can be recognized. The round shape is caused by the orientation of the depicted slice, as it transversely cuts the bundles. A $3 \mathrm{~d}$ visualization of the spatial distribution of blood vessels as well as fiber bundles within the brain stem was generated via a segmentation of the according structures in the volume. To this end, the gray value-based region-growing tool Magic Wand could be used due to the distinct gray values of the considered features. Especially the air-filled blood vessels could be easily segmented by manually defining specific seeding points. By adjusting the gray value range, a large part of the fiber bundles was segmented via the Magic Wand as well. The result of this segmentation is depicted in Fig. 7.9. For a localization of the blood vessels as well as fiber bundles with respect to the measured part of the brain stem as well as each other, the segmentation in combination with a volume rendering of the entire reconstruction is shown in (a). In the accompanying individual representations of the segmented features it can be recognized that the majority of fibers run vertically through the depicted volume, whereas the blood vessels seem to form a circular pattern.

Resolution The resolution reached in the single datasets was estimated via a Fourier shell correlation (cf. section 1.5) of the central $1000^{3}$ voxels in the reconstructed volumes. By obtaining two independent datasets, using every second projection from the tomographic scan, the Fourier shell correlation was calculated 

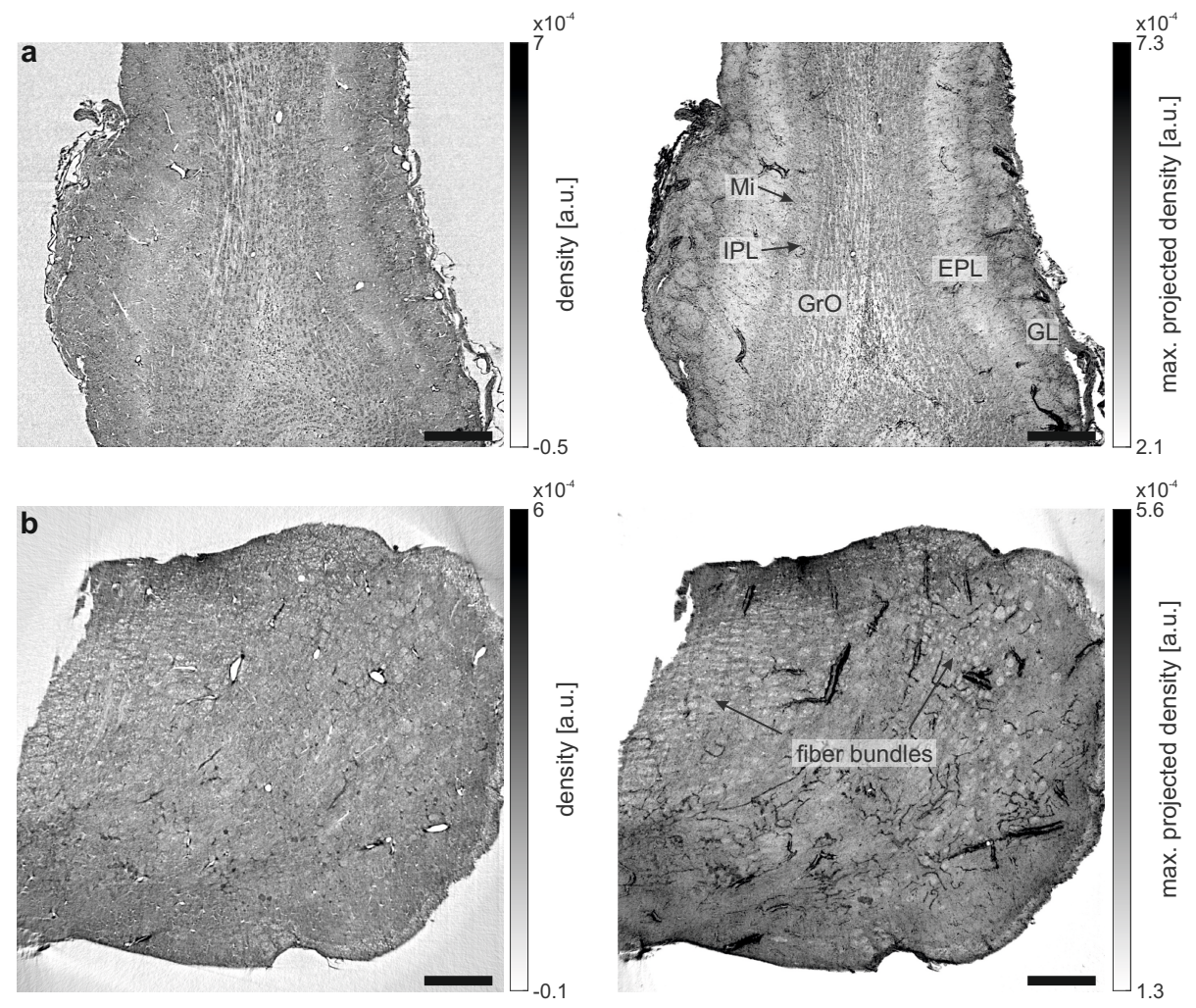

Figure 7.8: Tomographic results obtained for the olfactory bulb (OB) as well as brain stem of a mouse brain prepared after the EOS method. (a) The virtual slice through the reconstructed volume of the olfactory bulb (left) as well as the maximum intensity projection over 31 consecutive slices (right) reveal the typical layers of the olfactory bulb at cellular resolution: glomerular layer $(G L)$, external plexiform layer $(E P L)$, mitral cell layer (Mi), internal plexiform layer (IPL) and the granule cell layer of the $\mathrm{OB}(\mathrm{GrO})$. (b) In the virtual slice of the brain stem and the maximum intensity projection on the right, different features as the air-filled blood vessels as well as transversely cut fiber bundles, which can be recognized as the round structures with the slightly lower electron density compared to the surrounding tissue, are clearly resolved. Scale bars: $200 \mu \mathrm{m}$

according to eqn. (1.97). The intersection with the $1 / 2$-bit threshold curve (1.98) determines the maximum spatial frequency that contains enough information for interpretation of the data (cf. Fig. 7.10). The corresponding half-period resolutions are listed in Tab. 7.3. The comparison shows that the highest resolution was reached for the samples with the smallest sample dimensions and hence lowest absorption (olfactory bulb and brain stem), which indicates the dependence of the resolution on the noise level in the reconstructed volumes. 
a

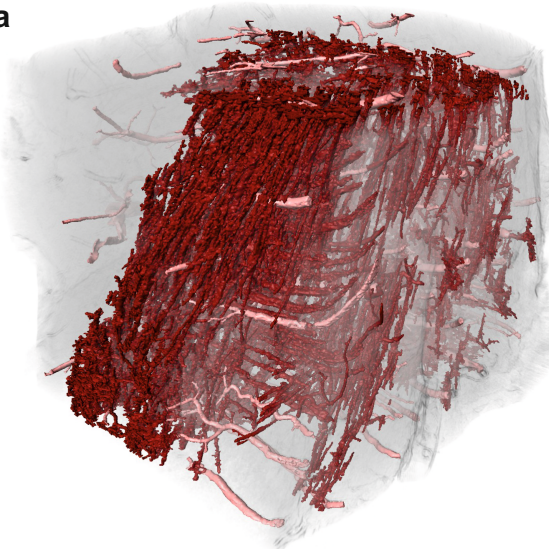

C

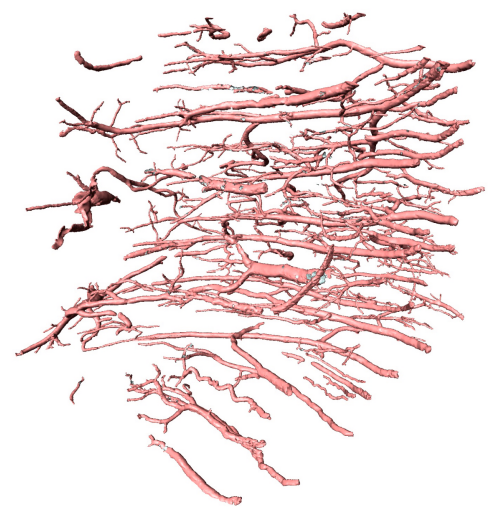

b
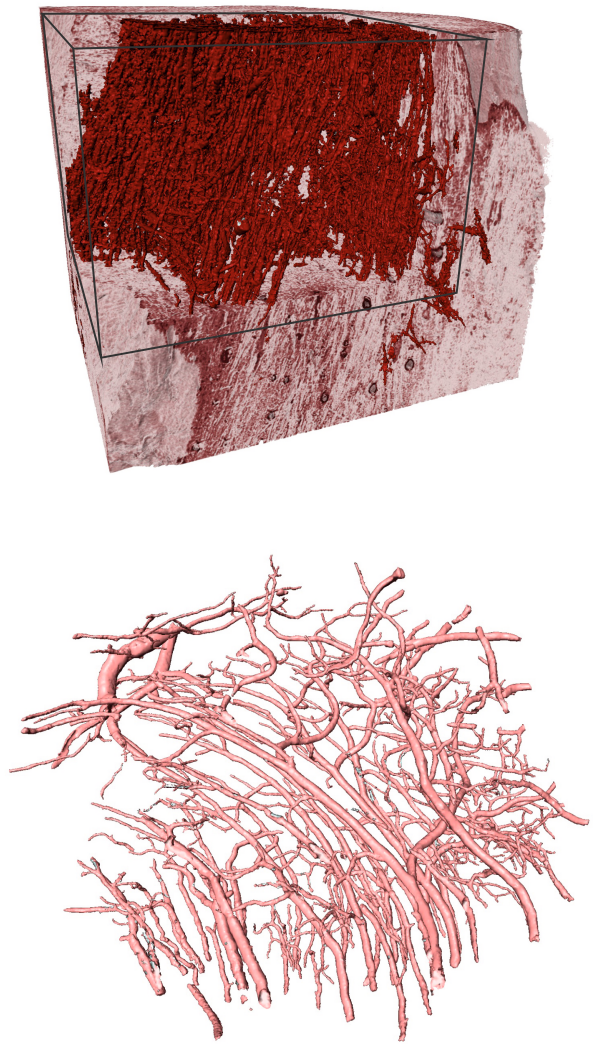

Figure 7.9: $3 d$ visualization of the brain stem of a mouse prepared after the EOS method. (a) Within the reconstruction volume, depicted in transparent gray, distinct features as the blood vessels as well as fiber bundles can be localized. (b) The segmentation of a part of the fiber bundles combined with a volume rendering of the brain stem clearly shows the approximate course of the fibers within the tissue and hence the direction of the signal transduction. (c) The (semi-)automatic segmentation of the larger blood vessels in the volume shows the spatial distribution of these structures within the brain stem.

In summary, the previous section showed that the evaporation-of-solvent method can be advantageously used to reveal the $3 \mathrm{~d}$ cytoarchitecture of a mouse brain at a compact laboratory setup. It provides the necessary contrast enhancement while the general structure of the tissue is preserved, enabling the observation of typical features known from classical histological sectioning in three dimensions with a resolution allowing for the tracking of single cells. 

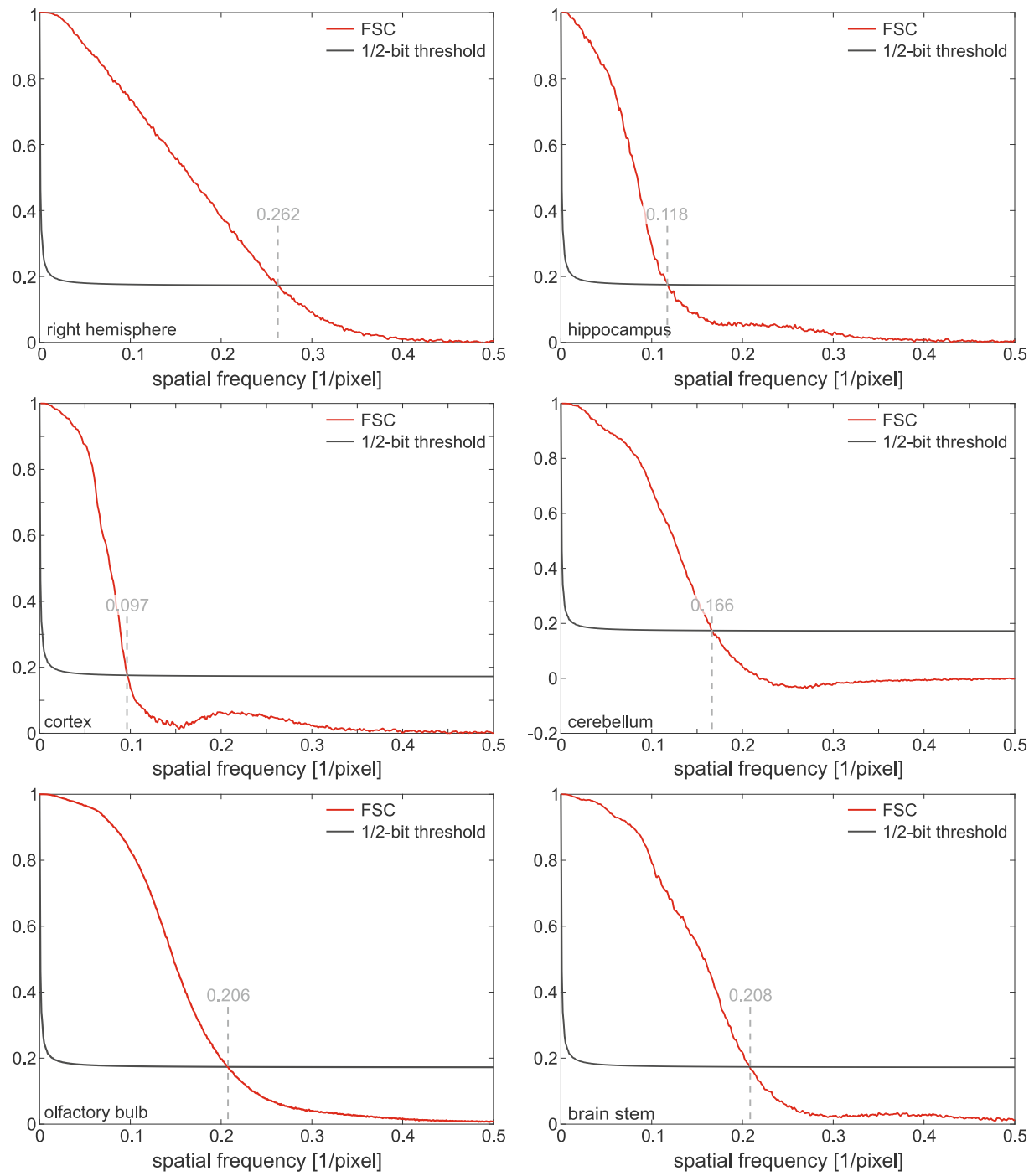

Figure 7.10: Fourier shell correlations of the central $1000^{3}$ voxels of the tomographic reconstructions for brain tissues prepared after the EOS method and the 1/2-bit threshold curves to determine the spatial resolution via the intersection. The corresponding halfperiod resolutions are listed in Tab. 7.3. 


\begin{tabular}{llc}
\hline & Figure & half-period resolution \\
\hline right hemisphere & 7.3 & $7.7 \mu \mathrm{m}$ \\
hippocampus & $7.5(\mathrm{a})$ & $4.0 \mu \mathrm{m}$ \\
cortex & $7.5(\mathrm{~b})$ & $4.8 \mu \mathrm{m}$ \\
cerebellum & 7.6 & $2.9 \mu \mathrm{m}$ \\
olfactory bulb & $7.8(\mathrm{a})$ & $2.3 \mu \mathrm{m}$ \\
brain stem & $7.8(\mathrm{~b})$ & $2.3 \mu \mathrm{m}$ \\
\hline
\end{tabular}

Table 7.3: Half-period resolutions for the different tomographic scans of brain tissue prepared after the EOS method, determined via a Fourier shell correlation of the central $1000^{3}$ voxels in the reconstructed volume.

\subsection{Mouse cerebellum embedded in ethanol}

\subsubsection{Methods}

\subsubsection{Sample preparation}

A wild type mouse, provided by the group of Frauke Alves (MPI for Experimental Medicine, Göttingen, Germany), was sacrificed and the brain was dissected and stored in $10 \%$ formalin for $24 \mathrm{~h}$ for fixation. Subsequently, the brain was embedded in $5 \%$ agarose in order to slice it into $500 \mu \mathrm{m}$ thick sections in the vibratome. For the exchange of water content, an ascending ethanol series was performed. In order to mount the sample in the setup and maintain experimental conditions, a $1 \mathrm{~mm}$ punch was taken from the region of the cerebellum and placed into a Kapton tube with $1 \mathrm{~mm}$ diameter, which was glued to a sample holder. Prior to placing the sample in the tube, it was filled with fresh ethanol. To prevent drying of the sample during the measurement, the tube was sealed with 2-component glue (cf. Fig. 7.11).

\subsubsection{Experimental parameters}

Experiments were carried out at the GINIX endstation at a photon energy of $8 \mathrm{keV}$. A waveguide containing both straight and tapered channels with air as guiding layer was placed in the focal spot of the KB-mirrors. During the experiment, a straight channel proved most stable in terms of the resulting illumination, providing a flux in the order of $10^{9} \mathrm{ph} / \mathrm{s}$. The 'Pirra' was positioned at a sourceto-detector distance $z_{02}=5.05 \mathrm{~m}$. For the experiments, the sample was positioned at a source-to-sample distance $z_{01}=145 \mathrm{~mm}$, leading to an effective pixel size 

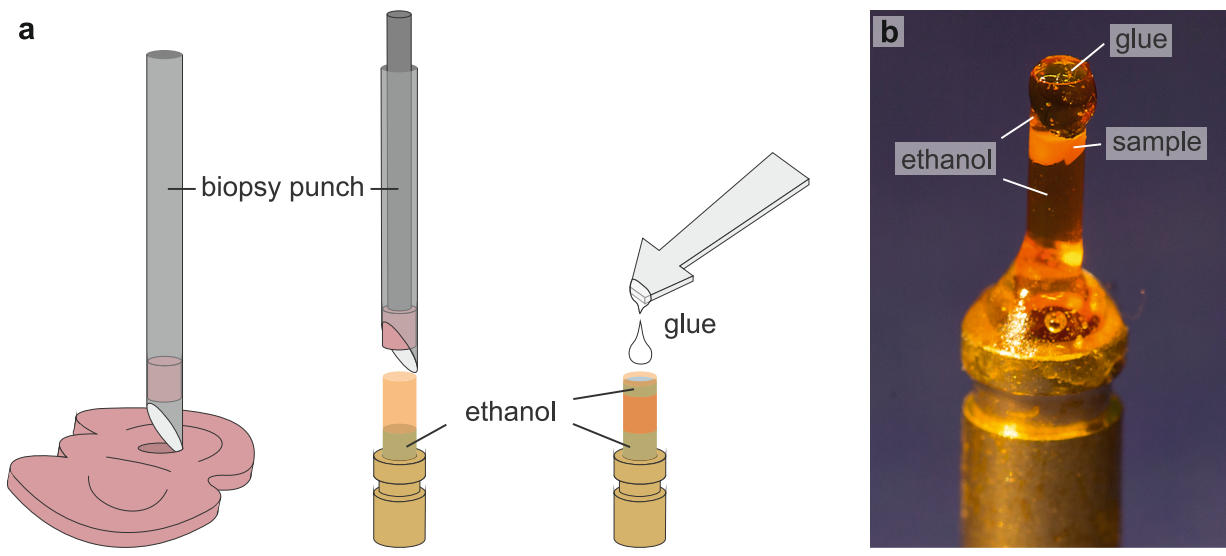

Figure 7.11: Sample preparation for the brain slice embedded in ethanol. (a) After exchanging the water content by ethanol, a $1 \mathrm{~mm}$ biopsy punch was taken from the resulting brain slice and placed in a $1 \mathrm{~mm}$ Kapton tube filled with ethanol. To prevent drying of the sample during the measurement, the tube was sealed with 2-component glue. (b) Photograph of the resulting liquid chamber, in which the sample, located near the center, as well as the sealing via the 2-component glue can be recognized.

$p_{\text {eff }}=187 \mathrm{~nm}$ and a field of view of $383 \times 383 \mu^{2}$. To reduce artifacts in the reconstructed phase distributions, tomograms were recorded at four propagation distances. Each measurement was carried out by acquiring 1500 projections over an angular range of $180^{\circ}$ with an exposure time of $0.15 \mathrm{~s}$. The experimental parameters are summarized in Tab. 7.4.

\subsubsection{Results}

Figure 7.12(a) shows an exemplary empty-beam corrected projection of the acquired tomographic dataset. The orientation of the projection is chosen such that already in this projection, the typical layers of the cerebellum can be clearly distinguished, especially the mono-cellular Purkinje cell layer in the center of the projection, which comprises the large cell bodies of the Purkinje cells. The CTFbased approach for a weakly absorbing object was used for phase retrieval with regularization parameters $\frac{\delta}{\beta}=50$ and $\alpha_{2}=0$, resulting in a quantitative reconstruction with significantly lower phase values obtained for the cell-rich granular layer compared to the low-cell molecular layer, as depicted in (b). In order to compensate for the different magnifications at the four propagation distances, the single projections were scaled to the same effective pixel size and aligned to each other in Fourier space. 


\begin{tabular}{lc}
\hline & experimental setting \\
\hline GINIX run & 56 \\
energy & $8 \mathrm{keV}$ \\
detector & 'Pirra' \\
$z_{01, \text { min }}$ & $145 \mathrm{~mm}$ \\
$z_{02}$ & $5.05 \mathrm{~m}$ \\
$p_{\text {eff }}$ & $187 \mathrm{~nm}$ \\
field of view & $383 \times 383 \mathrm{um}^{2}$ \\
Fresnel number $F$ & 0.0016 \\
& 'Hercules II' \\
waveguide & (straight channel) \\
number of distances & 4 \\
angular range & {$[0,180]^{\circ}$} \\
number of projections & 1500 \\
exposure time & $0.15 \mathrm{~s}$ \\
CTF $\frac{\delta}{\beta}$ & 50 \\
CTF $\alpha_{2}$ & 0 \\
\hline
\end{tabular}

Table 7.4: Experimental parameters used for imaging of a $1 \mathrm{~mm}$ punch from an unstained mouse cerebellum embedded in ethanol.

Prior to the tomographic reconstruction via the Matlab implementation of the filtered backprojection with a standard Ram-Lak filter, the simple ring-removal algorithm described in section 3.2.2 was applied on each sinogram. Two exemplary slices through the obtained volume are shown in Fig. 7.13(a) and (b). In order to increase the signal-to-noise ratio, all slices were filtered with a Gaussian function with a standard deviation of 1 pixel. Within the slices, the different layers of the cerebellum, including the single cells within these layers, are clearly resolved. Even sub-cellular details can be recognized as, e.g., the thick branches of the dendritic tree of the Purkinje cells close to the cell body as well as to some extent also the nucleolus within these cell bodies and the inner structure of the granule cells. Compared to the results obtained on a hydrated brain slice (cf. chapter 5), the cells seem to be visualized at higher contrast, which can be quantified by regarding the Weber contrast values. For the Purkinje cell layer this yields $C_{\mathrm{PCL}} \simeq 0.99$, whereas the granular cell layer has an approximate contrast of $C_{\mathrm{GL}} \simeq 0.34$. Hence, in comparison to the hydrated brain slice $\left(C_{\mathrm{PCL}} \simeq 0.5\right.$ and $\left.C_{\mathrm{GL}} \simeq 0.19\right)$ overall contrast is enhanced, though not as much as in the case of tissue stained with radiocontrast agents. Note, however, that factors as different noise levels due to varying waveguide performance and exposure times and differences in processing, as, e.g., the amount of high-pass filtering needed to reduce low-frequency artifacts 

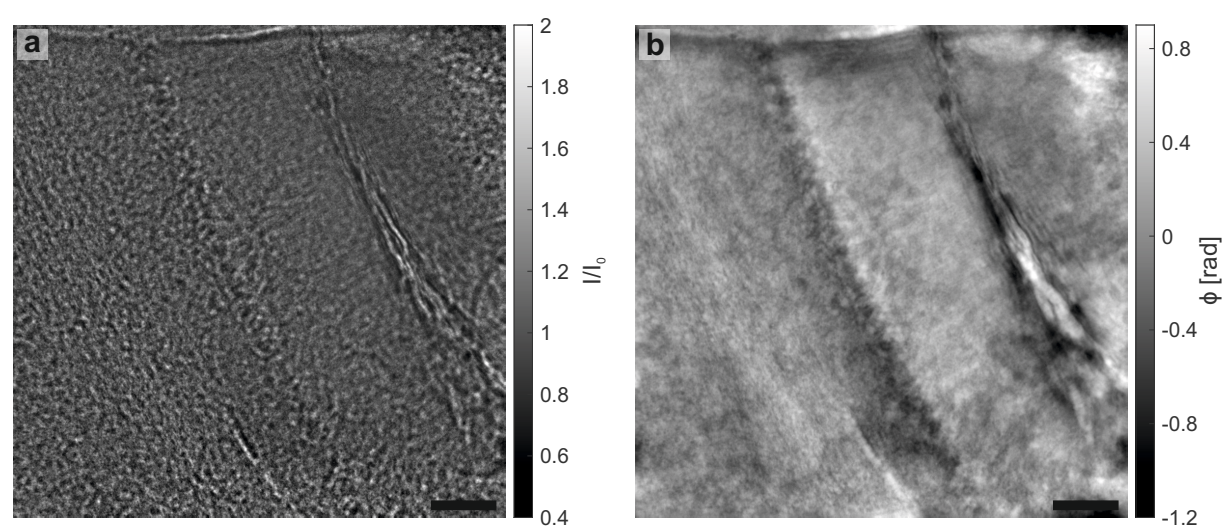

Figure 7.12: Exemplary projection from the tomographic scan of a $1 \mathrm{~mm}$ punch from an unstained mouse cerebellum embedded in ethanol. (a) Empty-beam corrected projection, in which the typical layers of the cerebellum are already visible to some extent, especially the mono-cellular Purkinje cell layer with the large cell bodies. (b) Reconstructed projection using the CTF-based approach for weakly absorbing objects with four propagation distances and the regularization parameters $\frac{\delta}{\beta}=50$ and $\alpha_{2}=0$. A clear density difference between the cell-rich granular layer and the low-cell molecular layer can be observed due to their significantly different gray values. Scale bars: $50 \mu \mathrm{m}$

caused by a changing illumination (cf. section 5.3.2), are not included in this estimation and hence, total values are only an approximation.

To obtain a better impression on the $3 \mathrm{~d}$ shape of the Purkinje cell layer, a gray value-based segmentation was performed in Avizo, using the region-growing tool Magic Wand. The result is depicted in Fig. 7.13(c), showing the typical flat shape of the Purkinje cells oriented in parallel. Despite the higher contrast compared to the hydrated brain slice, the Purkinje cells can be segmented at approximately the same amount of detail, which is probably due to the shrinking of the sample upon dehydration. Disregarding the influence of different mouse types on the cell size, this shrinking can be best visualized in the direct comparison between the hydrated as well as the ethanol-embedded brain slice, as depicted in Fig. 7.19. An estimation of the cell body sizes via manually determining their diameters for a few selected cells yields $\sim 15.8 \mu \mathrm{m}$ for the Purkinje cells in the hydrated and $\sim 14.7 \mu \mathrm{m}$ in the ethanol-embedded brain slice. For the granular layer, cell body sizes of $\sim 6.3 \mu \mathrm{m}$ and $\sim 4.4 \mu \mathrm{m}$, respectively, were determined. Hence, especially in the granular cell layer, a significant shrinking of the cell bodies due to the dehydration process can be observed. Note, however, that the determined size depends on the selected cells as well as the manual definition of the cell borders, which in some cases was challenging due to the lack of contrast. Therefore, the absolute 

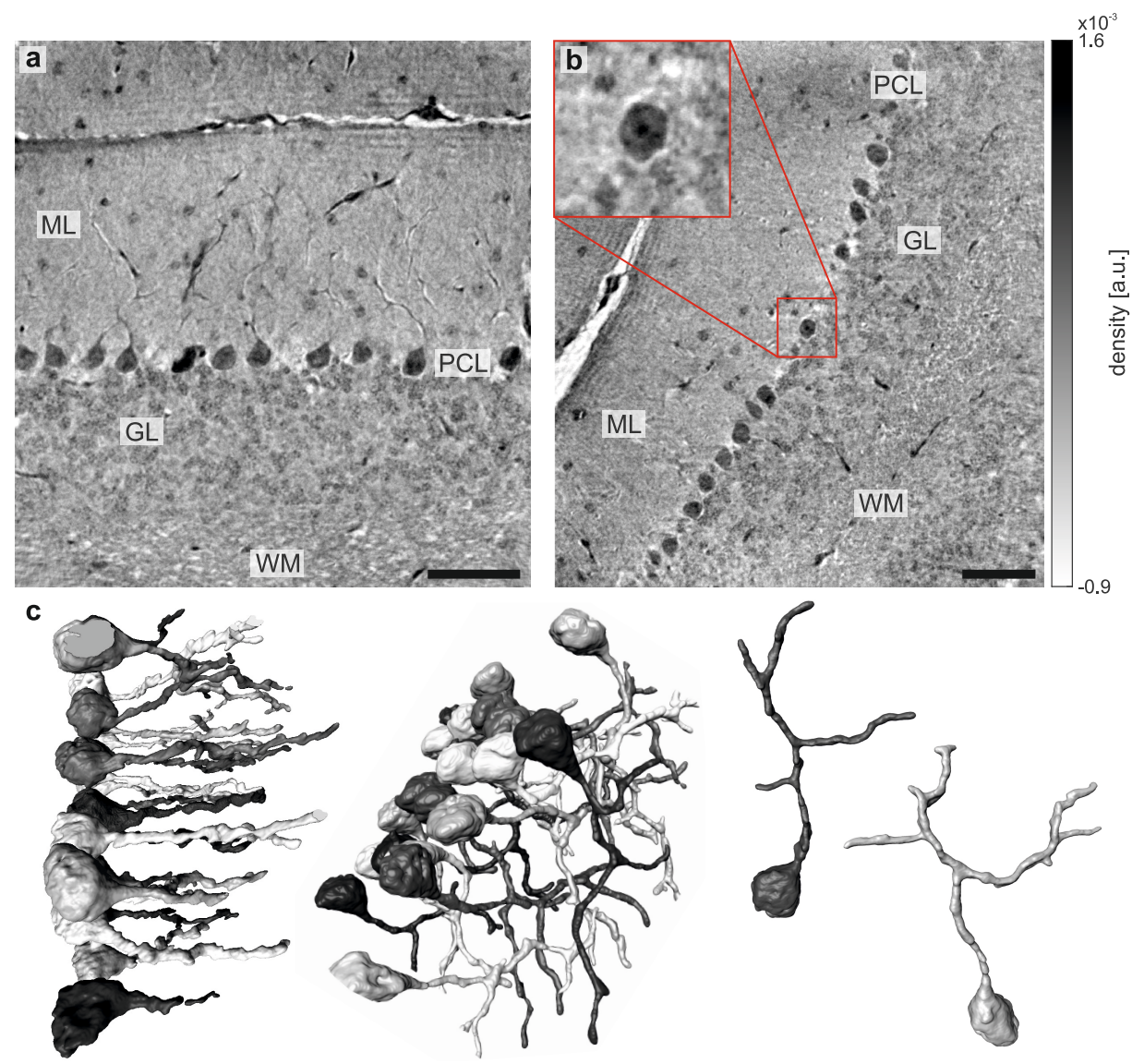

Figure 7.13: $3 d$ visualization of the $1 \mathrm{~mm}$ punch from an ethanol-embedded mouse cerebellum. $(a, b)$ Exemplary slices through the reconstructed density, showing the cellrich granular layer $(G L)$, the low-cell molecular layer $(M L)$, the mono-cellular Purkinje cell layer (PCL) and the white matter (WM). Within the large Purkinje cell bodies, subcellular details as the nucleolus can be recognized to some extent (cf. the inset in (b)). (b) The segmentation of the Purkinje cells shows the typical flat shape of these cells as well as the beginning of the highly branched dendritic tree. Scale bars: $50 \mu \mathrm{m}$

values are only relatively rough estimates.

Resolution As in the case of the hydrated unstained brain slice, the resolution was both estimated via the FSC criterion, leading to a measure of the overall quality of the experiment, as well as via an error function fit to an edge in the reconstructed slices, yielding the approximate image blur of the system. The FSC 


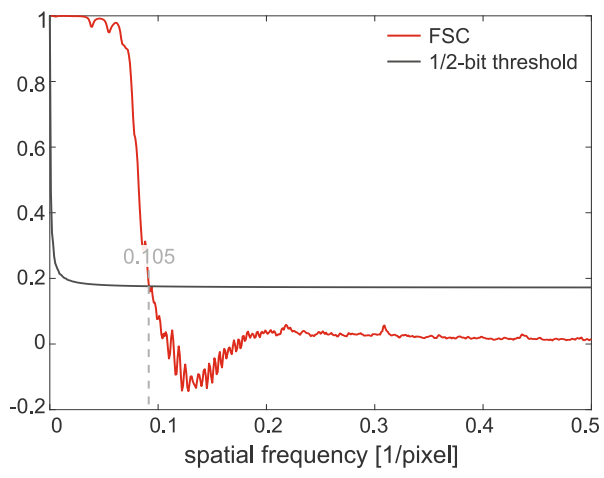

Figure 7.14: Fourier shell correlation of the central $1000^{3}$ voxels of the tomographic reconstruction of a mouse cerebellum embedded in ethanol, together with the 1/2-bit threshold curve. The intersection between these two curves determines the maximum spatial frequency which is resolved in the dataset.

for the central $1000^{3}$ voxels of the reconstructed $3 \mathrm{~d}$ density distribution, together with the 1/2-bit threshold curve for determining the maximum spatial frequency that can be resolved, is depicted in Fig. 7.14. The intersection at 0.092 cycles/pixel corresponds to a half period resolution of $1.02 \mu \mathrm{m}$. The error function fit to the profile along the edge between selected Purkinje cells and the surrounding tissue yields a FWHM of $\sim 800 \mathrm{~nm}$ and hence a half-period resolution of $\sim 400 \mathrm{~nm}$, showing that the high noise level and low contrast in the reconstructed density significantly limit the resolution.

\subsection{Mouse cerebellum embedded in paraffin}

\subsubsection{Methods}

\subsubsection{Sample preparation}

A wild type mouse, provided by the group of Frauke Alves (MPI for Experimental Medicine, Göttingen, Germany), was sacrificed and the brain was dissected and stored in $10 \%$ formalin for $24 \mathrm{~h}$ for fixation. Afterwards, the brain was embedded in paraffin. To this end, water content was removed via an ascending ethanol series $(60 \%$ : $1 \times 1.5 \mathrm{~h}, 75 \%$ : $2 \times 1.5 \mathrm{~h}, 96 \%$ : $2 \times 1.5 \mathrm{~h}, 100 \%: 2 \times 1.5 \mathrm{~h})$, which was subsequently exchanged by xylene $(2 \times 1.5 \mathrm{~h})$, enabling the infiltration of the tissue by molten paraffin wax $\left(\sim 60^{\circ} \mathrm{C}\right)$. After complete infiltration $(2 \times 1.5 \mathrm{~h})$, the tissue was transferred into a mold with fresh paraffin, which was subsequently 

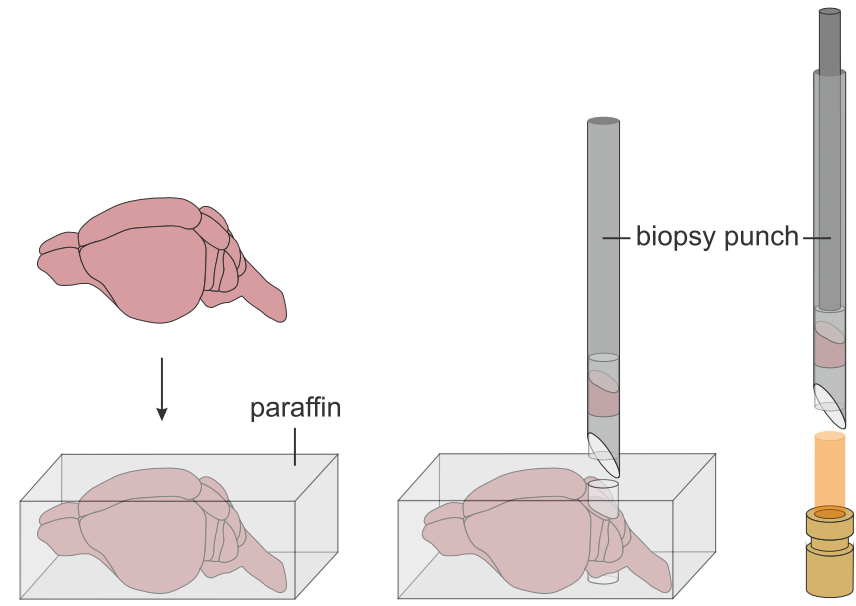

Figure 7.15: Sample preparation for the paraffin-embedded sample. An entire mouse brain was embedded in paraffin and a $1 \mathrm{~mm}$ biopsy punch was taken from the region of the cerebellum. For the mounting in the experimental setup, the punch was squeezed into a $1 \mathrm{~mm}$ Kapton tube which was glued to a sample holder.

hardened at room temperature. In order to mount the sample in the setup, a $1 \mathrm{~mm}$ biopsy punch was taken from the region of the cerebellum and squeezed into a $1 \mathrm{~mm}$ Kapton tube glued to a sample holder (cf. Fig. 7.15). Due to the stability of the sample, no additional sealing of the Kapton tube was necessary as opposed to the preparation in ethanol, described in the previous section.

\subsubsection{Experimental parameters}

Experiments were carried out at the GINIX endstation with a photon energy of $8 \mathrm{keV}$. As in the case of the ethanol-embedded sample, a waveguide with both straight and tapered channels etched into a silicon wafer was used during the experiment, with the straight channels providing the most stable illumination at a flux of $\sim 10^{9} \mathrm{ph} / \mathrm{s}$. For the detection of the photons, the 'Pirra' was installed at a sourceto-sample distance $z_{02}=5.05 \mathrm{~m}$, whereas the sample was placed at $z_{01}=145 \mathrm{~mm}$, leading to a magnification $M \simeq 35$ and an effective pixel size $p_{\text {eff }}=187 \mathrm{~nm}$. This resulted in a field of view of $383 \times 383 \mu^{2}$. In order to allow for phase retrieval with minimum artifacts, tomographic scans were recorded at four propagation distances. For each tomogram, 1500 projection over an angular range of $180^{\circ}$ were acquired with an exposure time of $0.1 \mathrm{~s}$ per projection. A summary of the experimental parameters can be found in Tab. 7.5. 


\begin{tabular}{lc}
\hline & experimental setting \\
\hline GINIX run & 56 \\
energy & $8 \mathrm{keV}$ \\
detector & 'Pirra' \\
$z_{01, \text { min }}$ & $145 \mathrm{~mm}$ \\
$z_{02}$ & $5.05 \mathrm{~m}$ \\
$p_{\text {eff }}$ & $187 \mathrm{~nm}$ \\
field of view & $383 \times 383 \mathrm{\mu m}^{2}$ \\
Fresnel number $F$ & 0.0016 \\
& 'Hercules II' \\
waveguide & $($ straight channel) \\
number of distances & 4 \\
angular range & {$[0,180]^{\circ}$} \\
number of projections & 1500 \\
exposure time & $0.1 \mathrm{~s}$ \\
CTF $\frac{\delta}{\beta}$ & 50 \\
CTF $\alpha_{2}$ & 0 \\
\hline
\end{tabular}

Table 7.5: Experimental parameters used for imaging of a $1 \mathrm{~mm}$ punch from an unstained mouse cerebellum embedded in paraffin.

\subsubsection{Results}

One exemplary projection of the acquired tomogram is depicted in Fig. 7.16(a), with the corresponding reconstructed phase map in (b). For the reconstruction, the CTF approach for weakly absorbing objects and the regularization parameters $\frac{\delta}{\beta}=50$ and $\alpha_{2}=0$ was used. In order to consider all four propagation distances, the single projections were again scaled to the same effective pixel size and aligned to each other in Fourier space. Low-frequency artifacts, caused by a changing illumination over time, were reduced by applying a high-pass filter on each acquired projection prior to phase retrieval. Apart from cracks in the paraffin, visible as bright features, no specific objects can be recognized in the $2 \mathrm{~d}$ projection and hence a tomographic reconstruction is needed to gain insight into the inner structure of the sample.

Ring artifacts in the reconstructed volume, obtained via the standard filtered backprojection implemented in Matlab, were reduced according to the simple ringremoval algorithm described in section 3.2.2. Two exemplary slices through the three-dimensional density distribution are depicted in Fig. 7.17(a) and (b). In order to increase the signal-to-noise ratio at the cost of resolution, a Gaussian filter with a standard deviation of 1 pixel was applied. Again, the typical layers of the 

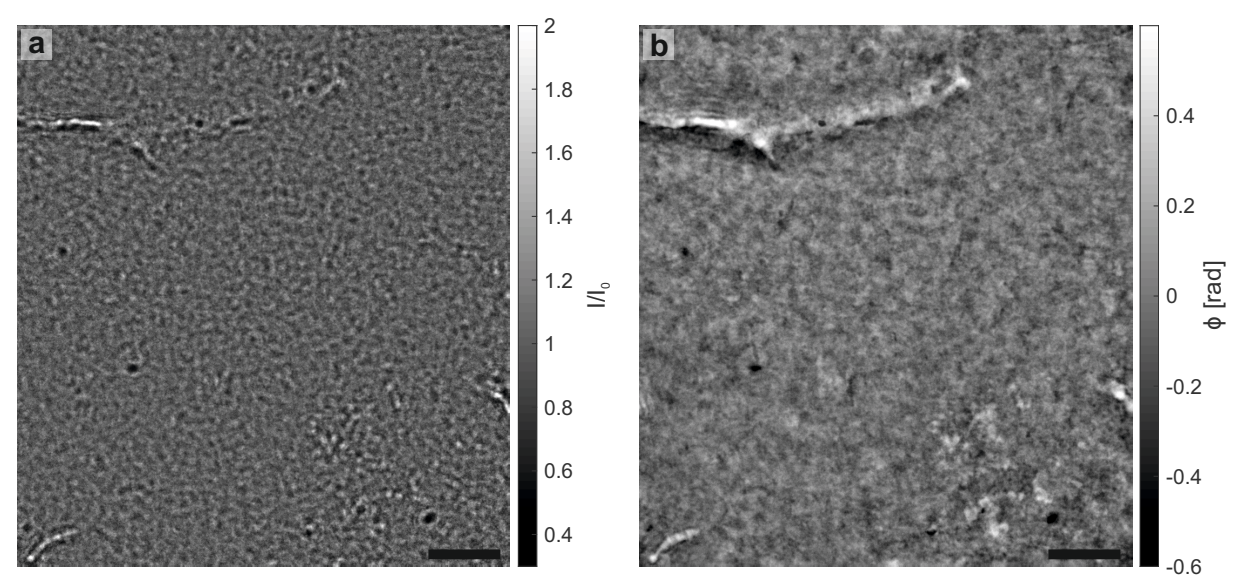

Figure 7.16: Exemplary projection from the tomographic scan of a paraffin-embedded mouse cerebellum. (a) Raw empty-beam corrected projection, in which cracks within the paraffin can be recognized as bright features. Note that in order to reduce low-frequency artifacts due to a changing illumination, a high-pass filter was applied. (b) Reconstructed phase map obtained via the CTF-based algorithm for weakly absorbing objects using four propagation distances as well as the regularization parameters $\frac{\delta}{\beta}=50$ and $\alpha_{2}=0$. Scale bars: $50 \mu \mathrm{m}$

cerebellum are clearly resolved, namely the cell-rich granular layer, the low-cell molecular layer, the interjacent Purkinje cell layer and the white matter containing large axon bundles. Even within the cells, and especially the large Purkinje cells, sub-cellular details as the nucleoli and nuclei are visible. However, already by visual inspection it is evident that the embedding in paraffin in contrast to ethanol does not lead to an increase in overall tissue contrast but rather an increase in these sub-cellular structures, while the soma and dendrites of the large Purkinje cells are depicted in lower contrast compared to the ethanol-embedded and even the hydrated tissue (cf. Fig. 7.19). To quantify this effect, the Weber contrast values were estimated both for the Purkinje cell and the granular layer, yielding $C_{\mathrm{PCL}} \simeq 0.2$ and $C_{\mathrm{GL}} \simeq 0.56$, respectively. Hence, compared to the hydrated as well as ethanol-embedded tissue, the paraffin embedding leads to a significant decrease in contrast within the soma of the Purkinje cell layer, whereas in the granular layer, contrast is superior to both techniques. Moreover, sub-cellular structures as the nucleoli and even the nuclei of the Purkinje cells can be observed at incomparable contrast and detail. This makes the paraffin embedding the ideal preparation technique to visualize cellular distributions in three dimensions. Note, however, that, as previously mentioned, the absolute contrast values also depend on factors as varying noise levels or different processing steps involved in the tomographic 

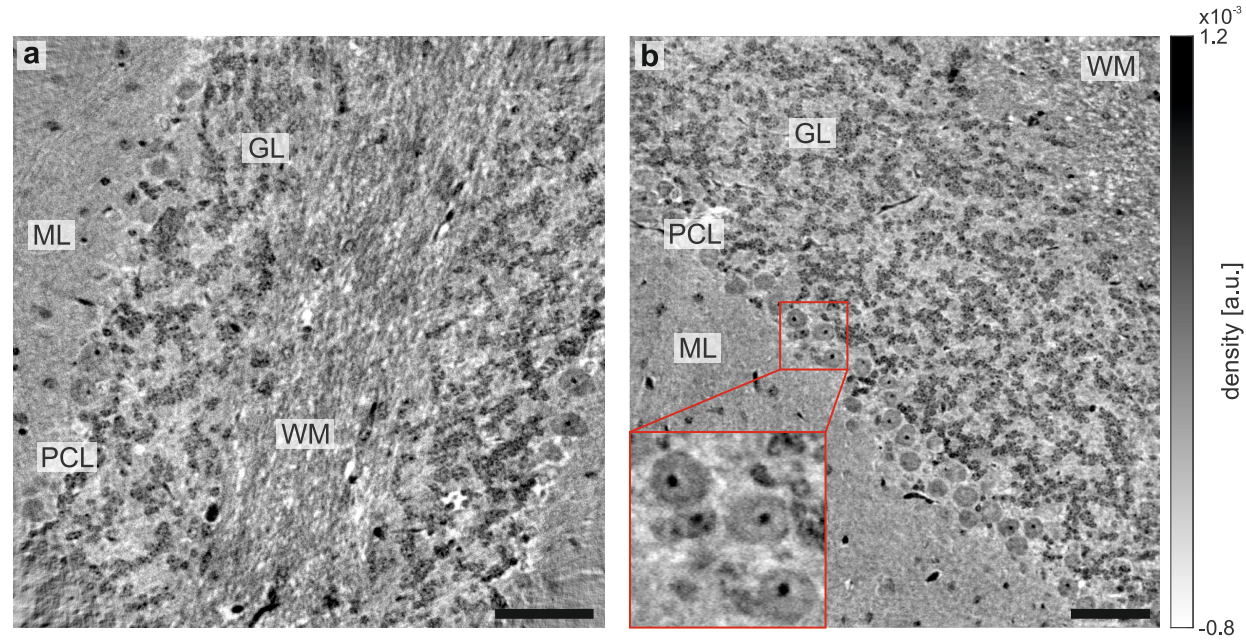

C
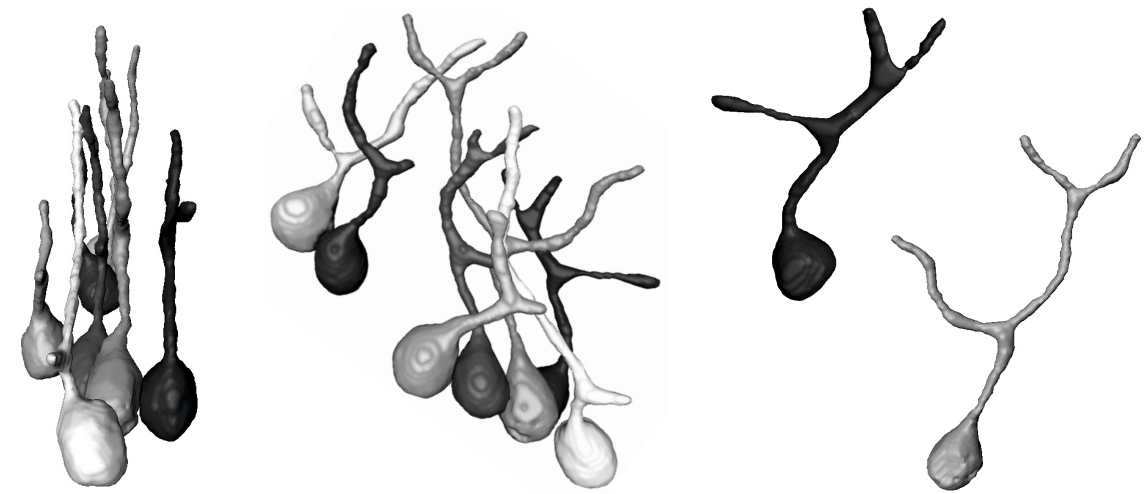

Figure 7.17: $3 d$ visualization of the reconstructed volume from the paraffin-embedded mouse cerebellum. (a,b) Exemplary slices through the reconstructed density distribution, showing the typical layers of the cerebellum (granular layer (GL), molecular layer (ML), Purkinje cell layer (PCL) and white matter (WM)). In contrast to the hydrated as well as ethanol-embedded sample, contrast within the cell bodies of the Purkinje cells is low with respect to the surrounding tissue, whereas nucleoli are depicted at high contrast (e.g., in the inset). (c) Segmentation of the Purkinje cell layer. The Purkinje cells show the expected two-dimensional shape and parallel orientation. However, due to the low contrast, only a small part of the dendritic tree can be visualized. Scale bars: $50 \mu \mathrm{m}$

reconstruction. Therefore, the exact values are only an approximation and the results should be regarded as an indication of the contrast development in different embedding media.

To obtain a better impression on the $3 \mathrm{~d}$ shape of the Purkinje cell layer, a cellular segmentation was performed for a selected number of cells. To this end, the 
gray value-based region-growing tool Magic Wand was used in Avizo by manually defining seeding points within these cells and adjusting the gray value range accordingly. The result is depicted in Fig. 7.17(c), showing the typical flat shape of the parallel oriented cells. However, compared to the ethanol-embedded sample, the dendritic tree is resolved at lower detail, which can be explained by the weaker contrast of these structures. Moreover, the tissue shrinkage, which is likely to limit the visualization of finer dendrites in the ethanol-embedded sample, also occurred during paraffin embedding. A rough estimate of the cell sizes in the Purkinje cell as well as granular layer, based on a manual selection and evaluation of specific cells, yields diameters of $\sim 14.7 \mu \mathrm{m}$ and $\sim 4 \mu \mathrm{m}$, respectively, comparable to the ethanol-embedded sample.

Resolution Analogous to the hydrated and ethanol-embedded unstained brain slices, the resolution of the scan was both estimated via the FSC criterion, yielding the overall experimental quality, as well as an error function fit to an edge along a strong feature, approximately resulting in the system blur for the given experimental parameters. The FSC of the central $1000^{3}$ voxels as well as the $1 / 2$ threshold curve are depicted in Fig. 7.18, yielding a half-period resolution of $930 \mathrm{~nm}$. Since the contrast for the large Purkinje cells is comparatively low, the high-contrast nucleoli within these cells were taken into consideration for the edge steepness determination, resulting in a FWHM of $\sim 620 \mathrm{~nm}$. Hence, the half-period resolution is given by $\sim 310 \mathrm{~nm}$. The fact that the resolution estimated via the edge steepness is a factor of 3 better compared to the FSC criterion again indicates the large influence of noise in the given reconstruction.

\subsection{Summary}

In this chapter, contrast enhancement in propagation-based imaging of neuronal tissue via embedding of the sample in a medium with a lower density compared to water, namely air, ethanol and paraffin, was evaluated. The preparation after the evaporation-of-solvent method, resulting in dried but generally structure preserved samples, enables the resolution of the three-dimensional cytoarchitecture of a mouse brain at the laboratory setup. Typical features as the different layers of the cerebellum, the olfactory bulb or the barrel fields of the cortex can be visualized at approximately the same detail as in standard histological sections. Compared to the hydrated brain slice measured at the synchrotron, an increase in tissue 


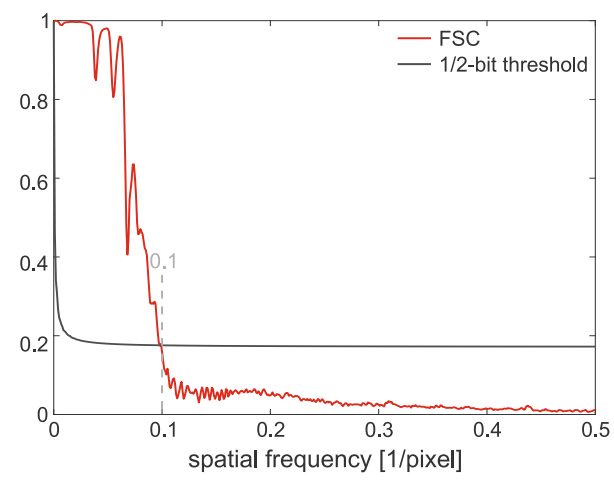

Figure 7.18: Fourier shell correlation of the central $1000^{3}$ voxels of the tomographic reconstruction of a mouse cerebellum embedded in paraffin, together with the 1/2-bit threshold curve. The intersection between these two curves determines the spatial frequency for which enough information was collected for interpretation.

\begin{tabular}{lccc}
\hline & half-period res. (FSC/edge) & contrast in PCL & contrast in GL \\
\hline hydrated & $809 \mathrm{~nm} / \sim 555 \mathrm{~nm}$ & $\sim 0.60$ & $\sim 0.19$ \\
ethanol & $1.02 \mu \mathrm{\mu m} / \sim 400 \mathrm{~nm}$ & $\sim 0.99$ & $\sim 0.34$ \\
paraffin & $930 \mathrm{~nm} / \sim 310 \mathrm{~nm}$ & $\sim 0.20$ & $\sim 0.56$ \\
EOS & $2.8 \mu \mathrm{m} /-$ & $\sim 0.65$ & $\sim 0.36$ \\
\hline
\end{tabular}

Table 7.6: Comparison of the achieved resolutions as well as contrast values in the Purkinje cell and granular layer of a mouse cerebellum embedded in different media.

contrast can be reached, despite the lower contrast transfer in the direct-contrast compared to the holographic regime (see Tab. 7.6). Due to the large differences in electron density between tissue and air, phase retrieval in the holographic regime is challenging and hence, imaging of samples prepared in accordance with the EOS protocol was limited to the laboratory and the corresponding resolution levels.

In order to reach higher resolutions and enable imaging in the holographic regime, ethanol and paraffin wax were evaluated as embedding media at the synchrotron. Due to the dehydration process involved in both preparation techniques, a significant amount of tissue shrinkage occurred, as can be seen in Fig. 7.19 in comparison to the hydrated brain slice. Apart from that, the embedding in ethanol leads to an increase in overall tissue contrast (see Tab. 7.6). The electron density differences are small enough so that the assumptions for the derivation of the CTF are still valid, enabling a sharp and quantitative reconstruction of the phase. Within the slices, sub-cellular details as the dendritic tree or the nucleolus of the Purkinje cells 

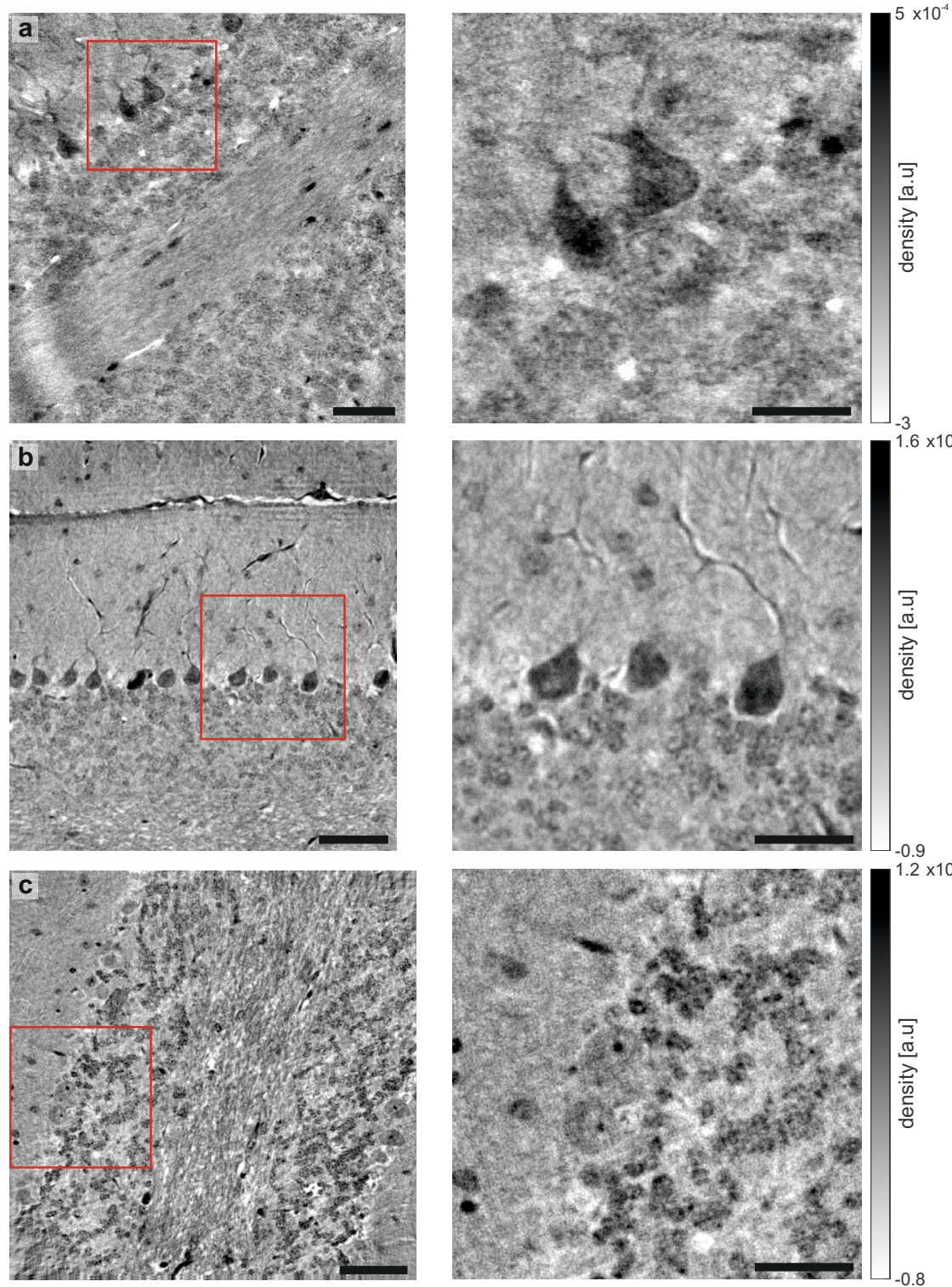

Figure 7.19: Comparison between the different embedding media used for experiments at the GINIX setup. Overall contrast in the hydrated brain slice (a) is low for the small granule cells, but the cell bodies and to some extent even the dendritic tree of the Purkinje cells can be visualized at relatively high contrast. The exchange of water content by ethanol (b) leads to an overall increase in contrast whereas in the paraffin-embedded tissue (c) the cell bodies of the Purkinje cells show a considerably decreased contrast. For the cell nuclei/nucleoli within the layers, it is, however, superior with respect to the other preparations. Scale bars: $50 \mu \mathrm{m}$ (left) and $25 \mu \mathrm{m}$ (right) 
as well as the inner structure of the granule cells can be to some extent resolved. The larger contrast compared to the hydrated brain slice also leads to an increase in resolution according to the fit of an error function to the edge between Purkinje cells and the surrounding tissue (see Tab. 7.6). Note that the differences in resolution determined via the FSC criterion are mainly caused by different numbers of projections and most importantly a better waveguide performance in the case of the hydrated brain slice.

The embedding in paraffin does not increase overall tissue contrast but rather emphasizes the cell nucleoli, whereas features as the soma or the dendritic tree reveal a decreased contrast compared to the ethanol-embedded and even hydrated tissue (see Tab. 7.6 as well as Fig. 7.19). Hence, this embedding procedure is especially well-suited for the visualization of three-dimensional cell distributions based on the location of the nuclei. Despite the low contrast in the surrounding parts of the cell, features as the dendritic tree of the Purkinje cells can still be resolved, though not as detailed as in the ethanol-embedded sample. One further advantage of the embedding in paraffin is the increased stability of the sample, both with respect to internal changes during the measurement, as, e.g., bubble formation, as well as mounting. This could explain the higher resolution for this sample, if considering the edge steepness (see Tab. 7.6).

Note the steps involved in the sample preparation for the different embedding media are standard steps in paraffin embedding, which is usually performed in order to generate histological sections and hence, the tomographic results could be potentially combined with histological studies. Even the dried sample can be rehydrated after the measurement and subsequently prepared for sectioning [167]. 


\section{Quantitative 3d histology of human cerebellum}

The ability to increase the contrast of unstained samples from the central nervous system by exchanging the surrounding medium makes it possible to examine neuropathological samples, in particular samples from the human brain obtained during routine autopsy, by means of phase-contrast tomography, as these are usually embedded in paraffin after fixation. Therefore, the complex cytoarchitecture of human brain can be studied in three dimensions at isotropic resolution without the need for slicing of the specimen and subsequent examination under a light microscope. This enables studying of the $3 \mathrm{~d}$ cellular distributions as well as possible changes due to neurological diseases like multiple sclerosis or Alzheimer's disease. In order to fully exploit the potential of phase-contrast tomography for $3 \mathrm{~d}$ virtual histology, automatic segmentation algorithms are necessary to facilitate the interpretation and quantification of the obtained volumes.

Several approaches have already been introduced to implement this for data recorded with synchrotron radiation, e.g., by Dyer et al., using manual training of an object classifier to obtain probability maps for neurons and specific sample features as blood vessels, as shown for osmium-stained mouse cortex [42] or by Hieber et al., using a Frangi filtering step to segment the large Purkinje cells in unstained human cerebellum embedded in paraffin [69].

In this chapter, a human cerebellum embedded in paraffin is studied both at the synchrotron as well as the laboratory setup at varying resolution and field of view. To quantify the 3d cytoarchitecture, a workflow based on the Spherical Hough transform $[74,133]$ is presented, which enables the automatic segmentation and localization of the small cells in the molecular as well as granular layer. From these results, local density distributions or statistical measures as the pair correlation function can be computed.

Note that the majority of the results presented in the following are published in a shorter form in [168] and that the corresponding figures are based on those from the paper. 


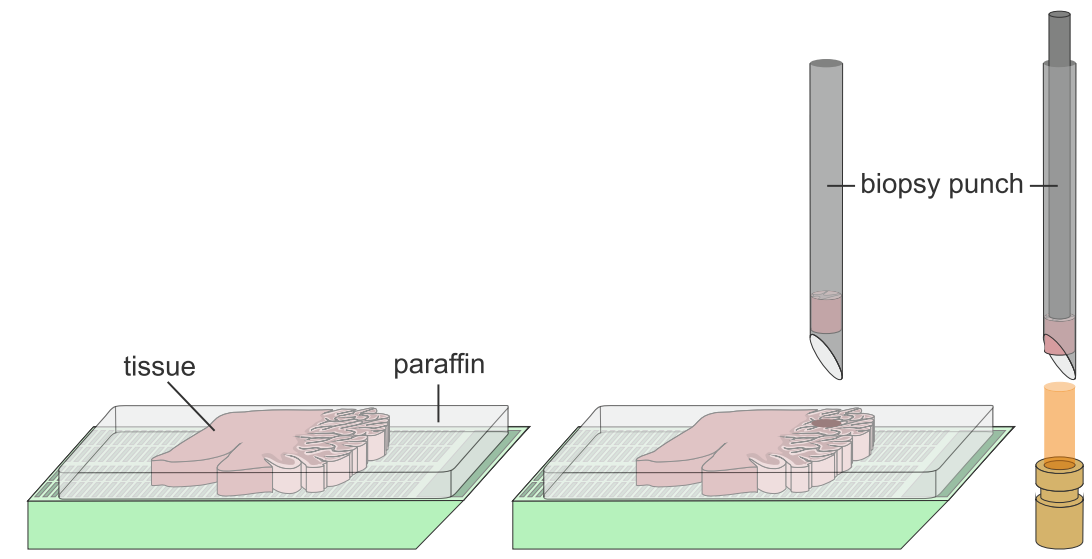

Figure 8.1: Sample preparation for a human cerebellum embedded in paraffin. Formalinfixed and paraffin-embedded cerebellar tissue was obtained during routine autopsy and a $1 \mathrm{~mm}$ biopsy punch was taken from it and squeezed into a Kapton tube glued to a sample holder.

\subsection{Sample preparation and experimental parameters}

Sample preparation Formalin-fixed and paraffin-embedded cerebellar tissue was obtained during routine autopsy and provided by the group of Christine Stadelmann-Nessler (Institute for Neuropathology, University Medical Center, Göttingen, Germany). For experiments, a $1 \mathrm{~mm}$ punch was taken from the embedded tissue and squeezed into a $1 \mathrm{~mm}$ Kapton tube glued to a sample holder (see Fig. 8.1).

GINIX setup The tomographic experiments at the GINIX setup were carried out both in parallel- as well as cone-beam geometry at photon energies of $13.8 \mathrm{keV}$ and $8 \mathrm{keV}$, respectively. For the parallel-beam geometry, enabling an overview of the sample at relatively large field of view but moderate resolution, the focusing optics, namely the KB-mirrors and the waveguide, were removed from the setup and the low divergent illumination of the undulator, monochromatized by the channel-cut crystal, was used for imaging. As the resolution in this configuration is limited by the detector, the 'Argos' was chosen as the detection device. Note that in contrast to the 'Argos' camera described in section 4.1, a slightly modified version was used, consisting of a $2472 \times 3296$ pixels CCD chip with an effective pixel size $p_{\text {eff }}=550 \mathrm{~nm}$ and a field of view of $1.36 \times 1.81 \mathrm{~mm}^{2}$. In the current 
setup, a fast shutter is located in the beam path that allows for blocking of the x-rays at times when no image acquisition takes place, e.g., during readout of the detector, thus reducing the radiation dose of the sample. The maximum opening of this shutter, however, limits the effective field of view in the vertical direction to approximately $630 \mu \mathrm{m}$, as can be seen in Fig. 8.2.

For the tomographic scan, 1000 projections over $180^{\circ}$ were acquired at three propagation distances, of which the largest was $z_{12}=50 \mathrm{~mm}$, leading to a minimum Fresnel number of 0.067 and hence imaging in the near holographic regime. The exposure time per projection was $0.01 \mathrm{~s}$.

In the cone-beam geometry, enabling the zoom into specific regions of interest, a straight waveguide etched into a silicon wafer was placed in the focal spot produced by the KB-mirrors, providing a flux in the order of $10^{9} \mathrm{ph} / \mathrm{s}$. As in the case of the ethanol as well as paraffin-embedded samples, the resulting illumination of this straight channel proved to be more stable compared to a tapered device. The 'Pirra' was positioned at a source-to-detector distance $z_{02}=5.05 \mathrm{~m}$, whereas the sample was located at $z_{01}=145 \mathrm{~mm}$, leading to an effective pixel size $p_{\text {eff }}=187 \mathrm{~nm}$ and a field of view of $383 \times 383 \mu^{2}$. For the tomographic scan, 1500 projections were acquired over an angular range of $180^{\circ}$ with an exposure time of $0.2 \mathrm{~s}$. To reduce artifacts in the reconstructed phase distributions, tomograms were recorded at four propagation distances. All experimental parameters for scans carried out at the GINIX endstation are summarized in Tab. 8.1.

Laboratory setup For the experiments carried out at the laboratory setup, the inverse geometry was used in order to achieve cellular resolution. To this end, the 'Argos' detector was placed at a source-to-detector distance $z_{02}=0.186 \mathrm{~m}$ while the source-to-sample distance was set to $158.75 \mathrm{~mm}$, leading to a magnification of 1.17 and an effective pixel size $p_{\text {eff }}=0.46 \mu \mathrm{m}$ with a field of view of $1.15 \times 1.53 \mathrm{~mm}^{2}$. The source was operated at an acceleration voltage of $40 \mathrm{kV}$ and an electron spot size of $10 \times 40 \mathrm{\mu m}^{2}$, enabling a power of $57 \mathrm{~W}$. In order to increase photon flux, the electron beam was slightly deflected towards the exit window (cf. Fig. 4.5).

The tomographic scan was carried out by recording 1000 projection over an angular range of $180^{\circ}$ with an exposure time of $50 \mathrm{~s}$ each. A summary of the experimental parameters is listed in Tab. 8.1. 


\begin{tabular}{lccc}
\hline & P10 parallel & P10 cone-beam & laboratory \\
\hline GINIX run & 67 & 56 & - \\
energy $[\mathrm{keV}]$ & 13.8 & 8 & $0-40 / 9.25$ \\
detector & 'Argos' & 'Pirra' & 'Argos' \\
$z_{01, \min }[\mathrm{mm}]$ & - & 145 & 158.75 \\
$z_{02}[\mathrm{~m}]$ & - & 5.05 & 0.186 \\
$z_{12, \max }[\mathrm{mm}]$ & 50 & 4905 & 27.25 \\
$p_{\text {eff }}[\mathrm{nm}]$ & 550 & 187 & 461 \\
field of view $\left[\mathrm{Hm}^{2}\right]$ & $1360 / 633$ & $383 \times 383$ & $1152 \times 1530$ \\
Fresnel number $F$ & 0.067 & 0.0016 & 0.069 \\
waveguide & - & (straight channel) & - \\
number of distances & 3 & 4 & 1 \\
angular range $\left[{ }^{\circ}\right]$ & {$[0,180]$} & {$[0,180]$} & {$[0,180]$} \\
number of projections & 1000 & 1500 & 1000 \\
exposure time $[\mathrm{s}]$ & 0.01 & 0.2 & 50 \\
BAC $\alpha$ & - & - & 0.01 \\
BAC $(\gamma \cdot F)$ & - & - & 0.15 \\
CTF $\frac{\delta}{\beta}$ & 30 & 50 & - \\
CTF $\alpha_{2}$ & 0 & 0 & - \\
\hline
\end{tabular}

Table 8.1: Overview of the experimental parameters for imaging of a $1 \mathrm{~mm}$ punch from a human cerebellum embedded in paraffin, measured both at the GINIX endstation as well as the laboratory setup.

\subsection{Results}

\subsubsection{Tomographic imaging at the GINIX setup}

Parallel geometry Measurements in parallel-beam geometry were carried out in order to obtain an overview of the sample at relatively large field of view and moderate half-period resolution of $1.0 \mu \mathrm{m}$, as determined via the corresponding FSC curve (not shown). A typical empty image obtained in this configuration is depicted in Fig. 8.2(a), showing the restricted field of view due to the limited opening of the fast shutter as well as small structures within the otherwise smooth illumination. These structures correspond to dust particles that are most likely located on a Kapton window in the beam path. Normally, they simply divide out in the empty-beam correction, as they are both present in the empty image as well as the acquired projections. However, due to sub-pixel shifts of the beam with respect to the setup, the location of these dust particles slightly changes during the scan, leading to artifacts in the empty-beam corrected projections, as depicted in Fig. 

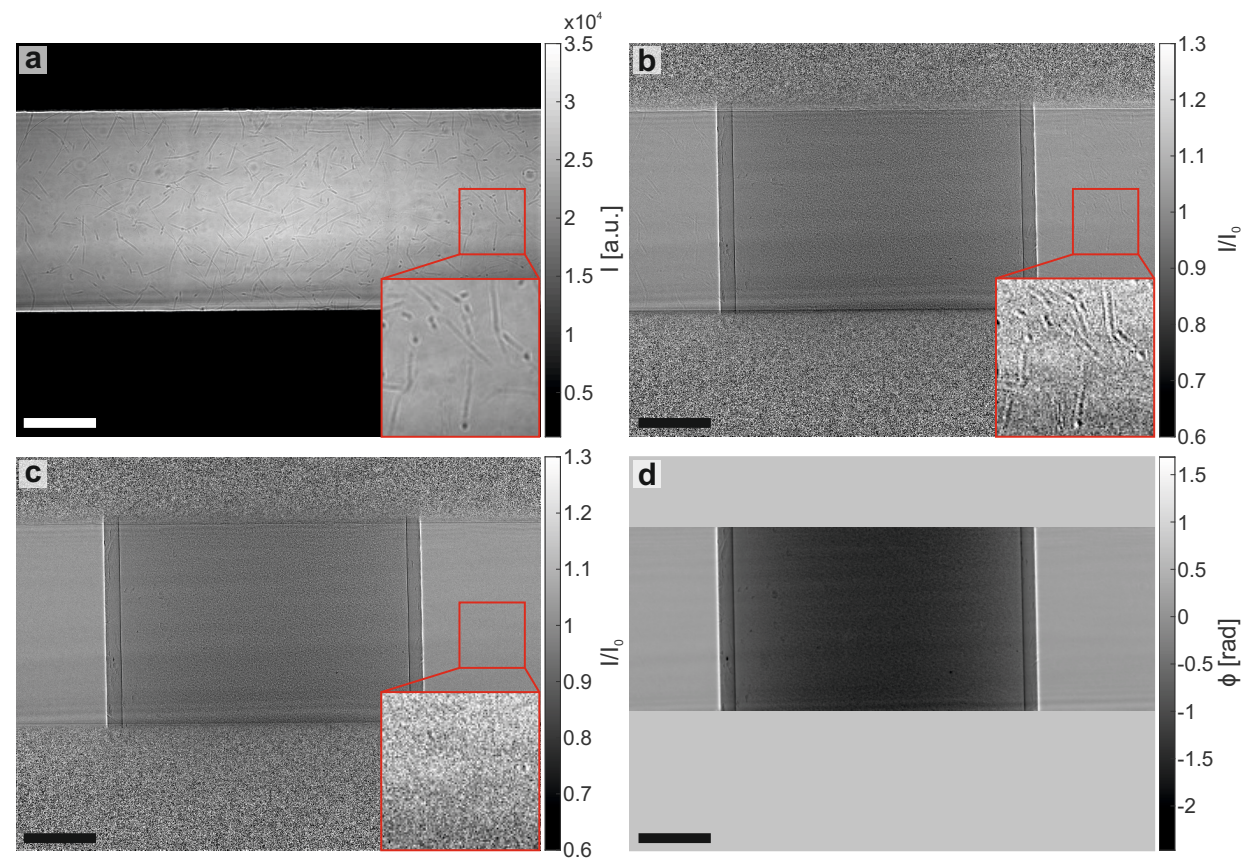

Figure 8.2: Exemplary projection image obtained in the parallel-beam setup at the GINIX endstation. (a) Typical empty image in which dust particles that are located on a Kapton window in the beam path are visible. (b) Empty-beam corrected projection of the sample. Sub-pixel shifts of the beam position with respect to the setup lead to small variations in the dust particle positions and hence an imperfect empty-beam correction. The gray value range in the inset is limited to $[0.95,1.05]$ to emphasize the effect. (c) By aligning the empty image to an empty region in the acquired projection, the artifacts can be reduced. (d) CTF-based reconstruction of the illuminated part of the projection. Due to the violation of the homogeneous object assumption, phase-contrast effects are not inverted at the interface between Kapton and air as the $\frac{\delta}{\beta}$-ratio was adapted with respect to the paraffin-embedded tissue in the center. Scale bars: $250 \mu \mathrm{m}$

8.2(b). As the position of the artifacts remains similar throughout the scan, they will result in severe ring artifacts in the tomographic reconstruction. To overcome this problem, the empty image and an empty region within the projections were aligned via a cross-correlation in Fourier space [59], resulting in the corrected image depicted in Fig. 8.2(c). The artifacts due to the varying dust particle positions are clearly removed and only slight low-frequency variations along the horizontal direction remain.

Phase contrast in this near holographic regime is mainly visible as edge enhancement, especially at the interface between the Kapton tube and air. The phase distribution in the illuminated part of the projection was reconstructed via the 
CTF-based approach for a weakly absorbing object with regularization parameters $\frac{\delta}{\beta}=30$ and $\alpha_{2}=0$, resulting in the image shown in Fig. 8.2(d). The residual edge enhancement is due to the varying electron density differences between Kapton and air at the edge of the sample as well as paraffin and soft tissue in the center, leading to a violation of the homogeneous object assumption.

The tomographic reconstruction was carried out via the Matlab implementation of the filtered backprojection with a standard Ram-Lak filter. One exemplary slice through the $3 \mathrm{~d}$ density distribution with an orientation parallel to the Purkinje cell layer is depicted in Fig. 8.3(a). The imperfect inversion of the interference fringes around the Kapton tube during phase retrieval leads to artifacts in the reconstructed volume in the form of edge enhancement, whereas the paraffin-embedded tissue in the center shows no comparable effects, proving the appropriate choice of regularization parameters with respect to this part of the sample. Within the tissue, the typical layers of the cerebellum, namely the cell-rich granular layer, the low-cell molecular layer, the mono-cellular Purkinje cell layer as well as the white matter consisting of large axon bundles, are resolved at cellular detail. Within the large Purkinje cells, even sub-cellular details as the dendritic tree as well as the nucleolus and to some extent the nucleus can be visualized, as shown in the upper inset on the right. However, the inner structure of the small granule cells is not resolved and neighboring cells in close proximity are not clearly separated (cf. lower inset).

To obtain an impression on the three-dimensional shape of the Purkinje cell layer, a semi-automatic segmentation was performed, analogous to the previous chapters. To this end, the region growing-based Magic Wand tool was used for the segmentation of the main part of a selected number of Purkinje cells by manually defining seeding points within the volume. Due to the relatively low contrast of the dendritic tree with respect to the surrounding tissue (cf. section 7.3), a manual refinement was necessary, as fine structures, which were not segmented automatically but nevertheless belonged to the respective cells, could be identified based on visual inspection. The resulting $3 \mathrm{~d}$ visualization of the main part of the Purkinje cell layer is depicted in Fig. 8.3(b). The typical shape of the layer, consisting of the flat Purkinje cells with a highly branched dendritic tree arranged in a parallel manner, is clearly visible. Note that for a large number of cells, the segmentation is rather limited by the edge of the Kapton tube instead of the resolution of the tomographic reconstruction. Hence, by repeating the measurement for a different punch from the human cerebellum, in which a larger part of the molecular layer and thus the dendritic tree of the Purkinje cells is contained, the three-dimensional 

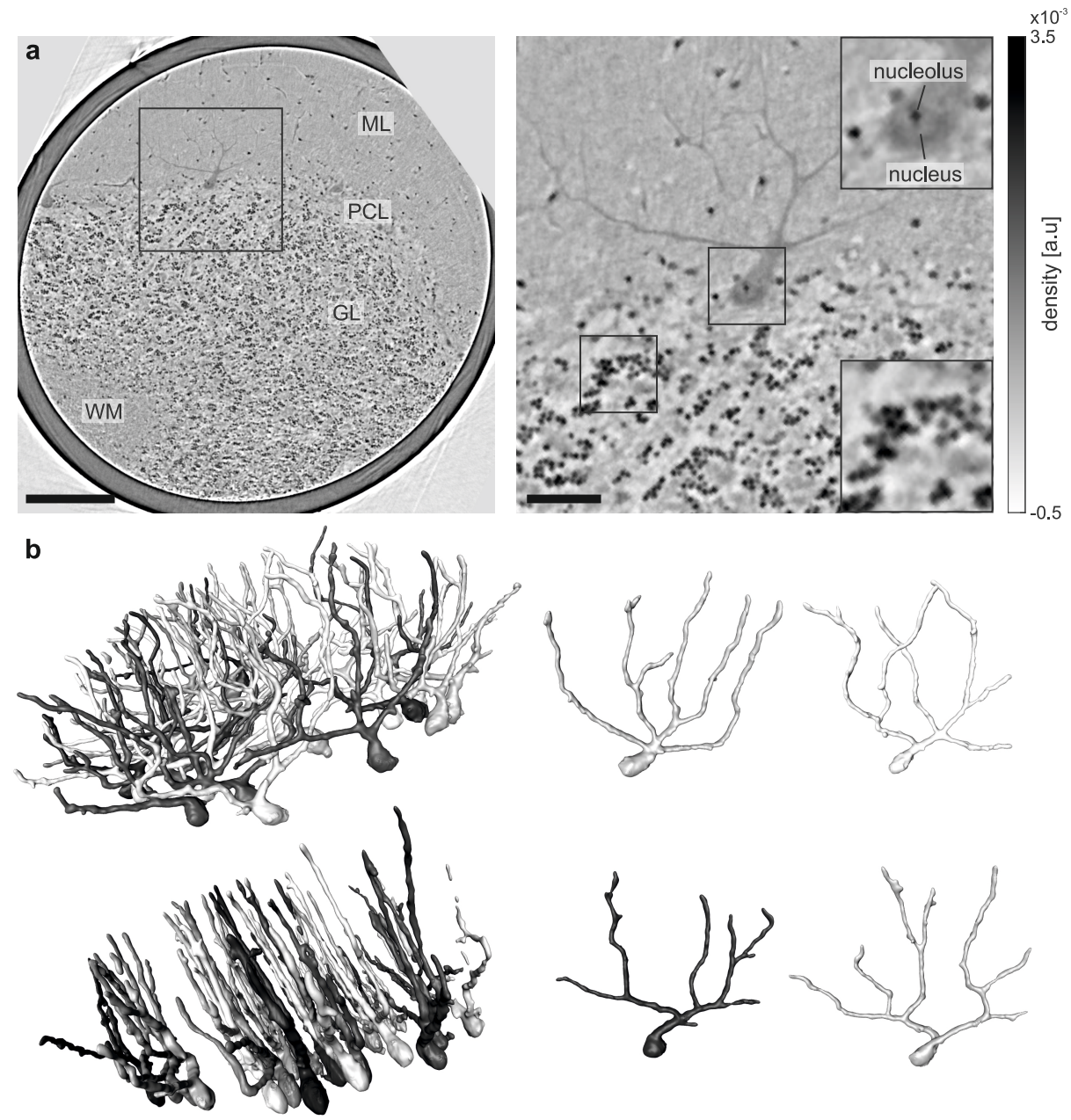

Figure 8.3: $3 d$ visualization of the tomographic results recorded in the parallel-beam geometry. (a) Exemplary virtual slice with an orientation parallel to the Purkinje cell layer. The granular layer (GL), molecular layer (ML), Purkinje cell layer (PCL) and white matter $(W M)$ can be visualized at cellular detail. Within the large Purkinje cells, the dendritic tree and the nucleolus as well as to some extent the nucleus are visible, whereas the inner structure of the small granule cells is not resolved. (b) The semiautomatic rendering of a major part of the Purkinje cells reveals their typical flat shape with a highly arbored dendritic tree as well as their parallel arrangement. Scale bars: $200 \mu \mathrm{m}$ (a, left) and $50 \mu \mathrm{m}$ (a, right) 
visualization could be further expanded.

Cone-beam geometry To obtain results at higher resolution and zoom into specific regions of interest, additional experiments were carried out in cone-beam geometry with an effective pixel size of $187 \mathrm{~nm}$, leading to a field of view of $381 \times 381 \mathrm{\mu m}^{2}$. Phase retrieval on the individual projections was performed via the CTF-based approach for weakly absorbing objects, using the regularization parameters $\frac{\delta}{\beta}=50$ and $\alpha_{2}=0$. As in the previous experiments carried out in cone-beam geometry, the projections acquired at different propagation distances were rescaled and aligned to each other prior to the phase-retrieval step. To reduce ring artifacts, the simple ring-removal algorithm described in section 3.2.2 was applied to the sinograms. Subsequently, the tomographic reconstruction was carried out via the Matlab implementation of the filtered backprojection with a standard Ram-Lak filter. Two slices through the resulting density distribution with an orientation parallel to the Purkinje cell layer are depicted in Fig. 8.4, showing the interface between the cell-rich granular layer and the low-cell molecular layer as well as single cells of the Purkinje cell layer and their large dendritic trees protruding into the molecular layer. Note that the position of the slice depicted on the left corresponds approximately to the magnified region shown in Fig. 8.3 and thus, the region-of-interest measurement can be set in relation to the large overview scan.

Sub-cellular details as the nucleolus as well as nucleus of the Purkinje cells can be clearly resolved and even the inner structure of the small granule cells as well as cells in the molecular layer can be visualized to some extent, as depicted in the lower inset on the left as well as in Fig. 8.13. Compared to the parallel-beam setup, the small granule cells are separated more clearly, enabling an unambiguous segmentation of these cells based on visual inspection. However, in contrast to the Purkinje cells, the vast number of cells in the granular layer impedes the manual selection of seeding points for the region growing-based segmentation tool used so far. Therefore, an automatic approach for the detection and localization of these small cells is required in order to quantitatively analyze the distribution of cells within the obtained tomographic dataset.

\subsubsection{Automated cellular segmentation in the synchrotron results}

As the cells in the granular and mostly also in the molecular layer have a round shape, an algorithm detecting (imperfect) spheres of varying radius in a three- 

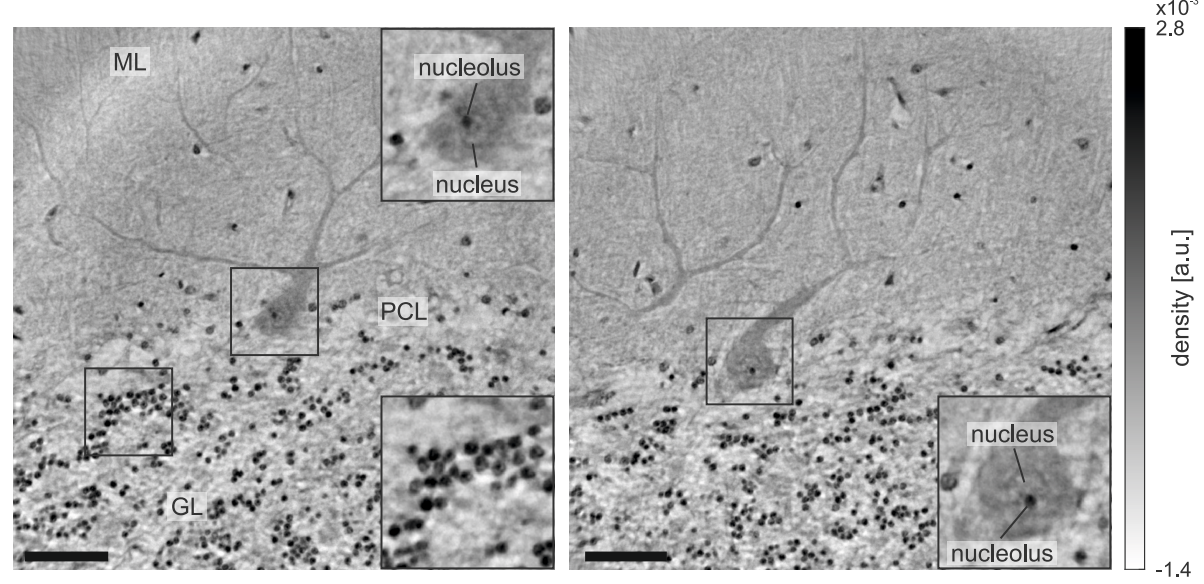

Figure 8.4: Virtual slices through the reconstructed volume from the measurements in cone-beam geometry. The interface between the cell-rich granular layer as well as low-cell molecular layer and single cells from the Purkinje cell layer can be resolved at sub-cellular detail, revealing structures as the dendritic tree as well as nucleolus and nucleus of the Purkinje cells. The position of the slice depicted on the left corresponds approximately to the magnified region shown in Fig. 8.3(a). Compared to the parallel-beam setup, the small granule cells are separated more clearly and even their inner structure is to some extent resolved. Scale bars: $50 \mu \mathrm{m}$

dimensional volume is a promising approach for the automatic detection and localization of these cells. One example of such an algorithm is the spherical Hough transform [74, 133], explained below in detail, which builds a so-called voting matrix in which centers of spherical shapes are represented as local maxima.

\subsubsection{Spherical Hough transform for 3d segmentation}

The implementation of the spherical Hough transform used in this thesis was provided by Luke Xie [133, 193]. For simplicity, the process of building the accumulation matrix is first regarded in $2 \mathrm{~d}$, but the implementation in $3 \mathrm{~d}$ is analogous. Consider the $2 \mathrm{~d}$ image depicted in Fig. 8.5(a), showing a circular object within a $2 \mathrm{~d}$ matrix. In a first step, the edge of the object is detected, e.g., by calculating the magnitude of the gradient vectors and defining a threshold value. As the gradient is maximal at the edge of the object, this will give an approximation of the outer shape. Due to the curvature of the circle, the corresponding gradient vectors are either pointing towards the center of the circle or away from it. This property is used for building the accumulation matrix. For each point on the selected edge, a line segment is added to the matrix in the direction of the gradient vector (see 
a

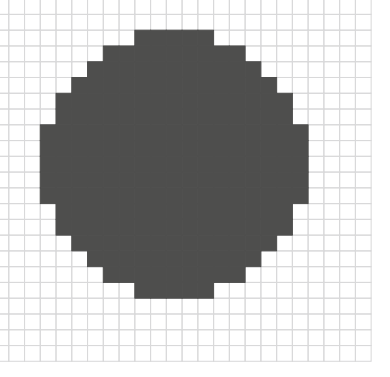

b

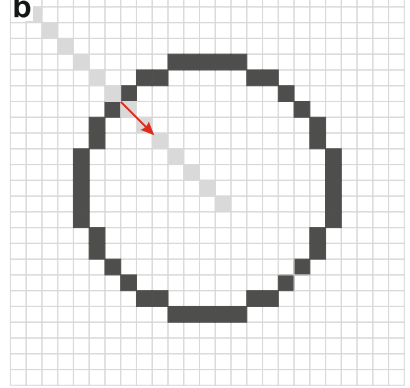

C

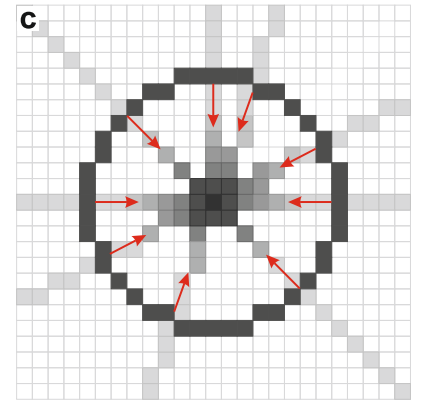

Figure 8.5: Construction of the accumulation array of the spherical Hough transform in $2 d$. (a) The raw image is represented by a circle lying within a $2 d$ array of pixels. (b) In a first step, the edge of the circle is determined via the gradient of the image. For each point on this edge, a line is plotted in the direction of the corresponding gradient vector, with a length equal to twice the maximum possible radius. Each line is additionally weighted by the corresponding magnitude of the gradient vector. (c) Repeating this for all points on the edge of the circle leads to the accumulation matrix with a maximum at the position of the central point.

the sketch in Fig. 8.5(b)). The length can be set manually and should correspond to the maximum expected diameter of the circles in the image. To give stronger edges a greater influence in the accumulation matrix, each line is weighted by the corresponding magnitude of the gradient vector. Performing this procedure for all pixels on the edge of a perfect circle leads to an intersection of the line segments and hence a maximum of the accumulation array in the center (cf. Fig. 8.5(c)). Therefore, the intensity values in the accumulation array of an arbitrary image represent the probability of a center point of a circle located at the corresponding position and for several circles present in the image, several maxima occur.

Before proceeding with the detection of these peaks in the accumulation array, its building process for the given three-dimensional data of a human cerebellum is shown. To this end, a small subvolume of 200 voxels $^{3}$ is considered, which is located within the granular layer, with one exemplary slice through this subvolume depicted in Fig. 8.6(a). Note that although each of the following steps is carried out in $3 \mathrm{~d}$, the individual steps are only visualized in $2 \mathrm{~d}$ sections for reasons of clarity. The magnitude of the gradient vectors is shown in (b), clearly revealing the edges of the cells due to the large density changes at the interface to the surrounding tissue. By defining a threshold based on visual inspection, the pixels belonging to the edge can be selected, as depicted in (c). For each of these pixels, a weighted line segment is added to the accumulation matrix, resulting in the image in $(d)$, with positions of the cell centers clearly visible as maxima. Single isolated pixels 

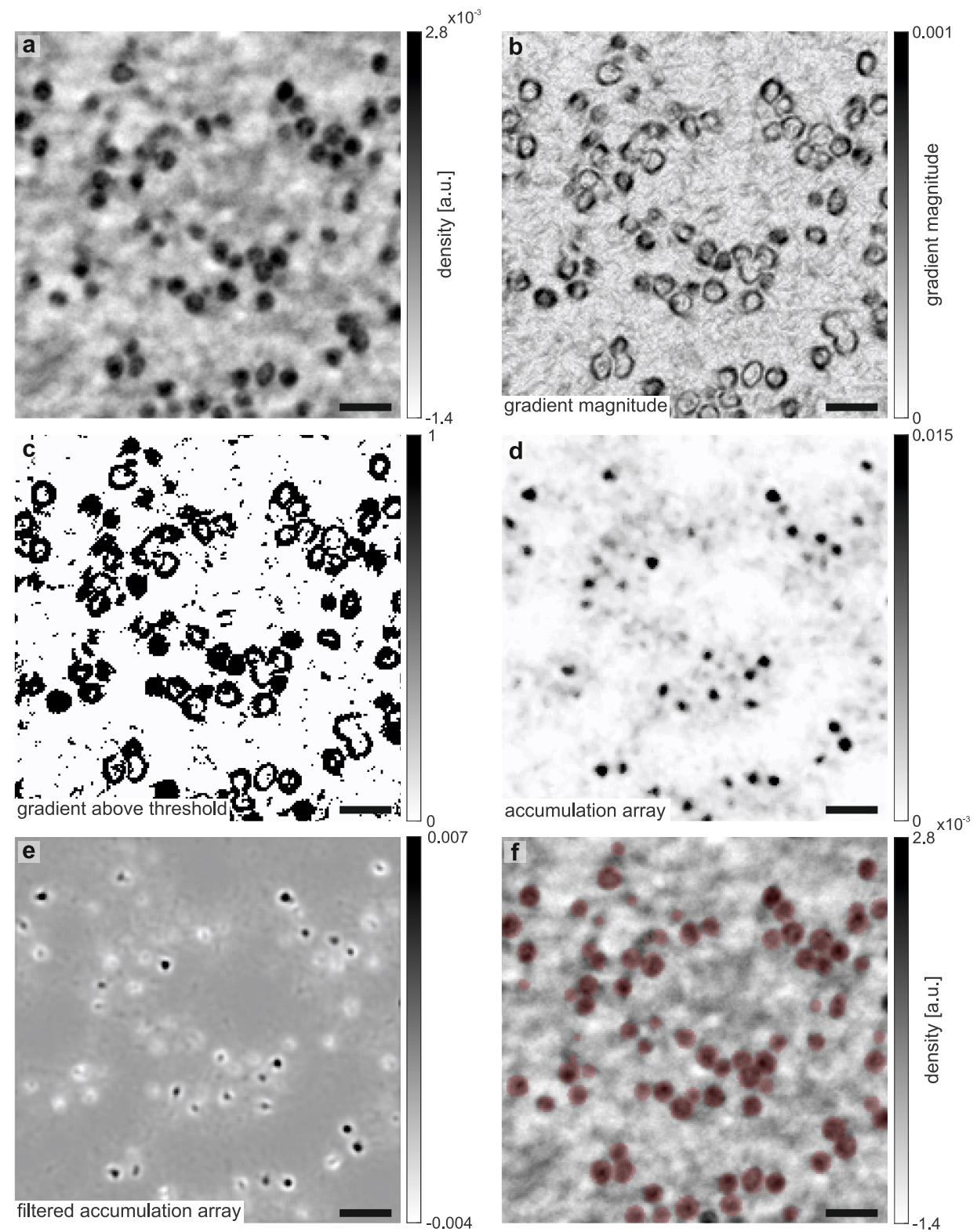

Figure 8.6: Visualization of the spherical Hough transform at one exemplary slice, shown in (a). (b) Corresponding magnitude of the gradient vectors, revealing the edges of the cells. (c) Pixels within the gradient image that lie above a specified threshold value are selected for building the accumulation array. (d) Resulting accumulation array with maxima at the location of the cell centers. (e) The application of a Laplacian of Gaussian filter on the accumulation array leads to an isolation of the maxima. (f) Resulting segmentation superimposed on the slice. Scale bars: $10 \mu \mathrm{m}$ 
in the surrounding tissue, which were likewise interpreted as edge pixels due to their large gradient magnitude, only lead to a non-zero background in the resulting accumulation matrix and hence have no negative influence on the localization of spherical structures. To detect the peaks in the accumulation array, the following steps are subsequently carried out:

- The accumulation matrix is thresholded, i.e., all pixels that have an intensity larger than $10 \%$ of the maximum value are set to 1 , while the rest is set to 0 .

- A Laplacian of Gaussian (LoG) filter [104] is applied to the accumulation matrix to clearly isolate local peaks from each other by filling the intermediate space with negative values (cf. Fig. 8.6).

- Each region within this filtered accumulation matrix that has a positive value and is simultaneously marked as 1 in the thresholded accumulation matrix is interpreted as a sphere. The location of the sphere center is determined by calculating the weighted centroid of the corresponding region in the original accumulation matrix.

The radius of the sphere is determined via the so-called signature curve $f(r)$, where $r$ is the distance to the center of the sphere and $f(r)$ is calculated via the averaged dot product between the gradient and radial vector, normalized by its length [133]. The calculation is carried out as follows: The gradient of a pixel $(i, j)$, located in the neighborhood of the central pixel of the sphere $\left(i_{c}, j_{c}\right)$, is given by $\mathbf{V}(i, j)$, while the radial vector is defined as $\mathbf{q}(i, j)=\left(i-i_{c}, j-j_{c}\right)$. For the determination of the signature curve, a sequence of discrete $r$ values is selected with fixed interval $\Delta r$, representing varying distances to the sphere center. The signature curve is then given by

$$
f(r)=\frac{\sum_{(i, j)} \frac{\mathbf{V}(i, j) \cdot \mathbf{q}(i, j)}{|\mathbf{q}(i, j)|}}{\sum_{i, j} 1}
$$

where the summation is carried out over all pixels $(i, j)$ that satisfy

$$
|| \mathbf{q}(i, j)|-r|<\frac{\Delta r}{2}
$$

The position of the maximum of this signature curve corresponds to the edge of the sphere and hence its radius.

For the application of the spherical Hough transform on data obtained at the GINIX setup in cone-beam geometry, the volume was first divided into multiple 
subvolumes in order to decrease computation time. Several parameters had to be chosen manually, namely the gradient threshold to select relevant pixels for building the accumulation matrix, the range of possible radii, the minimal intensity value of the detected sphere centers, the multiple radii tolerance $(0.1 \rightarrow$ several possible radii, $1 \rightarrow$ only 'principal' radius), and the size of the LoG filter. By changing the size of this filter, deviations from a perfect sphere can be accounted for, as in this case, the individual line segments do not necessarily intersect in a single center pixel, leading to a scattering of possible center positions in the accumulation matrix. By choosing a larger filter radius, those scattered maxima can be combined to one. In order to have the same conditions for all subvolumes, the gradient threshold as well as the minimal center intensity value were set to an absolute value chosen based on visual inspection.

For the segmentation of the small cells within the granular as well as molecular layer, the parameters listed in Tab. 8.2 were chosen, leading to the results depicted in Figs. 8.6(f) and 8.7(a) for a small subset as well as the entire volume, respectively. The position of the detected spheres and the cells coincide very well,

\begin{tabular}{lc}
\hline parameter & setting \\
\hline radius range [pixels] & $3-10$ \\
gradient threshold & $3.4 \cdot 10^{-4}$ \\
filter radius [pixels] & 3 \\
multiple radius tolerance & 1 \\
object center intensity threshold & 0 \\
\hline
\end{tabular}

Table 8.2: Parameters chosen for the spherical Hough transform in order to automatically detect all cells in the cone-beam measurement at the GINIX setup.

according to visual inspection, which indicates the applicability of the spherical Hough transform for the automated cell segmentation in the human cerebellum. In total, approximately 42,000 cells could be located automatically, again proving the non-feasibility of a manual segmentation. Note that the performance of the algorithm seemed to improve by increasing the signal-to-noise ratio at the cost of resolution via resampling by a factor of 2 as well as a Gaussian filter with a standard deviation of 0.3 pixels. Multiple segmentation of the same cell, mainly due to an overlap between the subvolumes, was avoided by merging automatically detected cells with a distance smaller than $2 \cdot(\min$ (radius $)-0.5)$ pixels. The subtraction of 0.5 pixels from the minimum radius accounts for the fact that only integer values for the radii can be determined by the algorithm.

The algorithm does not distinguish between different cell types and thus blood 

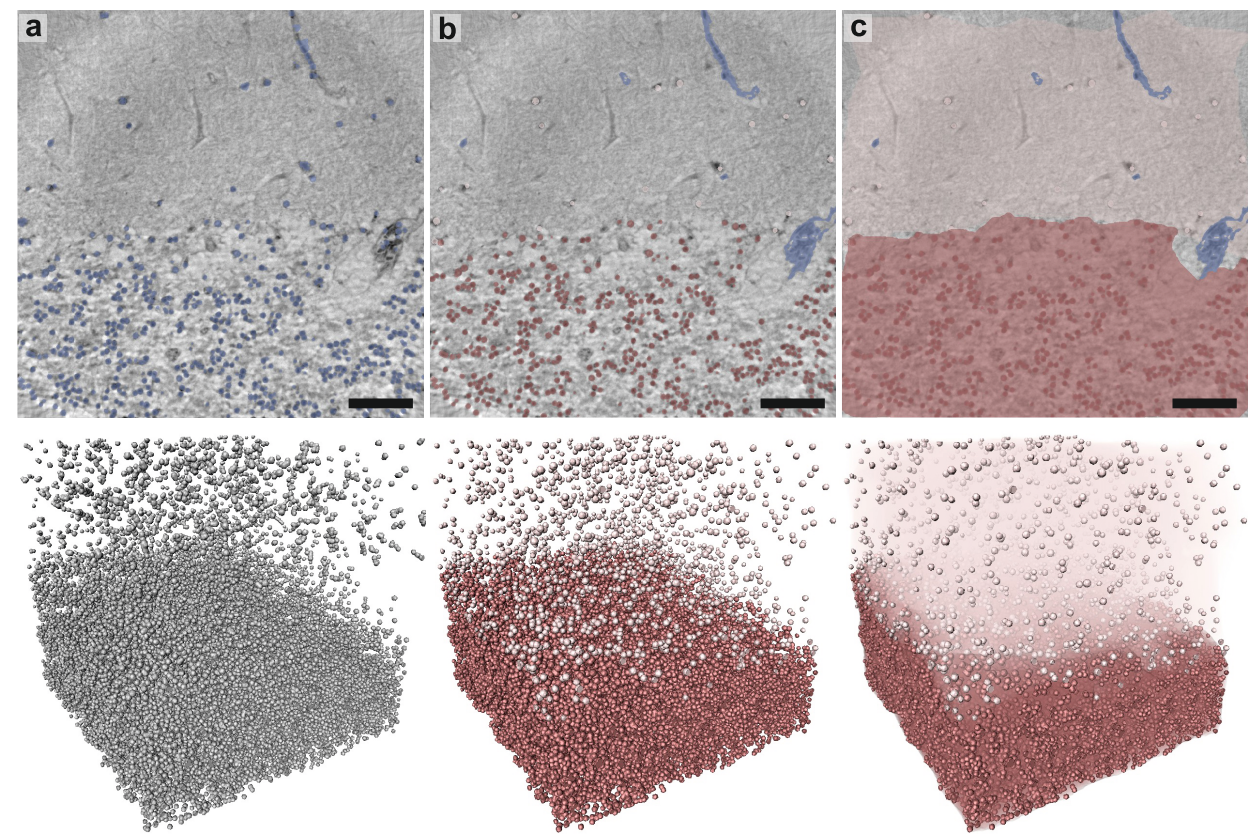

Figure 8.7: Results of the automated segmentation procedure, shown in an exemplary $2 d$ slice through the reconstruction volume (top panels) as well as in a $3 d$ view (bottom panels). (a) Superposition of all cells detected by the algorithm (blue) and the corresponding virtual slice through the volume. (b) After removal of blood vessels (blue), a separation into molecular layer (light red) and granular layer (dark red) was carried out by defining a threshold value for the mean distance to the 35 nearest neighbors of each cell. (c) An estimation for the volume of each layer was obtained by determining a hull around the respective cells. Scale bars: $50 \mu \mathrm{m}$

cells, which are contained in the blood vessels and are of similar size as the cells in the granular layer (cf. Fig. 8.8), were detected as well. To account for this, a semi-automatic segmentation of these vessels was performed in Avizo, using the region growing-based tool Magic Wand, by manually defining several seeding points within the vessels, and the corresponding cells were removed from the automatic result, as depicted in Fig. 8.7(b). Additionally, a separation into the low-cell molecular layer and the cell-rich granular layer was performed based on the mean distance to the 35 nearest neighbors. The threshold for the division was chosen based on visual inspection, leading to approximately 40,000 cells in the granular and 1,700 cells in the molecular layer. The volume of each of the layers, which will be used for the calculation of the cell density in section 8.2.2.3, was determined via the function boundary implemented in Matlab. It builds a $3 \mathrm{~d}$ hull enveloping all cells contained within the respective layer and enables the choice of a shrinking 

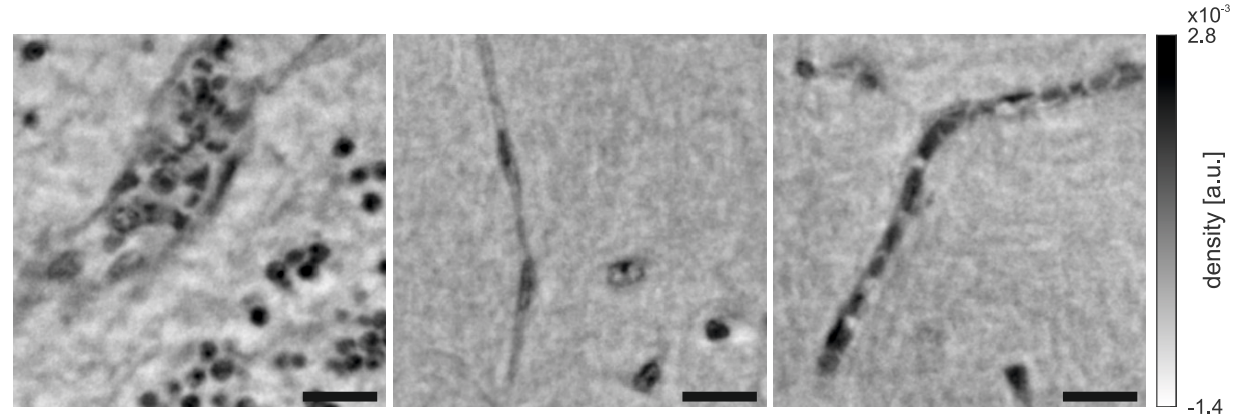

Figure 8.8: Exemplary blood vessels found in the reconstructed volume. The blood cells within the vessels are well resolved and therefore also found by the automatic cell segmentation algorithm. Scale bars: $15 \mu \mathrm{m}$

factor, where 0 gives a convex hull and 1 gives a compact boundary. In this case, the shrinking factor was set to the standard value of 0.5 . The resulting volumes, together with the cells within the two layers, are depicted in Fig. 8.7(c), both in an exemplary $2 \mathrm{~d}$ slice through the volume as well as for the entire dataset in $3 \mathrm{~d}$. By combining the output of the automated segmentation in the granular as well as molecular layer with a semi-automatic segmentation of the Purkinje cells within the reconstructed volume, which was performed analogously to the parallel-beam setup, a $3 \mathrm{~d}$ visualization of all the different cells within the cerebellum can be obtained, as depicted in Fig. 8.9. Different properties of the cerebellar layers are immediately visible, e.g., the smaller size of the cells within the molecular and granular layer compared to the large Purkinje cells, the significantly higher cell density in the granular layer compared to the molecular layer as well as the flat shape of the parallel oriented Purkinje cells with their highly arbored dendritic tree protruding into the molecular layer. Note that the two individual Purkinje cells are the same cells as depicted in Fig. 8.3(b) for the parallel-beam setup (top left and bottom right) and that the size of the segmented dendritic tree is mainly limited by the field of view of the dataset.

\subsubsection{Performance of the algorithm}

The performance of the segmentation algorithm was quantified via a comparison to a manual segmentation used as ground truth in several subvolumes of the whole dataset. To this end, the precision $p$ and recall $r$ were determined for each of the subvolumes. The precision is a measure of how many of the automatically detected cells correspond to real cells in the volume and it is calculated by the ratio between 
a
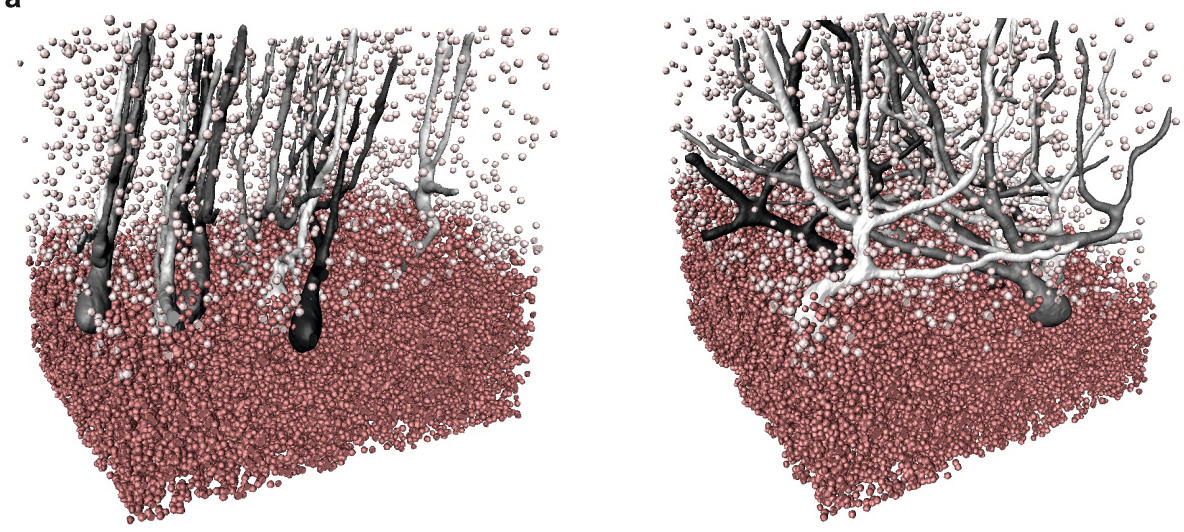

b
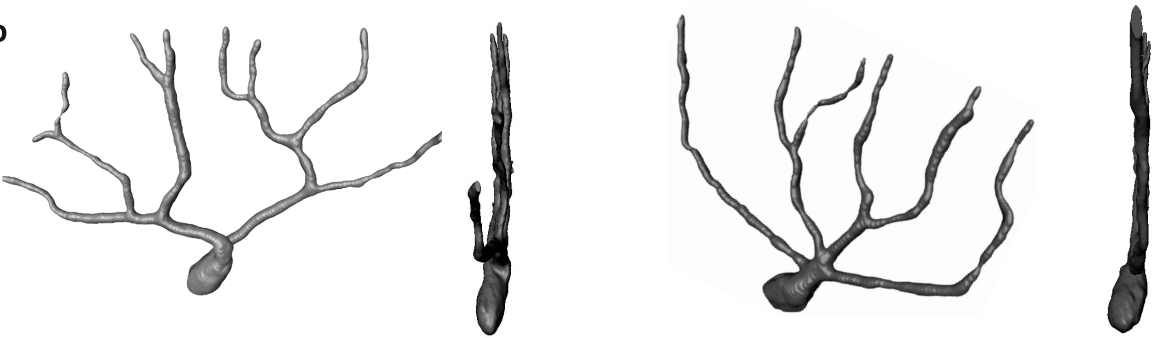

Figure 8.9: Cellular segmentation of the dataset obtained in cone-beam geometry at the synchrotron. (a) Each cell within the granular layer (dark red), the molecular layer (light red) and the Purkinje cell layer (shades of gray) can be visualized in $3 d$. For the large Purkinje cells, a semi-automatic approach was used for the segmentation, whereas the small cells in the granular and molecular layer could be located automatically via the spherical Hough transform. (b) In addition, two individual Purkinje cells are depicted, both front and side view, again showing the typical flat shape of this cell type.

the number of correctly segmented cells and the total number of segmented cells. The recall indicates how many of the real cells in the volume were detected by the algorithm and it is determined by the ratio between the number of correctly segmented cells and the total number of real cells in the volume (cf. Fig. 8.10).

The performance of the algorithm was quantified separately for the granular and molecular layer. Within the granular layer, two subvolumes with a size of $100^{3}$ voxels were randomly selected for manual segmentation, each containing $\sim 180$ cells. The position of these subvolumes with respect to the entire layer is indicated in Fig. 8.11(a). A comparison between the manually and automatically detected cells is shown in (b-d), both in $2 \mathrm{~d}$ slices and in 3d. Already by visual inspection, the agreement between these two segmentations is evident. The precision and recall within the two subvolumes yield $(p, r)=(1,1)$ and $(p, r)=(0.994,1)$ 

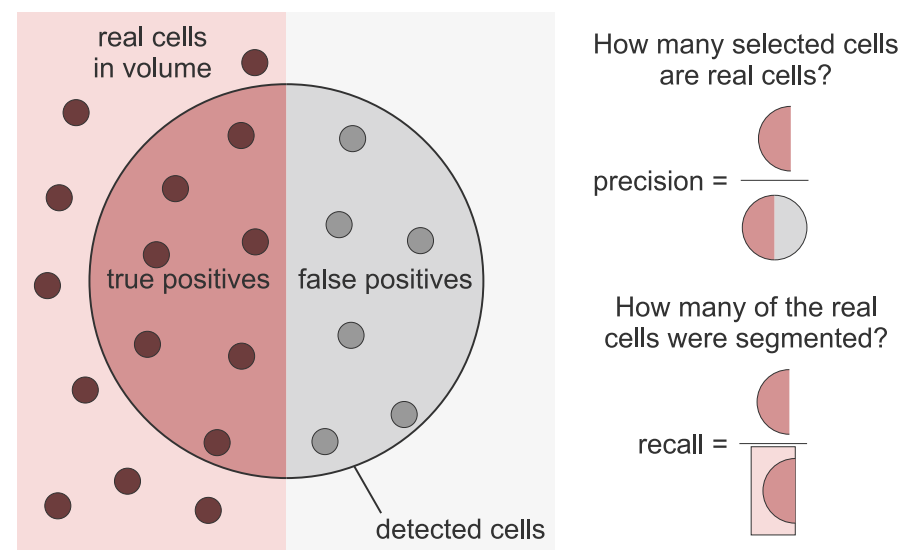

How many of the real cells were segmented?

Figure 8.10: Definition of precision and recall. The precision is a measure of how many of the automatically detected cells correspond to real cells in the volume and it is calculated by the ratio between the number of true positive findings and the total number of detected cells. The recall indicates how many of the real cells in the volume were detected automatically by the algorithm and it is determined by the ratio between the number of true positive findings and the total number of real cells in the volume. The drawing is based on [29].

for subvolume 1 and 2, respectively. In the molecular layer three subvolumes with twice the side length compared to granular layer $\left(200^{3}\right.$ voxels $)$ were chosen for manual segmentation due to the lower cell density, each containing only $\sim 20-30$ cells. The location of these subvolumes with respect to the entire layer is depicted in Fig. 8.12(a). The comparison between the manual and automatic segmentation, shown in (b) and (c) in $2 \mathrm{~d}$ slices as well as in $3 \mathrm{~d}$, yield precision and recall values of $(p, r)=(0.9,1)$ (subvolume 1$),(p, r)=(0.88,1)$ (subvolume 2$)$ and $(p, r)=(0.9,0.95)$ (subvolume 3$)$. Hence, the mean precision and recall obtained for the granular and molecular layer are $(p, r)=(0.994,1)$ and $(p, r)=(0.89,0.98)$, respectively, indicating an almost perfect performance of the automated segmentation algorithm, especially in the granular layer. The slightly worse performance within the molecular layer can be attributed to the diversity in cell size and shape compared to the more uniform distribution within the granular layer, which makes it more challenging to find one set of parameters for all cells (cf. Fig. 8.13). The nonetheless high accuracy of the automatic cell segmentation algorithm enables the determination of a variety of statistical measures based on the structural arrangement of ten thousands of cells within the three-dimensional volume, as shown for selected examples in the following section. 
a

C

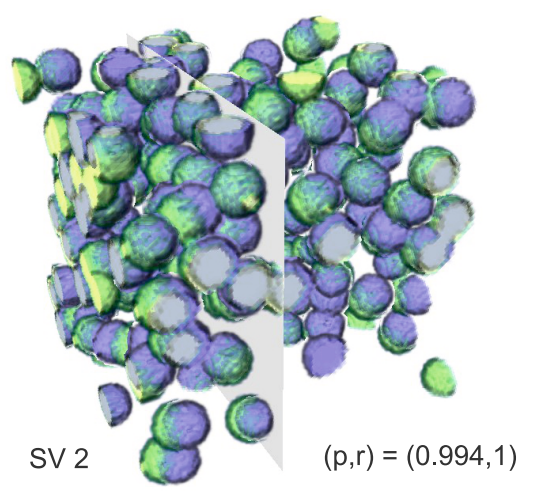

manual segmentation

automatic segmentation
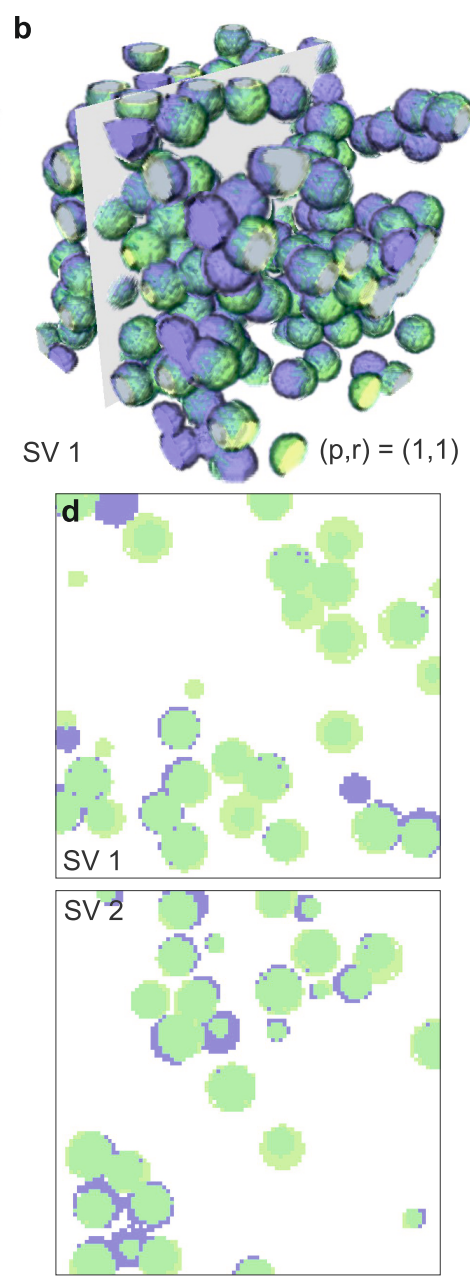

Figure 8.11: Comparison between manual and automatic segmentation results for the granular layer in the cone-beam dataset obtained at the GINIX setup. (a) Position of two randomly selected subvolumes $S V 1$ and $S V 2$ with volumes of $100^{3}$ voxels with respect to the entire granular layer. For these two subvolumes, a manual segmentation was performed and used as ground truth. (b-d) Comparison between the results of the automatic workflow (blue) and the manually obtained results (green). The positions of the $2 d$ slices in (d) are indicated in the volume renderings in (b) and (c), respectively. The performance of the automatic segmentation procedure was evaluated via the precision and recall, which were obtained for each subvolume separately and resulted in $(p, r)=(1,1)$ in subvolume 1 and $(p, r)=(0.994,1)$ in subvolume 2 . 


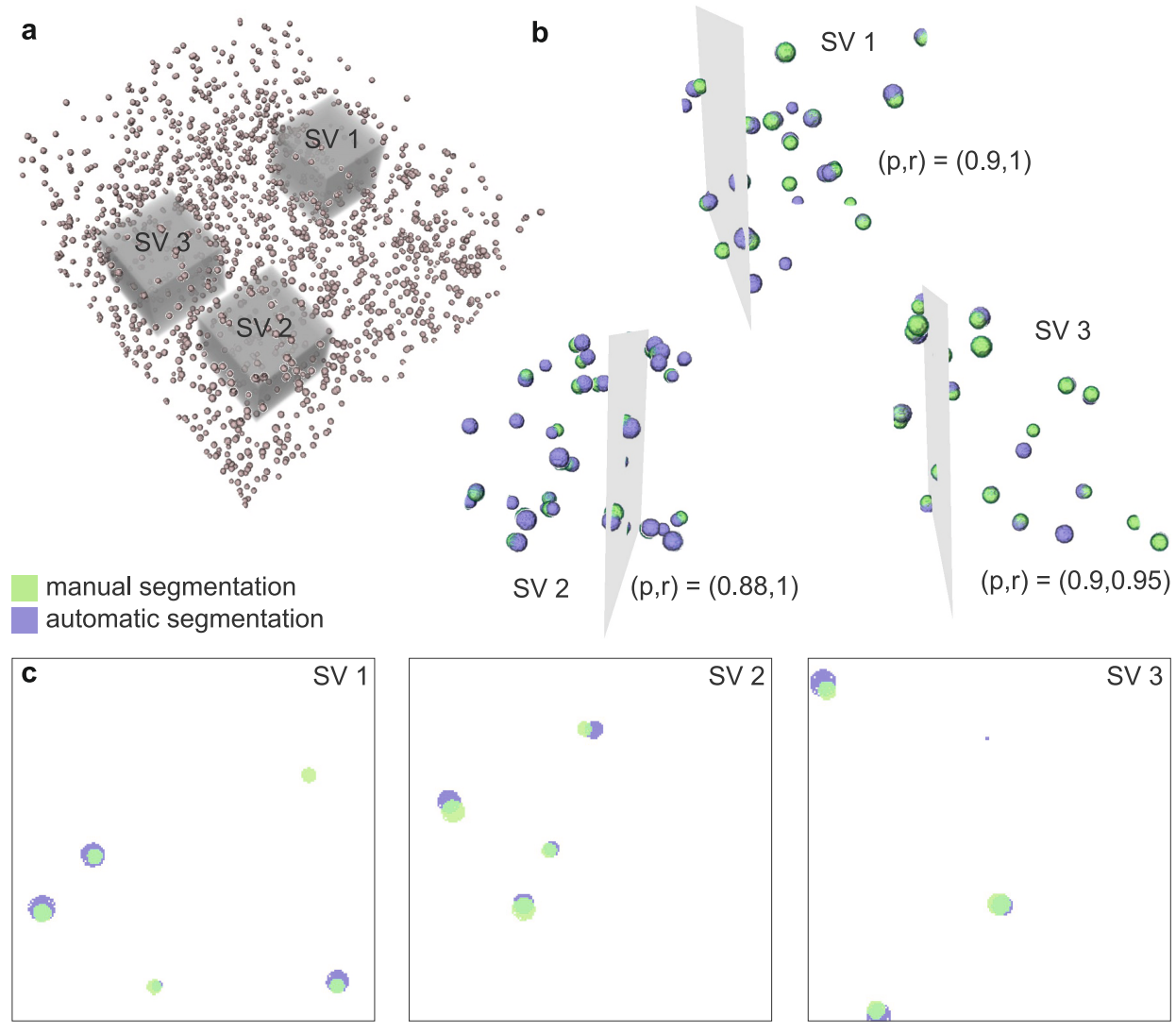

Figure 8.12: Comparison between manual and automatic segmentation results for the molecular layer. (a) For the manual segmentation, three randomly chosen subvolumes from the molecular layer were used with twice the side length compared to the granular layer $\left(200^{3}\right.$ voxels) due to the lower cell density and hence reduced statistics. $(b, c)$ Comparison between the manually segmented (green) and automatically detected cells (blue). The positions of the $2 d$ slices shown in (c) are depicted in the $3 d$ representations in (b). The precision and recall within the three subvolumes yield $(p, r)=(0.9,1)$ in subvolume $1,(p, r)=(0.88,1)$ in subvolume 2 and $(p, r)=(0.9,0.95)$ in subvolume 3 . Hence, the performance within the molecular layer is slightly worse compared to the granular layer, which can be mainly attributed to the larger diversity in cell size and shape (see Fig. 8.13).

\subsubsection{Statistical measures}

The cellular density in the molecular as well as granular layer was determined via the ratio between the total number of cells and the volume estimated for each layer, yielding $\rho=9.9 \cdot 10^{4} \mathrm{~mm}^{-3}$ and $\rho=2.7 \cdot 10^{6} \mathrm{~mm}^{-3}$, respectively, which is in good agreement with manual cell counting in $2 \mathrm{~d}$ histological sections [3]. Note 


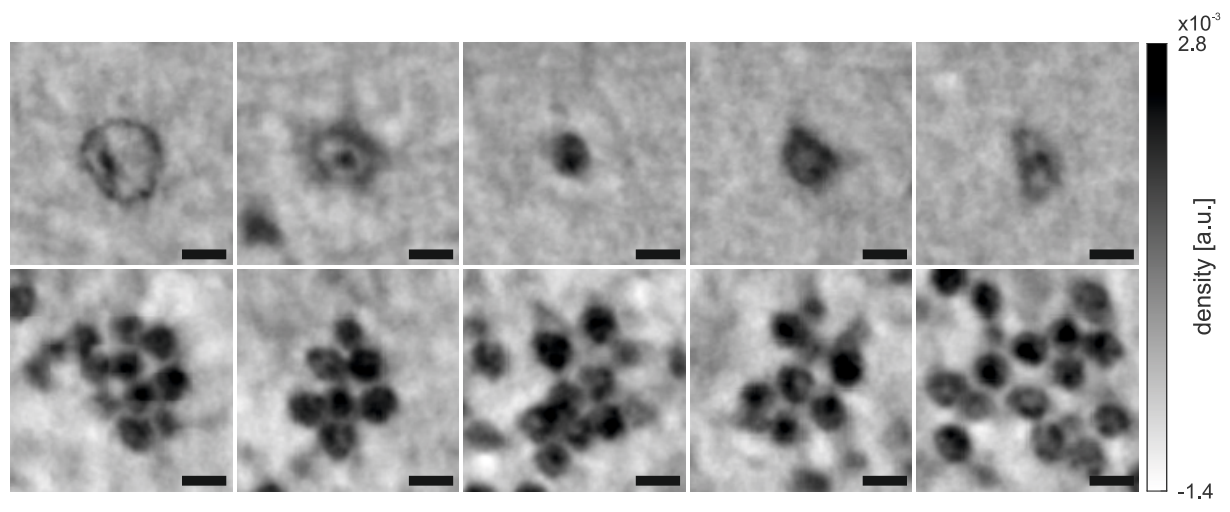

Figure 8.13: Comparison of cell types found in the molecular and granular layer of the cerebellum. Upper row: Examples of different cells in the molecular layer, indicating the diversity occurring in this layer. Lower row: Examples of cells in the granular layer, showing a more uniform distribution of cell shapes and sizes. Scale bars: $5 \mu \mathrm{m}$

that this corresponds to the average density of the two layers. A local density estimation was obtained via a convolution between the cell center positions and a sphere with varying radius, normalized by the respective volume of the sphere, as depicted in Fig. 8.14 for the granular layer. The example for the smallest sphere radius and thus the smallest volume for the determination of the local density shows large variations throughout the layer with distinct hotspots, indicating a clustering of the cells. With increasing radius, the differences in local cell density are vanishing, leading to an almost uniform distribution within the granular layer. However, the cell density is found to decrease considerably towards the molecular layer, indicating a smooth transition between the two layers.

From the positions of the cells within the volume, several statistical measures were obtained (cf. Fig. 8.15). The distribution of nearest-neighbor distances was determined by calculating all cell-to-cell distances and finding the minimum for each individual cell. The resulting histograms for the molecular and granular layer are shown in (a) and (b), revealing mean distances of $9.7 \pm 0.8 \mu \mathrm{m}$ (95\% confidence bounds) and $4.00 \pm 0.02 \mu \mathrm{m}$ with standard deviations $\sigma=7.3 \pm 0.8 \mu \mathrm{m}$ and $\sigma=0.45 \pm 0.02 \mu \mathrm{m}$, respectively. Considering the mean radius of cellular nuclei in the two layers (2.4 $\mathrm{mm}$ in the molecular and $2.1 \mu \mathrm{m}$ in the granular layer), this indicates that within the granular layer most of the cells have at least one neighboring cell in direct contact, whereas in the molecular layer the distance to the nearest cell is several cell radii larger.

By determining the mean gray value within the volume of a single cell, a measure 

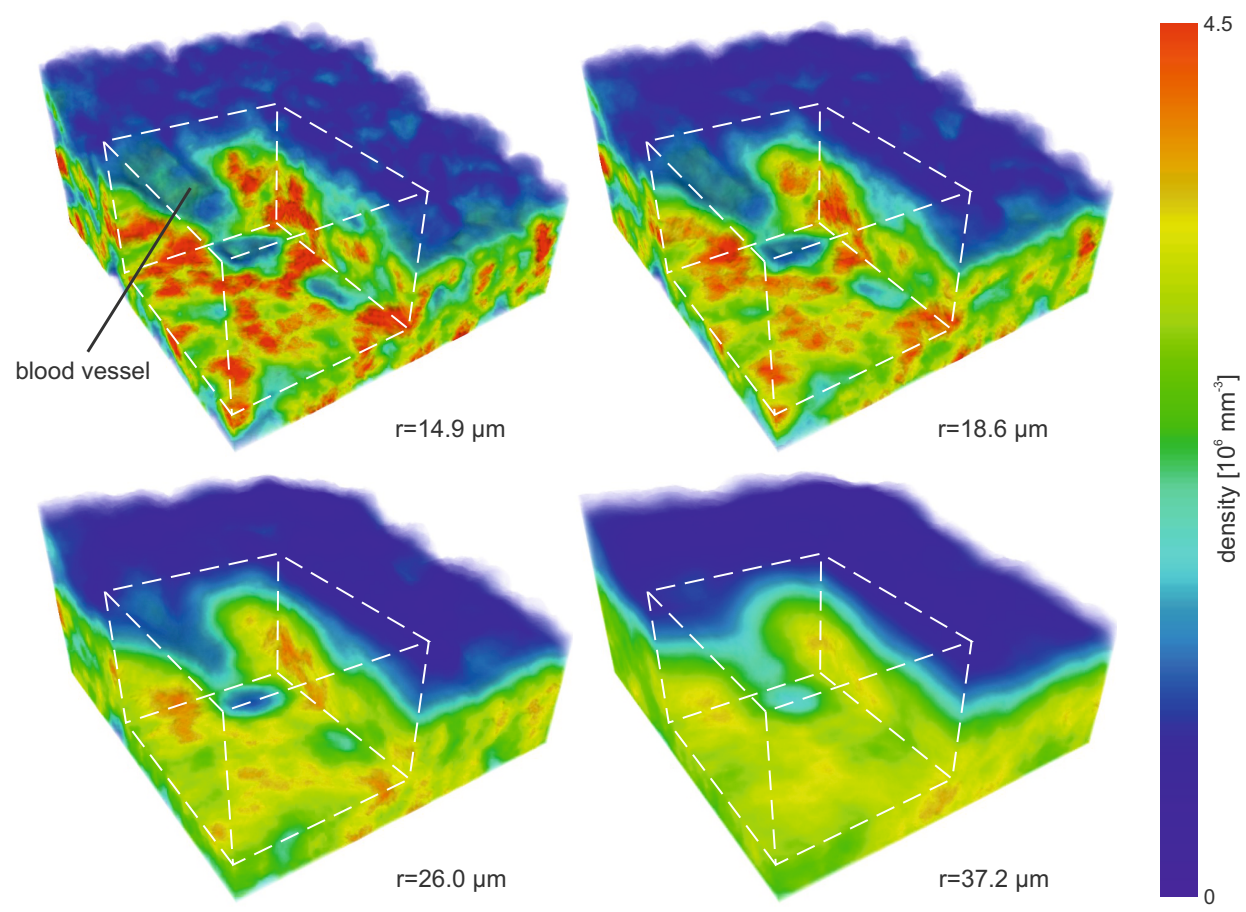

Figure 8.14: Local cell density distribution within the granular layer of the cerebellum, which was obtained via a convolution between the cell center positions and a sphere with varying radius, normalized by the respective volume of the sphere. The example for a sphere radius of $14.9 \mu \mathrm{m}$ shows distinct hotspots in density and hence indicates a clustering of cells within this layer. With increasing radius, the density distribution becomes more homogeneous. However, towards the interface between the granular and molecular layer, the cell density decreases considerably, indicating a gradual transition between the two layers.

of electron density can be obtained, as the reconstruction value is proportional to the real part of the refractive index. To this end, the gray values of the voxels within each cell were integrated and divided by the corresponding cell volume. The resulting histograms are shown in Fig. 8.15(c). A Gaussian fit to the mean gray values of the molecular layer yields $(1.17 \pm 0.02) \cdot 10^{-3}$ with standard deviation $\sigma=(2.9 \pm 0.2) \cdot 10^{-4}$, whereas for the granular layer a sum of two Gaussian functions fitted the data best, leading to $(1.24 \pm 0.01) \cdot 10^{-3}$ and $(1.40 \pm 0.02) \cdot 10^{-3}$ with standard deviations $\sigma_{1}=(1.6 \pm 0.1) \cdot 10^{-4}$ and $\sigma_{2}=(3.4 \pm 0.1) \cdot 10^{-4}$ in an approximate ratio of 30:70. The occurrence of two peaks in the histogram might be explained by different cell types in the granular layer with slightly different electron densities or artifacts due to the discrete representation of spheres with 

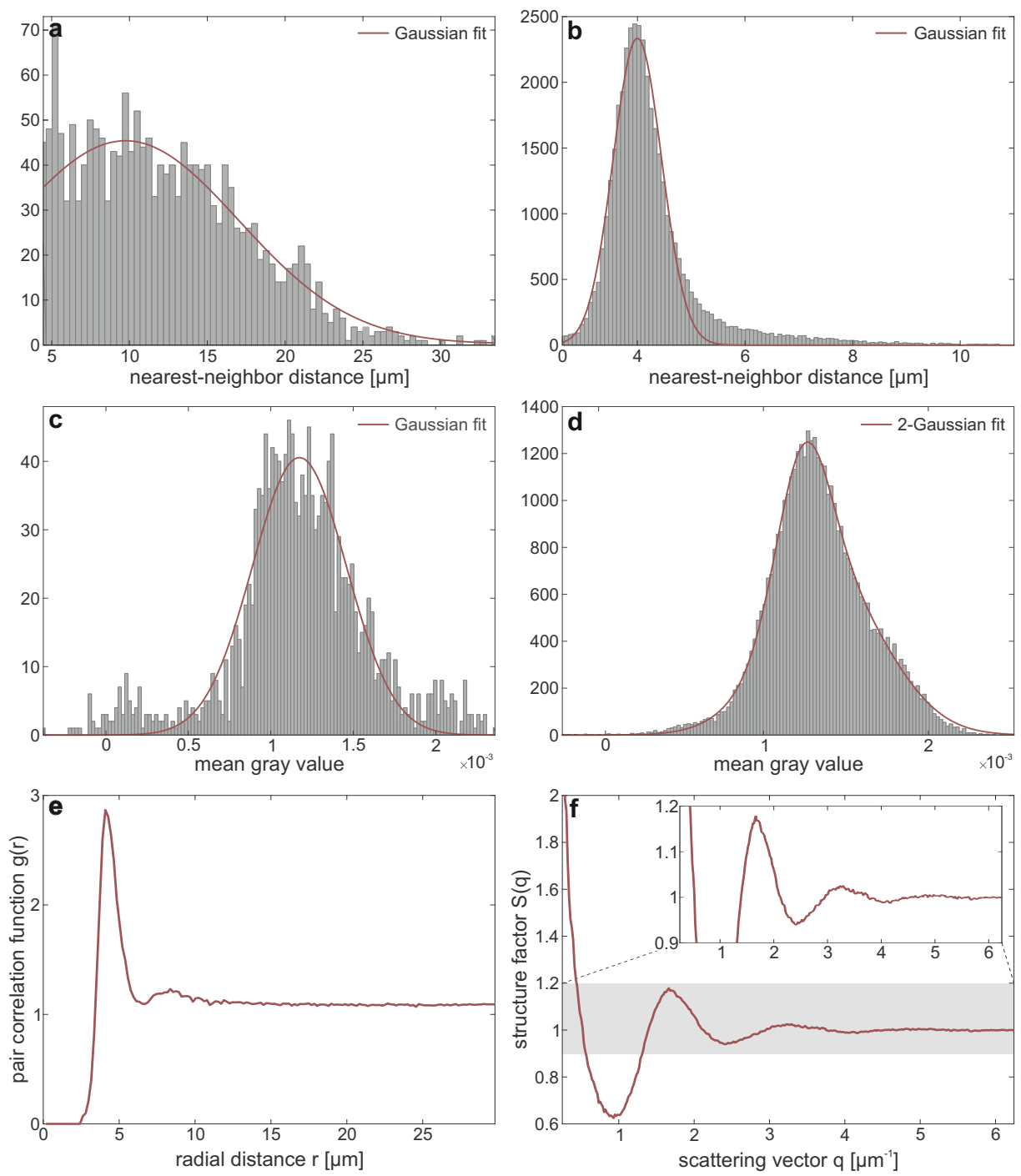

Figure 8.15: Statistical measures obtained from the automatically determined cell positions. (a,b) Histograms showing the nearest-neighbor distances in the molecular and granular layer, respectively. The Gaussian fits reveal mean nearest-neighbor distances of $9.7 \pm 0.8 \mu \mathrm{m}$ and $4.00 \pm 0.02 \mu \mathrm{m}$ (95\% confidence interval). (c,d) Histograms showing the mean gray value within the automatically detected cell volumes in the molecular and granular layer, respectively. A Gaussian fit leads to $(1.09 \pm 0.02) \cdot 10^{-3}$ in the molecular layer, whereas for the granular layer the shape is best fitted by a 2-Gaussian function with peak values of $(1.10 \pm 0.01) \cdot 10^{-3}$ and $(1.24 \pm 0.02) \cdot 10^{-3}$ in an approximate ratio of 30:70. (e) Pair correlation function of the cells in the granular layer, revealing two distinct peaks at $4.16 \pm 0.04 \mu \mathrm{m}$ and $8.5 \pm 0.3 \mu \mathrm{m}$. (f) Structure factor of the cells in the granular layer, corresponding to the Fourier transform of the pair correlation function. 
radii in the range of few pixels. In general, the comparison between the results for the molecular and granular layer indicates that the cells of the latter have a slightly higher density.

As a last step, the structural organization of the cells in the granular layer was further quantified. To this end, the pair correlation function $g(r)$ was computed, which is a measure for the probability of finding a cell at a certain distance $r$ from a given cell. It relates the bulk density $\rho^{\text {bulk }}$ to the average density of cells at distance $r$ via $\rho(r)=g(r) \rho^{\text {bulk }}[23]$ and is hence given by

$$
g(r)=\frac{N(r)}{4 \pi r^{2} \mathrm{~d} r \rho^{\mathrm{bulk}}},
$$

where $N(r)$ is the mean number of cells in a shell with width $\mathrm{d} r$ at radial distance $r$.

The pair correlation function was calculated by counting the number of cells in a spherical shell with radius $r$ and a width of 0.5 pixels around a single cell in the granular layer and averaging the resulting curve over all cells. Note that the maximum radius that was taken into account was limited by the distance of the considered cell to the edge of the layer. The resulting correlation function is depicted in Fig. 8.15(e), with two distinct peaks at $4.16 \pm 0.04 \mu \mathrm{m}$ and $8.5 \pm 0.3 \mu \mathrm{m}$, indicating a strong short-range order. The fact that the distance to the nearest neighbor is significantly smaller than the mean inter-nuclei distance $r_{m}=\rho^{-1 / 3}=7.2 \mu \mathrm{m}$, computed from the density, also points to a local clustering of the cells.

The coordination number is a measure for the mean number of neighbors within a distance $r$ from each cell and it is given by the integral of the pair correlation function [23]

$$
n_{\text {coord }}(r)=4 \pi \rho \int_{0}^{r} g\left(r^{\prime}\right) r^{\prime 2} \mathrm{~d} r^{\prime} .
$$

By executing the integration until the first minimum in the pair correlation function and hence over the first coordination shell, the mean number of nearest neighbors of each cell was determined. In contrast to an efficiently packed non-associated fluid, yielding a coordination number of 12 [11], the mean number of nearest neighbors of the present structure is approximately 5, again indicating an organization in small clusters. For clarity, a summary of the obtained statistical parameters is listed in Tab. 8.4 at the end of the chapter. 
Under the assumption of identical cells, the structure factor $S(q)$ is given by [43]

$$
S(q)=\frac{1}{N}\left|\sum_{k} e^{i \mathbf{q} \cdot \mathbf{r}_{k}}\right|^{2}
$$

where $N$ is the number of cells within the volume, $\mathbf{q}$ the scattering vector and $\mathbf{r}_{k}$ the position of the $k$-th cell. The structure factor and pair correlation function are related via Fourier transform, yielding

$$
S(q)-1=\frac{4 \pi \rho}{q} \int_{\mathbb{R}} r(g(r)-1) \sin (q r) \mathrm{d} r
$$

for an isotropic material [43]. For the calculation of the structure factor, the angular average over the $3 \mathrm{~d}$ Fourier transform of the array containing the center positions of each sphere was computed, resulting in the curve depicted in Fig. 8.15(f).

\subsubsection{Tomographic imaging at the laboratory setup}

Phase retrieval for the laboratory data was performed with the BAC approach with regularization parameters $\alpha=0.01$ and $(\gamma \cdot F)=0.15$. In contrast to the CTF approach used for the parallel-beam GINIX setup, the BAC algorithm can better account for the violation of the homogeneous object assumption and hence, edge enhancement at the Kapton-air interface is reduced while the inner part of the sample remains sharp. In order to increase the signal-to-noise ratio, all projections were resampled by a factor of 2 , leading to twice the effective pixel size in the reconstructed volume. Prior to the tomographic reconstruction via the FDK implementation of the ASTRA toolbox, the wavelet-based ring-removal algorithm was applied on the sinograms to reduce ring artifacts in the resulting virtual slices. One exemplary slice through the reconstructed density is depicted in Fig. 8.16. Note that it was filtered with a Gaussian function with a standard deviation of 1 pixel to further increase the signal-to-noise ratio. It shows that also in the laboratory dataset single cells are resolved in all layers of the paraffinembedded human cerebellum. The magnified region on the right, corresponding to the slices in Figs. 8.3(a) (right panel) and 8.4 (left panel) from the synchrotron measurements, also reveals a Purkinje cell, including the thick branches of its large dendritic tree. Due to the cellular resolution within the granular as well as molecular layer, the developed workflow for automated cell segmentation based on the spherical Hough transform was also applied on the laboratory data. 


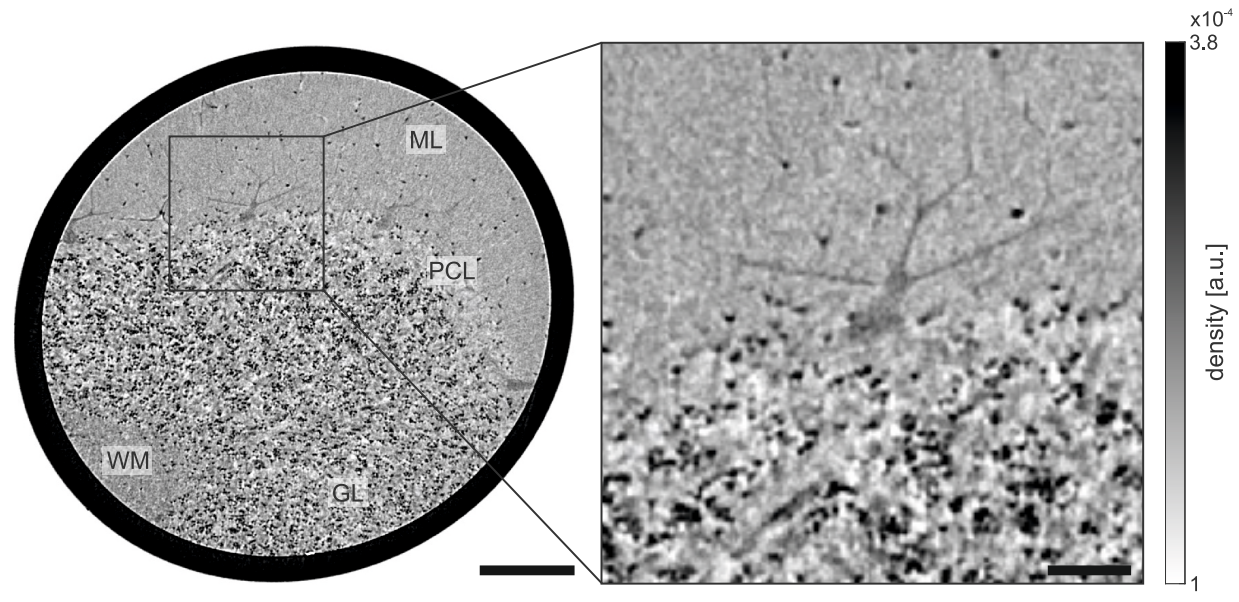

Figure 8.16: Virtual slices through the reconstructed volume from the laboratory dataset. The interface between the cell-rich granular layer and the low-cell molecular layer is clearly visible. Compared to the cone-beam measurement at the GINIX setup, a larger field of view is accessible while the resolution is still sufficient for single cell identification. Within the large Purkinje cells, even sub-cellular details as the dendritic tree are to some extent resolved. Note that the magnified region on the right corresponds to the slices shown in Figs. 8.3(a) (right panel) and 8.4 (left panel) from the synchrotron measurements in parallel- and cone-beam geometry, respectively. Scale bars: $200 \mu \mathrm{m}$ (left) and $50 \mu \mathrm{m}$ (right)

\subsubsection{Automated cellular segmentation in the laboratory results}

Spherical Hough transform for automated segmentation For the application of the spherical Hough transform on the laboratory data, the parameters listed in Tab. 8.3 were chosen based on visual inspection within a small subvolume of the data. Note that due to the large pixel size in the resampled dataset, the

\begin{tabular}{lc}
\hline parameter & setting \\
\hline radius range [pixels] & $1-6$ \\
gradient threshold & $3.1 \cdot 10^{-5}$ \\
filter radius [pixels] & 3 \\
multiple radius tolerance & 1 \\
object center intensity threshold & $1.6 \cdot 10^{-4}$ \\
\hline
\end{tabular}

Table 8.3: Parameters chosen for the spherical Hough transform in order to automatically detect the cells in the molecular and granular layer in the measurement at the laboratory setup. 

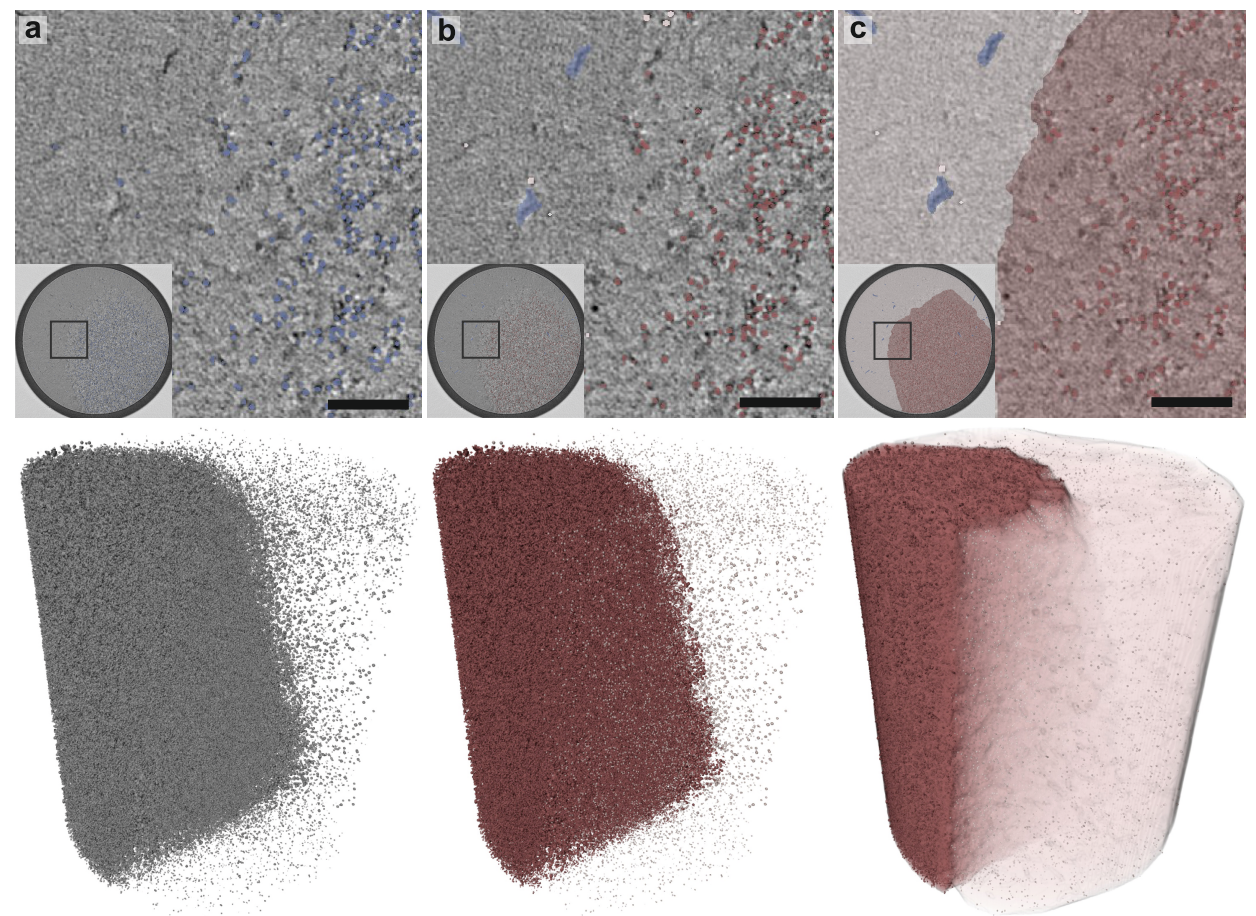

Figure 8.17: Results of the automated segmentation procedure for the laboratory data. (a) Output of the segmentation algorithm prior to additional processing steps, along with the corresponding volume rendering showing the $3 d$ spatial distribution. For better visibility, a $250 \times 250 \mathrm{\mu m}^{2}$ region, as indicated in the inset, is shown at higher magnification. (b) After semi-automatic removal of the cells contained in the blood vessels (blue), the segmentation results were divided into the molecular (light red) and granular layer (dark red) based on a visually defined threshold for the mean distance to the 35 nearest neighbors of each cell. (c) The volume of the two layers was determined via a $3 d$ envelope around the respective cells. Scale bars: $50 \mu \mathrm{m}$

cell radii were below 2 pixels and therefore not detectable. Thus, for the cellular segmentation the volume had to be reconstructed at full resolution with a subsequent low-pass filtering step to increase the signal-to-noise ratio. The resulting segmentation is shown in Fig. 8.17(a). Despite the inferior data quality compared to the synchrotron, the positions of the detected spheres and the cells seem to coincide very well. In total, the algorithm was able to detect approximately 1.8 million cells within the reconstructed field of view. As previously, cells contained within blood vessels were segmented as well and had to be removed via a semiautomatic segmentation of the vessels based on the Magic Wand tool in Avizo, leading to the results in (b). Additionally, a separation into the cell-rich granular 
a
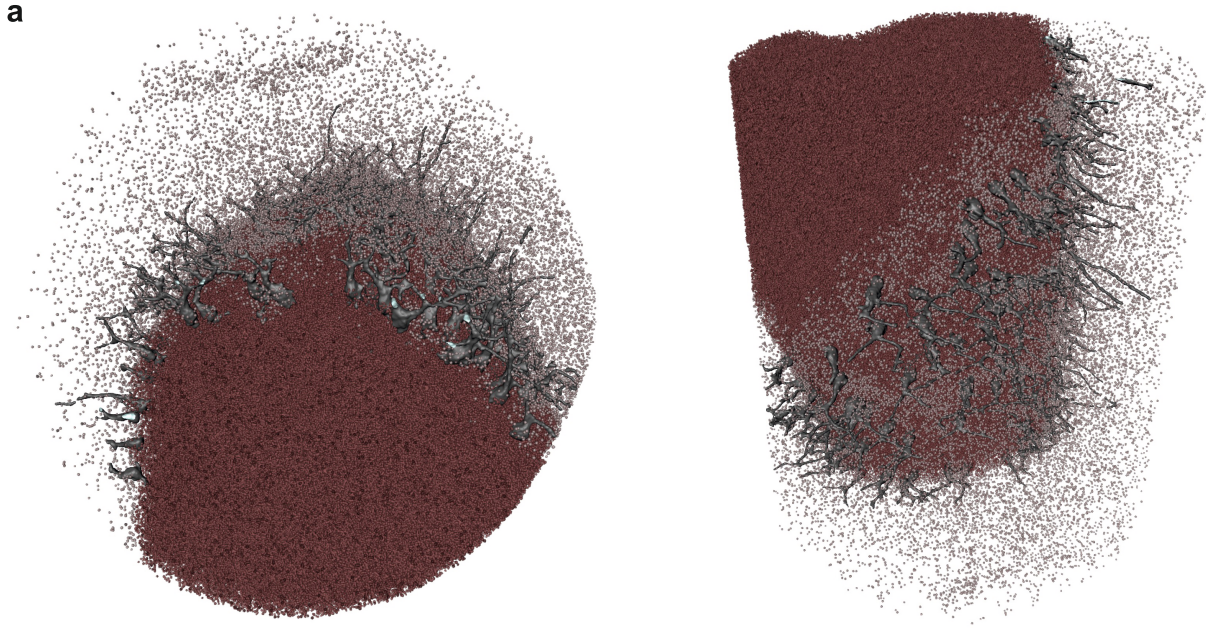

b
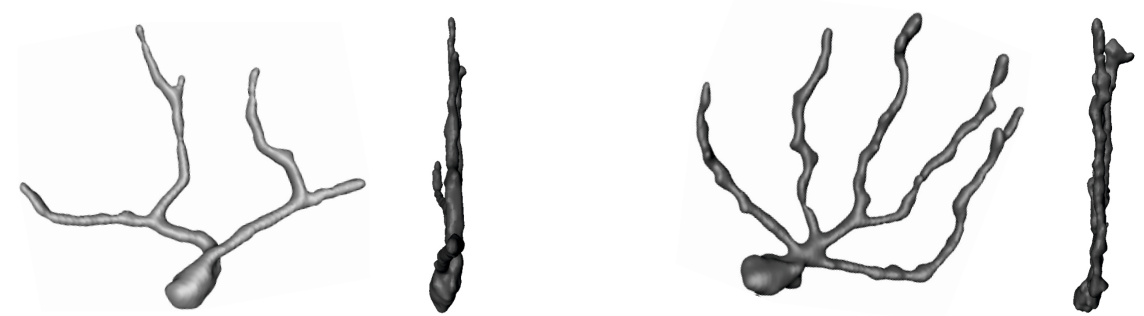

Figure 8.18: Cellular segmentation within the dataset obtained at the laboratory setup. (a) Each cell in the cerebellum can be visualized in 3d, including the cells in the granular layer (dark red), the molecular layer (light red) and the Purkinje cell layer (gray). (b) In addition, two individual Purkinje cells are depicted, both front and side view, again showing the typical flat shape of this cell type. Note that the cells correspond to those shown in Figs. 8.3(b) and 8.9(b).

and low-cell molecular layer was performed based on the mean distance to the 35 nearest neighbors, analogous to the synchrotron dataset. Again, the threshold for the division was based on visual inspection. The volume of each of the layers was determined via the function boundary implemented in Matlab and the standard shrinking factor 0.5, as depicted in Fig. 8.17(c).

By combining the results of the automated segmentation within the molecular and granular layer with a semi-automatic segmentation of the cells in the Purkinje cell layer via the Magic Wand tool, which was manually refined for two exemplary cells, a $3 \mathrm{~d}$ visualization of all cells within the measured portion of the human cerebellum can be obtained, as depicted in Fig. 8.18. The size differences between the cells in the molecular and granular layer and the Purkinje cells as well as the 
large cell density within the granular layer and the flat shape of the Purkinje cells become immediately apparent. Data quality at the laboratory is even high enough to segment the large branches of the Purkinje cells at comparable detail as in the synchrotron datasets (cf. Figs. 8.3(b) and 8.9(b)).

Performance of the algorithm In order to quantify the performance of the segmentation algorithm, the precision and recall were also determined for the laboratory dataset. Compared to the synchrotron dataset, a manual segmentation proved to be challenging due to the lower resolution and signal-to-noise ratio. Hence, the datasets obtained at both setups were manually aligned to each other and the result obtained from the automatic segmentation in the synchrotron dataset was considered as ground truth, which is justified by its high precision and recall. In Figs. 8.19 and 8.20 the position of the synchrotron dataset with respect to the laboratory data is indicated and the comparison between the datasets is depicted both in $2 \mathrm{~d}$ slices and in a $3 \mathrm{~d}$ volume rendering. The precision and recall for the laboratory dataset were estimated at $(p, r)=(0.71,0.72)$ for the molecular layer and $(p, r)=(0.85,0.93)$ for the granular layer. This shows that especially in the granular layer the performance of the algorithm is remarkably high and comparable to previous results obtained for osmium-stained tissue at synchrotron sources with a parallel-beam geometry [42].

Statistical measures The high precision and recall allows for a statistical analysis of the cellular distribution obtained from the laboratory dataset, analogous to the results of the cone-beam measurement at the GINIX setup.

Around 26,000 cells were identified automatically in the molecular layer, whereas the algorithm found approximately 1,760,000 cells in the granular layer, resulting in densities of $7.4 \cdot 10^{4} \mathrm{~mm}^{-3}$ and $3.4 \cdot 10^{6} \mathrm{~mm}^{-3}$, respectively. The deviation from the synchrotron results can be attributed to the lower precision and recall in the molecular layer, while in the granular layer, where precision and recall are high in both cases, it might be explained by the different probing volumes, as the field of view in the synchrotron data is much smaller compared to the laboratory dataset. This can be further quantified by regarding the local density within the granular layer, which was again obtained via a convolution of the cell center positions and spheres with varying radius, as depicted in Fig. 8.21. As for the synchrotron dataset (cf. Fig. 8.14), a clustering of the cells can be recognized as local density hotspots in the example with the smallest sphere of radius $13.8 \mu \mathrm{m}$. With increasing radius, the local density distribution becomes more homogeneous in the 
a

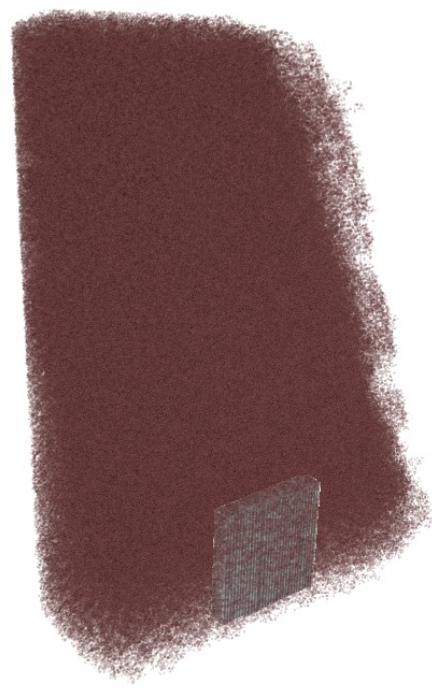

b

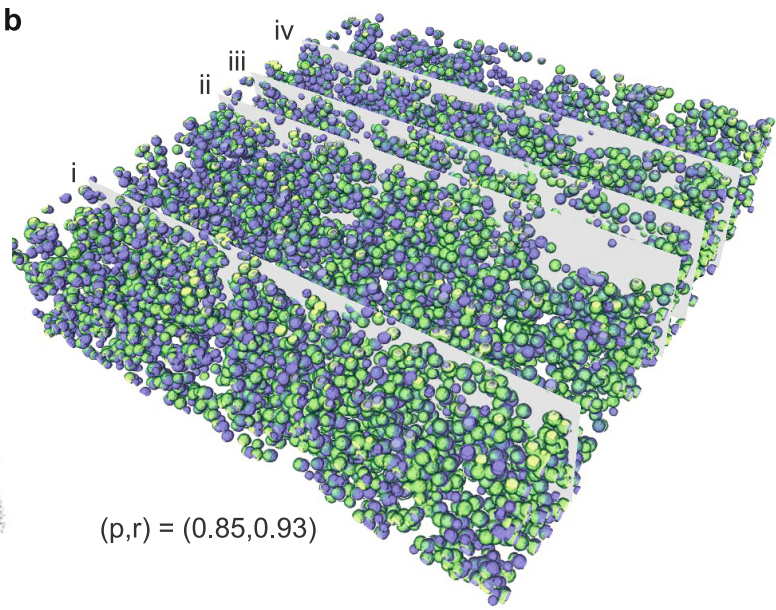

ground truth from SR data automatic segmentation
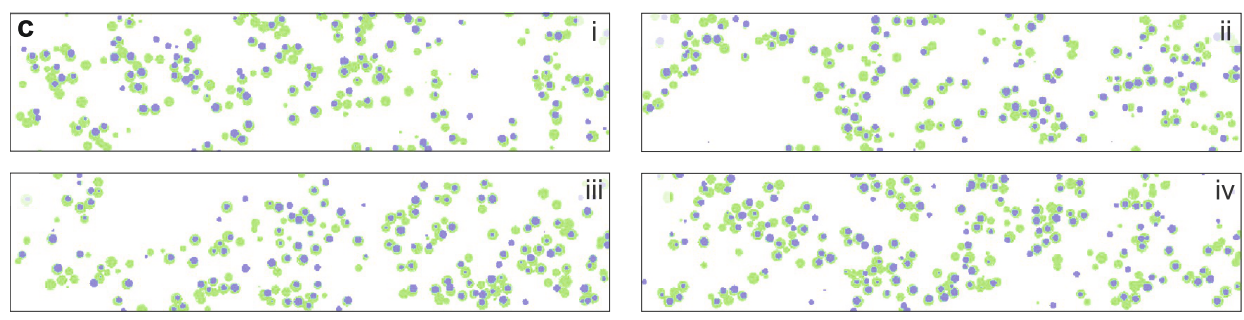

Figure 8.19: Comparison between the automatic segmentation result for the granular layer obtained in the laboratory as well as synchrotron dataset. (a) The position of the synchrotron measurement with respect to the laboratory data is indicated by the box in the respective cellular segmentation. (b) Comparison between the synchrotron results (green) and the segmentation results obtained for the laboratory data (blue) in $3 d$. (c) Slices through the rendering of the results from the synchrotron and the laboratory datasets, with the positions of the individual slices indicated in (b). Precision and recall were estimated at $(p, r)=(0.85,0.93)$, showing the good performance of the algorithm despite the lower data quality.

center of the layer, while it decreases significantly towards the interface to the molecular layer. On the one hand this indicates a smooth transition between the two layers, as in the synchrotron dataset, but on the other hand it also shows that the synchrotron dataset, which was measured at the edge between these two layer, comprises a less dense part of the granular layer, explaining the difference in overall density between the two datasets. By determining the cell density in the corresponding subvolume of the laboratory dataset, resulting in $2.9 \cdot 10^{6} \mathrm{~mm}^{-3}$, 
a

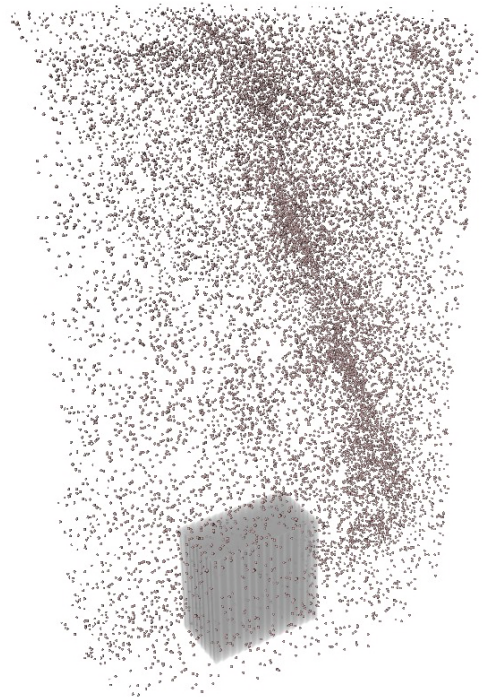

b

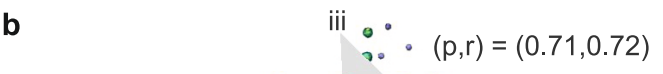

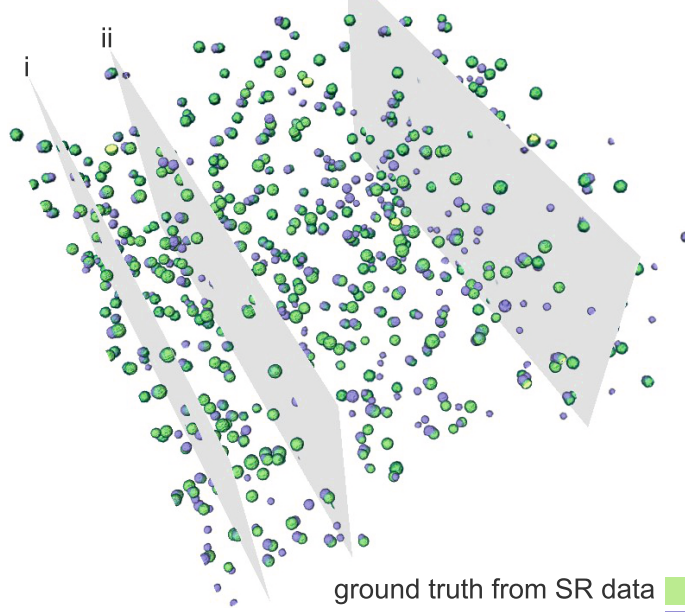
automatic segmentation
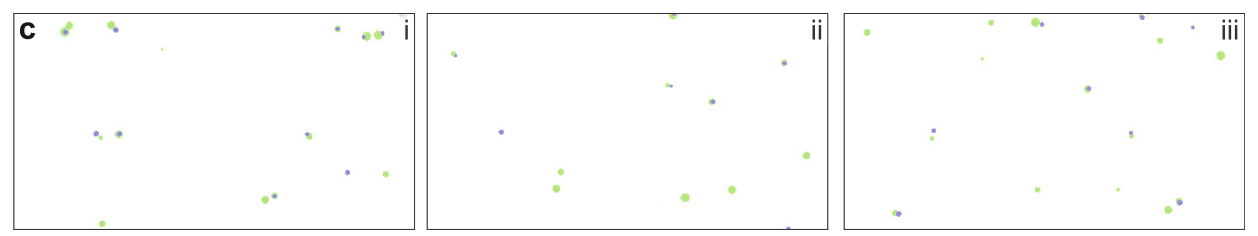

Figure 8.20: Evaluation of the performance of the automatic segmentation algorithm in the molecular layer of the laboratory dataset, with the synchrotron result considered as ground truth. (a) The position of the synchrotron dataset with respect to the laboratory data is indicated by the box in the cellular segmentation of the molecular layer. (b) Comparison between the segmentation results obtained from the synchrotron (green) and the laboratory data (blue) in 3d. (c) Slices through these segmentation results at the positions indicated in (b). Precision and recall were estimated at $(p, r)=(0.71,0.72)$.

this assumption can be confirmed. The remaining deviation by about $11 \%$ can be attributed to the difference in the precision and recall values for this layer.

From the segmentation results the same statistical measures as for the synchrotron dataset were determined (cf. Fig. 8.22). The distribution of nearest-neighbor distances in the molecular and the granular layer is shown in (a) and (b), revealing a mean nearest-neighbor distance of $8.6 \pm 0.4 \mu \mathrm{m}$ (95\% confidence bounds) with standard deviation $\sigma=7.8 \pm 0.4 \mu \mathrm{m}$ in the molecular layer, while the results from the granular layer could be best fitted by a sum of two Gaussian functions with peaks at $3.93 \pm 0.02 \mu \mathrm{m}$ and $2.64 \pm 0.02 \mu \mathrm{m}$ with standard deviations $\sigma_{1}=0.77 \pm 0.03 \mu \mathrm{m}$ and $\sigma_{2}=0.35 \pm 0.04 \mu \mathrm{m}$ at an approximate weight ratio of $86: 14$. The mean cell radii determined for the molecular and granular layer are 

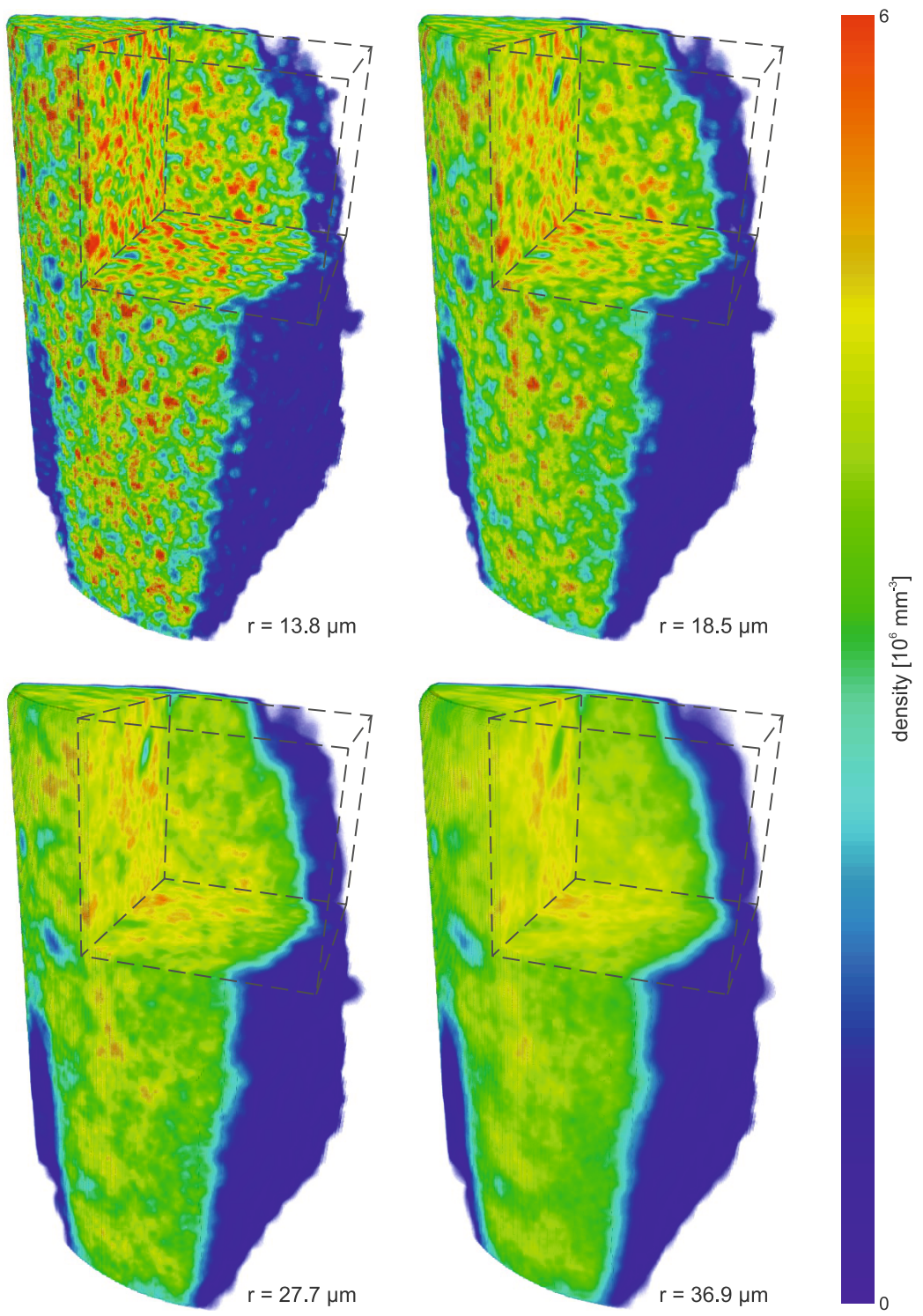

Figure 8.21: Local cell density distribution within the granular layer of the cerebellum measured at the laboratory setup. As in the synchrotron dataset, a clustering of the cells can be recognized due to the formation of local hotspots throughout the layer. With increasing sphere radius these differences are averaged out, leading to a more homogeneous density distribution which decreases significantly towards the interface between the molecular and granular layer. 

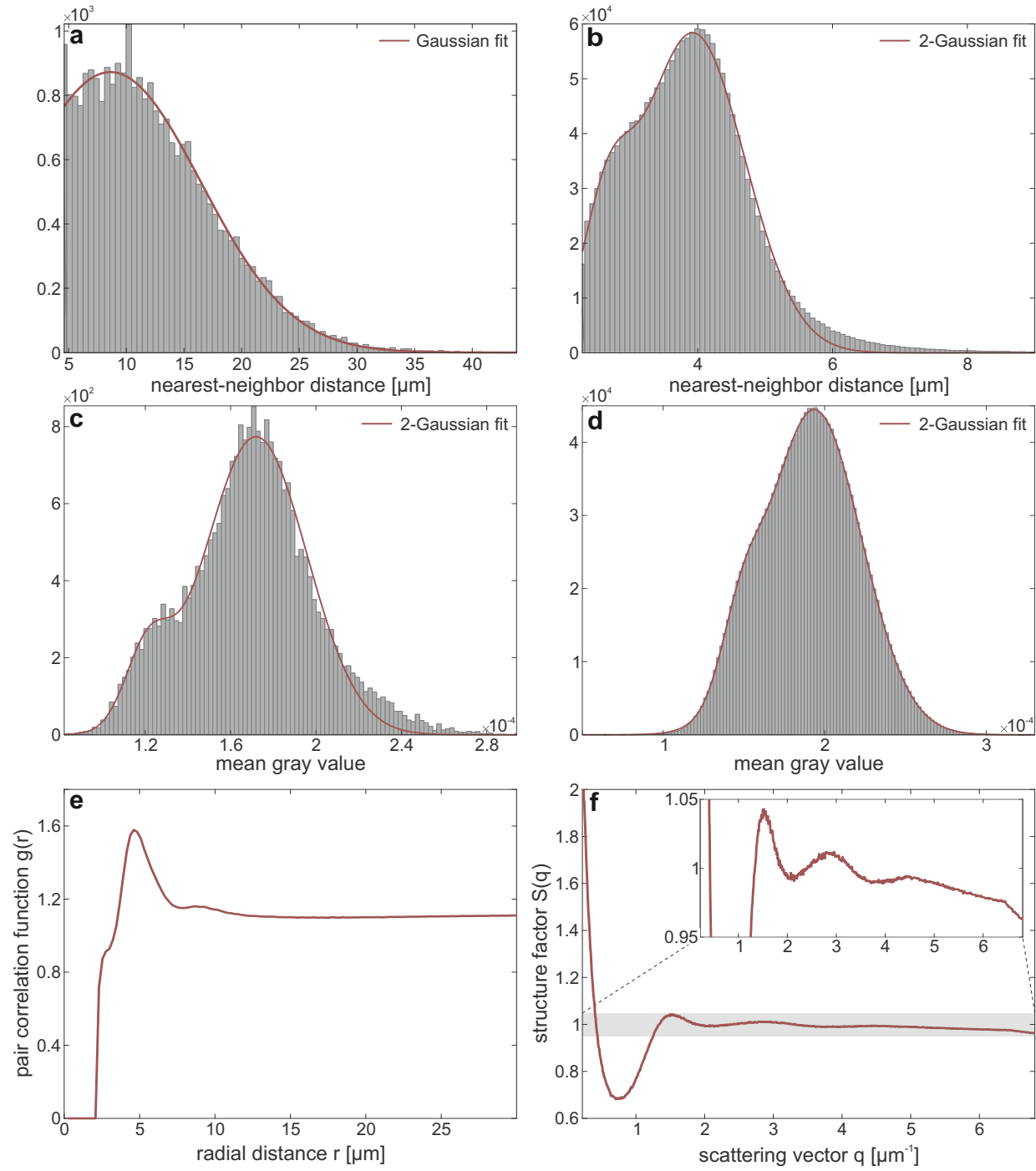

Figure 8.22: Statistical measures obtained from the laboratory dataset. (a,b) Histograms of the nearest-neighbor distances in the molecular and granular layer, respectively. The Gaussian fit reveals a mean distance of $8.6 \pm 0.4 \mu \mathrm{m}$ (95\% confidence interval) in the molecular layer, while in the granular layer a sum of two Gaussian functions with peaks at $3.93 \pm 0.02 \mu \mathrm{m}$ and $2.64 \pm 0.02 \mu \mathrm{m}$ fitted the data best (appr. ratio 86:14). (c,d) Histograms of the mean cellular gray values from the molecular and granular layer. A 2Gaussian fit reveals peaks at $(1.72 \pm 0.01) \cdot 10^{-4}$ and $(1.21 \pm 0.02) \cdot 10^{-4}$ (appr. ratio 90:10), and $(1.936 \pm 0.001) \cdot 10^{-4}$ and $(1.483 \pm 0.001) \cdot 10^{-4}$ (appr. ratio 90:10) for the molecular and granular layer, respectively. (e) Pair correlation function of the cells in the granular layer, with two principle peaks at $4.74 \pm 0.04 \mu \mathrm{m}$ and $8.7 \pm 0.1 \mu \mathrm{m}$ and a minor modulation at $2.50 \pm 0.05 \mu \mathrm{m}$. (f) Structure factor of the granular layer. 
$1.6 \mu \mathrm{m}$ and $1.5 \mu \mathrm{m}$, respectively, which indicates that in contrast to the molecular layer, cells in the granular layer have at least one neighboring cell in more or less direct contact, analogous to the synchrotron results. An estimate for the relative electron density is given by the mean gray value within the volume of the single cells, as depicted in Fig. 8.22(c) and (d). Note that due to the broad bremsspectrum of the laboratory source as well as the mixed contrast due to the superposition of attenuation as well as phase effects in the process of contrast formation, the obtained density distribution only corresponds to effective values. However, as this applies equally for the entire sample, a relative comparison between the mean electron density values within the cells of the two layers is still justified. For the molecular layer, a sum of two Gaussian functions fitted the data best, revealing peaks at $(1.72 \pm 0.01) \cdot 10^{-4}$ and $(1.21 \pm 0.02) \cdot 10^{-4}$ with standard deviations $\sigma_{1}=(2.4 \pm 0.1) \cdot 10^{-5}$ and $\sigma_{2}=(1.1 \pm 0.2) \cdot 10^{-5}$ and an approximate weight ratio of 90:10. A fit of two Gaussian functions to the results from the granular layer yields mean gray values of $(1.936 \pm 0.001) \cdot 10^{-4}$ and $(1.483 \pm 0.001) \cdot 10^{-4}$ with standard deviations $\sigma_{1}=(2.92 \pm 0.01) \cdot 10^{-5}$ and $\sigma_{2}=(1.23 \pm 0.02) \cdot 10^{-5}$ in an approximate weight ratio of 90:10, indicating that the cells of the granular layer show a slightly higher electron density.

The pair correlation function as well as structure factor of the entire granular layer are shown in Fig. 8.22(e) and (f). In the correlation function three maxima at $2.50 \pm 0.05 \mu \mathrm{m}, 4.74 \pm 0.04 \mu \mathrm{m}$ and $8.7 \pm 0.1 \mu \mathrm{m}$ are visible, the first of which is only a very small modulation. This again indicates a clustering of the cells in this layer, which is already visible in the local density shown in Fig. 8.21. Note that the peak at the smallest radial distance has only a hardly recognizable counterpart in the synchrotron dataset (cf. Fig. 8.15(e)). Hence, it is either a property of the inner part of the granular layer, which lies outside the field of view of the synchrotron dataset, or it is an artifact of falsely detected cells due to the lower precision and recall in the laboratory dataset.

The coordination number can be estimated at 7-8, depending on the definition of the first minimum, and it is thus larger compared to the coordination number obtained for the synchrotron dataset. One reason could be the higher cellular density in the granular layer. As the mean distance to the nearest neighbor is comparable in both datasets, a higher density can only be explained by a larger number of cells within the considered volume which might result in a larger number of close neighbors. A summary of the determined statistical parameters, also in comparison to those obtained at the GINIX setup in cone-beam geometry, is given in Tab. 8.4 . 


\begin{tabular}{|c|c|c|}
\hline & GINIX setup & laboratory setup \\
\hline \multirow{2}{*}{$\begin{array}{l}\text { density in ML } \\
\text { density in GL }\end{array}$} & $9.9 \cdot 10^{4} \mathrm{~mm}^{-3}$ & $7.4 \cdot 10^{4} \mathrm{~mm}^{-3}$ \\
\hline & $2.7 \cdot 10^{6} \mathrm{~mm}^{-3}$ & $\begin{array}{c}3.4 \cdot 10^{6} \mathrm{~mm}^{-3} \\
\left(2.9 \cdot 10^{6} \mathrm{~mm}^{-3}\right)\end{array}$ \\
\hline \multirow{2}{*}{$\begin{array}{l}\text { mean radius in } \mathrm{ML} \\
\text { mean radius in } \mathrm{GL}\end{array}$} & $2.4 \mu \mathrm{m}$ & $1.6 \mu \mathrm{m}$ \\
\hline & $2.1 \mu \mathrm{m}$ & $1.5 \mu \mathrm{m}$ \\
\hline \multirow{2}{*}{$\begin{array}{l}\text { mean NND in } \mathrm{ML} \\
\text { mean NND in GL }\end{array}$} & $9.7 \pm 0.8 \mu \mathrm{m}$ & $8.6 \pm 0.4 \mu \mathrm{m}$ \\
\hline & $4.00 \pm 0.02 \mu \mathrm{m}$ & $\begin{array}{l}2.64 \pm 0.02 \mu \mathrm{m} \\
3.93 \pm 0.02 \mu \mathrm{m}\end{array}$ \\
\hline \multirow{2}{*}{$\begin{array}{l}\text { mean gray value in } \mathrm{ML} \\
\text { mean gray value in } \mathrm{GL}\end{array}$} & $(1.17 \pm 0.02) \cdot 10^{-3}$ & $\begin{array}{l}(1.21 \pm 0.02) \cdot 10^{-4} \\
(1.72 \pm 0.01) \cdot 10^{-4}\end{array}$ \\
\hline & $\begin{array}{l}(1.24 \pm 0.01) \cdot 10^{-3} \\
(1.40 \pm 0.02) \cdot 10^{-3}\end{array}$ & $\begin{array}{l}(1.483 \pm 0.001) \cdot 10^{-4} \\
(1.936 \pm 0.001) \cdot 10^{-4}\end{array}$ \\
\hline peaks positions in $\mathrm{PCF}$ & $\begin{array}{c}4.16 \pm 0.04 \mu \mathrm{m} \\
8.5 \pm 0.3 \mu \mathrm{m}\end{array}$ & $\begin{array}{c}2.50 \pm 0.05 \mu \mathrm{m} \\
4.74 \pm 0.04 \mu \mathrm{m} \\
8.7 \pm 0.1 \mu \mathrm{m}\end{array}$ \\
\hline first coord. number & 5 & $7-8$ \\
\hline
\end{tabular}

Table 8.4: Statistical measures obtained for the molecular (ML) and granular layer (GL) of the $1 \mathrm{~mm}$ punch from a human cerebellum, measured both at the GINIX as well as laboratory setup. Abbreviations stand for nearest-neighbor distance (NND) and pair correlation function $(\mathrm{PCF})$.

\subsection{Summary}

The results presented in this chapter show that propagation-based phase-contrast tomography can be used for $3 \mathrm{~d}$ virtual histology of unstained human cerebellum embedded in paraffin. Results obtained at the synchrotron setup both in paralleland cone-beam geometry provide $3 \mathrm{~d}$ volumes with isotropic resolution and subcellular detail as the nuclei and nucleoli as well as dendritic tree of the large Purkinje cells. The size of the dendritic tree was mainly limited by the field of view of the tomographic measurement and not the resolution. In the parallel-beam geometry, a larger field of view could be easily enabled by implementing a fast shutter with a wider opening of the slits. Even at an optimized compact laboratory source, a data quality high enough for single cell segmentation could be reached, comparable to the parallel-beam setup at the synchrotron, making this method available for a broad range of studies without the need to apply for beamtime at a heavily occupied large-scale facility.

The embedding in paraffin enables subsequent histological studies with specific 
staining agents to combine the x-ray results, providing an overview of the $3 \mathrm{~d}$ electron density of the entire sample, with information on specific cells within this volume and to put the histological findings into perspective [69]. As no further staining methods as, e.g., staining with osmium tetroxide [42], was used, contrast is entirely based on the electron density differences and the results can be used for a quantitative comparison between different tissue types.

The presented workflow for automatic segmentation of the small cells present in the molecular and granular layer of the cerebellum is easily applicable and requires only a small set of parameters to be chosen. It is a straightforward approach which can be used without further training steps being necessary. The parameters needed for the algorithm to work properly could be chosen on a small subvolume based on visual inspection and subsequently applied on the whole dataset, as data quality was homogeneous throughout the entire volume. The obtained results indicate a strong short-range order within the densely packed granular layer, leading to a local clustering of cells accompanied by characteristic position correlations as quantified by pair correlation functions.

The comparison between the performance of the algorithm and a manual segmentation showed an almost perfect precision and recall in the case of the synchrotron dataset, especially in the granular layer, proving that the developed workflow can be used for high-quality automatic cell segmentation. Even in the lower resolution laboratory dataset, it provided a very precise segmentation, comparable to results obtained at the synchrotron [42]. Again, performance in the cell-dense granular layer with homogeneous size and shape distribution was better compared to the diverse molecular layer.

Previous findings for cellular density in the granular layer, based on manual cell counting in $2 \mathrm{~d}$ histological sections [3], correspond very well to the densities reported here. In contrast to the manual approach, the identification of cell positions was carried out in three dimensions, which enables a high precision determination of local cell densities throughout the entire volume. Note, however, that in the case of the manual counting a distinction between granule cells and glial cells, both present in the granular layer, was made. This may be implemented into the automatic workflow as well, e.g., by determining measures as density and volume of each cell, which are easily accessible by the presented approach, and correlating differences in these measures to specific cell types. For a more precise evaluation of the cell volume, one could even consider extending the presented approach so that the identified cell positions are used as seeding points for a region-growing algorithm, estimating the exact cell shape based on gray values instead of assuming 
a perfect sphere.

The automatic localization of the cells in the granular and molecular layer and the subsequent statistical analysis enables novel approaches to correlate tissue function with structure and to possibly identify biomarkers for diagnostic purposes. Importantly, the capability to study cellular distributions in $3 \mathrm{~d}$ allows for a precise quantification of nearest-neighbor distances or pair correlation functions, which can only be deduced from thin histological sections by assuming statistically homogeneous and isotropic cell distributions [78]. Among the possibility to describe the evaluated tissue based on the presented statistical measures, this also enables biomedical studies aiming at changes in cellular distribution between different individuals, e.g., in the course of neurodegenerative diseases. The presented workflow can be easily applied on a large number of samples, especially due to the good performance at a compact laboratory source, which makes it possible to carry out such studies without the need for beamtime at a synchrotron facility. 


\section{Outlook: 3d histology of neurodegenerative diseases}

The following chapter gives an outlook on the application of propagation-based phase-contrast tomography for imaging of structural changes occurring during neurodegenerative diseases, in particular at the examples of multiple sclerosis [22], Alzheimer's disease [119, 137] and a mouse model for ischemic stroke [37].

\subsection{Multiple sclerosis}

Multiple sclerosis (MS) is a chronic inflammatory disease of the central nervous system which results in lesions of demyelination both in gray and white matter of the brain. Symptoms include impairment of fine motor or coordination tasks or cognitive-behavioral dysfunction which can be attributed to cerebellar abnormalities. Within the cerebellum both gray and white matter demyelination can be observed and especially in progressive multiple sclerosis cortical demyelination, mainly in the form of lesions, occurs [131].

Analogous to the approach described in the previous chapter, the influence of multiple sclerosis on the cellular organization within the cerebellum is considered in the following by combining propagation-based phase-contrast tomography of paraffin-embedded human cerebellum both from healthy controls and patients with multiple sclerosis with the automated cell segmentation workflow based on the spherical Hough transform.

Methods Formalin-fixed and paraffin-embedded cerebellar tissue was obtained from nine healthy control patients, including the patient from the previous chapter which will be denoted as CTR 5 in the following, and six patients with multiple sclerosis. The samples were provided by the group of Christine Stadelmann-Nessler (Institute for Neuropathology, University Medical Center, Göttingen, Germany). A list linking the sample names used within this thesis to the respective patients can be found in Tab. 9.1. For mounting in the experimental setup, a $1 \mathrm{~mm}$ punch 


\begin{tabular}{cccccc}
\hline CTR 1 & CTR 2 & CTR 3 & CTR 4 & CTR 5 & CTR 6 \\
\hline 67/16-6 & 69/16-6 & $26 / 16-6$ & $54 / 16-6$ & $128 / 16-6$ & $65 / 15-6$ \\
\hline \multicolumn{7}{c}{ MS 1 } & MS 2 & MS 3 & MS 4 & MS 5 & MS 6 \\
\hline $120 / 06-11$ & T8/00-4 & $113 / 13-52$ & $136 / 09-39$ & $220 / 91-15$ & $36 / 14-62$ \\
\hline
\end{tabular}

Table 9.1: Patients corresponding to the sample names used in the course of this section. The nomenclature is patient number/year of autopsy-tissue block number.

was taken from the tissue and squeezed into a $1 \mathrm{~mm}$ Kapton tube which was glued to a sample holder, analogous to the sample preparation described in the previous chapter (cf. Fig. 8.1).

Tomographic experiments were carried out at the GINIX setup in cone-beam geometry. The experimental parameters for the specimen CTR 5 were already described in the previous section and a summary can be found in Tab. 8.1. For the remaining samples, the energy was set to $13.8 \mathrm{keV}$ and a crossed multilayer waveguide was placed in the focal spot of the KB-mirrors, providing a flux of $\sim 8 \cdot 10^{8} \mathrm{ph} / \mathrm{s}$. The 'Pirra' was located at a source-to-detector distance of $5.05 \mathrm{~m}$. For each measurement the source-to-sample distance was set to $z_{01}=145 \mathrm{~mm}$ and tomograms were recorded at 4 propagation distances by acquiring 1000 projections over an angular range of $180^{\circ}$ with an exposure time of $0.5 \mathrm{~s}$. For an overview, the experimental parameters are summarized in Tab. 9.2.

Results The processing steps to reconstruct the $3 \mathrm{~d}$ density distribution for sample CTR 5 can be found in the previous chapter. For the remaining samples, phase retrieval was carried out on the individual empty-beam corrected projections via the CTF-based algorithm for a weakly absorbing object with regularization parameters $\frac{\delta}{\beta}=30$ and $\alpha_{2}=0$. In order to take all propagation distances with varying magnification and field of view into account, projections were rescaled to the smallest effective pixel size and aligned to each other via a cross-correlation in Fourier space. Prior to tomographic reconstruction with the Matlab implementation of the filtered backprojection and a standard Ram-Lak filter, the simple ring-removal algorithm was applied to the sinograms. Additionally, low-frequency variations due to broad vertical stripes in the waveguide illumination were compensated following the approach described in section 5.3.2. In three of the measured control patients ('13/15-6', '30/16-6' and '57/10-6'), granule cell layer autolysis occurred prior to fixation, which can be recognized in a substantial cell degenera- 


\begin{tabular}{lc}
\hline & experimental setting \\
\hline GINIX run & $62 / 69(\mathrm{CTR} 1$ \& 2) \\
energy & $13.8 \mathrm{keV}$ \\
detector & 'Pirra' \\
$z_{01, \text { min }}$ & $145 \mathrm{~mm}$ \\
$z_{02}$ & $5.05 \mathrm{~m}$ \\
$p_{\text {eff }}$ & $187 \mathrm{~nm}$ \\
field of view & $383 \times 383 \mathrm{um}^{2}$ \\
Fresnel number $F$ & 0.0016 \\
waveguide & multilayer \\
number of distances & $(d=81 \mathrm{~nm})$ \\
angular range & 4 \\
number of projections & {$[0,180]^{\circ}$} \\
exposure time & 1000 \\
CTF $\frac{\delta}{\beta}$ & $0.5 \mathrm{~s}$ \\
CTF $\alpha_{2}$ & 30 \\
\hline
\end{tabular}

Table 9.2: Experimental parameters used for imaging of paraffin-embedded human cerebellum from both healthy controls and patients with multiple sclerosis.
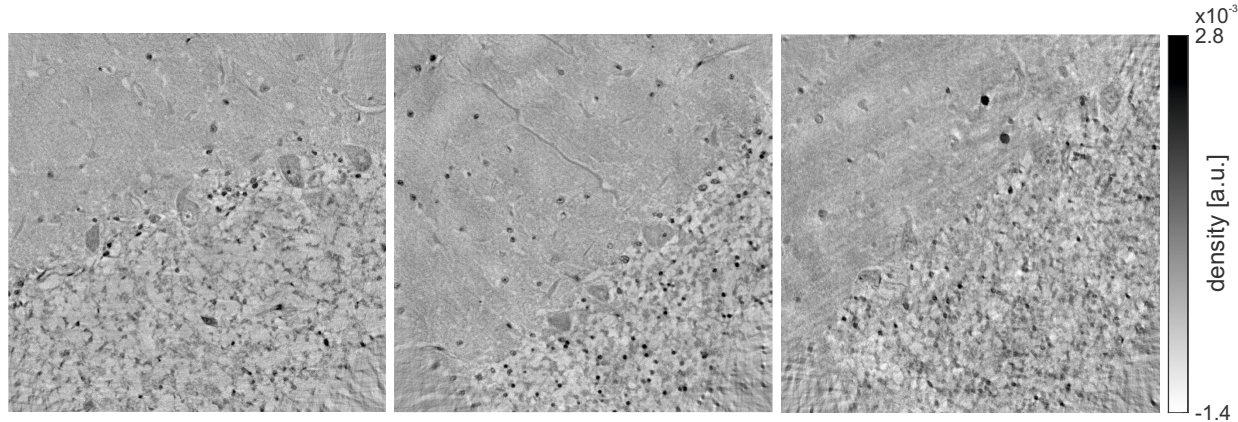

Figure 9.1: Virtual slices through the reconstructed volumes of paraffin-embedded human cerebellum from three control patients, in which substantial granule cell layer autolysis occurred prior to fixation, resulting in considerable cell degeneration.

tion (cf. Fig. 9.1) [155]. Hence, these samples were excluded from the subsequent quantitative analysis. Slices through the reconstructed density distributions of the remaining samples are depicted in Figs. 9.2 and 9.3, showing the transition between the cell-rich granular and low-cell molecular layer in each dataset.

For the quantification of cell densities and their structural organization, the automated segmentation workflow based on the spherical Hough transform (cf. section 

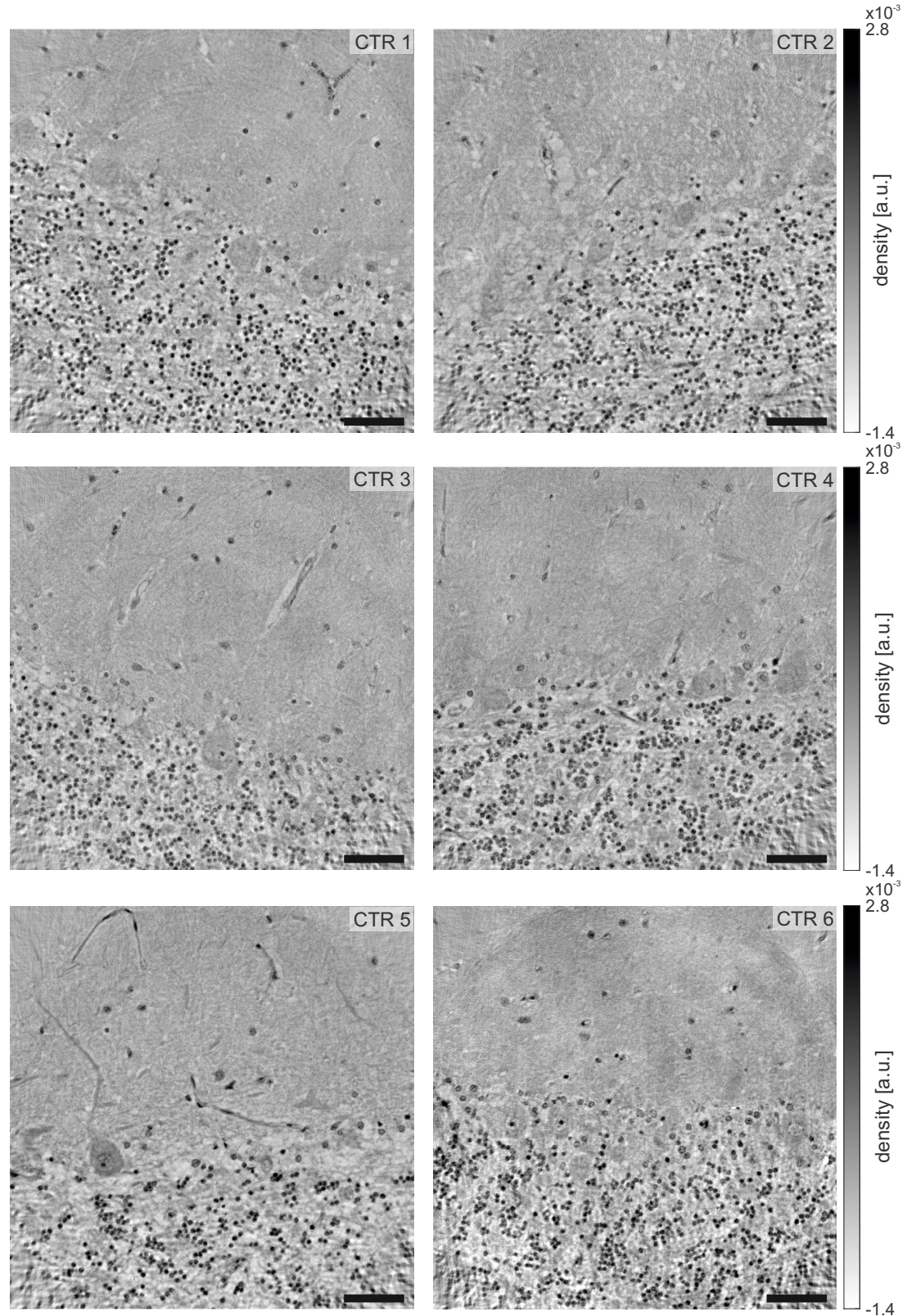

Figure 9.2: Virtual slices through the reconstructed volumes of paraffin-embedded human cerebellum from healthy control patients. In all datasets, the interface between the molecular and granular layer is included in the reconstruction volume, enabling the quantification of cell densities and spatial distributions in both layers. Scale bars: $50 \mu \mathrm{m}$ 

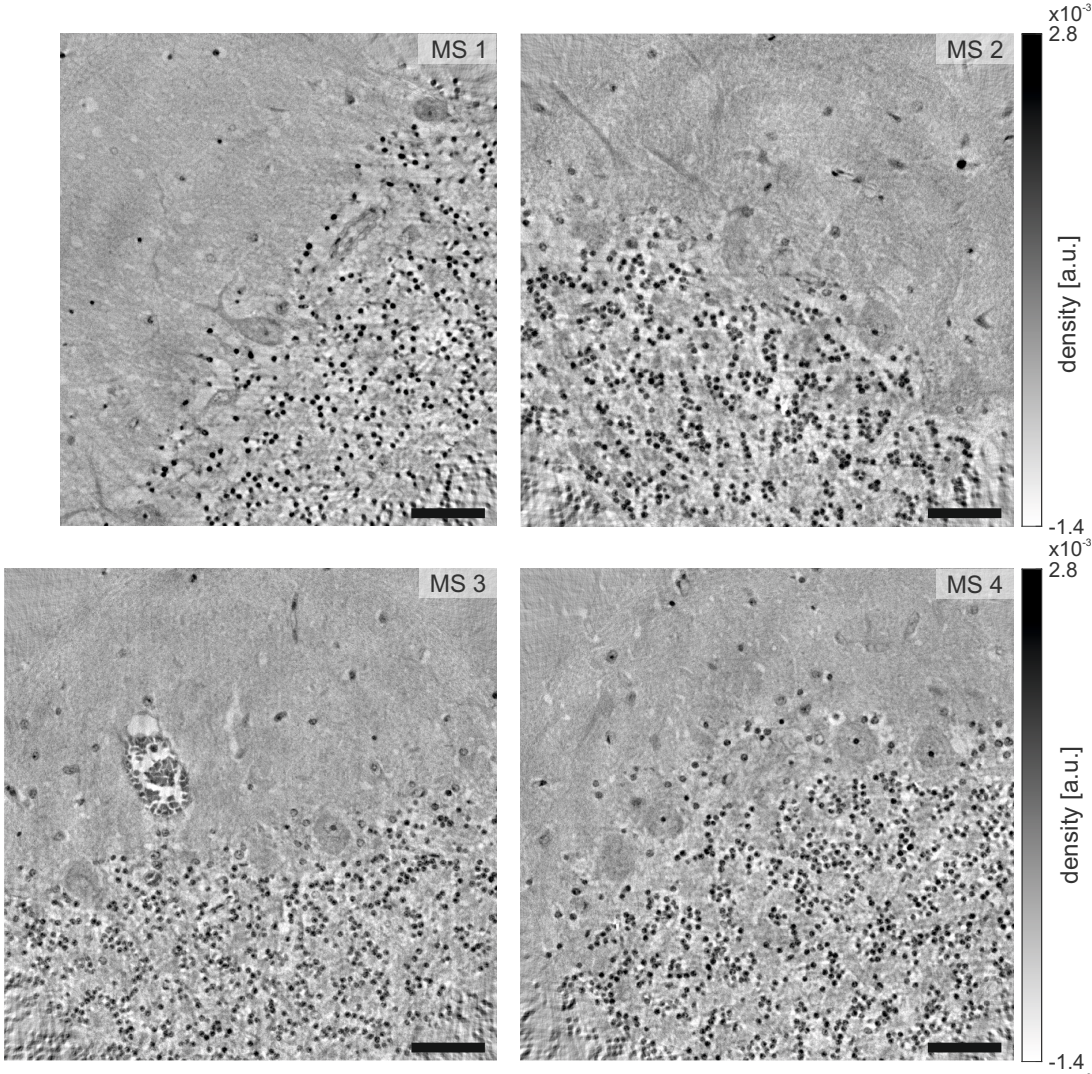

10
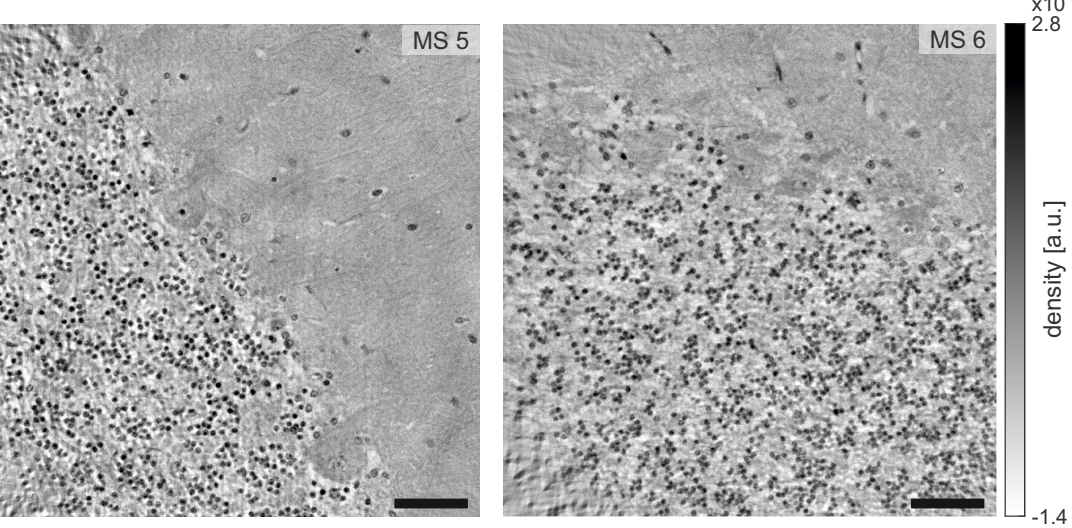

Figure 9.3: Virtual slices through the reconstructed volumes of paraffin-embedded human cerebellum from patients with multiple sclerosis. Also in these specimens, the interface between the molecular and granular layer was imaged in order to compare the results on cell densities and spatial distributions in both layers with those from the healthy control group. Scale bars: $50 \mu \mathrm{m}$ 
8.2.2) was applied to each of the reconstructed volumes. The parameters for the Hough transform were selected based on a small subvolume, leading to the values listed in Tab. 9.3. It is evident that throughout the different samples, the two most

\begin{tabular}{lccccc}
\hline & RR & GT & FR & MRT & OCIT \\
\hline CTR 1 & $3-10$ & $2.9 \cdot 10^{-4}$ & 3 & 1 & 0 \\
CTR 2 & $3-10$ & $3.1 \cdot 10^{-4}$ & 3 & 1 & 0 \\
CTR 3 & $3-10$ & $2.1 \cdot 10^{-4}$ & 3 & 1 & 0 \\
CTR 4 & $3-12$ & $1.9 \cdot 10^{-4}$ & 4 & 1 & $0.55 \cdot 10^{-4}$ \\
CTR 5 & $3-10$ & $3.4 \cdot 10^{-4}$ & 3 & 1 & 0 \\
CTR 6 & $3-10$ & $3.3 \cdot 10^{-4}$ & 3 & 1 & $0.7 \cdot 10^{-4}$ \\
MS 1 & $3-10$ & $2.9 \cdot 10^{-4}$ & 3 & 1 & $0.4 \cdot 10^{-4}$ \\
MS 2 & $3-10$ & $2.9 \cdot 10^{-4}$ & 3 & 1 & $0.4 \cdot 10^{-4}$ \\
MS 3 & $3-10$ & $2.4 \cdot 10^{-4}$ & 3 & 1 & $0.4 \cdot 10^{-4}$ \\
MS 4 & $3-10$ & $2.8 \cdot 10^{-4}$ & 3 & 1 & $0.4 \cdot 10^{-4}$ \\
MS 5 & $3-10$ & $2.9 \cdot 10^{-4}$ & 3 & 1 & $0.3 \cdot 10^{-4}$ \\
MS 6 & $3-10$ & $4.3 \cdot 10^{-4}$ & 3 & 1 & $0.25 \cdot 10^{-4}$ \\
\hline
\end{tabular}

Table 9.3: Radius range $(R R)$ in pixels, gradient threshold value $(G T)$, filter radius (FR) in pixels, multiple radius tolerance (MRT) and object center intensity threshold (OCIT) chosen for the spherical Hough transform in order to automatically detect all cells in the reconstructed volumes of paraffin-embedded human cerebellum from both healthy controls and patients with multiple sclerosis.

important factors which have to be set for the individual datasets are the gradient and object center intensity thresholds. The other settings remain constant, except for sample CTR 4, which further simplifies the selection of appropriate parameters for the segmentation of cells in structurally similar samples. The resulting $3 \mathrm{~d}$ cellular distributions are depicted in Figs. 9.4 and 9.5, showing that for all samples, a similar ratio between the molecular and granular layer was within the field of view which enables a quantitative comparison between the individual datasets.

For each of the measurements the mean density within the molecular and granular layer was determined by the ratio between the number of cells contained in each layer and the corresponding layer volume. As in the previous chapter, the volume of each layer was estimated via the function boundary implemented in Matlab with the standard shrinking factor 0.5. The resulting density values in the molecular as well as granular layer are shown in Fig. 9.6. Note that for better visibility, each data point was shifted laterally compared to its predecessor. Within the molecular layer no clear differences between the two patient groups can be recognized which is confirmed by the mean density values, yielding $\bar{\rho}_{\mathrm{CTR}}=(1.2 \pm 0.2) \cdot 10^{5} \mathrm{~mm}^{-3}$ and $\bar{\rho}_{\mathrm{MS}}=(1.4 \pm 0.2) \cdot 10^{5} \mathrm{~mm}^{-3}$ (standard error of the mean $\left.(\mathrm{SEM})\right)$ for the control 

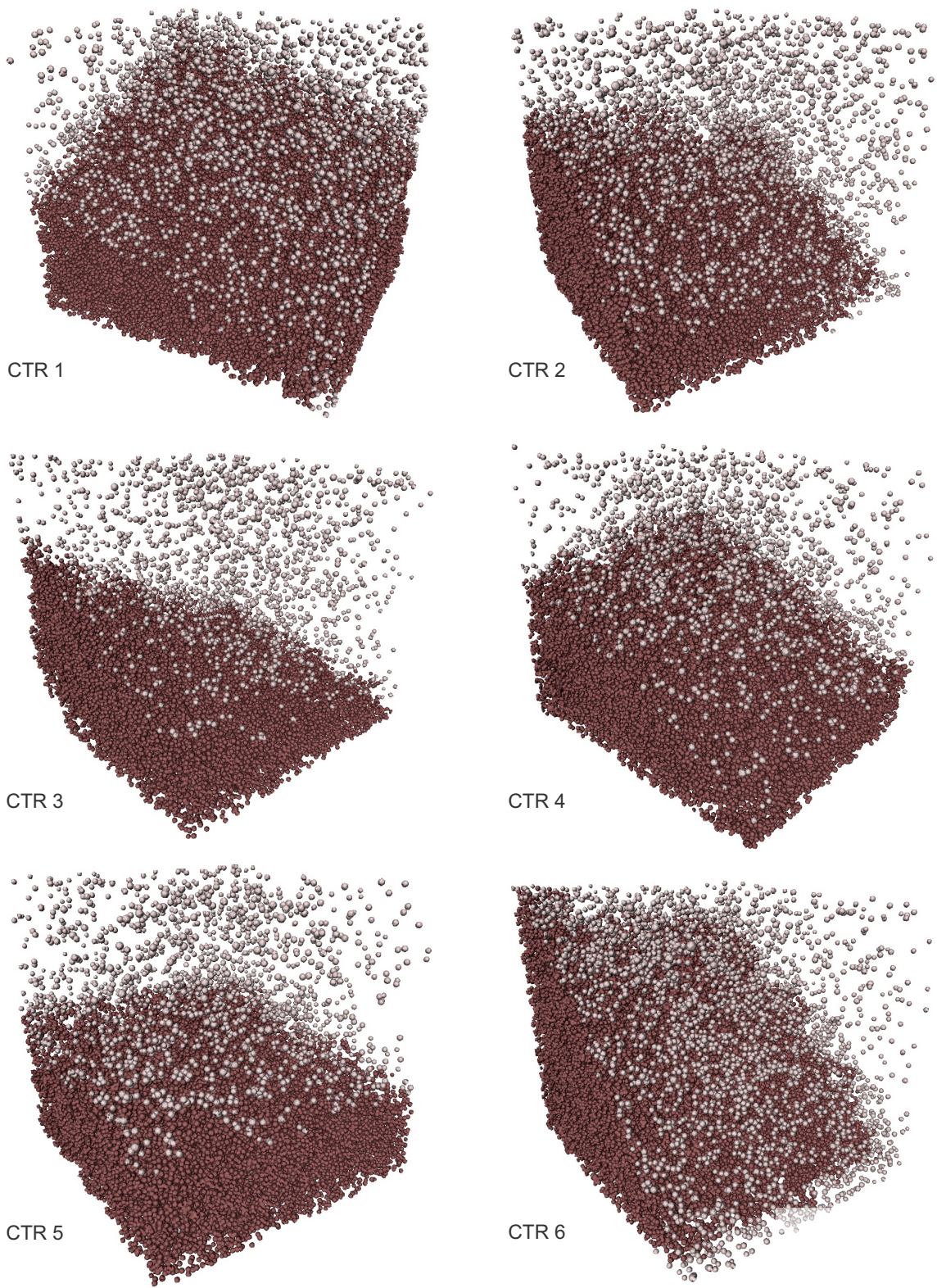

Figure 9.4: $3 d$ visualization of the cellular distributions within the molecular (light red) and granular layer (dark red) of paraffin-embedded human cerebellum from healthy control patients. The cellular positions were obtained via the automated workflow described in section 8.2.2, which is based on the spherical Hough transform. 

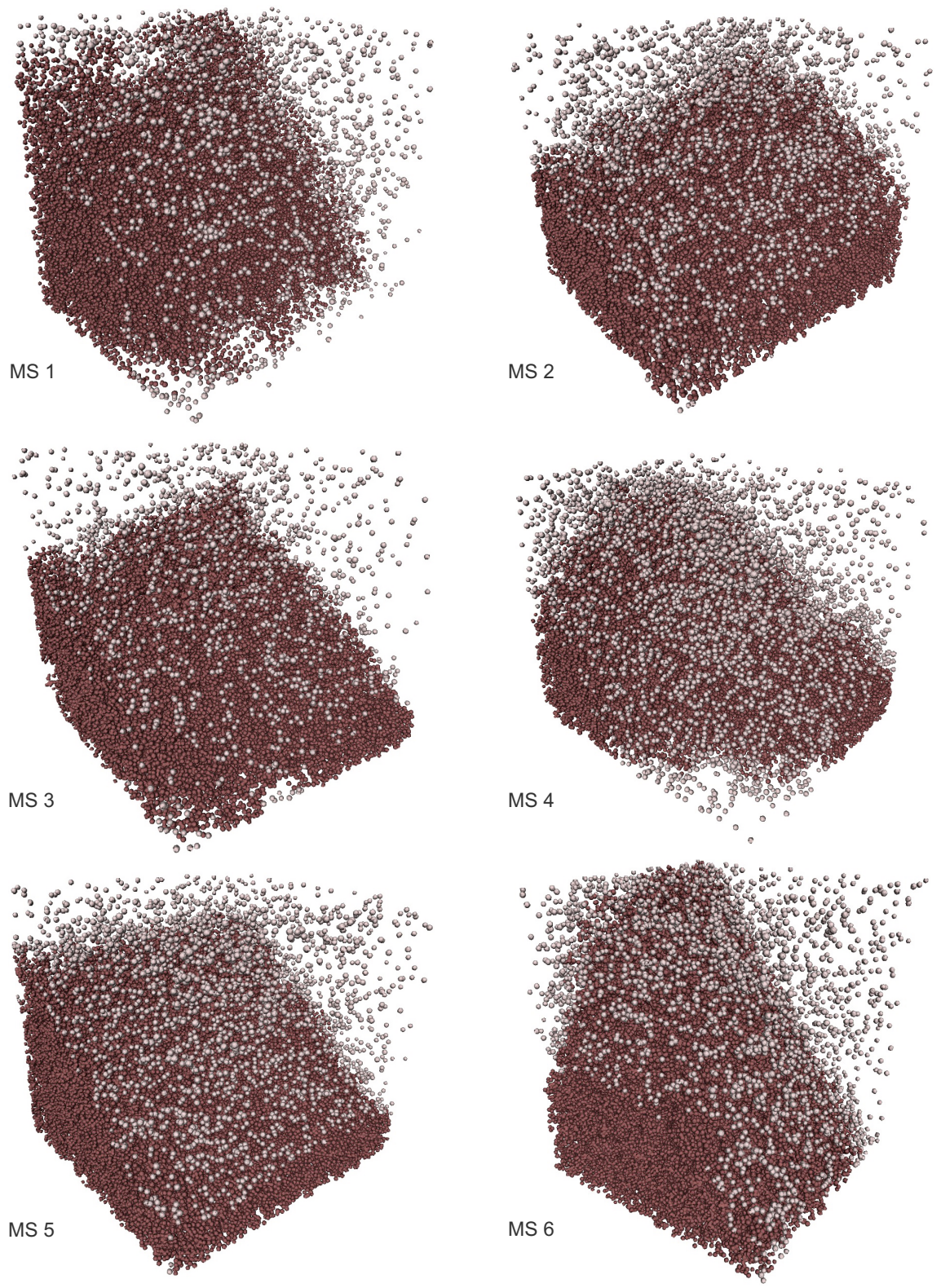

Figure 9.5: $3 d$ visualization of the cell distributions within the molecular (light red) and granular layer (dark red) of paraffin-embedded human cerebellum from patients with multiple sclerosis. Again, the automated workflow presented in section 8.2.2 was used for the detection of the cells. 

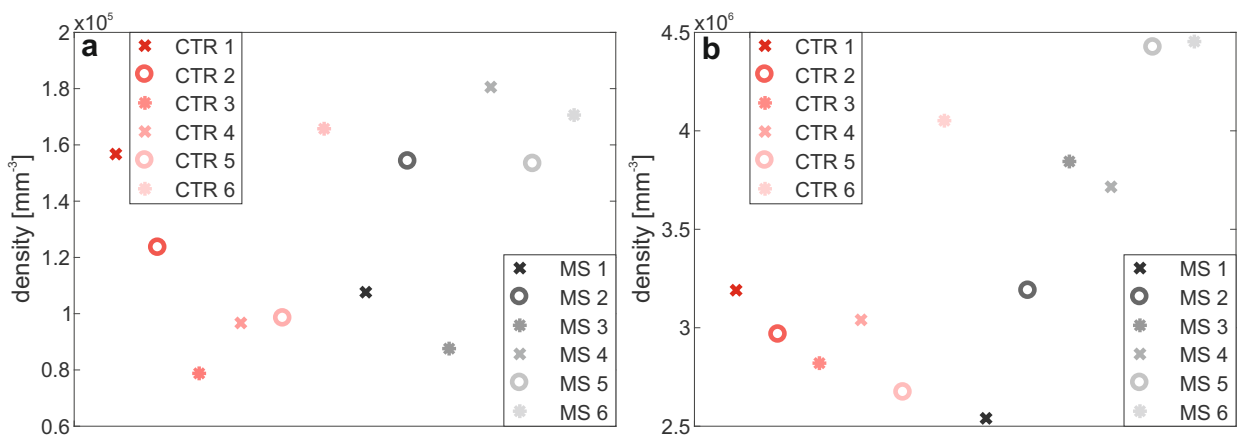

Figure 9.6: Cell densities in the cerebellum obtained for the two patient groups. For better visibility, each data point was shifted laterally compared to its predecessor. (a) Cell densities in the molecular layer. Between the two groups, no significant difference in cell densities can be recognized, as can additionally be seen in the mean density values, yielding $\bar{\rho}_{C T R}=(1.2 \pm 0.2) \cdot 10^{5} \mathrm{~mm}^{-3}$ (standard error of the mean) for the control patients and $\bar{\rho}_{M S}=(1.4 \pm 0.2) \cdot 10^{5} \mathrm{~mm}^{-3}$ for the patients with multiple sclerosis. (b) Cell densities in the granular layer. In contrast to the molecular layer, there is a clear trend towards higher cell densities in patients with multiple sclerosis, apart from one outlier in each patient group (CTR 6 and MS 1). The mean densities yield $\bar{\rho}_{C T R}=(3.1 \pm 0.2) \cdot 10^{6} \mathrm{~mm}^{-3}$ for the control patients and $\bar{\rho}_{M S}=(3.7 \pm 0.3) \cdot 10^{6} \mathrm{~mm}^{-3}$ for patients with multiple sclerosis. By excluding the two outliers CTR 6 and MS 1, leading to $\bar{\rho}_{C T R}=(2.9 \pm 0.1) \cdot 10^{6} \mathrm{~mm}^{-3}$ and $\bar{\rho}_{M S}=(3.9 \pm 0.3) \cdot 10^{6} \mathrm{~mm}^{-3}$, the difference becomes even clearer.

patients and those with multiple sclerosis, respectively. The cell densities in the granular layer show a slightly different behavior in which a trend towards higher cell densities in patients with multiple sclerosis can be recognized. Although each group comprises one outlier (CTR 6 and MS 1), this visual impression can be confirmed by the mean density values, yielding $\bar{\rho}_{\mathrm{CTR}}=(3.1 \pm 0.2) \cdot 10^{6} \mathrm{~mm}^{-3}$ for the control patients and $\bar{\rho}_{\mathrm{MS}}=(3.7 \pm 0.3) \cdot 10^{6} \mathrm{~mm}^{-3}(\mathrm{SEM})$ for patients with multiple sclerosis. By excluding the outliers, resulting in $\bar{\rho}_{\mathrm{CTR}}=(2.9 \pm 0.1) \cdot 10^{6} \mathrm{~mm}^{-3}$ and $\bar{\rho}_{\mathrm{MS}}=(3.9 \pm 0.3) \cdot 10^{6} \mathrm{~mm}^{-3}$ (SEM), respectively, the difference becomes even clearer, yielding an increase in granule cell density of approximately $35 \%$ for patients with multiple sclerosis with respect to healthy controls.

To compare the structural organization of granule cells in both patient groups, the pair correlation function was calculated from the corresponding cell positions, leading to the results depicted in Fig. 9.7. Note that for better visibility, the curves were shifted vertically by 1 compared to their predecessor. The general shape of the pair correlation functions is similar for all patients, showing two distinct maxima. Only in patient MS 1, which already had a significantly lower granule cell density compared to the remaining patients of the group, a clear deviation from 

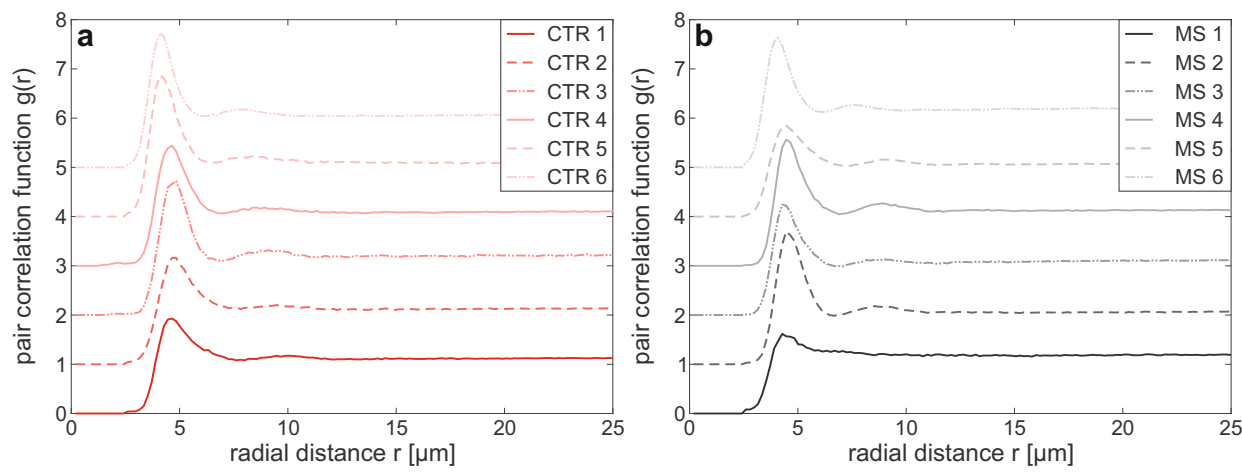

Figure 9.7: Pair correlation functions for the granular layer of human cerebellum, both in healthy controls and patients with multiple sclerosis. For better visibility, all curves were vertically shifted by 1 compared to their predecessor. (a) Pair correlation functions of the healthy control patients. The general shape of the pair correlation function is similar for all patients, showing two distinct maxima at approximately once and twice the medium cell diameter. (b) Pair correlation functions of the patients with multiple sclerosis. Apart from patient MS 1, which already showed a significantly different granule cell density (cf. Fig. 9.6(b)), the general shape is very similar to the healthy control patients.

this shape can be recognized. The mean peak positions in the healthy controls are $4.4 \pm 0.1 \mu \mathrm{m}$ and $9.1 \pm 0.3 \mu \mathrm{m}$ (SEM), corresponding to approximately once and twice the mean cell diameter $(4.30 \pm 0.05 \mu \mathrm{m})$, and for patients with multiple sclerosis mean peak positions at $4.4 \pm 0.1 \mu \mathrm{m}$ and $8.9 \pm 0.3 \mu \mathrm{m}$ (SEM) could be found, excluding patient MS 1 for the second peak, which is also consistent with once and twice the mean cell diameter $(4.21 \pm 0.09 \mu \mathrm{m})$. Hence, based on the pair correlation functions no significant differences between the two patient groups exist for the structural organization of the granule cell layer.

The mean number of nearest neighbors of each cell was determined via the first coordination number (cf. section 8.2.2.3), leading to $5.9 \pm 0.3$ in the group of healthy controls and $6.2 \pm 0.8$ (SEM) for the patients with multiple sclerosis. Due to the large deviation of the pair correlation function of patient MS 1 compared to the rest of the group, the corresponding coordination number (2.93) differs significantly and should be omitted from the analysis, leading to a corrected value of $6.9 \pm 0.5$ (SEM). Hence, a slightly larger mean number of nearest neighbors can be found for patients with multiple sclerosis compared to healthy controls, although a strong scattering within the groups can be observed.

Summary In this section, the application of propagation-based phase-contrast tomography on paraffin-embedded human cerebellum from both healthy controls 
and patients with multiple sclerosis was shown in combination with automated cell segmentation. In each of the acquired datasets, the segmentation of the small cells within the granular and molecular layer of the cerebellum could be successfully carried out by using the workflow described in section 8.2 .2 , with the performance being judged based on visual inspection. The resemblance of the different samples leads to similar parameters for the application of the spherical Hough transform, which facilitates the manual determination of these parameters and enables the use of this workflow also by inexperienced researchers.

The only apparent difference between the considered patient groups lies in the obtained cell densities in the granular layer and to some extent also the coordination numbers, while the overall structural organization seems to remain approximately constant according to the pair correlation function, with the exception of one sample from the group of multiple sclerosis patients. The reason for the cell density differences is still unclear at the moment and further investigations have to be carried out, e.g., by subsequent histological experiments with specific staining agents. In addition, a larger number of patients from each group should be studied to increase the statistical relevance of the experiment and confirm the assumption of a larger cell density in patients with multiple sclerosis.

Due to the large amount of information contained in the individual datasets, further structural parameters could be obtained, e.g., by analyzing the Purkinje cell densities as well as changes in the shape of these cells, which might indicate additional differences between the two patient groups.

\subsection{Alzheimer's disease}

Alzheimer's disease is an age-related neurodegenerative disease which leads to memory loss and a degradation of cognitive functions. The pathology is characterized by an intraneuronal aggregation of neurofibrillary tangles due to an abnormal hyperphosphorylation of tau protein, formation of extracellular plaques from amyloid- $\beta$ protein and massive neuronal death. These hallmarks occur in specific areas of the brain of which the hippocampus is one of the earliest to be affected [114].

In the following, the capabilities of propagation-based phase-contrast tomography for the $3 \mathrm{~d}$ visualization of this characteristic pathology are evaluated on paraffinembedded human hippocampus from a patient with Alzheimer's disease which was measured both at the synchrotron and at the laboratory setup. 
Methods Formalin-fixed and paraffin-embedded hippocampal tissue from a patient with Alzheimer's disease ('201/09-15') was obtained during autopsy and provided by the group of Christine Stadelmann-Nessler (Institute for Neuropathology, University Medical Center, Göttingen, Germany). As in the case of the paraffinembedded cerebellar tissue, a $1 \mathrm{~mm}$ punch was taken for the experiments and squeezed into a Kapton tube which was glued to a sample holder (cf. Fig. 8.1). Tomographic experiments were carried out both at the GINIX setup as well as the laboratory. For the experiments at the laboratory, the setup was used in inverse geometry with the detector 'Argos' placed at a source-to-detector distance $z_{02}=0.186 \mathrm{~m}$. The source was operated at an acceleration voltage of $40 \mathrm{kV}$ and an electron spot size of $10 \times 40 \mathrm{\mu m}^{2}$ with an electron beam power of $57 \mathrm{~W}$. In order to increase photon flux, the electron beam was slightly deflected towards the exit window (cf. Fig. 4.5). The sample was located at $z_{01}=158.75 \mathrm{~mm}$, leading to an effective pixel size of $p_{\text {eff }}=0.46 \mu \mathrm{m}$ and a field of view of $1.15 \times 1.53 \mathrm{~mm}^{2}$. For the tomographic scan, 1000 projections were recorded over an angular range of $180^{\circ}$ with an exposure time of $50 \mathrm{~s}$. In order to image the entire biopsy punch, three tomograms were acquired at adjacent vertical positions along the sample.

At the GINIX setup the energy was set to $8 \mathrm{keV}$ and a straight waveguide etched into a silicon wafer was placed in the focal spot of the KB-mirrors, providing a flux in the order of $10^{9} \mathrm{ph} / \mathrm{s}$. The 'Pirra' was placed in a source-to-detector distance $z_{02}=5.05 \mathrm{~m}$ while the sample was located at $z_{01}=145 \mathrm{~mm}$, leading to an effective pixel size of $p_{\text {eff }}=187 \mathrm{~nm}$ with a corresponding field of view of $383 \times 383 \mu^{2}$. For the tomographic scan, 1500 projections were acquired at four propagation distances over an angular range of $180^{\circ}$ with an exposure time of $0.1 \mathrm{~s}$. To increase the effective field of view, tomograms were recorded at two adjacent positions within the sample. A summary of all experimental parameters can be found in Tab. 9.4.

Results Phase retrieval on projections acquired at the laboratory setup was carried out with the BAC algorithm and the regularization parameters $\alpha=0.01$ and $(\gamma \cdot F)=0.15$. Prior to phase retrieval, all projections were resampled by a factor of 2 in order to increase the signal-to-noise ratio. Ring removal was performed via the wavelet-based correction algorithm and tomographic reconstruction was carried out via the FDK implementation of the ASTRA toolbox. To further increase the signal-to-noise ratio all slices were filtered with a Gaussian function with a standard deviation of 0.7 pixels. The reconstructed volumes from the three individual tomographic scans were manually aligned to each other and subsequently merged in Avizo. 


\begin{tabular}{lcc}
\hline & laboratory & GINIX setup \\
\hline GINIX run & - & 56 \\
energy $[\mathrm{keV}]$ & $0-40 / 9.25$ & 8 \\
detector & 'Argos' & 'Pirra' \\
$z_{01, \min }[\mathrm{mm}]$ & 158.75 & 145 \\
$z_{02}[\mathrm{~m}]$ & 0.186 & 5.05 \\
$p_{\text {eff }}[\mathrm{nm}]$ & 461 & 187 \\
field of view $\left[\mathrm{\mu m}^{2}\right]$ & $1152 \times 1530$ & $383 \times 383$ \\
Fresnel number $F$ & 0.069 & 0.0016 \\
& & 'Hercules II' \\
waveguide & - & 4 \\
number of distances & 1 & {$[0,180]$} \\
angular range $\left[{ }^{\circ}\right]$ & {$[0,180]$} & 1500 \\
number of projections & 1000 & 0.1 \\
exposure time $[\mathrm{s}]$ & 50 & - \\
BAC $\alpha$ & 0.01 & - \\
BAC $(\gamma \cdot F)$ & 0.15 & 50 \\
CTF $\frac{\delta}{\beta}$ & - & 0 \\
CTF $\alpha_{2}$ & - & \\
\hline
\end{tabular}

Table 9.4: Experimental parameters used for imaging of a $1 \mathrm{~mm}$ punch from a paraffinembedded human hippocampus from a patient with Alzheimer's disease.

For data acquired at the GINIX setup, phase retrieval was carried out via the CTFbased algorithm for weakly absorbing objects with the regularization parameters $\frac{\delta}{\beta}=50$ and $\alpha_{2}=0$. In order to take all propagation distances into account, the single projections were resampled to the smallest pixel size and subsequently aligned via a cross-correlation in Fourier space. Prior to tomographic reconstruction via the filtered backprojection implemented in Matlab with a standard Ram-Lak filter, the simple ring-removal algorithm was applied on the sinograms. Low-frequency artifacts due to the unstable waveguide illumination were reduced by following the approach in section 5.3.2. In this case, too, the individual reconstructed volumes were manually aligned and merged in Avizo.

Two exemplary slices through the resulting volumes are depicted in Fig. 9.8. Within the slices, the granular layer of the dentate gyrus can be recognized as well as calcified blood vessels and amyloid plaques which are visible as electron-dense structures. The plaques are located in the molecular layer of the dentate gyrus, which is consistent with the findings of previous histological studies [32, 145], and closely follow the shape of the granular layer. In addition, calcified blood vessels, which can be identified as an accumulation of electron-dense deposits in the vascular 

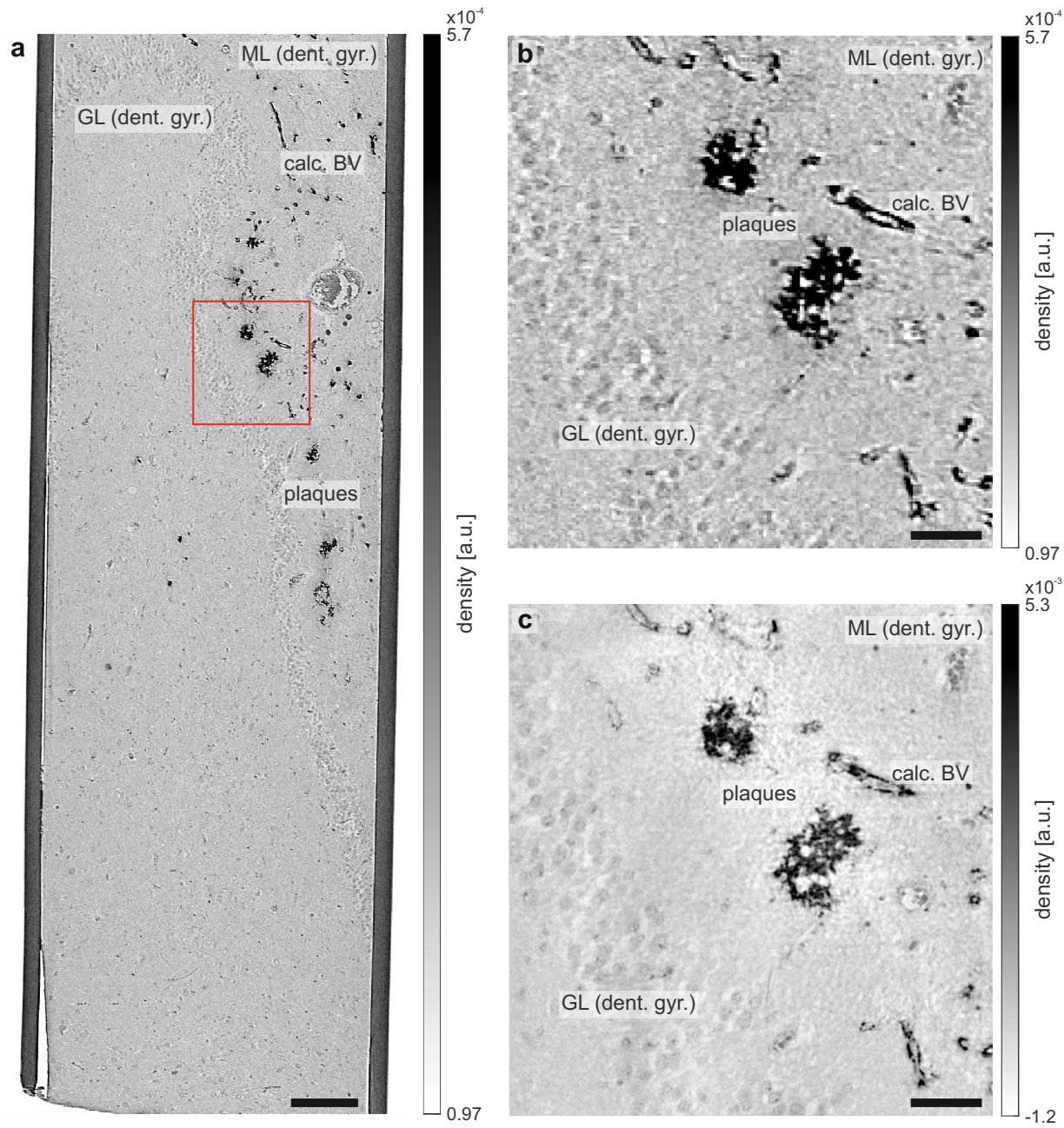

Figure 9.8: Visualization of the reconstructed volumes of a $1 \mathrm{~mm}$ punch from a paraffinembedded human hippocampus of a patient with Alzheimer's disease. (a) Virtual slice through the dataset obtained at the laboratory setup. In order to enlarge the field of view, three tomograms were acquired at adjacent positions in height. Within the slice, features as the granular $(G L)$, molecular $(M L)$ and polymorphic layer of the dentate gyrus, as well as calcified blood vessels and amyloid plaques, which typically occur in patients with Alzheimer's disease, can be recognized. (b) Magnified region from the overview shown in (a), which corresponds to the field of view of the synchrotron dataset. (c) Virtual slice through the dataset obtained at the synchrotron, showing the cells in the granular layer as well as the calcified blood vessels and the amyloid plaques at higher detail. Scale bars: $200 \mu \mathrm{m}(\mathrm{a})$ and $50 \mu \mathrm{m}(\mathrm{b}, \mathrm{c})$ 
wall, are also exclusively found in the molecular layer in close proximity to the amyloid plaques. To better visualize the structural organization of these specific features, a semi-automatic segmentation was carried out in Avizo. For the amyloid plaques and calcified and non-calcified blood vessels the region growing-based Magic Wand tool was used by manually determining seeding points within these structures, whereas the cells in the granular layer were labeled manually in each slice via the threshold-based Brush tool which only marks pixels within a manually selected region that lie in a specified gray value range. The segmentation result for the overview scan from the laboratory setup is shown in Fig. 9.9(a). The cell-rich band of the granular layer is depicted in light gray, enclosing the polymorphic layer on the left while the molecular layer is located on the right. It is evident that within the polymorphic layer only non-calcified blood vessels can be found whereas in the molecular layer the majority of blood vessels exhibits electron-dense deposits in the vascular wall. Amyloid plaques are exclusively found in the molecular layer in close proximity to the granular layer, following its general shape. The same behavior can be recognized in the segmentation results from the synchrotron, depicted in Fig. 9.9(b) and (c). The amyloid plaques are located in the molecular layer, enclosed by the cells of the granular layer and the calcified blood vessels.

Summary In this section, imaging results of a paraffin-embedded human hippocampus from a patient with Alzheimer's disease obtained both at the laboratory and the synchrotron were presented. In both datasets, amyloid plaques and calcified blood vessels are clearly visible due to the considerably larger electron density compared to the surrounding tissue. Both features are exclusively found in the molecular layer of the dentate gyrus, which is in good agreement with previous histological studies $[32,87,145,186]$. While the occurrence of amyloid plaques is one of the hallmarks of Alzheimer's disease, the link between Alzheimer's disease and the calcification of blood vessels in the hippocampus is not as clear. Wegiel et. al found a similar occurrence of calcifications in patients and an age-matched control group [186], whereas Kockelkoren et. al observed a significantly higher prevalence of hippocampal calcifications in patients with cognitive problems, which, however, were not necessarily caused by Alzheimer's disease [87]. Hence, a larger number of samples both from patients as well as age-matched controls would be required to further study the link between hippocampal calcifications and the occurrence or progression of Alzheimer's disease.

While the amyloid plaques as one of the hallmarks of Alzheimer's disease could 
a

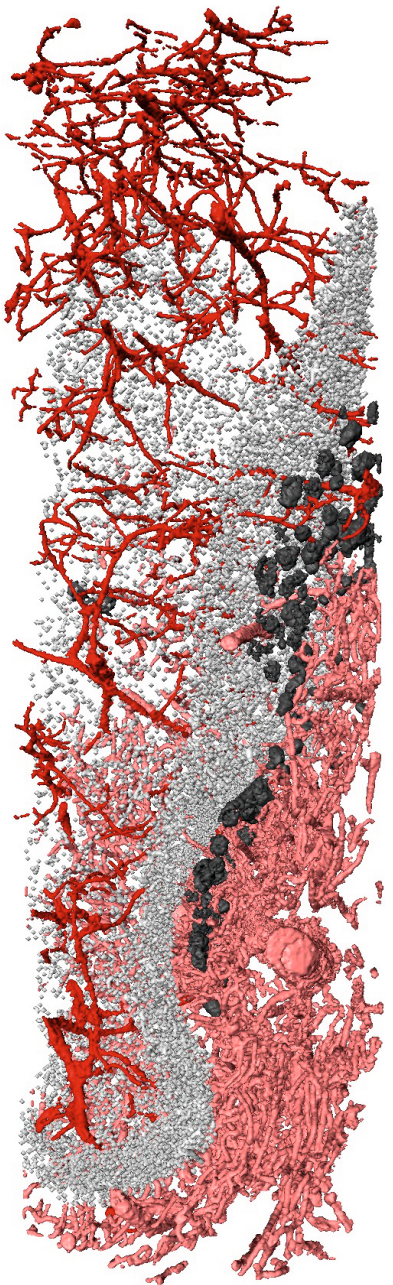

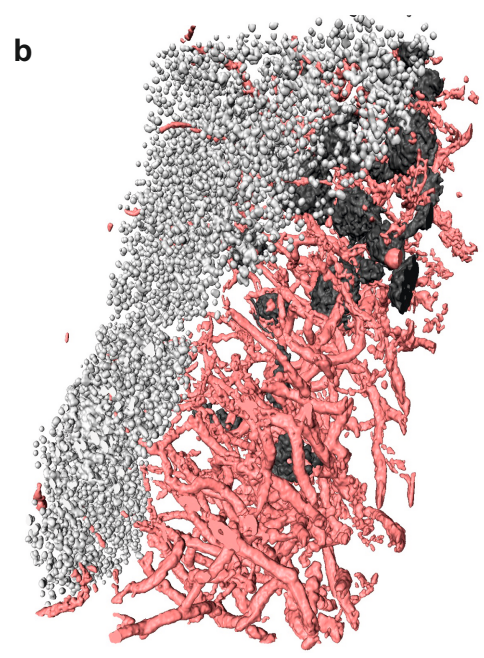

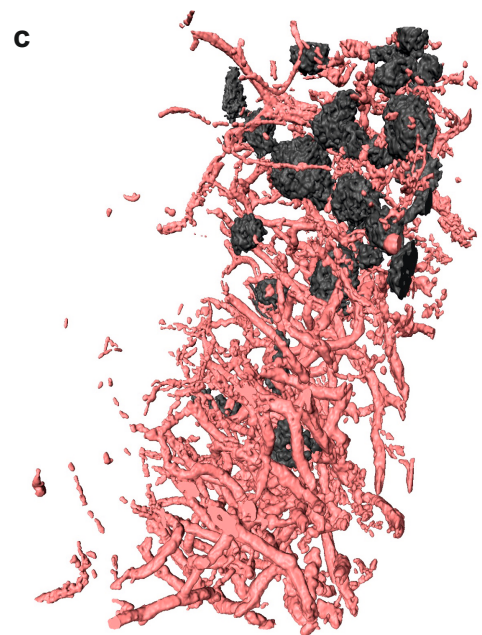

Figure 9.9: $3 d$ visualization of the $1 \mathrm{~mm}$ punch from a paraffin-embedded human hippocampus of a patient with Alzheimer's disease. (a) Segmentation of the reconstructed density distribution of the entire $1 \mathrm{~mm}$ punch obtained from three adjacent tomograms acquired at the laboratory setup. The cells of the granular layer are shown in light gray, whereas blood vessels are depicted in red, calcified blood vessels in light red and the amyloid plaques in dark gray. This segmentation shows that the amyloid plaques are located in close proximity to both the granular layer as well as the calcified blood vessels, whereas close to non-calcified blood vessels, no plaques can be recognized. (b,c) Segmentation of the synchrotron dataset both with the cells of the granular layer (b) and without (c). Also in this more detailed visualization of the plaques in relation to the calcified blood vessels, the close proximity between these two becomes apparent. 
be observed in the measurements, neurofibrillary tangles were not resolved. Since these tangles start to appear in the CA1 region of the hippocampus [154], the absence can most likely be explained by the position of the biopsy punch as it included the dentate gyrus and therefore the CA4 region (cf. section 6.2.1). Hence, in order to also image neurofibrillary tangles, further measurements have to be carried out on biopsy punches from the CA1 region of the hippocampus.

\subsection{Ischemic stroke}

Stroke is the second leading cause of mortality in the world with a crude death rate of 85 in 100,000 in the year 2015 [121]. It is either caused by vessel occlusions (ischemic stroke), which accounts for $85 \%$ of all cases, or primary intracerebral bleeding (hemorrhagic stroke). An ischemic stroke, which is most often caused by embolisms, is defined as a blood flow reduction which is severe enough to alter cellular function. For brain tissue, which is especially sensitive to ischemia, even brief periods of reduced blood flow can start a complex chain of events which may eventually lead to cellular death [192].

In the following, propagation-based phase-contrast tomography at the laboratory setup is used to image the alterations in brain tissue due to ischemic stroke in a mouse model. In order to compare the affected tissue with tissue from a healthy control, ischemia is induced exclusively in the left hemisphere, leaving the right hemisphere of the brain for comparison.

Methods Sample preparation was carried out following the approach described in detail in [39]. Ischemia was induced in the left hemisphere of a wild type mouse brain by temporary occluding the respective middle cerebral artery for 45 minutes. 24 hours after induction of ischemia, the animal was intraperitoneally injected with chloral hydrate $(420 \mathrm{mg} / \mathrm{kg}$ body weight) and transcardially perfused with $4 \%$ paraformaldehyde (PFA). The brain was dissected, postfixed in PFA and subsequently embedded in paraffin. Note that the sample was provided by the group of Thorsten Döppner (Department of Neurology, University Medical Center, Göttingen, Germany). For the experiments, excess paraffin was removed around the brain and an overview scan was recorded in cone-beam geometry. The sample was mounted in the setup by fixing it on a flat sample holder with hematocrit. Subsequently, the affected area was identified in the reconstructed volume and biopsy punches were taken from this area as well as the corresponding counterpart from the right hemisphere and squeezed into a Kapton tube which was glued to a 
sample holder (cf. Fig. 7.15). To control for the location of the punches, a second overview scan was recorded afterwards.

To image the entire brain, the laboratory setup was used in cone-beam geometry with the 'Talos' detector located at a source-to-detector distance $z_{02}=1.52 \mathrm{~m}$. The setup was operated at an acceleration voltage of $70 \mathrm{kV}$ and an electron spot size of $10 \times 40 \mathrm{\mu m}^{2}$ which enabled a power of $100 \mathrm{~W}$. By deflecting the electron beam slightly towards the exit window, the photon flux was increased (cf. Fig. 4.5). A filter consisting of a $25 \mu \mathrm{m}$ nickel foil and a $35 \mu \mathrm{m}$ silver foil was used for pre-hardening of the beam, leading to a main energy at the In- $K_{\alpha}$ emission line at $24.2 \mathrm{keV}$ [91, 170]. The sample was located $110 \mathrm{~mm}$ behind the source spot, resulting in an effective pixel size of $5.44 \mu \mathrm{m}$ and a corresponding field of view of $8.36 \times 10.58 \mathrm{~mm}^{2}$. For the tomographic scans, 1000 projections were recorded over an angular range of $183^{\circ}$ to account for the opening angle of the divergent beam. The exposure time was1.8 s per projection. Note that in order to increase the signal-to-noise ratio three images were acquired at each angular position and averaged in the subsequent data analysis.

The biopsy punches were measured in inverse geometry in order to reach the highest possible resolution and obtain results at cellular detail. By imaging the small $1 \mathrm{~mm}$ punches instead of zooming into the corresponding regions of the intact paraffin-embedded mouse brain, absorption by sample parts outside the field of view can be reduced, resulting in a higher signal-to-noise ratio in the reconstructed volumes. The 'Argos' detector was placed at a source-to-detector distance of $0.183 \mathrm{~m}$ and the acceleration voltage was decreased to $40 \mathrm{kV}$, enabling a maximum power of $57 \mathrm{~W}$. The source spot settings were chosen as in the cone-beam geometry. The sample was located at a source-to-sample distance of $158.75 \mathrm{~mm}$, resulting in an effective pixel size of $0.47 \mu \mathrm{m}$ with a field of view of $1.15 \times 1.53 \mathrm{~mm}^{2}$. Tomographic measurements were carried out by acquiring 1000 projections over $180^{\circ}$ with an exposure time of $50 \mathrm{~s}$. In order to image the entire biopsy punches, three tomograms at adjacent vertical positions along the sample were recorded both for the punch from the left and the right hemisphere. For a better overview, all experimental parameters are listed in Tab. 9.5.

Results Phase retrieval for the measurements in cone-beam geometry was performed via the BAC algorithm with the regularization parameters $\alpha=0.03$ and $(\gamma \cdot F)=0.16$. Prior to tomographic reconstruction with the FDK implementation of the ASTRA toolbox, the simple ring-removal algorithm (cf. section 3.2.2) was applied on the sinograms. The signal-to-noise ratio in the reconstructed volume 


\begin{tabular}{lcc}
\hline & cone-beam & inverse \\
\hline energy $[\mathrm{keV}]$ & $0-70 / 24.2$ & $0-40 / 9.25$ \\
detector & 'Talos' & 'Argos' \\
$z_{01}[\mathrm{~mm}]$ & 110 & 158.75 \\
$z_{02}[\mathrm{~m}]$ & 1.52 & 0.183 \\
$p_{\text {eff }}[\mathrm{\mu m}]$ & 5.44 & 0.47 \\
field of view $\left[\mathrm{mm}^{2}\right]$ & $8.36 \times 10.58$ & $1.15 \times 1.53$ \\
e-spot size $\left[\mathrm{\mu m}^{2}\right]$ & $10 \times 40$ & $10 \times 40$ \\
e-beam power $[\mathrm{W}]$ & 100 & 57 \\
Fresnel number $F$ & 5.66 & 0.078 \\
angular range $\left[{ }^{\circ}\right]$ & {$[0,183]$} & {$[0,180]$} \\
number of projections & 1000 & 1000 \\
exposure time $[\mathrm{s}]$ & $3 \times 1.8$ & 50 \\
BAC $\alpha$ & 0.03 & 0.01 \\
BAC $(\gamma \cdot F)$ & 0.16 & 0.16 \\
\hline
\end{tabular}

Table 9.5: Experimental parameters used for imaging of a paraffin-embedded mouse brain with an ischemic stroke induced in the left hemisphere.

was increased by filtering each two-dimensional slice with a Gaussian function with a 1-pixel standard deviation. To compare the overview scans recorded before and after taking the biopsy punches, the scans were manually aligned to each other in Avizo. For the measurements in inverse geometry, phase retrieval was likewise performed with the BAC algorithm, using the regularization parameters $\alpha=0.01$ and $(\gamma \cdot F)=0.16$. Prior to the phase-retrieval step, the projections were resampled by a factor of 2 to increase the signal-to-noise ratio. Ring artifacts were reduced via the wavelet-based approach described in section 3.2.2 and the tomographic reconstruction was again carried out via the FDK implementation of the ASTRA toolbox. Subsequently, the signal-to-noise ratio was further improved by filtering the reconstructed virtual slices with a Gaussian function with a standard deviation of 1 pixel. The single tomograms recorded at adjacent positions along the sample were aligned and merged in Avizo. Afterwards they were scaled to the size of the overview scans and manually aligned to these scans so that the position of the punches could be set in relation to the entire brain. This makes it possible to identify similar brain regions within the two punches using the results of the overview scan, which is necessary for a fair comparison.

The results from the overview scans are depicted in Fig. 9.10. A volume rendering of the entire brain prior to taking the biopsy punches is shown in (a). The outer shape of the brain can be clearly recognized as well as a slight swelling of the 
a

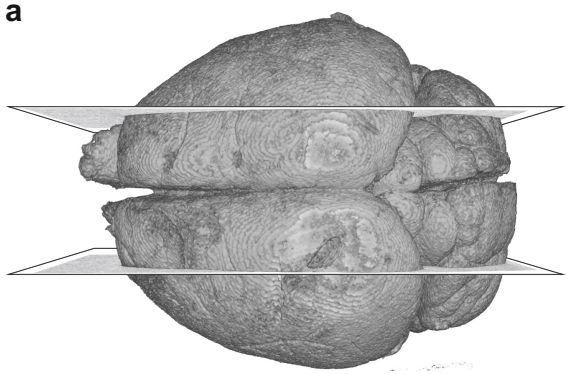

b

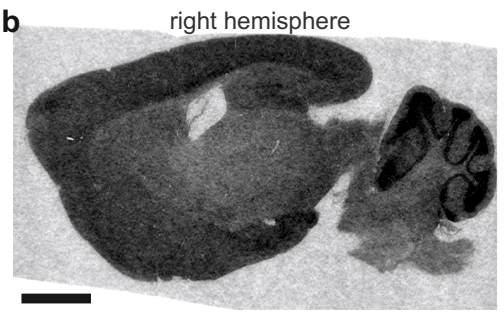

left hemisphere

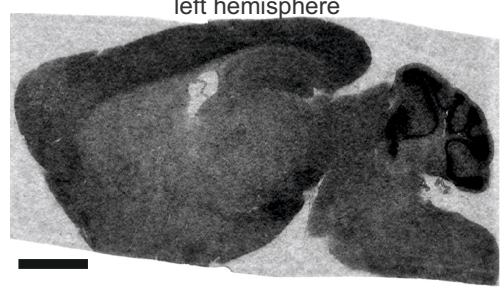

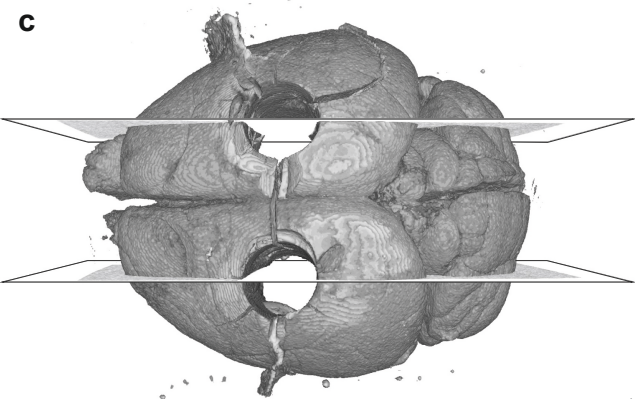

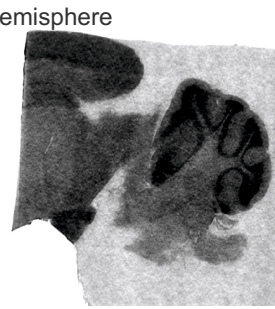

$\times 10^{-4}$

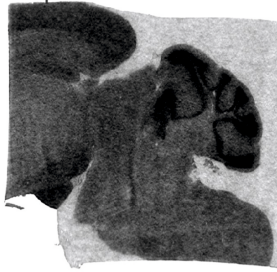

d

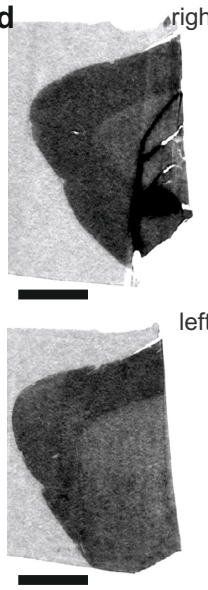

right hemisphere

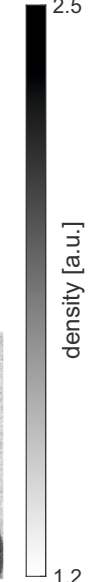

left hemisphere

1.2

Figure 9.10: Overview scan of the paraffin-embedded mouse brain with an ischemic stroke, both before and after taking a $1 \mathrm{~mm}$ punch from each of the hemispheres. (a) Volume rendering of the intact brain, in which the stroke was induced in the left hemisphere. A slight swelling of this hemisphere can be recognized in the $3 d$ visualization. Two planes mark the positions of the sagittal slices depicted in (b), which lie symmetrically around the center line of the brain. (b) Sagittal slices through the brain from both the right and the left hemisphere. In the slice from the left hemisphere, the stroke can be recognized as a slightly lower density in the affected areas. (c) Volume rendering of the brain after taking the two punches. A crack, which occurred after taking the second punch, can be recognized in the center of the brain. (d) Two sagittal slices at approximately the same position as in (b). The location of the punches can be clearly recognized, enabling a better comparison between the two high resolution measurements in Fig. 9.11. Scale bars: $1 \mathrm{~mm}$

left hemisphere caused by the ischemic stroke. The two planes lying symmetrically around the center line of the brain indicate the position of the sagittal slices in (b). By comparing these slices from the left and right hemisphere, the effect of the stroke can be recognized as a slightly lower overall tissue density in the affected areas. A volume rendering of the overview scan recorded after taking the biopsy 
punches is depicted in (c). The location of the punches is clearly visible as two cylindrical holes through the left and right hemispheres. As those punches lie approximately symmetrical around the center line of the brain, similar regions from the left and right hemisphere were measured in the high resolution scans, enabling a comparison between affected and healthy tissue. In the center of the brain, a crack running approximately perpendicular to the center line can be recognized which occurred after taking the second punch. The planes again indicate the positions of the two sagittal slices in (d), coinciding with those shown in (b) for a better comparison.

The results from the high resolution measurements are depicted in Fig. 9.11. In (a) and (b) sagittal slices through the $3 \mathrm{~d}$ density distributions of the punches from the right and left hemispheres are shown. The positions of these slices correspond to those in Fig. 9.10(b) and (d) from the overview scans. Within the slices, typical features as the cortex of the mouse brain, part of the pyramidal cell layer of the hippocampus, the ventricle as well as the striatum can be identified. The direct comparison between the left and right hemispheres confirm the results from the overview scan as the overall tissue density, especially in the striatum, seems to be lower in the left hemisphere which can be recognized at the slightly lighter gray values. However, when considering the enlarged views of the regions indicated by the rectangles, depicted in (c) and (d), it actually becomes apparent that within the punch of the left hemisphere, an increase in cell density occurs which at first sight contradicts the fact that an ischemic stroke leads to neuronal cell loss. As propagation-based imaging is sensitive to electron density differences only, the differentiation between cell types in unstained tissue is mainly limited to differences in shape and cells of similar shape are not distinguishable. Hence, the observed cells are not necessarily neurons and the higher cell density may be explained by the inflammatory response of the brain following an ischemic stroke which leads to an activation of microglia and astrocytes and an infiltration of neutrophils [79, 184]. This might explain the observed increase in cell density in the affected regions.

Summary In this section, propagation-based phase-contrast tomography at a laboratory source was used for imaging of a mouse brain with an ischemic stroke induced in the left hemisphere. The setup was used both in cone-beam as well as inverse geometry, enabling the measurement of the entire brain before and after taking two biopsy punches from the left and right hemisphere which were subsequently recorded at cellular detail. This combination of geometries allowed on the one hand the determination of regions of interest so that the punches could be 


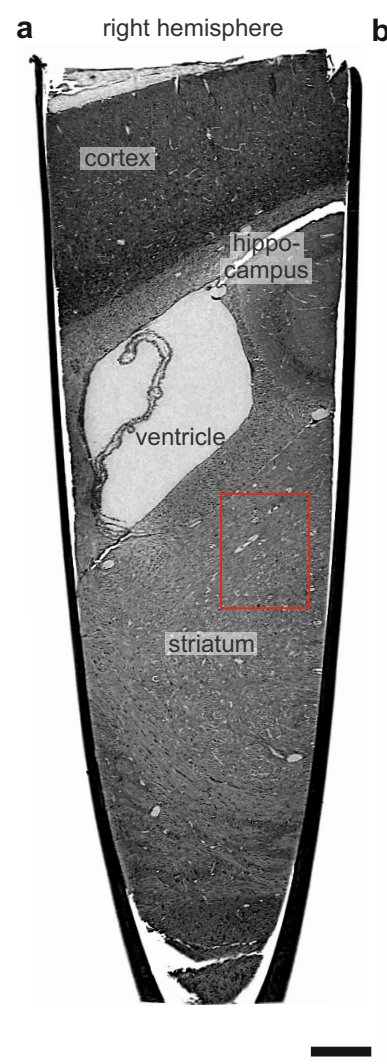

b left hemisphere
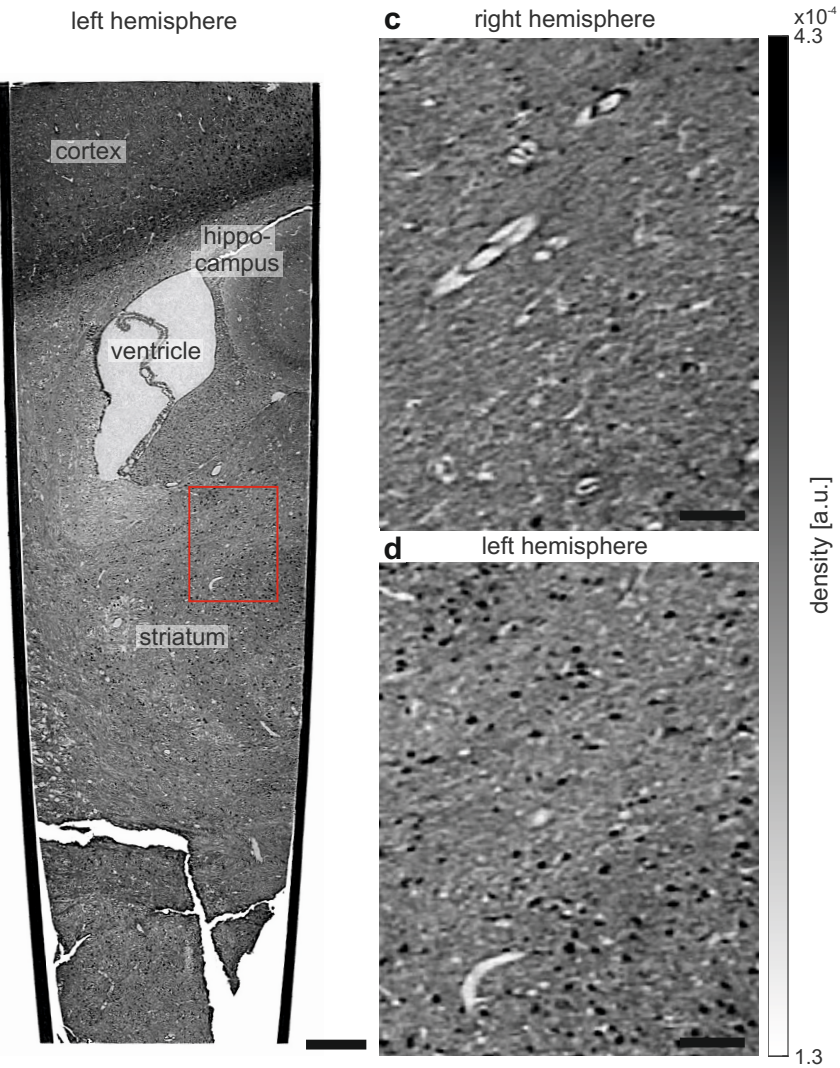

Figure 9.11: Slices through the reconstructed density of $1 \mathrm{~mm}$ biopsy punches from both the right and left hemisphere of a paraffin-embedded mouse brain with an ischemic stroke measured in inverse geometry. (a) Sagittal slice through the right hemisphere at approximately the same position as in Fig. 9.10(b) and (d). The field of view of the measurement was increased by recording three tomograms at adjacent positions in height. Typical features as the cortex, hippocampus, ventricle and striatum can be recognized. (b) Corresponding sagittal slice through the left hemisphere. Compared to the counterpart from the right hemisphere, overall tissue density, especially in the region of the striatum, seems to be lower. (c) Magnified region of the slice through the right hemisphere depicted in (a). The position of this region is marked by a rectangle. Few cells can be recognized as dark spots, while blood vessels exhibit light gray values. (d) Magnified view of the region marked by the rectangle in (b). Despite the lower overall tissue density, the number of cells is significantly increased compared to the right hemisphere. Scale bars: $200 \mu \mathrm{m}(\mathrm{a}, \mathrm{b})$ and $50 \mu \mathrm{m}(c, d)$ 
positioned properly while on the other hand the exact locations of the two punches could be verified afterwards. Additionally, the reconstructed densities of the high resolution scans could be set in relation to the entire brain by manually aligning the scans to each other, enabling the identification of similar regions within the two punches, which would not have been possible without the overview scan.

Both in the overview scan as well as the high resolution measurements of the biopsy punches the affected area could be identified due to a lower overall tissue density visible as lighter gray values in the corresponding regions. However, within the punch from the left hemisphere, a higher cell density could be identified in the region of the striatum which contradicts the neuronal cell loss expected from ischemic stroke. To answer the question whether the increased cell density is caused by the inflammatory response and hence activation of microglia and astrocytes as well as infiltration of neutrophils, the x-ray experiments could be combined with subsequent histological experiments with specific staining agents.

Additionally, a larger number of specimens should be studied to increase the statistical relevance of the experiment and the time lapse between the initiation of the stroke and the dissection of the brains could be varied in order to study the time course of the suspected inflammatory response to the induced ischemia. 



\section{Conclusion}

In this thesis, propagation-based phase-contrast tomography was used to perform $3 \mathrm{~d}$ virtual histology of neuronal tissue, providing insights into mm-sized specimens with (sub-)cellular resolution. To this end, different combinations of experimental setups, phase-retrieval algorithms and sample preparations were evaluated and optimized for the respective applications. Already in hydrated tissue, presenting a preparation technique in close proximity to the native state, tomographic experiments at the GINIX setup enabled the resolution of single cells in mm-sized tissue from a mouse cerebellum. One decisive factor for this was the increased contrast transfer due to image formation in the holographic regime. The small cells in the granular and molecular layer as well as the larger Purkinje cells and to some extent even their dendritic tree could be visualized in 3d. However, contrast was not sufficient to unequivocally decipher the cytoarchitecture or connectivity and hence, contrast enhancement via different preparation techniques was evaluated. One possibility to increase tissue contrast is provided by staining with radiocontrast agents. In this thesis, standard preparation protocols from electron microscopy and histology were evaluated, namely by imaging a strip of mouse retina stained with osmium tetroxide as well as a brain slice from the region of the hippocampus stained with silver according to the Golgi-Cox technique. Sub-cellular details like the different components of the retinal photoreceptor cells or the dendritic tree of hippocampal pyramidal cells were imaged at superior contrast and resolution. The neuronal cells could be semi-automatically segmented based on gray value differences, allowing for the visualization of their three-dimensional shape as well as structural organization with respect to the surrounding tissue. Even at the laboratory, the combination of an optimized geometry and suitable phase retrieval enabled a contrast and resolution high enough to image single cells at sub-cellular detail with a comparably large field of view. Hence, questions of neuron distribution and spatial organization, crude shape and orientation can already be addressed at the laboratory. Note that by enhancing the contrast via metal staining, imaging of neuronal specimens is either restricted to small sample sizes due to the large absorption of the respective contrast agents or to imaging of specific sparsely stained features. 
In order to increase overall tissue contrast at moderate absorption, the influence of embedding media with a lower density compared to water was evaluated as a second sample preparation approach. The largest difference in electron density was achieved by exchanging the water content of the samples by air. The development of the evaporation-of-solvent method by Jürgen Goldschmidt enabled this exchange while preserving the general structure of the sample. Imaging of different parts of a mouse brain at the laboratory source led to unprecedented data quality with micrometer resolution comparable to classical histology, showing important hallmarks of the mouse brain cytoarchitecture at cellular detail, including the different layers of the cerebellum, the barrel field of the cortex as well as the hippocampal formation or olfactory bulb. Data quality was high enough for a semi-automatic segmentation of large axon bundles and blood vessels as well as single cells within the different layers of the cerebellum, enabling the visualization of their three-dimensional spatial distribution. Due to the strong differences in electron density, phase retrieval in the holographic regime is challenging, impeding imaging of these samples at higher resolution at the synchrotron so far. Other reconstruction methods like near-field ptychography may overcome these limitations but yield further restrictions and disadvantages. In the current setting, embedding of the samples in ethanol and paraffin proved to be an appropriate choice to increase overall tissue contrast in a mouse cerebellum for measurements at the GINIX setup, with the latter being especially suited to decipher the threedimensional cytoarchitecture as nuclei and nucleoli were considerably enhanced with respect to the surrounding tissue. In addition, the increased stability of the sample with regard to internal changes as well as mounting yields a potential increase in resolution.

The ability to enhance the contrast of unstained samples by embedding them in paraffin enabled the study of neuropathological samples that are usually prepared in this way, especially samples from the human brain obtained during routine autopsy. Tomographic experiments on human cerebellum were performed at the GINIX setup both in parallel- and cone-beam geometry, providing 3d reconstructions with varying resolution and field of view which enabled the non-destructive visualization of sub-cellular details as the nuclei and nucleoli as well as dendritic tree of the large Purkinje cells. Also at the laboratory setup, single cells were resolved in all layers of the cerebellum and even the thick branches of the dendritic tree of the Purkinje cells could be recognized. By developing a workflow based on the spherical Hough transform, the small cells of the molecular and granular layer could be localized automatically, showing a high accuracy both for the syn- 
chrotron and laboratory results. It enabled the segmentation of several thousands to millions of cells, respectively, as well as a subsequent statistical analysis based on their spatial distribution.

The application of phase-contrast tomography for the $3 \mathrm{~d}$ visualization of different neurological diseases was demonstrated in first experiments on multiple sclerosis and Alzheimer's disease in human patients as well as ischemic stroke in a mouse model, showing alterations in $3 \mathrm{~d}$ cellular distributions compared to healthy controls or the evolution of disease-specific hallmarks. These studies could be extended in the future by increasing the number of samples in order to further emphasize differences between affected and healthy tissue or by combining the results of the $\mathrm{x}$ ray experiments with subsequent histological studies using specific staining agents to correlate the visualization of the entire electron density distribution with more specialized information, e.g., on different cell types.

In the course of this thesis it was shown that embedding in different media leads to an overall increase in tissue contrast suitable to resolve the underlying cytoarchitecture while staining with metals can be advantageously used to increase contrast in small sub-cellular details as the dendritic tree of pyramidal neurons. From this it can be concluded that the future goal of deciphering the three-dimensional connectivity between nerve cells with the help of x-ray phase-contrast tomography requires staining with suitable radiocontrast agents as this preparation approach can potentially provide the resolution and contrast necessary to resolve the synaptic connections between the dendrites and the axon terminals of the corresponding neighboring cells. In the experiments presented in this thesis, incomplete staining due to a leakage of silver molecules during the embedding procedure or tomographic acquisition as well as an insufficient system resolution, sample motion and imperfect phase retrieval prevented the visualization of these connections. Therefore, future experiments have to be optimized with respect to these multiple factors, e.g., by improving the mechanical stability of the setup as well as the waveguide performance, allowing for faster acquisitions with less motion artifacts and a higher signal-to-noise ratio, by adapting the sample preparation so that a complete and stable staining of specific features of interest can be reached or by applying phase-retrieval algorithms posing less restrictions on the sample.

In conclusion, 3d virtual histology of tissues from the central nervous system could be successfully performed with propagation-based phase-contrast tomography both at the laboratory as well as synchrotron, visualizing the $3 \mathrm{~d}$ cytoarchitecture of mm-sized samples with varying resolution and field of view. Automated cellular segmentation of several thousands to millions of cells within the 
reconstructed volumes allowed for the determination of the three-dimensional local density distribution and provided insights into their structural organization based on concepts borrowed from condensed matter physics. 


\section{Appendix}

\section{A.1 Fourier transform and its properties}

\section{A.1.1 Definition}

Throughout the present thesis, the Fourier transform $\mathcal{F}[f]$ of an integrable function $f \in L^{1}\left(\mathbb{R}^{n}\right)$ is defined as

$$
\hat{f}(\mathbf{k})=\mathcal{F}[f](\mathbf{k})=\frac{1}{(2 \pi)^{n / 2}} \int_{\mathbb{R}^{n}} f(\mathbf{r}) e^{-i \mathbf{k} \cdot \mathbf{r}} \mathrm{d} \mathbf{r} .
$$

Accordingly, the inverse Fourier transform is given by

$$
f(\mathbf{r})=\mathcal{F}^{-1}[\hat{f}](\mathbf{r})=\frac{1}{(2 \pi)^{n / 2}} \int_{\mathbb{R}^{n}} \hat{f}(\mathbf{k}) e^{i \mathbf{k} \cdot \mathbf{r}} \mathrm{d} \mathbf{k} .
$$

\section{A.1.2 Linearity}

The Fourier transform is a linear operation

$$
\mathcal{F}[a f+b g]=a \mathcal{F}[f]+b \mathcal{F}[b]
$$

\section{A.1.3 Pseudo-differential-operators}

A differentiation in real space leads to a multiplication in reciprocal space [125]

$$
\left(\frac{\partial^{m}}{\partial x^{m}}+\frac{\partial^{n}}{\partial y^{n}}\right)^{p}=\mathcal{F}_{\perp}^{-1}\left[\left(i k_{x}\right)^{m}+\left(i k_{y}\right)^{n}\right]^{p} \mathcal{F}_{\perp},
$$

with $m, n, p \in \mathbb{Z} \backslash\{0\}$. Note that for negative values of $m$ and $n$, this operation inverts the differentiation in real space. 


\section{A.1.4 Convolution theorem}

The Fourier transform of the convolution

$$
f(\mathbf{r}) \star g(\mathbf{r}):=\int_{\mathbb{R}^{n}} f\left(\mathbf{r}_{0}\right) g\left(\mathbf{r}-\mathbf{r}_{0}\right) \mathrm{d} \mathbf{r}_{0}
$$

is given by the product of their individual Fourier transforms

$$
\mathcal{F}[f(\mathbf{r}) \star g(\mathbf{r})]=(2 \pi)^{n / 2} \mathcal{F}[f(\mathbf{r})] \mathcal{F}[g(\mathbf{r})]
$$

and vice versa

$$
\mathcal{F}[f(\mathbf{r})] \mathcal{F}[g(\mathbf{r})]=\frac{1}{(2 \pi)^{n / 2}} \mathcal{F}[f(\mathbf{r}) \star g(\mathbf{r})]
$$

\section{A.1.5 Fourier transform of the Dirac Delta distribution}

The Dirac Delta distribution $\delta_{D}$, defined as

$$
\delta_{D}(\mathbf{r})=\left\{\begin{array}{ll}
+\infty, & \mathbf{r}=\mathbf{0} \\
0, & \mathbf{r} \neq \mathbf{0}
\end{array},\right.
$$

yields

$$
\int_{\mathbb{R}^{n}} \delta_{D}(\mathbf{r}) \mathrm{d} \mathbf{r}=1
$$

and applied to a compactly supported continuous function $f(\mathbf{r})$

$$
\int_{\mathbb{R}^{n}} \delta_{D}\left(\mathbf{r}-\mathbf{r}_{0}\right) f(\mathbf{r}) \mathrm{d} \mathbf{r}=f\left(\mathbf{r}_{0}\right) .
$$

It satisfies the scaling property

$$
\begin{aligned}
\int_{\mathbb{R}^{n}} \delta_{D}(\alpha \mathbf{r}) \mathrm{d} \mathbf{r} & =\int_{\mathbb{R}^{n}} \delta_{D}(\mathbf{u}) \frac{1}{|\alpha|} \mathrm{d} \mathbf{u} \\
\Rightarrow \delta_{D}(\alpha \mathbf{r}) & =\frac{\delta_{D}(\mathbf{r})}{|\alpha|}
\end{aligned}
$$


for $\alpha \neq 0$.

The Fourier transform of the Dirac Delta distribution results in

$$
\begin{aligned}
\mathcal{F}\left[\delta_{D}(\mathbf{r})\right] & =\frac{1}{(2 \pi)^{n / 2}} \int_{\mathbb{R}^{n}} \delta_{D}(\mathbf{r}) e^{-i \mathbf{k} \cdot \mathbf{r}} \mathrm{d} \mathbf{r} \\
& =\frac{1}{(2 \pi)^{n / 2}} e^{-i \mathbf{k} \cdot \mathbf{0}} \\
& =\frac{1}{(2 \pi)^{n / 2}} .
\end{aligned}
$$

Equivalently, the Fourier transform of $\mathbf{1}$ leads to a Delta distribution [18]

$$
\begin{aligned}
\mathcal{F}[\mathbf{1}] & =\frac{1}{(2 \pi)^{n / 2}} \int_{\mathbb{R}} e^{-i \mathbf{k} \cdot \mathbf{r}} \mathrm{d} \mathbf{r} \\
& =(2 \pi)^{n / 2} \delta_{D}(\mathbf{k}) .
\end{aligned}
$$

\section{A.2 Step-by-step derivation of the contrast transfer function}

The step-by-step derivation of the contrast transfer function closely follows [195] and [58]. The wave field $\psi_{\omega}\left(\mathbf{r}_{\perp}, 0\right)$ in the exit plane of the object is given by the product of the probe $P_{\omega}\left(\mathbf{r}_{\perp}\right)$ (1.63) and object transmission function $O_{\omega}\left(\mathbf{r}_{\perp}\right)$ (1.64)

$$
\begin{aligned}
\psi_{\omega}\left(\mathbf{r}_{\perp}, 0\right) & =e^{i k d} P_{\omega}\left(\mathbf{r}_{\perp}\right) e^{-i k \bar{\delta}_{\omega}\left(\mathbf{r}_{\perp}\right)-k \bar{\beta} \omega\left(\mathbf{r}_{\perp}\right)} \\
& =: e^{i k d} P_{\omega}\left(\mathbf{r}_{\perp}\right) e^{i \phi_{\omega}\left(\mathbf{r}_{\perp}\right)-\xi_{\omega}\left(\mathbf{r}_{\perp}\right)}
\end{aligned}
$$

To obtain the wave field at distance $z$ behind the object, the exit wave is propagated with the real space Fresnel propagator $H^{(F)}(1.37)$ via a convolution in real space. For simplicity, this operation is carried out in one dimension, without loss of generality along $x$, but due to the equivalence of the derivation, the transition to two dimensions is trivial. The propagated wave field is given by

$$
\psi_{\omega}(x, z)=\psi_{\omega}(x, 0) \star H^{(F)}(x, z)=\frac{1}{(i \lambda z)^{1 / 2}} \int_{\mathbb{R}} \mathrm{d} x^{\prime} e^{\frac{i \pi\left(x-x^{\prime}\right)^{2}}{\lambda z}} \psi_{\omega}\left(x^{\prime}\right),
$$

where $\psi_{\omega}\left(x^{\prime}\right):=\psi_{\omega}\left(x^{\prime}, 0\right)$ was defined.

The intensity is the only measurable quantity of the wave field and is therefore considered in the following. It can be calculated via the absolute square of the propagated wave field $I_{\omega}(x, z)=\left|\psi_{\omega}(x, z)\right|^{2}=\psi_{\omega}(x, z) \cdot \psi_{\omega}^{*}(x, z)$. The Fourier 
transform of the intensity yields

$$
\begin{aligned}
\hat{I}_{\omega}\left(k_{x}, z\right) & =\frac{1}{\lambda z} \frac{1}{\sqrt{2 \pi}} \int_{\mathbb{R}} \mathrm{d} x e^{-i k_{x} x} \int_{\mathbb{R}} \mathrm{d} x^{\prime} e^{\frac{i \pi\left(x-x^{\prime}\right)^{2}}{\lambda z}} \psi_{\omega}\left(x^{\prime}\right) \int_{\mathbb{R}} \mathrm{d} x^{\prime \prime} e^{-\frac{i \pi\left(x-x^{\prime \prime}\right)^{2}}{\lambda z}} \psi_{\omega}^{*}\left(x^{\prime \prime}\right) \\
& =\frac{1}{\lambda z} \frac{1}{\sqrt{2 \pi}} \int_{\mathbb{R}} \mathrm{d} x e^{-i k_{x} x} \int_{\mathbb{R}^{2}} \mathrm{~d} x^{\prime} \mathrm{d} x^{\prime \prime} e^{\frac{i \pi\left(\left(x-x^{\prime}\right)^{2}-\left(x-x^{\prime \prime}\right)^{2}\right)}{\lambda z}} \psi_{\omega}\left(x^{\prime}\right) \psi_{\omega}^{*}\left(x^{\prime \prime}\right) .
\end{aligned}
$$

Multiplying the quadratic terms leads to

$$
\hat{I}_{\omega}\left(k_{x}, z\right)=\frac{1}{\lambda z} \frac{1}{\sqrt{2 \pi}} \int_{\mathbb{R}} \mathrm{d} x e^{-i k_{x} x} \int_{\mathbb{R}^{2}} \mathrm{~d} x^{\prime} \mathrm{d} x^{\prime \prime} e^{\frac{i \pi\left(x^{\prime 2}-2 x^{\prime} x-x^{\prime \prime 2}+2 x^{\prime \prime} x\right)}{\lambda z}} \psi_{\omega}\left(x^{\prime}\right) \psi_{\omega}^{*}\left(x^{\prime \prime}\right) .
$$

Exploiting the integral definition of the Dirac Delta distribution (A.16) and isolating the quadratic terms in the exponential function, transforms this expression to

$$
\begin{aligned}
\hat{I}_{\omega}\left(k_{x}, z\right) & =\frac{1}{\lambda z} \frac{1}{\sqrt{2 \pi}} \int_{\mathbb{R}^{2}} \mathrm{~d} x^{\prime} \mathrm{d} x^{\prime \prime} e^{\frac{i \pi\left(x^{\prime 2}-x^{\prime \prime 2}\right)}{\lambda z}} \psi_{\omega}\left(x^{\prime}\right) \psi_{\omega}^{*}\left(x^{\prime \prime}\right) \underbrace{\int_{\mathbb{R}} \mathrm{d} x e^{\frac{2 i \pi x}{\lambda z}\left(x^{\prime \prime}-x^{\prime}-\frac{\lambda z k_{x}}{2 \pi}\right)}}_{\lambda z \delta_{D}\left(x^{\prime \prime}-x^{\prime}-\frac{\lambda z k_{x}}{2 \pi}\right)} \\
& =\frac{1}{\sqrt{2 \pi}} \int_{\mathbb{R}^{2}} \mathrm{~d} x^{\prime} \mathrm{d} x^{\prime \prime} e^{\frac{i \pi\left(x^{\prime 2}-x^{\prime \prime 2}\right)}{\lambda z}} \psi_{\omega}\left(x^{\prime}\right) \psi_{\omega}^{*}\left(x^{\prime \prime}\right) \delta_{D}\left(x^{\prime \prime}-x^{\prime}-\frac{\lambda z k_{x}}{2 \pi}\right) .
\end{aligned}
$$

Executing the integral over $x^{\prime \prime}$ and using eqn. (A.10) yields

$$
\hat{I}_{\omega}\left(k_{x}, z\right)=\frac{1}{\sqrt{2 \pi}} \int_{\mathbb{R}} \mathrm{d} x^{\prime} e^{\frac{i \pi\left(x^{\prime 2}-\left(x^{\prime}+\frac{\lambda z k_{x}}{2 \pi}\right)^{2}\right)}{\lambda z}} \psi_{\omega}\left(x^{\prime}\right) \psi_{\omega}^{*}\left(x^{\prime}+\frac{\lambda z k_{x}}{2 \pi}\right) .
$$

By again multiplying and rearranging the quadratic terms, the expression simplifies to

$$
\begin{aligned}
\hat{I}_{\omega}\left(k_{x}, z\right) & =\frac{1}{\sqrt{2 \pi}} \int_{\mathbb{R}} \mathrm{d} x^{\prime} e^{\frac{-i \pi\left(\frac{\lambda z k_{x} x^{\prime}}{\pi}+\frac{\lambda^{2} z^{2} k_{x}^{2}}{4 \pi^{2}}\right)}{\lambda z}} \psi_{\omega}\left(x^{\prime}\right) \psi_{\omega}^{*}\left(x^{\prime}+\frac{\lambda z k_{x}}{2 \pi}\right) \\
& =e^{-\frac{i \lambda z k_{x}^{2}}{4 \pi}} \frac{1}{\sqrt{2 \pi}} \int_{\mathbb{R}} \mathrm{d} x^{\prime} e^{-i k_{x} x^{\prime}} \psi_{\omega}\left(x^{\prime}\right) \psi_{\omega}^{*}\left(x^{\prime}+\frac{\lambda z k_{x}}{2 \pi}\right) .
\end{aligned}
$$


A plane wave illumination with intensity $I_{0}$ leads to

$$
\frac{\hat{I}_{\omega}\left(k_{x}, z\right)}{I_{0}}=e^{-\frac{i \lambda z k_{x}^{2}}{4 \pi}} \frac{1}{\sqrt{2 \pi}} \int_{\mathbb{R}} \mathrm{d} x^{\prime} e^{-i k_{x} x^{\prime}} O_{\omega}\left(x^{\prime}\right) O_{\omega}^{*}\left(x^{\prime}+\frac{\lambda z k_{x}}{2 \pi}\right) .
$$

With the assumption of a weak absorption $\left(\xi_{\omega}\left(x^{\prime}\right) \ll 1\right)$ and slowly varying phase $\left(\left|\Delta \phi_{\omega}\left(x^{\prime}\right)\right|=\left|\phi_{\omega}\left(x^{\prime}\right)-\phi_{\omega}\left(x^{\prime}+\frac{\lambda z k_{x}}{2 \pi}\right)\right| \ll 1\right)$, the product of the object transmission function can be approximated by a first order Taylor expansion

$$
\begin{aligned}
& O_{\omega}\left(x^{\prime}\right) O_{\omega}^{*}\left(x^{\prime}+\frac{\lambda z k_{x}}{2 \pi}\right)= e^{i\left(\phi_{\omega}\left(x^{\prime}\right)-\phi_{\omega}\left(x^{\prime}+\frac{\lambda z k_{x}}{2 \pi}\right)\right)-\left(\xi_{\omega}\left(x^{\prime}\right)+\xi_{\omega}\left(x^{\prime}+\frac{\lambda z k_{x}}{2 \pi}\right)\right)} \\
& \simeq 1+i\left(\phi_{\omega}\left(x^{\prime}\right)-\phi_{\omega}\left(x^{\prime}+\frac{\lambda z k_{x}}{2 \pi}\right)\right) \\
&-\left(\xi_{\omega}\left(x^{\prime}\right)+\xi_{\omega}\left(x^{\prime}+\frac{\lambda z k_{x}}{2 \pi}\right)\right) .
\end{aligned}
$$

Inserting this approximated expression into eqn. (A.28) and introducing $\chi=\frac{\lambda z k_{x}^{2}}{4 \pi}$ yields

$$
\begin{aligned}
\frac{\hat{I}_{\omega}\left(k_{x}, z\right)}{I_{0}}=e^{-i \chi} \frac{1}{\sqrt{2 \pi}} & \left\{\int_{\mathbb{R}} \mathrm{d} x^{\prime} e^{-i k_{x} x^{\prime}}\right. \\
+ & i \int_{\mathbb{R}} \mathrm{d} x^{\prime} e^{-i k_{x} x^{\prime}}\left(\phi_{\omega}\left(x^{\prime}\right)-\phi_{\omega}\left(x^{\prime}+\frac{\lambda z k_{x}}{2 \pi}\right)\right) \\
& \left.-\int_{\mathbb{R}} \mathrm{d} x^{\prime} e^{-i k_{x} x^{\prime}}\left(\xi_{\omega}\left(x^{\prime}\right)+\xi_{\omega}\left(x^{\prime}+\frac{\lambda z k_{x}}{2 \pi}\right)\right)\right\} .
\end{aligned}
$$

The first term in this expression corresponds to the Fourier transform of 1, leading to a Delta distribution (cf. eqn. (A.16)). As the phase factor $e^{-i \chi}$ is equal to unity when the Delta distribution deviates from 0 , it can be neglected, leading to

$$
\begin{aligned}
\frac{\hat{I}_{\omega}\left(k_{x}, z\right)}{I_{0}}= & \sqrt{2 \pi} \delta_{D}\left(k_{x}\right)+e^{-i \chi} \frac{1}{\sqrt{2 \pi}}\left\{i \int_{\mathbb{R}} \mathrm{d} x^{\prime} e^{-i k_{x} x^{\prime}}\left(\phi_{\omega}\left(x^{\prime}\right)-\phi_{\omega}\left(x^{\prime}+\frac{\lambda z k_{x}}{2 \pi}\right)\right)\right. \\
& \left.-\int_{\mathbb{R}} \mathrm{d} x^{\prime} e^{-i k_{x} x^{\prime}}\left(\xi_{\omega}\left(x^{\prime}\right)+\xi_{\omega}\left(x^{\prime}+\frac{\lambda z k_{x}}{2 \pi}\right)\right)\right\} .
\end{aligned}
$$

The first summands in the remaining integrals correspond to the Fourier transforms of the phase $\phi_{\omega}\left(x^{\prime}\right)$ and attenuation coefficient $\xi_{\omega}\left(x^{\prime}\right)$, respectively. Hence, 
the equation can be rewritten as

$$
\begin{aligned}
\frac{\hat{I}_{\omega}\left(k_{x}, z\right)}{I_{0}}= & \sqrt{2 \pi} \delta_{D}\left(k_{x}\right)+i e^{-i \chi}\left\{\hat{\phi}_{\omega}\left(k_{x}\right)-\frac{1}{\sqrt{2 \pi}} \int_{\mathbb{R}} \mathrm{d} x^{\prime} e^{-i k_{x} x^{\prime}} \phi_{\omega}\left(x^{\prime}+\frac{\lambda z k_{x}}{2 \pi}\right)\right\} \\
& -e^{-i \chi}\left\{\hat{\xi}_{\omega}\left(k_{x}\right)+\frac{1}{\sqrt{2 \pi}} \int_{\mathbb{R}} \mathrm{d} x^{\prime} e^{-i k_{x} x^{\prime}} \xi_{\omega}\left(x^{\prime}+\frac{\lambda z k_{x}}{2 \pi}\right)\right\} .
\end{aligned}
$$

Inserting the substitution $x:=x^{\prime}+\frac{\lambda z k_{x}}{2 \pi}$ with $\mathrm{d} x=\mathrm{d} x^{\prime}$ also enables the expression of the second terms in the form of Fourier transforms

$$
\begin{aligned}
\frac{\hat{I}_{\omega}\left(k_{x}, z\right)}{I_{0}}= & \sqrt{2 \pi} \delta_{D}\left(k_{x}\right)+i e^{-i \chi}\left\{\hat{\phi}_{\omega}\left(k_{x}\right)-e^{i \frac{\lambda z k_{x}^{2}}{2 \pi}} \frac{1}{\sqrt{2 \pi}} \int_{\mathbb{R}} \mathrm{d} x e^{-i k_{x} x} \phi_{\omega}(x)\right\} \\
& -e^{-i \chi}\left\{\hat{\xi}_{\omega}\left(k_{x}\right)+e^{i \frac{\lambda z k_{x}^{2}}{2 \pi}} \frac{1}{\sqrt{2 \pi}} \int_{\mathbb{R}} \mathrm{d} x e^{-i k_{x} x} \xi_{\omega}(x)\right\} \\
= & \sqrt{2 \pi} \delta_{D}\left(k_{x}\right)+i e^{-i \chi}\left\{\hat{\phi}_{\omega}\left(k_{x}\right)-e^{2 i \chi} \hat{\phi}_{\omega}\left(k_{x}\right)\right\} \\
& -e^{-i \chi}\left\{\hat{\xi}_{\omega}\left(k_{x}\right)-e^{2 i \chi} \hat{\xi}_{\omega}\left(k_{x}\right)\right\}
\end{aligned}
$$

With the definitions of the trigonometric functions via a combination of complex exponentials

$$
\sin x=\frac{e^{i x}-e^{-i x}}{2 i} \text { and } \quad \cos x=\frac{e^{i x}+e^{-i x}}{2},
$$

the Fourier transformed intensity can be rewritten as

$$
\begin{aligned}
\frac{\hat{I}_{\omega}\left(k_{x}, z\right)}{I_{0}} & =\sqrt{2 \pi} \delta_{D}\left(k_{x}\right)+\frac{-e^{-i \chi}+e^{i \chi}}{i} \hat{\phi}_{\omega}\left(k_{x}\right)-\left(e^{-i \chi}+e^{i \chi}\right) \hat{\xi}_{\omega}\left(k_{x}\right) \\
& =\sqrt{2 \pi} \delta_{D}\left(k_{x}\right)+2 \sin \chi \hat{\phi}_{\omega}\left(k_{x}\right)-2 \cos \chi \hat{\xi}_{\omega}\left(k_{x}\right),
\end{aligned}
$$

known as the contrast transfer function (CTF).

\section{A.3 Fringe visibility in the Young interferometer}

The following section is based on [125]. To derive an expression for the intensity pattern of the Young interferometer depicted in Fig. 1.7, the path difference $\Delta$ between two rays starting from the point $s\left(s_{x}\right)$ in the source plane, where $s_{x}$ is the coordinate perpendicular to the optical axis, and terminating in point $d\left(d_{x}\right)$ in the detection plane, passing through pinhole $p_{1}$ or pinhole $p_{2}$, respectively, is 
considered. According to Fig. 1.7 the path difference is given by

$$
\begin{aligned}
\Delta\left(s_{x}, d_{x}\right)= & r_{s p_{2}}+r_{p_{2} d}-r_{s p_{1}}-r_{p_{1} d} \\
= & \sqrt{r_{S P}^{2}+\left(\frac{b}{2}+s_{x}\right)^{2}}+\sqrt{r_{P D}^{2}+\left(\frac{b}{2}+d_{x}\right)^{2}} \\
& -\sqrt{r_{S P}^{2}+\left(\frac{b}{2}-s_{x}\right)^{2}}-\sqrt{r_{P D}^{2}+\left(\frac{b}{2}-d_{x}\right)^{2}}
\end{aligned}
$$

Assuming that $b, s_{x}$ and $d_{x}$ are much smaller compared to the distance $r_{S P}$ between source and screen and the distance $r_{P D}$ between screen and pinhole, this can be approximated via a first order Taylor expansion

$$
\begin{aligned}
\Delta\left(s_{x}, d_{x}\right) \simeq & r_{S P}+\frac{\left(\frac{b}{2}+s_{x}\right)^{2}}{2 r_{S P}}+r_{P D}+\frac{\left(\frac{b}{2}+d_{x}\right)^{2}}{2 r_{P D}} \\
& -r_{S P}-\frac{\left(\frac{b}{2}-s_{x}\right)^{2}}{2 r_{S P}}-r_{P D}-\frac{\left(\frac{b}{2}-d_{x}\right)^{2}}{2 r_{P D}} \\
= & \frac{b s_{x}}{r_{S P}}+\frac{b d_{x}}{r_{P D}}
\end{aligned}
$$

In the next step, this path difference is converted to a phase shift $\Delta \phi\left(s_{x}, d_{x}\right)$ between the wave going through pinhole $p_{1}$ and the wave going through pinhole $p_{2}$. The phase shift can be expressed as the ratio between the path difference $\Delta\left(s_{x}, d_{x}\right)$ and the mean wave length $\bar{\lambda}$ of the quasi-monochromatic illumination multiplied by $2 \pi$

$$
\Delta \phi\left(s_{x}, d_{x}\right)=2 \pi \frac{\Delta}{\bar{\lambda}} \simeq \frac{2 \pi b}{\bar{\lambda}}\left(\frac{s_{x}}{r_{S P}}+\frac{d_{x}}{r_{P D}}\right)
$$

If the two pinholes are of the same size and shape, the amplitudes of the two wave fields will be the same and they can be expressed in dependence of each other via $\psi_{1}\left(s_{x}, d_{x}\right)$ and $\psi_{2}\left(s_{x}, d_{x}\right)=\psi_{1}\left(s_{x}, d_{x}\right) e^{i \Delta \phi\left(s_{x}, d_{x}\right)}$, respectively. Thus, the intensity distribution in the detection plane produced by a point radiator located at $s_{x}$ is 
given by

$$
\begin{aligned}
I\left(s_{x}, d_{x}\right)= & \left|\psi_{1}\left(s_{x}, d_{x}\right)+\psi_{2}\left(s_{x}, d_{x}\right)\right|^{2} \\
\simeq & \mid \psi_{1}\left(s_{x}, d_{x}\right)+\psi_{1}\left(s_{x}, d_{x}\right) e^{\left.i \frac{2 \pi b}{\bar{\lambda}}\left(\frac{s_{x}}{r_{S P}}+\frac{d_{x}}{r_{P} D}\right)\right|^{2}} \\
= & \psi_{1}\left(s_{x}, d_{x}\right) \psi_{1}^{*}\left(s_{x}, d_{x}\right)+\psi_{1}\left(s_{x}, d_{x}\right) \psi_{1}^{*}\left(s_{x}, d_{x}\right) e^{i \frac{2 \pi b}{\bar{\lambda}}\left(\frac{s_{x}}{r_{S P}}+\frac{d_{x}}{r_{P D}}\right)} \\
& +\psi_{1}\left(s_{x}, d_{x}\right) \psi_{1}^{*}\left(s_{x}, d_{x}\right)+\psi_{1}\left(s_{x}, d_{x}\right) \psi_{1}^{*}\left(s_{x}, d_{x}\right) e^{-i \frac{2 \pi b}{\bar{\lambda}}\left(\frac{b s_{x}}{r_{S P}}+\frac{b d_{x}}{r_{P D}}\right)} \\
= & 2 I_{0}\left\{1+\cos \left[\frac{2 \pi b}{\bar{\lambda}}\left(\frac{s_{x}}{r_{S P}}+\frac{d_{x}}{r_{P D}}\right)\right]\right\}
\end{aligned}
$$

Note that the intensity $I_{0}$ was introduced, which would be the measured intensity if either of the two pinholes would have been blocked.

To obtain a measure for the intensity in the detection plane, resulting from the extended incoherent uniform quasi-monochromatic line source $S$, the single wave field components produced by each point radiator in the source are incoherently superimposed, i.e., the intensity contributions are added up. By using the definitions of the trigonometric functions via sums of complex exponentials given in eqn. (A.36), this can be expressed as

$$
\begin{aligned}
I\left(d_{x}\right) & =\int_{s_{x}=-l / 2}^{s_{x}=l / 2} I\left(s_{x}, d_{x}\right) \mathrm{d} s_{x} \\
& =\int_{s_{x}=-l / 2}^{s_{x}=l / 2} 2 I_{0}\left(1+\frac{e^{i \frac{2 \pi b}{\lambda}\left(\frac{s_{x}}{r_{S P}}+\frac{d_{x}}{r_{P D}}\right)}+e^{-i \frac{2 \pi b}{\lambda}\left(\frac{s_{x}}{r_{S P}}+\frac{d_{x}}{r_{P D}}\right)}}{2}\right) \mathrm{d} s_{x} \\
& =2 I_{0} l+\frac{I_{0} r_{S P} \bar{\lambda}}{2 i \pi b}\left[e^{\frac{i 2 \pi b d_{x}}{\bar{\lambda} r_{P D}}}\left(e^{\frac{i \pi b l}{\bar{\lambda} r_{S P}}}-e^{-\frac{i \pi b l}{\bar{\lambda} r_{S P}}}\right)+e^{\frac{-2 i \pi b d_{x}}{\bar{\lambda} r_{P D}}}\left(-e^{-\frac{i \pi b l}{\bar{\lambda} r_{S P}}}+e^{\frac{i \pi b l}{\bar{\lambda} r_{S P}}}\right)\right] \\
& =2 I_{0} l+\frac{2 I_{0} r_{S P} \bar{\lambda}}{\pi b} \sin \left(\frac{\pi b l}{\bar{\lambda} r_{S P}}\right) \cos \left(\frac{2 \pi b d_{x}}{\bar{\lambda} r_{P D}}\right) .
\end{aligned}
$$

A common measure for coherence is the visibility $V$ of the interference fringes in the detection plane

$$
V:=\frac{I_{\max }-I_{\min }}{I_{\max }+I_{\min }}
$$


as defined by Michelson [106]. The maximum value for the intensity is given at $\cos \left(\frac{2 \pi b d_{x}}{\bar{\lambda} r_{P D}}\right)=1$, provided only the absolute values of the sine function are considered, whereas the minimum value is reached at $\cos \left(\frac{2 \pi b d_{x}}{\bar{\lambda} r_{P D}}\right)=-1$

$$
\begin{aligned}
& I_{\max }=2 I_{0} l+\frac{2 I_{0} r_{S P} \bar{\lambda}}{\pi b}\left|\sin \left(\frac{\pi b l}{\lambda r_{S P}}\right)\right|, \\
& I_{\min }=2 I_{0} l-\frac{2 I_{0} r_{S P} \bar{\lambda}}{\pi b}\left|\sin \left(\frac{\pi b l}{\lambda r_{S P}}\right)\right| .
\end{aligned}
$$

Inserting this into the expression for the fringe visibility leads to

$$
V=\frac{|\sin \varrho|}{\varrho}
$$

where the effective variable $\varrho=\frac{\pi b l}{\bar{\lambda} r_{S P}}$ was introduced. Hence, the fringe visibility is a function of source size $l$, feature distance $b$, wavelength $\bar{\lambda}$ and distance $r_{S P}$ between the source and the screen.

\section{A.4 Regularization in the CTF-based reconstruction}

To account for the suppression of low frequencies in the CTF-based phase retrieval for a pure phase object, the results are compared with the results of the MBA, as in the direct-contrast regime the contrast transfer function for a pure phase object and the near-field TIE, used for the derivation of the MBA algorithm, are equivalent (cf. section 1.3.2). For a single propagation distance, the two reconstructions are only comparable if the sine functions in the filter are reduced prior to adding the regularization parameter $\alpha\left(\mathbf{k}_{\perp}\right)$. Hence, the regularization is changed from a Tikhonov-like regularization

$$
\frac{1}{\left|\mathbf{k}_{\perp}\right|^{2}} \rightarrow \frac{\left|\mathbf{k}_{\perp}\right|^{2}}{\left|\mathbf{k}_{\perp}\right|^{4}+\alpha}
$$

to a regularization of the form

$$
\frac{1}{\left|\mathbf{k}_{\perp}\right|^{2}} \rightarrow \frac{1}{\left|\mathbf{k}_{\perp}\right|^{2}+\alpha}
$$


as used in the MBA approach. Although this is not a valid operation in the case of multiple propagation distances due to the sum over the single sine functions multiplied with the measured intensity images, an approximated phase for low spatial frequencies is nevertheless reconstructed with this modified algorithm

$$
\hat{\phi}_{\omega}^{\prime}\left(\mathbf{r}_{\perp}\right)=\mathcal{F}_{\perp}^{-1}\left[\frac{\sum_{m=1}^{N} \mathcal{F}_{\perp}\left[I_{\omega}^{(\exp )}\left(\mathbf{r}_{\perp}, z_{m}\right)-1\right]}{\sum_{m=1}^{N} 2 \sin \chi_{m}+\alpha\left(\mathbf{k}_{\perp}\right)}\right],
$$

in order to reproduce the results obtained by the MBA algorithm. By combining the two solutions in Fourier space via

$$
\phi_{\text {comb. }}\left(\mathbf{k}_{\perp}\right)=\hat{\phi}_{\omega}^{\prime}\left(\mathbf{k}_{\perp}\right) \cdot f\left(\left|\mathbf{k}_{\perp}\right|\right)+\hat{\phi}_{\omega}\left(\mathbf{k}_{\perp}\right) \cdot\left(1-f\left(\left|\mathbf{k}_{\perp}\right|\right)\right),
$$

where $f\left(\left|\mathbf{k}_{\perp}\right|\right)$ is defined as in eqn. (2.30), the reconstructions in Fig. A.1(d-f) were obtained. The comparison shows that by introducing the 'MBA-like' filtering for low spatial frequencies, quantitative reconstructions with a comparable performance as the homogeneous object approach can be obtained, justifying the rather empirical introduction of the modified filter. However, as both approaches lead to comparable results and the derivation of the homogeneous object approach is mathematically more correct and does not rely on the combination of two separate reconstructions, it was used as the standard method throughout this thesis. 

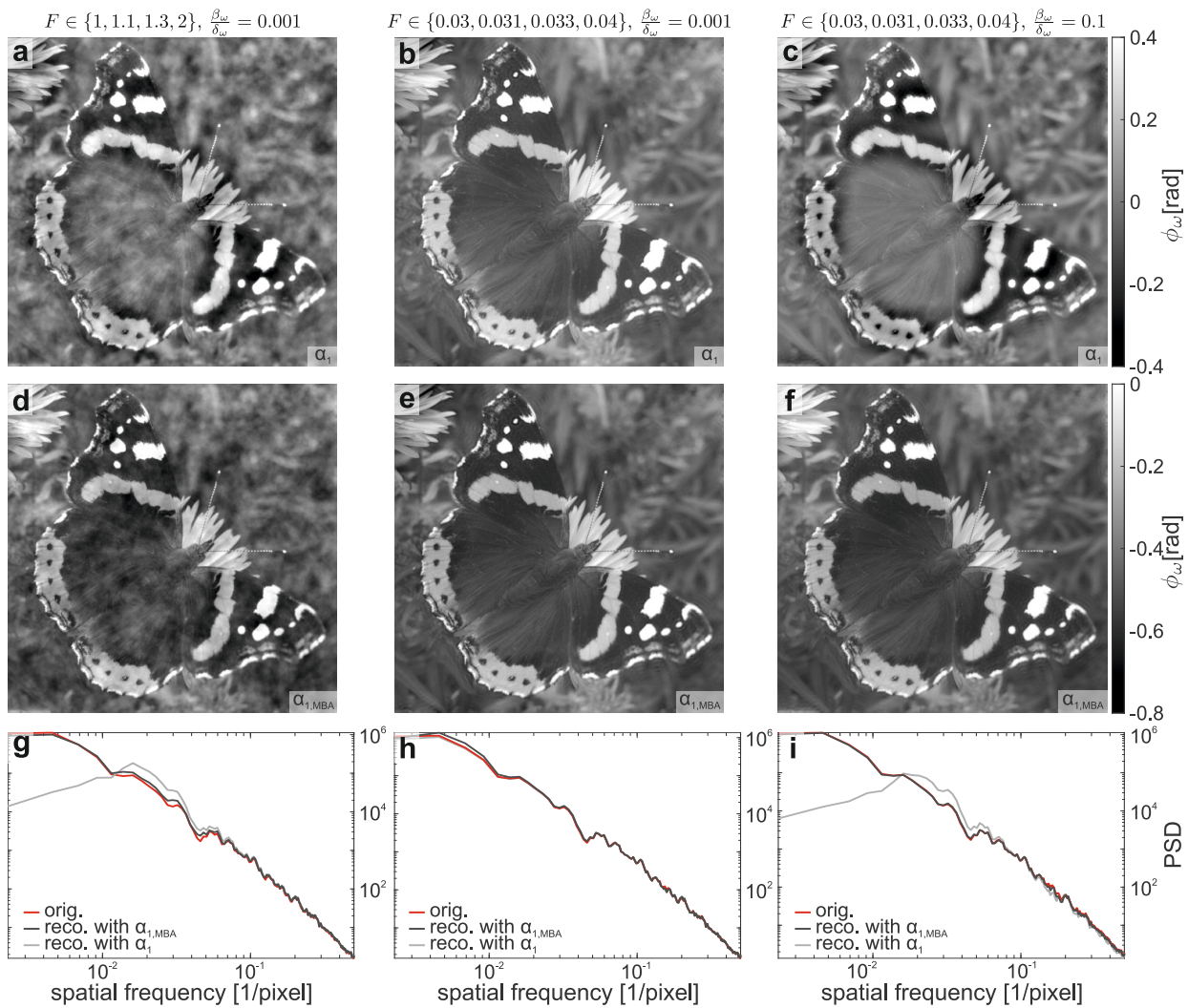

Figure A.1: Effect of the 'MBA-like' filtering in the pure phase CTF approach. (a-c) Reconstruction of the phase for the same combination of Fresnel numbers and $\frac{\delta}{\beta}$-ratios as in section 2.2.2, obtained by using the original Tikhonov-like regularization. The resulting images lack quantitativity due to the high-pass effect of the corresponding filter. (d-f) By exploiting an 'MBA-like' regularization for small spatial frequencies and combining both approaches in Fourier space, quantitativity can be regained and the corresponding reconstructions yield more accurate results with respect to the original data, as shown in the azimuthally averaged power spectral densities in ( $g$-i). 


\section{A.5 Main processing steps in the tomographic reconstruction}

\section{Tomographic reconstruction of laboratory datasets}

read in data and perform empty-beam correction

optional: resample projections

remove hot pixels

remove outliers (image, [threshold=2], [filterrange=5]) To remove hot pixels, a $2 d$ median filtering is performed on the image within the range specified by filterrange. Subsequently, the standard deviation $\sigma$ of the difference between the original and the filtered image is determined. Each pixel in the original image that deviates by more than threshold $\cdot \sigma$ from the filtered image is replaced by the filtered value.

reconstruct intensity in object plane for each projection via BAC BAC (projection, $\mathrm{F}$, settings)

Possible settings are the regularization parameters $\alpha$ and $y$ and padding values to reduce artifacts occuring at the edge of the projection.

In order to choose $\alpha$, the MBA can be applied in a first step via $\mathrm{MBA}$ (I1, F, mbasettings),

where $\alpha$ is chosen such that edge enhancement is removed at the cost of blurring.

optional: perform ring removal

ring remove(sinogram, ringparameter, [range=full])

The ringparameter determines the extent of the low-pass filtering of the profile and if a range is passed, the ring removal is only applied on a restricted part of the sinogram.

ring remove wavelet (sinogram, settings)

The settings include the wavelet basis, the number of wavelet decomposition steps and the standard deviation of the Gaussian filter.

determine roll angle (section 4.5) and tilt all projections accordingly

determine rotation axis shift by minimizing artifacts in reconstructed slice (section 3.2.1)

perform tomographic reconstruction

astraFDK (projections, angles, $z_{01}, z_{02}$,psize, eff. psize, settings) Possible settings for the tomographic reconstruction are: output_size to determine the slice dimensions, short_scan to weight projections if angular range is smaller than $360^{\circ}$,

num_slices to restrict reconstruction volume to specified number of slices and offset to change position of the central slice in the reconstruction.

Figure A.2: Main processing steps in Matlab involved in the tomographic reconstruction of the acquired dataset at the laboratory setup JuLiA. 


\title{
Tomographic reconstruction of synchrotron datasets
}

\author{
read in data and perform empty-beam correction \\ remove hot pixels \\ remove_outliers (image, [threshold=2], [filterrange=5]) \\ rescale and align images from different propagation distances \\ rescale defocus series astig(images to align, $\mathrm{F}_{\mathrm{x}}, \mathrm{F}_{\mathrm{y}}, \mathrm{M}_{\mathrm{x}}, \mathrm{M}_{\mathrm{y}}$, settings) \\ This function rescales and aligns the different projections to each other via a cross-correlation in \\ Fourier space. As an output, the aligned images as well as rescaled Fresnel numbers are given. \\ The suffix_astig indicates that different magnifications in horizontal and vertical direction can \\ be taken into account. The optional settings include CTF parameters for a single distance \\ reconstruction of the individual projections, high-pass and low-pass filter parameters, $2 d$ median \\ filtering or the specification of a region of interest for the alignment \\ reconstruct phase via CTF for weakly absorbing objects \\ CTFrec_astig (aligned_projections, $\mathrm{F}_{\mathrm{x}}, \mathrm{F}_{\mathrm{y}}$, settings) \\ Possible settings are delta beta and lim2, the cutoff frequency and the \\ width of the transition between the two regularization regimes. Additionally, padding of \\ the projections can be performed to reduce artifacts originating from truncation. \\ determine roll angle (section 4.5) and tilt all projections accordingly \\ determine rotation axis shift by minimizing \\ artifacts in reconstructed slice (section 3.2.1) \\ optional: reduction of low-frequency artifacts according to section 5.3.2

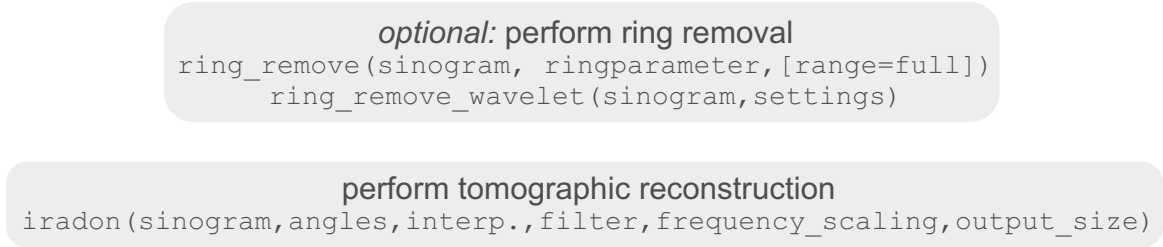

Figure A.3: General processing chain in Matlab for data acquired at the GINIX endstation. 



\section{Bibliography}

[1] J. Als-Nielsen and D. McMorrow. Elements of Modern X-ray Physics. Wiley, 2011.

[2] K. Amunts, C. Lepage, L. Borgeat, H. Mohlberg, T. Dickscheid, Ma.-É. Rousseau, S. Bludau, P.-L. Bazin, L. B. Lewis, A.-M. Oros-Peusquens, et al. Bigbrain: an ultrahigh-resolution 3d human brain model. Science, 340(6139): 1472-1475, 2013.

[3] B. B. Andersen, H. J. G. Gundersen, and B. Pakkenberg. Aging of the human cerebellum: a stereological study. J. Comp. Neurol., 466(3):356-365, 2003.

[4] Andor. Zyla 4.2 plus scmos, $2018 . \quad$ URL http://www. andor.com/scientific-cameras/neo-and-zyla-scmos-cameras/ zyla-42-plus-scmos. [Online; accessed 12-March-2018].

[5] J. C. Andrews, F. Meirer, Y. Liu, Z. Mester, and P. Pianetta. Transmission x-ray microscopy for full-field nano imaging of biomaterials. Microsc. Res. Tech., 74(7):671-681, 2011.

[6] F. A. C. Azevedo, L. R. B. Carvalho, L. T. Grinberg, J. M. Farfel, R. E. L. Ferretti, R. E. P. Leite, R. Lent, S. Herculano-Houzel, et al. Equal numbers of neuronal and nonneuronal cells make the human brain an isometrically scaled-up primate brain. J. Comp. Neurol., 513(5):532-541, 2009.

[7] M. Bartels. Cone-beam x-ray phase contrast tomography of biological samples Optimization of contrast, resolution and field of view. $\mathrm{PhD}$ thesis, Universität Göttingen, 2013.

[8] M. Bartels, V. H. Hernandez, M. Krenkel, T. Moser, and T. Salditt. Phase contrast tomography of the mouse cochlea at microfocus x-ray sources. Appl. Phys. Lett., 103(8):083703, 2013.

[9] M. Bartels, M. Krenkel, P. Cloetens, W. Möbius, and T. Salditt. Myelinated mouse nerves studied by x-ray phase contrast zoom tomography. J. Struct. Biol., 192(3):561-568, 2015.

[10] M. Bartels, M. Krenkel, J. Haber, R. N. Wilke, and T. Salditt. X-ray holo- 
graphic imaging of hydrated biological cells in solution. Phys. Rev. Lett., 114:048103, 2015.

[11] M. C. Bellissent-Funel. Hydration Processes in Biology: Theoretical and Experimental Approaches. A]: [NATO ASI series. IOS Press, 1999.

[12] M. Berger. Xcom: photon cross sections database. http://www.nist.gov/pml/data/xcom/index.cfm, 2010.

[13] S. Berujon, H. Wang, and K. Sawhney. X-ray multimodal imaging using a random-phase object. Phys. Rev. A, 86(6):063813, 2012.

[14] S. Bérujon, E. Ziegler, R. Cerbino, and L. Peverini. Two-dimensional x-ray beam phase sensing. Phys. Rev. Lett., 108(15):158102, 2012.

[15] N. Bevins, J. Zambelli, K. Li, Z. Qi, and G.-H. Chen. Multicontrast xray computed tomography imaging using talbot-lau interferometry without phase stepping. Med. Phys., 39(1):424-428, 2012.

[16] Leica Biosystems. An introduction to specimen preparation, 2018. URL https://www.leicabiosystems.com/pathologyleaders/ an-introduction-to-specimen-preparation/. [Online; accessed 07March-2018].

[17] M. Born, E. Wolf, and A. B. Bhatia. Principles of Optics: Electromagnetic Theory of Propagation, Interference and Diffraction of Light. Cambridge University Press, 2000.

[18] R. N. Bracewell. The Fourier transform and its applications. McGraw-Hill Series in Electrical and Computer Engineering. McGraw-Hill, 1986.

[19] A. A. T. Bui and R. K. Taira. Medical Imaging Informatics. Springer US, 2009.

[20] M. Busse, M. Müller, M. A. Kimm, S. Ferstl, S. Allner, K. Achterhold, J. Herzen, and F. Pfeiffer. Three-dimensional virtual histology enabled through cytoplasm-specific x-ray stain for microscopic and nanoscopic computed tomography. PNAS, 115(10):2293-2298, 2018.

[21] T. Buzug. Computed Tomography : From Photon Statistics to Modern ConeBeam CT. Springer-Verlag, 2008.

[22] A. Cedola, A. Bravin, I. Bukreeva, M. Fratini, A. Pacureanu, A. Mittone, L. Massimi, P. Cloetens, P. Coan, G. Campi, et al. X-ray phase contrast tomography reveals early vascular alterations and neuronal loss in a multiple sclerosis model. Sci. Rep., 7(1):5890, 2017.

[23] D. Chandler. Introduction to Modern Statistical Mechanics. Oxford University Press, 1987. 
[24] H.-Y. Chen, S. Hoffmann, and T. Salditt. X-ray beam compression by tapered waveguides. Appl. Phys. Lett., 106(19):194105, 2015.

[25] N. Chicherova, S. E. Hieber, G. Schulz, A. Khimchenko, C. Bikis, P. C. Cattin, and B. Müller. Automatic histology registration in application to xray modalities. volume 9967, page 996708. International Society for Optics and Photonics, 2016.

[26] C. C. Chien, P. Y. Tseng, H. H. Chen, T. E. Hua, S. T. Chen, Y. Y. Chen, W. H. Leng, C. H. Wang, Y. Hwu, G. C. Yin, K. S. Liang, F. R. Chen, Y. S. Chu, H. I. Yeh, Y. C. Yang, C. S. Yang, G. L. Zhang, J. H. Je, and G. Margaritondo. Imaging cells and sub-cellular structures with ultrahigh resolution full-field x-ray microscopy. Biotechnol. Adv., 31(3):375-386, 2013.

[27] P. Cloetens. Contribution to Phase Contrast Imaging, Reconstruction and Tomography with Hard Synchrotron Radiation. PhD thesis, 1999.

[28] P. Cloetens, W. Ludwig, J. Baruchel, D. Van Dyck, J. Van Landuyt, J. P. Guigay, and M. Schlenker. Holotomography: Quantitative phase tomography with micrometer resolution using hard synchrotron radiation x rays. Appl. Phys. Lett., 75(19):2912-2914, 1999.

[29] Wikimedia Commons. File:precisionrecall.svg — wikimedia commons, the free media repository, 2016. URL https://commons.wikimedia.org/w/ index.php?title=File: Precisionrecall. svg\&oldid=220068947. [Online; accessed 22-February-2018].

[30] Wikimedia Commons. File:purkinjecell.jpg — wikimedia commons, the free media repository, 2017. URL https://commons.wikimedia.org/w/index. php?title=File: PurkinjeCell. jpg\&oldid=254050874. [Online; accessed 9-February-2018].

[31] J. W. Cooley and J. W. Tukey. An algorithm for the machine calculation of complex fourier series. Mathematics of computation, 19(90):297-301, 1965.

[32] B. J. Crain and P. C. Burger. The laminar distribution of neuritic plaques in the fascia dentata of patients with alzheimer's disease. Acta Neuropathol., 76(1):87-93, 1988.

[33] B. De Man, J. Nuyts, P. Dupont, G. Marchal, and P. Suetens. Metal streak artifacts in x-ray computed tomography: a simulation study. IEEE Transactions on Nuclear Science, 46(3):691-696, 1999.

[34] Y. De Witte, M. Boone, J. Vlassenbroeck, M. Dierick, and L. Van Hoorebeke. Bronnikov-aided correction for x-ray computed tomography. J. Opt. Soc. Am. A, 26(4):890-894, 2009. 
[35] Deutsches Elektronen-Synchrotron DESY. Petra iii - facility information, 2018. URL http://photon-science.desy.de/facilities/petra_ iii/facility_information/index_eng.html. [Online; accessed 12-March2018].

[36] M. Dierolf, A. Menzel, P. Thibault, P. Schneider, C. M. Kewish, R. Wepf, O. Bunk, and F. Pfeiffer. Ptychographic x-ray computed tomography at the nanoscale. Nature, 467(7314):436, 2010.

[37] M. Dobrivojević, I. Bohaček, I. Erjavec, D. Gorup, and S. Gajović. Computed microtomography visualization and quantification of mouse ischemic brain lesion by nonionic radio contrast agents. Croat. Med. J., 54(1):3-11, 2013.

[38] H.-U. Dodt, U. Leischner, A. Schierloh, N. Jährling, C. P. Mauch, K. Deininger, J. M. Deussing, M. Eder, W. Zieglgänsberger, and K. Becker. Ultramicroscopy: three-dimensional visualization of neuronal networks in the whole mouse brain. Nat. Methods, 4(4):331, 2007.

[39] T. R. Doeppner, G. P. H. Dietz, M. El Aanbouri, J. Gerber, O. W. Witte, M. Bähr, and J. Weise. Tat-bcl-xl improves survival of neuronal precursor cells in the lesioned striatum after focal cerebral ischemia. Neurobiol. Dis., 34(1):87-94, 2009.

[40] A. E. Dorr, J. P. Lerch, S. Spring, N. Kabani, and R. M. Henkelman. High resolution three-dimensional brain atlas using an average magnetic resonance image of 40 adult c57bl/6j mice. NeuroImage, 42(1):60-69, 2008.

[41] J. Dudak, J. Zemlicka, J. Karch, M. Patzelt, J. Mrzilkova, P. Zach, Z. Hermanova, J. Kvacek, and F. Krejci. High-contrast x-ray micro-radiography and micro-ct of ex-vivo soft tissue murine organs utilizing ethanol fixation and large area photon-counting detector. Sci. Rep., 6:30385, 2016.

[42] E. L. Dyer, W. G. Roncal, J. A. Prasad, H. L. Fernandes, D. Gürsoy, V. De Andrade, K. Fezzaa, X. Xiao, J. T. Vogelstein, C. Jacobsen, et al. Quantifying mesoscale neuroanatomy using x-ray microtomography. eNeuro, 4(5):ENEURO-0195, 2017.

[43] T. Egami and S. J. L. Billinge. Underneath the Bragg Peaks: Structural Analysis of Complex Materials. Pergamon materials series. Elsevier Science \& Technology Books, 2003.

[44] L. A. Feldkamp, L. C. Davis, and J. W. Kress. Practical cone-beam algorithm. J. Opt. Soc. Am. A, 1(6):612-619, 1984.

[45] J. R. Fienup. Phase retrieval algorithms: a comparison. Appl. Opt., 21(15): 2758-2769, 1982. 
[46] The Center for X-Ray Optics. X-ray interactions with matter, 2010. URL http://henke.lbl.gov/optical_constants/. [Online; accessed 03January-2018].

[47] M. Frigo and S. G. Johnson. Fftw: An adaptive software architecture for the fft. In Acoustics, Speech and Signal Processing, 1998. Proceedings of the 1998 IEEE International Conference on, volume 3, pages 1381-1384. IEEE, 1998.

[48] D. Gabor et al. A new microscopic principle. Nature, 161(4098):777-778, 1948.

[49] R. W. Gerchberg and W. O. Saxton. A Practical Algorithm for the Determination of Phase from Image and Diffraction Plane Pictures. Optik, 35(2): 237-246, 1972.

[50] K. Giewekemeyer. A study on new approaches in coherent $x$-ray microscopy of biological specimens. PhD thesis, Universität Göttingen, 2011.

[51] K. Giewekemeyer, S. P. Krüger, S. Kalbfleisch, M. Bartels, C. Beta, and T. Salditt. X-ray propagation microscopy of biological cells using waveguides as a quasipoint source. Phys. Rev. A, 83(2):023804, 2011.

[52] P. F. Goldsmith. Quasioptical Systems: Gaussian Beam Quasioptical Propogation and Applications. IEEE Press Series on RF and Microwave Technology. Wiley, 1998.

[53] J. W. Goodman. Introduction to Fourier Optics. Roberts \& Company, 2005. ISBN 0974707724.

[54] G. Grant. How the 1906 nobel prize in physiology or medicine was shared between golgi and cajal. Brain Res. Rev., 55(2):490-498, 2007.

[55] H. S. Green and E. Wolf. A scalar representation of electromagnetic fields. Proc. Phys. Soc., Section A, 66(12):1129, 1953.

[56] A. Groso, R. Abela, and M. Stampanoni. Implementation of a fast method for high resolution phase contrast tomography. Opt. Express, 14(18):81038110, 2006.

[57] A. Groso, M. Stampanoni, R. Abela, P. Schneider, S. Linga, and R. Müller. Phase contrast tomography: An alternative approach. Appl. Phys. Lett., 88: 214104, 2006.

[58] J. P. Guigay. Fourier transform analysis of fresnel diffraction patterns and in-line holograms. Optik, 49(1):121-125, 1977.

[59] M. Guizar-Sicairos, S. T. Thurman, and J. R. Fienup. Efficient subpixel image registration algorithms. Optics letters, 33(2):156-158, 2008. 
[60] M. Guizar-Sicairos, J. J. Boon, K. Mader, A. Diaz, A. Menzel, and O. Bunk. Quantitative interior x-ray nanotomography by a hybrid imaging technique. Optica, 2(3):259-266, 2015.

[61] E. Gureyev, T, Y. I. Nesterets, A. W. Stevenson, P. R. Miller, A. Pogany, and S. W. Wilkins. Some simple rules for contrast, signal-to-noise and resolution in in-line x-ray phase-contrast imaging. Opt. Express, 16(5):3223-3241, 2008.

[62] J. Hagemann and T. Salditt. Divide and Update: Towards Single-Shot Object and Probe Retrieval for Near-Field Holography. Opt. Express, 25(18): 20953-20968, 2017.

[63] J. Hagemann, A.-L. Robisch, D. R. Luke, C. Homann, T. Hohage, P. Cloetens, H. Suhonen, and T. Salditt. Reconstruction of wave front and object for inline holography from a set of detection planes. Opt. Express, 22 (10):11552-11569, 2014.

[64] C. K. Hagen, P. R. T. Munro, M. Endrizzi, P. C. Diemoz, and A. Olivo. Low-dose phase contrast tomography with conventional x-ray sources. Med. Phys., 41(7):070701, 2014.

[65] G. Harauz and M. van Heel. Exact filters for general geometry three dimensional reconstruction. In Proceedings of the IEEE Computer Vision and Pattern Recognition Conf, volume 73, pages 146-156, 1986.

[66] O. Hemberg, M. Otendal, and H. M. Hertz. Liquid-metal-jet anode electronimpact x-ray source. Appl. Phys. Lett., 83(7):1483-1485, 2003.

[67] B. L. Henke, E. M. Gullikson, and J. C. Davis. X-Ray Interactions: Photoabsorption, Scattering, Transmission, and Reflection at $\mathrm{E}=50-30,000 \mathrm{eV}$, $\mathrm{Z}=1$-92. At. Data Nucl. Data Tables, 54(2):181-342, 1993.

[68] S. Herculano-Houzel. The human brain in numbers: a linearly scaled-up primate brain. Front. Hum. Neurosci., 3:31, 2009.

[69] S. E. Hieber, C. Bikis, A. Khimchenko, G. Schweighauser, J. Hench, N. Chicherova, G. Schulz, and B. Müller. Tomographic brain imaging with nucleolar detail and automatic cell counting. Sci. Rep., 6:32156, 2016.

[70] Sarah Hoffmann-Urlaub. X-ray waveguide optics: Beyond straight channels. PhD thesis, Universität Göttingen, 2016.

[71] Sarah Hoffmann-Urlaub, Philipp Höhne, Mike Kanbach, and Tim Salditt. Advances in fabrication of x-ray waveguides. Microelectron. Eng., 164:135$138,2016$.

[72] M. Holler, A. Diaz, M. Guizar-Sicairos, P. Karvinen, E. Färm, E. Härkönen, 
M. Ritala, A. Menzel, J. Raabe, and O. Bunk. X-ray ptychographic computed tomography at $16 \mathrm{~nm}$ isotropic 3d resolution. Sci. Rep., 4:3857, 2014.

[73] C. Homann, T. Hohage, J. Hagemann, A.-L. Robisch, and T. Salditt. Validity of the empty-beam correction in near-field imaging. Phys. Rev. A, 91:013821, 2015.

[74] P. V. C. Hough. Method and means for recognizing complex patterns. U.S. Patent 3,069,654, 1962.

[75] G. N. Hounsfield. Computerized transverse axial scanning (tomography): Part 1. description of system. Brit. J. Radiol., 46(552):1016-1022, 1973.

[76] M. R. Howells, T. Beetz, H. N. Chapman, C. Cui, J. M. Holton, C. J. Jacobsen, J. Kirz, E. Lima, S. Marchesini, H. Miao, D. Sayre, D. A. Shapiro, J. C. H. Spence, and D. Starodub. An assessment of the resolution limitation due to radiation-damage in X-ray diffraction microscopy. J. Electron. Spectrosc. Relat. Phenom., 170(1-3):4-12, 2009.

[77] C. Imig and B. H. Cooper. 3d analysis of synaptic ultrastructure in organotypic hippocampal slice culture by high-pressure freezing and electron tomography. In Synapse Development, pages 215-231. Springer, 2017.

[78] Y. Jiao, H. Berman, T.-R. Kiehl, and S. Torquato. Spatial organization and correlations of cell nuclei in brain tumors. PloS one, 6(11):e27323, 2011.

[79] R. Jin, G. Yang, and G. Li. Inflammatory mechanisms in ischemic stroke: role of inflammatory cells. J. Leukoc. Biol., 87(5):779-789, 2010.

[80] J. T. Johnson, M. S. Hansen, I. Wu, L. J. Healy, C. R. Johnson, G. M. Jones, M. R. Capecchi, and C. Keller. Virtual histology of transgenic mouse embryos for high-throughput phenotyping. PLOS Genet., 2(4):e61, 2006.

[81] A. C. Kak and M. Slaney. Principles of Computerized Tomographic Imaging. Classics in Applied Mathematics. Society for Industrial and Applied Mathematics, 2001.

[82] S. Kalbfleisch. A Dedicated Endstation for Waveguide-based X-Ray Imaging. PhD thesis, Universität Göttingen, 2012.

[83] R. A. Ketcham. New algorithms for ring artifact removal. Proc. SPIE, 6318: 63180O-63180O-7, 2006.

[84] A. Khimchenko, H. Deyhle, G. Schulz, G. Schweighauser, J. Hench, N. Chicherova, C. Bikis, S. E. Hieber, and B. Müller. Extending twodimensional histology into the third dimension through conventional micro computed tomography. NeuroImage, 139:26-36, 2016. 
[85] J. Kim, N. Kwon, S. Chang, K.-T. Kim, D. Lee, S. Kim, S. J. Yun, D. Hwang, J. W. Kim, Y. Hwu, G. Margaritondo, J. H. Je, and I. J. Rhyu. Altered branching patterns of purkinje cells in mouse model for cortical development disorder. Sci. Rep., 1:122, 2011.

[86] P. Kirkpatrick and A. V. Baez. Formation of optical images by x-rays. JOSA, 38(9):766-774, 1948.

[87] R. Kockelkoren, B. Jill, P. T. M. Willem, J. Hendrikse, P. A. de Jong, A. M. Rozemuller, and H. L. Koek. Hippocampal calcification on computed tomography in relation to cognitive decline in memory clinic patients: A casecontrol study. PloS one, 11(11):e0167444, 2016.

[88] M. Krenkel. Cone-beam x-ray phase-contrast tomography for the observation of single cells in whole organs. $\mathrm{PhD}$ thesis, Universitäet Göettingen, 2015.

[89] M. Krenkel, M. Bartels, and T. Salditt. Transport of intensity phase reconstruction to solve the twin image problem in holographic x-ray imaging. Opt. Express, 21(2):2220-2235, 2013.

[90] M. Krenkel, A. Markus, M. Bartels, C. Dullin, F. Alves, and T. Salditt. Phase-contrast zoom tomography reveals precise locations of macrophages in mouse lungs. Sci. Rep., 5:09973, 2015.

[91] M. Krenkel, M. Töpperwien, C. Dullin, F. Alves, and T. Salditt. Propagation-based phase-contrast tomography for high-resolution lung imaging with laboratory sources. AIP Adv., 6(3):035007, 2016.

[92] M. Krenkel, M. Toepperwien, F. Alves, and T. Salditt. Three-dimensional single-cell imaging with X-ray waveguides in the holographic regime. Acta Crystallogr. A, 73(4):282-292, 2017.

[93] S. P. Krüger. Optimization of waveguide optics for lensless x-ray imaging. PhD thesis, Universität Göttingen, 2010.

[94] S. P. Krüger, K. Giewekemeyer, S. Kalbfleisch, M. Bartels, H. Neubauer, and T. Salditt. Sub-15 nm beam confinement by twocrossed x-ray waveguides. Opt. Express, 18(13):13492-13501, 2010.

[95] S. P. Krüger, H. Neubauer, M. Bartels, S. Kalbfleisch, K. Giewekemeyer, P. J. Wilbrandt, M. Sprung, and T. Salditt. Sub-10 nm beam confinement by Xray waveguides: design, fabrication and characterization of optical properties. J. Synchrotron Rad., 19(2):227-236, 2012.

[96] A. Kyrieleis, V. Titarenko, M. Ibison, T. Connolley, and P. J. Withers. Region-of-interest tomography using filtered backprojection: assessing the practical limits. J. Microsc., 241(1):69-82, 2011. 
[97] National Physical Laboratory. Mechanical properties of materials, 2017. URL http://www.kayelaby.npl.co.uk/general_physics/2_2/2_ 2_1.html. [Online; accessed 15-February-2018].

[98] S. Lagomarsino, A. Cedola, P. Cloetens, S. Di Fonzo, W. Jark, G. Soullie, and C. Riekel. Phase contrast hard x-ray microscopy with submicron resolution. Appl. Phys. Lett., 71(18):2557-2559, 1997.

[99] D. H. Larsson, W. Vågberg, A. Yaroshenko, A. Ö. Yildirim, and H. M. Hertz. High-resolution short-exposure small-animal laboratory x-ray phase-contrast tomography. Sci. Rep., 6:39074, 2016.

[100] A. Li, H. Gong, B. Zhang, Q. Wang, C. Yan, J. Wu, Q. Liu, S. Zeng, and Q. Luo. Micro-optical sectioning tomography to obtain a high-resolution atlas of the mouse brain. Science, 330(6009):1404-1408, 2010.

[101] Z. Li, T. Zhang, Z. Lin, C. Hou, J. Zhang, Y. Men, H. Li, and J. Gao. Lgl1 is required for olfaction and development of olfactory bulb in mice. PloS one, 11(9):e0162126, 2016.

[102] A. S. Marathay and G. B. Parrent. Use of scalar theory in optics. JOSA, 60 (2):243-245, 1970.

[103] S. Marchesini. Invited article: A unified evaluation of iterative projection algorithms for phase retrieval. Rev. Sci. Instrum., 78(1):011301, 2007.

[104] D. Marr and E. Hildreth. Theory of edge detection. In Proc. R. Soc. Lond. $B$, volume 207, pages 187-217. The Royal Society, 1980.

[105] B. D. Metscher. Microct for comparative morphology: simple staining methods allow high-contrast $3 \mathrm{~d}$ imaging of diverse non-mineralized animal tissues. BMC Physiol., 9(1):11, 2009.

[106] A. A. Michelson. Studies in Optics. The Univ. of Chicago Science Series. University Press, 1927.

[107] S. Mikula and W. Denk. High-resolution whole-brain staining for electron microscopic circuit reconstruction. Nat. Methods, 12(6):541, 2015.

[108] S. Mikula, J. Binding, and W. Denk. Staining and embedding the whole mouse brain for electron microscopy. Nat. Methods, 9(12):1198, 2012.

[109] R. Mizutani and Y. Suzuki. X-ray microtomography in biology. Micron, 43 (2-3):104-115, 2012.

[110] W. Möbius, B. H. Cooper, W. A. Kaufmann, C. Imig, T. Ruhwedel, N. Snaidero, A. S. Saab, and F. Varoqueaux. Electron microscopy of the mouse central nervous system. In Methods Cell Biol., volume 96, pages 475-512. Elsevier, 2010. 
[111] A. Momose, T. Takeda, Y. Itai, and K. Hirano. Phase-contrast x-ray computed tomography for observing biological soft tissues. Nat. Med., 2(4): 473-475, 1996.

[112] W. D. Montgomery. Unitary operators in the homogeneous wave field. Opt. Lett., 6(7):314-315, 1981.

[113] K. S. Morgan, D. M. Paganin, and K. K. W. Siu. X-ray phase imaging with a paper analyzer. Appl. Phys. Lett., 100(12):124102, 2012.

[114] Y. Mu and F. H. Gage. Adult hippocampal neurogenesis and its role in alzheimer's disease. Mol. Neurodegener., 6(1):85, 2011.

[115] B. Münch, P. Trtik, F. Marone, and M. Stampanoni. Stripe and ring artifact removal with combined wavelet-fourier filtering. Opt. Express, 17(10):85678591, 2009.

[116] P. R. T. Munro, K. Ignatyev, R. D. Speller, and A. Olivo. Phase and absorption retrieval using incoherent x-ray sources. PNAS, 109(35):13922-13927, 2012.

[117] F. Natterer. The Mathematics of Computerized Tomography. Classics in Applied Mathematics. Society for Industrial and Applied Mathematics, 2001.

[118] H. Neubauer, S. Hoffmann, M. Kanbach, J. Haber, S. Kalbfleisch, S. P. Krüger, and T. Salditt. High aspect ratio x-ray waveguide channels fabricated by e-beam lithography and wafer bonding. J. Appl. Phys., 115(21): $214305,2014$.

[119] K. Noda-Saita, A. Yoneyama, Y. Shitaka, Y. Hirai, K. Terai, J. Wu, T. Takeda, K. Hyodo, N. Osakabe, T. Yamaguchi, et al. Quantitative analysis of amyloid plaques in a mouse model of alzheimer's disease by phasecontrast X-ray computed tomography. Neuroscience, 138(4):1205-1213, 2006.

[120] K. A. Nugent, T. E. Gureyev, D. F. Cookson, D. Paganin, and Z. Barnea. Quantitative Phase Imaging Using Hard X Rays. Phys. Rev. Lett., 77(14): 2961-2964, 1996.

[121] World Health Organization. Top 10 causes of death, 2018. URL http://www.who.int/gho/mortality_burden_disease/causes_death/ top_10/en/. [Online; accessed 03-March-2018].

[122] M. Osterhoff and T. Salditt. Coherence filtering of x-ray waveguides: analytical and numerical approach. New J. Phys., 13(10):103026, 2011.

[123] M. Otendal, T. Tuohimaa, U. Vogt, and H. M. Hertz. A 9 kev electron-impact liquid-gallium-jet x-ray source. Rev. Sci. Instrum., 79(1):016102, 2008. 
[124] C. H. Owen and W. J. Landis. Alignment of electron tomographic series by correlation without the use of gold particles. Ultramicroscopy, 63(1):27-38, 1996.

[125] D. Paganin. Coherent X-Ray Optics. Oxford Series on Synchrotron Radiation. OUP Oxford, 2013.

[126] D. Paganin and K. A. Nugent. Noninterferometric Phase Imaging with Partially Coherent Light. Phys. Rev. Lett., 80:2586-2589, 1998.

[127] D. Paganin, S. C. Mayo, T. E. Gureyev, P. R. Miller, and S. W. Wilkins. Simultaneous phase and amplitude extraction from a single defocused image of a homogeneous object. J. Microsc., 206(Pt 1):33-40, 2002.

[128] W. J. Palenstijn, K. J. Batenburg, and J. Sijbers. Performance improvements for iterative electron tomography reconstruction using graphics processing units (gpus). J. Struct. Biol., 176(2):250-253, 2011.

[129] E. Pannese. The black reaction. Brain Res. Bull., 41(6):343-349, 1996.

[130] D. L. Parker. Optimal short scan convolution reconstruction for fan beam ct. Med. Phys., 9(2):254-257, 1982.

[131] K. Parmar, C. Stadelmann, M. A. Rocca, D. Langdon, E. D'Angelo, M. D'Souza, J. Burggraaff, C. Wegner, J. Sastre-Garriga, A. Barrantes-Freer, et al. The role of the cerebellum in multiple sclerosis-150 years after charcot. Neuroscience \&3 Biobehavioral Reviews, 2018.

[132] E. Peli. Contrast in complex images. JOSA A, 7(10):2032-2040, 1990.

[133] T. Peng, A. Balijepalli, S. K. Gupta, and T. LeBrun. Algorithms for on-line monitoring of micro spheres in an optical tweezers-based assembly cell. $J$. Comput. Inf. Sci. Eng., 7(4):330-338, 2007.

[134] F. Pfeiffer, O. Bunk, C. David, M. Bech, G. Le Duc, A. Bravin, and P. Cloetens. High-resolution brain tumor visualization using threedimensional x-ray phase contrast tomography. Phys. Med. Biol., 52(23): 6923, 2007.

[135] F. Pfeiffer, C. Kottler, O. Bunk, and C. David. Hard X-Ray Phase Tomography with Low-Brilliance Sources. Phys. Rev. Lett., 98:108105, 2007.

[136] Photometrics. Prime 95b scientific cmos camera, 2018. URL https://www. photometrics . com/products/scmos/prime95B. [Online; accessed 09-April2018].

[137] B. R. Pinzer, M. Cacquevel, P. Modregger, S. A. McDonald, J. C. Bensadoun, T. Thuering, P. Aebischer, and M. Stampanoni. Imaging brain amyloid de- 
position using grating-based differential phase contrast tomography. NeuroImage, 61(4):1336-1346, 2012.

[138] J. Radon. Berichte über die verhandlungen der königlich sächsischen gesellschaft der wissenschaften zu leipzig. Mathem. Phys. Klasse, 69:262$266,1917$.

[139] A. G. Ramm and A. I. Katsevich. The Radon Transform and Local Tomography. Taylor \& Francis, 1996.

[140] W. Ribi, T. J. Senden, A. Sakellariou, A. Limaye, and S. Zhang. Imaging honey bee brain anatomy with micro-x-ray-computed tomography. $J$. Neurosci. Methods, 171(1):93-97, 2008.

[141] Rigaku. Microfocus rotating anode x-ray generator, 2018. URL https:// www.rigaku.com/en/products/protein/micromax007. [Online; accessed 16-March-2018].

[142] Rigaku. Ccd \& scmos detectors, 2018. URL https://www.rigakuoptics . com/detectors.php. [Online; accessed 12-March-2018].

[143] A.-L. Robisch and T. Salditt. Phase retrieval for object and probe using a series of defocus near-field images. Opt. Express, 21(20):23345-23357, 2013.

[144] J. M. Rodenburg and H. M. L. Faulkner. A phase retrieval algorithm for shifting illumination. Applied physics letters, 85(20):4795-4797, 2004.

[145] M. T. Roe, D. V. Dawson, C. M. Hulette, G. Einstein, and B. J. Crain. Microglia are not exclusively associated with plaque-rich regions of the dentate gyrus in alzheimer's disease. J. Neuropathol. Exp. Neurol., 55(3):366-371, 1996.

[146] A. Ruhlandt, M. Töpperwien, M. Krenkel, R. Mokso, and T. Salditt. Four dimensional material movies: High speed phase-contrast tomography by backprojection along dynamically curved paths. Sci. Rep., 7(1):6487, 2017.

[147] T. Salditt, K. Giewekemeyer, C. Fuhse, S. P. Kruger, R. Tucoulou, and P. Cloetens. Projection phase contrast microscopy with a hard x-ray nanofocused beam: Defocus and contrast transfer. Phys. Rev. B, 79(18):184112-6, 2009.

[148] T. Salditt, M. Osterhoff, M. Krenkel, R. N. Wilke, M. Priebe, M. Bartels, S. Kalbfleisch, and M. Sprung. Compound focusing mirror and X-ray waveguide optics for coherent imaging and nano-diffraction. J. Synchrotron Rad., 22(4):867-878, 2015.

[149] T. Salditt, T. Aspelmeier, and S. Aeffner. Biomedical Imaging: Principles 
of Radiography, Tomography and Medical Physics. De Gruyter Textbook Series. Walter de Gruyter GmbH, 2017.

[150] J. Schindelin, I. Arganda-Carreras, E. Frise, V. Kaynig, M. Longair, T. Pietzsch, S. Preibisch, C. Rueden, S. Saalfeld, B. Schmid, J.-Y. Tinevez, D. J. White, V. Hartenstein, K. Eliceiri, P. Tomancak, and A. Cardona. Fiji: an open-source platform for biological-image analysis. Nat. Methods, 9(7):676, 2012.

[151] G. Schmahl, D. Rudolph, B. Niemann, and O. Christ. Zone-plate X-ray microscopy. Q. Rev. Biophys., 13(3):297-315, 1980.

[152] G. Schulz, T. Weitkamp, I. Zanette, F. Pfeiffer, F. Beckmann, C. David, S. Rutishauser, E. Reznikova, and B. Müller. High-resolution tomographic imaging of a human cerebellum: comparison of absorption and grating-based phase contrast. J. R. Soc. Interface, 7(53):1665-1676, 2010.

[153] DESY Photon Science. P10 coherence beamline user guide, 2016. URL http://photon-science.desy.de/sites/site_photonscience/ content/e58/e176720/e177229/e178737/e179091/e179098/e269721/ P10_User_Guide_2016_eng.pdf. [Online; accessed 16-March-2018].

[154] A. Serrano-Pozo, M. P. Frosch, E. Masliah, and B. T. Hyman. Neuropathological alterations in alzheimer disease. CSH Perspect. Med., 1(1):a006189, 2011.

[155] D. Sheedy, A. Harding, M. Say, J. Stevens, and J. J. Kril. Histological assessment of cerebellar granule cell layer in postmortem brain; a useful marker of tissue integrity? Cell Tissue Bank., 13(4):521-527, 2012.

[156] A. Siegel and H. N. Sapru. Essential Neuroscience. Point (Lippincott Williams \& Wilkins). Wolters Kluwer Health/Lippincott Williams \& Wilkins, 2 edition, 2011.

[157] J. Sijbers and A. Postnov. Reduction of ring artefacts in high resolution micro-ct reconstructions. Phys. Med. Biol., 49(14):N247, 2004.

[158] S. W. Smith. The Scientist and Engineer's Guide to Digital Signal Processing. California Technical Pub., 1997.

[159] M. Stampanoni, R. Mokso, F. Marone, J. Vila-Comamala, S. Gorelick, P. Trtik, K. Jefimovs, and C. David. Phase-contrast tomography at the nanoscale using hard x rays. Phys. Rev. B, 81(14):140105, 2010.

[160] M. Stockmar, P. Cloetens, I. Zanette, B. Enders, M. Dierolf, F. Pfeiffer, and P. Thibault. Near-field ptychography: phase retrieval for inline holography using a structured illumination. Sci. Rep., 3:1927, 2013. 
[161] T. Takeda, T. Kunii, R. Sirai, T. Ohizumi, H. Maruyama, K. Hyodo, A. Yoneyama, K. Ueda, et al. Ethanol fixed brain imaging by phase-contrast x-ray technique. volume 425, page 022004. IOP Publishing, 2013.

[162] M. Tanaka, K. Yamaguchi, T. Tatsukawa, Theis. M., K. Willecke, and S. Itohara. Connexin43 and bergmann glial gap junctions in cerebellar function. Front. Neurosci., 2:225-233, 2008.

[163] M. R. Teague. Deterministic phase retrieval: a green's function solution. J. Opt. Soc. Am., 73(11):1434-1441, 1983.

[164] A. Tkachuk, F. Duewer, Ho. Cui, M. Feser, S. Wang, and W. Yun. X-ray computed tomography in zernike phase contrast mode at $8 \mathrm{kev}$ with 50$\mathrm{nm}$ resolution using cu rotating anode x-ray source. Z. Kristallogr. Cryst. Mater., 222(11/2007):650-655, 2007.

[165] M. Töpperwien, M. Krenkel, K. Müller, and T. Salditt. Phase-contrast tomography of neuronal tissues: from laboratory-to high resolution synchrotron ct. Proc. SPIE, 9967:99670T, 2016.

[166] M. Töpperwien, M. Krenkel, F. Quade, and T. Salditt. Laboratory-based x-ray phase-contrast tomography enables $3 \mathrm{D}$ virtual histology. Proc. SPIE, 9964:99640I, 2016.

[167] M. Töpperwien, M. Krenkel, D. Vincenz, F. Stöber, A. M. Oelschlegel, J. Goldschmidt, and T. Salditt. Three-dimensional mouse brain cytoarchitecture revealed by laboratory-based x-ray phase-contrast tomography. Sci. Rep., 7:42847, 2017.

[168] M. Töpperwien, F. van der Meer, C. Stadelmann, and T. Salditt. Threedimensional virtual histology of human cerebellum by x-ray phase-contrast tomography. PNAS, 2018.

[169] L. Turner, B. Dhal, J. Hayes, A. Mancuso, K. Nugent, D. Paterson, R. Scholten, C. Tran, and A. Peele. X-ray phase imaging: Demonstration of extended conditions for homogeneous objects. Opt. Express, 12(13):2960 $2965,2004$.

[170] M. Töpperwien, R. Gradl, D. Keppeler, M. Vassholz, A. Meyer, R. Hessler, K. Achterhold, B. Gleich, M. Dierolf, F. Pfeiffer, T. Moser, and T. Salditt. Propagation-based phase-contrast x-ray tomography of cochlea using a compact synchrotron source. Sci. Rep., 8:4922, 2018.

[171] K. Uesugi, M. Hoshino, and N. Yagi. Comparison of lens-and fiber-coupled ccd detectors for x-ray computed tomography. Journal of synchrotron radiation, 18(2):217-223, 2011. 
[172] W. Vågberg, J. C. Larsson, and H. M. Hertz. Removal of ring artifacts in microtomography by characterization of scintillator variations. Opt. Express, 25(19):23191-23198, 2017.

[173] W. van Aarle, W. J. Palenstijn, J. De Beenhouwer, T. Altantzis, S. Bals, K. J. Batenburg, and J. Sijbers. The astra toolbox: A platform for advanced algorithm development in electron tomography. Ultramicroscopy, 157:35-47, 2015.

[174] W. van Aarle, W. J. Palenstijn, J. Cant, E. Janssens, F. Bleichrodt, A. Dabravolski, J. De Beenhouwer, K. J. Batenburg, and J. Sijbers. Fast and flexible x-ray tomography using the astra toolbox. Opt. Express, 24(22): 25129-25147, 2016.

[175] M. van Heel. Similarity measures between images. Ultramicroscopy, 21(1): 95-100, 1987.

[176] M. van Heel and M. Schatz. Fourier shell correlation threshold criteria. J. Struct. Biol., 151(3):250-262, 2005.

[177] V. Van Nieuwenhove, J. De Beenhouwer, T. De Schryver, L Van Hoorebeke, and J. Sijbers. Affine deformation correction in cone beam computed tomography. In Fully Three-Dimensional Image Reconstruction in Radiology and Nuclear Medicine, pages 182-185, 2015.

[178] M. Vassholz, B. Koberstein-Schwarz, A. Ruhlandt, M. Krenkel, and T. Salditt. New x-ray tomography method based on the $3 \mathrm{~d}$ radon transform compatible with anisotropic sources. Phys. Rev. Lett., 116:088101, 2016.

[179] Georg Thieme Verlag. Römpp online, 2006. URL https://roempp. thieme. de/roempp4.0/do/data/RD-05-01878. [Online; accessed 15-February2018].

[180] Georg Thieme Verlag. Römpp online, 2013. URL https://roempp.thieme. de/roempp4.0/do/data/RD-23-00264. [Online; accessed 15-February2018].

[181] Georg Thieme Verlag. Römpp online, 2015. URL https://roempp. thieme. de/roempp4.0/do/data/RD-12-01679. [Online; accessed 15-February2018].

[182] D. G. Voelz and M. C. Roggemann. Digital simulation of scalar optical diffraction: revisiting chirp function sampling criteria and consequences. Appl. Opt., 48(32):6132-6142, 2009.

[183] K. von Sternberg and J. V. von Krombholz. Bericht über die Versammlung deutscher Naturforscher und Aerzte in Prag im September 183\%. Bericht 
über die Versammlung der Gesellschaft Deutscher Naturforscher und Ärzte. Druck und Papier von Gottlieb Haase Söhne, 1838.

[184] Q. Wang, X. N. Tang, and M. A. Yenari. The inflammatory response in stroke. J. Neuroimmunol., 184(1):53-68, 2007.

[185] E. Weckert, K. Balewski, W. Brefeld, W. Decking, W. Drube, H. Franz, P. Gürtler, U. Hahn, J. Pflüger, H. Schulte-Schrepping, et al. Petra iii: A new high brilliance synchrotron radiation source at desy. AIP Conf. Proc., 705(1):73-76, 2004.

[186] J. Wegiel, I. Kuchna, T. Wisniewski, M. de Leon, B. Reisberg, T. Pirttila, T. Kivimaki, and T. Lehtimaki. Vascular fibrosis and calcification in the hippocampus in aging, alzheimer disease, and down syndrome. Acta Neuropathol., 103(4):333-343, 2002.

[187] U. Weierstall, Q. Chen, J. C. H. Spence, M. R. Howells, M. Isaacson, and R. R. Panepucci. Image reconstruction from electron and X-ray diffraction patterns using iterative algorithms: experiment and simulation. Ultramicroscopy, 90(2-3):171-195, 2002.

[188] T. Weitkamp, A. Diaz, C. David, F. Pfeiffer, M. Stampanoni, C. Cloetens, and E. Ziegler. X-ray phase imaging with a grating interferometer. Opt. Express, 13(16):6296-6304, 2005.

[189] R. N. Wilke, M. Priebe, M. Bartels, K. Giewekemeyer, A. Diaz, P. Karvinen, and T. Salditt. Hard x-ray imaging of bacterial cells: nano-diffraction and ptychographic reconstruction. Opt. Express, 20(17):19232-19254, 2012.

[190] S. W. Wilkins, T. E. Gureyev, D. Gao, A. Pogany, and A. W. Stevenson. Phase-contrast imaging using polychromatic hard X-rays. Nature, 384(6607): 335-338, 1996.

[191] E. Wolf. A scalar representation of electromagnetic fields: Ii. Proc. Phys. Soc., 74(3):269, 1959.

[192] T. M. Woodruff, J. Thundyil, S.-C. Tang, C. G. Sobey, S. M. Taylor, and T. V. Arumugam. Pathophysiology, treatment, and animal and cellular models of human ischemic stroke. Mol. Neurodegener., 6(1):11, 2011.

[193] L. Xie. Spherical hough transform for 3d images. https://de.mathworks.com/matlabcentral/fileexchange/ 48219-spherical-hough-transform-for-3d-images, 2014.

[194] Y. Yang, R. Heine, Y. Cheng, C.-Ch. Wang, Y.-F. Song, and T. Baumbach. Approaching quantitative zernike phase contrast in full-field transmission 
hard x-ray microscopy: Origin and reduction of artifacts. Appl. Phys. Lett., 105(9):094101, 2014.

[195] S. Zabler, P. Cloetens, J.-P. Guigay, J. Baruchel, and M. Schlenker. Optimization of phase contrast imaging using hard x rays. Rev. Sci. Instrum., 76(7):073705, 2005.

[196] A. Zamir, P. C. Diemoz, F. A. Vittoria, C. K. Hagen, M. Endrizzi, and A. Olivo. Edge illumination x-ray phase tomography of multi-material samples using a single-image phase retrieval algorithm. Opt. Express, 25(10): 11984-11996, 2017.

[197] I. Zanette, S. Lang, A. Rack, M. Dominietto, M. Langer, F. Pfeiffer, T. Weitkamp, and B. Müller. Holotomography versus x-ray grating interferometry: A comparative study. Appl. Phys. Lett., 103(24), 2013.

[198] I. Zanette, T. Zhou, A. Burvall, U. Lundström, D. H. Larsson, M.-C. Zdora, P. Thibault, F. Pfeiffer, and H. M. Hertz. Speckle-based x-ray phase-contrast and dark-field imaging with a laboratory source. Phys. Rev. Lett., 112(25): 253903, 2014.

[199] M.-C. Zdora, P. Thibault, T. Zhou, F. J. Koch, J. Romell, S. Sala, A. Last, C. Rau, and I. Zanette. X-ray phase-contrast imaging and metrology through unified modulated pattern analysis. Phys. Rev. Lett., 118(20):203903, 2017.

[200] T. Zikmund, M. Novotná, M. Kavková, M. Tesařová, M. Kaucká, B. Szarowská, I. Adameyko, E. Hrubá, M. Buchtová, E. Dražanová, et al. High-contrast differentiation resolution $3 \mathrm{~d}$ imaging of rodent brain by $\mathrm{x}$-ray computed microtomography. J. Instrum., 13(02):C02039, 2018. 



\section{List of publications}

- M. Töpperwien, F. v. d. Meer, C. Stadelmann and T. Salditt. Three-dimensional virtual histology of human cerebellum by X-ray phase-contrast tomography. PNAS, 2018.

- M. Töpperwien, M. Krenkel, D. Vincenz, F. Stöber, A. M. Oelschlegel, J. Goldschmidt and T. Salditt. Three-dimensional mouse brain cytoarchitecture revealed by laboratory-based x-ray phase-contrast tomography. Sci. Rep., 7:42847, 2017.

- M. Töpperwien, R. Gradl, D. Keppeler, M. Vassholz, A. Meyer, R. Hessler, K. Achterhold, B. Gleich, M. Dierolf, F. Pfeiffer, T. Moser, and T. Salditt. Propagation-based phase-contrast x-ray tomography of cochlea using a compact synchrotron source. Sci. Rep., 8:4922, 2018.

- M. Töpperwien, M. Krenkel, F. Quade, and T. Salditt. Laboratory-based x-ray phase-contrast tomography enables $3 \mathrm{D}$ virtual histology. Proc. SPIE, 9964:99640I, 2016.

- M. Töpperwien, M. Krenkel, K. Müller, and T. Salditt. Phase-contrast tomography of neuronal tissues: from laboratory-to high resolution synchrotron CT. Proc. SPIE, 9967:99670T, 2016.

- M. Töpperwien, M. Krenkel, T. Ruhwedel, W. Möbius, A. Pacureanu, P. Cloetens, and T. Salditt. Phase-contrast tomography of sciatic nerves: image quality and experimental parameters. J. Phys: Conf. Ser., 849:12001, 2017.

- M. Töpperwien, M. Priebe and T. Salditt. Actin bundles cross-linked with $\alpha$ actinin studied by nanobeam X-ray diffraction. Eur. Biophys. J., 45:383-392, 2016 .

- M. Krenkel, M. Töpperwien, M. Bartels, P. Lingor, D. Schild and T. Salditt. X-ray phase contrast tomography from whole organ down to single cells. Proc. SPIE, 9212:92120R, 2014.

- A.D. Surowka, M. Töpperwien, M. Bernhardt, J.D. Nicolas, M. Osterhoff, T. Salditt, D. Adamek, M. Szczerbowska-Boruchowska. Combined in-situ imaging of structural organization and elemental composition of substantia nigra neurons in the elderly. Talanta, 161:368-376, 2016. 
- M. Krenkel, M. Töpperwien, C. Dullin, F. Alves and T. Salditt. Propagationbased phase-contrast tomography for high-resolution lung imaging with laboratory sources. AIP Advances, 6:035007, 2016.

- A. Ruhlandt, M. Töpperwien, M. Krenkel, R. Mokso, and T. Salditt. Four dimensional material movies: High speed phase-contrast tomography by backprojection along dynamically curved paths. Sci. Rep., 7:6487, 2017.

- M. Krenkel, M. Töpperwien, F. Alves, and T. Salditt. Three-dimensional single-cell imaging with $\mathrm{X}$-ray waveguides in the holographic regime. Acta Crystallogr. A, 73:282-292, 2017.

- M. Reichardt, J. Frohn, M. Töpperwien, J.-D. Nicolas, A. Markus, F. Alves, and T. Salditt. Nanoscale holographic tomography of heart tissue with x-ray waveguide optics. Proc. SPIE, 10391:1039105, 2017.

- E. Carboni, J.-D. Nicolas, M. Töpperwien, C. Stadelmann-Nessler, P. Lingor and T. Salditt. Imaging of neuronal tissues by x-ray diffraction and x-ray fluorescence microscopy: evaluation of contrast and biomarkers for neurodegenerative diseases. Biomed. Opt. Express, 8:4331-4347, 2017

- Y. Gutiérrez, D. Ott, M. Töpperwien, T. Salditt, and C. Scherber. X-Ray Computed Tomography and its Potential in Ecological Research: A Review of Studies and Optimization of Specimen Preparation. Ecology and Evolution, 2018

- M.-T. Weil, S. Heibeck, M. Töpperwien, S. t. Dieck, T. Ruhwedel, T. Salditt, M. C. Rodicio, J. Morgan, K.-A. Nave, W. Möbius, and H. Werner. Axonal ensheathment in the nervous system of lamprey: Implications for the evolution of myelinating glia. Journal of Neuroscience, 2018 


\section{Danksagung}

An dieser Stelle möchte ich mich bei allen Personen bedanken, die zum Gelingen dieser Doktorarbeit beigetragen haben.

Mein besonderer Dank gilt Prof. Dr. Tim Salditt, der es mir ermöglicht hat, meine Doktorarbeit über dieses interessante Thema zu schreiben und mir mit vielen Ratschlägen und Ideen zur Seite stand. Die interessanten fachlichen Diskussionen sowie seine Begeisterungsfähigkeit haben entscheidend zum Gelingen dieser Arbeit und zur Förderung meiner Motivation im Verlauf der letzten Jahre beigetragen. Ich danke ihm außerdem für das entgegengebrachte Vertrauen, welches es mir ermöglicht hat, eigene Ideen entwickeln und umsetzen zu können.

Weiterhin möchte ich mich bei Dr. Florian Rehfeldt für die Betreuung meiner Arbeit im Rahmen des Thesis Committees bedanken sowie bei Prof. Dr. Christine Stadelmann-Nessler, welche meine Arbeit nicht nur durch die Teilnahme am Thesis Committee unterstützt sondern durch unsere gemeinsame Zusammenarbeit entscheidend zu den erreichten Ergebnissen beigetragen hat. Insbesondere die unkomplizierte Bereitstellung der humanen Proben sowie interessante fachliche Diskussionen und die gemeinsame Entwicklung von Ideen für weitere Projekte haben diese Arbeit sehr bereichert. Ich bedanke mich außerdem bei Prof. Dr. Sarah Köster, Prof. Dr. Tobias Moser sowie Prof. Dr. Matthias Krüger für die Beteiligung an meiner Prüfungskomission.

Mein besonderer Dank gilt Dr. Martin Krenkel, der mich für das Thema Phasenkontrast-Tomographie begeistert hat und mir mit viel Rat und Tat zur Seite stand. Unsere Zusammenarbeit sowohl während meiner Masterarbeit also auch zu Beginn meiner Promotion wird mir immer in sehr guter Erinnerung bleiben.

Des weiteren möchte ich mich beim dritten Mitglied des Total-Tollen-Tomo-Teams, Dr. Aike Ruhlandt, für viele interessante Diskussionen und Gespräche sowie die schöne Zeit im gemeinsamen Büro bedanken. Außerdem danke ich Malte Vaßholz für eine angenehme und konstruktive Zusammenarbeit sowie seine Unterstützung sowohl im Labor als auch während zahlreicher Messzeiten an der GINIX und Dr. Johannes Hagemann, der mir in vielen Diskussionen und Softwarefragen zur Seite stand. Mein Dank gilt auch Dr. Anna-Lena Robisch und Marina Eckermann, die insbesondere in den letzten Monaten meiner Promotion viele Aufgaben übernom- 
men haben, was es mir ermöglichte diese Arbeit in Ruhe zum Abschluss zu bringen. Bei Jan Goeman und Dr. Markus Osterhoff bedanke ich mich für die hervorragende Unterstützung in allen IT-Angelegenheiten. Weiterhin danke ich Jochen Herbst für seine große Hilfsbereitschaft bei sämtlichen praktischen Problemen, welche im Laufe dieser Promotion auftraten, sowie die Herstellung von Probenhaltern für die Messzeiten. Mein Dank gilt außerdem Bastian Hartmann, Mike Kanbach, Peter Luley und Peter Nieschalk für die technische Unterstützung im JuLiA Labor sowie vor und während der Messzeiten an der GINIX. Mike Kanbach danke ich außerdem für die Unterstützung beim Zurechtschneiden zahlreicher Proben für die verschiedenen Messzeiten sowie seinen Einsatz bei der Wellenleiterherstellung.

Bei Julia Scherber, Susanne Hengst und Kristin Müller möchte ich mich für die Hilfe bei den Probenpräparationen bedanken. Dr. Sarah Hoffmann-Urlaub und Dr. Hsin-Yi Chen danke ich für die Weiterentwicklung der Röntgenwellenleiter, welche einen entscheidenden Anteil an den Ergebnissen dieser Arbeit hatten. Mein Dank gilt außerdem Sabine Balder, Kerstin Pluschke, Michaela Ständer, Eva Hetzel und Christine Wilke-Feist für ihre Unterstützung in administrativen Fragen.

Insgesamt möchte ich mich bei allen Mitgliedern des Instituts für Röntgenphysik für die gemeinsame Zeit sowie angenehme Atmosphäre innerhalb der Gruppe bedanken. Ich finde es schön, dass wir auch außerhalb des Instituts viele gemeinsame Aktivitäten unternommen haben (insbesondere seien hier die allwöchentlichen Thanners-Abende erwähnt), was für einen sehr schönen Ausgleich zum Arbeitsalltag geführt hat.

Bei Dr. Jürgen Goldschmidt und seiner Arbeitsgruppe bedanke ich mich herzlich für die tolle Zusammenarbeit und Präparation und Bereitstellung der getrockneten Maushirne. Außerdem danke ich Dr. Benjamin Cooper, Prof. Dr. Frauke Alves und ihrer Arbeitsgruppe, insbesondere Dr. Andrea Markus, Prof. Dr. Paul Lingor und seiner Arbeitsgruppe, insbesondere Dr. Eleonora Carboni, PD Dr. Thorsten Döppner und Felix Quade für die Präparation und Bereitstellung der weiteren Proben. Ein besonderer Dank gilt auch Dr. Michael Sprung und seinem Team für eine hervorragende Unterstützung während der GINIX Messzeiten sowie zahlreiche Ideen zur Verbesserung und Weiterentwicklung des Aufbaus.

Zuletzt möchte ich meinen Eltern für ihre Unterstützung, ihren Rat und ihre aufbauenden Worte danken.

Die Forschungsarbeit wurde von der Deutschen Forschungsgemeinschaft durch den SFB 755 Nanoscale Photonic Imaging und das Exzellenzcluster 171 Mikroskopie im Nanometerbereich und Molekularphysiologie des Gehirns unterstützt. 


\section{Curriculum Vitae}

\section{Personal Information}

Name:

Born:

Nationality:

\section{Studies}

since $02 / 2015$

$2009-2014$

09/2012-01/2013

$10 / 2012-12 / 2014$

$10 / 2009-09 / 2012$

06/2009
Mareike Töpperwien

22 December 1989 in Buxtehude

German

Doctoral student in physics,

Institut für Röntgenphysik,

Group of Prof. Dr. Tim Salditt

Georg-August-Universität, Göttingen

Scholarship from "Studienstiftung des Deutschen Volkes"

Exchange semester (ERASMUS) at Universiteit van Amsterdam

Master of Science in Physics

Georg-August-Universität, Göttingen

Title of thesis: „X-ray phase-contrast tomography of neuronal tissues"

Bachelor of Science in Physics

Georg-August-Universität, Göttingen

Title of thesis: „X-ray structure analysis of actin suspensions"

Abitur, Halepaghen-Schule Buxtehude 